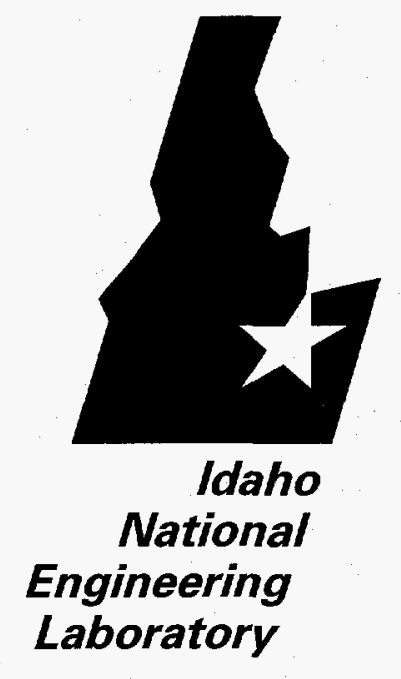

INEL-95/0355

September 1995

Process Modeling for the Integrated Thermal Treatment System (ITTS) Study

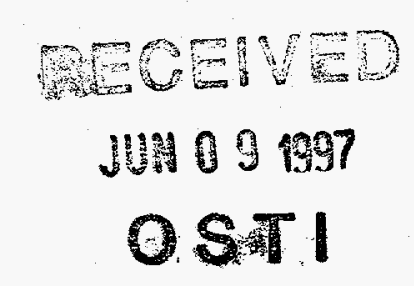

K. H. Liebelt

B. W. Brown

W. J. Quapp

JU 091997 
INEL-95/0355

\title{
Process Modeling for the Integrated Thermal Treatment System (ITTS) Study
}

\author{
K. H. Liebelt \\ B. W. Brown \\ W. J. Quapp
}

September 1995

Idaho National Engineering Laboratory

Lockheed Martin Idaho Technologies Company

Idaho Falls, ID 83415

Prepared for the

U.S. Department of Energy

Idaho Operations Office

Under DOE Idaho Field Office

Contract No. DE-AC07-94ID13223 

Process Modeling for the Integrated Thermal Treatment Systems (ITTS) Study

INEL-95/0335

Prepared by

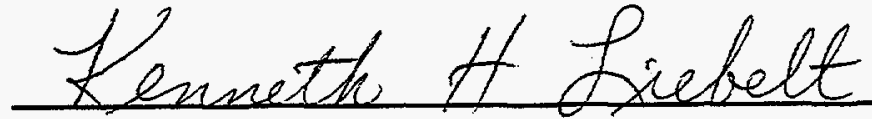

K. H. Liebelt, Advisory Engineer/Scientist
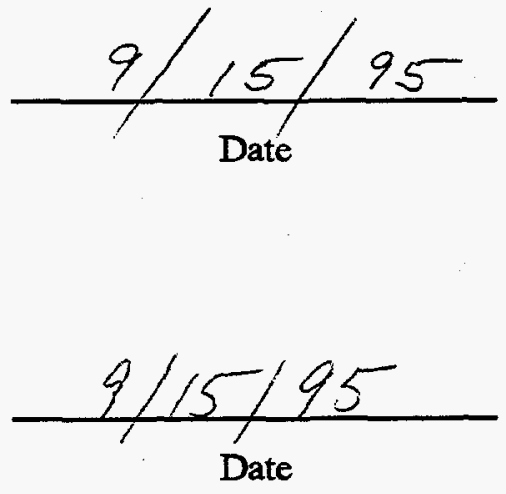

Approved by

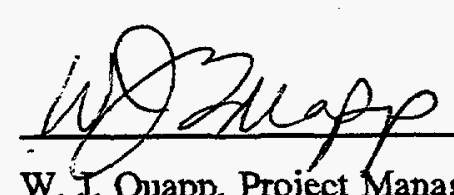

w. T. Quapp, Project Manager/Consulting Engineer Materials and Processing

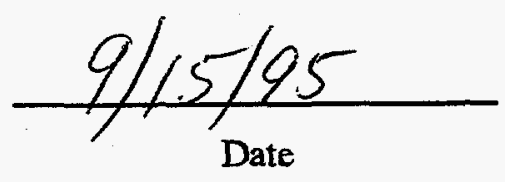




\begin{abstract}
This report describes the process modeling done in support of the integrated thermal treatment system (ITTS) study, Phases 1 and 2. ITTS consists of an integrated systems engineering approach for uniform comparison of widely varying thermal treatment technologies proposed for treatment of the contacthandled mixed low-level wastes (MLLW) currently stored in the U. S.

Department of Energy complex. In the overall study, 19 systems were evaluated. Preconceptual designs were developed that included all of the various subsystems necessary for a complete installation, from waste receiving through to primary and secondary stabilization and disposal of the processed wastes. Each system included the necessary auxiliary treatment subsystems so that all of the waste categories in the complex were fully processed. The objective of the modeling task was to perform mass and energy balances of the major material components in each system. Modeling of trace materials, such as pollutants and radioactive isotopes, were beyond the present scope. The modeling of the main and secondary thermal treatment, air pollution control, and metal melting subsystems was done using the ASPEN PLUS process simulation code, Version 9.1-3. These results were combined with calculations for the remainder of the subsystems to achieve the final results, which included offgas volumes, and mass and volume waste reduction ratios.
\end{abstract}




\section{DISCLAMIER}

Portions of this document may be illegible in electronic image products. Images are produced from the best available original document. 


\section{DISCLAIMER}

This report was prepared as an account of work sponsored by an agency of the United States Government. Neither the United States Government nor any agency thereof, nor any of their employees, make any warranty, express or implied, or assumes any legal liability or responsibility for the accuracy, completeness, or usefulness of any information, apparatus, product, or process disclosed, or represents that its use would not infringe privately owned rights. Reference herein to any specific commercial product, process, or service by trade name, trademark, manufacturer, or otherwise does not necessarily constitute or imply its endorsement, recommendation, or favoring by the United States Government or any agency thereof. The views and opinions of authors expressed herein do not necessarily state or reflect those of the United States Government or any agency therenf. 


\section{CONTENTS}

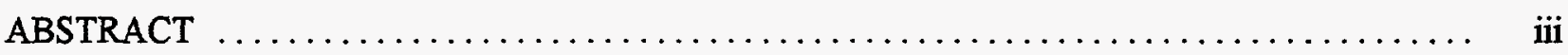

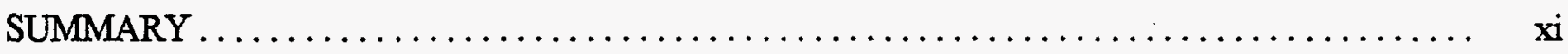

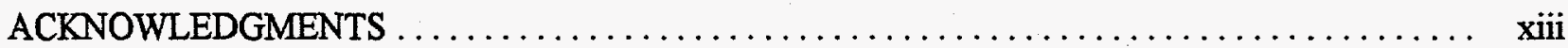

ABBREVIATIONS AND ACRONYMS $\ldots \ldots \ldots \ldots \ldots \ldots \ldots \ldots \ldots \ldots \ldots \ldots \ldots \ldots \ldots \ldots$

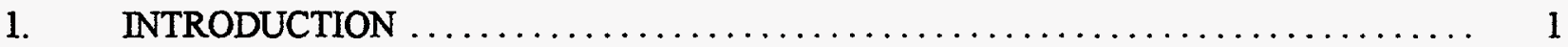

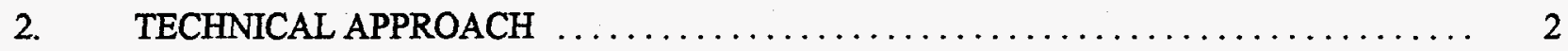

3. INPUT WASTE CHARACTERISTICS $\ldots \ldots \ldots \ldots \ldots \ldots \ldots \ldots \ldots \ldots \ldots \ldots \ldots \ldots$

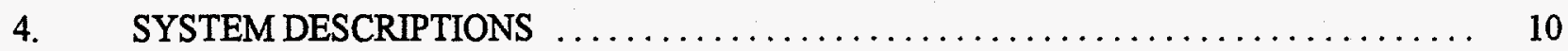

4.1 System A-1: Conventional Rotary Kiln, Air Combustion Gas, Dry-Wet

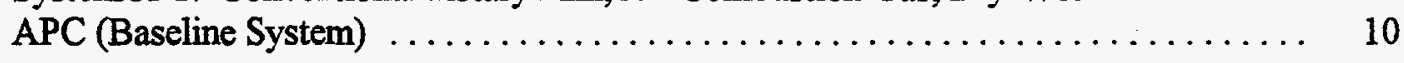

4.2 System A-2: Conventional Rotary Kiln, Oxygen Combustion Gas . . . . . . . . . 13

4.3 System A-3: Conventional Rotary Kiln, Wet Air Pollution

Control Subsystem $\ldots \ldots \ldots \ldots \ldots \ldots \ldots \ldots \ldots \ldots \ldots \ldots \ldots \ldots \ldots \ldots \ldots$

4.4 System A-4: Conventional Rotary Kiln, $\mathrm{CO}_{2}$ Retention $\ldots \ldots \ldots \ldots \ldots \ldots \ldots$

4.5 System A-5: Conventional Rotary Kiln, Polymer Stabilization ........... 14

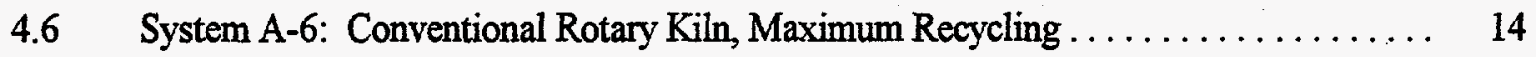

$4.7 \quad$ System A-7: Slagging Rotary Kiln $\ldots \ldots \ldots \ldots \ldots \ldots \ldots \ldots \ldots \ldots \ldots \ldots \ldots$

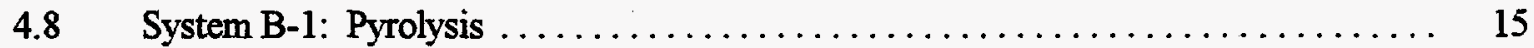

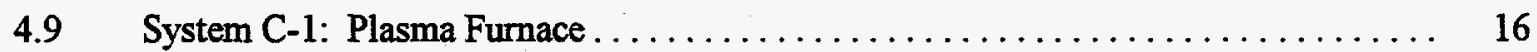

4.10 System C-2: Plasma Furnace, $\mathrm{CO}_{2}$ Retention $\ldots \ldots \ldots \ldots \ldots \ldots \ldots \ldots \ldots \ldots \ldots \ldots$

4.11 System C-3: Plasma Gasification $\ldots \ldots \ldots \ldots \ldots \ldots \ldots \ldots \ldots \ldots \ldots \ldots \ldots$

4.12 System D-1: $\mathrm{CO}_{2}$ Retention $\ldots \ldots \ldots \ldots \ldots \ldots \ldots \ldots \ldots \ldots \ldots \ldots \ldots \ldots \ldots \ldots \ldots$

4.13 System E-1: Thermal Desorption $\ldots \ldots \ldots \ldots \ldots \ldots \ldots \ldots \ldots \ldots \ldots \ldots$

4.14 System F-1: Molten Salt Oxidation $\ldots \ldots \ldots \ldots \ldots \ldots \ldots \ldots \ldots \ldots \ldots \ldots \ldots \ldots \ldots \ldots \ldots \ldots \ldots$

4.15 System G-1: Molten Metal Waste Destruction $\ldots \ldots \ldots \ldots \ldots \ldots \ldots \ldots \ldots$

4.16 System $H-1$ : Steam Gasification $\ldots \ldots \ldots \ldots \ldots \ldots \ldots \ldots \ldots \ldots \ldots \ldots \ldots \ldots \ldots \ldots \ldots \ldots \ldots$ 
4.17 System J-1: Joule-Heated Vitrification $\ldots \ldots \ldots \ldots \ldots \ldots \ldots \ldots \ldots \ldots \ldots, 23$

4.18 System K-1: Thermal Desorption and Mediated Electrochemical Oxidation ........ 23

4.19 System L-1: Thermal Desorption and Supercritical Water Oxidation ........... 25

5. MODELING OF MAIN TREATMENT SUBSYSTEMS-DESCRIPTIONS AND

RESULTS $\ldots \ldots \ldots \ldots \ldots \ldots \ldots \ldots \ldots \ldots \ldots \ldots \ldots \ldots \ldots \ldots \ldots \ldots \ldots \ldots \ldots \ldots, 47$

$5.1 \quad$ Background $\ldots \ldots \ldots \ldots \ldots \ldots \ldots \ldots \ldots \ldots \ldots \ldots \ldots \ldots \ldots \ldots, 47$

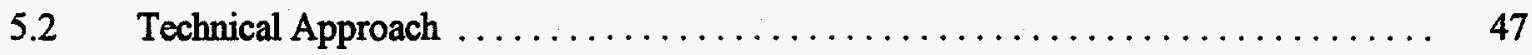

6. OVERALL MASS AND ENERGY BALANCES $\ldots \ldots \ldots \ldots \ldots \ldots \ldots \ldots \ldots \ldots \ldots \ldots$

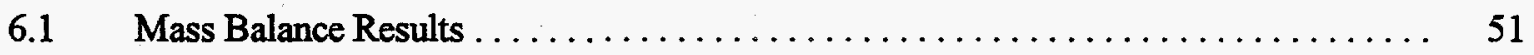

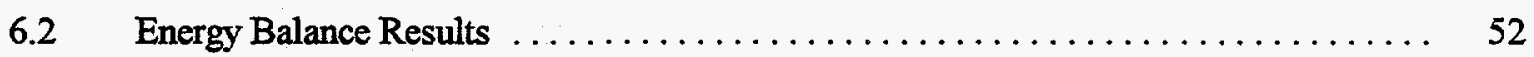

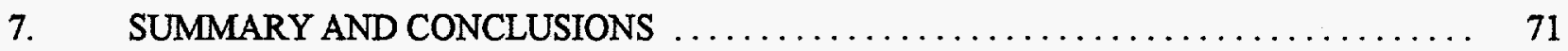

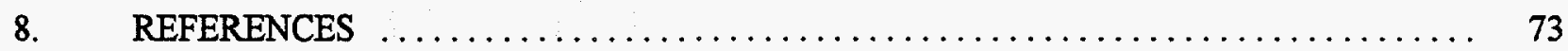

Appendix A-Model Process Flow Diagrams (MPFDS) and Assumption and Energy

Balance Tables . . . . . . . . . . . . . . .

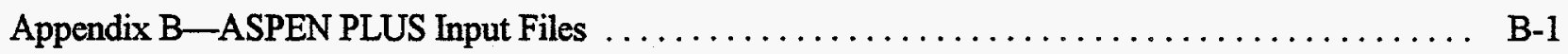

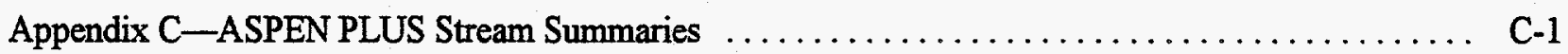

\section{FIGURES}

4-1. Rotary Kiln: PFD for Overall System (Systems A-1 through A-6) $\ldots \ldots \ldots \ldots \ldots \ldots \ldots$

4-2. Rotary Kiln: PFD for incineration (Systems A-1AND A-2) and Dry and Wet Air

Pollution Control Subsystems (Systems A-1 and A-2) . . . . . . . . . . . . . . . 28

4-3. Rotary Kiln: PFD for Dry and Wet Air Pollution Control Subsystem with $\mathrm{CO}_{2}$

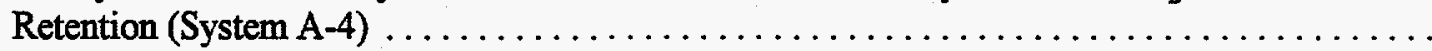

4-4. Rotary Kiln: PFD for Dry and Wet Air Pollution Control Subsystem with Salt Recovery (System A-6) ........................................

4-5. Slagging Rotary Kiln: PFD for Main Thermal Treatment, Air Pollution Control, and Primary Stabilization Subsystems (System A-7) $\ldots \ldots \ldots \ldots \ldots \ldots \ldots \ldots \ldots \ldots$

4-6. Pyrolysis: PFD for Incineration, Air Pollution Control, and Primary Stabilization Subsystems (System B-1) 
4-7. Plasma Furnace: PFD for Incineration, Air Pollution Control, and Primary

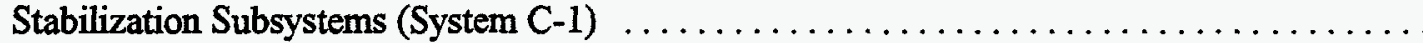

4-8. Plasma Furnace, $\mathrm{CO}_{2}$ Retention: PFD for Main Thermal Treatment, Air Pollution

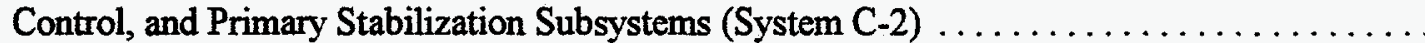

4-9. Plasma Gasification: PFD for Main Thermal Treatment, Air Pollution Control, and

Primary Stabilization Subsystems (System C-3) . . . . . . . . . . . . . . . . . . .

4-10. System D, Fixed Hearth with $\mathrm{CO}_{2}$ Retention: PFD for Incineration and Air Pollution

Control Subsystems (System D-1)

4-11. Thermal Desorption: PFD for Incineration, Thermal Desorber, and Air Pollution

Control Subsystems (System E-1) . . . . . . . . . . . . . . . . . . . . .

4-12. Molten Salt Oxidation: PFD for Main Thermal Treatment, Air Pollution Control, and

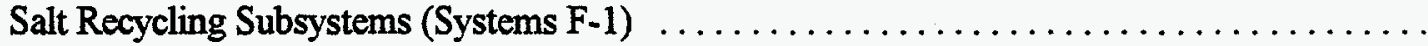

4-13. Molten Salt Oxidation: PFD for Primary Stabilization Subsystem (System F-1) $\ldots \ldots \ldots$

4-14. Molten Metal Waste Destruction: PFD for Main Thermal Treatment, Air Pollution Control, and Primary Stabilization Subsystems (System G-1) $\ldots \ldots \ldots \ldots \ldots \ldots \ldots \ldots \ldots \ldots \ldots$

4-15. Steam Gasification: PFD for Primary Stabilization Subsystem (System H-1) . . . . . . . .

4-16. Joule-heated Vitrification: PFD for Main Thermal Treatment, Air Pollution Control,

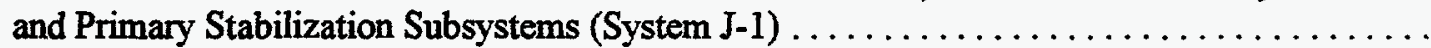

4-17. Thermal Desorption and Mediated Electrochemical Oxidation: PFD for Main Thermal Treatment and Air Pollution Control Subsystems (System K-1) . . . . . . . . .

4-18. Thermal Desorption and Mediated Electrochemical Oxidation: PFD for Organic

Liquid Waste Treatment Subsystem (System K-1)

4-19. Thermal Desorption and Supercritical Water Oxidation: PFD for Main Thermal

Treatment and Air Pollution Control Subsystems (System L-1) $\ldots \ldots \ldots \ldots \ldots \ldots$

4-20. Thermal Desorption and Supercritical Water Oxidation: PFD for Organic Liquid

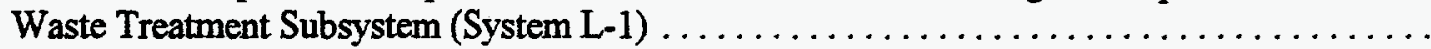

\section{TABLES}

2-1. Systems Included in the Integrated Thermal Treatment System Study $\ldots \ldots \ldots \ldots \ldots \ldots$

3-1A. Elemental Compositions in the Physical Waste Categories in the DOE Complex ....... 6

3-1B. Elemental Compositions in the Physical Waste Categories in the DOE Complex ....... 7

3-1C. Elemental Compositions in the Physical Waste Categories in the DOE Complex ........ 8

3-2. Waste Treatment Processes, and Waste Quantities and ITTS Flow Rates of Each ....... 9 
6-1. Overall Mass Balances and Summary Results for the ITTS Study Process Modeling

A-1. ASSUMPTIONS and ENERGY BALANCES for BASELINE ROTARY KILN, System A-1

A-2. ASSUMPTIONS and ENERGY BALANCES for ROTARY KILN W/ OXYGEN, System A-2 .

A-3. ASSUMPTIONS and ENERGY BALANCES for ROTARY KILN W/ WET APC,

System A-3.

A-4. ASSUMPTIONS and ENERGY BALANCES for ROTARY KILN W/ $\mathrm{CO}_{2}$

RETENTION, System A-4

A-5. ASSUMPTIONS and ENERGY BALANCES for ROTARY KILN W/ POLYMER

STABILIZATION, System A-5

A-6. ASSUMPTIONS and ENERGY BALANCES for ROTARY KILN W/ MAXIMUM

RECYCLING, System A-6

A-7. ASSUMPTIONS and ENERGY BALANCES for SLAGGING ROTARY KILN,

System A-7.

A-8. ASSUMPTIONS and ENERGY BALANCES for PYROLYSIS, System B-1

A-9. ASSUMPTIONS and ENERGY BALANCES for PLASMA FURNACE, System C-1

A-10. ASSUMPTIONS and ENERGY BALANCES for PLASMA FURNACE W/ $\mathrm{CO}_{2}$

RETENTION, System C-2

A-11. ASSUMPTIONS and ENERGY BALANCES for PLASMA FURNACE W/ STEAM

GASIFICATION, System C-3 .

A-12. ASSUMPTIONS and ENERGY BALANCES for FIXED-HEARTH PYROLYZER W/ $\mathrm{CO}_{2}$ RETENTION, System D-1

A-13. ASSUMPTIONS and ENERGY BALANCES for THERMAL DESORPTION, System E-1

A-14. ASSUMPTIONS and ENERGY BALANCES for MOLTEN SALT OXIDATION, System F-1

A-15. ASSUMPTIONS and ENERGY BALANCES for MOLTEN METAL DESTRUCTION, System G-1 .

A-16. ASSUMPTIONS and ENERGY BALANCES for STEAM GASIFICATION, System H-1

A-17. ASSUMPTIONS and ENERGY BALANCES for JOULE-HEATED VITRIFICATION, System J-1 
A-18. ASSUMPTIONS and ENERGY BALANCES for THERMAL DESORBER W/MEO,

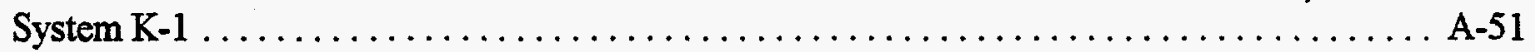

A-19. ASSUMPTIONS and ENERGY BALANCES for THERMAL DESORBER W/ SCWO, System L-1 


\section{SUMMARY}

This report describes the process modeling done in support of the integrated thermal treatment system (ITTS) study, Phases 1 and 2. ITTS consists of an integrated systems engineering approach for uniform comparison of widely varying thermal treatment technologies proposed for the treatment of contact-handled mixed low-level waste (MLLW) currently stored in the U. S. Department of Energy complex. In the overall study, 19 systems were evaluated. Preconceptual designs were developed that included all of the various subsystems necessary for a complete installation, from waste receiving through to primary and secondary stabilization and disposal of the processed wastes. Each system included the necessary auxiliary treatment subsystems so that all of the waste categories in the complex were fully processed. The main thermal treatment options considered were rotary kiln incinerators, pyrolysis, plasma furnace, fixed-hearth thermal desorption, molten salt oxidation, molten metal waste destruction, steam gasification, Joule-heated vitrification, mediated electrochemical oxidation, and supercritical water oxidation.

The study required knowledge of these mass and energy flows in order to compare the advantages and disadvantages of the systems. The amounts and types of end products, and to a lesser effect the energy used, determine the cost of each system. The objective of the process modeling task was to perform mass and energy balances of the major material components in each system. Modeling of trace materials, such as pollutants and radioactive isotopes, and minor amounts of energy consumption, were beyond the present scope and were generally not tracked.

For Phase 2 of the study, the modeling of the main and secondary thermal treatment, main air pollution control, and metal melting subsystems was done using the ASPEN PLUS process simulation code, Version 9.1-3. This code performs steady-state solutions of engineering processes. Each model consisted of some 8 to 20 unit operations. Some steps in the processes could be modeled very simply since only major material components were being tracked. Feed rates were obtained from knowledge of the elemental composition of the waste, combined with the projected operating life of the facilities. The code was able to predict the products from each step in the process while simultaneously converging on the correct amount of fuel, oxidant, cooling water, and other parameters to meet the desired operating conditions.

The code results presented consist of the required amounts of process inputs (such as fuel, oxidant, and cooling water); energy inputs and outputs for each of the unit operations; the amounts and compositions of the intermediate streams and final products (such as offgas and processed wastes); and the mass and volume waste reduction ratios for each of the systems. These results were combined with updated Phase 1 calculations for the remainder of the subsystems to arrive at the overall system mass balances. The remaining subsystems included metal decontamination, lead recovery, mercury amalgamation, aqueous waste treatment, and special wastes.

The complete ITTS comparison process consisted of many other considerations besides the mass and energy balance results, including operational requirements, conceptual design layouts, planning lifecycle cost estimates, and identification of technologies requiring development, which are reported elsewhere. 


\section{ACKNOWLEDGMENTS}

The authors acknowledge the leadership and guidance of Carl Cooley of the U. S. Department of Energy; and the support of Daryoush Bahar, Fred Feizollahi, Andrew Smith, Ben Teheranian, Julia Vetromile, and others of Morrison Knudsen Environmental Services. 


\section{ABBREVIATIONS AND ACRONYMS}

$\begin{array}{ll}\text { APC } & \text { air pollution control } \\ \text { ASCII } & \text { American Standard Code for Information Interchange } \\ \text { atm } & \text { atmosphere } \\ \text { BDAT } & \text { best demonstrated available technology } \\ \text { Btu } & \text { British thermal unit } \\ \text { CEM } & \text { continuous emissions monitoring } \\ \text { CFR } & \text { Code of Federal Regulations } \\ \text { DOE } & \text { U.S. Department of Energy } \\ \text { EPA } & \text { U.S. Environmental Protection Agency } \\ \text { F\&ORs } & \text { functional and operational requirements } \\ \text { FAD } & \text { functional allocation diagram } \\ \text { GOCO } & \text { government owned and contractor operated } \\ \text { HEPA } & \text { high-efficiency particulate air (filter) } \\ \text { hr } & \text { hour } \\ \text { INEL } & \text { Idaho National Engineering Laboratory } \\ \text { ITTS } & \text { integrated thermal treatment system } \\ \text { lb } & \text { pound } \\ \text { LLW } & \text { low-level (radioactive) waste } \\ \text { MEO } & \text { mediated electrochemical oxidation } \\ \text { MIT } & \text { Massachusetts Institute of Technology } \\ \text { MK } & \text { Morrison Knudsen Corporation, Engineering, Construction \& Environmental Group } \\ \text { MLLW } & \text { mixed low-level (radioactive) waste } \\ \text { MPFD } & \text { model process flow diagram } \\ \text { MSO } & \text { molten salt oxidation } \\ \text { nCi } & \text { nanoCurie } \\ \text { NO } & \text { nitrous oxide } \\ \text { ORNL } & \text { Oak Ridge National Laboratory } \\ \text { PAN } & \text { passive/active neutron } \\ \text { PCB } & \text { polychlorinated biphenyls } \\ \text { PFD } & \text { process flow diagram } \\ \text { PLCC } & \text { planning life-cycle cost } \\ \text { RCRA } & \text { Resource Conservation and Recovery Act } \\ \text { RTR } & \text { real-time radiography } \\ \text { SCC } & \text { secondary combustion chamber } \\ \text { SCWO } & \text { supercritical water oxidation } \\ \text { SGS } & \text { segmented gamma scanning } \\ \text { SNR } & \text { sorting not required } \\ \text { SR } & \text { sorting required } \\ \text { TCLP } & \text { toxicity characteristic leaching procedure } \\ \text { TDS } & \text { total dissolved solids } \\ \text { TRU } & \text { transuranic } \\ \text { TSCA } & \text { Toxic Substances Control Act } \\ \text { VOCs } & \text { volatile organic compounds } \\ & \\ & \end{array}$




\section{Process Modeling \\ for the \\ Integrated Thermal Treatment System (ITTS) Study}

\section{INTRODUCTION}

The U.S. Department of Energy's (DOE's) Environmental Management Office of Technology Development has commissioned an integrated thermal treatment system (ITTS) study to assess alternative systems for treating contact-handled mixed low-level radioactive waste (MLLW) and alpha-MLLW (10 $\leq$ $\mathrm{nCi} / \mathrm{g}$ of TRU $\leq 100$ ). The MLLW in the DOE complex consists of organic and inorganic solids and liquids comprising a wide variety of materials contaminated with radioactive substances. Treatment is needed that will destroy the organic material. Other operations are needed to stabilize the treatment residues, inorganic materials, and radionuclides prior to disposal in a MLLW disposal facility.

Regulations promulgated by both DOE and the U.S. Environmental Protection Agency (EPA) govern the storage, treatment, and disposal of these wastes.

The purpose of the ITTS study is to conduct a systematic engineering evaluation of a variety of MLLW treatment system alternatives. Preconceptual designs, consisting of process flow diagrams (PFDs), facility layouts, equipment lists, and material mass balances, have been developed, and the relative merits and life-cycle costs for each treatment alternative identified. The study also identified the research and development, demonstrations, and testing and evaluation needed to assure performance of the unit operations in the most promising alternative systems.

Thermal treatment ${ }^{\mathrm{b}}$ is the most effective technique.for destruction of toxic organic materials. Incineration, ${ }^{c}$ a form of thermal treatment, has been designated by the EPA as the best demonstrated available technology (BDAT) for destroying a number of these organic waste constituents.

Phase 1 of the ITTS study focused on establishing a baseline understanding of well-developed thermal treatment technologies, namely a conventional rotary kiln incinerator with six variations, a fixedhearth controlled-air incinerator, an indirectly-heated pyrolyzer, a thermal desorber for inorganic residue with a rotary kiln for the combustible waste fraction, and a plasma hearth melter system. The variations were used to examine the effects of combustion gas, air pollution control system design, and stabilization technology for the treatment residues on system performance and costs. Phase 2 addressed more innovative technologies such as one-step processing, gasification, low-temperature oxidation, molten salt oxidation, supercritical water oxidation, and molten metal destruction of wastes.

The systems evaluated were required to treat all waste stored in the DOE complex. This requirement established the need for several treatment lines within each system to accommodate the range

b. As defined by EPA in 40 CFR 260.10 , p. 10, "Thermal treatment means the treatment of hazardous waste in a device which uses elevated temperature as the primary means to change the chemical, physical, or biological character or composition of the hazardous waste. Examples of thermal treatment processes are incineration, molten salt, pyrolysis, calcination, wet air oxidation, and microwave discharge."

c. As defined by EPA in 40 CFR 260.10 , p. 10 , "Incinerator means any enclosed device that (1) uses controlled flame combustion and neither meets the criteria for classification as a boiler, sludge dryer, or carbon regeneration unit, nor is listed as an industrial furnace, or (2) meets the definition of an infrared incinerator or plasma arc incinerator." 
of MLLW encountered. Section 3 provides information on the specific composition of the wastes in the complex, and as used in the study and for modeling.

Portions of this report summarize material discussed in detail in the ITTS Phase 1 and Phase 2 reports ${ }^{1,2}$. Due to the large amount of information involved in describing the 19 systems involved in this study that descriptive material has not been reproduced here in its entirety. However, this report does contain a full description of the process modeling task. For that portion of the work done using the process simulation code, complete input files are included in the appendices to reproduce the numerical results presented.

\section{TECHNICAL APPROACH}

A key to accurate evaluation of the thermal treatment systems is using an integrated systems engineering approach that provides a uniform basis for comparing the merits of widely varying treatment alternatives. The systems considered in the study consist of all facilities, equipment, and methods needed for treating and disposing of the MLLW currently stored in the DOE complex. Steps within the treatment process include waste receiving, characterization, sizing, main thermal treatment, secondary treatments, air pollution control, primary and secondary stabilization of the waste residue, and eventual disposal. This approach more accurately evaluates systems such as plasma melters or electric arc furnaces that have a higher initial cost but produce less residue for disposal.

The focus of the ITTS study is on innovative and cost-effective treatment systems that minimize the short- and long-term adverse impacts on the worker and public environment, health, and safety. Two examples are the use of contaminated soil, when available, for vitrification of process residues and the use of $\mathrm{CO}_{2}$ absorbent materials for absorption (or delayed release) of process offgas discharged to the atmosphere.

For Phase 1, various combinations of the incinerator subsystem, the air pollution control subsystem, and the waste stabilization subsystem were considered. Incinerator subsystems considered were rotary kiln, plasma arc furnace, fixed-hearth furnace, and fluidized bed incinerator. Air pollution control subsystem designs included both wet and dry technologies. Waste stabilization subsystem options included concrete, polymer, and glass- or vitrified soil-based final waste forms. A panel of engineers with diverse experience and technical backgrounds in incineration and stabilization was convened to reduce the number of possible choices to a number which could be evaluated in detail within the time and resources available. Ten systems were selected for the Phase 1 effort. A similar selection process was used for the more innovative technologies of Phase 2. The system types for both phases and the designations of them used in this study are listed in Table 2-1.

Since the public is often concerned with stack emissions, specific attention was focused on alternative air pollution control unit designs. The intent was to specify a configuration that would provide better emission performance (by an order of magnitude) than required to meet current EPA standards. Where research, development, demonstration, testing, and evaluation activities were expected to be necessary to verify component performance for this application, those costs were included.

A second major concern of the public is disposal of hazardous solid residues, especially when radioactive. Thermal treatment alone will not render the wastes nonhazardous; the residues will contain hazardous materials as defined by the Resource Conservation and Recovery Act (RCRA), as well as radionuclides. Under EPA regulations, residues might have to be stabilized before disposal if leachability standards are not met. To ensure RCRA compliance and to provide long-term isolation of residues, the baseline process chosen for primary residue stabilization was molten glass or soil-based stabilization, referred to as vitrification. If done correctly, vitrification provides the greatest protection 
Table 2-1. Systems Included in the Integrated Thermal Treatment System Study.

System Description

Designation

Rotary kiln with air for combustion and dry/wet APC - baseline system

Phase 1 Phase 2

Rotary kiln with oxygen for combustion and dry/wet APC

A-1

Rotary kiln with air for combustion and wet APC

A-2

Rotary kiln with oxygen for combustion and $\mathrm{CO}_{2}$ retention

A-3

Rotary kiln with air for combustion and polymer stabilization

A-4

Rotary kiln with air for combustion and maximum recycling

A-5

Slagging rotary kiln

Indirectly-heated pyrolyzer with oxygen and dry/wet APC

A-6

Plasma hearth furnace with air and dry/wet APC

B-1

Plasma furnace, $\mathrm{CO}_{2}$ retention

Plasma gasification

Fixed-hearth furnace with oxygen and $\mathrm{CO}_{2}$ retention

D-1

Debris desorption and grouting with rotary kiln for oxidation of combustibles

E-1

Molten salt oxidation

Molten metal waste destruction

Steam gasification

Joule-heated vitrification

Thermal desorption and mediated electrochemical oxidation

$\mathrm{K}-1$

Thermal desorption and supercritical water oxidation

L-1

against future environmental releases following disposal and also provides a margin against more stringent future release standards. Vitrification binds the hazardous inorganic residues (glass or metal oxides) and the radioactive contaminants (also metal oxides) into a solid solution of rock-like material. DOE is currently supporting considerable research in the field of waste vitrification to improve the process engineering and understand the physical, chemical, and thermal treatment requirements to assure high performance. It is also expected that vitrification of residues may result in disposal cost savings and reduced public apprehension.

Variations in the stabilization process included polymers and cement. Some waste residues, such as salts resulting from the neutralization of acidic combustion gases and volatile incinerator fly ash, were stabilized in a second process using a polymer because they vaporize at vitrification temperatures, which can approach $3000^{\circ} \mathrm{F}$. Mercury is another material that cannot be vitrified because of its volatility. In this study, amalgamation was used for mercury since it is listed by the EPA as the BDAT.

As part of the design process, functional and operational requirements, flow sheets and mass balances, and conceptual equipment layouts were developed for each system. Mass balances were performed to account for all materials treated or used in the processes. All secondary residues were processed in accordance with regulatory requirements and the final volumes for disposal were estimated. Transportation and disposal cost estimates were applied to the disposal volume of each system as part of the planning level life-cycle cost (PLCC) estimate. Simplified system energy balances were conducted to 
determine the requirements and thereby assure that no system was excessively energy intensive. Costs have been estimated assuming the system is government owned and contractor operated (GOCO).

Initial mass and energy balances were done for Phase 1 by simpler, less rigorous methods. For Phase 2 , it was decided to model the systems with the ASPEN PLUS process simulation code ${ }^{3}$. Besides the of this code (and other similar codes) in having wide industrial acceptance, it provides numerous capabilities not only for the present task (macro-scale mass and energy balances), but also for addressing the inevitable 'what if scenarios and other possible expanded analyses. These capabilities include (but are not limited to) a large material database, no restrictions on system configuration (multiple feedback loops), availability of all types of unit operation models, simultaneous convergence on the user's design specifications, sensitivity studies, cost predictions, plotting, and report generation. Switching to ASPEN PLUS required that the Phase 1 results be recalculated, but, as discussed in the following section, Phase 2 used an expanded waste database compared to Phase 1 , which alone would have required a re-calculation of the Phase 1 results in order to compare all systems on an equal basis.

\section{INPUT WASTE CHARACTERISTICS}

Information on the composition of the entire contact-handled MLLW in the DOE complex was collected and summarized as part of the ITTS effort ${ }^{4}$. Certain waste streams, such as the Hanford tank waste and the Rocky Flats Plant solar pond liquids, were excluded from the ITTS database because these waste streams are being addressed using other processes that are more suitable than thermal treatment.

Initially, for the Phase 1 effort, a subset of the applicable DOE waste inventory was used. This consisted of the inventories at the ten largest sites, and amounted to about half of the total. For Phase 2 the database was expanded to that for the 20 largest sites, essentially $100 \%$ of the applicable waste, in order to better represent the likely (overall average) waste stream. While the smaller database in Phase 1 included all types of waste in the DOE complex, the relative proportions were not fully representative of that in the total inventory. Because the database was changed, even without a change in the method for calculating the mass and energy balances, it would have been necessary to recalculate the Phase 1 results in order to present a consistent comparison between all systems. Only the Phase 2 input waste characteristics (quantities, composition, and proposed ITTS feed rates) are discussed here. The following procedure applied to both phases, however.

First, the waste was characterized into 56 physical categories, such as, concrete, inorganic labpacks, paper, etc. The composition of each category was then defined in terms of 23 'elements'. The 'elements' consisted of chemical elements, chemical compounds $\left(\mathrm{NO}, \mathrm{SO}_{2}\right.$, and water), general chemical categories (glass forming inerts and bulk metal), and the radioisotope U-238. The matrix of physical categories and 'elements' are shown in Table 3-1 (A through C), as derived from Reference (4).

Next, the physical categories were condensed into nine groups based on the waste treatment process (subsystem) to which the material would be sent. ${ }^{2}$ For Phase 1 , the treatment processes were designated $\mathrm{A} 1, \mathrm{~A} 2$, and $\mathrm{B}$ through $\mathrm{H}$ as also shown in Table 3-1. Table 3-2 relates the treatment processes to the condensed physical categories. Later, for two of the Phase 2 systems, K-1 and L-1, treatment process Al (combustible waste) was subdivided to separate the 'organic liquids' from the remainder, which is also shown in Table 3-2. The main thermal treatment process for each of the 19 systems was defined as that which processed the major organic portion of the combustible and noncombustible wastes.

Finally, these quantities of waste were converted to design feed rates for each process. ${ }^{2}$ Design feed rates (nominal capacities) were based on a 20 -year operations life, and adjusted for assumed plant availability, number of shifts per day, amount of secondary waste, and smallest available equipment. 
Table 3-2 shows those feed rates used for the processes modeled with ASPEN PLUS, and for the other subsystem calculations as well.

For the ASPEN PLUS modeling, it was necessary to assign specific chemical compositions to the two general chemical categories, glass forming inerts and bulk metal, in order to take advantage of the material property data available in the code. However, precise definitions of the compositions were not necessary (and, in fact, were not known) since it was only necessary to model only a few of the many possible chemical reactions, for the present task. Given this, the physical properties of major interest were enthalpy and density, which do not vary greatly for reasonable selections. Thus, for the glass forming inerts it was assumed that the composition was a $50 / 50$ mixture of $\mathrm{Al}_{2} \mathrm{O}_{3}$ and $\mathrm{SiO}_{2}$, two of the most common constituents of glass; and, for the bulk metal the simple assumption was made that it consisted of $100 \%$ pure iron ( $\mathrm{Fe}$ ), the most common structural material. (Depending on the system under consideration and its known or expected operating characteristics, the iron was either assumed to be chemically inert or allowed to oxidize when chemically favored.)

Solid residues from the systems are stabilized by a combination of vitrification, polymer solidification, and grouting. Vitrification additives, if required, are introduced as part of the feedstock to the systems. Soil found at the INEL has been identified as a good additive material when mixed in approximately a 2-to-1 ratio with the ash from the thermal treatment processes ( 2 parts ash to 1 part soil), as discussed in Section 4.1 under primary stabilization. Even though contaminated soil from environmental restoration programs would likely be used as the additive, it is not considered as a waste for the purposes of this study.

The use of contaminated soil for the vitrification process provided extra value in those processes.

The composition of the soil was obtained from Reference (5). Only the eight most prevalent materials from the measured composition were used in the modeling. The "as used" formulation is found in the ASPEN PLUS stream summary results (for those systems which use soil) in Appendix C as the stream labeled 'SOIL'. Most notably, the composition includes $10 \%$ moisture and $12.8 \%$ calcium carbonate $\left(\mathrm{CaCO}_{3}\right)$, which decomposes at about $1500^{\circ} \mathrm{F}$ (below vitrification temperatures). In the modeling, the flow rate of the moist, as-received feed soil is calculated (using an ASPEN PLUS design specification) to form the 2-to-1 mixture. After considering the loss of moisture and $\mathrm{CO}_{2}$ (from the decomposition of $\mathrm{CaCO}_{3}$ ) from the raw soil, the final ratio of ash-to-processed soil becomes 2.37-to-1. 


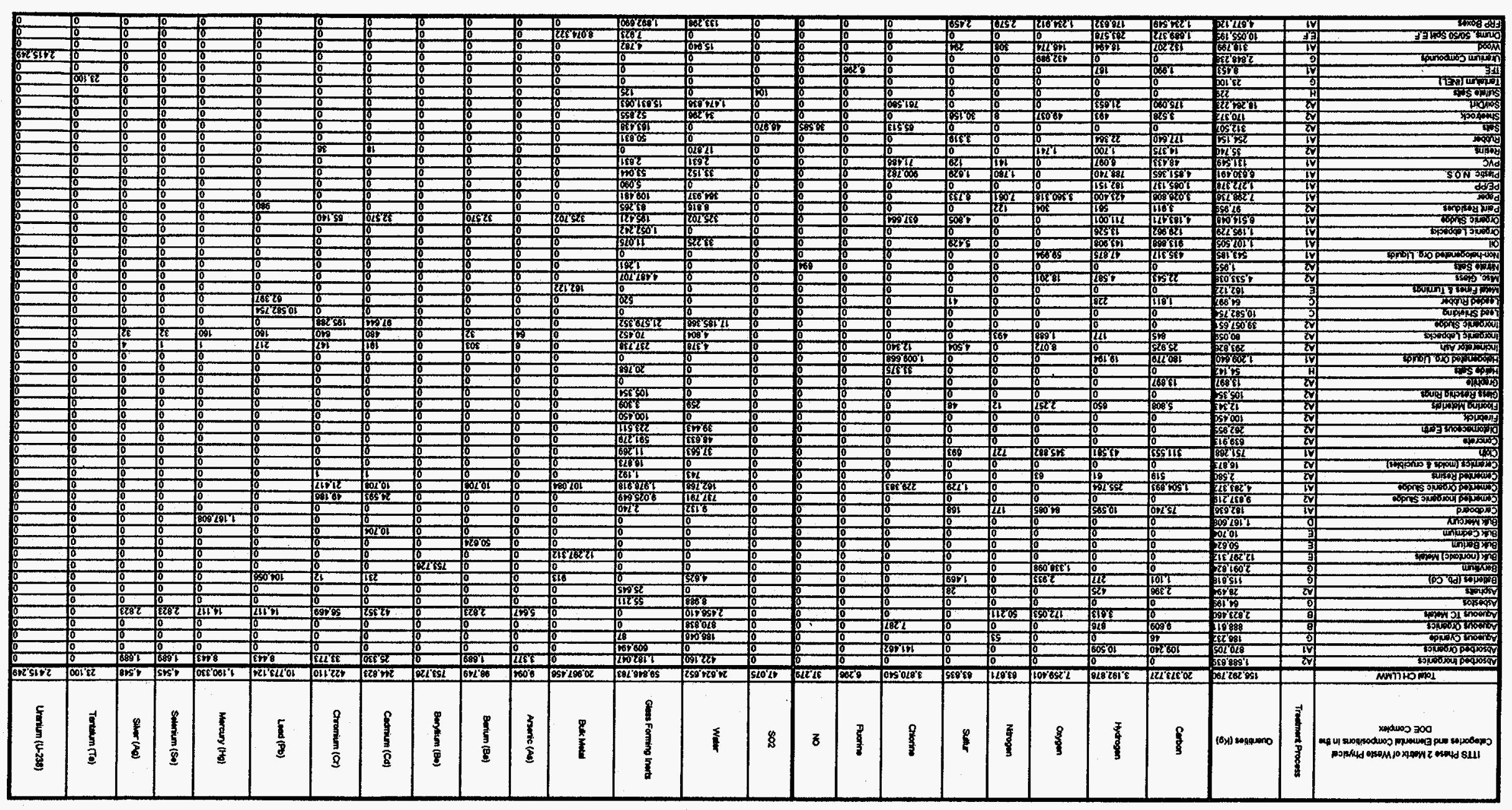


Table 3-1B. Elemental Compositions in the Physical Waste Categories in the DOE Complex.

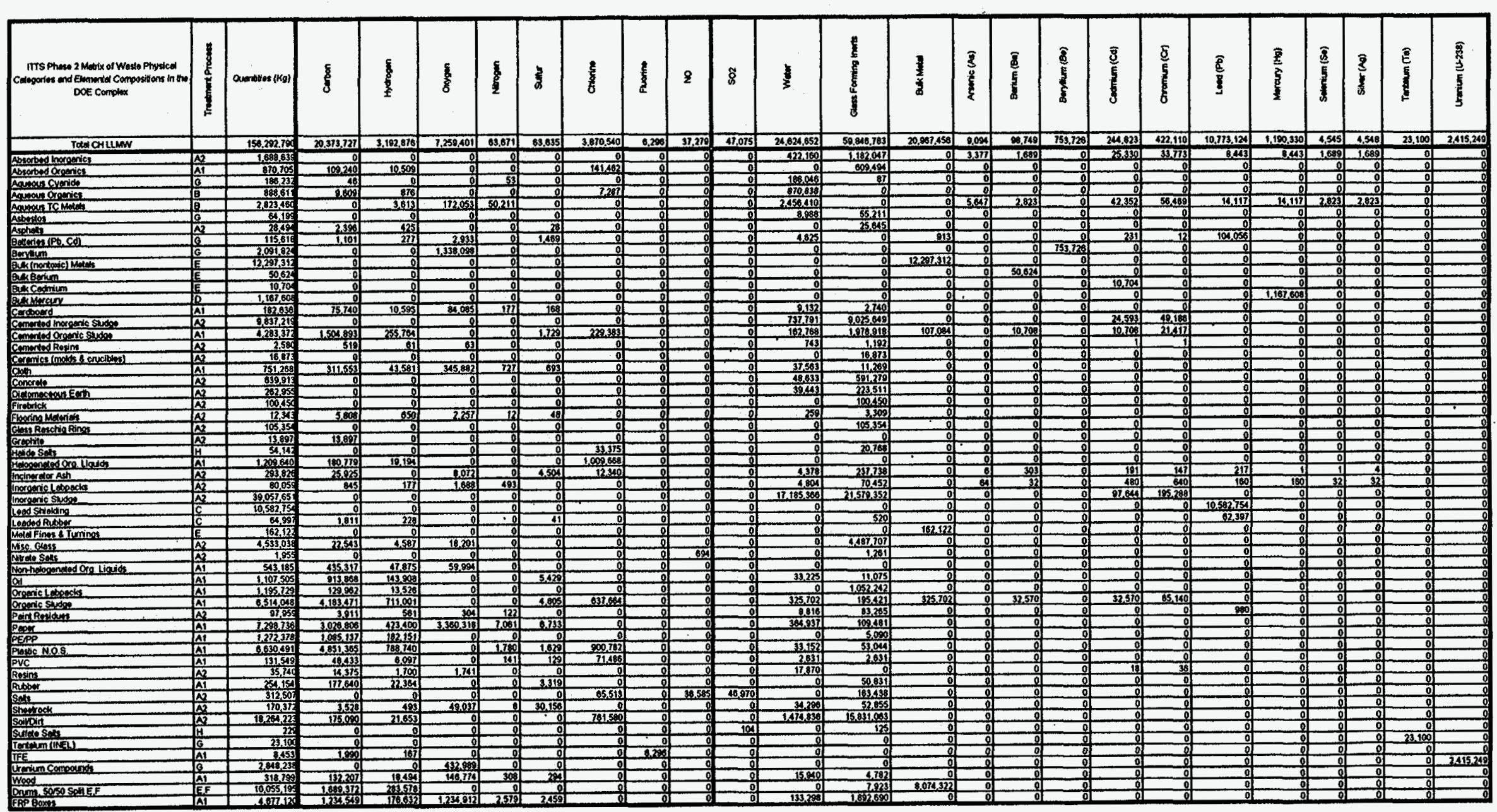




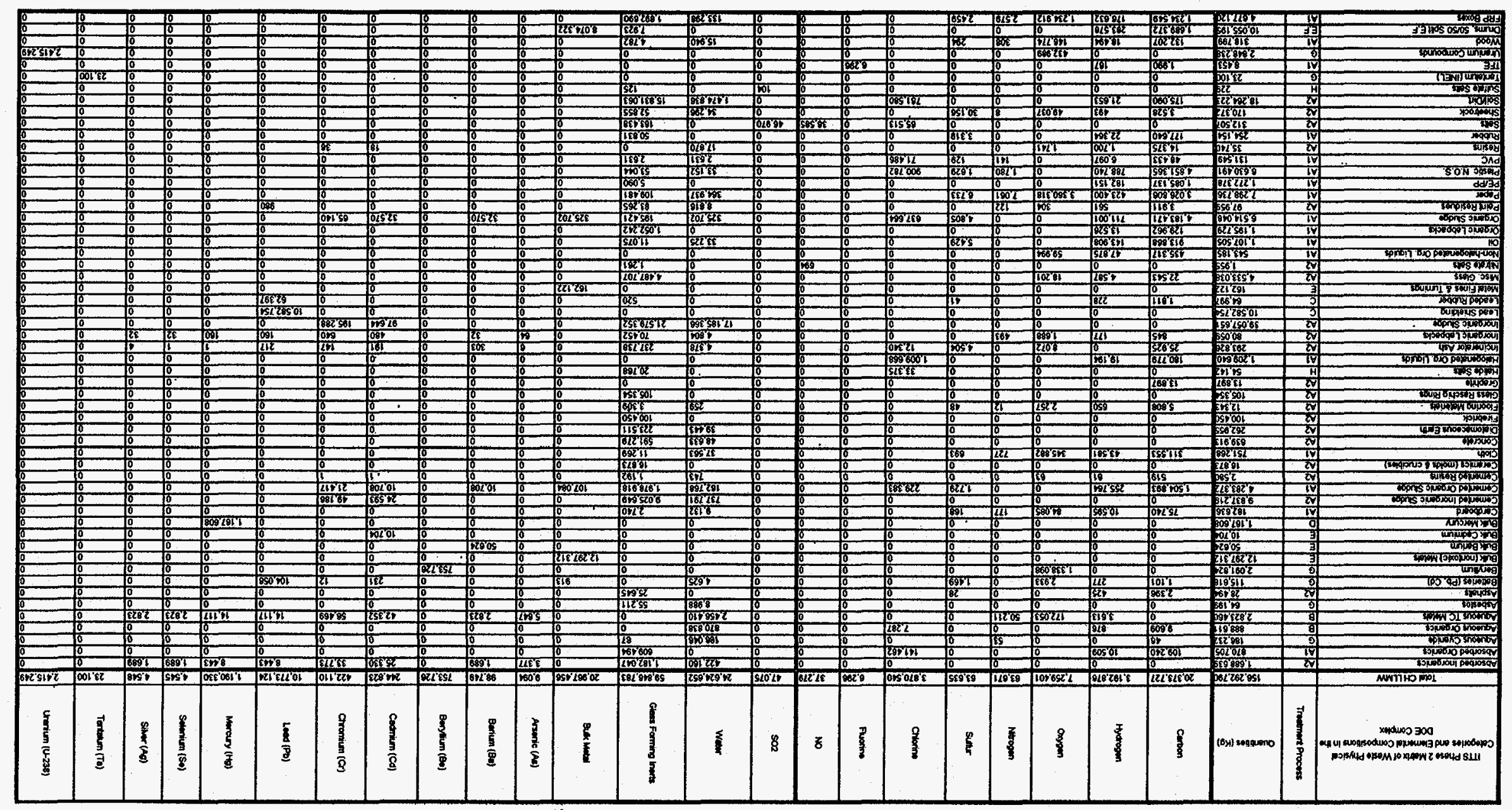

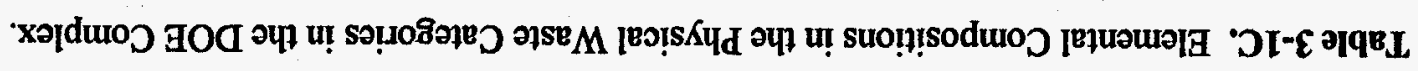


Table 3-2. Waste Treatment Processes, and Waste Quantities and ITTS Flow Rates of Each.

\begin{tabular}{|c|c|c|c|}
\hline Designator & Treatment Process/Waste Type & $\begin{array}{l}\text { Quantity } \\
\text { (kg) }\end{array}$ & $\begin{array}{c}\text { Feed Rate } \\
(\mathrm{lb} / \mathrm{hr})\end{array}$ \\
\hline Al & $\begin{array}{l}\text { Combustible Waste } \\
\text { Organic Liquids }=2,860,331 \mathrm{~kg}, 50.71 \mathrm{lb} / \mathrm{hr} \\
\text { Other }=34,389,440 \mathrm{~kg}, 609.71 \mathrm{lb} / \mathrm{hr}\end{array}$ & $37,249,771$ & 660.4 \\
\hline A2 & Noncombustible Waste & $75,556,048$ & 1339.6 \\
\hline B & $\begin{array}{l}\text { Aqueous Waste } \\
\text { (Systems K-1 and L-1) }\end{array}$ & $3,712,071$ & $80^{\dagger}$ \\
\hline $\mathrm{C}$ & Lead Recovery & $10,647,751$ & $26^{\dagger}$ \\
\hline $\mathrm{D}$ & Mercury Recovery & $1,167,608$ & $50^{\dagger}$ \\
\hline $\mathrm{E}$ & $\begin{array}{l}\text { Metal Melter } \\
\text { (Metal w/ Entrained Contamination and } 50 \% \text { of Metal } \\
\text { Drums) }\end{array}$ & $17,548,360$ & 149 \\
\hline $\mathbf{F}$ & $\begin{array}{l}\text { Metal Decontamination } \\
\text { (Metal Drums w/ Surface Contamination, } 50 \% \text { of Total) }\end{array}$ & $5,027,598$ & $468^{\dagger}$ \\
\hline G & Special Wastes & $5,329,211$ & $153^{\dagger}$ \\
\hline $\mathrm{H}$ & Polymer Stabilization(Halide and Sulfide Salts) & 54,372 & $1^{\dagger, t}$ \\
\hline TOTAL & & $156,292,790$ & 2927 \\
\hline Processes 1 & $\begin{array}{l}\text { resently modeled with process simulation code. } \\
\text { r polymer stabilization depends on system requirements. }\end{array}$ & & \\
\hline
\end{tabular}




\section{SYSTEM DESCRIPTIONS}

This section briefly describes the 19 proposed thermal treatment systems. Each system design consists of all structures, buildings, and equipment needed to accomplish the functional and operational requirements (F\&ORs), as fully described in References (1) and (2). Systems A-1 through A-7 are based on conventional rotary kiln technology. System B-1 uses an indirectly-heated, starved-air incinerator that operates in a pyrolysis mode. Systems C-1 through C-3 use a plasma-arc furnace. System D-1 is a fixedhearth, controlled-air incinerator distinguished by the addition of a $\mathrm{CO}_{2}$ retention system. System E-1 uses a rotary kiln for treatment of combustibles combined with an indirectly-heated rotary calciner used for the thermal desorption of debris. System F-1 uses molten salt oxidation (MSO). System G-1 involves molten metal waste destruction. System H-1 uses steam gasification. System J-1 uses Joule-heated vitrification. System K-1 involves thermal desorption and mediated electrochemical oxidation (MEO). System L-1 uses thermal desorption and supercritical water oxidation (SCWO).

System A-1 is the baseline system. For the sake of brevity, System A-1 is described more fully than the rest of the systems. The discussions of the other systems focus on their differences from the baseline system. Abbreviated descriptions of the treatment subsystems within each system are presented. The preconceptual design process flow diagrams (PFDs) for the main thermal treatment processes, Figures 4-1 through 4-19, are included for later comparison (Section 5) with the model process flow diagrams (MPFDs) created within ASPEN PLUS. (For convenience in reading the text, the figures for this section are located at the end of the section.)

\subsection{System A-1: Conventional Rotary Kiln, Air Combustion Gas, Dry-Wet APC (Baseline System)}

The majority of the technologies in this system use proven equipment that is commercially available. The system employs a rotary kiln incinerator using air as combustion gas. This is similar to treatment systems used by DOE at the TSCA incinerator at Oak Ridge National Laboratory (ORNL), Oak. Ridge, Tennessee. The air pollution control (APC) subsystem is based on dry filtration followed by wet offgas scrubbing. The Scientific Ecology Group (SEG) incineration facility at Oak Ridge uses this general approach in its APC. Solid residues from the system are stabilized by a combination of vitrification and polymer solidification. System A-1 has twelve subsystems, which are described below.

Receiving and preparation. This subsystem has cranes and forklift trucks to unload waste containers from incoming vehicles. The physical state of the waste in containers is identified by a realtime radiography (RTR) unit. The wastes are classified either as sorting required (SR) waste or sorting not required (SNR) waste. A passive/active neutron (PAN) assay unit determines the level of TRU contamination of the waste. A segmented gamma scanning (SGS) unit is used to assay beta and gamma radioactivity. A computer software and bar code scanning unit records and tracks the waste. Containers of SNR waste are moved directly to the thermal treatment subsystem. If the container of SR waste has restricted items, it is passed through a sorting train. The container is decapped by a saw mounted on a gantry robot. After decapping, the container is emptied on a sorting table equipped with master-slave and hydraulic manipulators used for removing restricted materials, such as large pieces of metal and lead, and mercury containers. The segregated waste is sent to the appropriate treatment subsystems. For purposes of this study, approximately $50 \%$ of the waste was assumed to require sorting.

Main thermal treatment This subsystem (incineration) receives combustible and noncombustible solids, sludges, and organic liquids. Some of the sludge from the aqueous waste treatment subsystem is also fed to the unit for drying and subsequent solidification with ash. Unless special precautions are taken, sludges have a tendency to form small surface-hardened balls during drying 
that resist complete destruction of organic material. This tendency can be countered by introducing the sludge into the rotary kiln as small particles ( $<1 / 4$ in. in diameter) or by introducing it with other wastes. The subsystem consists of a feed preparation shredder, a characterization unit, a natural gas and air-fired rotary kiln, a secondary combustion chamber (SCC), an air blower, and the associated combustion and feedstock preparation equipment. The size reduction unit shreds incoming waste in drums and boxes and feeds it to a series of transport bins and hoppers. Empty wooden boxes are also shredded, but empty metal containers are sent to the metal treatment subsystem. The contents of each hopper are sampled, characterized, and fed to the incinerator. Incineration takes place in a negative air pressure environment. The incinerator has a set of special graphite and steel seals designed to minimize air in-leakage. A metal housing around the incinerator unit provides secondary confinement.

Main thermal treatment APC. This subsystem has a dry gas filtration unit, a wet gas scrubbing unit, and a system for continuous emissions monitoring (CEM). In the dry gas filtration unit, the gas is partly quenched by water jets and filtered in either a baghouse or through high-temperature ceramic filters followed by high-efficiency particulate air (HEPA) filters. A charcoal or activated carbon filter is added in front of the HEPA filter to remove any trace quantities of mercury that might be present in the offgas. The wet offgas unit consists of a complete water quench, followed by hydrosonic and packed-bed scrubbers for removal of acid gas. A system to remove nitrogen oxides and dioxins is also included. The CEM unit monitors and records the quantities of $\mathrm{CO}, \mathrm{CO}_{2}, \mathrm{O}_{2}$, particulates, and other compounds discharged from the stack, to check compliance with air discharge permits. A continuous radiation sampling device is also included at the stack discharge.

Lead recovery. This subsystem has a decontamination train and an electrically-heated roasting oven. The decontamination train has mechanical devices, including saws, shears, and sanders, to cut the lead waste and to remove metal cladding from lead. Decontamination of lead takes place in scarfing and abrasive blasting booths. The oven is used to melt lead that cannot be decontaminated by mechanical means. The furnace APC subsystem has dry gas filtration similar to that of the APC subsystem provided for the rotary kiln.

Mercury amalgamation. This subsystem uses a retort followed by a condenser to reclaim mercury from contaminated solids. After mercury removal, the solids are sent to the primary stabilization subsystem. Offgas from the mercury condenser is treated in a secondary combustion chamber and a wetdry APC system similar to the incineration APC. Recovered elemental mercury is either recycled or amalgamated with zinc or copper in an amalgamation reactor.

Metal decontamination. This subsystem has a decontamination train. The decontamination train has size reduction tools (plasma torch, saw, and shear) and is provided with abrasive liquid blasting booths designed to remove entrained and surface contamination. It is assumed that dry ice blasting is used to reduce the quantity of liquid waste generated.

Metal melting. This subsystem includes a size reduction unit operation. Metals that cannot be decontaminated by mechanical means (surface blasting, grinding, etc.) are sent to this subsystem. In the induction melter, most radioactive material goes into the molten slag, which is cast in a container, cooled, and sent for inspection, assay, and shipment to storage or disposal facilities. Clean molten metal is poured into an ingot, cooled, and sent for recycling. The induction melter APC subsystem has a train for dry gas filtration similar to the APC subsystem provided for the thermal treatment unit.

Special waste treatment. It is assumed that there will be special wastes that require capabilities not included in the basic system. The treatment subsystem for special waste is located in a room equipped with a crane and all utilities needed for installing treatment systems for special wastes. 
Special treatment systems will be identified and provided on a case-by-case basis during facility operation. An equipment cost allowance of $\$ 3$ million is included in the cost estimates.

Aqueous waste treatment. This subsystem collects and treats input aqueous waste, which could include waste water having corrosive properties or contaminated with dissolved solids, suspended solids, organic compounds, or heavy metals. In addition, this subsystem treats the system's secondary aqueous wastes, such as the slurry from the APC subsystem scrubber blowdown, sludge from abrasive blasting in the metal decontamination subsystem, rinse water from container washdown, and water from equipment and floor drains.

The incoming aqueous waste is classified as having high levels of total organic carbon (TOC), high levels of total dissolved solids (TDS), or low levels of TDS, and then stored in the appropriate batch tank. The primary treatment train for high-TOC waste involves removing gross organics (using flotation thickeners or coalescers), filtering out suspended solids (using back flushable filters), removing dissolved organics (using carbon filtration or ozonators), and removing and polishing dissolved solids (using ion exchange). An alternative for treating high-TOC waste is to feed it to the thermal treatment subsystem. For liquids with high levels of TDS, the treatment train involves neutralization, filtering out suspended solids, and removing dissolved solids, probably by evaporation. For liquids with low levels of TDS, the primary treatment train consists of filtering out suspended solids, removing dissolved organics (using carbon filter or ozonators), and removing dissolved solids (by ion exchange).

Liquid wastes with mercury contamination are treated by precipitation and filtration using sulfurimpregnated carbon filters. Also, the ion exchange vessels will have mercury-selective resins to capture mercury.

In addition to the primary treatment trains, each waste type can be routed to or bypass a given treatment unit. The aqueous waste treatment subsystem concentrates all sludge waste produced by the various treatment trains and sends it to the stabilization subsystem. Spent ion exchange resin from treatment processes is dewatered and sent to the thermal treatment subsystem or to the stabilization subsystem.

Primary stabilization. This subsystem uses vitrification to convert the incinerator ash to a waste form suitable for disposal. In vitrification, soil (including contaminated soil from a DOE installation) or chemical additives ( $\mathrm{SiO}_{2}, \mathrm{Al}_{2} \mathrm{O}_{3}, \mathrm{Fe}_{2} \mathrm{O}_{3}$, etc.) are metered into the rotary kiln to act as glass formers. The additives are added in quantities proportional to the weight of expected bottom ash. In order to add the proper quantity, the input waste must be characterized well enough to predict the quantity of ash. The kiln mixes the soil with the ash and discharges the mixture into a storage hopper. The mixture is metered from the hopper into a melter furnace. The melter furnace vitrifies the ash and soil mixture and discharges the molten mixture into a container. The container is cooled, capped, and sent to a swiping and decontamination station. If surface contamination is found, the container is washed by high-pressure water jets. The inspected container is sent for assay, certification, and shipping to storage or disposal facilities. The melter furnace APC unit has a dry gas filtration train similar to the dry APC subsystem provided for the rotary kiln.

Research conducted at Idaho National Engineering Laboratory (INEL) has shown that the residue after combustion, when combined with about 40 to $50 \%$ soil, will form a good glass or ceramic waste form. Testing on bench-scale melters at INEL has shown that drying the soil before placing it in a melter results in less violent melter startup. Thus, the soil is introduced into the incinerator and dried. As an additional benefit, any organic carbon will be eliminated and carbonates will be disassociated. 
Secondary stabilization. This subsystem receives treated residues that are not suitable for processing by the primary stabilization subsystem, such as salts having a low melting point or fly ash with a salt concentration exceeding the limits specified for vitrification when it is the primary stabilization method. Stabilization of salts involves polymer encapsulation using sulfur cement, polyethylene, or polymerizing agents produced by Dow Chemical Company. The subsystem has a dryer that removes water from the incoming waste. The dried powder and polymer are metered into an extruder that heats and mixes the polymer with powdered waste. The extruder feeds the mixture into a drum. When filling is complete, the drum is capped and sent to a swiping and decontamination station. If surface contamination is detected, the container is washed by high-pressure water jets or blasts of dry ice. The inspected container is sent for assay, certification, and shipping to storage or disposal facilities. Bulk secondary waste, such as spent filters, is compacted and macro-encapsulated by grouting techniques.

Certify and ship. This subsystem characterizes the physical and radiological properties of the packaged waste to allow certification in accordance with transportation, storage, and disposal requirements. The containers of packaged waste for shipment are weighed. An RTR unit examines the container to ensure that the matrix is homogeneous and has no free water. If TRU or alpha contaminated waste is processed, the TRU concentration is measured by a PAN assay unit. An SGS unit is used to assay the beta and gamma radioactivity. After inspection, the waste is either sent to a temporary storage area or loaded onto a truck for offsite or onsite shipment.

\subsection{System A-2: Conventional Rotary Kiln, Oxygen Combustion Gas}

System A-2 is the same as System A-1 except that the incinerator is equipped to use commercially pure oxygen as the combustion gas. The APC subsystem has a smaller capacity, since oxygen combustion creates lower volumes and velocities of incineration offgas than does air combustion.

\subsection{System A-3: Conventional Rotary Kiln, Wet Air Pollution Control Subsystem}

System A-3 is the same as System A-1 except that the APC subsystem uses all wet filtration and cleaning techniques. The dry gas filtration unit (baghouse) is eliminated.

\subsection{System A-4: Conventional Rotary Kiln, $\mathrm{CO}_{2}$ Retention}

System A-4 is basically the same as System A-1 except that the incinerator uses oxygen as the combustion gas, and a different APC configuration is used. This APC subsystem treats offgas by the conventional dry filtration means and then removes carbon dioxide by absorption into lime in a fluidized bed. The water formed in the incinerator is condensed and sent to the aqueous waste treatment subsystem. The remaining gas is enriched with oxygen and rerouted to the incinerator for additional treatment of toxic materials. The $\mathrm{CO}_{2}$ retention concept is being developed at the Argonne National Laboratory. The lime (or dolomite) is recycled as many as ten times using a lime recovery system whose main component is a calciner. During recovery the calcium carbonate is reheated to release the $\mathrm{CO}_{2}$. The $\mathrm{CO}_{2}$ is then monitored and discharged to the atmosphere. Alternatively, the calcium carbonate can be calcined at a remote place, in which case the only offgas released to the atmosphere on a continuous basis close to the thermal treatment equipment is the inert gas that enters with the oxygen or leaks into the system. The $\mathrm{CaCO}_{3}$ could also be disposed as a solid, which would incur additional disposal costs. Compressed gas storage tanks are included for these gases.

System A-4's receiving and preparation subsystem is different from System A-1's in that bulk metals, slag, and tar formers need to be removed as part of that step. The capacity of System A-4's aqueous waste treatment subsystem is slightly larger than that of the baseline system in order to handle the water condensed from the offgas. 


\subsection{System A-5: Conventional Rotary Kiln, Polymer Stabilization}

System A-5 is the same as System A-1 except that the primary stabilization method uses polymers in place of vitrification. In this case, all solid residues are stabilized by polymers as described in Section 4.1, secondary stabilization. However, bottom ash and fly ash stabilized residues are kept separate for purposes of tracking transuranic activity.

\subsection{System A-6: Conventional Rotary Kiln, Maximum Recycling}

System A-6 is designed to minimize the volume of waste requiring disposal. The standard rotary kiln, which uses air as the combustion gas, is preceded by a feedstock preparation subsystem that maximizes decontamination to permit recycling of waste materials, containers, bulk metals, and process chemicals. Containers and some bulk metals are recovered, decontaminated, and recycled within the facility to the extent possible. Aqueous secondary waste streams, except for acid gas scrubber blowdown, are treated in an aqueous waste treatment subsystem. The blowdown from the acid gas scrubber is processed through a salt splitting system to produce a caustic and hydrochloric acid. The caustic can be recycled to the beginning of the wet section of the APC subsystem. Salt splitting is a specially-designed electrodialysis process that results in caustic being regenerated for reuse within the system. Activated carbon filters in the offgas line are recycled using a retorting process, which removes the mercury. HEPA filters in the offgas line use stainless steel cloth that is reusable after cleaning.

\subsection{System A-7: Slagging Rotary Kiln}

The slagging rotary kiln system accomplishes both incineration and vitrification in a single step. Combustible and noncombustible solids along with glass-forming material or contaminated soil are added to the kiln inlet. The kiln output is a vitrified slag that requires no further stabilization.

System A-7 does not have a metal melting subsystem, because metal with entrained contamination is fed to the slagging kiln and is embedded in the discharged slag. Also, since the kiln is a one-step oxidation-stabilization unit, a separate vitrification subsystem similar to the baseline system is not needed.

Main thermal treatment. The.main thermal treatment subsystem consists of a storage and characterization area, a rotary kiln fired by natural gas and air, an SCC, an air blower, and the associated combustion and feedstock transfer equipment. The thermal treatment subsystem receives combustible and noncombustible solids, metal with entrained contamination, sludge, and organic liquid. The slagging kiln performs the organic destruction and stabilization functions in one step. For more uniform and pufffree combustion, the slagging kiln selected for this study is designed to treat shredded waste.

The slagging rotary kiln is a commercial technology currently employed for hazardous waste treatment in Europe and in the United States. Some of the sludge from the aqueous waste treatment subsystem is also fed to the unit for drying and subsequent solidification with the slag. As the waste is fed into the kiln, necessary burner adjustments are made to maintain design levels of combustion and waste slagging. The kiln is equipped with graphite seals designed to minimize air leakage into or out of the kiln.

The typical temperature range for the slagging kiln is 1,500 to $2,500^{\circ} \mathrm{F}$, but can also be operated at lower temperatures in the ashing mode. The residence time of solids in the kiln is typically one hour. The destruction of solids in the slagging mode is expected to be enhanced relative to the ashing mode because of the increased heat transfer rate. The kiln has a seal at the waste inlet, the slag outlet, and the 
entrance to the attached SCC unit. A typical design includes a combination slag pot and SCC, which is attached to the kiln. The SCC is a refractory-lined vertical cylinder, operated at a combustion temperature of $2,500^{\circ} \mathrm{F}$. At the bottom of the SCC, a sloped opening provides an exit for the liquid slag (or ash, if operated in the ashing mode).

\subsection{System B-1: Pyrolysis}

System B-1 combines an indirect-fired, electrically-heated, rotary kiln pyrolyzer and a SCC using oxygen combustion gas with the vitrification unit. Electrical heating and burning in oxygen starvedpyrolysis minimizes production of offgas. The pyrolysis gas is oxidized with pure oxygen in the SCC, which is followed by a standard wet-dry APC subsystem. System B-1 uses a standard feedstock preparation subsystem. Solid residues from the pyrolyzer are treated by vitrification. An oxygen lance would be used in the vitrifier to assure burnout of carbon from the pyrolyzer. The offgas from the vitrification unit is fed to the pyrolyzer SCC inlet, thus eliminating a separate APC subsystem for the primary stabilization subsystem. Aqueous secondary waste streams are treated in an aqueous waste treatment subsystem. Organics recovered from aqueous treatment are recycled to the thermal treatment unit. Sludges resulting from precipitation and filtration are transferred to the primary stabilization subsystem or, if necessary, to the secondary stabilization subsystem. The treated water is recycled in the system, as required, for process use. Differences between this system and the baseline system are outlined below.

Receiving and preparation. The receiving and preparation subsystem is the same as that of the baseline system except that sorting requirements are substantially greater. This is due to limits on the noncombustible material that can be present in the pyrolyzer feed material. Most of the noncombustible bulk material needs to be separated from the waste before it can be fed into the pyrolyzer.

Main thermal treatment. This subsystem differs from that of the baseline system by integrating a pyrolyzer unit and SCC with the vitrification unit. Noncombustible waste is fed to a dryer along with soil or other additives, and then fed to the melter for vitrification. Combustible waste undergoes partial combustion by being heated in an oxygen-starved chamber. The resulting gases are burned in an SCC to which oxygen is added in stoichiometric proportions. The pyrolyzer operates at a temperature of $1,200^{\circ} \mathrm{F}$, and the SCC at a temperature of $2,200^{\circ} \mathrm{F}$. The ash from the pyrolyzer is fed to the vitrification unit. This ash is typically a char with a high carbon content, which could pose a problem for the melter. Oxygen must be supplied to the melter for combustion of the carbon. Burning carbon in the vitrifier will create gas pockets in the slag, which makes the waste form less dense. The melter offgas also goes to the SCC.

Main thermal treatment APC. As with System A-1, the APC subsystem is based on dry filtration followed by wet offgas scrubbing. However, this APC subsystem has a smaller capacity, since indirectly heated oxygen combustion creates lower volumes and velocities of offgas than does air combustion. The smaller quantity of offgas and the lower temperature created by pyrolysis also result in a smaller quantity of fly ash.

Primary stabilization. As indicated before, the vitrifier is part of the incineration subsystem. The primary stabilization subsystem performs only cooling and packaging of slag. 


\subsection{System C-1: Plasma Furnace}

System C-1 combines a plasma furnace with an SCC, both of which use air as the combustion gas. The plasma furnace performs three functions simultaneously: thermal treatment, vitrification, and metal melting. The SCC is followed by a standard dry/wet APC subsystem. The plasma furnace requires only that bulk lead and mercury be removed (for separate treatment) and that boxes, large metals, and debris be reduced in size to fit into the feed handling system and the plasma chamber; thus, feedstock preparation is minimal. The plasma furnace can accept bulk feed, including drums. This mode of operation is used only if the waste is adequately characterized to meet the RCRA permit restrictions. Contaminated soil or other glass or ceramic-forming additives are added to the furnace to produce a highly leach-resistant vitrified waste form. Solid residues from the plasma furnace, including radionuclides, can be drawn off in two streams: a molten glass stream containing the vitrified ash components, and a molten metal stream. Aqueous secondary waste streams are treated in an aqueous waste treatment subsystem. Organics recovered from aqueous waste treatment are recycled to the plasma furnace. Sludges resulting from precipitation and filtration are transferred to either the plasma furnace or, if necessary, to the secondary stabilization subsystem. Differences between this system and the baseline system are outlined below.

Receiving and preparation. System C-1 requires minimal processing during receiving and feedstock preparation. Bulk lead and mercury need to be removed and treated separately. Large pieces of bulk metal (steel, etc.) that require melting and other debris need only to be reduced in size sufficiently to fit the feed handling system and the plasma chamber.

Main thermal treatment. The main component of the incineration subsystem is a plasma furnace, which uses an electric arc. The arc produces a highly energized plasma that breaks the chemical bonds of waste materials. Plasma systems usually operate in a pyrolytic or starved-air mode in an attempt to minimize high-temperature formation of undesirable oxides of nitrogen. The offgases are burned in an SCC using air as the combustion gas.

Main thermal treatment APC. The APC subsystem has a smaller capacity than that of the baseline system, since the volume of offgas generated per unit mass of waste is smaller. (Even lower quantities of combustion gas could be obtained if oxygen were used instead of air.) It is likely that the capability to reduce levels of oxides of nitrogen in the offgas will be required.

Primary stabilization. An acceptable waste form is produced during incineration. The waste is slowly cooled in a slag chamber operating at an elevated temperature. After cooling, the waste form is moved to storage.

\subsection{System C-2: Plasma Furnace, $\mathrm{CO}_{2}$ Retention}

The plasma furnace with oxygen combustion and $\mathrm{CO}_{2}$ retention is an alternative to the conventional plasma furnace system (C-1). This system has been developed to study the effect of using oxygen for combustion and removing $\mathrm{CO}_{2}$ from the offgas resulting in discharge of a minimum amount of offgas to the environment.

The SCC is followed by a dry APC subsystem, modified to include carbon dioxide absorption of the offgas into lime, similar to System A-4. This system includes removal of chlorides, $\mathrm{CO}_{2}$, and water in the offgas in a fluidized bed. The remaining offgas, primarily oxygen, is recycled to the furnace. The $\mathrm{CO}_{2}$ is absorbed in lime to form calcium carbonate. A small bleed-off stream from the offgas recycled to the furnace is also discharged to the atmosphere. After a given retention period, the carbonate is calcined 
to release the $\mathrm{CO}_{2}$, allowing the recycle of the lime. The $\mathrm{CO}_{2}$ released from the calcining operation is monitored and discharged to the atmosphere.

\subsection{System C-3: Plasma Gasification}

This system is based on a conventional plasma furnace that operates in an oxygen-starved environment. It is designed to study the effect of operating a plasma furnace in a reducing mode and producing a synthesis gas that not only reduces the volume of offgas discharged to the environment, but produces an offgas that can be used for energy recovery. In this system, air is used as the torch gas, with steam added to the furnace to provide the oxidant.

The plasma furnace using steam performs two functions simultaneously: thermal treatment and vitrification. The plasma furnace requires that bulk lead and mercury be removed and treated separately and that bulk metal and large debris be reduced in size to fit into the feed handling system and the plasma chamber. The plasma furnace can accept shredded feed. Contaminated soil or other glass- or ceramicforming additives are added to the furnace to produce a leach-resistant vitrified waste form. Molten solid residues from the plasma furnace, including radionuclides, are drawn off as a vitrified material.

Since the thermal treatment process is a reduction reaction (i.e., oxygen starved environment), the plasma furnace exhaust contains synthesis gas (referred to as syngas) which is primarily $\mathrm{H}_{2}, \mathrm{CO}$, and $\mathrm{CO}_{2}$. The syngas contains impurities, such acidic gases and particulates, which must be removed. The syngas cleaning function is accomplished in an APC subsystem similar to the baseline system consisting of quenching, dry filtration, and wet scrubbing steps. The cleaned gas is either recovered by burning in a steam boiler or sent to a catalytic oxidation unit for conversion of $\mathrm{H}_{2}$ and $\mathrm{CO}$ to $\mathrm{H}_{2} \mathrm{O}$ and $\mathrm{CO}_{2}$ and subsequent release to the atmosphere. Fly ash recovered from the APC dry filtration step is recycled to the plasma furnace. Sludges containing salts resulting from the APC wet scrubbing step are transferred to the secondary stabilization subsystem. The treated water is recycled into the system, as required, for process use.

System C-3 does not have a metal melting subsystem because metals with entrained . contamination are melted in the plasma furnace and recycled when possible. Also, since the plasma furnace is a one-step oxidation-stabilization unit, a separate vitrification subsystem similar to the baseline system is not needed.

Main thermal treatment. The main component of the thermal treatment subsystem is a shredder and a plasma furnace, which uses a transfer electric arc contacting the slag layer as the anode. The furnace typically operates with a $3,000^{\circ} \mathrm{F}$ wall temperature and $1,800^{\circ} \mathrm{F}$ gas temperature. Heat is produced in the reactor chamber by the plasma torch. Steam is added to the chamber to provide an oxidant and to encourage the formation of carbon monoxide. The furnace operates in a reducing mode. The organics react with superheated steam, forming $\mathrm{CO}, \mathrm{CO}_{2}$, and $\mathrm{H}_{2}$. The offgas consists primarily of $\mathrm{H}_{2}, \mathrm{CO}$, and $\mathrm{CO}_{2}$. Metal will melt and sink to the bottom of the melt. Inorganic material forms vitrified slag, which is cooled into a stable waste form. The primary stabilization subsystem consists of collecting and cooling this metal-slag mixture.

\subsection{System D-1: $\mathrm{CO}_{2}$ Retention}

System D-1 combines a sub-stoichiometric, fixed-hearth primary combustor (frequently referred to as a controlled-air incinerator) with an SCC, both of which use oxygen as the combustion gas. This system uses a fluidized bed absorber to absorb the carbon dioxide in the lime after treatment in a conventional APC, similar to Systems A-4 and C-2. The incineration subsystem is preceded by a standard feedstock preparation subsystem in which special precautions are taken to remove large pieces of 
metal and other noncombustibles. Solid residues from the incineration subsystem are treated by vitrification or polymer solidification. Aqueous secondary waste streams are treated in an aqueous treatment subsystem. Organics recovered from aqueous waste treatment are recycled to the primary incinerator. Sludges resulting from precipitation and filtration are transferred to either the primary stabilization subsystem or, if necessary, to the secondary stabilization subsystem. Differences between this system and the previously discussed systems are outlined below.

Receiving and preparation. Receiving and preparation includes procedures to remove bulk metals and slag and tar formers.

Main thermal treatment. System D-1 employs a fixed-hearth incinerator in which waste is transported over a hearth by a ram feeder or other conventional type of feeder. A screw conveyor stirs the ash pile and eventually moves it to one or more ash ports. Combustible waste is heated in an oxygenstarved atmosphere, where it is volatilized and undergoes partial combustion. The resulting energybearing gases are burned in an SCC that runs on excess oxygen.

Aqueous waste treatment This subsystem handles the water condensed from the offgas. This subsystem is smaller than that of the baseline because liquid waste from the scrubber has been eliminated.

Primary stabilization. This subsystem is the same as in the baseline system except that noncombustible materials in the feed must be dried. Soil is mixed with ash coming from the incinerator. Dryers are needed to remove water from the soil, sludge, and other materials before vitrification.

\subsection{System E-1: Thermal Desorption}

System E-1 takes advantage of RCRA land disposal regulations that allow treatment of waste classified as debris by grouting only. Waste classified as process residues requires incineration. System E-1 is based on a standard rotary kiln incinerator that uses air as the combustion gas, and is smaller than the baseline unit. The kiln is preceded by a standard feedstock preparation subsystem and followed by a standard dry/wet APC subsystem. Ash from this system goes to a vitrification unit or, if small, to grouting. The incineration subsystem is standard except for a thermal desorption step parallel to the thermal treatment unit. The desorber separates volatile organic compounds (VOCs) from debris as defined by RCRA. Soil can also be treated by the parallel thermal desorber. Waste components vaporized in the thermal desorber are condensed to a liquid and sent to the rotary kiln. Solid residues from this system are stabilized by grouting. Solids from the desorber are shredded and microencapsulated by grouting. Aqueous secondary waste streams are treated in an aqueous waste treatment subsystem. Organics recovered from aqueous waste treatment are recycled to the rotary kiln. Sludges resulting from precipitation and filtration are transferred to either the primary stabilization subsystem or, if necessary, to the secondary stabilization subsystem. Differences from the baseline system are outlined below.

Receiving and preparation. The receiving and preparation subsystem is similar to that of the baseline system but more waste characterization and segregation are required.

Thermal desorption. This subsystem, which uses an indirectly-heated calciner for thermal desorption, separates VOCs from the feedstock before waste is fed to the stabilization subsystem. Waste components vaporized in the thermal desorber are treated in an APC consisting of a stripper and condensers. Captured organic liquids are sent to the incineration subsystem. Solid residues from the desorber are sent to a debris grouting subsystem. 
Debris grouting. The debris grouting subsystem stabilizes debris by mixing the shredded waste with grout consisting of cement, water, and sand. The mixture is poured into drums and allowed to cure. Once the grout has solidified, the drums are capped, washed, and moved to the certify and ship subsystem.

\subsection{System F-1: Molten Salt Oxidation}

The molten salt oxidation (MSO) subsystem is comprised of a thermal treatment subsystem, an APC, a salt recycle subsystem, and a primary stabilization subsystem with independent APC. Combustible waste is oxidized in a molten salt bed. The molten sodium carbonate acts as a catalyst for the oxidation of combustible waste. The bed also neutralizes halogenated acids and forms halogenated salts. Noncombustibles, such as metal and salt, collect in the bed and are removed by continuously transferring some of the salt bed into a collection vessel. The melt overflow from the thermal treatment subsystem is transferred to a salt recycling subsystem, where the ash is filtered out and sent to a primary stabilization subsystem and sodium carbonate salt is recovered and recycled back to the thermal treatment subsystem. An APC subsystem, which is based on dry filtration followed by wet scrubbing, is provided for treatment of the gas fumes from the MSO vessel. Fly ash from the APC subsystem is sent to a secondary stabilization subsystem where it is solidified with polymer. The fly ash consists mostly of salts. Since MSO can accept only combustible waste, all of the input noncombustible waste is sent directly to the primary stabilization subsystem. In the primary stabilization subsystem, noncombustible solids and ash are vitrified and packaged for certification and shipping.

The MSO system has fourteen major subsystems, including a salt recycle subsystem. Most of the subsystems are the same as the baseline system (A-1). Subsystems that are different from the baseline system are described below. Since the MSO thermal treatment unit processes only combustible waste, the sorting operations in the receiving and preparation subsystem are more extensive and, hence, the subsystem is larger than that included in the baseline system.

Main thermal treatment. The waste is size reduced and separated into combustible and noncombustible categories. The combustibles are processed in the molten salt oxidation subsystem, and the noncombustibles are processed in the primary stabilization subsystem. The combustible waste is size reduced to $1 / 8$ of an inch or smaller to transport through the feed system and to assure total combustion in the bath. The combustible waste and air/oxygen are mixed in a molten sodium carbonate $\left(\mathrm{Na}_{2} \mathrm{CO}_{3}\right)$ bed in an alumina-lined reactor. The molten bed is operated at temperatures of about 1,400 to $1,800{ }^{\circ} \mathrm{F}$ to maintain the melt viscosity. The moderate operating temperature limits the formation of nitrous oxide (NO). The offgas, principally $\mathrm{CO}_{2}$ and $\mathrm{H}_{2} \mathrm{O}$, is sent to the APC subsystem. Some of the melt is continuously removed to prevent the buildup of ash and other inerts in the reactor. Salt bath viscosity control requires that the ash fraction be kept below $20 \%$ by weight. The salt must remain fluid to facilitate transfer of the melt overflow and enhance oxidation of the organic waste. The salt overflow is sent to the salt recycling subsystem.

Main thermal treatment APC. The APC subsystem has a dry gas filtration unit, wet gas scrubbing unit, and a system for CEM. In the dry gas filtration unit, the gas is partially quenched by water jets and filtered through a baghouse to remove salt carryover. Special features have been included to clean salt cakes accumulated in the reactor exhaust pipes. A centrifugal wet scrubber collector is provided downstream of the baghouse to remove any salt fumes that might have escaped the baghouse. The scrubber is followed by a reheater and HEPA filters. The salt from the baghouse is sent to the secondary stabilization subsystem.

Salt recycling. In the salt recycling subsystem, the ash and salt mixture is cooled, crushed, and dissolved in a water tank. The mixture is then filtered, separating the ash from the dissolved salt. The ash 
slurry is dried and sent to the primary stabilization subsystem. The salt solution is sent to an evaporative crystallizer. The sodium carbonate will drop out of the solution in the form of crystals, because sodium carbonate has low solubility in water at room temperature. The salt crystal solution is routed to a centrifuge where the sodium carbonate salts are separated from the solution, later dried, and recycled back to the main thermal treatment subsystem. The supernate from the solution, which contains sodium chloride, is recycled to the dissolver tank or sent to the aqueous waste treatment subsystem for further treatment. The evaporated water collected from drying the ash sludge, sodium carbonate concentrate, and the evaporative crystallizer is condensed and sent to aqueous waste treatment or recycled to the dissolver tank.

Primary stabilization. The primary stabilization subsystem receives ash from the salt recycling subsystem and noncombustible waste feed from the receiving and preparation subsystem. The noncombustible waste feed is size reduced using a coarse shredder unit, which shreds waste and feeds it to a series of transport bins. A combined dryer and blender is added to reduce moisture in the waste feed entering the vitrifier. The vitrifier mixes soil with the ash, melts the mixture, and discharges a molten slag product into a waste container. The container of slag is cooled, capped, and sent to a

decontamination station. The offgas from the vitrifier is sent to the primary stabilization APC. which has dry/wet gas filtration units and a system for CEM. In the dry gas filtration unit, the gas is partially quenched by water jets and filtered through a baghouse followed by HEPA filters. The fly ash is sent back to the vitrifier. A charcoal or activated carbon filter is added in front of the HEPA filter to remove any trace quantities of mercury that might be present in the offgas. A wet scrubber removes acids and salts. Waste liquor is routed to aqueous waste treatment.

\subsection{System G-1: Molten Metal Waste Destruction}

The main thermal treatment unit in system G-1 is based on a molten metal waste destruction process developed by Molten Metal Technology (MMT) of Waltham, Massachusetts. The process employs a chemical reactor vessel containing a molten metal bath that thermally destroys incoming feed materials and converts them to their elemental forms. Solids and gaseous additives and catalysts (e.g., oxygen, slag formers, fluxing agents, lime, and carbon) are injected into the molten bath in order to separate radionuclides or other contaminants from the recoverable material in the feedstock. The recoverable material is sent for recycle and reuse.

According to the manufacturer, a key feature of the molten metal waste destruction process is the ability to accomplish, in a single train, three major steps: organic destruction, residue stabilization, and conversion of the recoverable material for either reuse at the plant or recycling at other facilities. While destroying the incoming waste, three phases form in the molten bath reactor: molten metal, vitreous slag, and syngas. Although for hazardous waste all three phases are claimed to be recycled, in the ITTS study only the syngas and molten metal are assumed to be recyclable. The slag is a radioactive waste which requires disposal as LLW. An APC subsystem purifies the syngas by a combination of dry quenching, dry particulate removal and wet acid gas scrubbing steps. The purified syngas is used for generating plant steam. Alternatively, syngas can be oxidized in a thermal oxidizer and discharged directly to the atmosphere. Molten metal is removed from the reactor, cast, and sent offsite for use in fabrication of waste containers and other devices that could be employed during waste management operations in the DOE complex. Molten slag containing radionuclides is removed from the reactor, poured into waste containers, and sent for disposal.

System G-1 has neither the metal melting nor the metal decontamination subsystems that are part of the baseline system. Feed normally processed by these subsystems is processed by the molten metal reactor and recycled. The sort unit operation in the receiving and preparation subsystem is smaller than in the baseline system. This is due to the ability of the process to accept a wide variety of the hazardous and 
toxic material in the feedstock. Also, since the molten metal reactor is a one-step reduction and stabilization unit, a separate vitrification subsystem similar to that of the baseline system is not needed.

Main thermal treatment The main component of the thermal treatment subsystem is a sealed molten metal bath reactor that operates at temperatures near $3,300^{\circ} \mathrm{F}$ in a reducing (oxygen starved) environment. The reactor is a pressurized induction-heated melter vessel with an enlarged head space designed for control and removal of the material from gaseous or molten phases. The bulk solids discharged from the shredder outlet are collected in air-tight transportable bins and set aside in the feed preparation area. On demand, a given waste bin is lifted and placed on top of a feed mechanism mounted above the reactor.

Experiments by MMT indicate that from the partitioning stand point, most of the radionuclides found in DOE MLLW may be categorized into four general groups: (1) uranium and transuranium, (2) technetium, (3) cesium and strontium, and (4) cobalt, nickel, and other isotopes with an atomic weight near iron. MMT tests, using hafnium as a surrogate element, predict that the elements in the first group, uranium and transuranium, will most likely oxidize and go into the slag phase. This means that metal contaminated with these elements can be cleaned with a high decontamination factor rendering the metal suitable for recycling within the nuclear industry.

The subsystem has a bulk material mixing station provided for preparing a homogenous mixture of waste and solid additives when such a mixture is needed. Gaseous additives and liquid wastes are fed to the reactor via tuyeres (pipes with spare nozzles) located in the bottom of the reactor.

The metal felt normally operates in a reducing atmosphere, with sub-stoichiometric addition of oxygen for syngas production. The syngas on top of the molten bath is generally composed of a combination of carbon monoxide $(\mathrm{CO})$, hydrogen $\left(\mathrm{H}_{2}\right)$, steam $\left(\mathrm{H}_{2} \mathrm{O}\right)$, and impurities such as acidic gases and particulates. Nitrogen gas is injected into the reactor to maintain the required pressure and maintain an inert environment in the head space, and to sweep the gas out of the reactor head space. The reactor exhaust is sent to the APC for treatment before reuse.

High-temperature instrumentation and computer models are needed to either measure or predict parameters needed to control the molten bath chemistry and metallurgy. Studies of reactor geometry to optimize input feed, reaction turbulence, refractory life, product discharge, and maintenance are also underway.

Main thermal treatment APC. The function of the APC is to remove impurities to a level that syngas can be safely burned. The APC subsystem accomplishes this function by a combination of dry quenching, dry particulate removal, and wet gas scrubbing steps. The dry quenching step consists of passing the syngas exhaust from the reactor through a fluidized bed cooling unit. The fluidized bed unit cools the syngas and drops the temperature from $3,300^{\circ} \mathrm{F}$ to approximately $400-300^{\circ} \mathrm{F}$. Silica sand or ceramic balls cooled by water-cooled coils are used as the fluidized medium. The sand in the fluidized bed cooler also acts as a cold trap filter and captures most of the volatilized metal escaping the reactor. Any waste residue from the fluidized bed is sent to the molten reactor for processing. Cooled syngas is processed through a cyclone separator, baghouse and HEPA filters to remove the solid particulates and fugitive bed media. A charcoal or activated carbon filter is added in front of the HEPA filter to remove any trace quantities of volatile organic compounds (VOCs) and mercury that might be present in the gas.

Particulate free syngas is then sent to the wet gas acid removal train. This train consists of a wet scrubber that is designed to remove dissolved acid precursors present in the syngas. An alkali scrubber is also available as backup to provide additional acid gas neutralization if necessary. Scrubber sludge is sent to the secondary stabilization subsystem where it is dried to powder and stabilized with polymer. 
The clean hydrogen and carbon monoxide rich syngas is sent to a steam boiler for energy recovery or burned in a catalytic oxidizer. The catalytic oxidation unit operates at $1500^{\circ} \mathrm{F}$, where carbon monoxide and hydrogen gas is burned and any trace VOCs are destroyed.

\subsection{System H-1: Steam Gasification.}

The steam gasification system is designed to accommodate conventional organic waste gasification technologies that convert the organic feedstock into ash and synthesis gas (or syngas). This concept not only minimizes the amount of gas discharged to the environment, but produces a gaseous product that can be used for energy recovery.

The main process line consists of a thermal treatment subsystem based on steam gasification, an APC subsystem, and a syngas oxidation subsystem. The main thermal treatment subsystem processes sorted combustible waste and superheated steam in an indirectly heated reactor. The heat in the reactor breaks down the organic compounds into their elemental forms. Steam reacts with the decomposed elements to form syngas and ash. An APC subsystem purifies the syngas by a combination of wet quenching, dry particulate removal, and wet acid gas scrubbing steps. The purified syngas is used for generating plant steam. Alternatively, syngas can be oxidized in a catalytic oxidizer and discharged directly to the atmosphere.

The thermal treatment unit has a low tolerance for accepting noncombustibles in the feed. Therefore, noncombustible waste, ash from the main thermal treatment subsystem, and fly ash from the APC subsystem are routed to the primary stabilization subsystem, which uses a vitrification furnace for waste stabilization. Contaminated soil or other glass- or ceramic-forming additives are added to the vitrifier to enhance the leach resistance of the final waste form. The APC scrubber liquor is sent to the aqueous waste treatment subsystem.

The steam gasification system has thirteen subsystems. The sort unit operation in the receiving and preparation subsystem is larger than that of the baseline system. Most of the subsystems are the same as those of the baseline system (A-1), with the exception of the main thermal treatment and the APC subsystems. These two subsystems are described below. The primary stabilization subsystem receives noncombustible waste feed from the receiving and preparation subsystem and fly ash from the main thermal treatment APC, and is the same as that for the molten salt oxidation system (F-1).

Main thermal treatment. The main component of the thermal treatment subsystem is a steam reforming reactor. The key function of the reactor is to mix the waste with steam to decompose the organic material in a high-temperature environment. The gasification reactor design is a fluidized bed vessel technology.

The waste feed must be reduced in size for more efficient turbulence and mixing during gasification in the reactor. Superheated steam enters the reactor through spargers at the bottom of the fluidized bed reactor and facilitates mixing. The reactor is heated by an indirect heat source. At operation temperatures of approximately 1,300 to $1,400^{\circ} \mathrm{F}$, and under reduced conditions, the organics react with superheated steam, forming syngas composed of $\mathrm{CO}, \mathrm{CO}_{2}, \mathrm{H}_{2}$, and $\mathrm{H}_{2} \mathrm{O}$ gases. The syngas is sent to the APC subsystem for purification and energy recovery. The bottom ash, solids, and some bed material removed from the bed are collected, cooled, and sent to the primary stabilization subsystem for vitrification.

Main thermal treatment APC. The APC subsystem is based on dry particulate filtration followed by wet acid scrubbing and final polishing of the syngas. In the dry filtration, the syngas passes through a cyclone unit, then a ceramic filter to remove coarse and fine particulates in the syngas. 


\subsection{System J-1: Joule-Heated Vitrification}

The Joule-heated vitrification system is designed around a conventional glass making melter. The intent is to use a Joule-heated melter in a one-step oxidation-vitrification application for treating both combustible and noncombustible waste. Several vendors have developed, or are in the process of developing, this approach.

In a typical process, glass- or ceramic-forming additives or contaminated soil are added to the vitrification unit. Oxygen is added to the melter to oxidize the organic compounds. The upper part of the melter is provided with a plenum to house gas that has formed during combustion. Ash from the combustion and the glass-forming material mix in the melter to form a homogeneous product. Offgas from the vitrification subsystem is sent to an APC subsystem. The APC subsystem uses dry filtration followed by wet offgas scrubbing. Descriptions of the main subsystems are presented below.

The Joule-heated vitrification system has eleven subsystems. Since the main thermal treatment of the system is a single-step oxidation and vitrification process, a separate primary stabilization subsystem is not needed. Most of the subsystems are the same as those of the baseline system (A-1), with the exception of the main thermal treatment subsystem.

Main thermal treatment. The heart of the main thermal treatment subsystem is a vitrifier, which performs organic destruction and waste stabilization in one step. Combustible and noncombustible waste are size reduced. Liquid waste will be fed on a continuous mode to reduce unexpected spikes and allow maximum throughput. A dryer has not been considered at this point in the design, but could be used to reduce moisture in slurry and soil fed to the melter. The vitrifier mixes the soil with the waste feed, melts the mixture, and discharges a moiten slag product into a waste container.

Glass handling. The glass handling subsystem consists of hardware necessary to remove molten glass from the melter, and cast the hot glass into a monolith.

\subsection{System K-1: Thermal Desorption and Mediated Electrochemical Oxidation}

The K-1 system is designed to accommodate flameless, low-temperature (less than $600^{\circ} \mathrm{F}$ ) technologies for processing DOE's MLLW. In this system the design objective is to heat up combustible and noncombustible solids to vaporize low-boiling-point VOCs. This study assumes that $10 \%$ of the organic waste is volatilized. The vaporized VOCs are condensed and captured in an organic liquid form. After heating and removing the VOCs, combustible and noncombustible solid residues are stabilized in a low-temperature process such as grouting. The organic liquid is destroyed in a MEO cell, which is a low temperature oxidation process using liquid electrolytes.

The heating device employed in this system is an indirectly heated thermal desorber designed to receive and heat combustible and noncombustible waste to about $600^{\circ} \mathrm{F}$. Vaporized compounds exiting the desorber are filtered in a sintered metal gas/solids separator. The gas is then cooled to remove any residual mercury in a heat exchanger. The condensed mercury is collected in a liquid container and transferred to a mercury amalgamation subsystem. A secondary heat exchanger condenses the liquid water and volatilized compounds into a liquid form. The condensed liquid is sent to a liquid organic destruction subsystem based on MEO technology. Solid residue from the desorber is transferred to the primary stabilization unit where residue is grouted.

In the liquid organic waste treatment subsystem, an MEO reactor converts the incoming organics to gaseous products. The offgas from MEO is treated by neutralization to remove chlorine, followed by catalytic oxidation. Aqueous secondary waste streams generated by the recycling of spent electrolyte are 
processed in a fractionator unit. Solids recovered are sent to secondary stabilization. The distillate is condensed, and the sulfuric acid is separated and recycled.

This system has all the thirteen subsystems included in the baseline system plus an additional liquid organic treatment subsystem. Most of the subsystems are the same as in the baseline system (A-1), with the exception of the main thermal treatment, the main thermal treatment APC, liquid organic treatment (MEO), and the primary stabilization subsystems. Also, the aqueous waste treatment subsystem no longer has the organic liquid waste treatment function. This function has been transferred to a new subsystem, the liquid organic waste treatment subsystem (the MEO process). Subsystems that are different from the baseline system are described below.

Main thermal treatment. The main thermal treatment subsystem consists of a feed preparation and characterization area, an indirectly heated thermal desorber, and the associated heating and feedstock transfer equipment. The thermal treatment subsystem receives shredded combustible and noncombustible solids and sludge. Organic liquid bypasses the desorber and is transferred directly to the organic liquid waste treatment subsystem. The rotary thermal desorber volatilizes organics and water at temperatures of 500 to $600^{\circ} \mathrm{F}$. The remaining solids, which includes organics that did not volatilize in the desorber, are stabilized by cement grouting in the primary stabilization subsystem. The temperature of the desorber is kept below the melting point of most plastic materials to avoid creating excessive chlorinated gas, but high enough so that it volatilizes most organic compounds.

Main thermal treatment APC. The APC subsystem uses dry sintered metal filtration, two stage condensation, carbon and HEPA filtration followed by catalytic oxidation of noncondensable gases. The main purpose of the APC system is to condense water and volatile organics for treatment in the organic liquid waste subsystem. Following the desorber, gas enters the metal filter for removal of particulates. The offgas is passed through a heat exchanger designed to condense mercury for transfer to the mercury amalgamation subsystem. The second stage heat exchanger condenses water, and volatile organics for treatment in the liquid organic waste treatment subsystem. The remaining offgas passes through carbon and HEPA filters. Noncondensable gas and remaining volatile organics such as methane are combusted in a catalytic oxidizer, before discharge to the atmosphere.

Liquid organic waste treatment. The liquid organic waste treatment subsystem consists of feed characterization and batching tanks, electrolyte circulation tanks, mediated electrochemical oxidation reactors, offgas treatment by neutralization and catalytic oxidation, and associated pumps and instruments. The liquid organic feed from the condenser following thermal desorption is blended with other liquid organic waste streams. Electrolyte is recycled from the electrochemical reactors. Sulfuric acid/cobalt sulfate electrolyte and water are continually supplied to the circulation tank. The ratio of input waste feed to recirculating electrolyte is approximately $2 \%$ by weight.

The MEO reactors operate at 50 to $60^{\circ} \mathrm{C}$ and atmospheric pressure. The electrolytic cells are packaged into modular units. An electrolyte solution of cobalt sulfate and sulfuric acid is recirculated through a series of cells in the MEO unit at rates of up to $100 \mathrm{gpm}$. Electrical energy is supplied to the anode and cathode. The oxidized metal mediator acts as the primary active oxidizer to destroy organics. The cobalt is transformed from $\mathrm{Co}^{2+}$ to $\mathrm{Co}^{3+}+\mathrm{e}^{-}$. The metal ion reacts with organic species or water to produce a reactive intermediate such as the hydroxyl radical, which also oxidizes any organic material. A side stream of the electrolyte solution is bled off to prevent buildup of inerts and to recycle sulfuric acid.

Offgas is generated at the cathode (primarily $\mathrm{H}_{2}$ ) and at the anode (primarily $\mathrm{CO}_{2}, \mathrm{Cl}_{2}$ from chlorocarbons and small amounts of $\mathrm{O}_{2}$ ). The anode stream is put through a caustic scrubber to convert the chlorine to hypochlorite. Both offgas streams are put through a catalytic oxidizer to react the hydrogen with air to form water and to destroy any volatile organics that may vaporize from the MEO 
reactor. Additional air must be added to the catalytic oxidizer to control the heat generated by the hydrogen oxidation reaction.

Primary stabilization. The primary stabilization subsystem receives desorbed solids from the main thermal treatment subsystem, and other solid waste from the receiving and preparation subsystem. The stabilization process may be classified as macroencapsulation, which meets EPA requirements for stabilizing solid wastes that fall under the debris rule.

\subsection{System L-1: Thermal Desorption and Supercritical Water Oxidation}

The thermal desorption step is similar to system K-1. The organic liquid is destroyed in a SCWO reactor, which is a low-temperature, high-pressure water oxidation process. Several vendors have developed or are in the process of developing this approach.

In the liquid organic waste treatment subsystem a SCWO reactor using the properties of supercritical water oxidizes organic waste to gaseous products. Supercritical water is water at a temperature above $705^{\circ} \mathrm{F}$ and a pressure above $218 \mathrm{~atm}$. Supercritical water has properties different from those of subcritical water. Organic compounds that are normally immiscible in water are miscible in supercritical water. Inorganic compounds, such as salts, are almost completely insoluble in supercritical water. These properties allow a quick and complete oxidation of organic waste in the reactor vessel. Hydrogen peroxide or pure oxygen is used as the oxidant in the reaction. Salts formed from the inorganic elements that are ubiquitous in most waste streams either precipitate out of the supercritical water or are removed as offgas. This gas is normally not acidic in nature and requires minimal further treatment before it can be discharged. Continuous emission monitoring is performed before the offgas is discharged. The precipitated salts are stabilized before disposal.

Most of the thirteen subsystems are the same as in the baseline system (A-1), or system K-1, with the exception of the main thermal treatment, the main thermal treatment APC, liquid organic waste treatment (SCWO), and the primary stabilization subsystems. Also, the aqueous waste treatment subsystem no longer treats organic liquids. This function has been transferred to a new subsystem, the liquid organic waste treatment subsystem (the SCWO process). However, the size of the aqueous waste treatment subsystem will remain the same as the baseline system because of the added duty of treating liquid waste effluent from the SCWO process. Subsystems that are different from the baseline system are described below.

Liquid organic waste treatment. The liquid organic waste treatment subsystem consists of feed characterization, feed and additive pressurization, the SCWO reactor, and two treatment trains for liquids/solid separation and offgas air pollution control. Input waste to this system consists of organic liquids and organic sludge. Organic sludge is filtered to an acceptable size for the SCWO process. Optimal performance is achieved when the maximum particle size in the waste stream is about $100 \mu \mathrm{m}$. Material exceeding the $100 \mu \mathrm{m}$ limit is sent to primary stabilization.

The SCWO reaction process consists of three subprocesses: feed preparation, reaction, and air pollution control. In the feed preparation step, the reactants are pressurized above $218 \mathrm{~atm}$ and heated above $705^{\circ} \mathrm{F}$. Products from the oxidation reaction include $\mathrm{H}_{2} \mathrm{O}, \mathrm{CO}_{2}$, and inorganic salt precipitates. Salts are separated from the aqueous phase in an agitated thin-film filter and evaporator. The inorganic salts are sent to the secondary stabilization subsystem while the offgas is further treated by activated carbon adsorption and HEPA filtration. Liquid from the thin-film filter is polished using activated carbon and ion-exchange resins, and is then tested. Next, it is either recycled or sent for further treatment to the aqueous waste treatment subsystem. 
Process Flow Diagrams of All Systems 


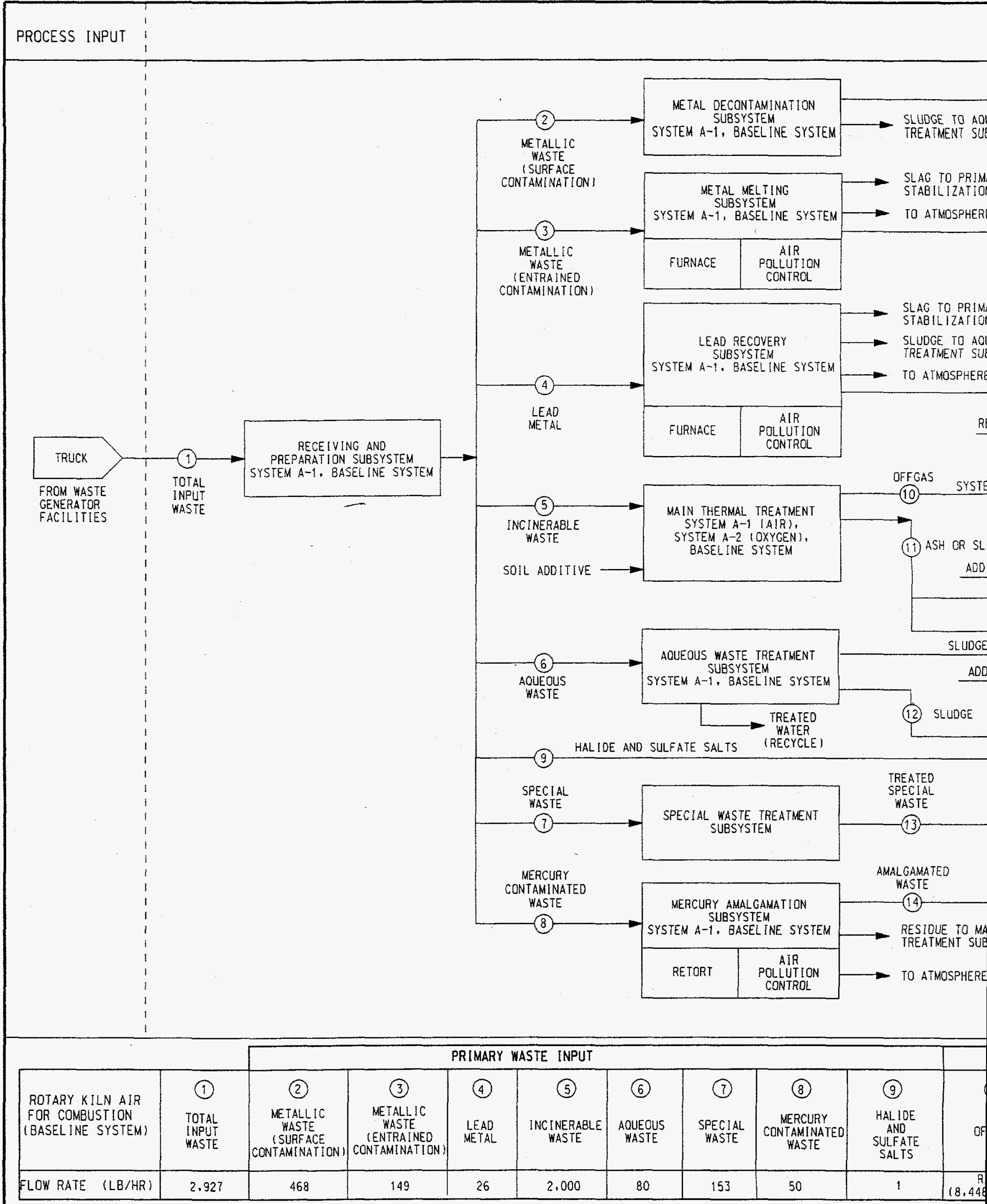

Figure 4-1. Rotary Kiln: PFD for Overall System (Systems A-1 through A-6). 


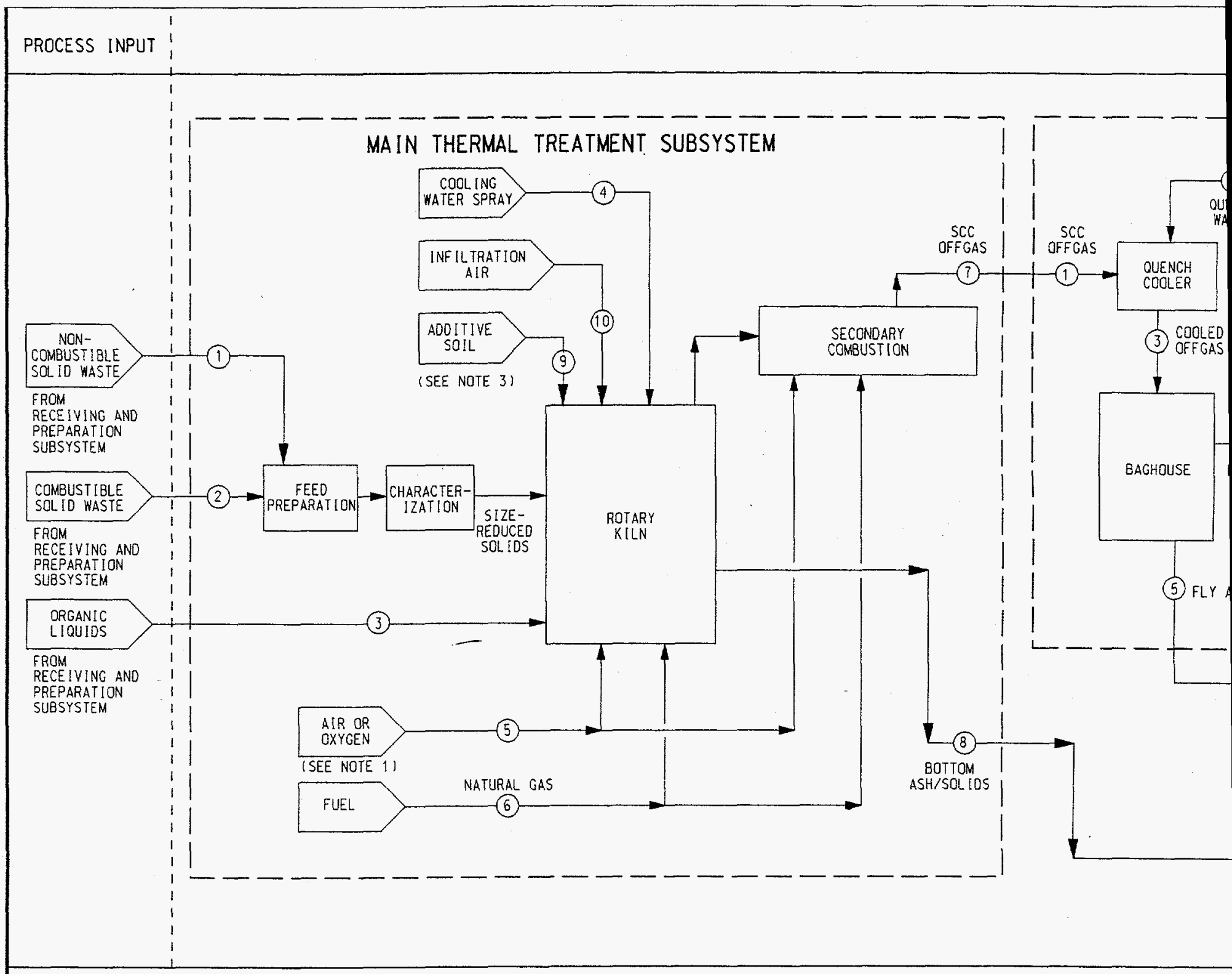

NDTES:

1. SYSTEM A-1 EMPLOYS AIR FOR COMBUSTION. SYSTEM A-2 EMPLOYS OXYGEN.

2. SECONOARY WASTE INPUT FROM VARIOUS SUBSYSTENS NOT SHOWN.

3. ADDITIVE SOIL $=50 \%$ OF INERT MATERIAL IN FEED.

4. INFILTRATION AIR INCLUDED WITH INPUT AIR UNLESS NOTEO.

5. TRACE METALS REMOVED FROM OFFGAS IN THE AIR POLLUTION CONTROL

SUBSYSTEM ARE ROUTED TO SECONOARY STABILIZATION AND NOT SHOWN ABOVE.

(SEE GENERAL MASS FLOW RATES).

\begin{tabular}{|c|c|c|c|c|}
\hline $\begin{array}{c}\text { MAIN THERMAL TREATMENT } \\
\text { SUBSYSTEM }\end{array}$ & $\begin{array}{c}\text { SORTED } \\
\text { NON-COMBUSTIBLE } \\
\text { SOL ID WASTE }\end{array}$ & $\begin{array}{c}\text { (2) } \\
\text { SORTED } \\
\text { COMBUSTIBLE } \\
\text { SOL ID WASTE }\end{array}$ & $\begin{array}{l}\text { (3) } \\
\text { ORGANIC } \\
\text { LIOUIDS }\end{array}$ & $\begin{array}{c}\text { (4) } \\
\text { COOLIN } \\
\text { WATER } \\
\text { SPRAY }\end{array}$ \\
\hline FLOW RATE (LB/HR) (SYSTEM A-1) & 1340 & 609 & 51 & 482 \\
\hline FLOW RATE (LB/HR) (SYSTEM A-2) & 1340 & 609 & 51 & 1,82 \\
\hline $\begin{array}{c}\text { MAIN THERMAL TREATMENT } \\
\text { AIR POLLUTION } \\
\text { CONTROL SUBSYSTEM }\end{array}$ & $\begin{array}{c}1 \\
\text { SCC } \\
\text { OFFGAS }\end{array}$ & $\begin{array}{l}\text { (2) } \\
\text { OUENCH } \\
\text { HATER }\end{array}$ & $\begin{array}{l}\text { (3) } \\
\text { COOLED } \\
\text { OFFGAS }\end{array}$ & $\begin{array}{l}(4) \\
\text { FILTER } \\
\text { OFFGA }\end{array}$ \\
\hline FLOW RATE (LB/HR) (SYSTEM A-1) & 21,420 & 10.221 & 31,641 & 31,3 \\
\hline FLOW RATE (LB/HR) (SYSTEM A-2) & 8,448 & 4,890 & 13.338 & 13. \\
\hline
\end{tabular}

Figure 4-2. Rotary Kiln: PFD for incineration (Systems A-1 and A-2) and Dry and Wet Air Pollution Control Subsystems (Systen 


\section{MAIN THERMAL TREATMENT AIR POLLUTION CONTROL SUBSYSTEM}
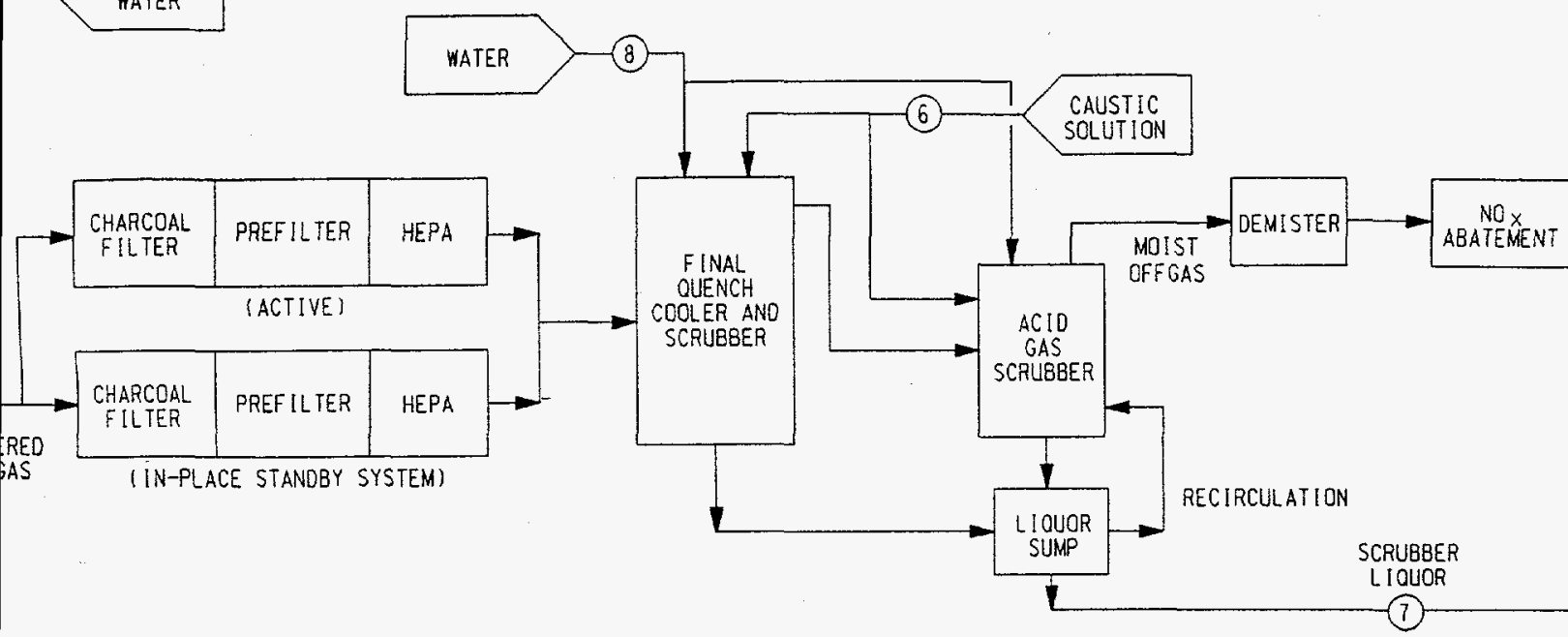

SCRUBBER LI QUOR

TO AOUEOUS WASTE TREATMENT SUESYSTEM

\begin{tabular}{|c|c|c|c|c|c|c|}
\hline \multicolumn{2}{|c|}{ (5) } & (6) & (7) & (8) & -9 & (10) \\
\hline AIR & OXYGEN & NATURAL GAS & $\begin{array}{l}\text { SCC } \\
\text { OFFGAS }\end{array}$ & $\begin{array}{c}\text { BOTTOM } \\
\text { ASH/SOL IDS }\end{array}$ & $\begin{array}{l}\text { ADDITIVE } \\
\text { SOIL }\end{array}$ & $\underset{\text { INF IL TRATION }}{\text { TIR }}$ \\
\hline 7859 & - & 9.082 & 21.420 & 1,196 & 498 & 2.695 \\
\hline - & 1,830 & 796 & 8,448 & 1,196 & 498 & 2.695 \\
\hline & & (6) & (7) & (8) & (9) & \\
\hline & & $\begin{array}{l}\text { CAUSTIC } \\
\text { SOLUT [ON } \\
\text { (20\% NCOH) }\end{array}$ & $\begin{array}{l}\text { SCRUBBER } \\
\text { LIOUOR }\end{array}$ & $\begin{array}{c}\text { SCRLBBER/ } \\
\text { COOLER } \\
\text { WATER }\end{array}$ & $\begin{array}{l}\text { TREATED } \\
\text { OFFGAS }\end{array}$ & \\
\hline & 39 & 156 & 13.474 & 2.181 & 20.200 & \\
\hline & & 156 & 9,996 & 2,181 & 5,378 & \\
\hline
\end{tabular}




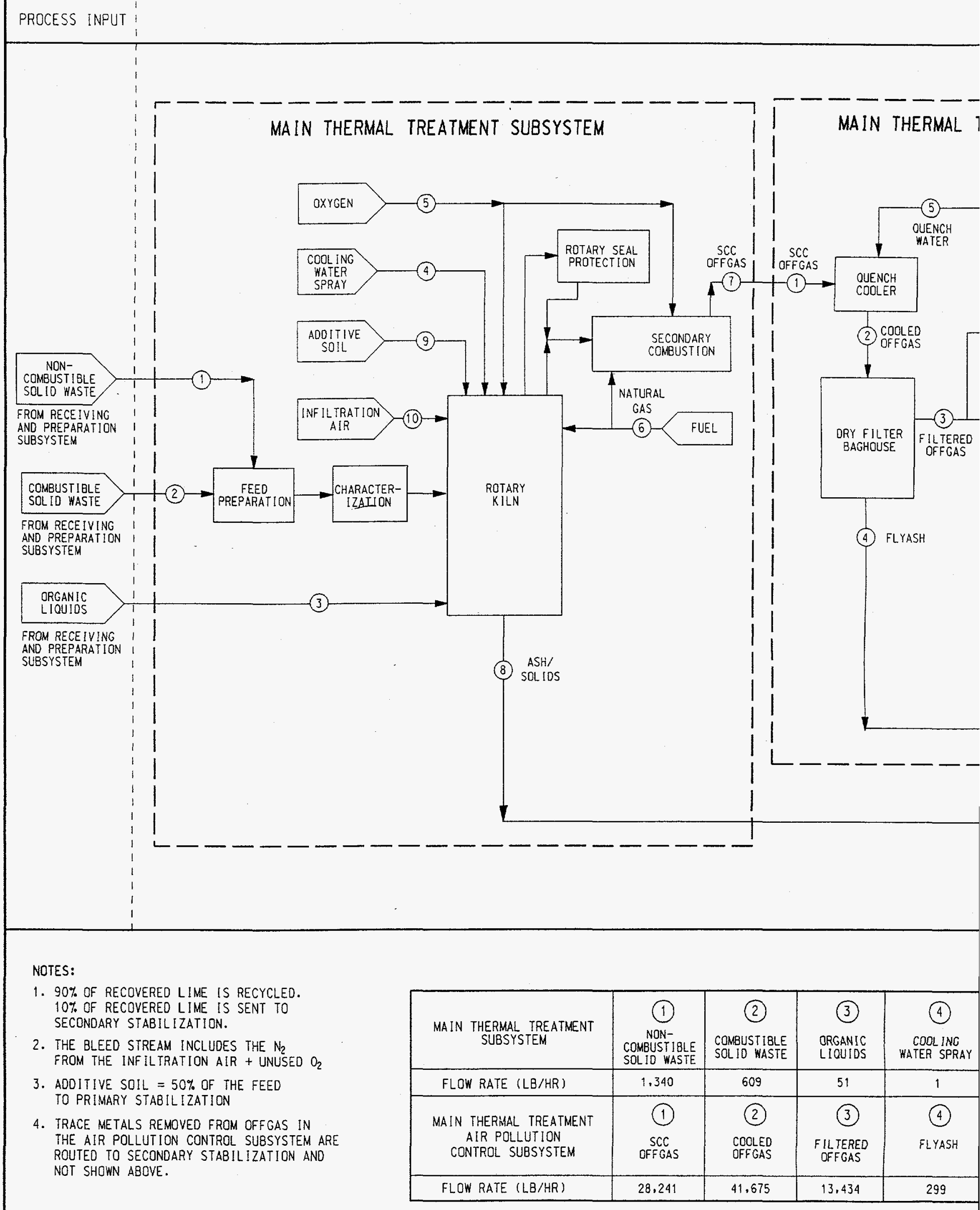

Figure 4-3. Rotary Kiln: PFD for Dry and Wet Air Pollution Control Subsystem with $\mathrm{CO}_{2}$ Retention (System A-4). 
TMENT AIR POLLUTION CONTROL SUBSYSTEM

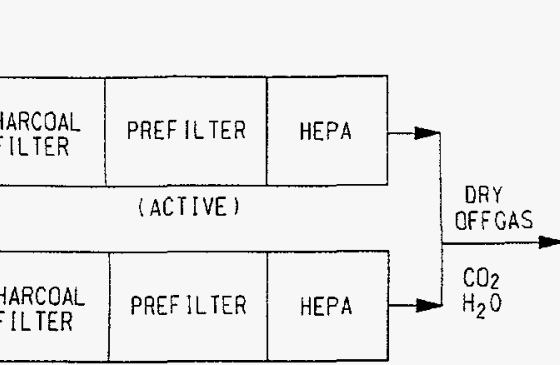

(IN-PLACE STANDEY SYSTEM)

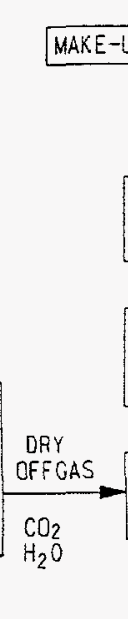

LLLAKER

SOLIDS

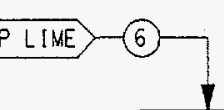

1

BAGHOUSE

BCUIDIZED
BED COL

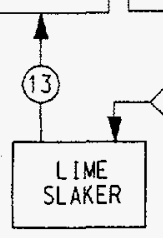

1 ABSCRBER

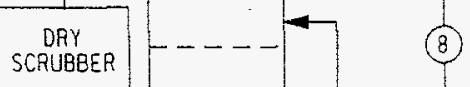

SCRUBBER
(11)

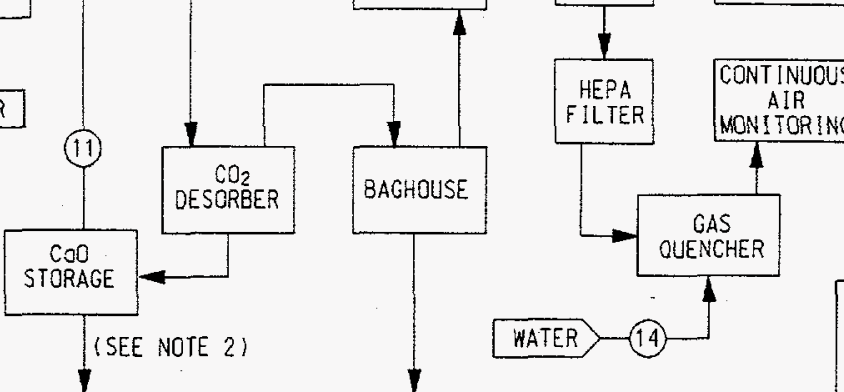

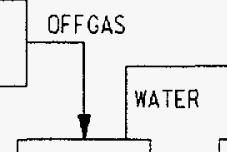
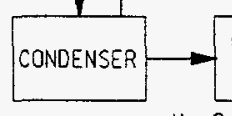
$\mathrm{N}_{2}, \mathrm{O}_{2} \quad \mathrm{~N}_{2}, \mathrm{O}_{2}$ MAIN STREAM $\left.\begin{array}{c}\mathrm{N}_{2}, \mathrm{O}_{2} \\ \text { SIDE } \\ \text { STREAM }\end{array}\right] \quad \mathrm{N}_{2}, \mathrm{O}_{2}$ MAIN ST<smiles>[C+]1CCCC1</smiles>

COMPRESSOR
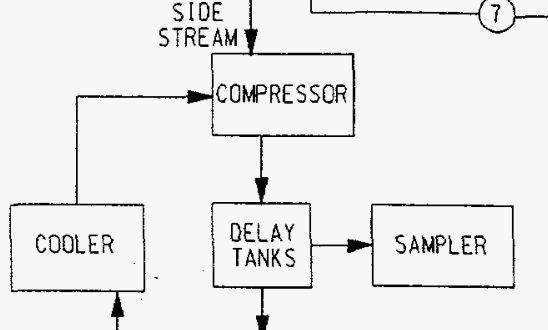

PRESSURE
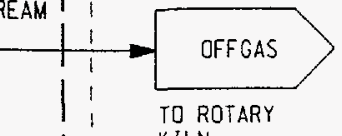

KILN

WASTE
WATER

To AQUEOUS WASTE TREATMENT SUBSYSTEM

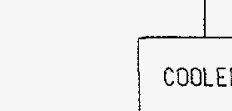

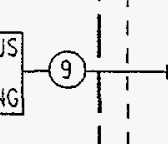

TREATED
OFFGAS TO ATMOSPHERE

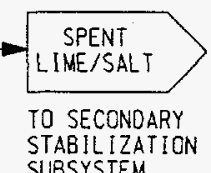

\begin{tabular}{|c|c|c|c|c|c|c|c|c|c|}
\hline $\begin{array}{l}5 \\
\text { OXYGEN }\end{array}$ & $\underset{\substack{\text { NATURAL } \\
\text { GAS }}}{(6)}$ & $\begin{array}{c}(7) \\
\text { SCC } \\
\text { DFFGAS }\end{array}$ & $\begin{array}{c}\text { (8) } \\
\text { ASH/SOLIDS }\end{array}$ & $\underbrace{9}_{\substack{\text { ADDITIVE } \\
\text { SOIL }}}$ & $\begin{array}{c}\text { 10 } \\
\text { INF IL TRATION } \\
\text { AIR }\end{array}$ & & & & \\
\hline 198 & 3,586 & 28,241 & 1.196 & 498 & 2.695 & & & & \\
\hline (5) & (6) & (7) & (8) & (9) & (10) & (11) & (12) & (13) & (14) \\
\hline $\begin{array}{l}\text { OUENCH } \\
\text { WATER }\end{array}$ & $\begin{array}{l}\text { MAKE-UP } \\
\text { LIME }\end{array}$ & $\begin{array}{c}\text { SCRUBBED } \\
\text { OFFGAS }\end{array}$ & $\mathrm{CACO}_{3}$ & $\begin{array}{l}\text { TREATED } \\
\text { OFFGAS }\end{array}$ & $\begin{array}{l}\text { SPENT LIME/ } \\
\text { SALT \& TRACE } \\
\text { METALS }\end{array}$ & $\begin{array}{c}\text { RECYCLE } \\
\text { LIME } \\
\text { (SEE NOTE 1) }\end{array}$ & $\begin{array}{l}\text { WASTE } \\
\text { WATER }\end{array}$ & $\begin{array}{c}\text { RECYCLED } \\
\text { LIME }\end{array}$ & WATER \\
\hline 41.376 & 374 & 20,460 & 6.664 & 6.127 & 434 & 3.362 & 15,653 & 57 & 925 \\
\hline
\end{tabular}




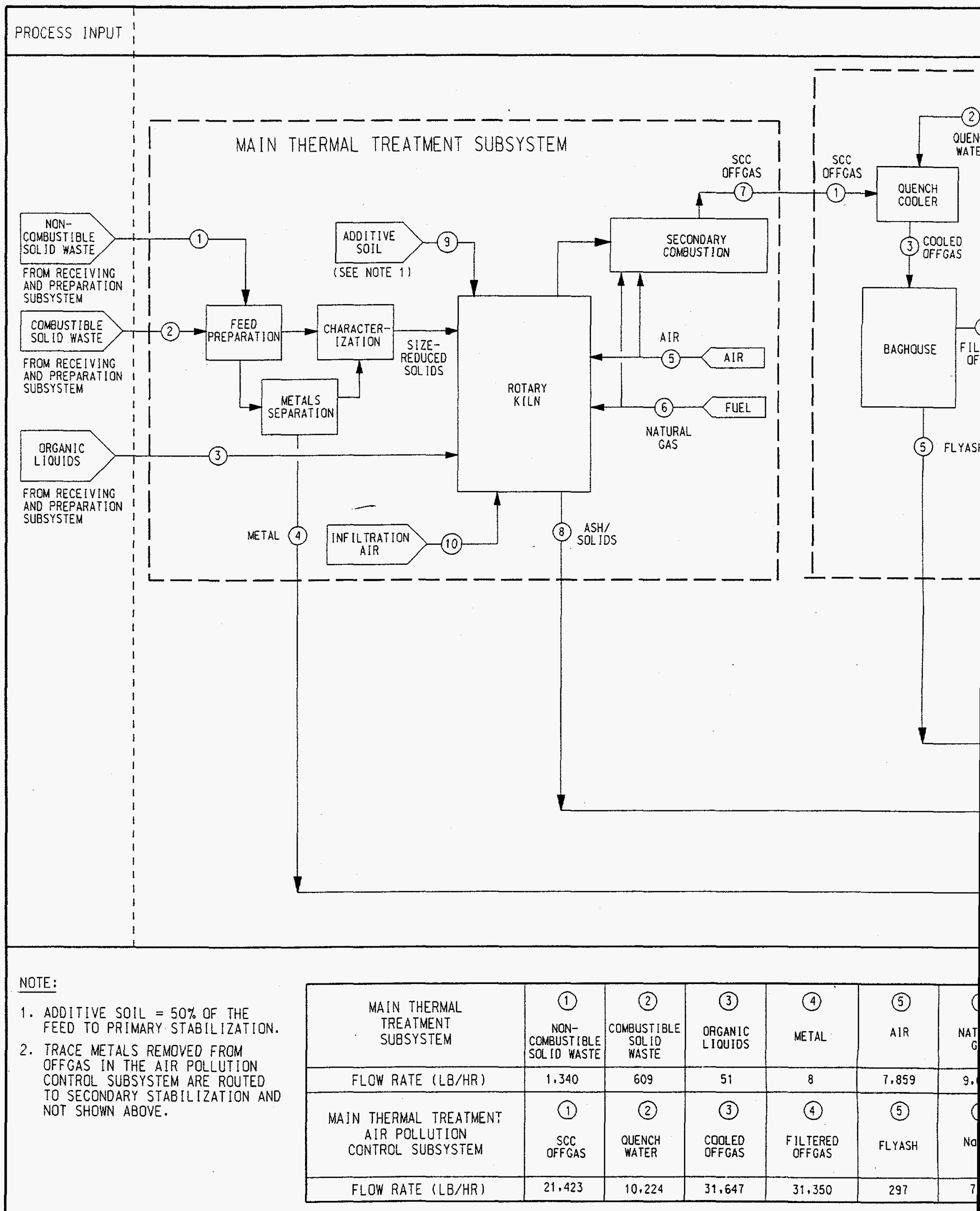

Figure 4-4. Rotary Kiln: PFD for Dry and Wet Air Pollution Control Subsystem with Salt Recovery (System A-6). 
MAIN THERMAL TREATMENT AIR POLLUTION CONTROL SUBSYSTEM

OUENCH

WATER

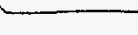

STAINLESS STEEL

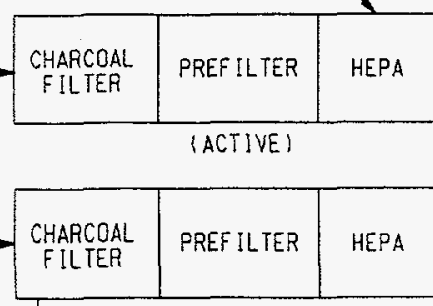

(IN-PLACE STANOBY SYSTEM)
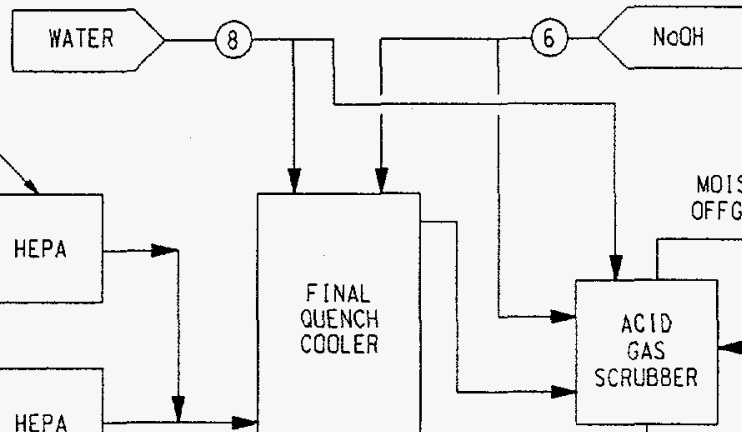

DRY-TREATED
OFFGAS
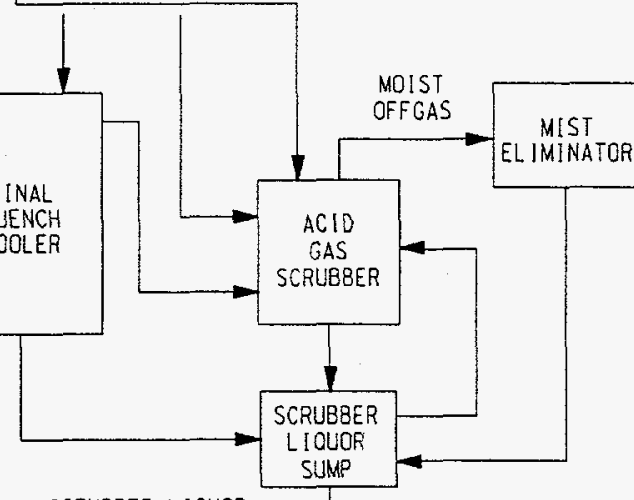

SCRUBBER L I OUDR

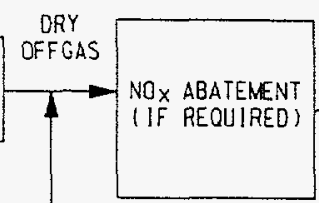

(10)
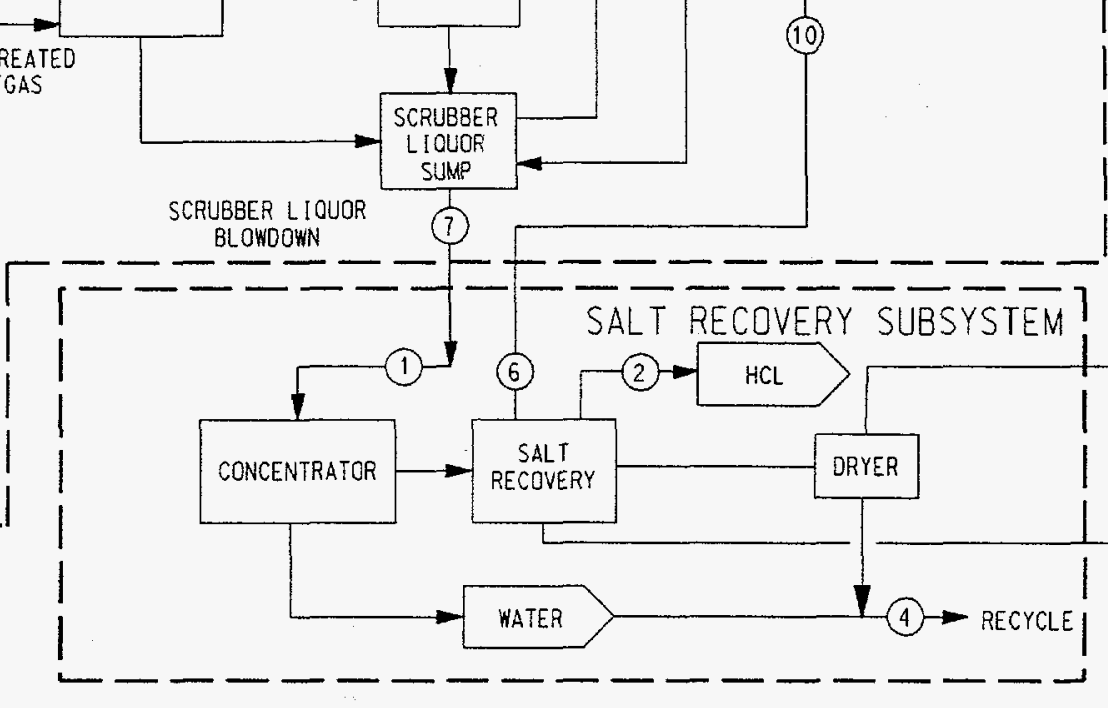

\begin{tabular}{|c|c|c|c|c|c|c|c|c|c|}
\hline $\begin{array}{c}(7) \\
\text { SCC } \\
\text { OFFGAS }\end{array}$ & $\begin{array}{c}\text { (8) } \\
\text { ASH' } \\
\text { SOLIOS }\end{array}$ & $\begin{array}{c}\text { (9) } \\
\text { ADDIIIVE } \\
\text { SOIL }\end{array}$ & $\begin{array}{c}\text { (10) } \\
\text { INF ILIRATION } \\
\text { AIR }\end{array}$ & $\begin{array}{l}\text { SAL T RECOVERY } \\
\text { SUBSYSTEM }\end{array}$ & $\begin{array}{c}\text { (1) } \\
\text { SCRUBBER } \\
\text { LIOUOR } \\
\text { BLOWDOWN }\end{array}$ & (2) & (3) & $\begin{array}{l}\text { (4) } \\
\text { MATER }\end{array}$ & $\begin{array}{l}\text { (5) } \\
\text { ORIED } \\
\text { SALTS }\end{array}$ \\
\hline 21,423 & 1,188 & 495 & 2.695 & FLOW RATE (LB/HR) & 13,480 & 140 & 78 & 13.226 & 0.24 \\
\hline $\begin{array}{c}\text { (1) } \\
\text { SCRUBBER } \\
\text { LIOURR } \\
\text { BLOWDOWN }\end{array}$ & $\begin{array}{l}\text { (8) } \\
\text { SCFUBBER/ } \\
\text { COOLER } \\
\text { WATER }\end{array}$ & $\begin{array}{c}\text { (9) } \\
\text { TREATED } \\
\text { OFFGAS }\end{array}$ & $\begin{array}{c}\text { (10) } \\
\text { OFFGAS }\end{array}$ & & $\begin{array}{c}\text { (6) } \\
\text { OFFGAS }\end{array}$ & & & & \\
\hline 13.480 & 2.259 & 20,241 & 38 & & 38 & & & & \\
\hline
\end{tabular}




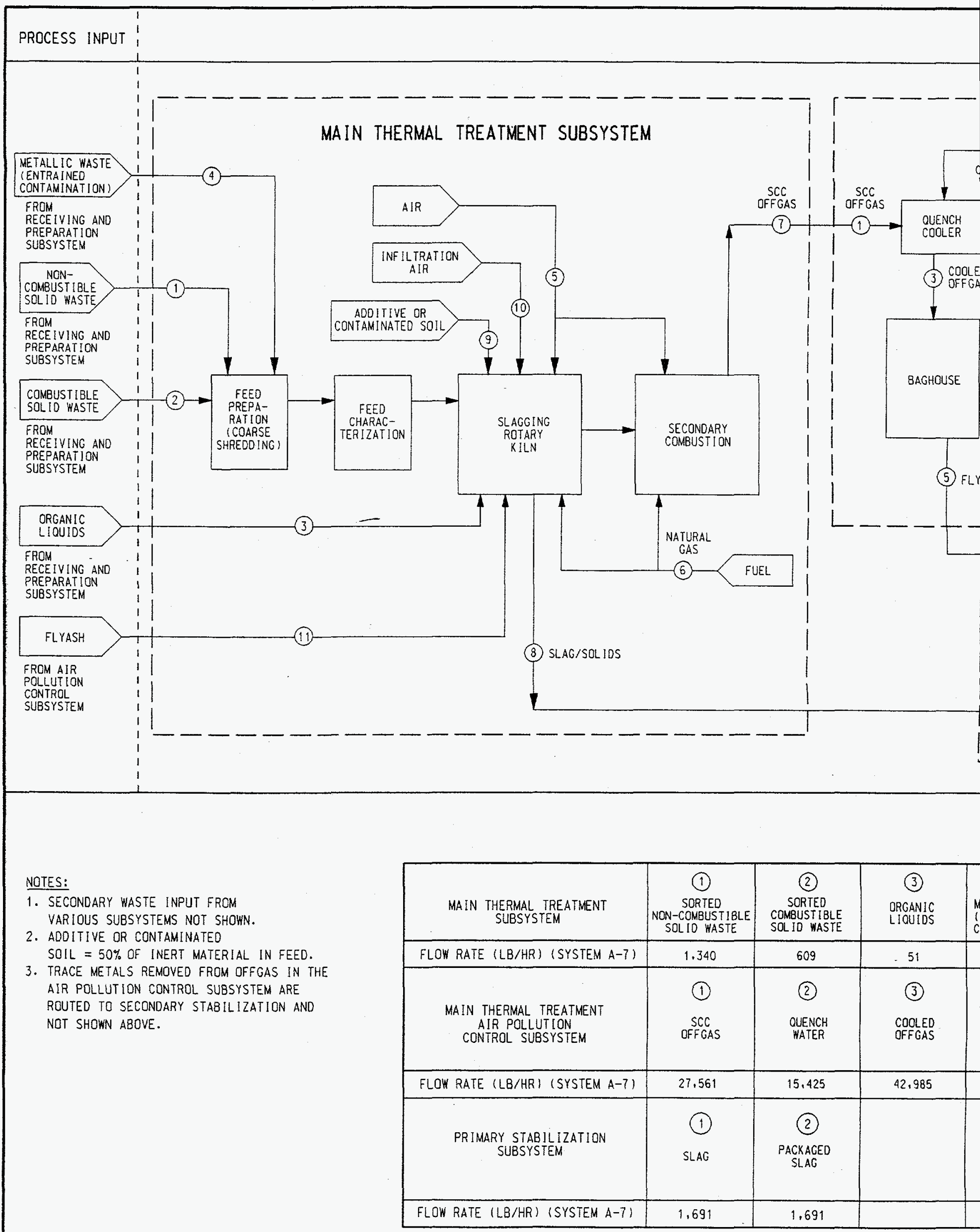

Figure 4-5. Slagging Rotary Kiln: PFD for Main Thermal Treatment, Air Pollution Control, and Primary Stabilization Subsyste 


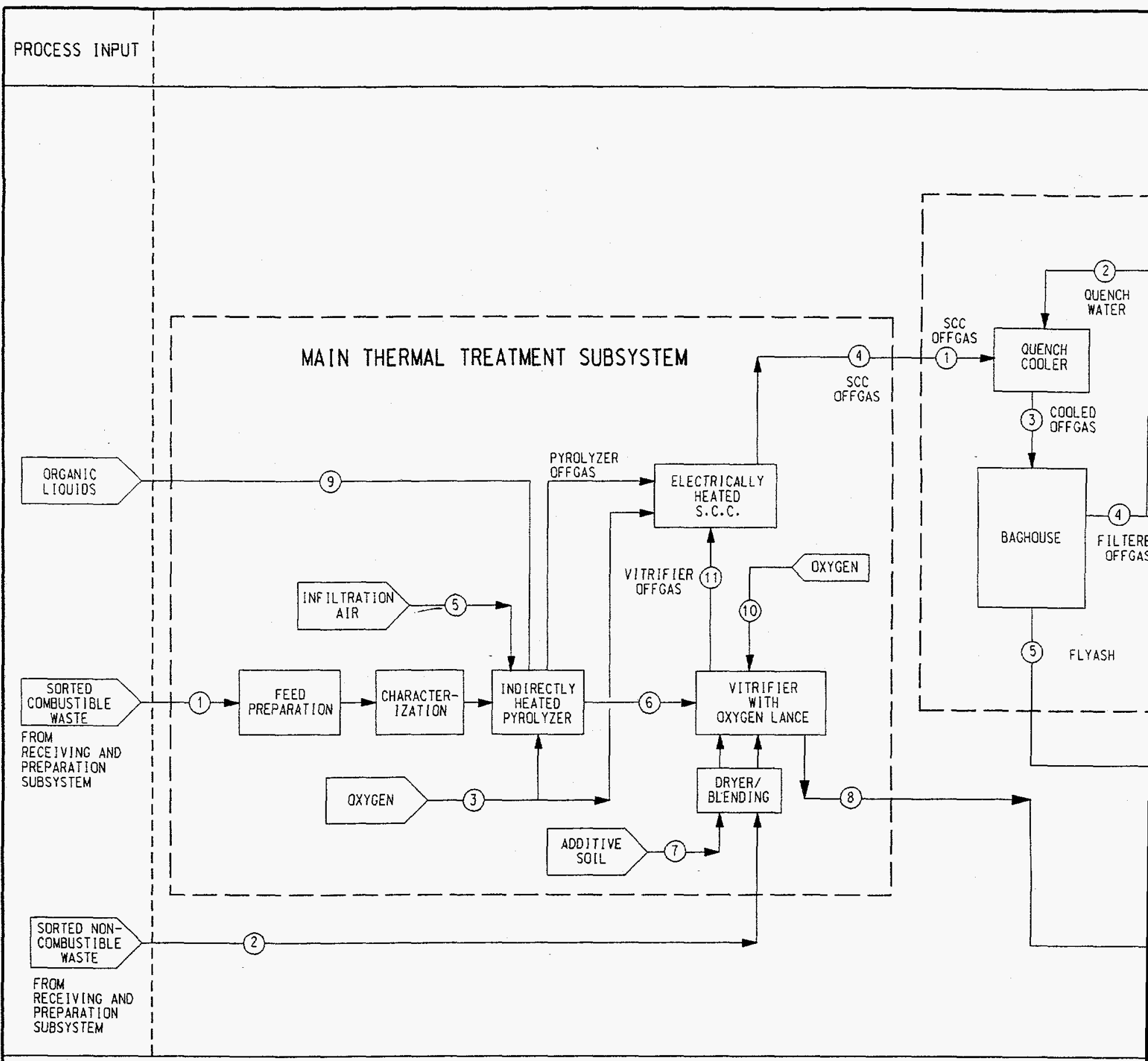

\section{NOTES:}

1. ALL INPUT SOLID WASTE MUST BE SORTED ANO CATEGORIZED AS EITHER COMBUSTIBLE OR NON-COMBUSTIBLE SOLIDS.

2. ADDITIVE 50IL $=50 \%$ OF THE FEED TO PRIMARY STABILIZATION.

3. TRACE METALS REMOVED FROM OFFGAS IN THE AIR POLLUTION CONTROL SUBSYSTEM ARE ROUTEO TO SECONOARY STABILIZATION AND NOT SHOWN ABOVE (SEE CENERAL MASS FLOW RATES).

\begin{tabular}{|c|c|c|}
\hline PRIMARY STABILIZATION SUBSYSTEM & $\begin{array}{l}\text { VITRIF IER } \\
\text { SL.AG }\end{array}$ & $\begin{array}{l}\text { PACKAGED } \\
\text { SLAG }\end{array}$ \\
\hline FLOW RATE( LB/HR) & 1,495 & 1,495 \\
\hline $\begin{array}{c}\text { MAIN THERMAL TREATMENT } \\
\text { SUBSYSTEM }\end{array}$ & $\begin{array}{c}1 \\
\text { SORTEO } \\
\text { COMBUSTIBLE } \\
\text { WASTE }\end{array}$ & $\begin{array}{l}\text { (2) } \\
\text { SORTED NON- } \\
\text { COMBUSTIBLE } \\
\text { WASTE }\end{array}$ \\
\hline FLOW RATE (LB/HR) & 609 & 1,340 \\
\hline $\begin{array}{c}\text { MAIN THERMAL TREATMENT } \\
\text { AIR POLLUTION } \\
\text { CONTROL SUBSYSTEM }\end{array}$ & $\begin{array}{l}\text { SCC } \\
\text { OFFGAS }\end{array}$ & $\begin{array}{l}2 \\
\text { OUENCH } \\
\text { WATER }\end{array}$ \\
\hline FLOW RATE (LB/HR) & 2.397 & 1,411 \\
\hline
\end{tabular}

Figure 4-6. Pyrolysis: PFD for Incineration, Air Pollution Control, and Primary Stabilization Subsystems (System B-1). 
MAIN THERMAL TREATMENT AIR POLLUTION CONTROL SUBSYSTEM

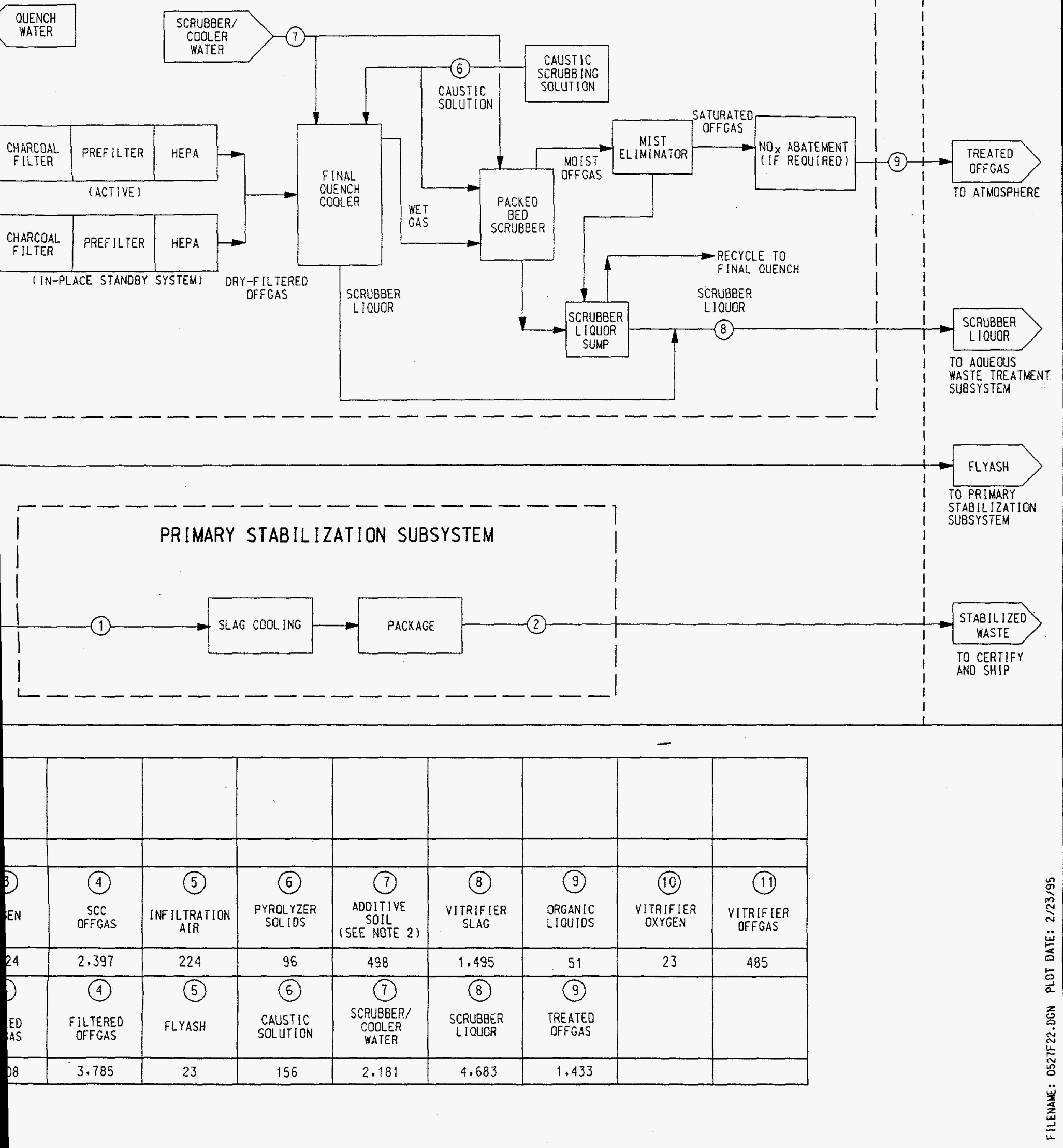




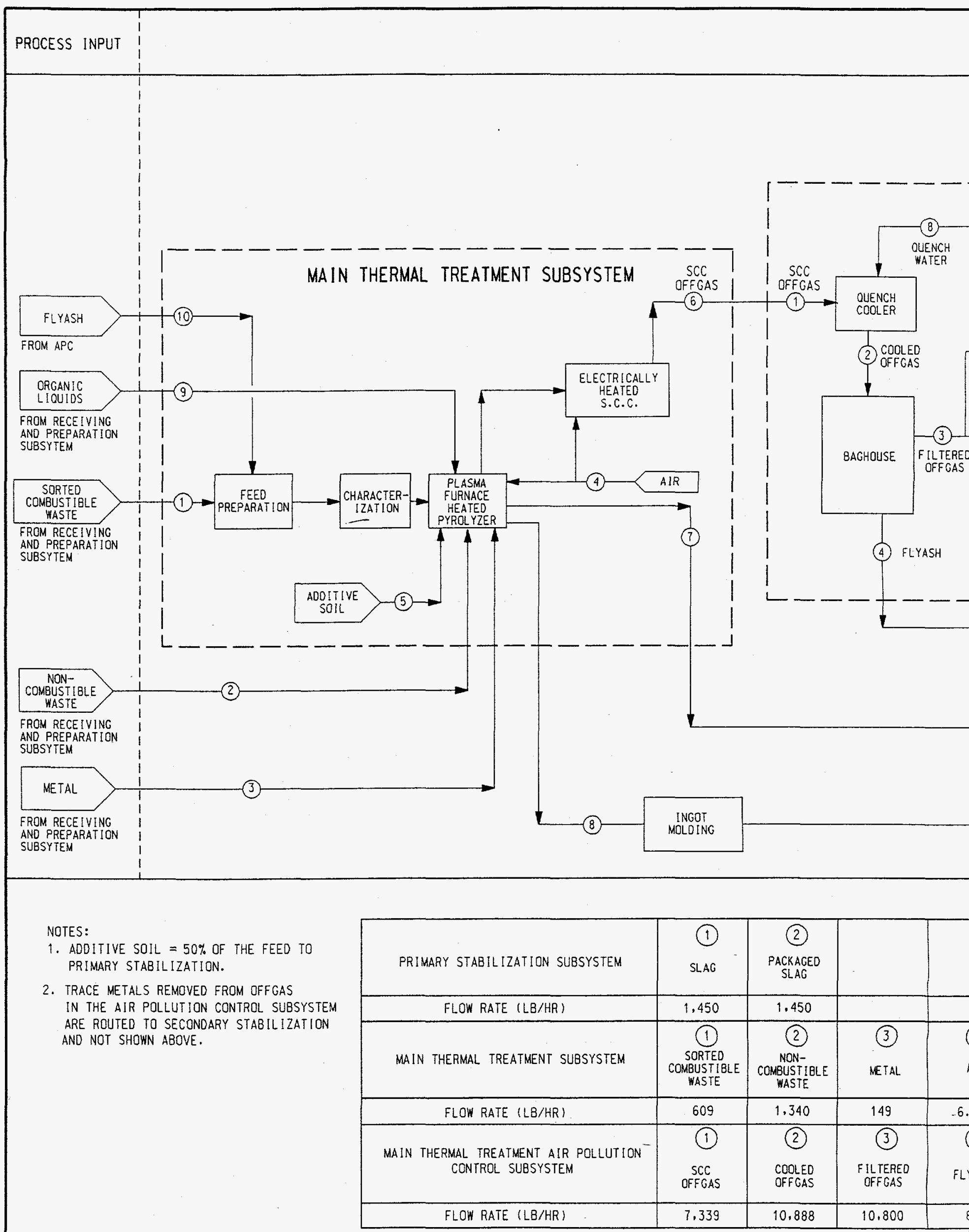

Figure 4-7. Plasma Furnace: PFD for Incineration, Air Pollution Control, and Primary Stabilization Subsystems (System C-1). 


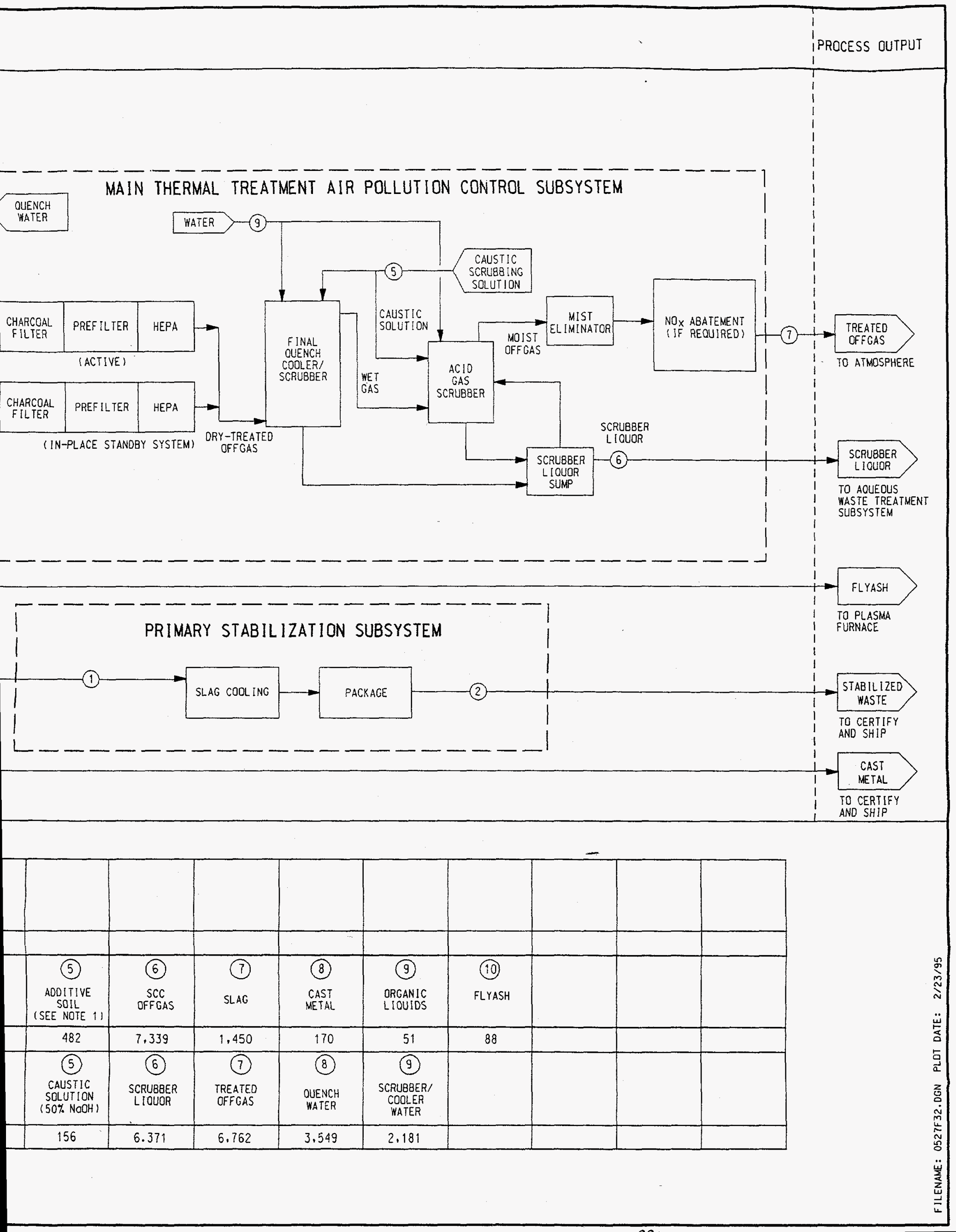




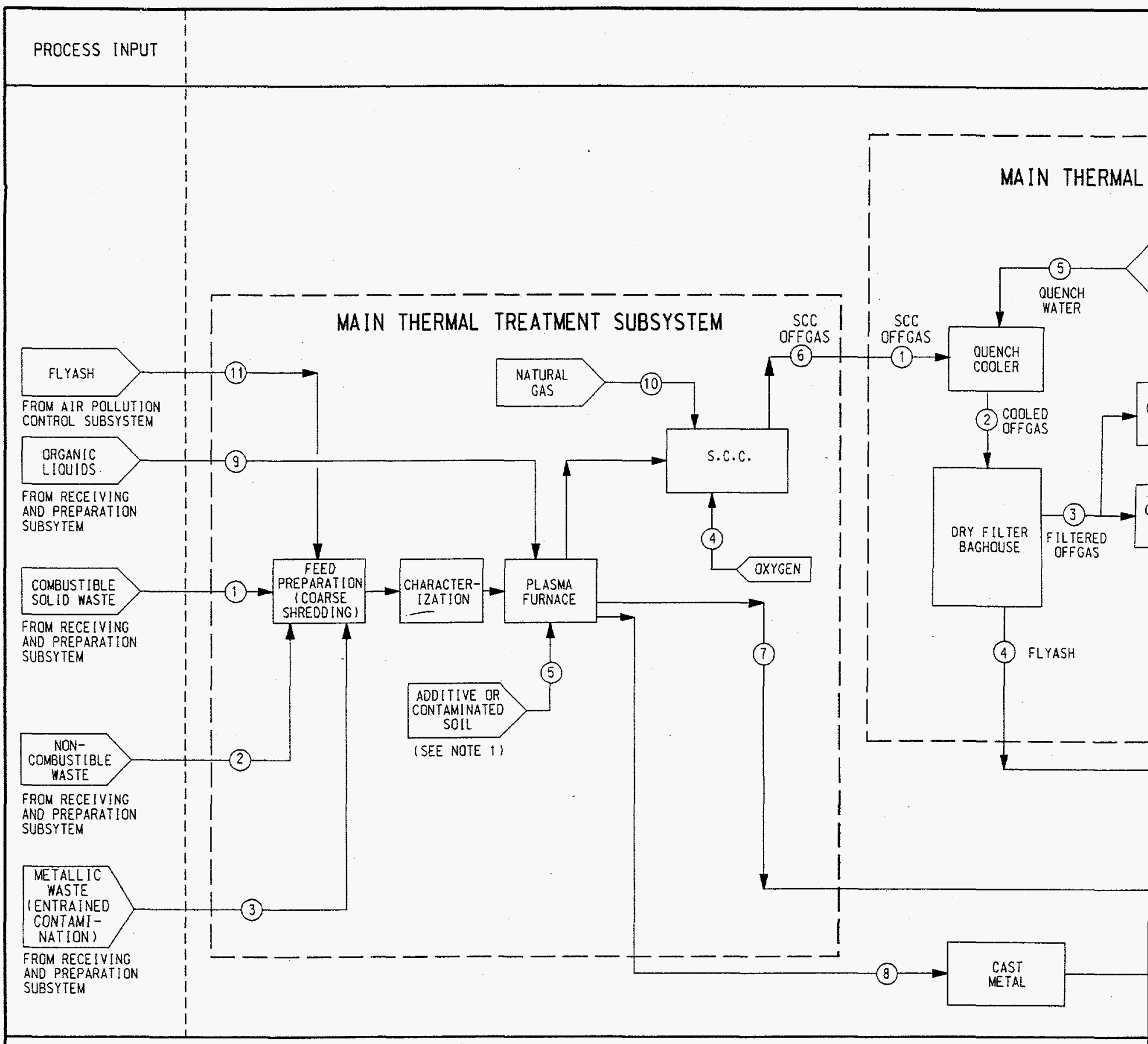

NOTES:

1. ADOITIVE OR CONTAMINATED SOIL $=50 \%$ OF THE FEED TD PRIMARY STABILIZATION.

2. 90\% OF RECOVERED LIME IS RECYCLED TD THE ABSORBER. $10 \%$ OF RECOVERED LIME IS SENT TO THE ORY SCRUBBER OR TO SECONDARY STABILIZATION.

3. TRACE METALS REMOVED FROM OFFGAS JN THE AIR POLLUTION CONTROL SUBSYSTEM ARE ROUTED TO SECONDARY STABILIZATION AND NDT SHOWN ABOVE.

\begin{tabular}{|c|c|c|c|}
\hline PRIMARY STABILIZATION SUBSYSTEM & SLAG & $\begin{array}{l}\text { PACKAGED } \\
\text { SLAG }\end{array}$ & \\
\hline FLOW RATE (LB/HR) & 1.450 & 1,450 & \\
\hline $\begin{array}{c}\text { MAIN THERMAL TREATMENT } \\
\text { SUBSYSTEM }\end{array}$ & $\begin{array}{l}\text { (1) } \\
\text { COMBUSTIBLE } \\
\text { SOL ID WASTE }\end{array}$ & $\begin{array}{l}\text { NON- } \\
\text { COMBUSTIBLE } \\
\text { WASTE }\end{array}$ & $\begin{array}{l}\text { (3) } \\
\text { METALLIC HASTE } \\
\text { IENTRAINED } \\
\text { CONTAMINATIONI }\end{array}$ \\
\hline FLOW RATE (LB/HR) & 609 & 1.340 & 149 \\
\hline $\begin{array}{c}\text { MAIN THERMAL TREATMENT } \\
\text { AIR POLLUTION } \\
\text { CONTROL SUBSYSTEM }\end{array}$ & $\begin{array}{l}\text { SCC } \\
\text { OFFGAS }\end{array}$ & $\begin{array}{l}\text { (2) } \\
\text { COOLED } \\
\text { OFFGAS }\end{array}$ & $\begin{array}{l}3 \\
\text { FILTERED } \\
\text { OFFGAS }\end{array}$ \\
\hline FLOW RATE (LB/HR) & 3.039 & 4,772 & 4,684 \\
\hline
\end{tabular}

Figure 4-8. Plasma Furnace, $\mathrm{CO}_{2}$ Retention: PFD for Main Thermal Treatment, Air Pollution Control, and Primary Stabilization 


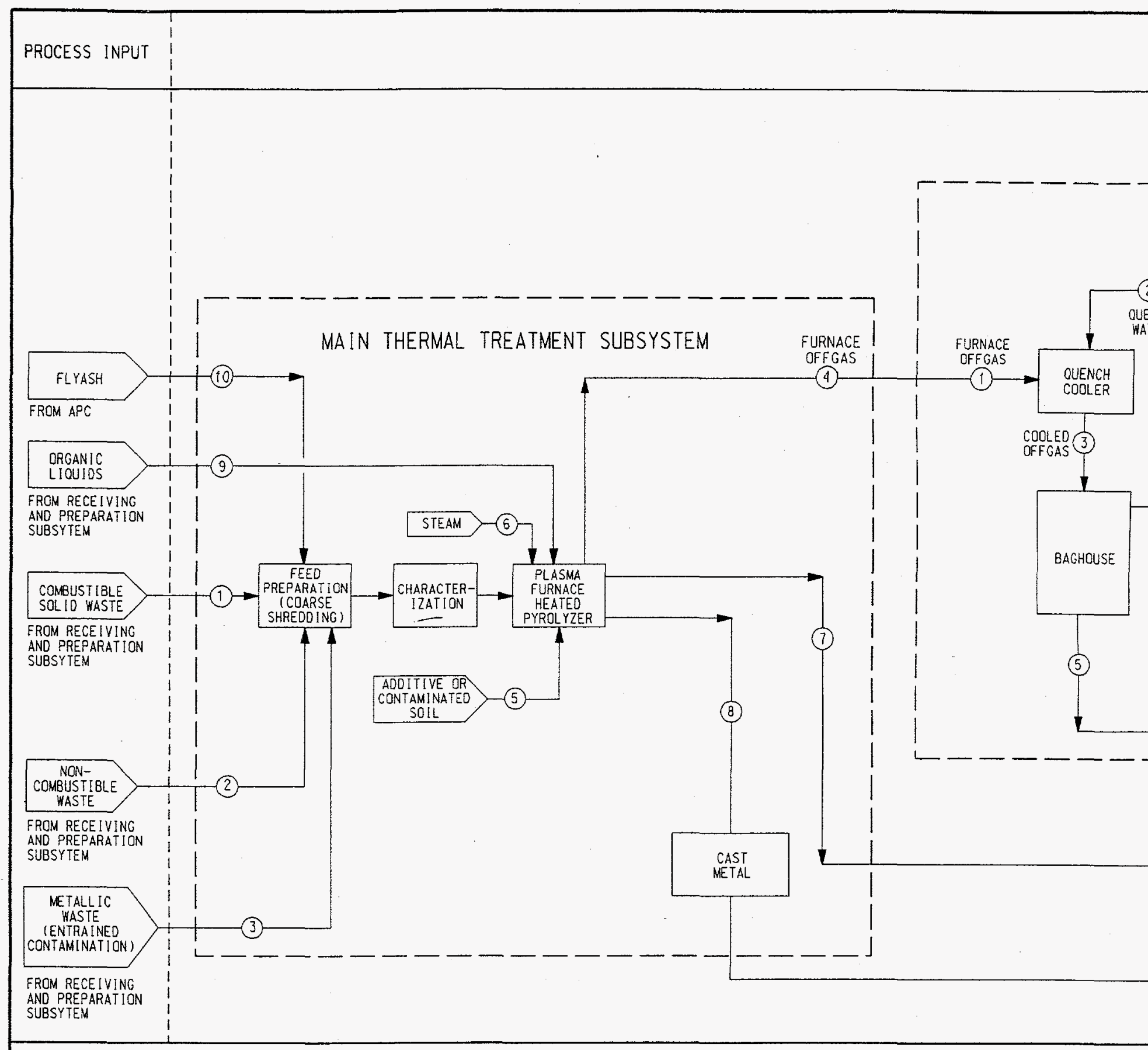

NOTE:

1. ADDIIIVE OR CONTAMINATED SOIL $=50 \%$ OF THE FEED TO PRIMARY STABILIZATION.

2. TRACE METALS REMOVED FROM OFFGAS IN THE AIR POLLUTION CONTROL SUBSYSTEM ARE ROUTED TO SECDNDARY STABILIZATION AND NOT SHOWN ABOVE.

\begin{tabular}{|c|c|c|c|c|}
\hline PRIMARY STABILIZATION SUBSYSTEM & (1) & $\begin{array}{l}\text { (2) } \\
\text { PACKAGED } \\
\text { SLAG }\end{array}$ & & \\
\hline FLOW RATE (LB/HR) & 1,452 & 1.452 & & \\
\hline $\begin{array}{c}\text { MAIN THERMAL TREATMENT } \\
\text { SUBSYSTEM }\end{array}$ & $\begin{array}{c}1 \\
\text { COMBUSTIBLE } \\
\text { SOL IO HASTE }\end{array}$ & $\begin{array}{c}\text { NON- } \\
\text { COMBUSTIBLE } \\
\text { WASTE }\end{array}$ & METAL & \\
\hline FLOW RATE (LB/HR) & 609 & 1,340 & 149 & 1 \\
\hline $\begin{array}{c}\text { MAIN THERMAL TREATMENT } \\
\text { AIR POLLUTION } \\
\text { CONTROL SUBSYSTEM }\end{array}$ & $\begin{array}{c}(1 \\
\text { FURNACE } \\
\text { OFFGAS }\end{array}$ & $\begin{array}{l}\text { (2) } \\
\text { OUENCH } \\
\text { WATER }\end{array}$ & $\begin{array}{l}\text { (3) } \\
\text { COOLED } \\
\text { OFFGAS }\end{array}$ & $\begin{array}{c}F I L \\
\text { OF }\end{array}$ \\
\hline FLOW RATE (LB/HR) & 1.159 & 1.189 & 2.348 & 2 \\
\hline
\end{tabular}

Figure 4-9. Plasma Gasification: PFD for Main Thermal Treatment, Air Pollution Control, and Primary Stabilization Subsysten 


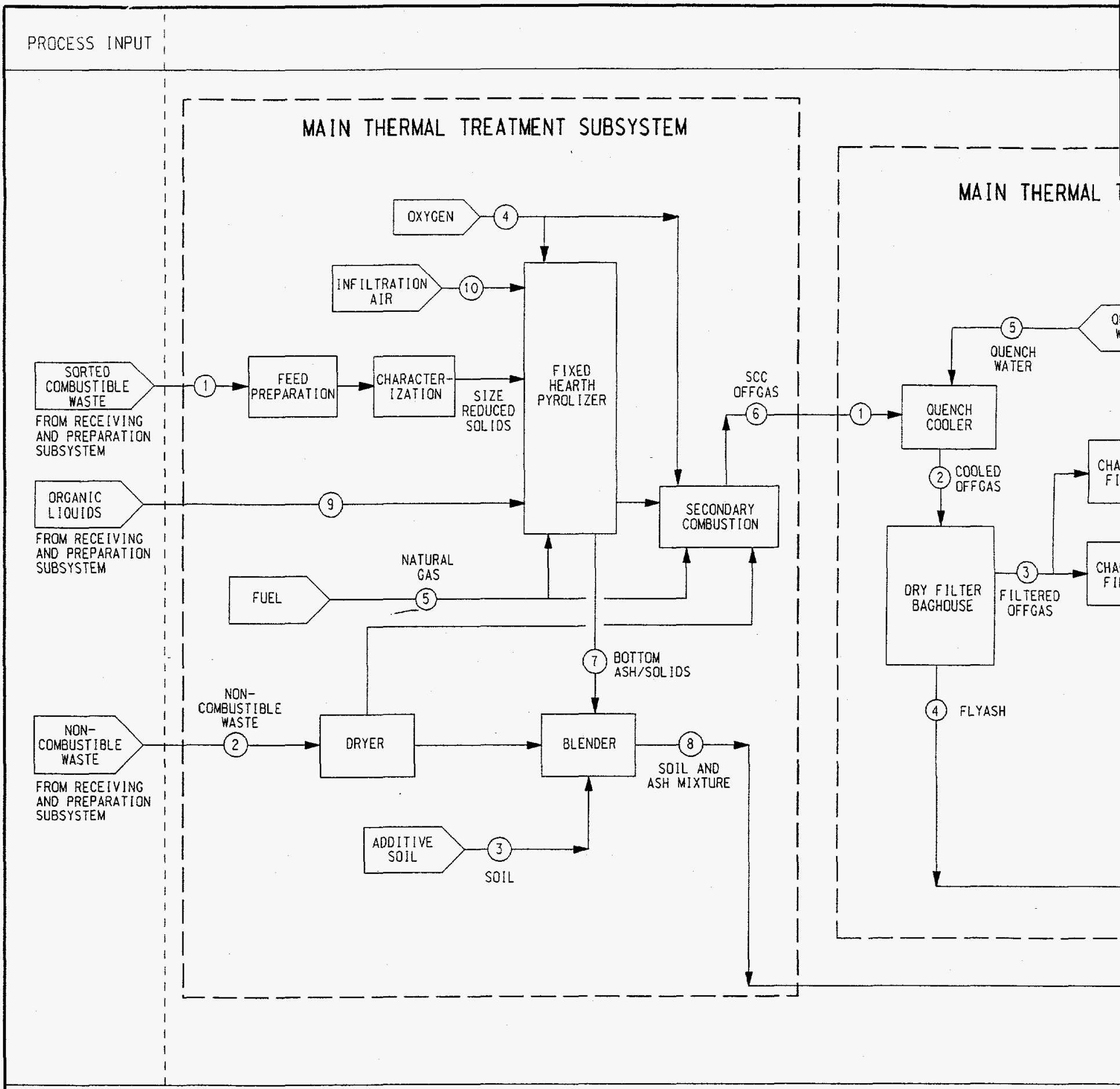

\section{NOIES:}

1. 10\% OF LIME IS DUMPED TO STABILIZATION.

2. $90 \%$ OF LIME IS RECYCLED.

3. ADOITIVE SOIL $=50 \%$ OF THE FEED TO PRIMARY STABILIZATION.

4. TRACE METALS REMOVED FROM OFFGAS IN THE AIR POLLUTION CONTROL SUBSYSTEM ARE ROUTED TO SECONDARY STABILIZATION ANO NOT SHOWN ABOVE.

\begin{tabular}{|c|c|c|c|c|c|}
\hline $\begin{array}{c}\text { MAIN THERMAL TREATMENT } \\
\text { SUBSYSTEM }\end{array}$ & $\begin{array}{l}\text { (1) } \\
\text { COMBUSTIBLE } \\
\text { WASTE }\end{array}$ & $\begin{array}{c}\text { (2) } \\
\text { NON- } \\
\text { COMBUSTIBLE } \\
\text { WASTE }\end{array}$ & $\begin{array}{l}\text { (3) } \\
\text { ADOITIVE } \\
\text { SOIL } \\
\text { (SEE NOTE 3) }\end{array}$ & $\begin{array}{l}\text { (4) } \\
\text { DXYGEN }\end{array}$ & $\begin{array}{l}5 \\
\text { NATURAL } \\
\text { GAS }\end{array}$ \\
\hline FLOW RATE (L8/HR) & 660 & 1,340 & 499 & 1.130 & 1 \\
\hline $\begin{array}{c}\text { MAIN THERMAL TREATMENT } \\
\text { AIR POLLUTION } \\
\text { CONTROL SUBSYSTEM }\end{array}$ & $\begin{array}{c}\text { SCC } \\
\text { OFFGAS }\end{array}$ & $\begin{array}{l}\text { (2) } \\
\text { CODLED } \\
\text { OFFGAS }\end{array}$ & $\begin{array}{l}3 \\
\text { FILTEREO } \\
\text { OFFGAS }\end{array}$ & FLYASH & $\begin{array}{l}\text { (5) } \\
\text { OUENCH } \\
\text { WATER }\end{array}$ \\
\hline FLOW RATE (LB/HR) & 6.196 & 10,421 & 10.397 & 24 & 4,225 \\
\hline
\end{tabular}

Figure 4-10. System D, Fixed Hearth with $\mathrm{CO}_{2}$ Retention: PFD for Incineration and Air Pollution Control Subsystems (Syste 


\section{ATMENT AIR POLLUTION CONTROL SUBSYSTEM}

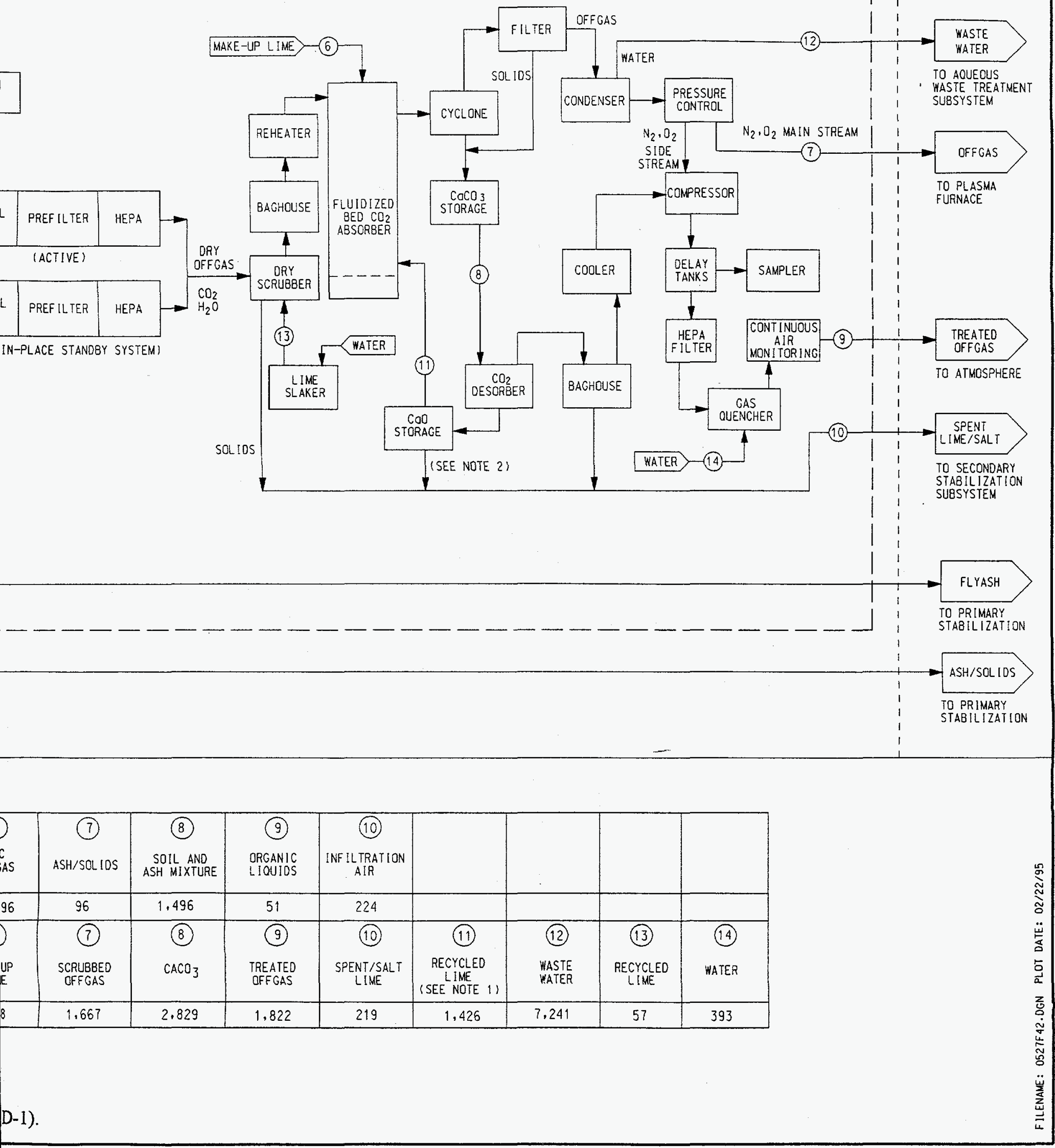




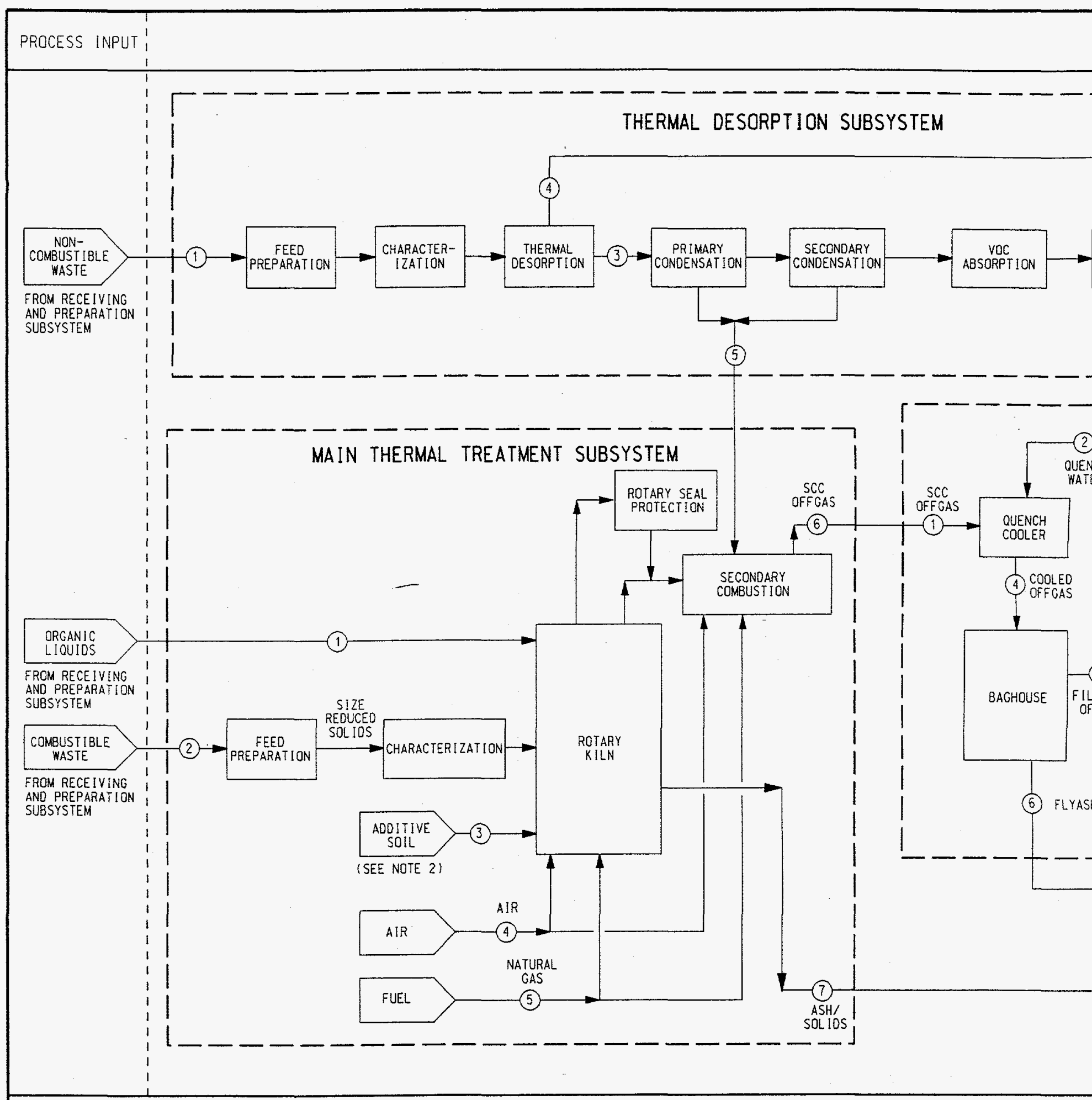

NOTES:

1. ASSUME GROUT TO WASTE RATES:2 TO 1

2. ADDITIVE SOIL $=50 \%$ OF THE FEED TO PRIMARY STABILIZATION.

3. TRACE METALS REMOVED FROM OFFGAS IN THE AIR POLLUTION CONTROL SUBSYSTEM ARE RQUTED TO SECONDARY STABILIZATION AND NOT SHOWN ABOVE.

\begin{tabular}{|c|c|c|c|c|}
\hline $\begin{array}{l}\text { THERMAL DESORPTION } \\
\text { SUBSYSTEM }\end{array}$ & $\begin{array}{c}\text { (1) } \\
\text { NON- } \\
\text { COMBUSTIBLE } \\
\text { WASTE }\end{array}$ & $\begin{array}{l}\text { (2) } \\
\text { TREATED } \\
\text { OFFGAS }\end{array}$ & (3) & $\begin{array}{c}\text { (4) } \\
\text { DEBRIS } \\
\text { TO } \\
\text { GROUTING }\end{array}$ \\
\hline FLOW RATE (LB/HR) & 1.340 & NEGL IGIBLE & 289 & 1.051 \\
\hline $\begin{array}{c}\text { MAIN THERMAL TREATMENT } \\
\text { AIR POLLUTION } \\
\text { CONTROL SUBSYSTEM }\end{array}$ & $\begin{array}{c}1 \\
\text { SCC } \\
\text { OFFGAS }\end{array}$ & $\begin{array}{l}\text { (2) } \\
\text { OUENCH } \\
\text { WATER }\end{array}$ & $\begin{array}{c}\text { (3) } \\
\text { SCRUBBER/ } \\
\text { COOLER } \\
\text { WATER }\end{array}$ & $\begin{array}{l}\text { (4) } \\
\text { COOLED } \\
\text { OFFGAS }\end{array}$ \\
\hline FLOW RATE (LB/HR) & 21.531 & 10,407 & 1.800 & 31.938 \\
\hline
\end{tabular}

Figure 4-11. Thermal Desorption: PFD for Incineration, Thermal Desorber, and Air Pollution Control Subsystems (System E-1) 


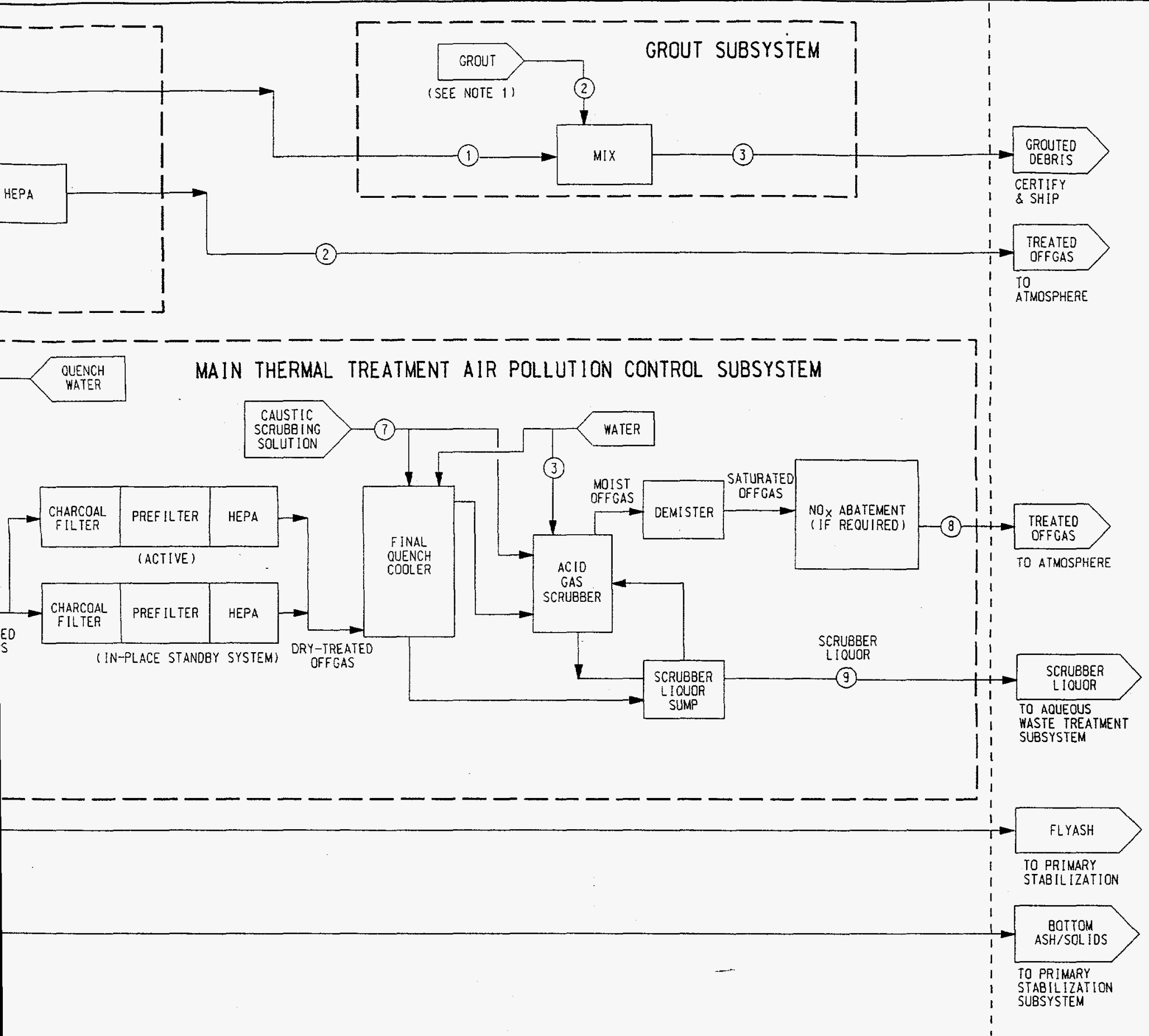

\begin{tabular}{|c|c|c|c|c|c|c|c|c|c|}
\hline \multicolumn{2}{|c|}{$\begin{array}{l}\text { MAIN THERMAL } \\
\text { TREATMENTSUBSYSTEM }\end{array}$} & $\begin{array}{c}1 \\
\text { ORGANIC } \\
\text { LIOUIOS }\end{array}$ & $\begin{array}{c}2 \\
\text { COMBUSTIBLE } \\
\text { KASTE }\end{array}$ & $\begin{array}{c}\text { (3) } \\
\text { ADOI IIVE } \\
\text { SOIL } \\
\text { (SEE NOTE 2) }\end{array}$ & $\begin{array}{l}\text { (4) } \\
\text { AIR }\end{array}$ & $\begin{array}{c}\text { (5) } \\
\text { NATURAL } \\
\text { GAS }\end{array}$ & $\begin{array}{c}\text { SCC } \\
\text { OFFGAS }\end{array}$ & $\begin{array}{c}\text { (7) } \\
\text { ASH/SOL IOS }\end{array}$ & \\
\hline & & 51 & 609 & 54 & 7.766 & 9,286 & 21.531 & 129 & \\
\hline $\begin{array}{c}\text { (6) } \\
\text { FLYASH }\end{array}$ & $\begin{array}{l}(7) \\
\text { CAUSTIC } \\
\text { SOLUTION } \\
150 \% \text { NOOH }\end{array}$ & $\begin{array}{c}\text { (8) } \\
\text { TREATED } \\
\text { OFFGAS }\end{array}$ & $\begin{array}{c}9 \\
\text { SCRUBBER } \\
\text { LIOUOR }\end{array}$ & & & & DEBRIS & GROUT & $\begin{array}{c}\text { (3) } \\
\text { GROUSED } \\
\text { MASTE }\end{array}$ \\
\hline 32 & 128 & 20.267 & 13,566 & & & & 1.051 & 2.102 & 3.969 \\
\hline
\end{tabular}




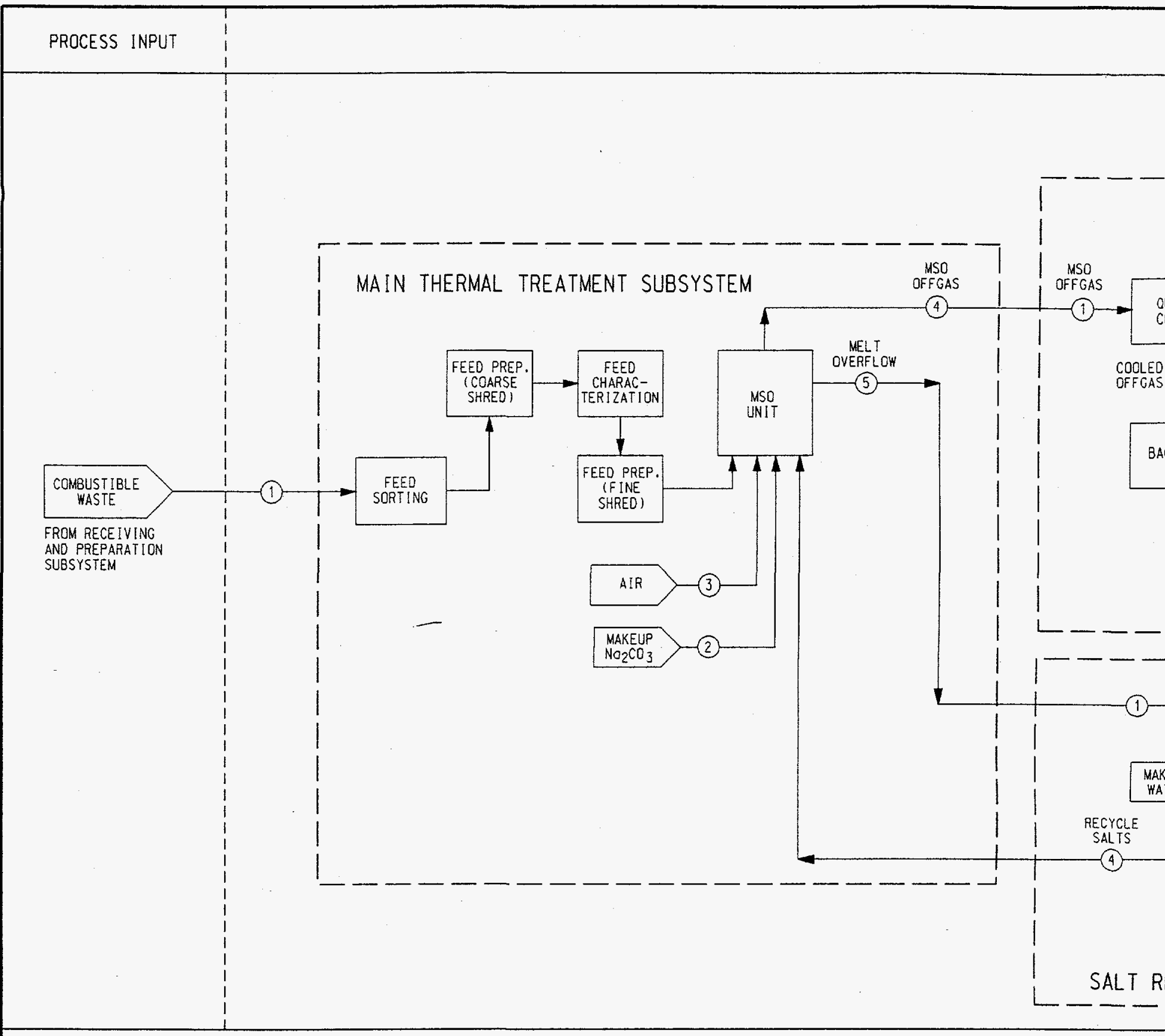

NOTE: TRACE METALS REMOVEO FROM OFFGAS IN THE AIR POLLUTION CONTROL SUBSYSTEM ARE ROUTED TO SECONDARY STABILIZATION AND NOT SHOWN ABOVE.

\begin{tabular}{|c|c|c|c|}
\hline $\begin{array}{l}\text { MAIN THERMAL TREATMENT } \\
\text { SUBSYSTEM- }\end{array}$ & $\begin{array}{c}\text { (1) } \\
\text { COMBUSTIBLE } \\
\text { WASTE }\end{array}$ & $\begin{array}{l}\text { (2) } \\
\text { MAKEUP } \\
\text { SOD IUM } \\
\text { CARBONATE }\end{array}$ & $\begin{array}{l}\text { (3) } \\
\text { AIR }\end{array}$ \\
\hline FLOW RATE (LB/HR) & 660 & 121 & 6.120 \\
\hline SALT RECYCLE SUBSYSTEM & $\begin{array}{l}\text { MELT } \\
\text { OVERFLOW }\end{array}$ & ASH & SALTS \\
\hline FLOW RATE (LB/HR) & 607 & .121 & 127 \\
\hline $\begin{array}{c}\text { MAIN THERMAL TREATMENT } \\
\text { AIR POLLUTION } \\
\text { CONTROL SLBSYSTEM }\end{array}$ & $\begin{array}{l}\text { MSO } \\
\text { OFFGAS }\end{array}$ & $\begin{array}{l}\text { (2) } \\
\text { QUENCH } \\
\text { WATER }\end{array}$ & $\begin{array}{c}\text { (3) } \\
\text { COOLED } \\
\text { OFFGAS }\end{array}$ \\
\hline FLOW RATE (LB/HR) & 6,653 & 2,114 & 8,767 \\
\hline
\end{tabular}

Figure 4-12. Molten Salt Oxidation: PFD for Main Thermal Treatment, Air Pollution Control, and Salt Recycling Subsystems ( 

-(2)
MAIN THERMAL TREATMENT

AIR POLLUTION CONTROL SUBSYSTEM

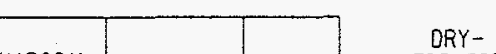

CHARCOAL
FILTER PREF ILTER HEPA $\longrightarrow \begin{gathered}\text { ORY- } \\ \text { TREATED } \\ \text { OFFGAS }\end{gathered}$

(ACTIVE)

CHARCOAL PREFILTER

FILTER

(IN-PLACE STANOBY SYSTEM)

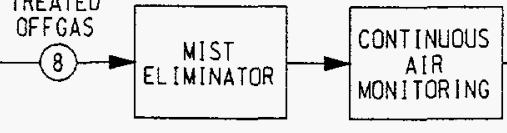

TREATED

OFFGAS

TO ATMOSPHERE

SALT/SOL IDS

(5)

SCRUBBER LIOUOR

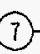

SCRUBBER LIOUOR

TO AOUEOUS WASTE TREATMENT

SALT/ SOLIDS

TO SECONDARY STABILIZATION

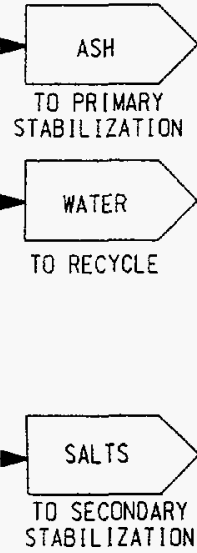

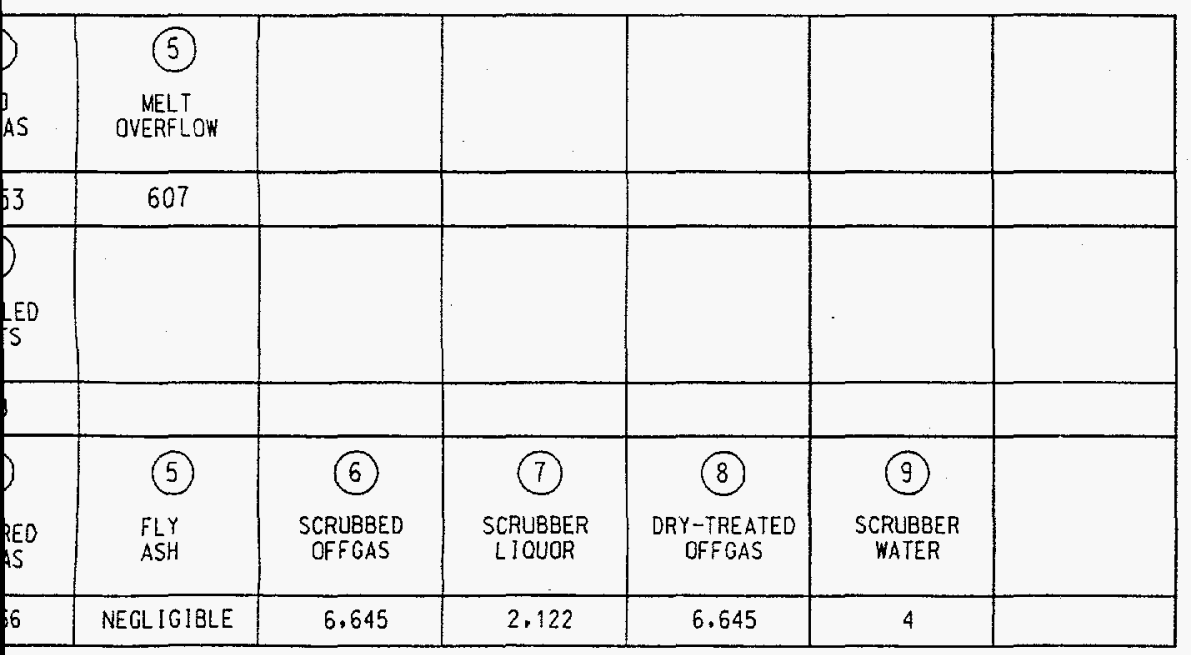

tem F-1) 


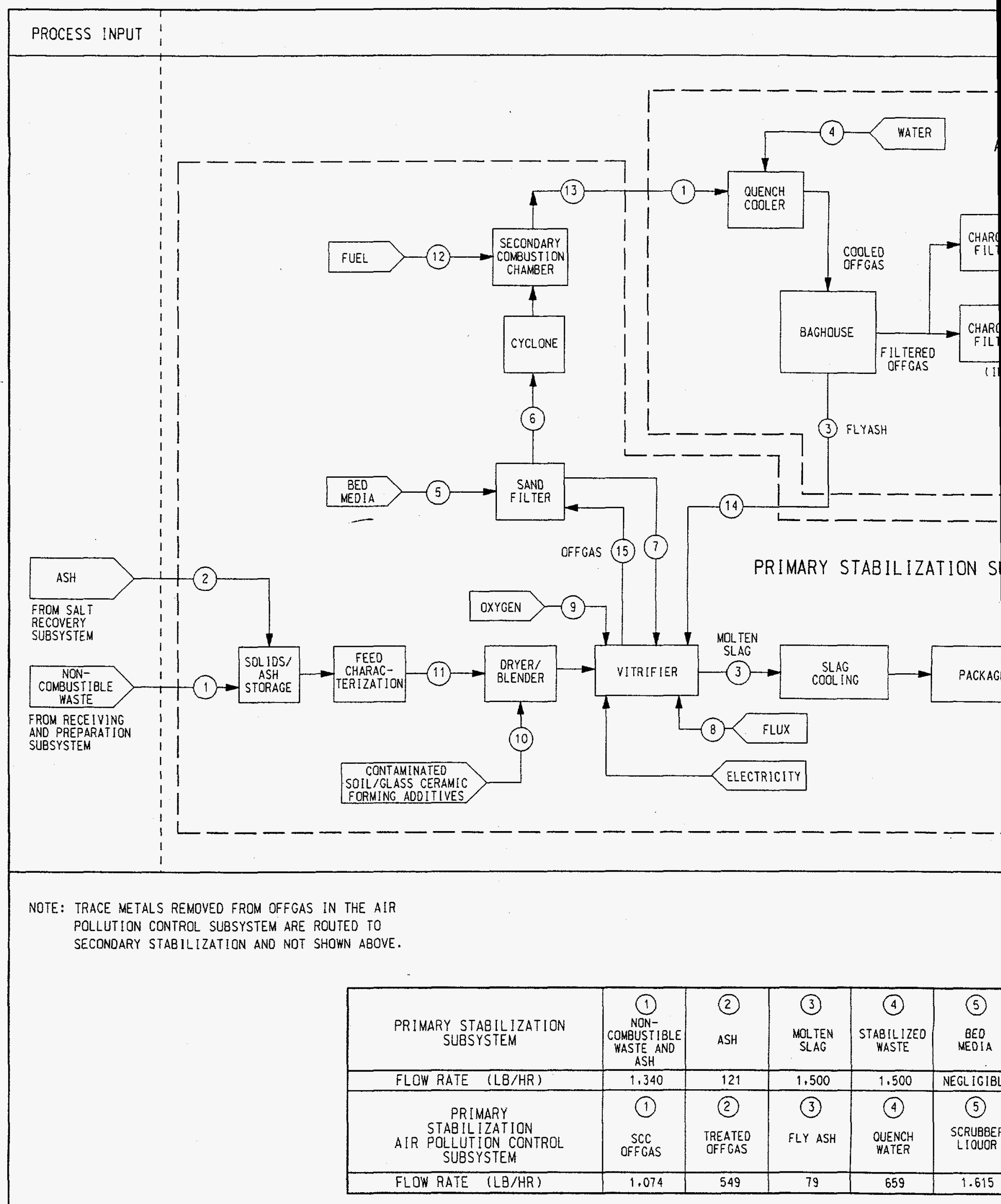

Figure 4-13. Molten Salt Oxidation: PFD for Primary Stabilization Subsystem (System F-1). 
PRIMARY STABILIZATION

POLLUTION CONTROL SUBSYSTEM

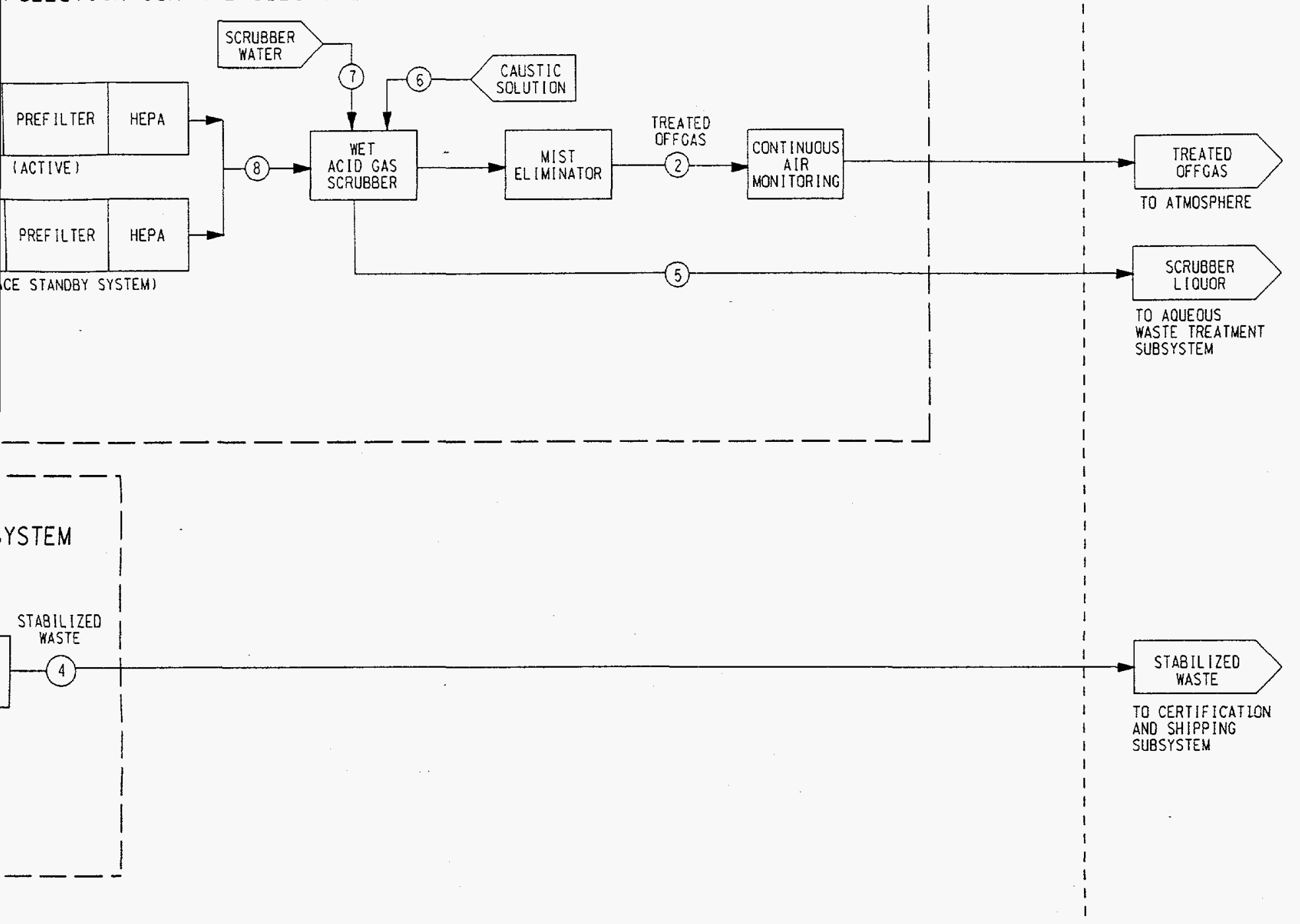

\begin{tabular}{|c|c|c|c|c|c|c|c|c|c|}
\hline OFFGAS & $\begin{array}{c}\text { (1) } \\
\text { SPENT } \\
\text { BEO MEDIA }\end{array}$ & (8) & $\begin{array}{l}\text { (9) } \\
\text { OXYGEN }\end{array}$ & $\begin{array}{l}\text { 10 } \\
\text { CONTAMINATED } \\
\text { SOIL/GLASS } \\
\text { CERAMIC FORMING } \\
\text { ADDITIVES }\end{array}$ & $\begin{array}{l}\text { (11) } \\
\text { VITRIF IER } \\
\text { FEED }\end{array}$ & $\begin{array}{l}\text { (12) } \\
\text { FUEL }\end{array}$ & $\begin{array}{l}\text { (13) } \\
\text { SCC } \\
\text { OFFGAS }\end{array}$ & $\begin{array}{l}\text { (14) } \\
\text { FLY ASH }\end{array}$ & $\begin{array}{l}\text { (15) } \\
\text { OFFGAS }\end{array}$ \\
\hline 554 & NEGLIGIBLE & NEGLIGIBLE & 15 & 500 & 1,461 & 520 & 1.074 & 79 & 554 \\
\hline (6) & (7) & (8) & & & & & & & \\
\hline $\begin{array}{l}\text { CAUSTIC } \\
\text { SOLUITION } \\
50 \% \text { NoOH }\end{array}$ & $\begin{array}{c}\text { SCRUBBER } \\
\text { HATER }\end{array}$ & $\begin{array}{l}\text { FULTERED } \\
\text { OFFGAS }\end{array}$ & & & & & & & \\
\hline 34 & 477 & 1.654 & & & & & & & \\
\hline
\end{tabular}




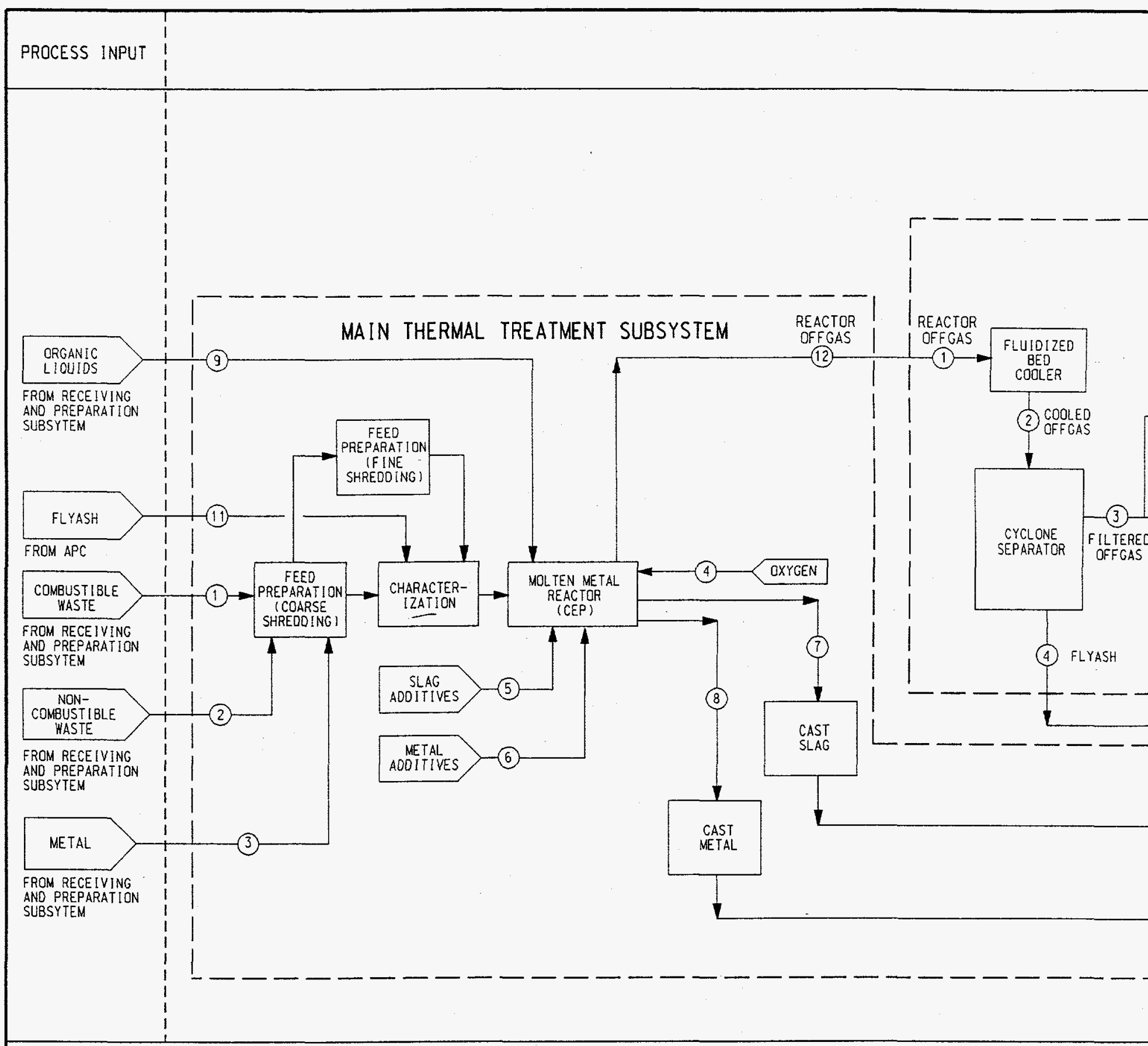

NOTE: TRACE METALS REMOVEO FROM OFFGAS IN THE AIR

POLLUTION CONTROL SUBSYSTEM ARE ROUTED TO SECONDARY

STABILIZATION AND NOT SHOWN ABOVE.

\begin{tabular}{|c|c|c|c|c|c|}
\hline $\begin{array}{c}\text { MAIN THERMAL TREATMENT } \\
\text { SUBSYSTEM }\end{array}$ & $\begin{array}{l}\text { (1) } \\
\text { COMBUSTIBLE } \\
\text { WASTE }\end{array}$ & $\begin{array}{l}\text { NON- } \\
\text { COMBUSTIBLE } \\
\text { WASTE }\end{array}$ & METAL & DXYGEN & SDDIT \\
\hline FLOW RATE (LB/HR) & 609 & 1.340 & 617 & 79 & 52 \\
\hline $\begin{array}{c}\text { MAIN THERMAL TREATMENT } \\
\text { AIR POLLUTION } \\
\text { CONTROL SUBSYSTEM }\end{array}$ & $\begin{array}{l}\text { (1) } \\
\text { REACTOR } \\
\text { OFFGAS }\end{array}$ & $\begin{array}{l}(2) \\
\text { COOLED } \\
\text { OFFGAS }\end{array}$ & $\begin{array}{l}3 \\
\text { FILTEREO } \\
\text { OFFGAS }\end{array}$ & FLYASH & \\
\hline FLOW RATE (LB/HR) & 1,467 & 1,467 & 1.084 & 383 & 10 \\
\hline
\end{tabular}

Figure 4-14. Molten Metal Waste Destruction: PFD for Main Thermal Treatment, Air Pollution Control, and Primary Stabilize 


\section{MAIN THERMAL TREATMENT AIR POLLUTION CONTROL SUBSYSTEM}

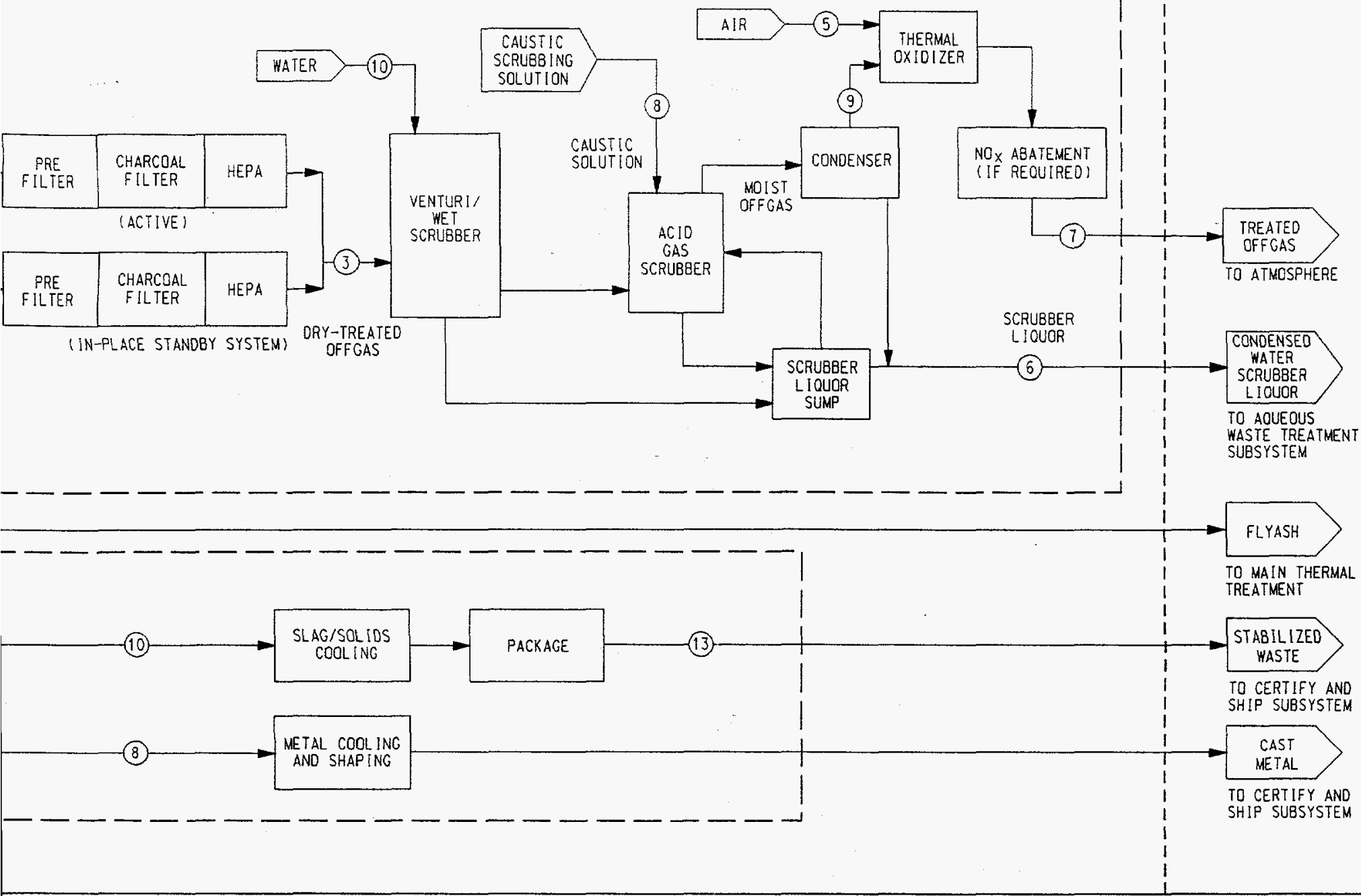

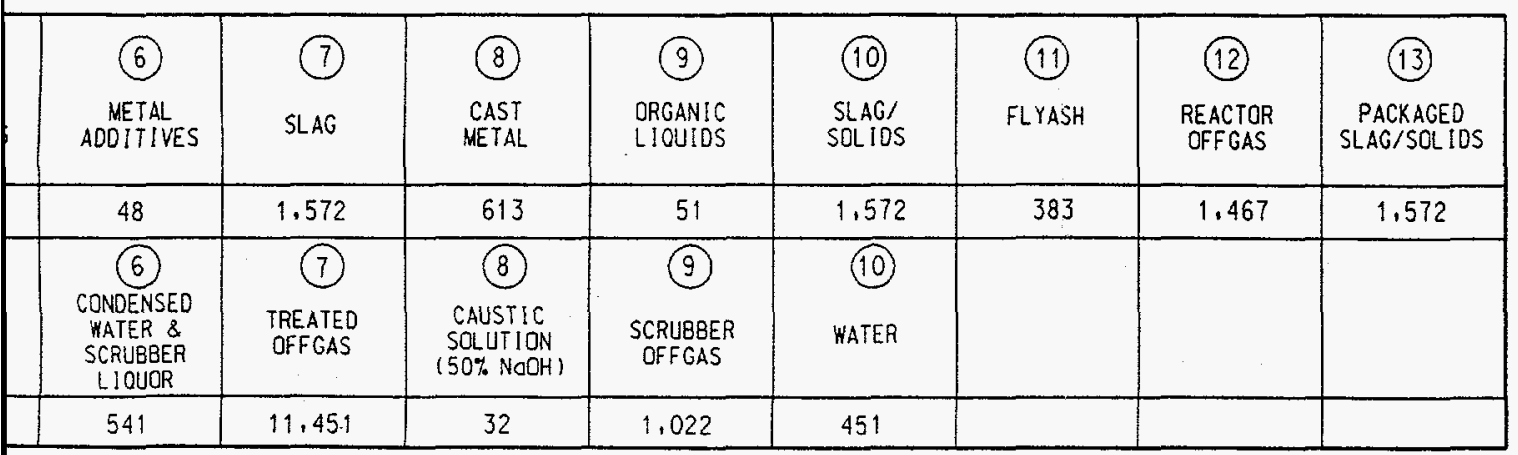




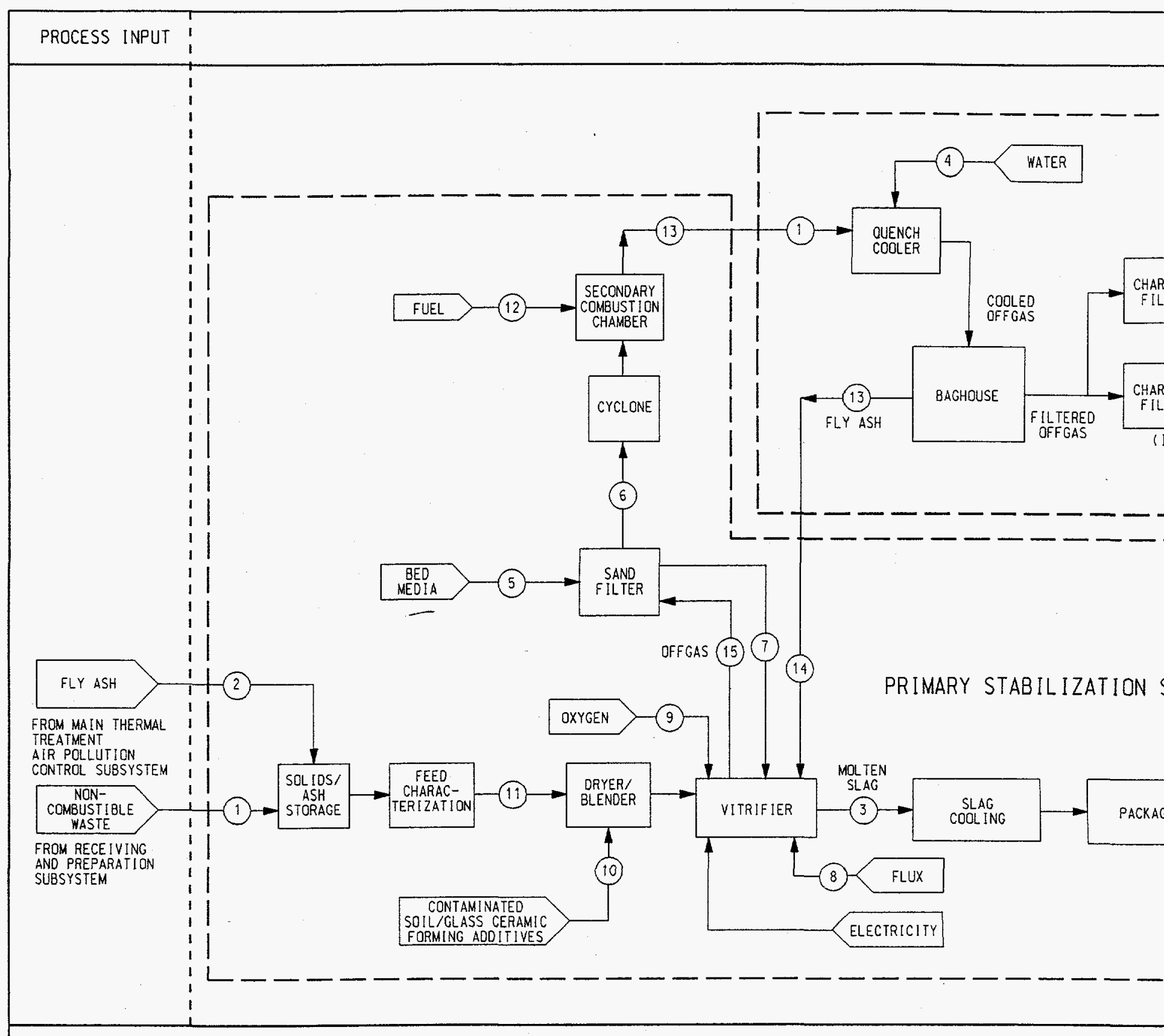

NOTE: TRACE METALS REMOVED FROM OFFGAS IN THE AIR POLLUTION CONTROL SUBSYSTEM ARE ROUTED TO SECONOARY STABILIZATION AND NOT SHOWN ABOVE.

\begin{tabular}{|c|c|c|c|c|c|c|}
\hline $\begin{array}{c}\text { PRIMARY STABILIZATION } \\
\text { SUBSYSTEM }\end{array}$ & $\begin{array}{c}1 \\
\text { NON- } \\
\text { COMBUSTIBLE } \\
\text { WASTE }\end{array}$ & $\begin{array}{l}2 \\
\text { FLY ASH }\end{array}$ & $\begin{array}{l}\text { (3) } \\
\text { MLLTEN } \\
\text { SLAG }\end{array}$ & $\begin{array}{l}\text { (4) } \\
\text { STABILIZED } \\
\text { HASTE }\end{array}$ & $\begin{array}{c}5 \\
\text { BED } \\
\text { MEDIA }\end{array}$ & \\
\hline FLOW RATE (LB/HR) & 1.340 & 150 & 1.493 & 1.493 & NEGLIGIBLE & \\
\hline $\begin{array}{c}\text { PRIMARY } \\
\text { STABILIZATION } \\
\text { AIR POLLUTION CONTROL } \\
\text { SUBSYSTEM }\end{array}$ & $\begin{array}{l}0 \\
\text { SCC } \\
\text { OFFGAS }\end{array}$ & $\begin{array}{l}(2) \\
\text { TREATED } \\
\text { OFFGAS }\end{array}$ & $\begin{array}{l}\text { (3) } \\
\text { FLY ASH }\end{array}$ & $\begin{array}{l}\text { (4) } \\
\text { QLENCH } \\
\text { HATER }\end{array}$ & $\begin{array}{c}5 \\
\text { SCRUB8ER } \\
\text { LLOUOR }\end{array}$ & $\begin{array}{l}6 \\
\text { CAUS } \\
\text { SOLU) } \\
150 \%\end{array}$ \\
\hline FLOW RATE (LB/HR) & 1,277 & 754 & 79 & 751 & 1.706 & \\
\hline
\end{tabular}

Figure 4-15. Steam Gasification: PFD for Primary Stabilization Subsystem (System H-1). 


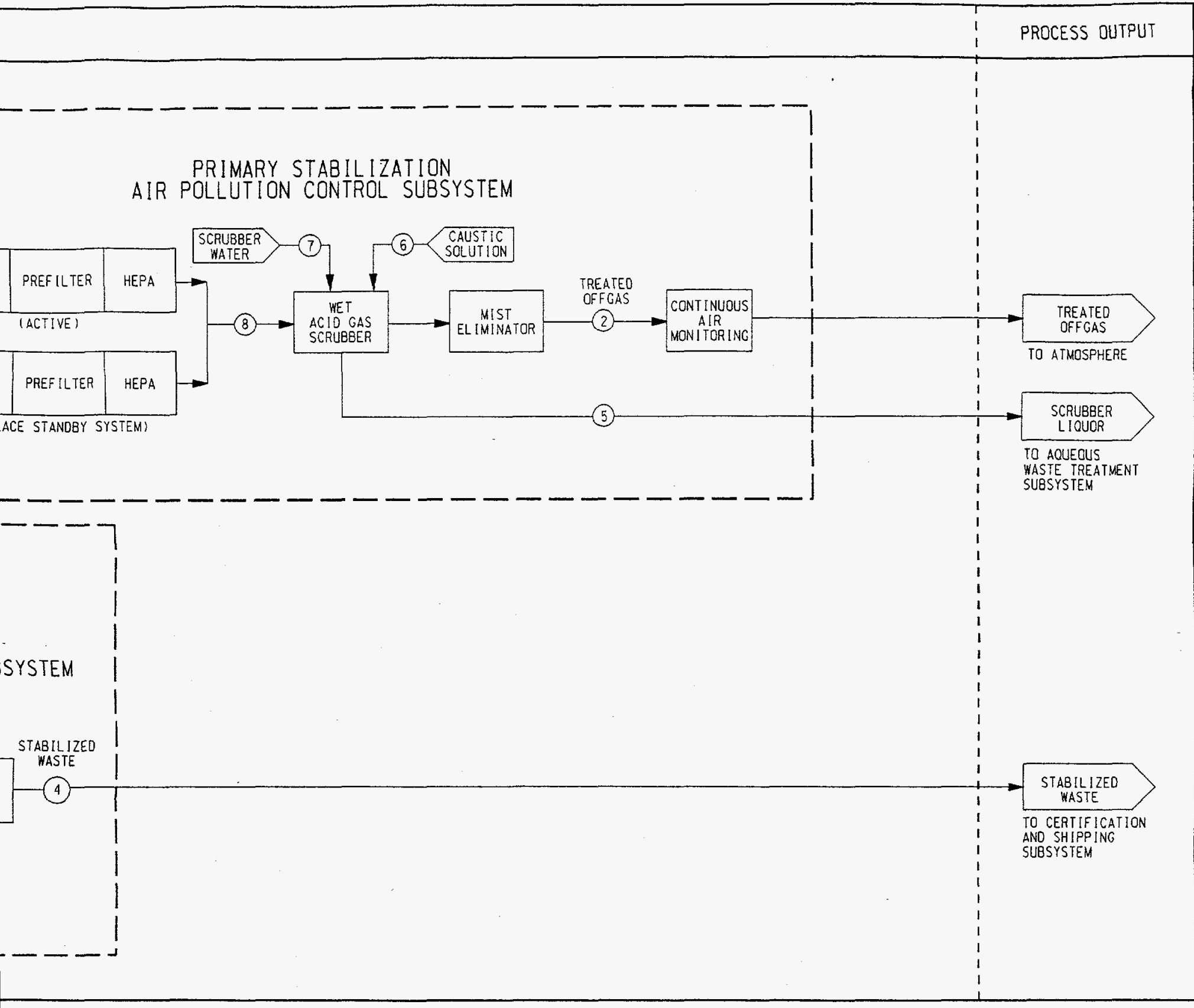

\begin{tabular}{|c|c|c|c|c|c|c|c|c|}
\hline $\begin{array}{c}\text { (7) } \\
\text { SPENT } \\
\text { BED MEDIA }\end{array}$ & (8) & $\begin{array}{l}\text { (9) } \\
\text { OXYGEN }\end{array}$ & $\begin{array}{l}\text { (10) } \\
\text { CONTAMINATED } \\
\text { SOIL/GLASS } \\
\text { CERAMIC FORMING } \\
\text { ADDITIVES }\end{array}$ & $\begin{array}{c}\text { (11) } \\
\text { VITRIFIER } \\
\text { FEED }\end{array}$ & (12) & $\begin{array}{l}\text { (13) } \\
\text { SCC } \\
\text { OFFGAS }\end{array}$ & $\begin{array}{l}\text { (14) } \\
\text { FLY ASH }\end{array}$ & $\begin{array}{l}\text { (15) } \\
\text { OFFGAS }\end{array}$ \\
\hline NEGL IGIBLE & NEGLIGIBLE & 118 & 498 & 1.490 & 586 & 1.277 & 79 & 691 \\
\hline $\begin{array}{c}\text { (7) } \\
\text { SCRUBBER } \\
\text { WATER }\end{array}$ & $\begin{array}{c}\text { (8) } \\
\text { FLLTERED } \\
\text { OFFGAS }\end{array}$ & & & & & & & \\
\hline 477 & 1,949 & & & & & & & \\
\hline
\end{tabular}




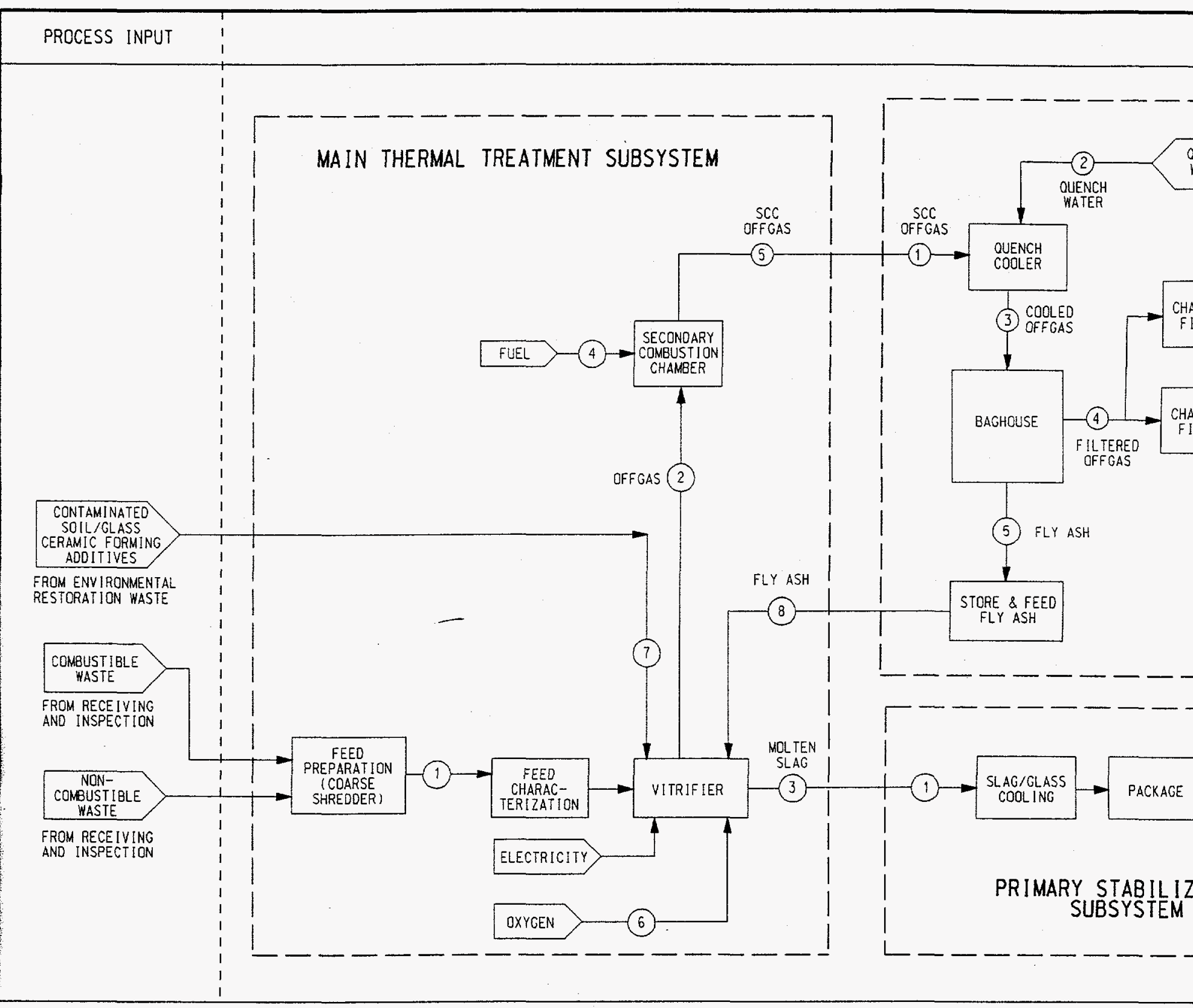

NOTE: TRACE METALS REMOVED FROM OFFGAS IN THE AIR POLLUTION CONTROL SUBSYSTEM ARE ROUTED TO SECONDARY STABILIZATION AND NOT SHOWN ABOVE.

\begin{tabular}{|c|c|c|c|c|c|c|}
\hline $\begin{array}{c}\text { MAIN THERMAL TREATMENT } \\
\text { SUBSYSTEM }\end{array}$ & $\begin{array}{c}\text { (1) } \\
\text { PREPARED } \\
\text { WASTE FEED }\end{array}$ & $\begin{array}{c}(2) \\
\text { OFFGAS }\end{array}$ & $\begin{array}{l}\text { (3) } \\
\text { MOLTEN } \\
\text { SLAG }\end{array}$ & $\begin{array}{l}\text { (4) } \\
\text { FUEL }\end{array}$ & $\begin{array}{l}\text { (5) } \\
\text { SCC } \\
\text { OFFGAS }\end{array}$ & $\begin{array}{l}6 \\
\text { OXYGE }\end{array}$ \\
\hline FLOW RATE (LB/HR) & 2.000 & 2,497 & 1,494 & 1.742 & 4,239 & 1,4 \\
\hline $\begin{array}{c}\text { MAIN THERMAL TREATMENT } \\
\text { AIR POLLUTIDN } \\
\text { CONTROL SUBSYSTEM }\end{array}$ & $\begin{array}{c}1 \\
\text { SCC } \\
\text { OFFGAS }\end{array}$ & $\begin{array}{l}\text { (2) } \\
\text { QUENCH } \\
\text { WATER }\end{array}$ & $\begin{array}{c}(3) \\
\text { COOLED } \\
\text { OFFGAS }\end{array}$ & $\begin{array}{c}\text { (4) } \\
\text { FILTERED } \\
\text { OFFGAS }\end{array}$ & $\begin{array}{l}\text { (5) } \\
F L Y \\
\text { ASH }\end{array}$ & $\begin{array}{l}\text { (6) } \\
\text { CAUST } \\
\text { SOLUTI } \\
150 \% \mathrm{NC}\end{array}$ \\
\hline FLOH RATE (LB/HR) & 4,239 & 2,293 & 6.523 & 6.449 & 79 & 154 \\
\hline
\end{tabular}

Figure 4-16. Joule-heated Vitrification: PFD for Main Thermal Treatment, Air Pollution Control, and Primary Stabilization Subsy 


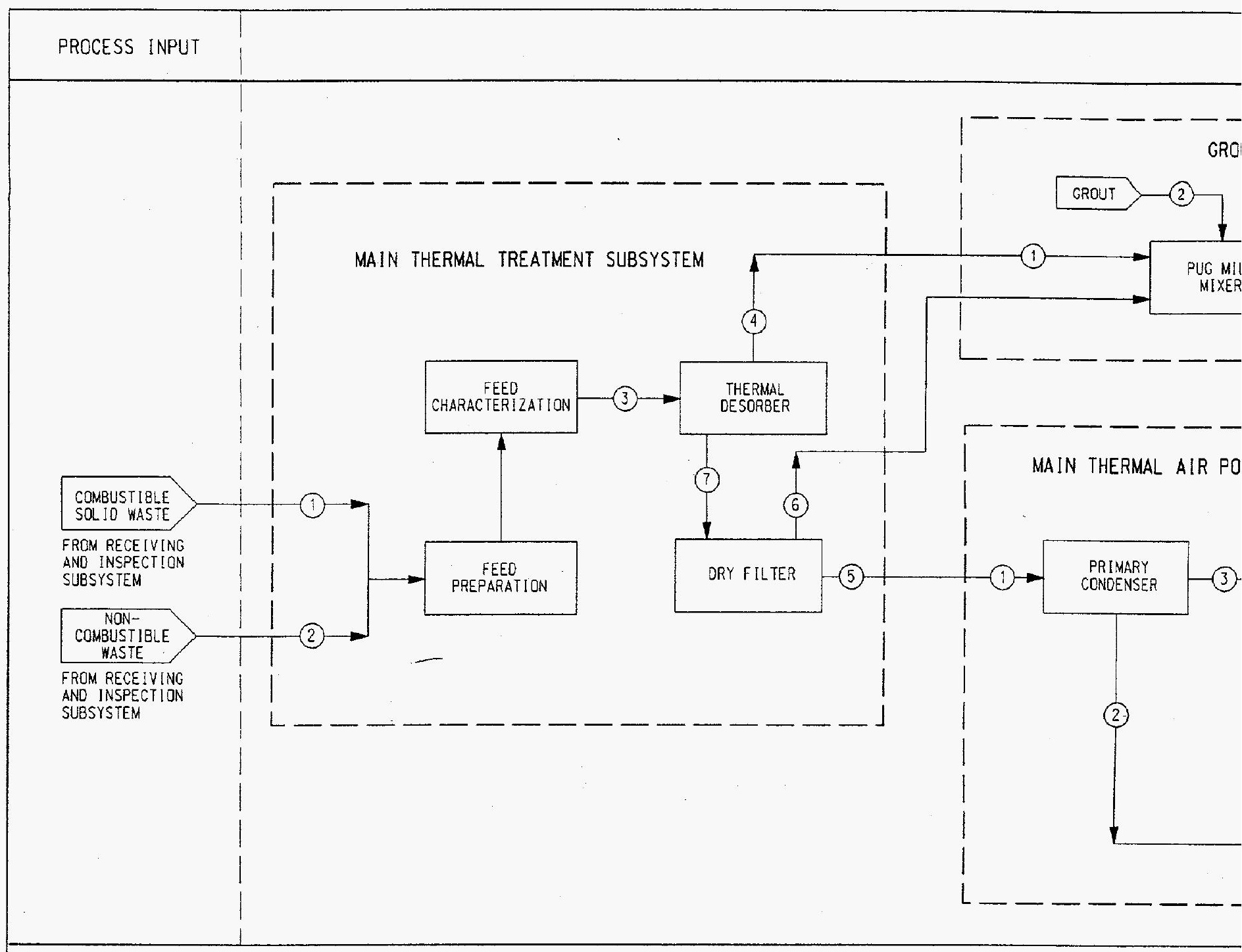

NOTE: TRACE METALS REMOVED FROM OFFGAS IN THE AIR POLLUTION CONTROL SUBSYSTEM ARE ROUTED TO SECONDARY STABILIZATION AND NOT SHOHN ABOVE.

\begin{tabular}{|c|c|c|c|}
\hline $\begin{array}{l}\text { MAIN THERMAL } \\
\text { TREATMENT } \\
\text { SUBSYSTEM }\end{array}$ & $\begin{array}{l}\text { COMBUSTIBLE } \\
\text { SOL ID WASTE }\end{array}$ & $\begin{array}{l}\text { NON- } \\
\text { CONBUSTIBLE } \\
\text { WASTE }\end{array}$ & \\
\hline FLOW RATE (LB/HR) & 609 & 1,340 & 1 \\
\hline $\begin{array}{l}\text { AIR POLLUTION } \\
\text { CONTROL } \\
\text { SUBSYSTEM }\end{array}$ & $\begin{array}{l}\text { (1) } \\
\text { THERMAL } \\
\text { OESORBER } \\
\text { OFFGAS }\end{array}$ & $\begin{array}{l}\text { (2) } \\
\text { LIOUID } \\
\text { MERCURY } \\
\text { WASTE }\end{array}$ & $\begin{array}{r}\text { UNCON } \\
\text { GA }\end{array}$ \\
\hline FLOW RATE (LB/HR) & 399 & NEGL IGIBLE & \\
\hline $\begin{array}{l}\text { GRQUT } \\
\text { STABILIZATION } \\
\text { SUBSYSTEM }\end{array}$ & $\begin{array}{l}\text { DESORBED } \\
\text { SOLIDS }\end{array}$ & GROUT & $\begin{array}{l}\text { GRO } \\
\text { WA }\end{array}$ \\
\hline FLOW RATE (LB/HR) & 1.550 & 3.100 & \\
\hline
\end{tabular}

Figure 4-17. Thermal Desorption and Mediated Electrochemical Oxidation: PFD for Main Thermal Treatment and Air Pol 


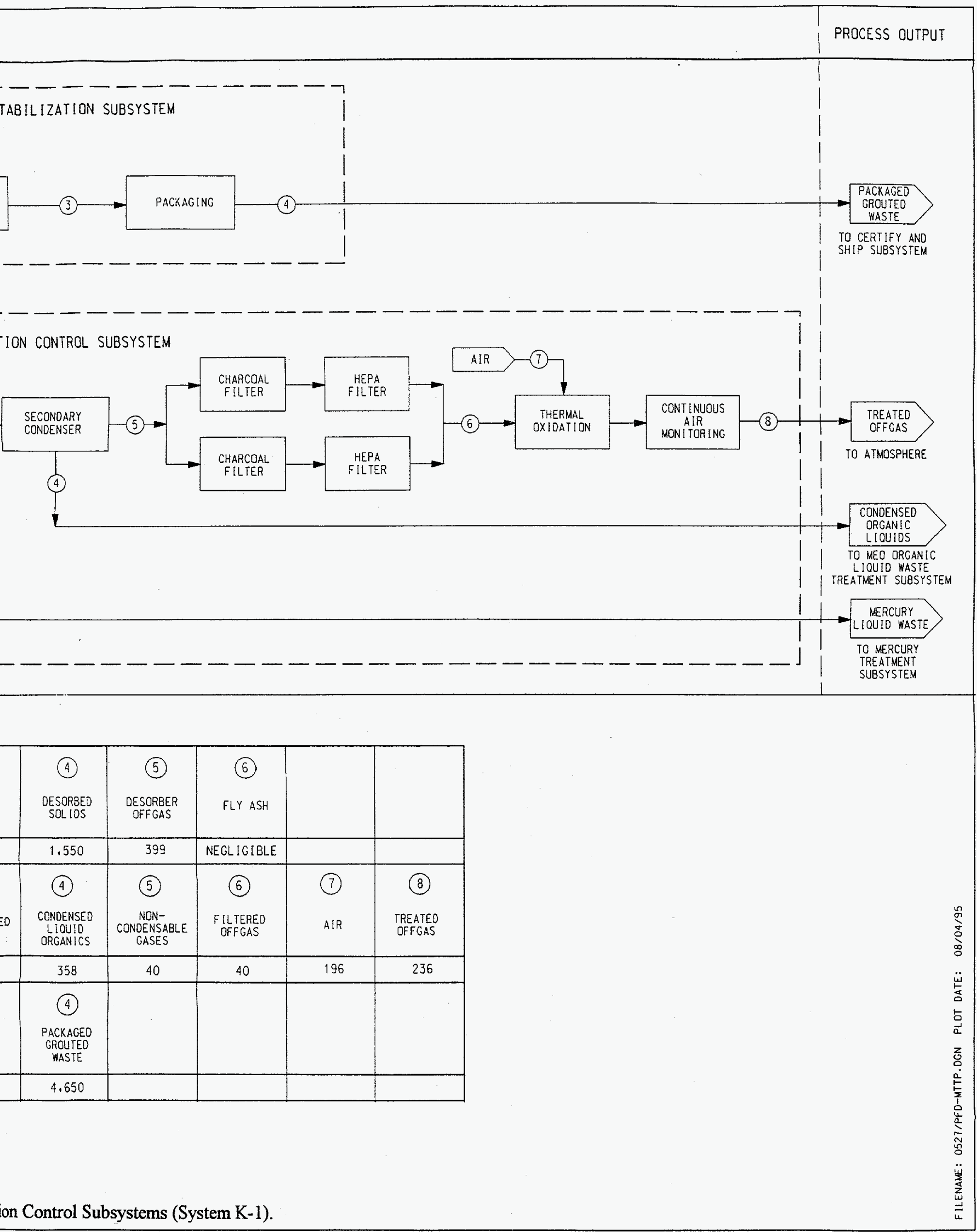




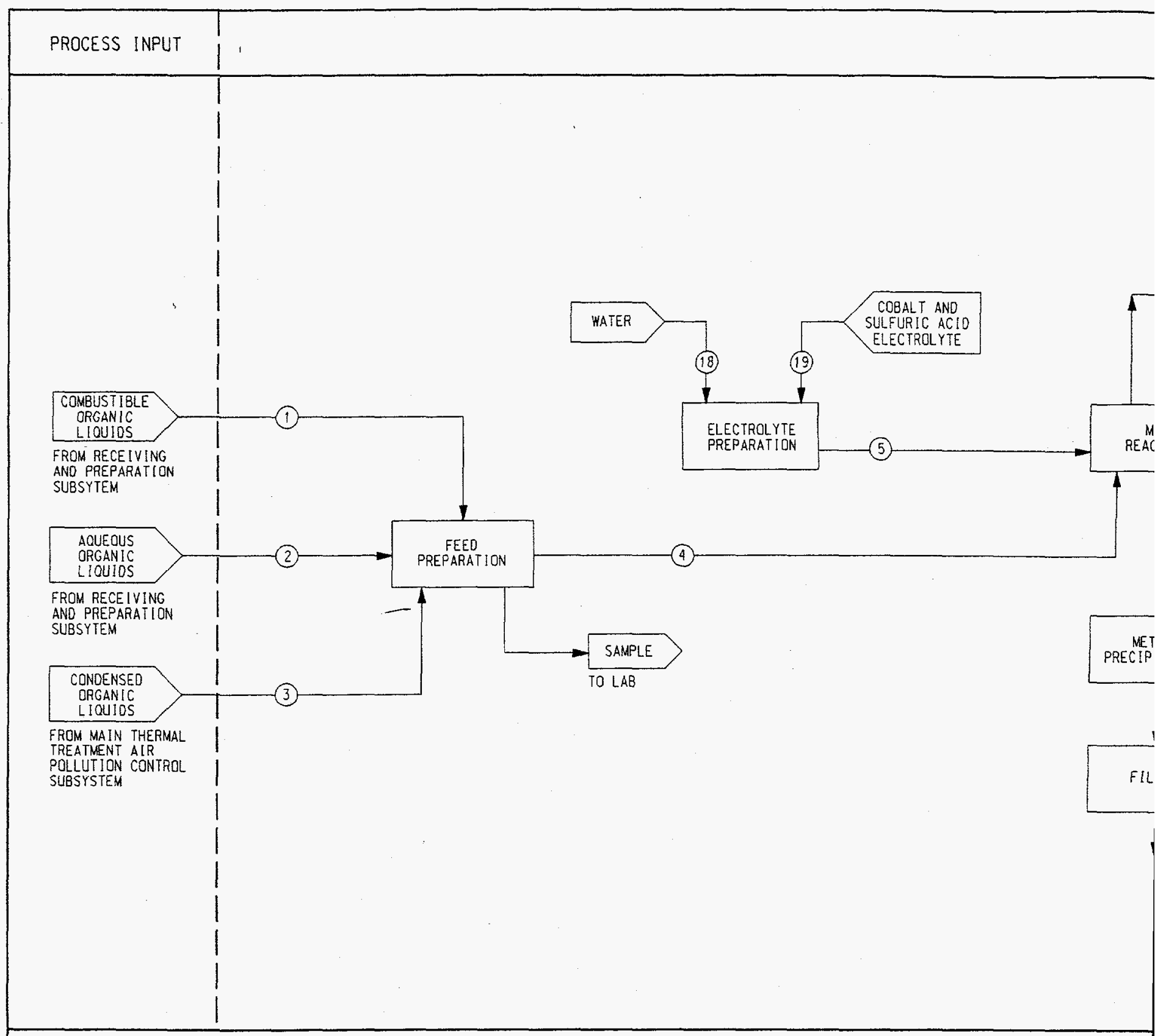

NOTE: TRACE METALS REMOVED FROM OFFGAS IN THE AIR POLLUTION CONTRCL SUBSYSTEM ARE ROUTED TO SECONDARY STABILIZATION AND NOT SHOWN ABOVE.

\begin{tabular}{|c|c|c|c|c|}
\hline $\begin{array}{c}\text { ORGANIC LIOUID } \\
\text { WASTE TREATMENT } \\
\text { SUBSYSTEM }\end{array}$ & $\begin{array}{c}\text { COMBUSTIQLE } \\
\text { ORGANIC } \\
\text { LIQUIOS }\end{array}$ & $\begin{array}{c}\text { AOUEOUS } \\
\text { ORGANIC } \\
\text { LIOUIDS }\end{array}$ & $\begin{array}{c}\text { CONOENSED } \\
\text { ORGANIC } \\
\text { LIOUIOS }\end{array}$ & $\begin{array}{c}\text { ORGANIC } \\
\text { LIOUIDS } \\
\text { FEED }\end{array}$ \\
\hline FLOW RATE (LB/HR) & 51 & 16 & 358 & 425 \\
\hline $\begin{array}{c}\text { ORGANIC LIOUID } \\
\text { WASTE TREATMENT } \\
\text { SUBSYSTEM }\end{array}$ & $\begin{array}{c}\text { TREATED } \\
\text { OFFGAS }\end{array}$ & $150 \%$ & 13 & 14 \\
\hline SOOH & SOLUTION: & FILTRATE & $\begin{array}{c}\text { HYPOCHLORI } \\
\text { SOLUTION }\end{array}$ \\
\hline FLOW RATE (LB/HR) & 3.170 & 62 & 1.058 & 950 \\
\hline
\end{tabular}

Figure 4-18. Thermal Desorption and Mediated Electrochemical Oxidation: PFD for Organic Liquid Waste Treatment Su 


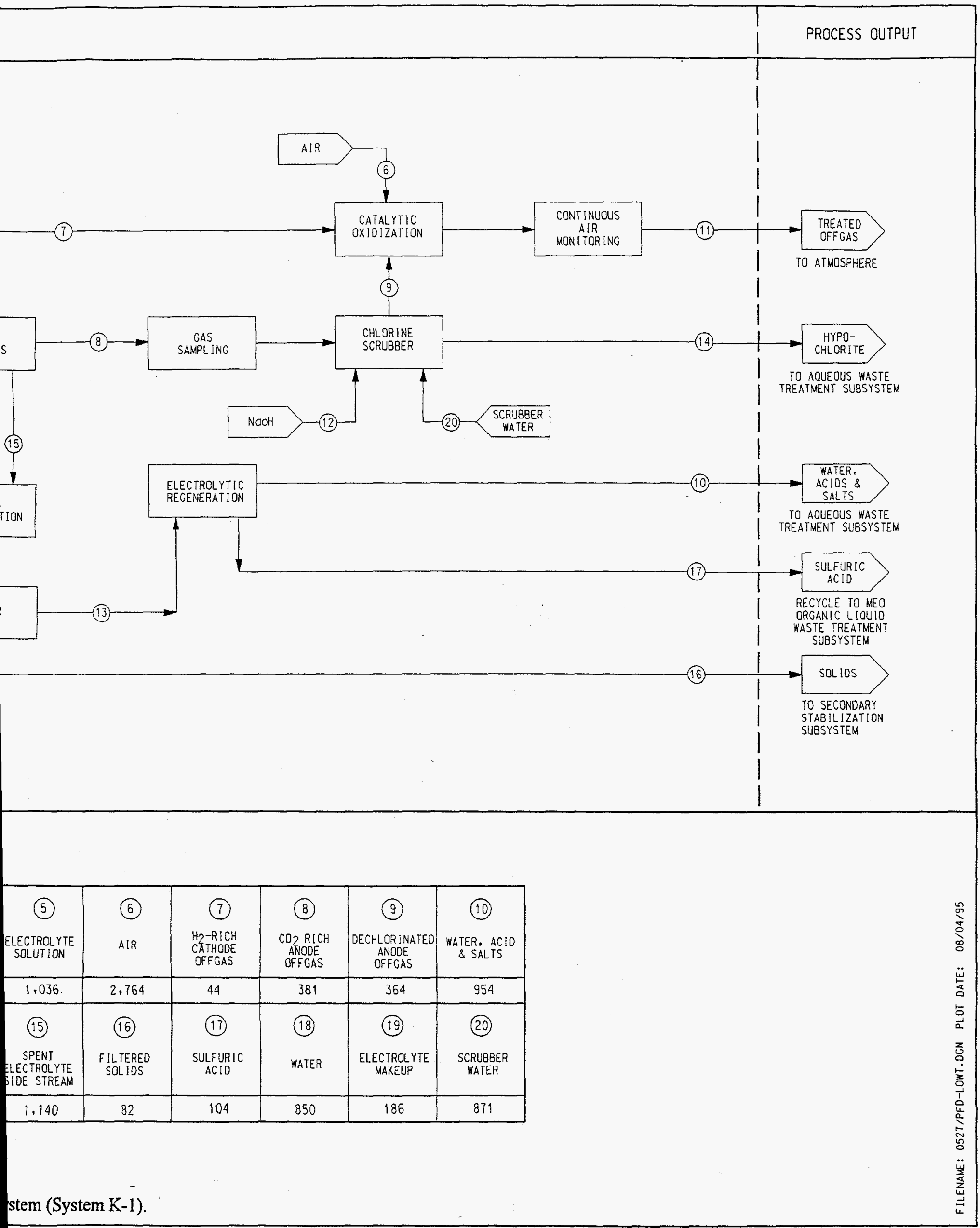




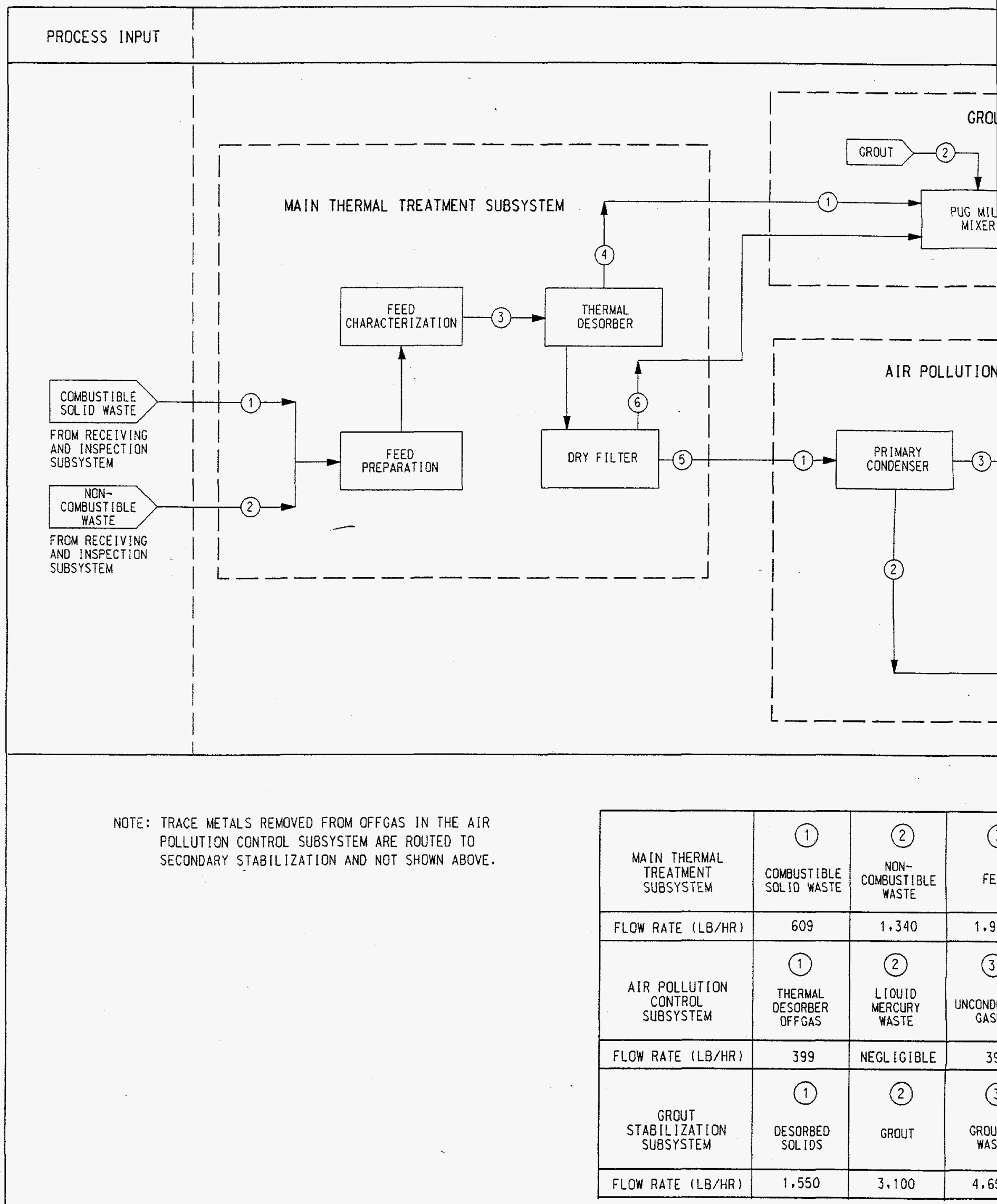

Figure 4-19. Thermal Desorption and Supercritical Water Oxidation: PFD for Main Thermal Treatment and Air Pollution 


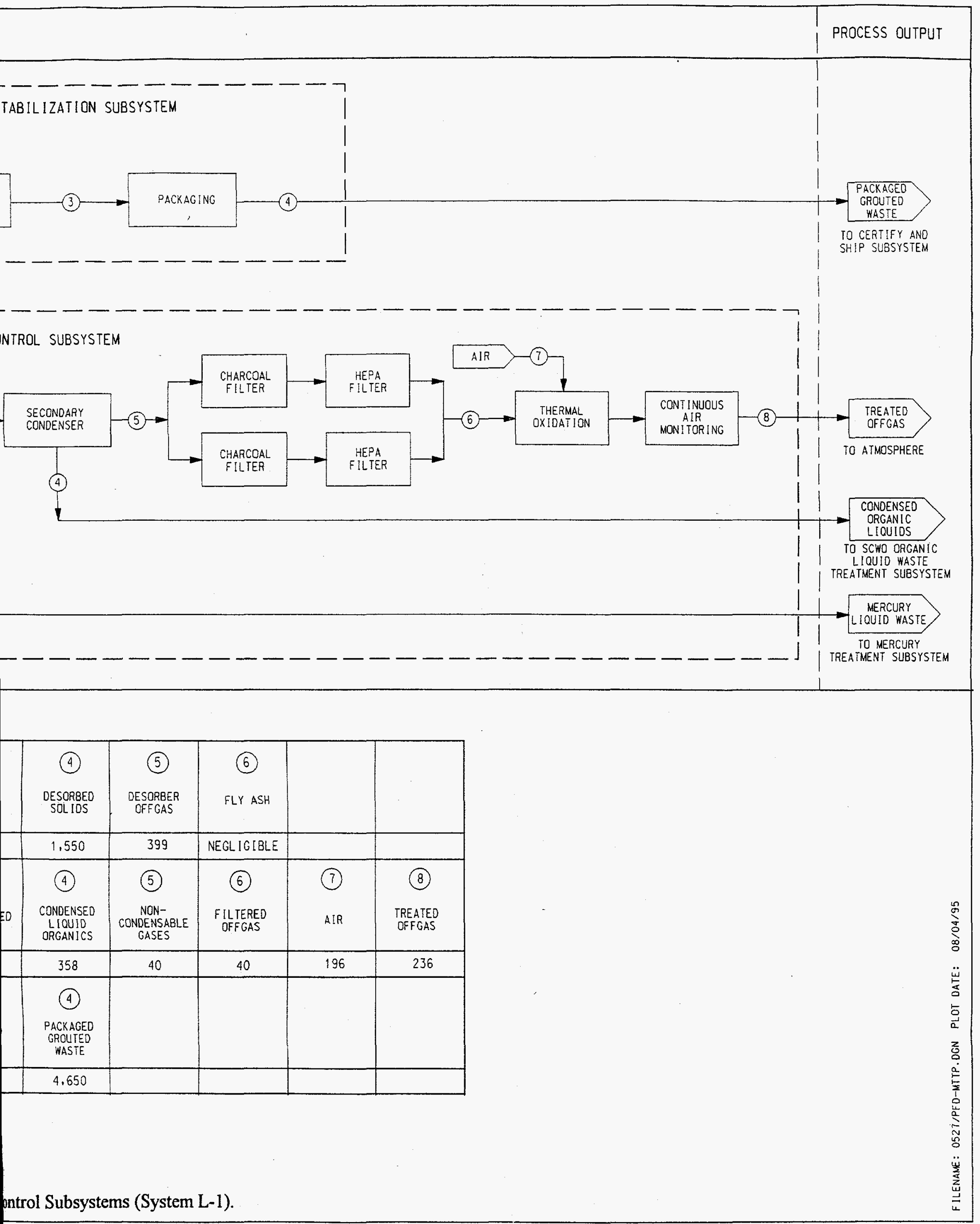




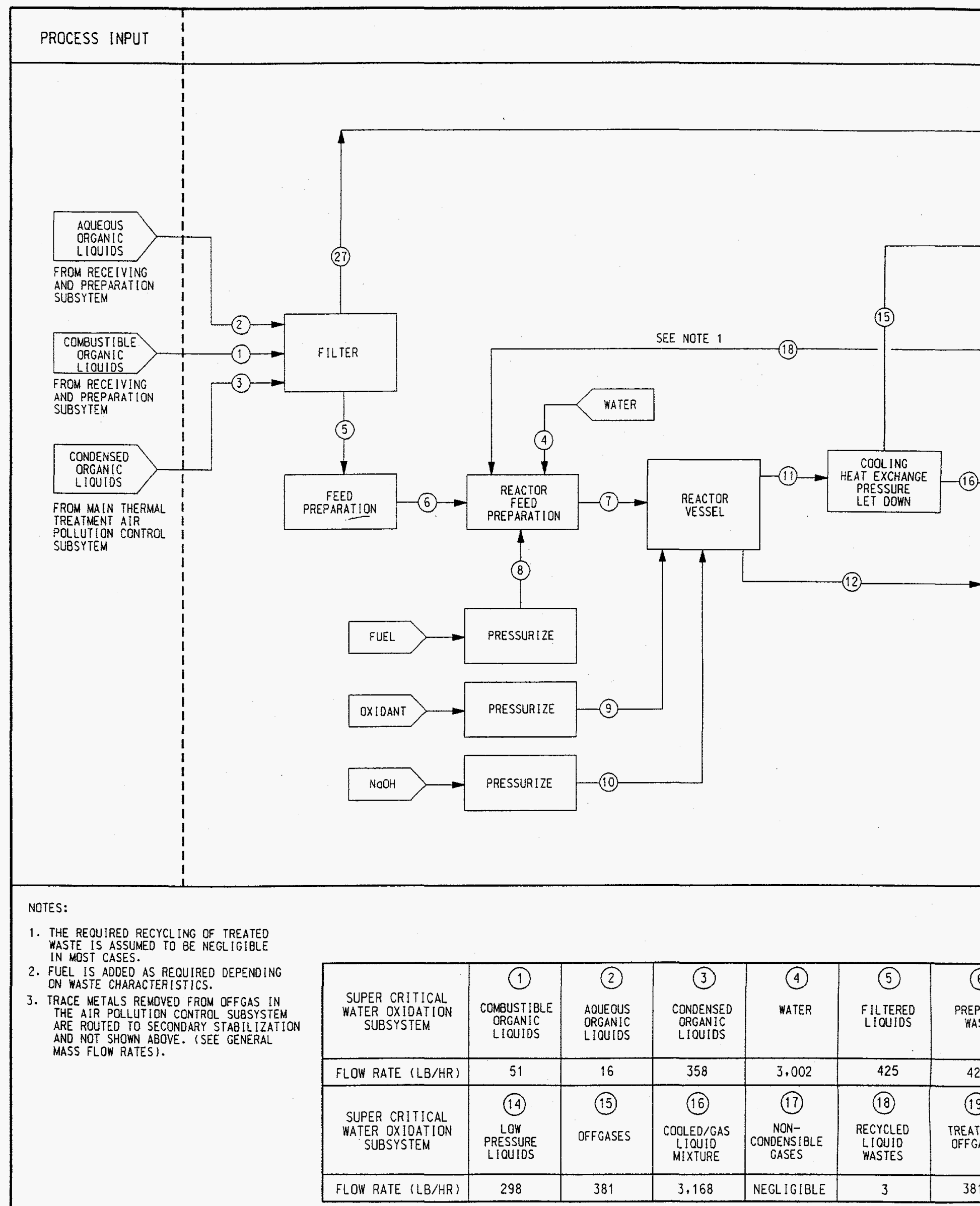

Figure 4-20. Thermal Desorption and Supercritical Water Oxidation: PFD for Organic Liquid Waste Treatment Subsystem (S 


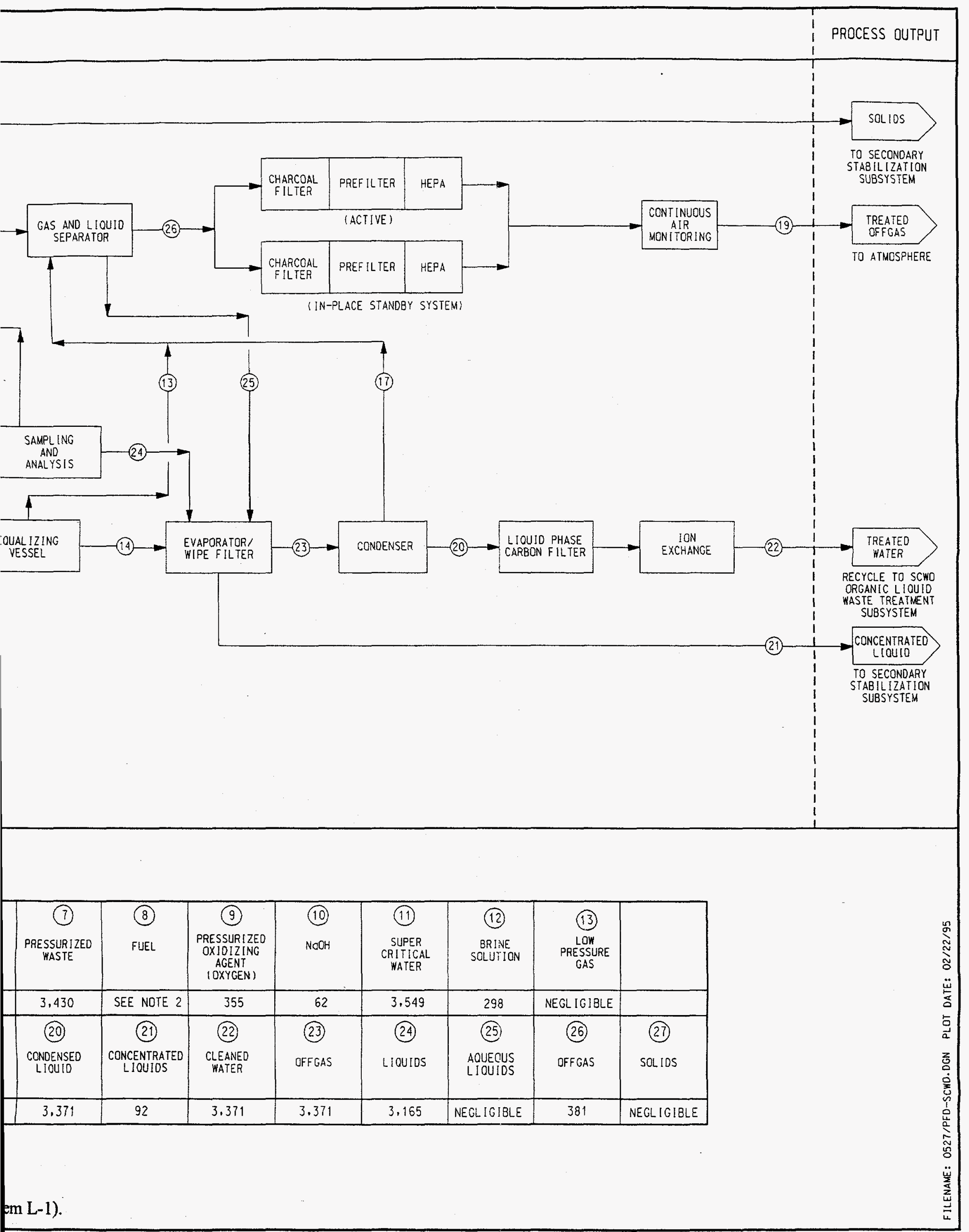




\section{MODELING OF MAIN TREATMENT SUBSYSTEMS - DESCRIPTIONS AND RESULTS}

\subsection{Background}

Initially, for Phase 1, mass and energy balances were done by simpler, less rigorous methods. Also, the database for calculating the input waste streams included only about half of the total contacthandled MLLW in the DOE complex. For Phase 2, the database was expanded to the full quantity, with the exception of the Hanford tank waste and the Rocky Flats Plant solar pond liquids, which are being addressed elsewhere using other processes that are more suitable than thermal treatment.

Further, for Phase 2, it was decided to model the systems with more robust analytical methods. The obvious choice was to select an existing, proven process simulation code from the many available in the private sector. The value of using this type of code over techniques developed specifically for the task at hand include (a) wide industrial acceptance, (b) proven capabilities to model essentially all types of unit operations, (c) large material data base, (d) capability to converge on complex design specifications, and (e) additional capabilities (such as economic studies or more detailed chemistry) which the project may wish to utilize beyond the present mass and energy balance calculations. The process simulation code chosen was ASPEN PLUS ${ }^{3}$, which is derived from the basic non-proprietary ASPEN code developed at MIT. Its capabilities include a large material database, no restrictions on system configuration (multiple feedback loops), availability of all types of unit operation models, simultaneous convergence on the user's design specifications, sensitivity analysis, cost predictions, plotting, report generation, and a large user support organization. In particular, ASPEN PLUS now includes an updated graphical user interface, which facilitates the construction of the model process flow diagrams (designated herein as MPFDs, as opposed to the process flow diagrams, PFDs, developed as part of the ITTS study and included in Section 4.), and an expert system to guide the user through the required numerical input. Other unique advantages were that INEL study participants had previous experience using ASPEN-based codes, and other organizations at the INEL were already licensed to use this particular product.

\subsection{Technical Approach}

The objective of the modeling was to achieve balances for the major input and output mass flows and the major energy flows in each unit operation. This allowed limiting the detail and complexity in the unit operation models (even though those capabilities existed in the code) and modeling of only the major streams and chemical species. The modeling objective was met to the extent possible within the available resources. The efficiency of the modeling task was significantly impacted by instabilities in the current version of the code, which was newly released at the time the work was performed. This being the case, the original intent, to include all of the subsystems within the ASPEN PLUS models has not yet been achieved. The subsystems not modeled were previously indicated in Table 3-2. They include the lead recovery, mercury recovery, metal decontamination, polymer stabilization, aqueous waste (except for systems K-1 and L-1), and special wastes subsystems. Thus, the only subsystems modeled with ASPEN PLUS were the main and secondary thermal treatment subsystems, their APC subsystems, and the metal melter subsystem for metals with entrained contamination. Full system models would facilitate the calculation of mass and energy balances for the systems (and would make the models more useful for other calculations as well).

Models were developed from the descriptions and figures provided in References (1) and (2), and with discussions with the authors and other contributors. After successful development of the Phase 2 models, similar models were developed for the Phase 1 systems. The modeling process involved several 
generations of models wherein the proper amount of detail was achieved, process refinements were incorporated, and a standardized modeling convention was developed to the extent possible for such varied systems.

The remainder of this section contains a general discussion of the models, applicable to all of the systems. Model-specific information is largely contained in the appendices. In particular, the MPFDs and assumption tables discussed below are contained in Appendix A.

Each of the ASPEN PLUS-generated MPFDs presented here should be compared with the corresponding PFD in Section 4 to better understand the simplifications incorporated into the modeling. Note that the MPFDs are not merely sketches of the models produced after the fact, but rather are derived directly from the actual flow sheets used within the code, upon which results are automatically printed after each run. Names of streams and unit operations were assigned in a consistent manner for all models to the extent possible. This was helpful because a major aspect of the ITTS study was to consider various combinations of the same basic subsystems. Names are somewhat cryptic due to an 8-character word size limitation in the code. Stream data are presented on "flags" attached to the streams, to conserve space. An option not chosen was to download abbreviated stream summary tables directly into the MPFDs. (Complete stream summaries are presented in Appendix C.) Information selected for display on the MPFDs include the temperature and total mass flow of each stream, and the heat duty of each unit operation (if non-zero). Pressure is not shown because, except for the high-pressure SCWO vessel used in system $L-1$, pressures were fixed at $1 \mathrm{~atm}$, and no pressure drops were calculated.

The code offered a selection of graphical icons for each type of unit operation. As in the naming, these also were selected in a consistent manner within and between systems based on function and for ease in interpreting the MPFDs. Each general category of unit operation was assigned a unique icon. In general, the thermal treatment, quench, and scrubber operations were modeled with reactor blocks which calculate chemical equilibrium based on minimization of Gibbs free energy (called RGIBBS blocks in the code), different icons being used for the high-temperature vs. the low-temperature operations. In using these type blocks it was necessary to be aware of which products were known not to form based on operational experience, even though predicted based on only equilibrium considerations. Their formation was then restricted in the input specifications. Other operations, such as the pyrolyzers, catalytic oxidizers, $\mathrm{CO} 2$ absorbers, the salt splitter (system A-6), and the gasifier (system H-1), among others, were modeled with RSTOIC blocks, which calculated only the specific stoichiometric reactions, and their extent, as input by the user. Other commonly used unit operations included physical separation processes (filters, etc.), flash units, and stream splitters for separating the products exiting the reactor blocks. Some systems required special operations, such as the pumps and compressors used for the supercritical water oxidation process in system L-1.

Stream duplicators (titled DUPL1, DUPL2) and "dummy units" (Q1, Q2) were used as modeling artifacts to include the effect of heat losses from certain units (as explained in the tables below) and are shown on the MPFDs for completeness, but do not represent actual stream flows or actual operational units within the ITTS systems.

The code requires specification of a component list of all the elements and compounds the user wishes to consider. This list was kept small but consistent in the models because of the limited objectives of the present task. If all possible chemical combinations were allowed, run times would become excessively long with little gain. Trace materials such as radionuclides and pollutants were generally not included in the list and, thus, not tracked by the models. Also, it was not the job here to predict the products generated in the various treatment schemes (especially the more exotic designs), but to develop a reasonable component list based on the information supplied by the designers and manufacturers of the systems. It is necessary in ASPEN PLUS to specify solid materials (here using -S suffix) separately from 
fluids, so that some components were specified twice. However, phase changes of minor constituents were not considered because it would not greatly affect the energy balances. For example, note that electrolytes are merely modeled as solids for simplicity. The complete list of elements and compounds used in the models were: $\mathrm{H}_{2}, \mathrm{O}_{2}, \mathrm{~N}_{2}, \mathrm{Cl}_{2}, \mathrm{~F}_{2}, \mathrm{NO}, \mathrm{SO}_{2}, \mathrm{H}_{2} \mathrm{O}, \mathrm{CO}_{2}, \mathrm{CO}, \mathrm{HCl}, \mathrm{CH}_{4}, \mathrm{~S}, \mathrm{As}, \mathrm{Cd}, \mathrm{Hg}, \mathrm{Se}, \mathrm{Fe}$, $\mathrm{SiO}_{2}, \mathrm{Al}_{2} \mathrm{O}_{3}, \mathrm{Fe}_{3} \mathrm{O}_{4}, \mathrm{Na}_{2} \mathrm{CO}_{3}, \mathrm{Na}_{2} \mathrm{SO}_{4}, \mathrm{H}_{2} \mathrm{SO}_{4}, \mathrm{H}_{2} \mathrm{O}-2, \mathrm{C}-\mathrm{S}, \mathrm{S}-\mathrm{S}, \mathrm{NaOH}-\mathrm{S}, \mathrm{NaCl}-\mathrm{S}, \mathrm{NaF}-\mathrm{S}, \mathrm{Fe}-\mathrm{S}, \mathrm{SiO}_{2}-\mathrm{S}$, $\mathrm{Al}_{2} \mathrm{O}_{3}-\mathrm{S}$, As-S, Ba-S, Cd-S, Cr-S, Pb-S, Se-S, Ag-S, CaCO 3 -S, MgO-S, Na 2 O-S, $\mathrm{K}_{2} \mathrm{O}-\mathrm{S}, \mathrm{Fe}_{3} \mathrm{O}_{4}-\mathrm{S}$, $\mathrm{CaO}-\mathrm{S}, \mathrm{CaCl}_{2}-\mathrm{S}, \mathrm{CaSO}_{4}-\mathrm{S}, \mathrm{Na}_{2} \mathrm{CO}_{3}-\mathrm{S}, \mathrm{Na}_{2} \mathrm{SO}_{4}-\mathrm{S}$, and $\mathrm{CoSO}_{4}$. A few of these were only used in special cases. (Note that compound $\mathrm{H} 2 \mathrm{O}-2$ was used as a second water stream within a single unit wherein the streams did not mix.)

There is not always a one-to-one correspondence between the modeling units and real system hardware. Sometimes two or more ASPEN PLUS units were required to model the processes occurring in a single piece of hardware. This was commonly done at the exit of a reactor. An example of this would be a plasma furnace which has several product streams. A second unit to perform the separation (block type SEP) was placed after the furnace in the model to split the products into as many separate streams as required, such as, molten metal, slag, and gases with entrained particulate as specified by the user in the input for the SEP unit.

In other cases, a number of unit operations were combined into a single modeling block. For example, this technique was applied in many instances to represent the uninterrupted sequence of filtering steps applied to the offgas, namely, the baghouse, charcoal filter, prefilter, and HEPA filter, between the quench step and scrubbing of the offgas. These were grouped into a single SEP unit (named BAGHOUSE) which separated the "volatile trace metals" from the fly ash and from the offgas. The definition of "volatile trace metals" is itself a modeling artifact. Grouping the low-concentration, highvolatility metals (mercury and three other metals, hence the stream name HGPLUS) together was a convenient way to handle their disposition from the system. Since they were of low concentration, it was not required to accurately predict their true partitioning in the system when only a mass and energy balance of the major components was being sought. But still, for modeling purposes, it was necessary that these components completely exit the model so that they did not build up within feedback loops and prevent convergence of the models. Grouping of the volatile trace metals into a single product stream is not meant to imply that a single physical process is used in the real system to collect them for subsequent processing.

Assumption and energy balance summaries. Each of the models required hundreds of input specifications. Some of this information, such as the material flow connections between unit operations, is best presented and understood in the MPFDs. Other information is easily understood in tabular form, such as a list of the material components defined for each model, so that one can simply refer to the input files in Appendix B (see below). For much of the remainder of the information, especially that unique to each model, it was useful to summarize the data in an individual table for each system. This served as a useful tool for reviewing results. The tables contain the calculated operating temperature and heat duty of each unit and summaries of the allowed (or disallowed) chemical and/or physical processes in each unit operation. For example, the extent to which a reaction will occur must often be specified. For the RGIBBS reactors, which calculate equilibrium based on the minimization of Gibbs free energy, reactions that were not expected to reach equilibrium (for example, the recombination of $\mathrm{C}$ and $\mathrm{H}_{2}$ into $\mathrm{CH}_{4}$ at room temperature) were restricted accordingly. (Alternatively, an RSTOIC block could have been used, but this would have required that each separate reaction stoichiometry and extent be specified.) Many physical "extents" also had to be specified, especially with respect to separation of products; for example, the percent carryover of ash with the exhaust gas (fly ash) in a rotary kiln, which was specified as a fixed value based on vendor-supplied information . 
Also contained in the assumption and energy balance tables is a description of the feedback loops. These are termed DESIGN SPECs in the code. Two examples are feedback loops which (1) calculate the amount of cooling water required to meet a specified outlet temperature, and (2) calculate the correct amount of gross soil (including moisture and $\mathrm{CaCO}_{3}$ ) needed by the kiln to achieve a 2-to-1 ratio of bottom ash to soil (for vitrification). The code converges on solutions to all of the DESIGN SPECs during a run.

One of the unit operations that was greatly simplified was the wet acid gas scrubber units found in the majority of the systems. Again, since only mass and energy balance information was sought, the process was modeled as an ordinary chemical reactor rather than as electrolytic reactions in a distillation column. The issue of cooling in the scrubbers was an important consideration to the ITTS results. Preliminarily, the ITTS concept was to add cooling water directly into the scrubbers to eliminate (or minimize) the need for heat exchangers, the idea being to avoid a potential high-cost maintenance item because of the radioactive nature of the waste. This concept was abandoned due to the resulting high water flow rates required. Instead, only enough water is added to assure that scrubber liquor contains no more than 5\% dissolved salts (TDS), taking no credit for the water vapor in the process stream. This greatly reduces the size requirements of the scrubbers and any downstream equipment, but necessitates the use of the heat exchangers. The MPFDs and the assumption and energy balance tables are contained in Appendix A.

Appendix B contains the ASCII text input files for all of the models (with filename extensions of ".INP"). Input files are the shortest means of documenting all of the information needed to recreate the numerical results, excluding any of the graphics. The code also produces ASCII text backup files ("*.BKP") that store all of the model information, and are transferable between computer systems.

Appendix C contains the detailed, ASPEN PLUS-generated stream summary results for each of the systems. These show the mass flow rates of each element/chemical in each stream, sub-divided into "MIXED" (gas or liquid phases) and "SOLID" (solid phase) substreams. (To conserve space, the computer output files in Appendices B and C have been slightly edited for this report.) 


\section{OVERALL MASS AND ENERGY BALANCES}

The system modeling using the ASPEN PLUS code was used to model only the most complex and energy intensive of the subsystems for the 19 thermal treatment systems in the ITTS study. For the mass balances, these results were combined with the results of other calculations for the remainder of the subsystems to arrive at overall mass balances. These data and others were then entered into a spreadsheet program and used to arrive at a variety of summary results for comparison of the systems. Most notably, a comparison is made of the amount of volume and mass waste reduction achieved in processing and stabilizing the wastes. Energy balances are presented in this section based only on the ASPEN PLUS results. Energy consumption in the other subsystems would be much smaller than in the subsystems modeled with the code. Using current energy prices, energy costs are also presented. All of the results presented here are also found in Reference (2).

\subsection{Mass Balance Results}

The following discussion refers to the results shown in the mass balance summaries of Table 6-1. Included in the table are all of the ASPEN PLUS results. The results of the mass balance calculations for those subsystems not modeled with ASPEN PLUS are taken from Reference (2). Those calculations had been done for the Phase 1 task (Reference 1), and in some cases the flow rates and processes were revised and recalculated for Phase 2. Certain of those subsystems recycle small amounts of material back to the main thermal treatment subsystem. Had all of the subsystems been incorporated into the ASPEN PLUS models, the feedback from these recycle streams could have been considered. However, this was not done (for reasons discussed previously) so that there is not complete closure in the mass balances. These effects, along with roundoff errors, are the cause of the small differences in input and output flow rates found in the table. The maximum overall mass balance error, as calculated in the spreadsheet, is less than $6 \mathrm{lb} / \mathrm{hr}(\sim 0.05 \%)$.

All mass balance results, along with estimated input and output material densities, were entered into a spreadsheet program (Ref. 6). Various summations and calculations of parameters of interest were then made, and are presented in Table 6-1. The size of the table is indicative of the complexity of the comparison process undertaken in the ITTS study.

Referring to Table 6-1, waste feed rates are the same for every system at $2927 \mathrm{lb} / \mathrm{hr}$. Soil is not included in this category because it was considered a process input rather than a waste input. No credit has been taken for the possible use of contaminated soil in the ITTS study. Process inputs required by the systems include soil, fuel, oxidant, water, and caustic. Process water will be supplied almost wholly by recycle of output from the aqueous waste subsystems. (Preliminary calculations have shown that by slightly adjusting system temperatures, for example, at the scrubber outlet, more or less water can be lost by evaporization to the offgas streams to achieve a net water usage of zero.) Water used for indirect cooling (cooling water) is not considered under the category of process water. Significant differences exist between systems for these process inputs. Including the process water, amounts vary from a low of $4,555 \mathrm{lb} / \mathrm{hr}$ for System C-2 (Plasma Furnace w/ $\mathrm{CO}_{2}$ Retention) to $45,698 \mathrm{lb} / \mathrm{hr}$ for System A-7 (Slagging Rotary Kiln). The process water portion of the process inputs ranges from $539 \mathrm{lb} / \mathrm{hr}$ for System G-1 (Molten Metal) to $18,649 \mathrm{lb} / \mathrm{hr}$ for System A-7 (Slagging Rotary Kiln).

System outputs fall into four categories: treated offgas, water for recycle, reclaimed solids and solid wastes. Offgas quantities are of interest because their potential impact on the environment and because of public perception. Offgas quantities range from a low of $792 \mathrm{lb} / \mathrm{hr}$ for System L-1 (Desorption w/ SCWO) to $26,437 \mathrm{lb} / \mathrm{hr}$ for System A-7 (Slagging Rotary Kiln). Reclaimed solids included decontaminated metal drums, lead ingots, and some recast metals of roughly $600 \mathrm{lb} / \mathrm{hr}$ for all systems (slightly less for System A-7). 
All solid wastes exit the systems in a stabilized form. (However, the special wastes are, by definition, undefined at this point.) Stabilization is achieved in a number of ways. Mercury is always amalgamated. The remainder of the waste is either vitrified, polymerized, or grouted. Mixing ratios for the stabilization materials were, for vitrification, approximately one part soil per two parts waste (see previous sections for exact ratio), for polymerization, a one-to-one ratio, and for grouting, two parts grout to one part waste. Densities for the materials are found in the table.

Two (of the many) measures of the suitability of each of these nineteen technologies is the amount of mass and volume reduction achieved in the waste compared to the initial material. The parameter used for both is the ratio of unprocessed input wastes to processed output wastes. Volume reduction ratios exceeded 1.00 in every case, indicating a net reduction. Values ranged from 1.07 for System K-1 (Desorption w/ MEO) to 4.27 for System G-1 (Molten Metal), and averaged 2.87 for all systems. Mass reduction ratios were as low as about 0.6 for systems $\mathrm{K}-1$ and L-1, meaning that more processed wastes left the facilities than raw wastes entered. The maximum mass reduction ratio was 1.73 for system G-1, and the average was 1.33 for all systems.

\subsection{Energy Balance Results}

Energy requirements (heat duties) are calculated by ASPEN PLUS as part of the process simulation. Since the subsystems modeled with the code included all of the most energy intensive unit operations, this represented the majority of the energy consumption associated with each of the systems. Energy consumption for the remainder of the subsystems are not included for the present results, which are shown in Table 6-2. The table includes only (prime) energy input requirements, and does not include any cooling requirements (such as the indirect cooling of the scrubbers). In the models, energy loss terms were included for those operations which were directly heated by methane burning. This was done because the products of combustion become part of the waste stream and affect the operation and size of all the downstream units, thus making it important to accurately estimate their magnitude. The losses were estimated to be $5 \%$ of the sensible heat added to the stream, as discussed on the assumption and energy balance tables in Appendix A. For other unit operations, loss terms were not added to the models but are included in the present table. Energy usage varied by an order of magnitude with the highest user being System A-6 (Rotary Kiln w/ Maximum Recycling) at 35.7 MMBtw/hr, and the lowest System B-1 (Pyrolyzer) at 3.35 MMBtu/hr.

Energy costs are also included on Table 6-2. Here the maximum and minimum vary by a factor of twenty, with the highest again being System A-1 at $\$ 33.0$ million over twenty years (1994 dollars), and the lowest, System A-5 (Rotary Kiln w/ Polymer Stabilization) at \$1.6 million. Although System A-5 uses almost three times as much primary energy as System B-1, its usage is predominately in the form of natural gas which is currently much cheaper than electricity. As discussed in Reference (2), where all costs are presented, the energy costs represented only a minor portion of the total lifecyle costs of any of the systems. 
Table 6-1. Overall Mass Balances and Summary Results for the ITTS Study Process Modeling.

\begin{tabular}{|c|c|c|c|c|c|c|c|c|c|}
\hline \multirow[b]{2}{*}{ Mass Flow (lb/hr) } & \multicolumn{9}{|c|}{ OVERALL MASS BALANCES FOR 19 ITTS SYSTEMS (including ASPEN modeling results) } \\
\hline & $\begin{array}{l}\text { A-1 Baseline } \\
\text { Rotary Kiln }\end{array}$ & $\begin{array}{l}\text { A-2 Rotary } \\
\text { Kiln w/ } \\
\text { Oxygen }\end{array}$ & $\begin{array}{l}\text { A-3 Rotary } \\
\text { Kiln w/ Wot } \\
\text { APC }\end{array}$ & $\begin{array}{l}\text { A-4 Rotary } \\
\text { Kiln w/ CO2 } \\
\text { Retention }\end{array}$ & $\begin{array}{l}\text { A-5 Rotary } \\
\text { Kin w/ Poly. } \\
\text { Stabilization }\end{array}$ & $\begin{array}{l}\text { A-6 Rotary } \\
\text { KIln w/ Max. } \\
\text { Recycling }\end{array}$ & $\begin{array}{l}\text { A-7 } \\
\text { Slagging } \\
\text { Rotary Kiln }\end{array}$ & $\begin{array}{l}\text { B-1 } \\
\text { Pyrolysis }\end{array}$ & $\begin{array}{l}\text { C-1 } \\
\text { Plasma } \\
\text { Furnace }\end{array}$ \\
\hline & \multicolumn{9}{|c|}{ WASTE INPUT } \\
\hline \multicolumn{10}{|l|}{$\begin{array}{l}\text { MAIN THERTWAL TREATIMENT } \\
\text { SUBSYSTEM (MTT) }\end{array}$} \\
\hline Combustible & 660.4 & 660.4 & 660.4 & 660.4 & 660.4 & 660.4 & 660.4 & 660.4 & 660.4 \\
\hline Noncombustible & $1,339.6$ & $1,339.6$ & $1,339.6$ & $1,339.6$ & $1,339.6$ & $1,339.6$ & $1,339.6$ & & $1,339.6$ \\
\hline Metal & & & & & & & 149 & & 149 \\
\hline Aqueous Liquid : & & & & & & & & & \\
\hline THERMALDESORBER & & & & & & & & & \\
\hline Combustible & & & & & & & & & \\
\hline Noncombustible & & & & & & & & & \\
\hline VITRIFIER & & & & & & & & & \\
\hline Combustible & & & & & & & & & \\
\hline Noncombustible & & & & & & & & 1,340 & \\
\hline Subtotal - MTT & 2,000 & 2,000 & 2,000 & 2,000 & 2,000 & 2,000 & 2,149 & 2,000 & 2,149 \\
\hline AUXILIARY SUBSYSTEMS & & & & & & & & & \\
\hline $\begin{array}{l}\text { Tetallo Waste (Surface } \\
\text { Contamination) }\end{array}$ & 468 & 468 & 468 & 468 & 468 & 468 & 468 & 468 & 468 \\
\hline $\begin{array}{l}\text { Metallic Waste (Entrained } \\
\text { Contamination) }\end{array}$ & 149 & 149 & 149 & 149 & 149 & 149 & & 149 & \\
\hline Mercury & 50 & 50 & 50 & 50 & 50 & 50 & 50 & 50 & 50 \\
\hline LeadMetal & 26 & 26 & 26 & 26 & 26 & 26 & 26 & 26 & 26 \\
\hline Aqueous Waste & 80 & 80 & 80 & 80 & 80 & 80 & 80 & 80 & 80 \\
\hline SpecialWaste & 153 & 153 & 153 & 153 & 153 & 153 & 153 & 153 & 153 \\
\hline Halide and Sulfide salts & & 1 & 1 & 1 & 1 & 1 & 1 & 1 & 1 \\
\hline Subtotal - Auxilliary & 927 & 927 & 927 & 827 & 927 & 927 & 778 & 927 & 778 \\
\hline $\begin{array}{l}\text { Contaminated soil (Can replace } \\
\text { Soll, below) }\end{array}$ & & & & & & & & & \\
\hline TOTAL WASTE INPUT & 97 & 89 & $3 \%$ & 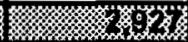 & $3 \%$ & \% & 30 & 84 & $83 \%$ \\
\hline
\end{tabular}




\begin{tabular}{|c|c|c|c|c|c|c|c|c|c|}
\hline \multirow[b]{2}{*}{ Mass Flow (b/hr) } & \multicolumn{9}{|c|}{ OVERALL MASS BALANCES FOR 19 ITTS SYSTEMS (including ASPEN modeling results) } \\
\hline & $\begin{array}{l}\text { A-1 Baseline } \\
\text { Rotary Kiln }\end{array}$ & $\begin{array}{l}\text { A-2 Rotary } \\
\text { Kiln w/ } \\
\text { Oxygen }\end{array}$ & $\begin{array}{l}\text { A-3 Rotary } \\
\text { Kiln w/ Wet } \\
\text { APC }\end{array}$ & $\begin{array}{l}\text { A-4 Rotary } \\
\text { Klin w/ CO2 } \\
\text { Retention }\end{array}$ & $\begin{array}{l}\text { A-5 Rotany } \\
\text { Kin w/ Poly. } \\
\text { Stabilization }\end{array}$ & $\begin{array}{l}\text { A-6 Rotary } \\
\text { Kiln w/ Max. } \\
\text { Recycling }\end{array}$ & \begin{tabular}{|l|} 
A-7 \\
Slagging \\
Rotary Kin
\end{tabular} & $\begin{array}{l}\text { 8-1 } \\
\text { Pyrolysis }\end{array}$ & \begin{tabular}{|l} 
C-1 \\
Plasma \\
Furnace
\end{tabular} \\
\hline & \multicolumn{9}{|c|}{ A. PROCESS INPUTS } \\
\hline \multicolumn{10}{|c|}{$\begin{array}{l}\text { MAIN THERMALTREATMENT } \\
\text { SUBSYSTEM (MTT) }\end{array}$} \\
\hline $\mathrm{H} 2 \mathrm{O}$ & 482 & 1,824 & 526 & & 663 & 485 & & & \\
\hline CH4 & 1 & & 1 & 261 & 1 & 1 & 568 & & \\
\hline 02 & & 1,830 & & 1,455 & & & & 290 & \\
\hline Alr & 7,859 & & 7,859 & & 7,878 & 7,859 & 18,326 & & 6,239 \\
\hline Air Inleakage & 2,695 & 2,695 & 2,695 & 2,695 & 2,695 & 2,695 & 2,695 & 224 & \\
\hline Soil & 498 & 498 & 370 & 498 & & 495 & 564 & & 482 \\
\hline \multicolumn{10}{|l|}{ VITRIFIER } \\
\hline \begin{tabular}{|l} 
Soil \\
\end{tabular} & & & & & & & & 498 & \\
\hline Water & 91 & 91 & 68 & 91 & & 90 & & & \\
\hline $\mathrm{CH} 4$ & 15 & 15 & 11 & 15 & & 15 & & & \\
\hline 02 & 197 & 197 & 146 & 197 & & 196 & & 23 & \\
\hline Air & 15 & 15 & 11 & 15 & & 15 & & & \\
\hline \multicolumn{10}{|l|}{$\operatorname{scc}$} \\
\hline $\mathrm{CH} 4$ & 418 & 137 & 419 & 355 & 424 & 418 & 208 & & \\
\hline 02 & & 658 & & 1,712 & & & & 834 & \\
\hline Air & 8,663 & & 8,691 & & 8,790 & 8,666 & 4,319 & & \\
\hline \multicolumn{10}{|l|}{ WATER } \\
\hline Primary Quench & 10221 & 4890 & & 13434 & 10354 & 10224 & 15425 & 1411 & 3549 \\
\hline Secondary Quench & & & & 926 & & & & & \\
\hline Primary Scrubber & 2,259 & 2,259 & 2,259 & & 2,259 & 2,259 & 2,259 & 2,259 & 2,259 \\
\hline \multirow{2}{*}{\multicolumn{10}{|c|}{ OTHER }} \\
\hline & & & & & & & & & \\
\hline $\mathrm{NaOH}$ & 78 & 78 & 76 & & 78 & 2 & 78 & 78 & 78 \\
\hline \multicolumn{10}{|l|}{$\mathrm{Na2} \mathrm{CO} 3$} \\
\hline $\mathrm{CaO}$ & & & & 374 & & & & & \\
\hline \multicolumn{10}{|l|}{ Electrolyte(water \& $\operatorname{CoSO} 4)$} \\
\hline \multirow{2}{*}{\multicolumn{10}{|c|}{$\begin{array}{l}\text { Input to Indirect Heater } \\
\text { Air to Catalutic Oxidizer }\end{array}$}} \\
\hline & & & & & & & & & \\
\hline \multicolumn{10}{|l|}{$\mathrm{CH} 4$} \\
\hline \multicolumn{10}{|l|}{ Air } \\
\hline Subtotal-MTT & 33,492 & 15,188 & 23,132 & 22,029 & 33,142 & 33,420 & 44,442 & 5,617 & 12,608 \\
\hline \multirow{2}{*}{\multicolumn{10}{|c|}{$\begin{array}{l}\text { AUXILIARY SUBSYSTEMS } \\
\text { METAL.MELTTNG (MM) }\end{array}$}} \\
\hline & & & & & & & & & \\
\hline Alr & 25 & 25 & 25 & 25 & 25 & 25 & 0 & 25 & $\overline{0}$ \\
\hline Water & 19 & 19 & 19 & 19 & 19 & 19 & 0 & 19 & $\overline{0}$ \\
\hline Soll + Flux & 10 & 10 & 10 & 10 & 10 & 10 & 0 & 10 & 0 \\
\hline Subtotal-MM & 64 & 84 & 64 & 64 & 64 & 34 & (0) & 64 & it \\
\hline
\end{tabular}




\begin{tabular}{|c|c|c|c|c|c|c|c|c|c|}
\hline \multirow[b]{2}{*}{ Mass Flow (b/hr) } & \multicolumn{9}{|c|}{ OVERALL MASS BALANCES FOR 19 ITTS SYSTEMS (including ASPEN modeling results) } \\
\hline & $\begin{array}{l}\text { A-1 Baseline } \\
\text { Rotary KIln }\end{array}$ & $\begin{array}{l}\text { A-2 Rotary } \\
\text { Kln w/ } \\
\text { Oxygen }\end{array}$ & $\begin{array}{l}\text { A-3 Rotary } \\
\text { Kilin w/ Wot } \\
\text { APC }\end{array}$ & $\begin{array}{l}\text { A-4 Rotany } \\
\text { Kin w/ CO2 } \\
\text { Retention }\end{array}$ & $\begin{array}{l}\text { A-5 Rolary } \\
\text { Kiln w/ Poly. } \\
\text { Stabllization }\end{array}$ & $\begin{array}{l}\text { A-6 Rotary } \\
\text { Kln W/ Max. } \\
\text { Recycling }\end{array}$ & \begin{tabular}{|l} 
A-7 \\
Slagging \\
Rotary Kiln
\end{tabular} & \begin{tabular}{|l} 
B-1 \\
Pyrolysis
\end{tabular} & \begin{tabular}{|l|} 
C-1 \\
Plasma \\
Furnace
\end{tabular} \\
\hline LEAD RECOVERY (PbR) & & & & & & & & & \\
\hline APC Water (use recycle) & 14 & 14 & 14 & 14 & 14 & 14 & T4 & 14 & $\overline{14}$ \\
\hline Fuel & & & & & & & & & \\
\hline Air & 21 & 21 & 21 & 21 & 21 & 21 & 21 & 21 & 21 \\
\hline Blast Water & 29 & 29 & 29 & 29 & 29 & 29 & 29 & 29 & 29 \\
\hline Soll & 0.26 & 0.26 & 0.26 & 0.26 & 0.26 & 0.26 & 0.26 & 0.26 & 0.26 \\
\hline Subtotal-PbR & 64.26 & 64.26 & 64.26 & 64.26 & 64.26 & 64.26 & 64.26 & 64.26 & 64.26 \\
\hline $\begin{array}{l}\text { AQUEOUSWASTE } \\
\text { TREATMENT (AQ) }\end{array}$ & & & & & & & & & \\
\hline Resins \& Activated Carbon & 810.96 & 600.96 & 199.56 & $\overline{942.6}$ & 827.1 & 798.36 & 1117.02 & 281.22 & 385.56 \\
\hline Addittves $(0.051 \mathrm{lb} / \mathrm{b}$ input') & & & & 0 & 0 & & & 0 & 0 \\
\hline Rinse Water for $2^{\circ}$ Stabllization & 403.25 & 298.25 & 97.55 & 469.07 & 411.32 & 396.95 & 556.28 & 138.38 & 190.55 \\
\hline Subtotal-AQ & 1214.208 & 600.96 & 199.56 & 942.6 & 827.1 & 798.36 & 1117.02 & 281.22 & 385.56 \\
\hline $\begin{array}{l}\text { MERCURY AMALGAMATTON } \\
(\mathrm{HgA})\end{array}$ & & & & & & & & & \\
\hline Additives $(0.1 \mathrm{lb} / \mathrm{b})$ & 5 & 5 & 5 & 5 & 5 & 5 & 5 & 5 & 5 \\
\hline Nitrogen $(0.22 \mathrm{lb} / \mathrm{lb})$ & 11 & 11 & $\frac{\pi}{11}$ & 11 & 11 & 11 & 11 & 11 & $\overline{11}$ \\
\hline Air $(0.60 \mathrm{lb} / \mathrm{hb})$ & 30 & 30 & 30 & 30 & 30 & 30 & 30 & 30 & 30 \\
\hline Water $(0.58 \mathrm{lb} / \mathrm{b})$ & 29 & 29 & 29 & 29 & 29 & 29 & 29 & 29 & 29 \\
\hline Subtotal-HgA & $\frac{5}{75}$ & 76 & $\frac{25}{75}$ & $\frac{25}{75}$ & 75 & $\frac{20}{75}$ & $-\frac{25}{76}$ & $\frac{20}{75}$ & $\frac{20}{75}$ \\
\hline TOTAL PROCESS INPUTS & 39896 & 4508 & $2 \times 325$ & \%3:165 & 3\%6 & 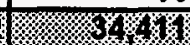 & $85 \% 696$ & 806 & 及 \\
\hline & & & & INTERNA & L SYSTEM & RECYCLE & & & \\
\hline $\begin{array}{l}\text { Scrubber Liquor from scrubber to } \\
\text { aqueous waste treatment }\end{array}$ & 13,436 & 9,936 & 3,246 & 15,630 & 13,705 & 13,226 & 18,537 & 4,607 & 6,346 \\
\hline $\begin{array}{l}\text { Sludge from } \mathrm{Pb} \text { recovery to } \\
\text { aqueous waste treatment }\end{array}$ & 0 & 0 & 0 & 0 & 0 & $\mathbf{0}$ & 0 & 0 & 0 \\
\hline $\begin{array}{l}\text { Resins from aqueous waste } \\
\text { treatment to thermal treatment }\end{array}$ & 824 & 611 & 203 & 958 & 841 & 812 & 1,136 & 286 & 392 \\
\hline $\begin{array}{l}\text { Ig amalgamation solids to thermal } \\
\text { treatment }\end{array}$ & 18 & 18 & 18 & 18 & 18 & 18 & 18 & 18 & 18 \\
\hline $\begin{array}{l}\text { Waler from metal decontaminalion } \\
\text { to recycte }\end{array}$ & 112 & 112 & 112 & 112 & 112 & 112 & 112 & 112 & 112 \\
\hline $\begin{array}{l}\text { TOTAL INTERNAL SYSTEM } \\
\text { RECYCLE (Not including recycle } \\
\text { to thermal trealment) }\end{array}$ & 13,548 & 10,048 & 3,358 & 15,742 & 13,817 & 13,338 & 18,649 & 4,719 & 6,458 \\
\hline TOTAL INPUT & $6 \%$ & 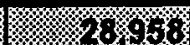 & - & \% & $60 \% 00$ & cows & 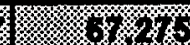 & 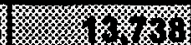 & \%, \\
\hline
\end{tabular}




\begin{tabular}{|c|c|c|c|c|c|c|c|c|c|}
\hline \multirow[b]{2}{*}{ Mass Flow (b/hr) } & \multicolumn{9}{|c|}{ OVERALL MASS BALANCES FOR 19 ITTS SYSTEMS (including ASPEN modeling results) } \\
\hline & $\begin{array}{l}\text { A-1 Basellne } \\
\text { Rotary Kln }\end{array}$ & $\begin{array}{l}\text { A-2 Rotary } \\
\text { KIn w/ } \\
\text { Oxygen }\end{array}$ & $\begin{array}{l}\text { A-3 Rotary } \\
\text { Kiln w/ Wet } \\
\text { APC }\end{array}$ & $\begin{array}{l}\text { A-4 Rotary } \\
\text { Kiln w/ CO2 } \\
\text { Retention }\end{array}$ & $\begin{array}{l}\text { A-5 Rotary } \\
\text { Kiln w/ Poly. } \\
\text { Stabilization }\end{array}$ & $\begin{array}{l}\text { A-6 Rotary } \\
\text { Kiln w/ Max. } \\
\text { Recycling }\end{array}$ & $\begin{array}{l}\text { A-7 } \\
\text { Slagging } \\
\text { Rotary Kiln }\end{array}$ & $\begin{array}{l}\text { B-1 } \\
\text { Pyrolysis }\end{array}$ & $\begin{array}{l}\text { C-1 } \\
\text { Plasma } \\
\text { Furnace }\end{array}$ \\
\hline & \multicolumn{9}{|c|}{ SYSTEM OUTPUT } \\
\hline & \multicolumn{9}{|c|}{ B. MAIN THERMAL TREATMENT (MTT) SUBSYSTEM OUTPUT } \\
\hline \multicolumn{10}{|l|}{ SOLIDS } \\
\hline Slag & 1,495 & 1,495 & 1,109 & 1,495 & & 1,485 & 1,691 & 1,495 & 1,450 \\
\hline \multicolumn{10}{|l|}{ Debris } \\
\hline Nonvitrified Ash & & & 277 & & 1,078 & & & & \\
\hline \multicolumn{10}{|l|}{ Metal } \\
\hline Trace Metals & 4 & & 4 & 4 & 4 & 4 & 4 & 4 & 4 \\
\hline \multicolumn{10}{|l|}{$\mathrm{Na} 2 \mathrm{CO} 3$} \\
\hline NaCV/NaF & & & & & & 0.24 & & & \\
\hline \multicolumn{10}{|l|}{ HCl (50\% in water) } \\
\hline $\mathrm{CaO}$ & & & & 317 & & & & & \\
\hline $\mathrm{CaCl} 2, \mathrm{CaSO} 4$ & & & & 113 & & & & & \\
\hline Subtotal-Solids & $\mathbf{1 , 4 9 9}$ & 1,499 & 1,390 & 1,929 & 1,082 & 1,637 & 1,696 & 1,499 & 1,624 \\
\hline \multicolumn{10}{|l|}{$\begin{array}{l}\text { Main Thermal Treatment } \\
\text { APC }\end{array}$} \\
\hline \multicolumn{10}{|l|}{ LIQUIDS \& DISSOLVED GASES } \\
\hline Scrubber Waler & 13,323 & 9,823 & 3,133 & 15,630 & 13,592 & 13,224 & 18,424 & 4,494 & 6,233 \\
\hline Dissolved salts & 113 & 113 & 113 & & 113 & 2 & 113 & 113 & 113 \\
\hline \multicolumn{10}{|l|}{ Dissolved gases } \\
\hline \multicolumn{10}{|l|}{02} \\
\hline \multicolumn{10}{|l|}{ N2 } \\
\hline $\mathrm{CO} 2$ & 23 & 47 & 5 & & 23 & & 35 & 74 & 17 \\
\hline \multirow{2}{*}{\multicolumn{10}{|c|}{ Electrolye(water \& COSO4) }} \\
\hline & & & & & & & & & \\
\hline \multicolumn{9}{|l|}{$\begin{array}{l}\text { Subtotal-Liquids \& Dissolved } \\
\text { Gases }\end{array}$} & 6.369 \\
\hline \multicolumn{10}{|l|}{ GASES } \\
\hline 02 & 1,625 & 1,387 & 1,627 & 156 & 1,632 & 1,627 & 1,489 & 16 & 258 \\
\hline N2 & 14,730 & 2,062 & 14,762 & 2,046 & 14,842 & 14,746 & 19,523 & 171 & 4,781 \\
\hline $\mathrm{CO} 2$ & $\frac{\mid 7,164}{2,364}$ & 1,569 & 2,378 & 2,929 & 2,352 & 2,387 & $\frac{10,366}{3,366}$ & 1167 & $\frac{1,236}{1,236}$ \\
\hline H2O vapor & 1,478 & 357 & 1,482 & 996 & 1,488 & 1,479 & 1,923 & 77 & 485 \\
\hline $\mathrm{SO2}$ & & & & & & & & & 2 \\
\hline Subtotal-Gases & 20,200 & 6,377 & 20,252 & 6,127 & 20,317 & 20,242 & 26,304 & 1,433 & 6,762 \\
\hline
\end{tabular}




\begin{tabular}{|c|c|c|c|c|c|c|c|c|c|}
\hline \multirow[b]{2}{*}{ Mass Flow (lb/hr) } & \multicolumn{9}{|c|}{ OVERALL MASS BALANCES FOR 19 ITTS SYSTEMS (including ASPEN modeling results) } \\
\hline & $\begin{array}{l}\text { A-1 Basellne } \\
\text { Rotary Kiln }\end{array}$ & $\begin{array}{l}\text { A-2 Rolary } \\
\text { Kln w/ } \\
\text { Oxygen }\end{array}$ & $\begin{array}{l}\text { A-3 Rotary } \\
\text { Kiln w/ Wet } \\
\text { APC }\end{array}$ & $\begin{array}{l}\text { A-4 Rolary } \\
\text { Kiln w/ CO2 } \\
\text { Retention }\end{array}$ & $\begin{array}{l}\text { A-5 Rotary } \\
\text { Kiln w/ Poly. } \\
\text { Stabilization }\end{array}$ & $\begin{array}{l}\text { A-6 Rotary } \\
\text { KIIn w/ Max. } \\
\text { Recycling }\end{array}$ & $\begin{array}{l}\text { A-7 } \\
\text { Slagging } \\
\text { Rotary Kin }\end{array}$ & $\begin{array}{l}\text { B-1 } \\
\text { Pyrolysis }\end{array}$ & $\begin{array}{l}\text { C-1 } \\
\text { Plasma } \\
\text { Fumace }\end{array}$ \\
\hline \multicolumn{10}{|l|}{ INDIRECT-FIRED EXHAUST } \\
\hline \\
\hline N2 & & & & & & & - & & \\
\hline \multicolumn{10}{|l|}{$\mathrm{CO2}$} \\
\hline \multicolumn{10}{|l|}{ H2O vapor } \\
\hline Subtotal - Indirect-fired exhaust & ol & of & 0] & of & 0 & of & of & of & 0 \\
\hline $\begin{array}{l}\text { TOTAL - MTT Subsystems } \\
\text { Output }\end{array}$ & 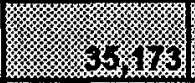 & 桑6\% & 4606 & s.og & 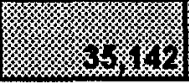 & 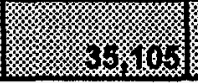 & \%om & 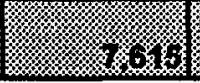 & $x+6$ \\
\hline & \multicolumn{9}{|c|}{ AUXILIARY SUBSYSTEM OUTPUTS } \\
\hline \multicolumn{10}{|l|}{ Metal Decontamination (MD) } \\
\hline Clean Melal & 463 & 463 & 463 & 463 & 463 & 463 & 463 & 463 & 463 \\
\hline Grit & 5 & 5 & 5 & 5 & 5 & 5 & 5 & 5 & 5 \\
\hline Water & 112 & 112 & 112 & 112 & 112 & 112 & 112 & 112 & 112 \\
\hline Subtotal - Metal Decon & 680 & 580 & 580 & 580 & 880 & 680 & 6801 & 680 & 680 \\
\hline \multicolumn{10}{|l|}{ Metal Melting } \\
\hline Clean Metal & 134 & 134 & 134 & 134 & 134 & 134 & 0 & 134 & 0 \\
\hline Treated Offgas & 46 & 46 & 46 & 46 & 46 & 46 & of & 46 & 0 \\
\hline Slag & 23 & 23 & 23 & 23 & 23 & 23 & 0 & 23 & 0 \\
\hline Subtotal - Metal Melting & 203 & 203 & 203 & 203 & 203 & 203 & of & 203 & 0 \\
\hline \multicolumn{10}{|l|}{ Lead Recovery } \\
\hline Clean Lead & 20 & 20 & 20 & 20 & 20 & 20[ & 20 & 201 & 20 \\
\hline Offgas & 35 & 35 & 35 & 35 & 35 & 35 & 35 & 35 & 35 \\
\hline Water & 29.7 & 29.7 & 29.7 & 29.7 & 29.7 & 29.7 & 29.7 & 29.7 & 29.7 \\
\hline Sludge & 0.3 & 0.3 & 0.3 & 0.3 & 0.3 & 0.3 & 0.3 & 0.3 & 0.3 \\
\hline Lead Ingots & 4.5 & 4.5 & 4.5 & 4.5 & 4.5 & 4.5 & 4.5 & 4.5 & 4.5 \\
\hline Slag & 0.78 & 0.78 & 0.78 & 0.78 & 0.78 & 0.78 & 0.78 & 0.78 & 0.78 \\
\hline Subtotal-Lead Recovery & 90.28 & 90.28 & 90.28 & 90.28 & 90.28 & 90.28 & 80.28 & 90.28 & 80.28 \\
\hline \multicolumn{10}{|l|}{ Primary Stabilization } \\
\hline Offgas & 318 & 318 & 236 & 318 & & 316 & - & & \\
\hline Subtotal - Primary Stab. & 318 & 318 & 236 & 318 & 0 & 316 & 01 & 의 & 0 \\
\hline
\end{tabular}




\begin{tabular}{|c|c|c|c|c|c|c|c|c|c|}
\hline \multirow[b]{2}{*}{ Mass Flow (ib/hr) } & \multicolumn{9}{|c|}{ OVERALL MASS BALANCES FOR 19 ITTS SYSTEMS (Including ASPEN modeling results) } \\
\hline & $\begin{array}{l}\text { A-1 Baseline } \\
\text { Rotary Klln }\end{array}$ & $\begin{array}{l}\text { A-2 Rotary } \\
\text { Kiln w/ } \\
\text { Oxygen }\end{array}$ & $\begin{array}{l}\text { A-3 Rotany } \\
\text { Kiln w/ Wet } \\
\text { APC }\end{array}$ & $\begin{array}{l}\text { A-4 Rotary } \\
\text { Kiln W/ CO2 } \\
\text { Retention }\end{array}$ & $\begin{array}{l}\text { A-5 Rotary } \\
\text { Kiln w/ Poly. } \\
\text { Stabilization }\end{array}$ & $\begin{array}{l}\text { A-6 Rotary } \\
\text { Kiln w/Max. } \\
\text { Recycling }\end{array}$ & \begin{tabular}{|l|} 
A-7 \\
Slagging \\
Rotary Kiln
\end{tabular} & \begin{tabular}{|l} 
B-1 \\
Pyrolysis
\end{tabular} & \begin{tabular}{|l|} 
C-1 \\
Plasma \\
Furnace
\end{tabular} \\
\hline Aqueous Waste Treatment & & & & & & & & & \\
\hline $\begin{array}{l}\text { Treated Water (0.9/716/16 } \\
\text { aqueous waste, } 0.899 \mathrm{lb} / \mathrm{b} \\
\text { scrubber liquor) }\end{array}$ & $12,157.12$ & $9,010.62$ & $2,996.31$ & $14,129.53$ & $12,398.96$ & $11,968.33$ & $16,742.92$ & $4,219.85$ & $5,783.21$ \\
\hline $\begin{array}{l}\text { Resins, Organics, Spent } \\
\text { Carbon }(0.061 \mathrm{lb} / \mathrm{b} \text { aqueous } \\
\text { waste, } 0.061 \mathrm{lb} / \mathrm{b} \text { scrubber liquor) }\end{array}$ & 824.476 & 610.976 & 202.886 & 958.31 & 840.885 & 811.666 & 1135.637 & 285.907 & 391.986 \\
\hline $\begin{array}{c}\text { Treated ofigas } \\
\text { (b/b aqueous waste) }\end{array}$ & 0.56 & 0.56 & 0.56 & 0.56 & 0.56 & 0.56 & 0.56 & 0.56 & 0.56 \\
\hline $\begin{array}{l}\text { Concentrated Liquids (0.00\% } \\
\text { Ib/b aqueous waste, } 0.10 \mathrm{lb} / \mathrm{lb} \\
\text { scrubber liquor) }\end{array}$ & $1,231.16$ & 881.16 & 212.16 & $1,563.56$ & $1,258.06$ & $1,321.16$ & $1,741.26$ & 348.26 & 522.16 \\
\hline Salts from Concentrated Liquids & 113.00 & 113.00 & 113.00 & 0.00 & 113.00 & 2.00 & 113.00 & 113.00 & 113.00 \\
\hline Rinse water from $2^{\circ}$ stablization & 403.25 & 298.25 & 97.55 & 469.07 & 411.32 & 396.95 & 556.28 & 138.38 & 1900.55 \\
\hline $\begin{array}{l}\text { Subtotal-Aqueous Waste } \\
\text { Treatment }\end{array}$ & $14,729.67$ & $10,616.32$ & $\mathbf{3 , 6 2 4 . 9 2}$ & $16,651.96$ & $14,611.46$ & $14,103.72$ & $19,733.38$ & $4,967.68$ & $6,810.92$ \\
\hline Mercury Amalgamation & & & & & & & & & \\
\hline $\begin{array}{l}\text { Amalgamated Waste } \\
\text { lb } \mathrm{b} \text { b input) }\end{array}$ & 10.5 & 10.5 & 10.5 & 10.5 & 10.5 & 10.5 & 10.5 & 10.5 & 10.5 \\
\hline Offgas (1.94 lb/b input) & 97 & 97 & 97 & 97 & 97 & 97 & 97 & 97 & 97 \\
\hline $\begin{array}{l}\text { Conlaminated Solds } \\
\text { (0.35 lb/b input) }\end{array}$ & 17.5 & 17.5 & 17.5 & 17.5 & 17.5 & 17.5 & 17.5 & 17.5 & 17.5 \\
\hline $\begin{array}{l}\text { subtotal- Mercury } \\
\text { Amalgamation }\end{array}$ & 125 & 126 & 126 & 125 & 125 & 125 & 125 & 125 & 126 \\
\hline Special Waste & 153 & 153 & 153 & 153 & 153 & 153 & 153 & 153 & 153 \\
\hline $\begin{array}{l}\text { SUBTOTAL - Auxiliary } \\
\text { Subsystem Outputs }\end{array}$ & 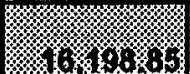 & \% & gropog & $60 / 2 \times 2$ & 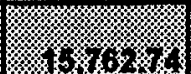 & 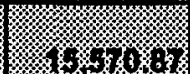 & 4060.606 & 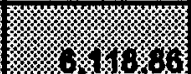 & $.868,20$ \\
\hline SUBTOTAL - Offgas & $20 \% 60656$ & 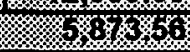 & Moth & 6.6.5. & $10 \% 55 \%$ & 1366 & 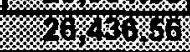 & 6 & 88694656 \\
\hline TOTAL OUTPUT & 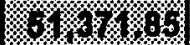 & 8895660 & 4860800 & 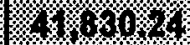 & $30.90 \% \%$ & $50666 \times 1$ & $69 x<60$ & 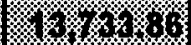 & $2,80 \times 1 \times 2$ \\
\hline $\begin{array}{l}\text { MASS BALANCE (Input - } \\
\text { Output) }\end{array}$ & 2.92 & 0.92 & 1.02 & 3.92 & 1.92 & 0.68 & 1.92 & 3.92 & 3.92 \\
\hline
\end{tabular}




\begin{tabular}{|c|c|c|c|c|c|c|c|c|c|}
\hline \multirow[b]{2}{*}{ Mass Flow (b/hr) } & \multicolumn{9}{|c|}{ OVERALL MASS BALANCES FOR 19 ITTS SYSTEMS (including ASPEN modeling results) } \\
\hline & $\begin{array}{l}\text { A-1 Baseline } \\
\text { Rotary Kln }\end{array}$ & $\begin{array}{l}\text { A-2 Rotary } \\
\text { Kiln w/ } \\
\text { Oxygen }\end{array}$ & $\begin{array}{l}\text { A-3 Rotary } \\
\text { Kiln w/ Wet } \\
\text { APC }\end{array}$ & $\begin{array}{l}\text { A-4 Rotary } \\
\text { Kiln w/ CO2 } \\
\text { Retention }\end{array}$ & $\begin{array}{l}\text { A-5 Rotary } \\
\text { Kiln w/ Poly. } \\
\text { Stabilization }\end{array}$ & $\begin{array}{l}\text { A-6 Rotary } \\
\text { Klin w/ Max. } \\
\text { Recycling }\end{array}$ & \begin{tabular}{|l} 
A-7 \\
Slagging \\
Rotary Kiln
\end{tabular} & \begin{tabular}{|l|} 
B-1 \\
Pyrolysis
\end{tabular} & \begin{tabular}{|l} 
C-1 \\
Plasma \\
Furnace \\
\end{tabular} \\
\hline $\begin{array}{l}\text { Volume Reduction Ratio } \\
\text { Calculation }\end{array}$ & & & & & & & & & \\
\hline Densifies (b/ft3) & & & & & & & & & \\
\hline Input Waste & 64 & 64 & 64 & 64 & 64 & 64 & 64 & 64 & 64 \\
\hline Soil & 67 & 67 & 67 & 67 & 67 & 67 & 67 & 67 & 67 \\
\hline Metal (Input) & 26 & 26 & 26 & 26 & 26 & 26 & 26 & 26 & 26 \\
\hline Metal (Output) & 480 & 480 & 480 & 480 & 480 & 480 & 480 & 480 & 480 \\
\hline Aqueous Liquid & 62 & 62 & 62 & 62 & 62 & 62 & 62 & 62 & 62 \\
\hline Slag & 187 & 187 & 187 & 187 & 187 & 187 & 187 & 187 & 187 \\
\hline Polymerized Salt & 80 & 80 & 80 & 80 & 80 & 80 & 80 & 80 & 80 \\
\hline Polymerized Ash/Metal/Salt & 94 & 94 & 94 & 94 & 94 & 94 & 94 & 94 & 94 \\
\hline Polymerized Lime & 96.5 & 96.5 & 96.5 & 96.5 & 96.5 & 96.5 & 96.5 & 96.5 & 96.5 \\
\hline Grouted Debris & 126.6 & 126.6 & 126.6 & 126.6 & 126.6 & 126.6 & 126.6 & 126.6 & 126.6 \\
\hline Stab Hg Amalgam & 732.96 & 732.96 & 732.96 & 732.96 & 732.96 & 732.96 & 732.96 & 732.96 & 732.96 \\
\hline Volumelric Flow Rate Calculation & & & & & & & & & \\
\hline Volume Solids In (fi3/hr) & 45.73 & 45.73 & 45.73 & 45.73 & 45.73 & 45.73 & 45.73 & 45.73 & 45.73 \\
\hline Mass Slag Out (1b/hr) & 151878 & 151878 & 1.13278 & 151878 & 2378 & 1.508 .78 & 1.691 .78 & 1518.78 & 1.450 .78 \\
\hline Mass Nonvitrified Ash out & 0.00 & 0 & 277 & $\frac{1,010.00}{0}$ & 1,078 & 0 & 0 & $\frac{1,010.10}{0}$ & $\frac{1,400.10}{0}$ \\
\hline Mass Special Waste (not stab) & 153.00 & 153.00 & 153.00 & 153.00 & 153.00 & 153.00 & 153.00 & 153.00 & 153.00 \\
\hline Mass Residues from MD & 5.00 & 5.00 & 5.00 & 5.00 & 5.00 & 5.00 & 5.00 & 5.00 & 5.00 \\
\hline Mass MTT Debris \& PbR Sludge & & & & & & & & & \\
\hline Out (lb/hr) & 0.30 & 0.30 & 0.30 & 0.30 & 0.30 & 0.30 & 0.30 & 0.30 & 0.30 \\
\hline Mass Metal/Pb Out (b/hr)clean & 621.50 & 621.50 & 621.50 & 621.50 & 621.50 & 629.50 & 487.50 & 621.50 & 657.50 \\
\hline Mass Salts Out (b/hr) & 118.00 & 118.00 & 118.00 & 118.00 & 118.00 & 7.24 & 118.00 & 118.00 & 118.00 \\
\hline Mass Lime Out (b/hr) & 0.00 & & & 317 & & & 0 & & 0 \\
\hline Mass Stab Hg Amalgam Out & 10.50 & 10.50 & 10.50 & 10.50 & 10.50 & 10.50 & 10.50 & 10.50 & 10.50 \\
\hline
\end{tabular}




\begin{tabular}{|c|c|c|c|c|c|c|c|c|c|}
\hline \multirow[b]{2}{*}{ Mass Flow $(\mathrm{lb} / \mathrm{hr})$} & \multicolumn{9}{|c|}{ OVERALL MASS BALANCES FOR 19 ITTS SYSTEMS (including ASPEN modeling results) } \\
\hline & $\begin{array}{l}\text { A-1 Baseline } \\
\text { Rotary Kiln }\end{array}$ & $\left\{\begin{array}{l}\text { A-2 Rotary } \\
\text { KIln w/ } \\
\text { Oxygen }\end{array}\right.$ & $\begin{array}{l}\text { A-3 Rotany } \\
\text { KIn w/ Wet } \\
\text { APC }\end{array}$ & $\begin{array}{l}\text { A-4 Rotary } \\
\text { Kaln w/ CO2 } \\
\text { Retention }\end{array}$ & $\begin{array}{l}\text { A-5 Rotary } \\
\text { KIln w/ Poly. } \\
\text { Stabilization }\end{array}$ & $\begin{array}{l}\text { A-6 Rotary } \\
\text { KIln w/ Max. } \\
\text { Recycling }\end{array}$ & $\begin{array}{l}\text { A-7 } \\
\text { Slagging } \\
\text { Rotary KIIn }\end{array}$ & $\begin{array}{l}\text { B-1 } \\
\text { Pyrolysis }\end{array}$ & $\begin{array}{l}\text { C-1 } \\
\text { Plasma } \\
\text { Fumace }\end{array}$ \\
\hline Volume Slag Out (ft3/hr) & 8.12 & 8.12 & 6.06 & 8.12 & 0.13 & 8.07 & 9.05 & 8.12 & 7.76 \\
\hline $\begin{array}{l}\text { Vo stab Nonvitritied Ash out } \\
\text { (ft3/hr) }\end{array}$ & 0.00 & 0.00 & 5.89 & 0.00 & 22.94 & 0.00 & 0.00 & 0.00 & 0.00 \\
\hline $\begin{array}{l}\text { Volume special Wastes (not } \\
\text { stabilized)(ti3/hr) }\end{array}$ & 2.39 & 2.39 & 2.39 & 2.39 & 2.39 & 2.39 & 2.39 & 2.39 & 2.39 \\
\hline Vol Stab MD Residues Out (ft $3 / \mathrm{hr})$ & 0.11 & 0.11 & 0.11 & 0.11 & 0.11 & 0.11 & 0.11 & 0.11 & 0.11 \\
\hline $\begin{array}{l}\text { Volume Stab MTT Debris \& PbR } \\
\text { Sludge Out (ft } 3 / \mathrm{hr} \text { ) }\end{array}$ & 0.01 & 0.01 & 0.01 & 0.01 & 0.01 & 0.01 & 0.01 & 0.01 & 0.01 \\
\hline Volume Metal/Pb Out ( $(\mathrm{t} 3 / \mathrm{hr})$ & 1.29 & 1.29 & 1.29 & 1.29 & 1.29 & 1.31 & 1.02 & 1.29 & 1.37 \\
\hline $\begin{array}{l}\text { Volume stabilized salts out } \\
\text { (ft } 3 / \mathrm{hr})\end{array}$ & 2.95 & 2.95 & 2.51 & 2.45 & 2.95 & 0.18 & 2.95 & 2.95 & 2.95 \\
\hline Volume Stabilized Lime Out (f $\mathrm{f} / \mathrm{hr})$ & 0.00 & 0.00 & 0.00 & 6.57 & 0.00 & 0.00 & 0.00 & 0.00 & 0.00 \\
\hline Vol Stab Hg Amalgam Out & 0.01 & 0.01 & 0.01 & 0.01 & 0.01 & 0.01 & 0.01 & 0.01 & 0.01 \\
\hline $\begin{array}{l}\text { Tolalvoluline solids outco } \\
\text { Disposal(fi3/hr) (includes 1:1 } \\
\text { polymer:ash, 1:1 polymer:salt, 2:1 } \\
\text { grout:debris) }\end{array}$ & 13.6 & 13.6 & 17.0 & 19.7 & 28.5 & 10.8 & 14.5 & 13.6 & 13.2 \\
\hline Volume Metal to Recycle ( $(\mathrm{t} 3 / \mathrm{hr})$ & 1.29 & 1.29 & 1.29 & 1.29 & 1.29 & 1.31 & 1.02 & 1.29 & 1.37 \\
\hline $\begin{array}{l}\text { Waste Volume Reduction Ratio } \\
\text { (in/Out) }\end{array}$ & 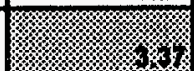 & 怒好 & 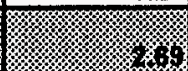 & (x) & 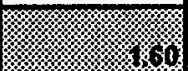 & 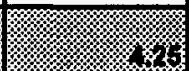 & 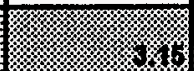 & 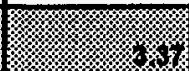 & 8 \\
\hline $\begin{array}{l}\text { Mass Processed Waste out } \\
(\mathrm{lb} / \mathrm{hr})\end{array}$ & & & & & & & & & \\
\hline Slag & 1518.78 & 1518.78 & 1132.78 & 1518.78 & 23.78 & 1508.78 & 1691.78 & 1518.78 & 1450.78 \\
\hline Stabilized Ash (1:1 polymer:ash) & 0.00 & 0.00 & 554.00 & 0.00 & 2156.00 & 0.00 & 0.00 & 0.00 & 0.00 \\
\hline Special Waste & 153.00 & 153.00 & 153.00 & 153.00 & 153.00 & 153.00 & 153.00 & 153.00 & 153.00 \\
\hline $\begin{array}{l}\text { stabilized MD Residue (1:T } \\
\text { polymer:resldue) }\end{array}$ & 10.00 & 10.00 & 10.00 & 10.00 & 10.00 & 10.00 & 10.00 & 10.00 & 10.00 \\
\hline $\begin{array}{l}\text { Stabilized Debris \& PBR Sludge. } \\
\text { (2:1 grout:debris) }\end{array}$ & 0.90 & 0.90 & 0.20 & 0.90 & 0.90 & 0.90 & 0.90 & 0.90 & 0.90 \\
\hline Stabilized Salts (1:1 polymer:salt) & 236.00 & 236.00 & 236.00 & 236.00 & 236.00 & 14.48 & 236.00 & 236.00 & 236.00 \\
\hline Stabllized Lime (1:1 polymer:lime) & 0.00 & 0.00 & 0.00 & 634.00 & 0.00 & 0.00 & 0.00 & 0.00 & 0.00 \\
\hline Hg Amalgam & 10.50 & 10.50 & 10.50 & 10.50 & 10.50 & 10.50 & 10.50 & 10.50 & 10.50 \\
\hline $\begin{array}{l}\text { Total Mass Processed Wastes } \\
\text { Out (lb/hr) }\end{array}$ & 1929.18 & 1929.18 & 2097.18 & 2563.18 & 2590.18 & 1697.66 & 2102.18 & 1929.18 & 1861.18 \\
\hline $\begin{array}{l}\text { Waste Mass Reduction Ratio } \\
\text { (In/Out) }\end{array}$ & 格 & 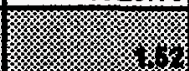 & 济滀 & 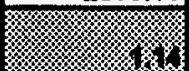 & 级 & 4 & ( & 学 & $\alpha$ \\
\hline
\end{tabular}




\begin{tabular}{|c|c|c|c|c|c|c|c|c|c|}
\hline \multirow[b]{2}{*}{ Mass Flow (lb/hr) } & \multicolumn{9}{|c|}{ OVERALL MASS BALANCES FOR 19 ITTS SYSTEMS (including ASPEN modeling results) } \\
\hline & $\mid \begin{array}{ll}\text { A-1 Baseline } \\
\text { Rotary Kiln }\end{array}$ & $\begin{array}{l}\text { A-2 Rotary } \\
\text { Kin w/ } \\
\text { Oxygen }\end{array}$ & $\begin{array}{l}\text { A-3 Rotary } \\
\text { Kiln w/ Wet } \\
\text { APC }\end{array}$ & $\begin{array}{l}\text { A-4 Rotary } \\
\text { Kiln w/ CO2 } \\
\text { Retention }\end{array}$ & $\begin{array}{l}\text { A-5 Rotary } \\
\text { Kiln w/ Poly. } \\
\text { Stabilization }\end{array}$ & $\begin{array}{l}\text { A-6 Rotary } \\
\text { Kiln w/ Max. } \\
\text { Recycling }\end{array}$ & \begin{tabular}{|l} 
A-7 \\
Slagging \\
Rotary Klin \\
\end{tabular} & $\begin{array}{l}\text { B-1 } \\
\text { Pyrolysis }\end{array}$ & $\begin{array}{l}\text { C-1 } \\
\text { Plasma } \\
\text { Fumace }\end{array}$ \\
\hline & \multicolumn{9}{|c|}{ Calculated Liquid Water Generation (lb/hr) } \\
\hline $\begin{array}{l}\text { Thputs to Aqueous Waste } \\
\text { Treatment System }\end{array}$ & & & & & & & & & \\
\hline Metal Decontamination Water & 112.00 & .112 .00 & 112.00 & 112.00 & 112.00 & 112.00 & 112.00 & 112.00 & 112.00 \\
\hline Lead Recovery Blast Waler & 29.70 & 29.70 & 29.70 & 29.70 & 29.70 & 29.70 & 29.70 & 29.70 & 29.70 \\
\hline Aqueous Waste & 80.00 & 80.00 & 80.00 & 80.00 & 80.00 & 80.00 & 80.00 & 80.00 & 80.00 \\
\hline Rinse Water from $2^{\circ}$ stabilization & 403.25 & 298.25 & 97.55 & 469.07 & 411.32 & 396.95 & 556.28 & 138.38 & 190.55 \\
\hline Scrubber water & $13,323.00$ & $9,823.00$ & $3,133.00$ & $15,630.00$ & $13,592.00$ & $13,224,00$ & $18,424.00$ & $4,494.00$ & $6,233.00$ \\
\hline $\begin{array}{l}\text { Totalmput o Aqueous } \\
\text { Treatment System (avallable for } \\
\text { recycle) }\end{array}$ & $13,947.95$ & $10,342,95$ & $3,452.25$ & $16,320.77$ & $14,225.02$ & $13,842.65$ & $19,201.98$ & $4,854,08$ & 6,645.25 \\
\hline Total Water Requirements & & & & & & & & & \\
\hline Water input to $1^{\circ}$ & 482.00 & $1,824,00$ & 526.00 & $\quad 1.00$ & 663.00 & 485.00 & 0.00 & 0.00 & 0.00 \\
\hline $1^{\circ}$ and $2^{\circ}$ Quench water & $10,221.00$ & $4,890.00$ & 0.00 & $14,360.00$ & $10,354.00$ & $10,224.00$ & $15,425.00$ & $1,411.00$ & $3,549.00$ \\
\hline $1^{\circ}$ and $2^{\circ}$ Scrubber water & $2,259.00$ & $2,259,00$ & $2,259.00$ & 0.00 & $2,259.00$ & $2,259.00$ & $2,259.00$ & $2,259.00$ & $\overline{2,259.00}$ \\
\hline Rinse Water for $2^{\circ}$ stabilization & 403.25 & 298.25 & 97.55 & 469.07 & 411.32 & 396.95 & 556.28 & 138.38 & 190.55 \\
\hline Metal Decontamination Water & 112.00 & 112.00 & 112.00 & 112.00 & 112.00 & 112.00 & 112.00 & 112.00 & 112.00 \\
\hline Mercury Amalgamation Water & 29.00 & 29.00 & 29.00 & 29.00 & 29.00 & 29.00 & 29.00 & 29.00 & 29.00 \\
\hline Lead Recovery, Blast and APC & 43.00 & 43.00 & 43.00 & 43.00 & 43.00 & 43.00 & 43.00 & 43.00 & 43.00 \\
\hline Total Water Requirements & $13,549.25$ & $9,455.25$ & $3,066.55$ & $15,014.07$ & $13,871,32$ & $13,548.95$ & $18,424,28$ & $3,992,38$ & 6,182.65 \\
\hline $\begin{array}{l}\text { Net Water ceneration Rate } \\
\text { (recycle - required) }\end{array}$ & 399 & 888 & 386 & 1,307 & 354 & 294 & 778 & 862 & 463 \\
\hline
\end{tabular}




\begin{tabular}{|c|c|c|c|c|c|c|c|c|c|c|}
\hline \multirow[b]{2}{*}{ Mass Flow (lb/hr) } & \multicolumn{10}{|c|}{ OVERALL MASS BALANCES FOR 19 ITTS SYSTEMS (Including ASPEN modeling results) } \\
\hline & $\begin{array}{l}\text { C-2 Plasma w/ } \\
\text { CO2 } \\
\text { Retention }\end{array}$ & $\begin{array}{l}\text { C-3 Plasma } \\
\text { w/ Steam } \\
\text { Gasification }\end{array}$ & \begin{tabular}{|l|} 
D-1 Fixed \\
Hearth \\
Pyrolyzer \\
\end{tabular} & \begin{tabular}{|l} 
E-1 \\
Thermal \\
Desorption
\end{tabular} & $\begin{array}{l}\text { F-1 } \\
\text { Molten Salt } \\
\text { Oxidation } \\
\end{array}$ & \begin{tabular}{|l|} 
G-1 Motten \\
Metal
\end{tabular} & $\begin{array}{l}\text { H-1 Steam } \\
\text { Gasification }\end{array}$ & $\begin{array}{l}\mathrm{J}-1 \text { Joule- } \\
\text { Heated } \\
\text { Vitrification }\end{array}$ & $\begin{array}{l}\text { K-1 } \\
\text { Desorption } \\
\text { w/ MEO }\end{array}$ & $\begin{array}{l}\text { L-1 } \\
\text { Desorption } \\
\text { w/ ScWO }\end{array}$ \\
\hline & \multicolumn{10}{|c|}{ WASTE INPUT } \\
\hline $\begin{array}{l}\text { MAIN THERMALL TREATMENT } \\
\text { SUBSYSTEM (MTT) }\end{array}$ & & & & & & & & & & \\
\hline Combustible & 660.4 & 660.4 & 660.4 & 660.4 & 660.4 & 660.4 & 660.4 & 660.4 & 50.7 & 50.7 \\
\hline Noncombustible & $1,339.6$ & $1,339.6$ & & & & 1,339.6 & & $1,339.6$ & & \\
\hline Metal & 149 & 149 & & & & 617 & & & & \\
\hline Aqueous Liquid & & & & & & & & & 16 & 16 \\
\hline$\frac{\text { THERMAL DESORBER }}{\text { Combusilible }}$ & & & & & & & & & 6097 & \\
\hline $\begin{array}{l}\text { Combuslible } \\
\text { Noncombustible }\end{array}$ & & & & 133966 & & & & & $\frac{609.7}{1340}$ & $\frac{609.7}{1340}$ \\
\hline VITRIFIER & & & & & & & & & $1,0+4$ & 1,340 \\
\hline Combustible & & & & & & & & & & \\
\hline Noncombustible & & & 1,340 & & 1,340 & & 1,340 & & & \\
\hline Subtotal-MTT & 2,149 & 2,149 & 2,000 & 2,000 & 2,000 & 2,617 & 2,000 & 2,000 & 2,016 & 2,016 \\
\hline AUXILIARY SUBSYSTEMS & & & & & & & & & & \\
\hline Metallic Waste (Surtace & & & & & & & & & & \\
\hline Contamination) & 468 & 468 & 468 & 468 & 468 & 0 & 468 & 468 & 468 & 468 \\
\hline Meallic Waste (Entrained & & & & & & & & & & \\
\hline Contamination) & & & 149 & 149 & 149 & & 149 & 149 & 149 & 149 \\
\hline Mercury & 50 & 50 & 50 & 50 & 50 & 50 & 50 & 50 & 50 & 50 \\
\hline Lead Metal & 26 & 26 & 26 & 26 & 26 & 26 & 26 & 26 & 26 & 26 \\
\hline Aqueous Waste & 80 & 80 & 80 & 80 & 80 & 80 & 80 & 80 & 64 & 64 \\
\hline Special Waste & 153 & 153 & 153 & 153 & 153 & 153 & 153 & 153 & 153 & 153 \\
\hline Halide and Sulfide salts & 1 & 1 & 1 & & 1 & & 1 & 1 & 1 & \\
\hline Subtotal - Auxiliary & 778 & 778 & 927] & 927 & 927 & 310 & 927 & 927 & 911 & 811 \\
\hline $\begin{array}{l}\text { Conlaminated Soll (Can replace } \\
\text { Soll, below) }\end{array}$ & & & & & & & & & & \\
\hline TOTAL WASTE INPUT & 87 & 8 & 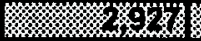 & zot & 30 & 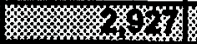 & 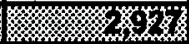 & 30 & $x$ & 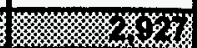 \\
\hline
\end{tabular}




\begin{tabular}{|c|c|c|c|c|c|c|c|c|c|c|}
\hline \multirow[b]{2}{*}{ Mass Flow $(\mathrm{lb} / \mathrm{hr})$} & \multicolumn{10}{|c|}{ OVERALL MASS BALANCES FOR 19 ITTS SYSTEMS (including ASPEN modelling results) } \\
\hline & $\begin{array}{l}\text { C-2 Plasma w/ } \\
\mathrm{CO} 2 \\
\text { Retention }\end{array}$ & $\begin{array}{l}\text { C-3 Plasma } \\
\text { w/ Steam } \\
\text { Gasification }\end{array}$ & $\begin{array}{l}\text { D-1 Fixed } \\
\text { Hearth } \\
\text { Pyrolyzer }\end{array}$ & \begin{tabular}{|l} 
E-1 \\
Thermal \\
Desorption
\end{tabular} & \begin{tabular}{|l} 
F-1 \\
Molten Salt \\
Oxidation
\end{tabular} & $\begin{array}{l}\text { G-1 Molten } \\
\text { Metal }\end{array}$ & $\begin{array}{l}\mathrm{H}-1 \quad \text { Steam } \\
\text { Gasification }\end{array}$ & $\begin{array}{l}\text { J-1 Joule- } \\
\text { Heated } \\
\text { Vitrification }\end{array}$ & \begin{tabular}{|l} 
K-1 \\
Desorption \\
W/ MEO
\end{tabular} & $\begin{array}{l}\text { L-1 } \\
\text { Desorption } \\
\text { w/ SCWO }\end{array}$ \\
\hline & \multicolumn{10}{|c|}{ A. PROCESS INPUTS } \\
\hline \multicolumn{11}{|l|}{$\begin{array}{l}\text { MAIN THERMAL TREATMENT } \\
\text { SUBSYSTEM (MTT) }\end{array}$} \\
\hline $\mathrm{H} 2 \mathrm{O}$ & & 60 & 142 & 910 & & & 446 & & & 3,033 \\
\hline \multicolumn{11}{|l|}{$\mathrm{CH} 4$} \\
\hline 02 & 1,225 & & 142 & & & 79 & & 1,414 & & 355 \\
\hline Air & & & & 7,766 & 6,120 & & & & & \\
\hline Air Inleakage & & & 224 & 2,695 & & & & & & \\
\hline Soil & 482 & 484 & & 54 & & 524 & & 498 & & \\
\hline \multicolumn{11}{|l|}{ VITRIFIER } \\
\hline Soll & & & 499 & & 500 & & 498 & & & \\
\hline \multirow{2}{*}{\multicolumn{11}{|c|}{$\frac{\text { Water }}{\mathrm{CH} 4}$}} \\
\hline & & & & & & & & & & \\
\hline 02 & & & 23 & 21 & 15 & & 118 & & & \\
\hline \multicolumn{11}{|l|}{ Air } \\
\hline \multicolumn{11}{|l|}{$\operatorname{SCC}$} \\
\hline $\mathrm{CH} 4$ & 36 & & 1 & 427 & 24 & & 27 & 80 & & \\
\hline 02 & 174 & & 988 & & & & & & & \\
\hline Air & & & 1982 & 8,858 & 496 & & 559 & 1,662 & & \\
\hline \multicolumn{11}{|l|}{ WATER } \\
\hline Primary Quench & 1732 & 1189 & 4225 & 10407 & 2114 & & & 2293 & & \\
\hline Secondary Quench & 427 & & 393 & & 659 & & 751 & & & \\
\hline Primany Scrubber & & 2,259 & & 1,864 & 4 & 467 & 1,765 & 2,254 & 902 & \\
\hline Secondary Scrubber & & & & & 494 & & 494 & & & \\
\hline \multicolumn{11}{|l|}{ OTHER } \\
\hline $\mathrm{NaOH}$ & & 78 & & 64 & 17 & 16 & 78 & 77 & 31 & 31 \\
\hline $\mathrm{Na2CO}$ & & & & & 121 & & & & & \\
\hline $\mathrm{CaO}$ & 173 & & 158 & & & 48 & & & & \\
\hline Electrolyte(water \& CoSO4) & & & & & & & & & 1,036 & \\
\hline \multicolumn{11}{|l|}{ Input to Indirect Heater } \\
\hline Air to Catalytic Oxidizer & & 10,254 & & & & 10,429 & 6,000 & & 2,960 & 196 \\
\hline $\mathrm{CH} 4$ & & & & 46 & & & 155 & & 59 & 59 \\
\hline Alr & & & & 948 & & & 3,215 & & 1,224 & 1,224 \\
\hline Subtotal - MTT & 4,249 & 14,324 & 8,777 & 34,074 & 10,564 & 11,563 & 14,106 & 8,278 & 8,212 & 4,898 \\
\hline AUXILIARY SUBSYSTEMS & & & & & & & & & & \\
\hline \multicolumn{11}{|l|}{ AUXTAL MELTING (MM) } \\
\hline Air & 0 & $\mathbf{0}$ & 25 & 25 & 25 & 0 & 25 & 25 & 25 & 25 \\
\hline Water & 0 & 0 & 19 & 19 & 19 & $\overline{0}$ & 19 & 19 & 19 & 19 \\
\hline Soll + Flux & $\overline{0}$ & $\overline{0}$ & 10 & 10 & 10 & $\overline{0}$ & 10 & 10 & 10 & 10 \\
\hline Subtotal - MM & 7 & 0 & 54 & 34 & 54 & 0 & 54 & 84 & 54 & 54 \\
\hline
\end{tabular}




\begin{tabular}{|c|c|c|c|c|c|c|c|c|c|c|}
\hline \multirow[b]{2}{*}{ Mass Flow (lb/hr) } & \multicolumn{10}{|c|}{ OVERALL MASS BALANCES FOR 19 ITTS SYSTEMS (including ASPEN modeling results) } \\
\hline & \begin{tabular}{|l|} 
C-2 Plasma w/ \\
CO2 \\
Retention \\
\end{tabular} & $\begin{array}{l}\text { C-3 Plasma } \\
\text { w/ Steam } \\
\text { Gasification }\end{array}$ & $\begin{array}{l}\text { D-1 Fixed } \\
\text { Hearth } \\
\text { Pyrolyzer }\end{array}$ & $\begin{array}{l}\text { E-1 } \\
\text { Thermal } \\
\text { Desorption }\end{array}$ & \begin{tabular}{|l|} 
F-1 \\
Molten Salt \\
Oxidation \\
\end{tabular} & $\begin{array}{ll}\text { G-1 } & \text { Molten } \\
\text { Metal } & \\
\end{array}$ & $\begin{array}{l}\text { H-1 Steam } \\
\text { Gasification }\end{array}$ & \begin{tabular}{|l|} 
J-1 Joule- \\
Heated \\
Vitifification \\
\end{tabular} & \begin{tabular}{|l|} 
K-1 \\
Desorption \\
W/ MEO
\end{tabular} & $\begin{array}{l}\text { L-1 } \\
\text { Desorption } \\
\text { W/ SCWO }\end{array}$ \\
\hline LEAD RECOVERY (PbR) & & & & & & & & & & \\
\hline APC Waler (use recycle) & 14 & 14 & 14 & 14 & 14 & 14 & 14 & 14 & 14 & 14 \\
\hline Fuel & & & & & & & & & & \\
\hline Air & 21 & 21 & 21 & 21 & 21 & 21 & 21 & 21 & 21 & 21 \\
\hline Blast Water & 29 & 29 & 29 & 29 & 29 & 29 & 29 & 29 & 29 & 29 \\
\hline Soll & 0.26 & 0.26 & 0.26 & 0.26 & 0.26 & 0.26 & 0.26 & 0.26 & 0.26 & 0.26 \\
\hline Subtotal -PbR & 64.26 & 64.26 & 64.26 & 64.26 & 64.26 & 64.26 & 64.26 & 64.26 & 64.26 & 64.26 \\
\hline $\begin{array}{l}\text { AQUEOUSWASTE } \\
\text { TREATMENT (AQ) }\end{array}$ & & & & & & & & & & \\
\hline Resins \& Aclivated Carbon & 167.1 & 181.44 & 438.6 & 816.54 & 228.12 & 37.14 & 187.44 & 337.02 & 59.94 & 209.7 \\
\hline Additives $(0.051 \mathrm{lb} / \mathrm{b}$ input) & 0 & 0 & 0 & 0 & $\therefore 0$ & 0 & 0 & 0 & 0 & 0 \\
\hline Rinse Water for $2^{\circ}$ Stabllization & 81.32 & 88.49 & 217.07 & 406.04 & 111.83 & 16.34 & 91.49 & 166.28 & 28.18 & 103.06 \\
\hline Subtotal -AQ & 167.1 & 181.44 & 438.6 & 816.64 & 228.12 & 37.14 & 187.44 & 337.02 & 59.94 & 209.7 \\
\hline $\begin{array}{l}\text { MERCURY AMALGAMATTON } \\
\text { (HGA) }\end{array}$ & & & & & & & & & & \\
\hline Addilives $(0.1 \mathrm{lb} / \mathrm{b})$ & 5 & 5 & 5 & 5 & 5 & 5 & 5 & 5 & 5 & 5 \\
\hline Nitrogen $(0.22 \mathrm{lb} / \mathrm{b} / \mathrm{b})$ & 11 & 11 & 11 & 11 & 11 & 11) & 11 & 11 & 11 & 11 \\
\hline Air $(0.60 \mathrm{lb} / \mathrm{b})$ & 30 & 30 & 30 & 30 & 30 & 30 & 30 & 30 & 30 & 30 \\
\hline Water $(0.58 \mathrm{lb} / \mathrm{b})$ & 29 & 29 & 29 & 29 & 29 & 29 & 29 & 29 & 29 & 29 \\
\hline Subtotal - HgA & 75 & 75 & 75 & 75 & 76 & 76 & 76 & 76 & 76 & 76 \\
\hline TOTAL PROCESS INPUTS & 2555 & 14645 & 9609 & 36084 & $80 \% 985$ & J678 & 84887 & 8606 & $6 \times 665$ & 301 \\
\hline & \multicolumn{10}{|c|}{ INTERNAL SYSTEM RECYCLE } \\
\hline $\begin{array}{l}\text { Scrubber Liquor from scrubber to } \\
\text { aqueous waste treatment }\end{array}$ & 2,705 & 2,944 & 7,230 & 13,529 & 3,722 & 539 & 3,044 & 5,537 & 935 & 3,431 \\
\hline $\begin{array}{l}\text { Sludge from } \mathrm{Pb} \text { recovery to } \\
\text { aqueous waste treatment }\end{array}$ & 0 & 0 & 0 & 0 & o. & 0 & o & o & 0 & o \\
\hline $\begin{array}{l}\text { Resins from aqueous waste } \\
\text { treatment to thermal treatment }\end{array}$ & 170 & 184 & 446 & 830 & 232 & 38 & 191 & 343 & 61 & 213 \\
\hline Hg amalgamation solids to thermal & 18 & 18 & 18 & 48 & 18 & 101 & 10 & 101 & 10 & 100 \\
\hline 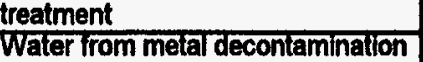 & 18 & 18 & & & & 18 & 18 & 100 & 18 & 18) \\
\hline to recycle & 112 & 112 & 112 & 112 & 112 & 0 & 112 & 112 & 112 & 112 \\
\hline $\begin{array}{l}\text { TOTAL INTERNAL. SYSTEM } \\
\text { RECYCLE (Not including recycle } \\
\text { to thermal treatment) }\end{array}$ & 2,817 & 3,056 & 7,342 & 13,641 & 3,834 & 539 & 3,156 & 5,649 & 1,047 & 3,543 \\
\hline TOTAL INPUT & 0000 & 2006 & 99680 & 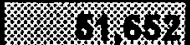 & 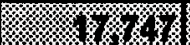 & 8,806 & \%30\% & 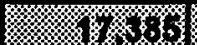 & 80940 & 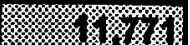 \\
\hline
\end{tabular}




\begin{tabular}{|c|c|c|c|c|c|c|c|c|c|c|}
\hline \multirow[b]{2}{*}{ Mass Flow (lb/hr) } & \multicolumn{10}{|c|}{ OVERALL. MASS BALANCES FOR 19 ITTS SYSTEMS (Including ASPEN modeling results) } \\
\hline & $\begin{array}{l}\text { C-2 Plasma w } \\
\text { CO2 } \\
\text { Retention }\end{array}$ & $\begin{array}{l}\text { C-3 Plasma } \\
\text { W/ Steam } \\
\text { Gasification }\end{array}$ & $\begin{array}{l}\text { D-1 Fixed } \\
\text { Hearth } \\
\text { Pyrolyzer }\end{array}$ & \begin{tabular}{|l} 
E-1 \\
Thermal \\
Desorption
\end{tabular} & \begin{tabular}{|l} 
F-1 \\
Molten Salt \\
Oxidation
\end{tabular} & $\begin{array}{l}\text { G-1 Molten } \\
\text { Metal }\end{array}$ & $\begin{array}{l}\text { H-1 } \\
\text { Gasification }\end{array}$ & $\begin{array}{l}\text { J-1 Joule- } \\
\text { Heated } \\
\text { Vitrification }\end{array}$ & \begin{tabular}{|l|} 
K-1 \\
Desorption \\
W/ MEO
\end{tabular} & $\begin{array}{l}\text { L-1 } \\
\text { Desorption } \\
\text { W/ SCWO }\end{array}$ \\
\hline & \multicolumn{10}{|c|}{ SYSTEM OUTPUT } \\
\hline & \multicolumn{10}{|c|}{ B. MAIN THERMAL TREATMENT (MTT) SUBSYSTEM OUTPUT } \\
\hline SOLIDS & & & & & & & & & & \\
\hline Slag & 1,450 & 1,452 & 1,496 & 161 & 1,500 & 1,572 & 1,493 & 1,494 & & \\
\hline Debris & & & & 1,051 & & & & & 1,550 & 1,550 \\
\hline Nonvitriffed Ash & & & & & & & & & & \\
\hline Metal & 170 & 170 & & & & 613 & & & & \\
\hline Trace Metals & 4 & 4 & 4 & 1 & 4 & 4 & 4 & 4 & 1 & \\
\hline $\mathrm{Na2CO3}$ & & & & & 40 & & & & & \\
\hline $\mathrm{NaCl} / \mathrm{NaF}$ & & & & & 87 & & & & & \\
\hline $\mathrm{HCl}(50 \%$ in water) & & & & & & & & & & \\
\hline $\mathrm{CaO}$ & 116 & & 102 & & & & & & & \\
\hline $\mathrm{CaCl} 2, \mathrm{CaSO} 4$ & 113 & & 113 & & & & & & & \\
\hline Subtotal - Solids & 1,853 & 1,626 & 1,715 & 1,213 & 1,631 & 2,189 & 1,497 & 1,498 & 1,551 & 1,551 \\
\hline $\begin{array}{l}\text { Main Thermal Treatment } \\
\text { APC }\end{array}$ & & & & & & & & & & \\
\hline LIQUIDS \& DISSOLVED GASES & & & & & & & & & & \\
\hline Scrubber Water & 2,705 & 2,831 & 7,230 & 13,436 & 3,697 & 516 & 2,931 & 5,424 & 890 & 3,386 \\
\hline Dissolved salls & & 113 & & 93 & 25 & 23 & 113 & 113 & 45 & 45 \\
\hline Dissolved gases & & & & & & & & & & \\
\hline 02 & 5 & & 1 & 2 & & & & 1 & & \\
\hline N2 & & & 10 & 13 & 4 & & & 3 & & \\
\hline $\mathrm{CO} 2$ & & 16 & & 23 & 11 & 1 & 8 & 41 & 14 & 30 \\
\hline $\mathrm{SO} 2$ & & & & & 1 & & & 1 & & \\
\hline Electrolyte(water \& CoSO4) & & & & & & & & & 1,036 & \\
\hline $\begin{array}{l}\text { Subtotal-Lquids \& DIssolved } \\
\text { Gases }\end{array}$ & 2,710 & 2,960 & 7,241 & 13,667 & 3,738 & 540 & 3,054 & 6,583 & 1,985 & 3,462 \\
\hline GASES & & & & & & & & & & \\
\hline 02 & 54 & 1,194 & 16 & 1,622 & 276 & 1,215 & 359 & 317 & 375 & 85 \\
\hline $\mathrm{N} 2$ & & 7,865 & 163 & 14,809 & 5,071 & 8,000 & 5,030 & 1,272 & 2,270 & 150 \\
\hline $\mathrm{CO2}$ & 1,352 & 1,237 & 1,244 & 2,351 & 1,330 & 1,351 & 1,306 & 1,402 & 308 & 292 \\
\hline $\mathrm{H} 2 \mathrm{O}$ vapor & 429 & 1,587 & 399 & 1,484 & 515 & 883 & 1,486 & 204 & 453 & 87 \\
\hline SO2 & & & & & & 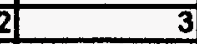 & & & & \\
\hline Subtotal-Gases & 1,836 & 11,886 & 1,822 & 20,267 & 7,194 & 11,452 & 8,183 & 3,197 & 3,406 & 614 \\
\hline
\end{tabular}




\begin{tabular}{|c|c|c|c|c|c|c|c|c|c|c|}
\hline & \multicolumn{10}{|c|}{ OVERALL MASS BALANCES FOR 19 ITTS SYSTEMS (including ASPEN modelling results) } \\
\hline Mass Flow (lb/hr) & \begin{tabular}{|l} 
C-2 Plasma w/ \\
CO2 \\
Retention
\end{tabular} & $\begin{array}{l}\text { C-3 Plasma } \\
\text { w/ Steam } \\
\text { Gasification }\end{array}$ & \multirow{2}{*}{$\begin{array}{l}\text { D-1 Flxed } \\
\text { Hearth } \\
\text { Pyrolyzer } \\
\end{array}$} & \multirow[t]{2}{*}{\begin{tabular}{|l|} 
E-1 \\
Thermal \\
Desorption \\
\end{tabular}} & \multirow[t]{2}{*}{\begin{tabular}{|l|} 
F-1 \\
Molten Salt \\
Oxidation
\end{tabular}} & \multirow[t]{2}{*}{$\begin{array}{l}\text { G-1 Molten } \\
\text { Metal }\end{array}$} & \multirow[t]{2}{*}{$\begin{array}{ll}\text { H-1 } & \text { Steam } \\
\text { Gasification }\end{array}$} & \multirow[t]{2}{*}{\begin{tabular}{|l|} 
J-1 Joule- \\
Heated \\
Vitrification \\
\end{tabular}} & \multirow[t]{2}{*}{\begin{tabular}{|l|} 
K-1 \\
Desorption \\
W/MEO \\
\end{tabular}} & $\begin{array}{l}\text { L-1 } \\
\text { Desorption } \\
\text { w/ ScWo }\end{array}$ \\
\hline INDIRECT-FIRED EXHAUST & & & & & & & & & & \\
\hline $\mathrm{O} 2$ & & & & 38 & & & 130 & & 49 & 49 \\
\hline N2 & & & & 728 & & & 2467 & & 939 & 939 \\
\hline $\mathrm{CO} 2$ & & & & 125 & & & 425 & & 162 & 162 \\
\hline H2O vapor & & & & 103 & & & 348 & & 133 & 133 \\
\hline Subtotal - Indirect-fired exhaust & $\mathbf{0}$ & o & o & 994 & 0 & o] & 3370 & o. & 1283 & 1283 \\
\hline $\begin{array}{l}\text { TOTAL - MTT Subsystems } \\
\text { Output }\end{array}$ & 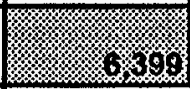 & 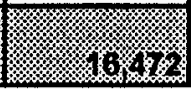 & 60168 & 36.041 & $12 x_{66}$ & 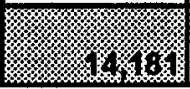 & 60.043 & 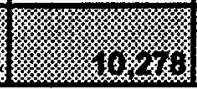 & .225 & 6810 \\
\hline & \multicolumn{10}{|c|}{ AUXILIARY SUBSYSTEM OUTPUTS } \\
\hline Metal Decontamination (MD) & & & & & & & & & & \\
\hline Clean Metal & 463 & 463 & 463 & 463 & 463 & 0 & 463 & 463 & 463 & 463 \\
\hline Grit & & & & & 5 & of & 5 & 5 & 5 & 5 \\
\hline Water & 112 & 112 & 112 & 112 & 112 & 0 & 112 & 112 & 112 & 112 \\
\hline Subtotal-Metal Decon & 680 & 680 & 580 & 680 & 680 & 0 & 680 & 680 & 680 & 680 \\
\hline Metal Melting & & & & & & & & & & \\
\hline Clean Metal & 0 & 0 & 134 & 134 & 134 & 0 & 134 & 134 & 134 & 134 \\
\hline Treated Offgas & 0 & 0 & 46 & 46 & 46 & 0 & 46 & 46 & 46 & 46 \\
\hline Slag & 0 & 0 & 23 & 23 & 23 & o & 23 & 23 & 23 & 23 \\
\hline Subtotal - Metal Melting & 0 & 0 & 203 & 203 & 203 & 0 & 203 & 203 & 203 & 203 \\
\hline Lead Recovery & & & & & & & & & & \\
\hline Clean Lead & 20 & 20 & 20 & 20 & 20 & 20 & 201 & 20 & 20 & 20 \\
\hline Offgas & 35 & 35 & 35 & 35 & 35 & 35 & 35 & 35 & 35 & 35 \\
\hline Water & 29.7 & 29.7 & 29.7 & 29.7 & 29.7 & 29.7 & 29.7 & 29.7 & 29.7 & 29.7 \\
\hline Sludge & 0.3 & 0.3 & 0.3 & 0.3 & 0.3 & 0.3 & 0.3 & 0.3 & 0.3 & 0.3 \\
\hline Lead Ingols & 4.5 & 4.5 & 4.5 & 4.5 & 4.5 & 4.5 & 4.5 & 4.5 & 4.5 & 4.5 \\
\hline Slag & 0.78 & 0.78 & 0.78 & 0.78 & 0.78 & 0.78 & 0.78 & 0.78 & 0.78 & 0.78 \\
\hline Subtotal-Lead Recovery & 90.28 & 90.28 & 90.28 & 90.28 & 90.28 & 90.28 & 90.28 & 90.28 & 80.28 & 90.28 \\
\hline Primary Stabilization & & & & & & & & & & \\
\hline Offgas & & & & 3 & & & & & & \\
\hline Subtotal - Primary Stab. & 0 & 0 & 0 & 34 & 0 & 0) & of & 0 & of & t \\
\hline
\end{tabular}




\begin{tabular}{|c|c|c|c|c|c|c|c|c|c|c|}
\hline \multirow[b]{2}{*}{ Mass Flow (b/hr) } & \multicolumn{10}{|c|}{ OVERALL MASS BALANCES FOR 19 ITTS SYSTEMS (Including ASPEN modeling results) } \\
\hline & $\begin{array}{l}\text { C-2 Plasma w/ } \\
\text { CO2 } \\
\text { Retention } \\
\end{array}$ & $\begin{array}{l}\text { C-3 Plasma } \\
\text { w/ Steam } \\
\text { Gasification } \\
\end{array}$ & $\begin{array}{l}\text { D-1 Fixed } \\
\text { Hearth } \\
\text { Pyrolyzer } \\
\end{array}$ & \begin{tabular}{|l} 
E-1 \\
Thermal \\
Desorption \\
\end{tabular} & \begin{tabular}{|l|} 
F-1 \\
Molten Salt \\
Oxidation \\
\end{tabular} & $\begin{array}{|ll|}\text { G-1 } & \text { Molten } \\
\text { Metal }\end{array}$ & $\begin{array}{ll}\text { H-1 } & \text { Steam } \\
\text { Gasification }\end{array}$ & $\begin{array}{l}\text { J-1 Joule- } \\
\text { Heated } \\
\text { Vitrification } \\
\end{array}$ & \begin{tabular}{|l} 
K-1 \\
Desorption \\
W/MEO \\
\end{tabular} & $\begin{array}{l}\text { L-1 } \\
\text { Desorption } \\
\text { W/ ScWO } \\
\end{array}$ \\
\hline Aqueous Waste Treatment & & & & & & & & & & \\
\hline $\begin{array}{l}\text { Trealed water (0.97/16/16 } \\
\text { aqueous waste, } 0.899 \mathrm{lb} / \mathrm{b} \\
\text { scrubber liquor) }\end{array}$ & $2,509.96$ & $2,724.82$ & $6,577.93$ & $12,240.73$ & 3,424.24 & 562.72 & $2,814.72$ & $5,055.92$ & 903.09 & $3,147.00$ \\
\hline $\begin{array}{l}\text { Resins, Organics, Spent } \\
\text { Carbon }(0.061 \mathrm{lb} / \mathrm{b} \text { aqueous } \\
\text { waste, } 0.061 \mathrm{lb} / \mathrm{b} \text { scrubber liquor) }\end{array}$ & 169.885 & 184.464 & 445.91 & 830.149 & 231.922 & 37.759 & 190.564 & 342.637 & 60.939 & 213.195 \\
\hline $\begin{array}{l}\text { Treated ofigas } \\
\text { Ib/b aqueous waste) }\end{array}$ & 0.56 & 0.56 & 0.56 & 0.56 & 0.56 & 0.56 & 0.56 & 0.56 & 0.448 & \\
\hline $\begin{array}{l}\text { Concentrated Liquids (0.00/ } \\
\text { lb/b aqueous waste, } 0.10 \mathrm{lb} / \mathrm{b} \\
\text { scrubber liguor) }\end{array}$ & 271.06 & 181.96 & 723.56 & 1.260 .46 & 347.76 & 31.46 & 191.96 & 441.26 & 48.95 & 298.55 \\
\hline Salts from Concentrated Liquids & 0.00 & 113.00 & 0.00 & 93.00 & 25.00 & 23.00 & 113.00 & 113.00 & 45.00 & 45.00 \\
\hline Rinse water from $2^{\circ}$ stabilization & 81.32 & 88.49 & 217.07 & 406.04 & 111.83 & 16.34 & 91.49 & 166.28 & 28.18 & 103.06 \\
\hline $\begin{array}{l}\text { Subtotal-Aqueous Waste } \\
\text { Treatment }\end{array}$ & $2,961.46$ & $3,204,80$ & $7,747.96$ & $14,424,90$ & $4,029.48$ & 655.50 & $3,310,80$ & $6,853.38$ & $1,068.43$ & $3,704.19$ \\
\hline Mercury Amalgamation & & & & & & & & & & \\
\hline $\begin{array}{l}\text { Amalgamated Waste } \\
\text { Ib/b input) }\end{array}$ & 10.5 & 10.5 & 10.5 & 10.5 & 10.5 & 10.5 & 10.5 & 10.5 & 10.5 & 10.5 \\
\hline Offgas (1.94 lb/b input) & 97 & 97 & 97 & 97 & 97 & 97 & 97 & 97 & 97 & 97 \\
\hline $\begin{array}{l}\text { Contaminated Solids } \\
(0.35 \mathrm{lb} / \mathrm{b} \text { input) }\end{array}$ & 17.5 & 17.5 & 17.5 & 17.5 & 17.5 & 17.5 & 17.5 & 17.5 & 17.5 & 17.5 \\
\hline $\begin{array}{l}\text { Subtotal-Mercury } \\
\text { Amalgamation }\end{array}$ & 125 & 125 & 125. & 125 & 125 & 125 & 125 & 125 & 125. & 125 \\
\hline Special Waste & 153 & 153 & 153 & 153 & 153 & 153 & 153 & 153 & 153 & 153 \\
\hline $\begin{array}{l}\text { SUBTOTAL-Auxillary } \\
\text { Subsystem Outputs }\end{array}$ & 3.899614 & $=4 \operatorname{css} 602$ & $8: 8690.24$ & 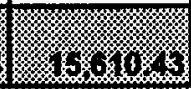 & 6,8028 & mox & $2: 1,462008$ & $8 \times 104660$ & $2,34692,3$ & 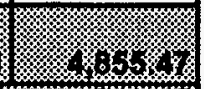 \\
\hline SUBTOTAL - Offgas & 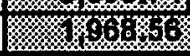 & K $80 \% 6 \%$ & 300056 & 16\% & 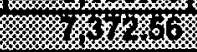 & 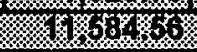 & 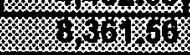 & 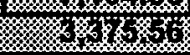 & $x$ & 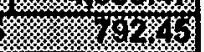 \\
\hline TOTAL OUTPUT & rowosk & $30 \% 60 \% 0$ & $696 \% \% 23$ & $6605 \%$ & 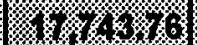 & $4<0.86$ & $60 \% 366 \times 36$ & W & 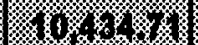 & 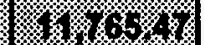 \\
\hline $\begin{array}{l}\text { MASS BALANCE (input - } \\
\text { Qutput) }\end{array}$ & 0.92 & 2.92 & 0.92 & 0.92 & 2.92 & 0.02 & 3.92 & 1.92 & 4.79 & 6.79 \\
\hline
\end{tabular}




\begin{tabular}{|c|c|c|c|c|c|c|c|c|c|c|}
\hline \multirow[b]{2}{*}{ Mass Flow (lb/hr) } & \multicolumn{10}{|c|}{ OVERALL MASS BALANCES FOR 19 ITTS SYSTEMS (including ASPEN modeling results) } \\
\hline & $\begin{array}{l}\text { C-2 Plasma w/ } \\
\text { CO2 } \\
\text { Retention }\end{array}$ & $\begin{array}{l}\text { C-3 Plasma } \\
\text { W/ Steam } \\
\text { Gasification }\end{array}$ & \begin{tabular}{|l|} 
D-1 Fixed \\
Hearth \\
Pyrolyzer
\end{tabular} & \begin{tabular}{|l|} 
E-1 \\
Thermal \\
Desorption
\end{tabular} & \begin{tabular}{|l|} 
F-1 \\
Molten Salt \\
Oxidation
\end{tabular} & $\mid \begin{array}{ll}\text { G-1 } & \text { Molten } \\
\text { Metal }\end{array}$ & $\begin{array}{l}\text { H-1 Steam } \\
\text { Gasification }\end{array}$ & \begin{tabular}{|l|}
$J-1$ Joule- \\
Heated \\
Vitrification \\
\end{tabular} & $\begin{array}{l}K-1 \\
\text { Desorption } \\
W / M E O\end{array}$ & $\begin{array}{l}\text { L-1 } \\
\text { Desorption } \\
\text { W/ SCWO }\end{array}$ \\
\hline \multicolumn{11}{|l|}{$\begin{array}{l}\text { Volume Reduction Ratio } \\
\text { Calculation }\end{array}$} \\
\hline \multicolumn{11}{|l|}{ Densities (lb/tit3) } \\
\hline Input Waste & 64 & 64 & 64 & 64 & 64) & 64 & 64 & 64 & 64 & 64 \\
\hline Soil & 67. & 67 & 67 & 67 & 67) & 67] & 67 & 67. & 67 & 67 \\
\hline Metal (Input) & 26 & 26 & 26 & 26 & 26 & 26 & 26 & 26 & 26 & 26 \\
\hline Metal (Output) & 480 & 480 & 480 & 480 & 480 & 480 & 480 & 480 & 480 & 480 \\
\hline Aqueous Liquid & 62 & 62 & 62 & 62 & 62 & 62 & 62 & 62 & 62 & 62 \\
\hline Slag & 187 & 187 & 187 & 187 & 187 & 187 & 187 & 187 & 187 & 187 \\
\hline Polymerized Salt & 80 & 80 & 80 & 80 & 80 & 80 & 80 & 80 & 80 & 80 \\
\hline Polymerized Ash/Metal/Salt & 94 & 94 & 94 & 94 & 94 & 94 & 94 & 94 & 94 & 94 \\
\hline Polymerized LIme & 96.5 & 96.5 & 96.5 & 96.5 & 96.5 & 96.5 & 96.5 & 96.5 & 96.5 & 96.5 \\
\hline Grouted Debris & 126.6 & 126.6 & 126.6 & 126.6 & 126.6 & 126.6 & 126.6 & 126.6 & 126.6 & 126.6 \\
\hline Stab Hg Amalgam & 732.96 & 732.96 & 732.96 & 732.96 & 732.96 & 732.96 & 732.96 & 732.96 & 732.96 & 732.96 \\
\hline \multicolumn{11}{|l|}{ Volumetric Flow Rale Calculation } \\
\hline Volume Solids $\ln (\mathrm{t}(\mathrm{B} / \mathrm{hr})$ & 45.73 & 45.73 & 45.73 & 45.73 & 45.73 & 45.73 & 45.73 & 45.73 & 45.73 & 45.73 \\
\hline Mass Slag Out (lb/hr) & $1,450.78$ & $1,452.78$ & $1,519.78$ & 184.78 & $1,523.78$ & $1,572.78$ & $1,516.78$ & $1,517.78$ & 23.78 & 23.78 \\
\hline Mass Nonvitifified Ash Out & & 0 & 0 & 0 & 0 & 0 & & 0 & 0 & \\
\hline Mass Special Waste (not stab) & 153.00 & 153.00 & 153.00 & 153.00 & 153.00 & 153.00 & 153.00 & 153.00 & 153.00 & 153.00 \\
\hline Mass Residues from MD & 5.00 & 5.00 & 5.00 & 5.00 & 5.00 & 0.00 & 5.00 & 5.00 & 5.00 & 5.00 \\
\hline Mass MTT Debris \& PbR Sludge & & & & & & 0301 & 0.301 & 0.30 & 155030 & 155030 \\
\hline$\frac{\text { Out (b/hr) }}{\text { Mass Metal/Pb Out (lb/hrrclean }}$ & $\begin{array}{r}0.30 \\
657.50\end{array}$ & $\begin{array}{r}0.30 \\
657.50\end{array}$ & $\frac{0.30}{621.50}$ & $\frac{1,051.30}{621.50}$ & \begin{tabular}{r|}
0.30 \\
621.50
\end{tabular} & $\begin{array}{r}0.30 \\
637.50\end{array}$ & 621.50 & $\frac{0.30}{621.50}$ & $\frac{1,000.00}{621.50}$ & $\frac{1,550.30}{621.50}$ \\
\hline Mass Salts Out (b/hr) & 118.00 & 118.00 & 118.00 & 95.00 & 157.00 & 28.00 & 118.00 & 118.00 & 129.00 & 47.00 \\
\hline Mass Lime Out (b/hr) & 116 & 0 & 102 & & & of & 0 & 0 & 0 & 0 \\
\hline Mass Stab Hg Amalgam out & 10.50 & 10.50 & 10.50 & 10.50 & 10.50 & 10.50 & 10.50 & 10.50 & 10.50 & 10.50 \\
\hline
\end{tabular}




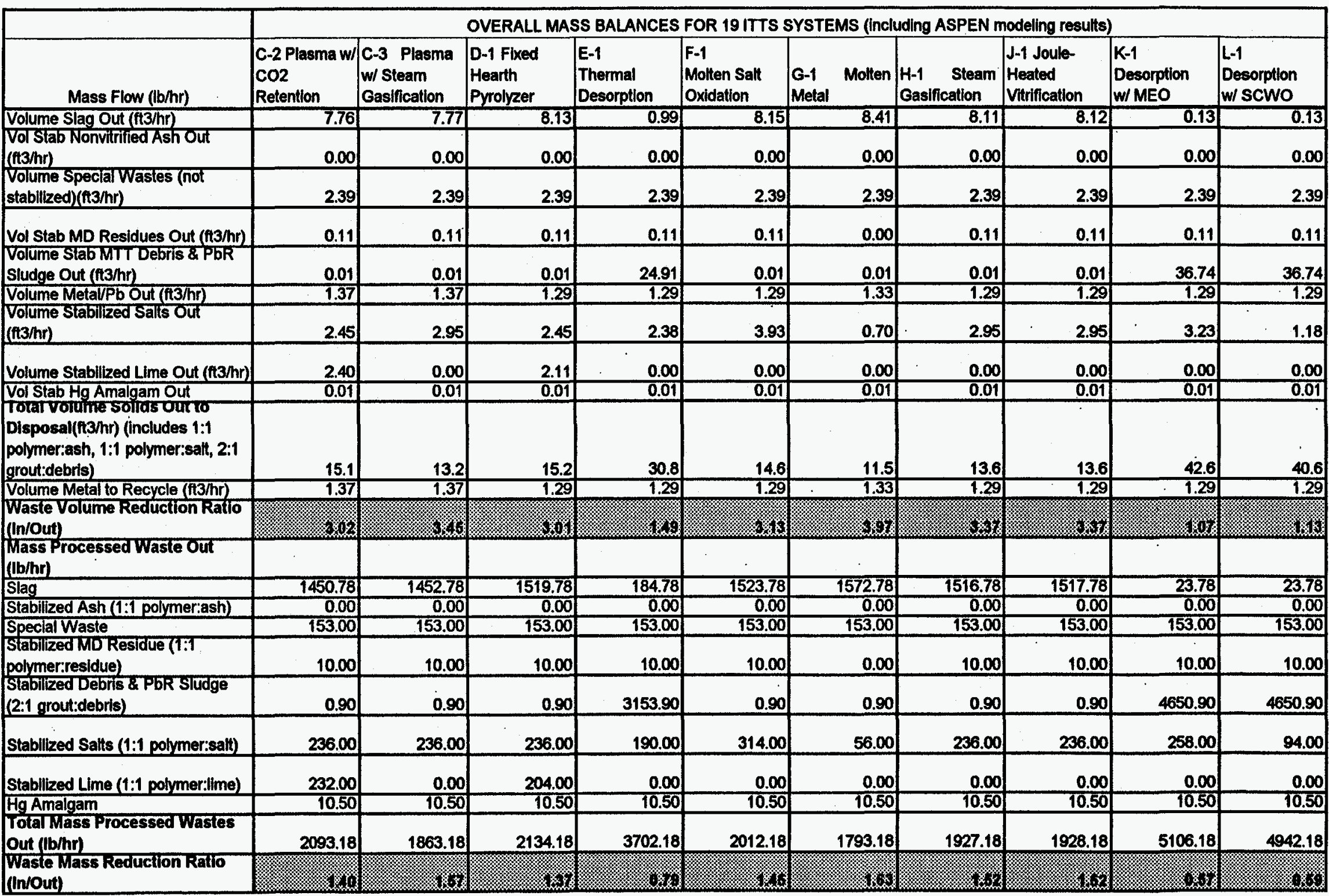




\begin{tabular}{|c|c|c|c|c|c|c|c|c|c|c|}
\hline \multirow[b]{2}{*}{ Mass Flow (lb/hr) } & \multicolumn{10}{|c|}{ OVERALL MASS BALANCES FOR 19 ITTS SYSTEMS (Including ASPEN modeling results) } \\
\hline & $\begin{array}{l}\text { C-2 Plasma w/ } \\
\mathrm{CO} 2 \\
\text { Retention }\end{array}$ & $\begin{array}{l}\text { C-3 Plasma } \\
\text { w/ Steam } \\
\text { Gasificalion } \\
\end{array}$ & $\begin{array}{l}\text { D-1 Fixed } \\
\text { Hearth } \\
\text { Pyrolyzer } \\
\end{array}$ & \begin{tabular}{|l} 
E-1 \\
Thermal \\
Desorption
\end{tabular} & \begin{tabular}{|l|} 
F-1 \\
Molten Salt \\
Oxidation \\
\end{tabular} & \begin{tabular}{|l} 
G-1 Molten \\
Metal
\end{tabular} & $\begin{array}{l}\text { H-1 Steam } \\
\text { Gasification }\end{array}$ & \begin{tabular}{|l|} 
J-1 Joule- \\
Heated \\
Vitrification
\end{tabular} & \begin{tabular}{|l|}
$K-1$ \\
Desorption \\
W/ MEO
\end{tabular} & $\begin{array}{l}\text { L-1 } \\
\text { Desorption } \\
\text { W/ SCWO }\end{array}$ \\
\hline & \multicolumn{10}{|c|}{ Calculated Liquid Water Generation (lb/hr) } \\
\hline \multicolumn{11}{|l|}{$\begin{array}{l}\text { Inputs to Aqueous Waste } \\
\text { Treatment System }\end{array}$} \\
\hline Metal Decontamination Water & 112.00 & 112.00 & 112.00 & 112.00 & 112.00 & 0.00 & 112.00 & 112.00 & 112.00 & 112.00 \\
\hline Lead Recovery Blast Water & 29.70 & 29.70 & 29.70 & 29.70 & 29.70 & 29.70 & 29.70 & 29.70 & 29.70 & 29.70 \\
\hline Aqueous Waste & 80.00 & 80.00 & 80.00 & 80.00 & 80.00 & 80.00 & 80.00 & 80.00 & 64.00 & 64.00 \\
\hline Rinse Water from $2^{\circ}$ stabilization & 81.32 & 88.49 & 217.07 & 406.04 & 111.83 & 16.34 & 91.49 & 166.28 & 28.18 & 103.06 \\
\hline Scrubber water & $2,705.00$ & $2,831.00$ & $7,230.00$ & $13,436.00$ & $3,697.00$ & 516.00 & $2,931.00$ & $5,424,00$ & 890.00 & $3,386,00$ \\
\hline $\begin{array}{l}\text { Treatment System (available for } \\
\text { recycle) }\end{array}$ & $3,008.02$ & 3,141.19 & $7,668.77$ & $14,063.74$ & $4,030.63$ & 642.04 & $3,244.19$ & $\mathbf{6 , 8 1 1 . 9 8}$ & $1,123.88$ & $3,694.76$ \\
\hline \multicolumn{11}{|l|}{ Total Water Requirements } \\
\hline Water input to $1^{\circ}$ & 0.00 & 60.00 & 142.00 & 910.00 & 0.00 & 0.00 & 446.00 & 0.00 & 0.00 & $3,033.00$ \\
\hline $1^{\circ}$ and $2^{\circ}$ Quench water & $2,159.00$ & $1,189.00$ & $4,618.00$ & $10,407.00$ & $2,773.00$ & 0.00 & 751.00 & $2,293.00$ & 0.00 & 0.00 \\
\hline $1^{\circ}$ and $2^{\circ}$ Scrubber water & 0.00 & 2.259 .00 & 0.00 & $1,864.00$ & 498.00 & 467.00 & $2,259.00$ & $2,254.00$ & 902.00 & 0.00 \\
\hline Rinse Water for $2^{\circ}$ stabilization & 81.32 & 88.49 & 217.07 & 406.04 & 111.83 & 16.34 & 91.49 & 166.28 & 28.18 & 103.06 \\
\hline Metal Decontamination Water & 112.00 & 112.00 & 112.00 & 112.00 & 112.00 & 0.00 & 112.00 & 112.00 & 112.00 & 112.00 \\
\hline Mercury Amalgamation Water & 29.00 & 29.00 & 29.00 & 29.00 & 29.00 & 29.00 & 29.00 & 29.00 & 29.00 & 29.00 \\
\hline Lead Recovery, Blast and APC & 43.00 & 43.00 & 43.00 & 43.00 & 43.00 & 43.00 & 43.00 & 43.00 & 43.00 & 43.00 \\
\hline Total Water Requirements & $2,424.32$ & $3,780.49$ & 6,161.07 & $13,771.04$ & $3,566.83$ & 655.34 & $3,731.49$ & $4,897.28$ & 1,114.18 & $3,320.06$ \\
\hline $\begin{array}{l}\text { Net Water Generation Rate } \\
\text { (recycle - required) }\end{array}$ & 684 & -639 & 2,508 & 293 & 464 & 87 & -487 & 916 & 10 & 375 \\
\hline
\end{tabular}




\section{SUMMARY AND CONCLUSIONS}

Nineteen systems encompassing several incineration design options were developed in the study. For Phase 2 of the study, the ASPEN PLUS process simulation code was used. However, only the three most complex and energy intensive subsystems were modeled with ASPEN PLUS for each of the systems: main and secondary thermal treatment, main air pollution control, and metal melting. The objective of the modeling was to obtain mass and energy balances of the major components entering and exiting the process. Modeling of trace materials, such as pollutants and radioactive isotopes, were beyond the present scope, and were generally not tracked. The modeling results yielded information on the types and quantities of input material needed to process the waste, as well as the nature and amounts of the output products generated by each of the processes. Because an all-inclusive process simulation code was used for this study, the existing models can be expanded to investigate (and automate the calculation of) other system characteristics, for example, more detailed chemical reactions, tracing of minor constituents such as pollutants and radioactive isotopes, and cost studies.

For those subsystems not analyzed with the code, other calculations, from the Phase 1 task (updated accordingly), were used to arrive at the mass balances. These were combined with the modeling results to arrive at the overall mass balances. The results are presented in Table 6-1. The resulting mass and volume reduction ratios for the wastes form a basis for comparison of the nineteen diverse technologies covered in the study. Volume reduction ratios exceeded 1.00 in every case, indicating a net reduction. Values ranged from 1.07 for System K-1 (Desorption w/ MEO) to 4.27 for System G-1 (Molten Metal), and averaged 2.87 for all systems. Mass reduction ratios were as low as about 0.6 for systems K-1 and L-1 (Desorption w/ SCWO), meaning that more processed wastes left the facilities than raw wastes entered. The maximum mass reduction ratio was 1.73 for system G-1, and the average was 1.33 for all systems.

Energy (heating) requirements for the most energy intensive unit operations are shown in Table 6-2. Energy usage varied by an order of magnitude with the highest being System A-6 (Rotary Kiln w/ Maximum Recycling) at 35.7 MMBtu/hr, and the lowest System B-1 (Pyrolyzer) at 3.35 MMBtu/hr.

Energy costs varied by a factor of twenty, with the highest again being System A-6 at $\$ 33.0$ million over twenty years (1994 dollars), and the lowest, System A-5 (Rotary Kiln w/ Polymer Stabilization) at \$1.6 million. Although System A-5 uses almost three times as much primary energy as the lowest consuming system, B-1, its usage is predominately in the form of natural gas which is currently much cheaper than electricity, and thus has the lowest costs. As discussed in Reference (2), where all costs are presented, the energy costs represented only a minor portion of the total lifecyle costs of any of the systems.

Even though the accuracy and reliability of the assumptions used in this study may be subject for debate, the fact that they were uniformly applied allows a direct comparison of the systems considered.

The mass and energy balance results were only one of many factors of interest to the ITTS study. The complete comparison process consisted of many other considerations, including operational requirements, conceptual design layouts, planning life-cycle cost estimates, and areas requiring further development. The overall comparison is reported in Reference (2). There, all factors are considered, and recommendations are made as to the preferred technology based on today's knowledge. 


\section{REFERENCES}

1. Feizollahi, F., W. J. Quapp, H. G. Hempill, F. J. Groffie, Integrated Thermal Treatment System Study - Phase 1 Results, EGG-MS-11211, July 1994

2. Feizollahi, F., W. J. Quapp, Integrated Thermal Treatment System Study, Phase 2 Results, Third Draft, January 1995

3. ASPEN PLUS, Version 9.1-3 for PC's, Cambridge, MA; Aspen Technology, Inc., 1994

4. Huebner, T. L., et al, Quantities and Characteristics of the Contact-Handled Low-Level Mixed Waste Streams in the DOE Complex, EGG-MS-11303, August 1994

5. L. L. Oden, et al, Baseline Tests for Arc Melter Vitrification of INEL Buried Wastes, Vol I: Facility Description and Summary Data Report, EGG-WTD-10981, Table 3-9(a), November 1993

6. Microsoft Corp., Microsoft Excel, Version 5.0, 1993. 

Appendix A

Model Process Flow Diagrams (MPFDs)

and

Assumption and Energy Balance Tables 
A-2 


\section{System A-1: Conventional Rotary Kiln, Air Combustion Gas, Dry-Wet APC}

(Baseline System)

Table A-1.

ASSUMPTIONS and ENERGY BALANCES for BASELINE ROTARY KIIN, System A-1

GENERAL COMMENTS: RGIBBS units calculate chemical and phase equilibrium with some restrictions, not all stated here. For detailed information refer to the ASPEN PLUS Release 9 input file. Chemical reactions and phase changes for some species are neglected. "Volatile" trace metals $\mathrm{As}, \mathrm{Cd}, \mathrm{Hg}$, and $\mathrm{Se}$ are allowed to change phase, while $\mathrm{Ba}, \mathrm{Cr}, \mathrm{Pb}$, and $\mathrm{Ag}$ are restricted to the solid phase. The inlet fuel mixture streams in this system are composed of $\mathrm{CH} 4$ and $20 \%$ excess air. Design Spec's on air and oxygen flow consider both free oxygen and that bound in organics.

\begin{tabular}{|c|c|c|c|}
\hline $\begin{array}{l}\text { Block Name } \\
\text { (ASPEN } \\
\text { Type) } \\
\end{array}$ & $\begin{array}{l}\text { Outlet } \\
\text { Temp (F) }\end{array}$ & $\begin{array}{l}\text { Heat Duty } \\
\text { (MMBtw/hr) }\end{array}$ & Description and Assumptions \\
\hline $\begin{array}{l}\text { KIIN } \\
\text { (RGIBBS) }\end{array}$ & 1600 & -0.361 & $\begin{array}{l}\text { Conventional rotary kiln. Accepts combustible and non- } \\
\text { combustible wastes. CH4-fueled with air as oxidant. Heat losses } \\
\text { assumed to be 5\% of the sensible heat of the products. Includes } \\
\text { fixed air leakage through seals. Chemical and phase equilibrium } \\
\text { of Fe and Fe3O4 are restricted. } \\
\text { Design Spec QKILN: Vary either inlet fuel mixture flow (if heat } \\
\text { is needed) or inlet water flow (if cooling is needed) such that heat } \\
\text { duty is } 5 \% \text { of that calculated for unit Q1. } \\
\text { Design Spec OXI: Vary inlet air flow in stream AIR such that } \\
\text { total inlet oxygen flow (minus that used by the CH4) is twice the } \\
\text { outlet oxygen flow (100\% excess). } \\
\text { Design Spec SOIIH2O: Vary inlet soil feed rate (substream } \\
\text { MIXED, } 10 \% \text { of total flow) such that it is } 1 / 30 \text { the magnitude of } \\
\text { the SLAG stream (ash-to-gross soil ratio of } 2: 1 \text { ). } \\
\text { Design Spec SOILSOL: Vary inlet soil feed rate (substream } \\
\text { CISOLID, } 90 \% \text { of total flow) such that it is } 1 / 3.333 \text { the magnitude } \\
\text { of the SLAG stream (ash-to-gross soil ratio of } 2: 1 \text { ). }\end{array}$ \\
\hline $\begin{array}{l}\text { DUPL1 } \\
\text { (DUPL) }\end{array}$ & 1600 & 0 & $\begin{array}{l}\text { A stream duplicator block used to send a copy of the KIIN outlet } \\
\text { stream to the dummy unit Q1. }\end{array}$ \\
\hline $\begin{array}{l}\text { Q1 } \\
\text { (RGIBBS) }\end{array}$ & 68 & -7.229 & $\begin{array}{l}\text { Dummy unit. This calculates chemical equilibrium at } 1600 \mathrm{~F} \text {, } \\
\text { identical to unit } \mathrm{KIN} \text {, but has an outlet temperature of } 68 \mathrm{~F} \text {, thus } \\
\text { yielding an estimate of the sensible heat of the products of unit } \\
\mathrm{KINN} \text {. }\end{array}$ \\
\hline $\begin{array}{l}\text { KILNSEP } \\
\text { (SEP) }\end{array}$ & 1600 & -0.485 & $\begin{array}{l}\text { 2-way separation of } \mathrm{KIIN} \text { products: } 80 \% \text { of metals and oxides go } \\
\text { to VITRIFY (via stream ASH). Remainder, including } 100 \% \text { of } \\
\text { volatile trace metals and vapors, sent to SCC. Stream ASH } \\
\text { flashed to } 68 \mathrm{~F} \text { to represent storage of material before use. }\end{array}$ \\
\hline $\begin{array}{l}\text { SCC } \\
\text { (RGIBBS) }\end{array}$ & 2200 & -0.790 & $\begin{array}{l}\text { Secondary combustion unit. CH4-fueled with air as oxidant. Heat } \\
\text { losses assumed to be } 5 \% \text { of the sensible heat of the products. } \\
\text { Chemical and phase equilibrium of Fe and Fe3O4 are restricted. } \\
\text { Design Spec QSCC: Vary inlet fuel mixture flow such that heat } \\
\text { duty is } 5 \% \text { of that calculated for unit Q2. }\end{array}$ \\
\hline $\begin{array}{l}\text { DUPL2 } \\
\text { (DUPL) }\end{array}$ & 2200 & 0 & $\begin{array}{l}\text { A stream duplicator block used to send a copy of the SCC outlet } \\
\text { stream to the dummy unit Q2. }\end{array}$ \\
\hline
\end{tabular}


Table A-1.

ASSUMPTIONS and ENERGY BALANCES for BASELINE ROTARY KILN, System A-1

GENERAL COMMENTS: RGIBBS units calculate chemical and phase equilibrium with some restrictions, not all stated here. For detailed information refer to the ASPEN PLUS Release 9 input file. Chemical reactions and phase changes for some species are neglected. "Volatile" trace metals As, Cd, $\mathrm{Hg}$, and $\mathrm{Se}$ are allowed to change phase, while $\mathrm{Ba}, \mathrm{Cr}, \mathrm{Pb}$, and $\mathrm{Ag}$ are restricted to the solid phase. The inlet fuel mixture streams in this system are composed of $\mathrm{CH}_{4}$ and $20 \%$ excess air. Design Spec's on air and oxygen flow consider both free oxygen and that bound in organics.

\begin{tabular}{|c|c|c|c|}
\hline $\begin{array}{l}\text { Block Name } \\
\text { (ASPEN } \\
\text { Type) }\end{array}$ & $\begin{array}{l}\text { Outlet } \\
\text { Temp (F) }\end{array}$ & $\begin{array}{l}\text { Heat Duty } \\
\text { (MMBtu/hr) }\end{array}$ & Description and Assumptions \\
\hline $\begin{array}{l}\text { Q2 } \\
\text { (RGIBBS) }\end{array}$ & 68 & -15.791 & $\begin{array}{l}\text { Dummy unit. This calculates chemical equilibrium at } 2200 \mathrm{~F} \text {, } \\
\text { identical to unit SCC, but has an outlet temperature of } 68 \mathrm{~F} \text {, thus } \\
\text { yielding an estimate of the sensible heat of the products of unit } \\
\text { SCC. }\end{array}$ \\
\hline $\begin{array}{l}\text { QUENCH } \\
\text { (RGIBBS) }\end{array}$ & 350 & 0 & $\begin{array}{l}\text { Quenching of the exhaust of the } \mathrm{SCC} \text {. No losses included. } \\
\text { Formation of } \mathrm{CH} 4, \mathrm{C}, \mathrm{S} \text { and } \mathrm{Cl} 2 \text { is restricted. Chemical and phase } \\
\text { equilibrium of } \mathrm{Fe} \text { and } \mathrm{Fe} 3 \mathrm{O} 4 \text { are restricted. } \\
\text { Design Spec QQUENCH: Vary inlet water flow rate to achieve } \\
\text { zero heat duty. }\end{array}$ \\
\hline $\begin{array}{l}\text { BAGHOUSE } \\
\text { (SEP) }\end{array}$ & 350 & -0.017 & $\begin{array}{l}\text { 3-way separation of QUENCH products. Vapors sent to SCRUB, } \\
\text { volatile trace metals exit system (via stream HGPLUS), and } \\
\text { remainder (soil + ash) recycles to VITRIFY (via FLYASH). } \\
\text { Stream FLYASH flashed to } 68 \text { F to represent storage of material } \\
\text { before use. }\end{array}$ \\
\hline $\begin{array}{l}\text { SCRUB } \\
\text { (RGIBBS) }\end{array}$ & 120 & -13.844 & $\begin{array}{l}\text { Wet acid scrubber. } \\
\text { Design Spec SCRUBH2O: Vary inlet water flow such that inlet } \\
\text { liquid water flow equals } 20 \text { times outlet dissolved salts ( } 5 \% \mathrm{TDS} \text { ), } \\
\text { with a minimum value equal to inlet } \mathrm{NaOH} \text { flow ( } 50 \% \text { soln). } \\
\text { Design Spec } \mathrm{NAOH} \text { : Vary inlet } \mathrm{NaOH} \text { flow such that inlet } \mathrm{NaOH} \\
\text { flow equals } 100 \text { times outlet } \mathrm{NaOH} \text { flow ( } 1 \% \text { excess). }\end{array}$ \\
\hline $\begin{array}{l}\text { L-V-SEP } \\
\text { (FLASH2) }\end{array}$ & 120 & 0 & $\begin{array}{l}\text { 2-way separation of SCRUB products. Flash calculation to } \\
\text { separate liquid and vapor. }\end{array}$ \\
\hline $\begin{array}{l}\text { VITRIFY } \\
\text { (RGIBBS) }\end{array}$ & 3000 & +1.491 & $\begin{array}{l}\text { Vitrifier. Heat duty does not include heat losses. Feed material } \\
\text { enters at } 68 \mathrm{~F} \text {. Chemical and phase equilibrium of } \mathrm{Fe} \text { and } \mathrm{Fe} 3 \mathrm{O} 4 \\
\text { are restricted. }\end{array}$ \\
\hline $\begin{array}{l}\text { L-V-SEP2 } \\
\text { (FLASH2) }\end{array}$ & 3000 & 0 & $\begin{array}{l}\text { 2-way separation of VITRIFY products. Flash calculation to } \\
\text { separate liquid and vapor. No vapor expected. }\end{array}$ \\
\hline $\begin{array}{l}\text { MELTER } \\
\text { (RGIBBS) }\end{array}$ & 3000 & +0.120 & $\begin{array}{l}\text { Metal melter. Accepts metals with fixed contamination. } \\
\text { Electrically heated with heat losses not included. }\end{array}$ \\
\hline
\end{tabular}


Table A-2.

ASSUMPTIONS and ENERGY BALANCES for ROTARY KILN W/ OXYGEN, System A-2

GENERAL COMMENTS: RGIBBS units calculate chemical and phase equilibrium with some restrictions, not all stated here. For detailed information refer to the ASPEN PLUS Release 9 input file. Chemical reactions and phase changes for some species are neglected. "Volatile" trace metals $\mathrm{As}, \mathrm{Cd}, \mathrm{Hg}$, and Se are allowed to change phase, while $\mathrm{Ba}, \mathrm{Cr}, \mathrm{Pb}$, and $\mathrm{Ag}$ are restricted to the solid phase. The inlet fuel mixture streams in this system are composed of CH4 and $20 \%$ excess oxygen. Design Spec's on air and oxygen flow consider both free oxygen and that bound in organics.

\begin{tabular}{|c|c|c|c|}
\hline $\begin{array}{l}\text { Block Name } \\
\text { (ASPEN } \\
\text { Type) }\end{array}$ & $\begin{array}{l}\text { Outlet } \\
\text { Temp (F) }\end{array}$ & $\begin{array}{l}\text { Heat Duty } \\
\text { (MMBtu/hr) }\end{array}$ & Description and Assumptions \\
\hline $\begin{array}{l}\text { KILN } \\
\text { (RGIBBS) }\end{array}$ & 1600 & -0.367 & $\begin{array}{l}\text { Conventional rotary kiln. Accepts combustible and non- } \\
\text { combustible wastes. CH4-fueled with oxygen as oxidant. Heat } \\
\text { losses assumed to be } 5 \% \text { of the sensible heat of the products. } \\
\text { Includes fixed air leakage through seals. Chemical and phase } \\
\text { equilibrium of Fe and Fe3O4 are restricted. } \\
\text { Design Spec QKIIN: Vary either inlet fuel mixture flow (if heat } \\
\text { is needed) or inlet water flow (if cooling is needed) such that heat } \\
\text { duty is 5\% of that calculated for unit Q1. } \\
\text { Design Spec OXI: Vary inlet oxygen flow in stream OXYGEN } \\
\text { such that total inlet oxygen flow (minus that used by the CH4) is } \\
\text { twice the outlet oxygen flow (100\% excess). } \\
\text { Design Spec SOIIH2O: Vary inlet soil feed rate (substream } \\
\text { MIXED, } 10 \% \text { of total flow) such that it is } 1 / 30 \text { the magnitude of } \\
\text { the SLAG stream (ash-to-gross soil ratio of } 2: 1 \text { ). } \\
\text { Design Spec SOILSOL: Vary inlet soil feed rate (substream } \\
\text { CISOLD, } 90 \% \text { of total flow) such that it is } 1 / 3.333 \text { the magnitude } \\
\text { of the SLAG stream (ash-to-gross soil ratio of } 2: 1 \text { ). }\end{array}$ \\
\hline $\begin{array}{l}\text { DUPL1 } \\
\text { (DUPL) }\end{array}$ & 1600 & 0 & $\begin{array}{l}\text { A stream duplicator block used to send a copy of the KIIN outlet } \\
\text { stream to the dummy unit } Q 1 \text {. }\end{array}$ \\
\hline $\begin{array}{l}\text { Q1 } \\
\text { (RGIBBS) }\end{array}$ & 68 & -7.340 & $\begin{array}{l}\text { Dummy unit. This calculates chemical equilibrium at } 1600 \mathrm{~F} \text {, } \\
\text { identical to unit } \mathrm{KILN} \text {, but has an outlet temperature of } 68 \mathrm{~F} \text {, thus } \\
\text { yielding an estimate of the sensible heat of the products of unit } \\
\text { KIIN. }\end{array}$ \\
\hline $\begin{array}{l}\text { KIINSEP } \\
\text { (SEP) }\end{array}$ & 1600 & -0.485 & $\begin{array}{l}\text { 2-way separation of KILN products: } 80 \% \text { of metals and oxides go } \\
\text { to VITRIFY (via stream ASH). Remainder, including } 100 \% \text { of } \\
\text { volatile trace metals and vapors, sent to SCC. Stream ASH } \\
\text { flashed to } 68 \mathrm{~F} \text { to represent storage of material. }\end{array}$ \\
\hline $\begin{array}{l}\text { SCC } \\
\text { (RGIBBS) }\end{array}$ & 2200 & -0.481 & $\begin{array}{l}\text { Secondary combustion unit. CH4-fueled with oxygen as oxidant. } \\
\text { Heat losses assumed to be } 5 \% \text { of the sensible heat of the products. } \\
\text { Chemical and phase equilibrium of } \mathrm{Fe} \text { and } \mathrm{Fe} 3 \mathrm{O} 4 \text { are restricted. } \\
\text { Design Spec QSCC: Vary inlet fuel mixture flow such that heat } \\
\text { duty is 5\% of that calculated for unit } \mathrm{Q} 2 \text {. }\end{array}$ \\
\hline $\begin{array}{l}\text { DUPL2 } \\
\text { (DUPL) }\end{array}$ & 2200 & 0 & $\begin{array}{l}\text { A stream duplicator block used to send a copy of the SCC outlet } \\
\text { stream to the dummy unit Q2. }\end{array}$ \\
\hline
\end{tabular}


Table A-2.

ASSUMPTIONS and ENERGY BALANCES for ROTARY KIIN W/ OXYGEN, System A-2

GENERAL COMMENTS: RGIBBS units calculate chemical and phase equilibrium with some restrictions, not all stated here. For detailed information refer to the ASPEN PLUS Release 9 input file. Chemical reactions and phase changes for some species are neglected. "Volatile" trace metals As, $\mathrm{Cd}, \mathrm{Hg}$, and $\mathrm{Se}$ are allowed to change phase, while $\mathrm{Ba}, \mathrm{Cr}, \mathrm{Pb}$, and $\mathrm{Ag}$ are restricted to the solid phase. The inlet fuel mixture streams in this system are composed of CH4 and $20 \%$ excess oxygen. Design Spec's on air and oxygen flow consider both free oxygen and that bound in organics.

\begin{tabular}{|c|c|c|c|}
\hline $\begin{array}{l}\text { Block Name } \\
\text { (ASPEN } \\
\text { Type) }\end{array}$ & $\begin{array}{l}\text { Outlet } \\
\text { Temp (F) }\end{array}$ & $\begin{array}{l}\text { Heat Duty } \\
\text { (MMBtu/hr) }\end{array}$ & Description and Assumptions \\
\hline $\begin{array}{l}\text { Q2 } \\
\text { (RGIBBS) }\end{array}$ & 68 & -9.614 & $\begin{array}{l}\text { Dummy unit This calculates chemical equilibrium at } 2200 \mathrm{~F} \text {, } \\
\text { identical to unit SCC, but has an outlet temperature of } 68 \mathrm{~F} \text {, thus } \\
\text { yielding an estimate of the sensible heat of the products of unit } \\
\text { SCC. }\end{array}$ \\
\hline $\begin{array}{l}\text { QUENCH } \\
\text { (RGIBBS) }\end{array}$ & 350 & 0 & $\begin{array}{l}\text { Quenching of the exhaust of the SCC. No losses included. } \\
\text { Formation of } \mathrm{CH} 4, \mathrm{C}, \mathrm{S} \text { and } \mathrm{Cl} 2 \text { is restricted. Chemical and phase } \\
\text { equilibrium of } \mathrm{Fe} \text { and } \mathrm{Fe} 3 \mathrm{O} 4 \text { are restricted. } \\
\text { Design Spec QQUENCH: Vary inlet water flow rate to achieve } \\
\text { zero heat duty. }\end{array}$ \\
\hline $\begin{array}{l}\text { BAGHOUSE } \\
\text { (SEP) }\end{array}$ & 350 & -0.017 & $\begin{array}{l}\text { 3-way separation of QUENCH products. Vapors sent to SCRUB, } \\
\text { volatile trace metals exit system (via stream HGPLUS), and } \\
\text { remainder (soil + ash) recycles to VITRIFY (via FLYASH). } \\
\text { Stream FLYASH flashed to } 68 \text { F to represent storage of material. }\end{array}$ \\
\hline $\begin{array}{l}\text { SCRUB } \\
\text { (RGIBBS) }\end{array}$ & 120 & -8.916 & $\begin{array}{l}\text { Wet acid scrubber. } \\
\text { Design Spec SCRUBH2O: Vary inlet water flow such that inlet } \\
\text { liquid water flow equals } 20 \text { times outlet dissolved salts ( } 5 \% \mathrm{TDS} \text { ), } \\
\text { with minimum value equal to inlet } \mathrm{NaOH} \text { flow ( } 50 \% \text { soln). } \\
\text { Design Spec } \mathrm{NAOH} \text { : Vary inlet } \mathrm{NaOH} \text { flow such that inlet } \mathrm{NaOH} \\
\text { flow equals } 100 \text { times outlet } \mathrm{NaOH} \text { flow ( } 1 \% \text { excess). }\end{array}$ \\
\hline $\begin{array}{l}\text { L-V-SEP } \\
\text { (FLASH2) }\end{array}$ & 120 & 0 & $\begin{array}{l}\text { 2-way separation of SCRUB products. Flash calculation to } \\
\text { separate liquid and vapor. }\end{array}$ \\
\hline $\begin{array}{l}\text { VITRIFY } \\
\text { (RGIBBS) }\end{array}$ & 3000 & +1.491 & $\begin{array}{l}\text { Vitrifier. Heat duty does not include heat losses. Feed material } \\
\text { enters at } 68 \mathrm{~F} \text {. Chemical and phase equilibrium of } \mathrm{Fe} \text { and } \mathrm{Fe} 3 \mathrm{O} 4 \\
\text { are restricted. }\end{array}$ \\
\hline $\begin{array}{l}\text { L-V-SEP2 } \\
\text { (FLASH2) }\end{array}$ & 3000 & 0 & $\begin{array}{l}\text { 2-way separation of VITRIFY products. Flash calculation to } \\
\text { separate liquid and vapor. No vapor expected. }\end{array}$ \\
\hline $\begin{array}{l}\text { MELTER } \\
\text { (RGIBBS) }\end{array}$ & 3000 & +0.120 & $\begin{array}{l}\text { Metal melter. Accepts metals with fixed contamination. } \\
\text { Electrically heated with heat losses not included. }\end{array}$ \\
\hline
\end{tabular}


Table A-3.

ASSUMPTIONS and ENERGY BALANCES for ROTARY KILN W/ WET APC, System A-3

GENERAL COMMENTS: RGIBBS units calculate chemical and phase equilibrium with some restrictions, not all stated here. For detailed information refer to the ASPEN PLÚS Release 9 input file. Chemical reactions and phase changes for some species are neglected. "Volatile" trace metals. As, $\mathrm{Cd}, \mathrm{Hg}$, and Se are allowed to change phase, while $\mathrm{Ba}, \mathrm{Cr}, \mathrm{Pb}$, and $\mathrm{Ag}$ are restricted to the solid phase. The inlet fuel mixture streams in this system are composed of CH4 and $20 \%$ excess air. Design Spec's on air and oxygen flow consider both free oxygen and that bound in organics.

\begin{tabular}{|c|c|c|c|}
\hline $\begin{array}{l}\text { Block Name } \\
\text { (ASPEN } \\
\text { Type) }\end{array}$ & $\begin{array}{l}\text { Outlet } \\
\text { Temp (F) }\end{array}$ & $\begin{array}{l}\text { Heat Duty } \\
\text { (MMBtu/hr) }\end{array}$ & Description and Assumptions \\
\hline $\begin{array}{l}\text { KILN } \\
\text { (RGIBBS) }\end{array}$ & 1600 & -0.362 & $\begin{array}{l}\text { Conventional rotary kiln. Accepts combustible and non- } \\
\text { combustible wastes. CH4-fueled with air as oxidant. Heat losses } \\
\text { assumed to be } 5 \% \text { of the sensible heat of the products. Includes } \\
\text { fixed air leakage through seals. Chemical and phase equilibrium } \\
\text { of Fe and Fe3O4 are restricted. } \\
\text { Design Spec QKILN: Vary either inlet fuel mixture flow (if heat } \\
\text { is needed) or inlet water flow (if cooling is needed) such that heat } \\
\text { duty is 5\% of that calculated for unit Q1. } \\
\text { Design Spec OXI: Vary inlet air flow in stream AIR such that } \\
\text { total inlet oxygen flow (minus that used by the CH4) is twice the } \\
\text { outlet oxygen flow (100\% excess). } \\
\text { Design Spec SOILH2O: Vary inlet soil feed rate (substream } \\
\text { MIXED, 10\% of total flow) such that it is } 1 / 30 \text { the magnitude of } \\
\text { the SLAG stream (ash-to-gross soil ratio of 2:1). } \\
\text { Design Spec SOILSOL: Vary inlet soil feed rate (substream } \\
\text { CISOLD, } 90 \% \text { of total flow) such that it is } 1 / 3.333 \text { the magnitude } \\
\text { of the SLAG stream (ash-to-gross soil ratio of } 2: 1 \text { ). }\end{array}$ \\
\hline $\begin{array}{l}\text { DUPL1 } \\
\text { (DUPL) }\end{array}$ & 1600 & 0 & $\begin{array}{l}\text { A stream duplicator block used to send a copy of the KIIN outlet } \\
\text { stream to the dummy unit } Q 1 \text {. }\end{array}$ \\
\hline $\begin{array}{l}\text { Q1 } \\
\text { (RGIBBS) }\end{array}$ & 68 & -7.242 & $\begin{array}{l}\text { Dummy unit. This calculates chemical equilibrium at } 1600 \mathrm{~F} \text {, } \\
\text { identical to unit KILN, but has an outlet temperature of } 68 \mathrm{~F} \text {, thus } \\
\text { yielding an estimate of the sensible heat of the products of unit } \\
\mathrm{KILN} \text {. }\end{array}$ \\
\hline $\begin{array}{l}\text { KILNSEP } \\
\text { (SEP) }\end{array}$ & 1600 & -0.450 & $\begin{array}{l}\text { 2-way separation of KILN products: } 80 \% \text { of metals and oxides go } \\
\text { to VITRIFY (via stream ASH). Remainder, including } 100 \% \text { of } \\
\text { volatile trace metals and vapors, sent to SCC. Stream ASH } \\
\text { flashed to } 68 \text { F to represent storage of material before use. }\end{array}$ \\
\hline $\begin{array}{l}\text { SCC } \\
\text { (RGIBBS) }\end{array}$ & 2200 & -0.793 & $\begin{array}{l}\text { Secondary combustion unit. CH4-fueled with air as oxidant. Heat } \\
\text { losses assumed to be 5\% of the sensible heat of the products. } \\
\text { Chemical and phase equilibrium of Fe and Fe3O4 are restricted. } \\
\text { Design Spec QSCC: Vary inlet fuel mixture flow such that heat } \\
\text { duty is 5\% of that calculated for unit Q2. }\end{array}$ \\
\hline $\begin{array}{l}\text { DUPL2 } \\
\text { (DUPL) }\end{array}$ & 2200 & 0 & $\begin{array}{l}\text { A stream duplicator block used to send a copy of the SCC outlet } \\
\text { stream to the dummy unit Q2. }\end{array}$ \\
\hline
\end{tabular}


Table A-3.

ASSUMPTIONS and ENERGY BALANCES for ROTARY KIIN W/ WET APC, System A-3

GENERAL COMMENTS: RGIBBS units calculate chemical and phase equilibrium with some restrictions, not all stated here. For detailed information refer to the ASPEN PLUS Release 9 input file. Chemical reactions and phase changes for some species are neglected. "Volatile" trace metals As, $\mathrm{Cd}, \mathrm{Hg}$, and Se are allowed to change phase, while $\mathrm{Ba}, \mathrm{Cr}, \mathrm{Pb}$, and $\mathrm{Ag}$ are restricted to the solid phase. The inlet fuel mixture streams in this system are composed of CH4 and $20 \%$ excess air. Design Spec's on air and oxygen flow consider both free oxygen and that bound in organics.

\begin{tabular}{|l|l|l|l|}
\hline $\begin{array}{l}\text { Block Name } \\
\text { (ASPEN } \\
\text { Type) }\end{array}$ & $\begin{array}{l}\text { Outlet } \\
\text { Temp (F) }\end{array}$ & $\begin{array}{l}\text { Heat Duty } \\
\text { (MMBtu/hr) }\end{array}$ & Description and Assumptions \\
\hline $\begin{array}{l}\text { Q2 } \\
\text { (RGIBBS) }\end{array}$ & 68 & -15.866 & $\begin{array}{l}\text { Dummy unit. This calculates chemical equilibrium at 2200 F, } \\
\text { identical to unit SCC, but has an outlet temperature of } 68 \mathrm{~F} \text {, thus } \\
\text { yielding an estimate of the sensible heat of the products of unit } \\
\text { SCC. }\end{array}$ \\
\hline $\begin{array}{l}\text { SCRUB } \\
\text { (RGIBBS) }\end{array}$ & 120 & -14.336 & $\begin{array}{l}\text { Wet APC system. Full quench/hydrosonic scrubber/packed tower. } \\
\text { Design Spec SCRUBH2O: Vary inlet water flow such that inlet } \\
\text { liquid water flow equals 20 times outlet dissolved salts (5\% TDS), } \\
\text { with a minimum value equal to inlet NaOH flow (50\% soln) } \\
\text { Design Spec NAOH: Vary inlet NaOH flow such that inlet NaOH } \\
\text { flow equals 100 times outlet NaOH flow (1\% excess). }\end{array}$ \\
\hline $\begin{array}{l}\text { SOLIDSEP } \\
\text { (SEP) }\end{array}$ & 120 & 0 & $\begin{array}{l}\text { 3-way separation of QUENCH products to identify breakdown of } \\
\text { components. Vapors sent to L-V-SEP, volatile trace metals exit } \\
\text { system (via stream HGPLUS); and remainder (soil + ash) exits } \\
\text { separately via FLYASH. }\end{array}$ \\
\hline $\begin{array}{l}\text { L-V-SEP } \\
\text { (FLASH2) }\end{array}$ & 120 & 0 & $\begin{array}{l}\text { 2-way separation of liquid/vapor SCRUB products. Flash } \\
\text { calculation to separate liquid and vapor. }\end{array}$ \\
\hline $\begin{array}{l}\text { VITRIFY } \\
\text { (RGIBBS) }\end{array}$ & 3000 & +1.116 & $\begin{array}{l}\text { Vitrifier. Heat duty does not include heat losses. Feed material } \\
\text { enters at 68 F. Chemical and phase equilibrium of Fe and Fe3O4 } \\
\text { are restricted. }\end{array}$ \\
\hline $\begin{array}{l}\text { L-V-SEP2 } \\
\text { (FLASH2) }\end{array}$ & 3000 & 0 & $\begin{array}{l}\text { 2-way separation of VITRIFY products. Flash calculation to } \\
\text { separate liquid and vapor. No vapor expected. }\end{array}$ \\
\hline $\begin{array}{l}\text { MELTER } \\
\text { (RGIBBS) }\end{array}$ & 3000 & $\begin{array}{l}\text { Metal melter. Accepts metals with fixed contamination. } \\
\text { Electrically heated with heat losses not included. }\end{array}$ \\
\hline
\end{tabular}


Table A-4.

ASSUMPTIONS and ENERGY BALANCES for ROTARY KIIN W/ $\mathrm{CO}_{2}$ RETENTION, System A-4

GENERAL COMMENTS: RGIBBS units calculate chemical and phase equilibrium with some restrictions, not all stated here. For detailed information refer to the ASPEN PLUS Release 9 input file. Chemical reactions and phase changes for some species are neglected. "Volatile" trace metals $\mathrm{As}, \mathrm{Cd}, \mathrm{Hg}$, and $\mathrm{Se}$ are allowed to change phase, while $\mathrm{Ba}, \mathrm{Cr}, \mathrm{Pb}$, and $\mathrm{Ag}$ are restricted to the solid phase. The inlet fuel mixture streams in this system are composed of CH4 and $20 \%$ excess oxygen. Design Spec's on air and oxygen flow consider both free oxygen and that bound in organics.

\begin{tabular}{|c|c|c|c|}
\hline $\begin{array}{l}\text { Block Name } \\
\text { (ASPEN } \\
\text { Type) } \\
\end{array}$ & $\begin{array}{l}\text { Outlet } \\
\text { Temp (F) }\end{array}$ & $\begin{array}{l}\text { Heat Duty } \\
\text { (MMBtu/hr) }\end{array}$ & Description and Assumptions \\
\hline $\begin{array}{l}\text { KIIN } \\
\text { (RGIBBS) }\end{array}$ & 1600 & -0.685 & $\begin{array}{l}\text { Conventional rotary kiln. Accepts combustible and non- } \\
\text { combustible wastes. CH4-fueled with oxygen as oxidant. Heat } \\
\text { losses assumed to be } 5 \% \text { of the sensible heat of the products. } \\
\text { Includes fixed air leakage through seals. Chemical and phase } \\
\text { equilibrium of Fe and Fe3O4 are restricted. } \\
\text { Design Spec QKILN1: Vary either inlet fuel mixture flow (if heat } \\
\text { is needed) or inlet water flow (if cooling is needed) such that heat } \\
\text { duty is } 5 \% \text { of that calculated for unit Q1. } \\
\text { Design Spec OXYGEN: Vary inlet oxygen flow in stream } \\
\text { OXYGEN such that total inlet oxygen flow (minus that used by } \\
\text { the CH4) is twice the outlet oxygen flow (100\% excess). } \\
\text { Design Spec SOILH2O: Vary inlet soil feed rate (substream } \\
\text { MIXED, 10\% of total flow) such that it is } 1 / 30 \text { the magnitude of } \\
\text { the SLAG stream (ash-to-gross soil ratio of } 2: 1 \text { ). } \\
\text { Design Spec SOILSOL: Vary inlet soil feed rate (substream } \\
\text { CISOLID, } 90 \% \text { of total flow) such that it is } 1 / 3.333 \text { the magnitude } \\
\text { of the SLAG stream (ash-to-gross soil ratio of } 2: 1 \text { ). }\end{array}$ \\
\hline $\begin{array}{l}\text { DUPL1 } \\
\text { (DUPL) }\end{array}$ & 1600 & 0 & $\begin{array}{l}\text { A stream duplicator block used to send a copy of the KILN outlet } \\
\text { stream to the dummy unit } \mathrm{Q} 1 \text {. }\end{array}$ \\
\hline $\begin{array}{l}\text { Q1 } \\
\text { (RGIBBS) }\end{array}$ & 68 & -13.704 & $\begin{array}{l}\text { Dummy unit. This calculates chemical equilibrium at } 1600 \mathrm{~F} \text {, } \\
\text { identical to unit KIIN, but has an outlet temperature of } 68 \mathrm{~F} \text {, thus } \\
\text { yielding an estimate of the sensible heat of the products of unit } \\
\text { KIIN. }\end{array}$ \\
\hline $\begin{array}{l}\text { KILNSEP } \\
\text { (SEP) }\end{array}$ & 1600 & -0.485 & $\begin{array}{l}\text { 2-way separation of KIIN products: } 80 \% \text { of metals and oxides go } \\
\text { to VITRIFY (via stream ASH). Remainder, including } 100 \% \text { of } \\
\text { volatile trace metals and vapors, sent to SCC. Stream ASH } \\
\text { flashed to } 68 \mathrm{~F} \text { to represent storage of material before use. }\end{array}$ \\
\hline $\begin{array}{l}\text { SCC } \\
\text { (RGIBBS) }\end{array}$ & 2200 & -1.031 & $\begin{array}{l}\text { Secondary combustion unit. CH4-fueled with oxygen as oxidant. } \\
\text { Heat losses assumed to be } 5 \% \text { of the sensible heat of the products. } \\
\text { Chemical and phase equilibrium of } \mathrm{Fe} \text { and Fe3O4 are restricted. } \\
\text { Design Spec QSCC: Vary inlet fuel mixture flow such that heat } \\
\text { duty is } 5 \% \text { of that calculated for unit Q2. }\end{array}$ \\
\hline $\begin{array}{l}\text { DUPL2 } \\
\text { (DUPL) }\end{array}$ & 2200 & 0 & $\begin{array}{l}\text { A stream duplicator block used to send a copy of the SCC outlet } \\
\text { stream to the dummy unit Q2. }\end{array}$ \\
\hline
\end{tabular}


Table A-4.

ASSUMPTIONS and ENERGY BALANCES for ROTARY KIIN W/ $\mathrm{CO}_{2}$ RETENTION, System A-4

GENERAL COMMENTS: RGIBBS units calculate chemical and phase equilibrium with some restrictions, not all stated here. For detailed information refer to the ASPEN PLUS Release 9 input file. Chemical reactions and phase changes for some species are neglected. "Volatile" trace metals $\mathrm{As}, \mathrm{Cd}, \mathrm{Hg}$, and $\mathrm{Se}$ are allowed to change phase, while $\mathrm{Ba}, \mathrm{Cr}, \mathrm{Pb}$, and $\mathrm{Ag}$ are restricted to the solid phase. The inlet fuel mixture streams in this system are composed of $\mathrm{CH} 4$ and $20 \%$ excess oxygen. Design Spec's on air and oxygen flow consider both free oxygen and that bound in organics.

\begin{tabular}{|c|c|c|c|}
\hline $\begin{array}{l}\text { Block Name } \\
\text { (ASPEN } \\
\text { Type) } \\
\end{array}$ & $\begin{array}{l}\text { Outlet } \\
\text { Temp (F) }\end{array}$ & $\begin{array}{l}\text { Heat Duty } \\
\text { (MMBtu/hr) }\end{array}$ & Description and Assumptions \\
\hline $\begin{array}{l}\text { Q2 } \\
\text { (RGIBBS) }\end{array}$ & 68 & -20.626 & $\begin{array}{l}\text { Dummy unit. This calculates chemical equilibrium at } 2200 \mathrm{~F} \text {, } \\
\text { identical to unit SCC, but has an outlet temperature of } 68 \mathrm{~F} \text {, thus } \\
\text { yielding an estimate of the sensible heat of the products of unit } \\
\text { SCC. }\end{array}$ \\
\hline $\begin{array}{l}\text { QUENCH } \\
\text { (RGIBBS) }\end{array}$ & 350 & 0 & $\begin{array}{l}\text { Quenching of the exhaust of the } \mathrm{SCC} \text {. No losses included. } \\
\text { Formation of } \mathrm{CH} 4, \mathrm{C}, \mathrm{S} \text { and } \mathrm{Cl} 2 \text { is restricted. Chemical and phase } \\
\text { equilibrium of } \mathrm{Fe} \text { and } \mathrm{Fe} 3 \mathrm{O} 4 \text { are restricted. } \\
\text { Design Spec QQUENCH: Vary inlet water flow rate to achieve } \\
\text { zero heat duty. }\end{array}$ \\
\hline $\begin{array}{l}\text { BAGHOUSE } \\
\text { (SEP) }\end{array}$ & 350 & -0.017 & $\begin{array}{l}\text { 3-way separation of QUENCH products. Vapors sent to SCRUB, } \\
\text { volatile trace metals exit system (via stream HGPLUS), and } \\
\text { remainder (soil + ash) recycles to VITRIFY (via FLYASH). } \\
\text { Stream FLYASH flashed to } 68 \text { F to represent storage of material } \\
\text { before use. }\end{array}$ \\
\hline $\begin{array}{l}\text { DRYSCRUB } \\
\text { (RGIBBS) }\end{array}$ & 350 & -0.096 & $\begin{array}{l}\text { Dry scrubber. Chemical equilibrium calculated for conversion of } \\
\mathrm{CaO} \text { to } \mathrm{CaSO} 4 \text { and } \mathrm{CaCl} 2 \text {, with all volatiles in the system } \\
\text { participating. SO2 is fully converted (not included in the product } \\
\text { list). Heat duty does not include any losses. Water used to slake } \\
\text { the lime is not included in the model. } \\
\text { Design Spec SCRUB: Vary the specified fractional flow split of } \\
\text { unit SPLIT (between } 0 \text { and } 0.1 \text { ) such that the inlet } \mathrm{CaO} \text { mole flow } \\
\text { to DRYSCRUB is equal to the inlet mole flow of the SO2 plus } \\
\text { half the inlet mole flow of the } \mathrm{HCl} \text {. }\end{array}$ \\
\hline $\begin{array}{l}\text { SCRUBSEP } \\
\text { (SEP) }\end{array}$ & 350 & 0 & $\begin{array}{l}\text { 2-way separation of DRYSCRUB products. Vapors sent to } \mathrm{CO} 2- \\
\text { ABS and remainder exits system. }\end{array}$ \\
\hline $\begin{array}{l}\text { CO2-ABS } \\
\text { (RSTOIC) }\end{array}$ & 1200 & +8.523 & $\begin{array}{l}\text { Restricted chemical reaction combining } \mathrm{CO} 2 \text { and } \mathrm{CaO} \text { to form } \\
\mathrm{CaCO} \text {, assuming } 100 \% \text { utilization of the } \mathrm{CO} 2 \text {. Heat duty does } \\
\text { not include any losses. }\end{array}$ \\
\hline $\begin{array}{l}\text { ABSSEP } \\
\text { (SEP) }\end{array}$ & 1200 & 0 & $\begin{array}{l}\text { 2-way separation of } \mathrm{CO} 2-\mathrm{ABS} \text { products. Vapors sent to FLASH. } \\
\text { Remainder, consisting only of } \mathrm{CaCO} \text {, sent to } \mathrm{CO} \text {-DES. }\end{array}$ \\
\hline $\begin{array}{l}\text { FLASH } \\
\text { (FLASH2) }\end{array}$ & 90 & -31.471 & $\begin{array}{l}1200 \mathrm{~F} \text { inlet stream flashed at } 90 \mathrm{~F} \text { to separate liquid and vapor. } \\
\text { Heat duty does not include any losses. Heat removed by an } \\
\text { unspecified indirect heat exchange process. }\end{array}$ \\
\hline $\begin{array}{l}\text { EX-SPLIT } \\
\text { (FSPLIT) }\end{array}$ & 90 & 0 & $\begin{array}{l}\text { A split of the vapor stream exiting FLASH (predominately } \\
\text { oxygen), with } 90 \% \text { recycled to PLENUM and } 10 \% \text { exiting system. }\end{array}$ \\
\hline
\end{tabular}


Table A-4.

ASSUMPTIONS and ENERGY BALANCES for ROTARY $\mathrm{KIIN} \mathrm{W/} \mathrm{CO}_{2}$ RETENTION, System A-4

GENERAL COMMENTS: RGIBBS units calculate chemical and phase equilibrium with some restrictions, not all stated here. For detailed information refer to the ASPEN PLUS Release 9 input file. Chemical reactions and phase changes for some species are neglected. "Volatile" trace metals $\mathrm{As}, \mathrm{Cd}, \mathrm{Hg}$, and Se are allowed to change phase, while $\mathrm{Ba}, \mathrm{Cr}, \mathrm{Pb}$, and $\mathrm{Ag}$ are restricted to the solid phase. The inlet fuel mixture streams in this system are composed of $\mathrm{CH} 4$ and $20 \%$ excess oxygen. Design Spec's on air and oxygen flow consider both free oxygen and that bound in organics.

\begin{tabular}{|c|c|c|c|}
\hline $\begin{array}{l}\text { Block Name } \\
\text { (ASPEN } \\
\text { Type) }\end{array}$ & $\begin{array}{l}\text { Outlet } \\
\text { Temp (F) }\end{array}$ & $\begin{array}{l}\text { Heat Duty } \\
\text { (MMBtu/hr) }\end{array}$ & Description and Assumptions \\
\hline $\begin{array}{l}\text { CO2-DES } \\
\text { (RGIBBS) }\end{array}$ & 1700 & +5.755 & $\begin{array}{l}\text { Desorption of the } \mathrm{CO} 2 \text { from } \mathrm{CaCO} \text {, producing } \mathrm{CaO} \text {. Heat duty } \\
\text { does not include heat losses. }\end{array}$ \\
\hline $\begin{array}{l}\text { CO2-SEP } \\
\text { (SEP) }\end{array}$ & 1700 & -1.326 & $\begin{array}{l}\text { 2-way separation of } \mathrm{CO} 2 \text {-DES products. } \mathrm{CO} 2 \text { is sent to } \\
\text { GASQUENC and remainder, consisting only of } \mathrm{CaO} \text {, sent to } \\
\text { SPLIT: } \mathrm{CaO} \text { (stream CAO-OUT) is flashed to } 68 \mathrm{~F} \text { at outlet to } \\
\text { represent storage of the product before use. }\end{array}$ \\
\hline $\begin{array}{l}\text { SPLIT } \\
\text { (FSPLIT) }\end{array}$ & 68 & 0 & $\begin{array}{l}\text { A split of the solids stream from the separator CO2-SEP, recycling } \\
90 \% \text { of the CaO back to CO2-ABS. The remaining } 10 \% \text { is split } \\
\text { between that going to DRYSCRUB for } \mathrm{Cl} 2 \text { and } \mathrm{S} \text { removal (1.5\%), } \\
\text { and the rest exiting system to stabilization ( } 8.5 \%) \text {. }\end{array}$ \\
\hline $\begin{array}{l}\text { CAOMIXER } \\
\text { (MIXER) }\end{array}$ & 68 & 0 & $\begin{array}{l}\text { Mixing of the recycled } \mathrm{CaO} \text { with make-up } \mathrm{CaO} \text {. } \\
\text { Design Spec } \mathrm{CAO} \text { : Vary inlet flow of make-up } \mathrm{CaO} \text { to achieve an } \\
\text { equal molar flow rate between the } \mathrm{CO} 2 \text { and the } \mathrm{CaO} \text { as they enter } \\
\text { the absorber (to produce } \mathrm{CaCO} \text { ). }\end{array}$ \\
\hline $\begin{array}{l}\text { GASQUENC } \\
\text { (RGIBBS) }\end{array}$ & 350 & 0 & $\begin{array}{l}\text { Quenching of the } 1700 \mathrm{~F} \mathrm{CO} 2 \text { exhaust stream with water. No } \\
\text { losses included. } \\
\text { Design Spec QQUENCH2: Vary inlet water flow rate to achieve } \\
\text { zero heat duty. }\end{array}$ \\
\hline $\begin{array}{l}\text { VITRIFY } \\
\text { (RGIBBS) }\end{array}$ & 3000 & +1.491 & $\begin{array}{l}\text { Vitrifier. Heat duty does not include heat losses. Feed material } \\
\text { enters at } 68 \mathrm{~F} \text {. Chemical and phase equilibrium of } \mathrm{Fe} \text { and } \mathrm{Fe} 3 \mathrm{O} 4 \\
\text { are restricted. }\end{array}$ \\
\hline $\begin{array}{l}\text { L-V-SEP2 } \\
\text { (FLASH2) }\end{array}$ & 3000 & 0 & $\begin{array}{l}\text { 2-way separation of VITRIFY products. Flash calculation to } \\
\text { separate liquid and vapor. No vapor expected. }\end{array}$ \\
\hline $\begin{array}{l}\text { MELTER } \\
\text { (RGIBBS) }\end{array}$ & 3000 & +0.120 & $\begin{array}{l}\text { Metal melter. Accepts metals with fixed contamination. } \\
\text { Electrically heated with heat losses not included. }\end{array}$ \\
\hline
\end{tabular}


A-12 


\section{System A-5: Conventional Rotary Kiln, Polymer Stabilization}

Table A-5.

Page 1 of 2

ASSUMPTIONS and ENERGY BALANCES for ROTARY KILN W/ POLYMER STABILIZATION, System

$$
\text { A-5 }
$$

GENERAL COMMENTS: RGIBBS units calculate chemical and phase equilibrium with some restrictions, not all stated here. For detailed information refer to the ASPEN PLUS Release 9 input file. Chemical reactions and phase changes for some species are neglected. "Volatile" trace metals $\mathrm{As}, \mathrm{Cd}, \mathrm{Hg}$, and Se are allowed to change phase, while $\mathrm{Ba}, \mathrm{Cr}, \mathrm{Pb}$, and $\mathrm{Ag}$ are restricted to the solid phase. The inlet fuel mixture streams in this system are composed of $\mathrm{CH} 4$ and $20 \%$ excess air. Design Spec's on air and oxygen flow consider both free oxygen and that bound in organics.

\begin{tabular}{|c|c|c|c|}
\hline $\begin{array}{l}\text { Block Name } \\
\text { (ASPEN } \\
\text { Type) } \\
\end{array}$ & $\begin{array}{l}\text { Outlet } \\
\text { Temp (F) }\end{array}$ & $\begin{array}{l}\text { Heat Duty } \\
\text { (MMBtu/hr) }\end{array}$ & Description and Assumptions \\
\hline $\begin{array}{l}\text { KILN } \\
\text { (RGIBBS) }\end{array}$ & 1600 & -0.365 & $\begin{array}{l}\text { Conventional rotary kiln. Accepts combustible and non- } \\
\text { combustible wastes. CH4-fueled with air as oxidant. Heat losses } \\
\text { assumed to be } 5 \% \text { of the sensible heat of the products. Includes } \\
\text { fixed air leakage through seals. Chemical and phase equilibrium } \\
\text { of Fe and Fe3O4 are restricted. } \\
\text { Design Spec QKIIN: Vary either inlet fuel mixture flow (if heat } \\
\text { is needed) or inlet water flow (if cooling is needed) such that heat } \\
\text { duty is } 5 \% \text { of that calculated for unit Q1. } \\
\text { Design Spec OXI: Vary inlet air flow in stream AIR such that } \\
\text { total inlet oxygen flow (minus that used by the CH4) is twice the } \\
\text { outlet oxygen flow (100\% excess). }\end{array}$ \\
\hline $\begin{array}{l}\text { DUPL1 } \\
\text { (DUPL) }\end{array}$ & 1600 & 0 & $\begin{array}{l}\text { A stream duplicator block used to send a copy of the } \mathrm{KIIN} \text { outlet } \\
\text { stream to the dummy unit } \mathrm{Q} 1 \text {. }\end{array}$ \\
\hline $\begin{array}{l}\text { Q1 } \\
\text { (RGIBBS) }\end{array}$ & 68 & -7.299 & $\begin{array}{l}\text { Dummy unit. This calculates chemical equilibrium at } 1600 \mathrm{~F} \text {, } \\
\text { identical to unit KILN, but has an outlet temperature of } 68 \mathrm{~F} \text {, thus } \\
\text { yielding an estimate of the sensible heat of the products of unit } \\
\mathrm{KILN} \text {. }\end{array}$ \\
\hline $\begin{array}{l}\text { KILNSEP } \\
\text { (SEP) }\end{array}$ & 1600 & 0 & $\begin{array}{l}\text { 2-way separation of KILN products: } 80 \% \text { of metals and oxides } \\
\text { exit system to polymer stabilization (via stream ASH). } \\
\text { Remainder, including } 100 \% \text { of volatile trace metals and vapors, } \\
\text { sent to SCC. }\end{array}$ \\
\hline $\begin{array}{l}\text { SCC } \\
\text { (RGIBBS) }\end{array}$ & 2200 & -0.806 & $\begin{array}{l}\text { Secondary combustion unit. CH4-fueled with air as oxidant. Heat } \\
\text { losses assumed to be } 5 \% \text { of the sensible heat of the products. } \\
\text { Chemical and phase equilibrium of } \mathrm{Fe} \text { and } \mathrm{Fe} 3 \mathrm{O} 4 \text { are restricted. } \\
\text { Design Spec QSCC: Vary inlet fuel mixture flow such that heat } \\
\text { duty is } 5 \% \text { of that calculated for unit Q2. }\end{array}$ \\
\hline $\begin{array}{l}\text { DUPL2 } \\
\text { (DUPL) }\end{array}$ & 2200 & 0 & $\begin{array}{l}\text { A stream duplicator block used to send a copy of the SCC outlet } \\
\text { stream to the dummy unit Q2. }\end{array}$ \\
\hline $\begin{array}{l}\text { Q2 } \\
\text { (RGIBBS) }\end{array}$ & 68 & -16.123 & $\begin{array}{l}\text { Dummy unit. This calculates chemical equilibrium at } 2200 \mathrm{~F} \text {, } \\
\text { identical to unit SCC, but has an outlet temperature of } 68 \mathrm{~F} \text {, thus } \\
\text { yielding an estimate of the sensible heat of the products of unit } \\
\text { SCC. }\end{array}$ \\
\hline
\end{tabular}


Table A-5.

ASSUMPTIONS and ENERGY BALANCES for ROTARY KILN W/ POLYMER STABILIZATION, System

$$
\text { A-5 }
$$

GENERAL COMMENTS: RGIBBS units calculate chemical and phase equilibrium with some restrictions, not all stated here. For detailed information refer to the ASPEN PLUS Release 9 input file. Chemical reactions and phase changes for some species are neglected. "Volatile" trace metals $\mathrm{As}, \mathrm{Cd}, \mathrm{Hg}$, and Se are allowed to change phase, while $\mathrm{Ba}, \mathrm{Cr}, \mathrm{Pb}$, and $\mathrm{Ag}$ are restricted to the solid phase. The inlet fuel mixture streams in this system are composed of CH4 and $20 \%$ excess air. Design Spec's on air and oxygen flow consider both free oxygen and that bound in organics.

\begin{tabular}{|l|l|l|l|}
\hline $\begin{array}{l}\text { Block Name } \\
\text { (ASPEN } \\
\text { Type) }\end{array}$ & $\begin{array}{l}\text { Outlet } \\
\text { Temp (F) }\end{array}$ & $\begin{array}{l}\text { Heat Duty } \\
\text { (MMBtu/hr) }\end{array}$ & Description and Assumptions \\
\hline $\begin{array}{l}\text { QUENCH } \\
\text { (RGIBBS) }\end{array}$ & 350 & 0 & $\begin{array}{l}\text { Quenching of the exhaust of the SCC. No losses included. } \\
\text { Formation of CH4, C, S and Cl2 is restricted. Chemical and phase } \\
\text { equilibrium of Fe and Fe3O4 are restricted. } \\
\text { Design Spec QQUENCH: Vary inlet water flow rate to achieve } \\
\text { zero heat duty. }\end{array}$ \\
\hline $\begin{array}{l}\text { BAGHOUSE } \\
\text { (SEP) }\end{array}$ & 350 & 0 & $\begin{array}{l}\text { 3-way separation of QUENCH products. Vapors sent to SCRUB, } \\
\text { volatile trace metals exit system (via stream HGPLUS), and } \\
\text { remainder (ash) exits to polymer stabilization (via FLYASH). }\end{array}$ \\
\hline $\begin{array}{l}\text { SCRUB } \\
\text { (RGIBBS) }\end{array}$ & 120 & -14.157 & $\begin{array}{l}\text { Wet acid scrubber. } \\
\text { Design Spec SCRUBH2O: Vary inlet water flow such that inlet } \\
\text { liquid water flow equals 20 times outlet dissolved salts (5\% TDS), } \\
\text { with a minimum value equal to inlet NaOH flow (S0\% soln). } \\
\text { Design Spec NAOH: Vary iniet NaOH flow such that inlet NaOH } \\
\text { flow equals 100 times outlet NaOH flow (1\% excess). }\end{array}$ \\
\hline $\begin{array}{l}\text { L-V-SEP } \\
\text { (FLASH2) }\end{array}$ & 120 & 0 & $\begin{array}{l}\text { 2-way separation of SCRUB products. Flash calculation to } \\
\text { separate liquid and vapor. }\end{array}$ \\
\hline $\begin{array}{l}\text { MELTER } \\
\text { (RGIBBS) }\end{array}$ & 3000 & +0.120 & $\begin{array}{l}\text { Metal melter. Accepts metals with fixed contamination. } \\
\text { Electrically heated with heat losses not included. }\end{array}$ \\
\hline
\end{tabular}


Table A-6.

ASSUMPTIONS and ENERGY BALANCES for ROTARY KILN W/ MAXIMUM RECYCLING, System A-6

GENERAL COMMENTS: RGIBBS units calculate chemical and phase equilibrium with some restrictions, not all stated here. For detailed information refer to the ASPEN PLUS Release 9 input file. Chemical reactions and phase changes for some species are neglected. "Volatile" trace metals $\mathrm{As}, \mathrm{Cd}, \mathrm{Hg}$, and $\mathrm{Se}$ are allowed to change phase, while $\mathrm{Ba}, \mathrm{Cr}, \mathrm{Pb}$, and $\mathrm{Ag}$ are restricted to the solid phase. The inlet fuel mixture streams in this system are composed of $\mathrm{CH} 4$ and $20 \%$ excess air. Design Spec's on air and oxygen flow consider both free oxygen and that bound in organics.

\begin{tabular}{|c|c|c|c|}
\hline $\begin{array}{l}\text { Block Name } \\
\text { (ASPEN } \\
\text { Type) } \\
\end{array}$ & $\begin{array}{l}\text { Outlet } \\
\text { Temp (F) }\end{array}$ & $\begin{array}{l}\text { Heat Duty } \\
\text { (MMBtu/hr) }\end{array}$ & Description and Assumptions \\
\hline $\begin{array}{l}\text { METALSEP } \\
\text { (SEP) }\end{array}$ & 68 & 0 & $\begin{array}{l}\text { Separation of bulk metals (all } \mathrm{Fe} \text { ) from the Combustible and } \\
\text { Noncombustible input waste streams for recycling. }\end{array}$ \\
\hline $\begin{array}{l}\text { KIIN } \\
\text { (RGIBBS) }\end{array}$ & 1600 & -0.362 & $\begin{array}{l}\text { Conventional rotary kiln. Accepts combustible and non- } \\
\text { combustible wastes and metals with fixed contamination (after } \\
\text { sorting). CH4-fueled with air as oxidant. Heat losses assumed to } \\
\text { be } 5 \% \text { of the sensible heat of the products. Includes fixed air } \\
\text { leakage through seals. } \\
\text { Design Spec QKIIN: Vary either inlet fuel mixture flow (if heat } \\
\text { is needed) or inlet water flow (if cooling is needed) such that heat } \\
\text { duty is } 5 \% \text { of that calculated for unit Q1. } \\
\text { Design Spec OXI: Vary inlet air flow in stream AIR such that } \\
\text { total inlet oxygen flow (minus that used by the CH4) is twice the } \\
\text { outlet oxygen flow (100\% excess). } \\
\text { Design Spec SOILH2O: Vary inlet soil feed rate (substream } \\
\text { MIXED, 10\% of total flow) such that it is } 1 / 30 \text { the magnitude of } \\
\text { the SLAG stream (ash-to-gross soil ratio of } 2: 1 \text { ). } \\
\text { Design Spec SOILSOL: Vary inlet soil feed rate (substream } \\
\text { CISOLID, } 90 \% \text { of total flow) such that it is } 1 / 3.333 \text { the magnitude } \\
\text { of the SLAG stream (ash-to-gross soil ratio of } 2: 1 \text { ). }\end{array}$ \\
\hline $\begin{array}{l}\text { DUPL1 } \\
\text { (DUPL) }\end{array}$ & 1600 & 0 & $\begin{array}{l}\text { A stream duplicator block used to send a copy of the KILN outlet } \\
\text { stream to the dummy unit Q1. }\end{array}$ \\
\hline $\begin{array}{l}\text { Q1 } \\
\text { (RGIBBS) }\end{array}$ & 68 & -7.231 & $\begin{array}{l}\text { Dummy unit. This calculates chemical equilibrium at } 1600 \mathrm{~F} \text {, } \\
\text { identical to unit } \mathrm{KINN} \text {, but has an outlet temperature of } 68 \mathrm{~F} \text {, thus } \\
\text { yielding an estimate of the sensible heat of the products of unit } \\
\text { KILN. }\end{array}$ \\
\hline $\begin{array}{l}\text { SCC } \\
\text { (RGIBBS) }\end{array}$ & 2200 & -0.79 & $\begin{array}{l}\text { 2-way separation of KILN products: } 80 \% \text { of metals and oxides go } \\
\text { to VITRIFY (via stream ASH). Remainder, including } 100 \% \text { of } \\
\text { volatile trace metals and vapors, sent to SCC. Stream ASH } \\
\text { flashed to } 68 \text { F to represent storage of material before use. } \\
\text { Secondary combustion unit. CH4-fueled with air as oxidant. Heat } \\
\text { losses assumed to be } 5 \% \text { of the sensible heat of the products. } \\
\text { Design Spec QSCC: Vary inlet fuel mixture flow such that heat } \\
\text { duty is } 5 \% \text { of that calculated for unit Q2. }\end{array}$ \\
\hline $\begin{array}{l}\text { DUPL2 } \\
\text { (DUPL) }\end{array}$ & 2200 & 0 & $\begin{array}{l}\text { A stream duplicator block used to send a copy of the SCC outlet } \\
\text { stream to the dummy unit Q2. }\end{array}$ \\
\hline
\end{tabular}


Table A-6.

ASSUMPTIONS and ENERGY BALANCES for ROTARY KILN W/MAXIMUM RECYCLING, System A-6

GENERAL COMMENTS: RGIBBS units calculate chemical and phase equilibrium with some restrictions, not all stated here. For detailed information refer to the ASPEN PLUS Release 9 input file. Chemical reactions and phase changes for some species are neglected. "Volatile" trace metals $\mathrm{As}, \mathrm{Cd}, \mathrm{Hg}$, and $\mathrm{Se}$ are allowed to change phase, while $\mathrm{Ba}, \mathrm{Cr}, \mathrm{Pb}$, and $\mathrm{Ag}$ are restricted to the solid phase. The inlet fuel mixture streams in this system are composed of $\mathrm{CH} 4$ and $20 \%$ excess air. Design Spec's on air and oxygen flow consider both free oxygen and that bound in organics.

\begin{tabular}{|c|c|c|c|}
\hline $\begin{array}{l}\text { Block Name } \\
\text { (ASPEN } \\
\text { Type) } \\
\end{array}$ & $\begin{array}{l}\text { Outlet } \\
\text { Temp (F) }\end{array}$ & $\begin{array}{l}\text { Heat Duty } \\
\text { (MMBtu/hr) }\end{array}$ & Description and Assumptions \\
\hline $\begin{array}{l}\text { Q2 } \\
\text { (RGIBBS) }\end{array}$ & 68 & -15.798 & $\begin{array}{l}\text { Dummy unit. This calculates chemical equilibrium at } 2200 \mathrm{~F} \text {, } \\
\text { identical to unit SCC, but has an outlet temperature of } 68 \mathrm{~F} \text {, thus } \\
\text { yielding an estimate of the sensible heat of the products of unit } \\
\text { SCC. }\end{array}$ \\
\hline $\begin{array}{l}\text { QUENCH } \\
\text { (RGIBBS) }\end{array}$ & 350 & 0 & $\begin{array}{l}\text { Quenching of the exhaust of the SCC. No losses included. } \\
\text { Formation of CH4, C, S and Cl2 is restricted. } \\
\text { Design Spec QQUENCH: Vary inlet water flow rate to achieve } \\
\text { zero heat duty. }\end{array}$ \\
\hline $\begin{array}{l}\text { BAGHOUSE } \\
\text { (SEP) }\end{array}$ & 350 & -0.017 & $\begin{array}{l}\text { 3-way separation of QUENCH products. Vapors sent to SCRUB, } \\
\text { volatile trace metals exit system (via stream HGPLUS), and } \\
\text { remainder (soil + ash) recycles to VITRIFY (via FLYASH). } \\
\text { Stream FLYASH flashed to } 68 \text { F to represent storage of material } \\
\text { before use. }\end{array}$ \\
\hline $\begin{array}{l}\text { SCRUB } \\
\text { (RGIBBS) }\end{array}$ & 120 & -13.85 & $\begin{array}{l}\text { Wet acid scrubber. } \\
\text { Design Spec SCRUBH2O: Vary inlet water flow such that inlet } \\
\text { liquid water flow equals } 20 \text { times outlet dissolved salts ( } 5 \% \text { TDS), } \\
\text { with a minimum value equal to inlet } \mathrm{NaOH} \text { flow ( } 50 \% \text { soln). } \\
\text { Design Spec NAOH: Vary inlet } \mathrm{NaOH} \text { flow such that total inlet } \\
\mathrm{NaOH} \text { flow (including recycled } \mathrm{NaOH} \text { ) equals } 100 \text { times outlet } \\
\mathrm{NaOH} \text { flow.(1\% excess). }\end{array}$ \\
\hline $\begin{array}{l}\text { L-V-SEP } \\
\text { (FLASH2) }\end{array}$ & 120 & 0 & $\begin{array}{l}\text { 2-way separation of SCRUB products. Flash calculation to } \\
\text { separate liquid and vapor. }\end{array}$ \\
\hline $\begin{array}{l}\text { SPLITTER } \\
\text { (RSTOIC) }\end{array}$ & 221 & 14.528 & $\begin{array}{l}\text { Splitting of the } \mathrm{NaCl} \text { into } \mathrm{HCl} \text { and } \mathrm{NaOH} \text { by electrolysis. Feed } \\
\text { material raised to } 221 \mathrm{~F} \text { to evaporate off water. Conversion } \\
\text { assumed at } 100 \% \text { of } \mathrm{NaCl} \text { and process assumed } 100 \% \text { efficient } \\
\text { with no heat losses included. }\end{array}$ \\
\hline $\begin{array}{l}\text { SALT-SEP } \\
\text { (SEP) }\end{array}$ & 221 & -14.905 & $\begin{array}{l}\text { Separation of splitter products: } \mathrm{HCl} \text { assumed taken off as a } 50 \% \\
\text { solution, flashed to } 68 \mathrm{~F} ; 98 \% \text { of } \mathrm{NaOH} \text { assumed recycled as a } \\
\text { pure component at } 68 \mathrm{~F} \text {; water ( } \& \text { remaining } \mathrm{NaOH} \text { ) for recycle, } \\
\text { flashed to } 68 \mathrm{~F} \text {; and the remainder, including other dry salts and } \\
\text { (predominantly) the gases formerly in solution. }\end{array}$ \\
\hline $\begin{array}{l}\text { VTRIFY } \\
\text { (RGIBBS) }\end{array}$ & 3000 & +1.485 & $\begin{array}{l}\text { Vitrifier. Heat duty does not include heat losses. Feed material } \\
\text { enters at } 68 \mathrm{~F} \text {. }\end{array}$ \\
\hline $\begin{array}{l}\text { L-V-SEP2 } \\
\text { (FLASH2) }\end{array}$ & 3000 & 0 & $\begin{array}{l}\text { 2-way separation of VITRIFY products. Flash calculation to } \\
\text { separate liquid and vapor. No vapor expected. }\end{array}$ \\
\hline
\end{tabular}


Table A-6.

ASSUMPTIONS and ENERGY BALANCES for ROTARY KILN W/MAXIMUM RECYCLING, System A-6

GENERAL COMMENTS: RGIBBS units calculate chemical and phase equilibrium with some restrictions, not all stated here. For detailed information refer to the ASPEN PLUS Release 9 input file. Chemical reactions and phase changes for some species are neglected. "Volatile" trace metals $\mathrm{As}, \mathrm{Cd}, \mathrm{Hg}$, and Se are allowed to change phase, while $\mathrm{Ba}, \mathrm{Cr}, \mathrm{Pb}$, and $\mathrm{Ag}$ are restricted to the solid phase. The inlet fuel mixture streams in this system are composed of $\mathrm{CH} 4$ and $20 \%$ excess air. Design Spec's on air and oxygen flow consider both free oxygen and that bound in organics.

\begin{tabular}{|l|l|l|l|}
\hline $\begin{array}{l}\text { Block Name } \\
\text { (ASPEN } \\
\text { Type) }\end{array}$ & $\begin{array}{l}\text { Outlet } \\
\text { Temp (F) }\end{array}$ & $\begin{array}{l}\text { Heat Duty } \\
\text { (MMBtu/hr) }\end{array}$ & Description and Assumptions \\
\hline $\begin{array}{l}\text { MELTER } \\
\text { (RGBBBS) }\end{array}$ & 3000 & +0.120 & $\begin{array}{l}\text { Metal melter. Accepts metals with fixed contamination. } \\
\text { Electrically heated with heat losses not included. }\end{array}$ \\
\hline
\end{tabular}


A-18 
Table A-7.

ASSUMPTIONS and ENERGY BALANCES for SLAGGING ROTARY KILN, System A-7

Page 1 of 2

GENERAL COMMENTS: RGIBBS units calculate chemical and phase equilibrium with some restrictions, not all stated here. For detailed information refer to the ASPEN PLUS Release 9 input file. Chemical reactions and phase changes for some species are neglected. "Volatile" trace metals $\mathrm{As}, \mathrm{Cd}, \mathrm{Hg}$, and Se are allowed to change phase, while $\mathrm{Ba}, \mathrm{Cr}, \mathrm{Pb}$, and $\mathrm{Ag}$ are restricted to the solid phase. The inlet fuel mixture streams in this system are composed of $\mathrm{CH} 4$ and $20 \%$ excess air. Design Spec's on air and oxygen flow consider both free oxygen and that bound in organics.

\begin{tabular}{|c|c|c|c|}
\hline $\begin{array}{l}\text { Block Name } \\
\text { (ASPEN } \\
\text { Type) }\end{array}$ & $\begin{array}{l}\text { Outlet } \\
\text { Temp (F) }\end{array}$ & $\begin{array}{l}\text { Heat Duty } \\
\text { (MMBtu/hr) }\end{array}$ & Description and Assumptions \\
\hline $\begin{array}{l}\text { KIIN } \\
\text { (RGIBBS) }\end{array}$ & 2500 & -1.021 & $\begin{array}{l}\text { Slagging rotary kiln. Accepts combustible and non-combustible } \\
\text { wastes and metals with fixed contamination. CH4-fueled with air } \\
\text { as oxidant. Heat losses assumed to be } 5 \% \text { of the sensible heat of } \\
\text { the products. Includes fixed air leakage through seals. Chemical } \\
\text { and phase equilibrium of Fe and Fe3O4 are restricted. Glass } \\
\text { forming inerts forced to melt. } \\
\text { Design Spec QKILN: Vary inlet fuel mixture flow such that heat } \\
\text { duty is } 5 \% \text { of that calculated for unit Q1. } \\
\text { Design Spec OXI: Vary inlet air flow in stream AIR such that } \\
\text { total iniet oxygen flow (minus that used by the CH4) is twice the } \\
\text { outlet oxygen flow (100\% excess). } \\
\text { Design Spec SOILH2O: Vary inlet soil feed rate (substream } \\
\text { MIXED, 10\% of total flow) such that it is } 1 / 30 \text { the magnitude of } \\
\text { the SLAG stream (ash-to-gross soil ratio of } 2: 1 \text { ). } \\
\text { Design Spec SOILSOL: Vary inlet soil feed rate (substream } \\
\text { CISOLID, } 90 \% \text { of total flow) such that it is } 1 / 3.333 \text { the magnitude } \\
\text { of the SLAG stream (ash-to-gross soil ratio of } 2: 1 \text { ). }\end{array}$ \\
\hline $\begin{array}{l}\text { DUPL1 } \\
\text { (DUPL) }\end{array}$ & 2500 & 0 & $\begin{array}{l}\text { A stream duplicator block used to send a copy of the } \mathrm{KIIN} \text { outlet } \\
\text { stream to the dummy unit } \mathrm{Q} 1 \text {. }\end{array}$ \\
\hline $\begin{array}{l}\text { Q1 } \\
\text { (RGIBBS) }\end{array}$ & 68 & -20.423 & $\begin{array}{l}\text { Dummy unit. This calculates chemical equilibrium at } 2500 \mathrm{~F} \text {, } \\
\text { identical to unit KINN, but has an outlet temperature of } 68 \mathrm{~F} \text {, thus } \\
\text { yielding an estimate of the sensible heat of the products of unit } \\
\text { KILN. }\end{array}$ \\
\hline $\begin{array}{l}\text { KIINSEP } \\
\text { (SEP) }\end{array}$ & 2500 & 0 & $\begin{array}{l}\text { 2-way separation of KILN products: } 80 \% \text { of metals and oxides } \\
\text { exit system (via stream SLAG). Remainder, including } 100 \% \text { of } \\
\text { volatile trace metals and vapors, sent to SCC. }\end{array}$ \\
\hline $\begin{array}{l}\text { SCC } \\
\text { (RGIBBS) }\end{array}$ & 2500 & -1.134 & $\begin{array}{l}\text { Secondary combustion unit. CH4-fueled with air as oxidant. Heat } \\
\text { losses assumed to be } 5 \% \text { of the sensible heat of the products. } \\
\text { Chemical and phase equilibrium of } \mathrm{Fe} \text { and } \mathrm{Fe} 3 \mathrm{O} 4 \text { are restricted. } \\
\text { Glass forming inerts forced to melt. } \\
\text { Design Spec QSCC: Vary inlet fuel mixture flow such that heat } \\
\text { duty is } 5 \% \text { of that calculated for unit Q2. }\end{array}$ \\
\hline $\begin{array}{l}\text { DUPL2 } \\
\text { (DUPL) }\end{array}$ & 2500 & 0 & $\begin{array}{l}\text { A stream duplicator block used to send a copy of the SCC outlet } \\
\text { stream to the dummy unit Q2. }\end{array}$ \\
\hline
\end{tabular}


Table A-7.

ASSUMPTIONS and ENERGY BALANCES for SLAGGING ROTARY KILN, System A-7

GENERAL COMMENTS: RGIBBS units calculate chemical and phase equilibrium with some restrictions, not all stated here. For detailed information refer to the ASPEN PLUS Release 9 input file. Chemical reactions and phase changes for some species are neglected. "Volatile" trace metals $\mathrm{As}, \mathrm{Cd}, \mathrm{Hg}$, and $\mathrm{Se}$ are allowed to change phase, while $\mathrm{Ba}, \mathrm{Cr}, \mathrm{Pb}$, and $\mathrm{Ag}$ are restricted to the solid phase. The inlet fuel mixture streams in this system are composed of CH4 and $20 \%$ excess air. Design Spec's on air and oxygen flow consider both free oxygen and that bound in organics.

\begin{tabular}{|l|l|l|l|}
\hline $\begin{array}{l}\text { Block Name } \\
\text { (ASPEN } \\
\text { Type) }\end{array}$ & $\begin{array}{l}\text { Outlet } \\
\text { Temp (F) }\end{array}$ & $\begin{array}{l}\text { Heat Duty } \\
\text { (MMBtu/hr) }\end{array}$ & Description and Assumptions \\
\hline $\begin{array}{l}\text { Q2 } \\
\text { RGIBBS) }\end{array}$ & 68 & -22.672 & $\begin{array}{l}\text { Dummy unit. This calculates chemical equilibrium at 2500 F, } \\
\text { identical to unit SCC, but has an outlet temperature of 68 F, thus } \\
\text { yielding an estimate of the sensible heat of the products of unit } \\
\text { SCC. }\end{array}$ \\
\hline $\begin{array}{l}\text { QUENCH } \\
\text { (RGIBBS) }\end{array}$ & 350 & 0 & $\begin{array}{l}\text { Quenching of the exhaust of the SCC. No losses included. } \\
\text { Formation of CH4, C, and S is restricted. Chemical and phase } \\
\text { equilibrium of Fe and Fe3O4 are restricted. Glass forming inerts } \\
\text { forced to solidify. } \\
\text { Design Spec QQUENCH: Vary inlet water flow rate to achieve } \\
\text { zero heat duty. }\end{array}$ \\
\hline $\begin{array}{l}\text { BAGHOUSE } \\
\text { (SEP) }\end{array}$ & 350 & 0 & $\begin{array}{l}\text { 3-way separation of QUENCH products. Vapors sent to SCRUB, } \\
\text { volatile trace metals exit System, and remainder (soil + ash) } \\
\text { recycles to KILN (via FLYASH). }\end{array}$ \\
\hline $\begin{array}{l}\text { SCRUB } \\
\text { (RGIBBS) }\end{array}$ & 120 & -20.019 & $\begin{array}{l}\text { Wet acid scrubber. } \\
\text { Design Spec SCRUBH2O: Vary inlet water flow such that inlet } \\
\text { liquid water flow equals 20 times outlet dissolved salts (5\% TDS), } \\
\text { with a minimum value equal to inlet NaOH flow (50\% soln). } \\
\text { Design Spec NAOH: Vary inlet NaOH flow such that inlet NaOH } \\
\text { flow equals 100 times outlet NaOH flow (1\% excess). }\end{array}$ \\
\hline $\begin{array}{l}\text { L-V-SEP } \\
\text { (FLASH2) }\end{array}$ & 120 & 0 & $\begin{array}{l}\text { 2-way separation of SCRUB products. Flash calculation to } \\
\text { separate liquid and vapor. }\end{array}$ \\
\hline
\end{tabular}




\section{System B-1: Pyrolysis}

Table A-8.

ASSUMPTIONS and ENERGY BALANCES for PYROLYSIS, System B-1

Page 1 of 2

GENERAL COMMENTS: RGIBBS units calculate chemical and phase equilibrium with some restrictions, not all stated here. For detailed information refer to the ASPEN PLUS Release 9 input file. Chemical reactions and phase changes for some species are neglected. "Volatile" trace metals $\mathrm{As}, \mathrm{Cd}, \mathrm{Hg}$, and Se are allowed to change phase, while $\mathrm{Ba}, \mathrm{Cr}, \mathrm{Pb}$, and $\mathrm{Ag}$ are restricted to the solid phase. No direct use of auxiliary fuel occurs in this system. Design Spec's on air and oxygen flow consider both free oxygen and that bound in organics.

\begin{tabular}{|c|c|c|c|}
\hline $\begin{array}{l}\text { Block Name } \\
\text { (ASPEN } \\
\text { Type) }\end{array}$ & $\begin{array}{l}\text { Outlet } \\
\text { Temp (F) }\end{array}$ & $\begin{array}{l}\text { Heat Duty } \\
\text { (MMBtw/hr) }\end{array}$ & Description and Assumptions \\
\hline $\begin{array}{l}\text { PYROLYZR } \\
\text { (RSTOIC) }\end{array}$ & 1200 & -0.742 & $\begin{array}{l}\text { Rotary kiln pyrolyzer. Accepts combustible wastes only. Indirect } \\
\text { fired, electrically heated to minimize offgas volume. Heat duty } \\
\text { (net heat output) does not include any heat losses. Model includes } \\
\text { fixed air leakage through seals as used in original Phase I report } \\
\text { (not same amount as in systems A1-A7). Only chemical or phase } \\
\text { reaction allowed is } 99 \% \text { conversion of carbon to CO, by use of } \\
\text { additional oxygen. } \\
\text { Design Spec OXYGEN: Vary inlet oxygen flow in stream } \\
\text { OXYGEN such that outlet mole flow of carbon minus two times } \\
\text { outlet mole flow of oxygen equals } 0.01 \text { (-0-\% excess oxygen). }\end{array}$ \\
\hline $\begin{array}{l}\text { PYRO-SEP } \\
\text { (SEP) }\end{array}$ & 1200 & -0.027 & $\begin{array}{l}\text { 2-way separation of PYROLYZR products: } 80 \% \text { of "ash" (solid } \\
\text { carbon and sulphur, metals, and inerts) including the solid volatile } \\
\text { trace metals go to VITRFY (via stream ASH). Remainder, } \\
\text { including } 100 \% \text { of the molten and gaseous volatile trace metals } \\
\text { and the gases and organic constituents, sent to SCC. Stream ASH } \\
\text { is flashed to } 68 \text { F to represent storage of material before use. }\end{array}$ \\
\hline $\begin{array}{l}\text { VITRIFY } \\
\text { (RGIBBS) }\end{array}$ & 3000 & +2.571 & $\begin{array}{l}\text { Vitrifier. Accepts non-combustible wastes. Use of an oxygen } \\
\text { lance assures.burnout of remaining carbon. No heat losses } \\
\text { included. Chemical and phase equilibrium of Fe and Fe3O4 are } \\
\text { restricted. } \\
\text { Design Spec O2-VIT: Vary the inlet oxygen flow such that total } \\
\text { inlet oxygen flow is six times the outlet oxygen flow ( } 20 \% \\
\text { excess). } \\
\text { Design Spec SOILH2O: Vary inlet soil feed rate (substream } \\
\text { MIXD, } 10 \% \text { of total flow) such that it is } 1 / 30 \text { the magnitude of } \\
\text { the SLAG stream (ash-to-gross soil ratio of } 2: 1 \text { ). } \\
\text { Design Spec SOILSOL: Vary inlet soil feed rate (substream } \\
\text { CISOLI, } 90 \% \text { of total flow) such that it is } 1 / 3.333 \text { the magnitude } \\
\text { of the SLAG stream (ash-to-gross soil ratio of } 2: 1 \text { ). }\end{array}$ \\
\hline $\begin{array}{l}\text { VIT-SEP } \\
\text { (SEP) }\end{array}$ & 3000 & 0 & $\begin{array}{l}\text { 2-way separation of VITRIFY products: } 80 \% \text { of "ash" (solid } \\
\text { carbon and sulphur, metals, and inerts) including the solid volatile } \\
\text { trace metals go to VITRIFY (via stream ASH). Remainder, } \\
\text { including } 100 \% \text { of the molten or gaseous volatile trace metals and } \\
\text { the gases and organic constituents, sent to SCC. }\end{array}$ \\
\hline $\begin{array}{l}\text { SCC } \\
\text { (RGIBBS) }\end{array}$ & 2200 & -5.258 & $\begin{array}{l}\text { Secondary combustion unit. Heat losses are not included in the } \\
\text { heat duty. Oxygen to be supplied in stoichiometric proportions. } \\
\text { Design Spec O2-SCC: Vary the inlet oxygen flow such that total } \\
\text { inlet oxygen flow is } 51 \text { times the outlet oxygen flow ( } 2 \% \text { excess). }\end{array}$ \\
\hline
\end{tabular}


Table A-8.

ASSUMPTIONS and ENERGY BALANCES for PYROLYSIS, System B-1

GENERAL COMMENTS: RGIBBS units calculate chemical and phase equilibrium with some restrictions, not all stated here. For detailed information refer to the ASPEN PLUS Release 9 input file. Chemical reactions and phase changes for some species are neglected. "Volatile" trace metals $\mathrm{As}, \mathrm{Cd}, \mathrm{Hg}$, and Se are allowed to change phase, while $\mathrm{Ba}, \mathrm{Cr}, \mathrm{Pb}$, and $\mathrm{Ag}$ are restricted to the solid phase. No direct use of auxiliary fuel occurs in this system. Design Spec's on air and oxygen flow consider both free oxygen and that bound in organics.

\begin{tabular}{|l|l|l|l|}
\hline $\begin{array}{l}\text { Block Name } \\
\text { (ASPEN } \\
\text { Type) }\end{array}$ & $\begin{array}{l}\text { Outlet } \\
\text { Temp (F) }\end{array}$ & $\begin{array}{l}\text { Heat Duty } \\
\text { (MMBtu/hr) }\end{array}$ & Description and Assumptions \\
\hline $\begin{array}{l}\text { QUENCH } \\
\text { (RGBBS) }\end{array}$ & 350 & 0 & $\begin{array}{l}\text { Quenching of the exhaust of the SCC. No losses included. } \\
\text { Fornation of CH4, C, S and C12 is restricted. } \\
\text { Design Spec QQUENCH: Vary inlet water flow rate to achieve } \\
\text { zero heat duty. }\end{array}$ \\
\hline $\begin{array}{l}\text { BAGHOUSE } \\
\text { (SEP) }\end{array}$ & 350 & -0.001 & $\begin{array}{l}\text { 3-way separation of QUENCH products. Vapors sent to SCRUB, } \\
\text { volatile trace metals exit system (via stream HGPLUS), and } \\
\text { remainder (soil + ash) recycles to VITRIFY (via FLYASH). } \\
\text { Stream FLYASH is flashed to 68 F to represent storage of } \\
\text { material before use. }\end{array}$ \\
\hline $\begin{array}{l}\text { SCRUB } \\
\text { (RGIBBS) }\end{array}$ & 120 & -2.608 & $\begin{array}{l}\text { Wet acid scrubber. Formation of CH4, C, and S is restricted. } \\
\text { Design Spec SCRUBH2O: Vary inlet water flow such that iniet } \\
\text { liquid water flow equals 20 times outlet dissolved salts (5\% TDS), } \\
\text { with a minimum value equal to inlet NaOH flow (50\% soln). } \\
\text { Design Spec NAOH: Vary inlet NaOH flow such that inlet NaOH } \\
\text { flow equals 100 times outlet NaOH flow (1\% excess). }\end{array}$ \\
\hline $\begin{array}{l}\text { L-V-SEP } \\
\text { (FLASH2) }\end{array}$ & 120 & 0 & $\begin{array}{l}\text { 2-way separation of SCRUB products. Flash calculation to } \\
\text { separate liquid and vapor. }\end{array}$ \\
\hline $\begin{array}{l}\text { MELTER } \\
\text { (RGIBBS) }\end{array}$ & 3000 & +0.120 & $\begin{array}{l}\text { Metal melter. Accepts metals with fixed contamination. } \\
\text { Electrically heated with heat losses not included. }\end{array}$ \\
\hline
\end{tabular}


Table A-9.

ASSUMPTIONS and ENERGY BALANCES for PLASMA FURNACE, System C-1

GENERAL COMMENTS: RGIBBS units calculate chemical and phase equilibrium with some restrictions as stated. For detailed information refer to the ASPEN PLUS Release 9 input file. Chemical reactions and phase changes for some species are neglected. "Volatile" trace metals $\mathrm{As}, \mathrm{Cd}, \mathrm{Hg}$, and Se are allowed to change phase, while $\mathrm{Ba}, \mathrm{Cr}, \mathrm{Pb}$, and $\mathrm{Ag}$ are restricted to the solid phase. No direct use of auxiliary fuel occurs in this system. Design Spec's on air and oxygen flow consider both free oxygen and that bound in organics.

\begin{tabular}{|c|c|c|c|}
\hline $\begin{array}{l}\text { Block Name } \\
\text { (ASPEN } \\
\text { Type) }\end{array}$ & $\begin{array}{l}\text { Outlet } \\
\text { Temp (F) }\end{array}$ & $\begin{array}{l}\text { Heat Duty } \\
\text { (MMBtu/hr) }\end{array}$ & Description and Assumptions \\
\hline $\begin{array}{l}\text { FEED-SEP } \\
\text { (SEP) }\end{array}$ & 70 & 0 & $\begin{array}{l}\text { 2-way separation of the feed material. SOLIDS stream goes to the } \\
\text { unit representing the vitrifier molten pool, while the organic-type } \\
\text { components go to the unit representing the vitrifier cover gas } \\
\text { region. Five percent of the carbon and sulfur is assumed to end up } \\
\text { trapped in the melt, and } 5 \% \text { of the solids are assumed to be carried } \\
\text { off with the vapors. }\end{array}$ \\
\hline $\begin{array}{l}\text { PLASMA } \\
\text { (RGIBBS) }\end{array}$ & 3000 & +2.934 & $\begin{array}{l}\text { Plasma furnace. Accepts the non-organic portion of the } \\
\text { combustible and non-combustible wastes and metals with fixed } \\
\text { contamination. The organic portion is assumed to be volatilized } \\
\text { by the plasma arc at a lower temperature and released to the } \\
\text { plenum over the melt. Heat duty does not include any losses or } \\
\text { the energy needed to heat and volatilize the organics. } \\
\text { Design Spec SOILH2O: Vary inlet soil feed rate (substream } \\
\text { MIXED, } 10 \% \text { of total flow) such that it is } 1 / 30 \text { the magnitude of } \\
\text { the SLAG stream (ash-to-gross soil ratio of } 2: 1 \text { ). } \\
\text { Design Spec SOILSOL: Vary inlet soil feed rate (substream } \\
\text { CISOLID, } 90 \% \text { of total flow) such that it is } 1 / 3.333 \text { the magnitude } \\
\text { of the SLAG stream (ash-to-gross soil ratio of } 2: 1 \text { ). }\end{array}$ \\
\hline $\begin{array}{l}\text { PLAS-SEP } \\
\text { (SEP) }\end{array}$ & 3000 & 0 & $\begin{array}{l}\text { 3-way separation of PLASMA products: } 100 \% \text { of metals exit } \\
\text { system (via stream METAL). Some Fe } 304 \text { is reduced to Fe. All } \\
\text { of the remaining solids go to the slag stream (SLAG). Any gases } \\
\text { produced, as well as } 100 \% \text { of volatile trace metals, are sent to unit } \\
\text { PLENUM. }\end{array}$ \\
\hline $\begin{array}{l}\text { PLENUM } \\
\text { (RGIBBS) }\end{array}$ & 1800 & -3.297 & $\begin{array}{l}\text { Plasma furnace plenum area. Organic constituents are volatilized } \\
\text { and released from the meit into the plenum. Air is injected for } \\
\text { complete combustion. The heat duty does not include any losses. } \\
\text { Formation of CH4 is restricted. } \\
\text { Design Spec AIR: Vary the inlet air flow (stream AIR) such that } \\
\text { the total incoming oxygen is six times the outlet oxygen flow } \\
\text { (20\% excess). }\end{array}$ \\
\hline $\begin{array}{l}\text { SCC } \\
\text { (RGIBBS) }\end{array}$ & 2200 & +0.975 & $\begin{array}{l}\text { Secondary combustion unit. Further combustion of the vapors at a } \\
\text { temperature to assure destruction of PCB's. Add air at up to } 20 \% \\
\text { excess, if needed. The heat duty does not include any losses. } \\
\text { Formation of CH4, C and S is restricted. } \\
\text { Design Spec AIR2: Vary the inlet air flow (stream AIR2) such } \\
\text { that the total incoming oxygen is six times the outlet oxygen flow } \\
\text { (20\% excess). }\end{array}$ \\
\hline
\end{tabular}


Table A-9.

ASSUMPTIONS and ENERGY BALANCES for PLASMA FURNACE, System C-1

GENERAL COMMENTS: RGIBBS units calculate chemical and phase equilibrium with some restrictions as stated. For detailed information refer to the ASPEN PLUS Release 9 input file. Chemical reactions and phase changes for some species are neglected. "Volatile" trace metals $\mathrm{As}, \mathrm{Cd}, \mathrm{Hg}$, and Se are allowed to change phase, while $\mathrm{Ba}, \mathrm{Cr}, \mathrm{Pb}$, and $\mathrm{Ag}$ are restricted to the solid phase. No direct use of auxiliary fuel occurs in this system. Design Spec's on air and oxygen flow consider both free oxygen and that bound in organics.

\begin{tabular}{|l|l|l|l|}
\hline $\begin{array}{l}\text { Block Name } \\
\text { (ASPEN } \\
\text { Type) }\end{array}$ & $\begin{array}{l}\text { Outlet } \\
\text { Temp (F) }\end{array}$ & $\begin{array}{l}\text { Heat Duty } \\
\text { (MMBtu/hr) }\end{array}$ & Description and Assumptions \\
\hline $\begin{array}{l}\text { QUENCH } \\
\text { RGBBS) }\end{array}$ & 350 & 0 & $\begin{array}{l}\text { Quenching of the exhaust vapors of the SCC. No losses included. } \\
\text { Formation of CH4, C and S is restricted. } \\
\text { Design Spec QQUENCH: Vary inlet water flow rate (QNCH2O) } \\
\text { to achieve zero.heat duty. }\end{array}$ \\
\hline $\begin{array}{l}\text { BAGHOUSE } \\
\text { (SEP) }\end{array}$ & 350 & -0.005 & $\begin{array}{l}\text { 3-way separation of QUENCH products, modeling the various } \\
\text { filters. Vapors sent to DRYSCRUB (via QNCHVAP), volatile } \\
\text { trace metals exit system (via HGPLUS), and remainder recycles to } \\
\text { PLASMA (via FL YASH). Stream FL YASH flashed to 68 F to } \\
\text { represent storage of material before use. }\end{array}$ \\
\hline $\begin{array}{l}\text { SCRUB } \\
\text { (RGIBBS) }\end{array}$ & 120 & -4.954 & $\begin{array}{l}\text { Wet acid scrubber. } \\
\text { Design Spec SCRUBH2O: Vary inlet water flow such that inlet } \\
\text { liquid water flow equals 20 times outlet dissolved salts (5\% TDS), } \\
\text { with a minimum value equal to inlet NaOH flow (50\% soln). } \\
\text { Design Spec NAOH: Vary inlet NaOH flow such that inlet NaOH } \\
\text { flow equals lo0 times outlet NaOH flow (1\% excess). }\end{array}$ \\
\hline $\begin{array}{l}\text { L-V-SEP } \\
\text { (FLASH2) }\end{array}$ & 120 & 0 & $\begin{array}{l}\text { 2-way separation of SCRUB products. Flash calculation to } \\
\text { separate liquid and vapor. }\end{array}$ \\
\hline
\end{tabular}


Table A-10.

ASSUMPTIONS and ENERGY BALANCES for PLASMA FURNACE W/ $\mathrm{CO}_{2}$ RETENTION, System C-2

GENERAL COMMENTS: RGIBBS units calculate chemical and phase equilibrium with some restrictions as stated. For detailed information refer to the ASPEN PLUS Release 9 input file. Chemical reactions and phase changes for some species are neglected. "Volatile" trace metals $\mathrm{As}, \mathrm{Cd}, \mathrm{Hg}$, and Se are allowed to change phase, while $\mathrm{Ba}, \mathrm{Cr}, \mathrm{Pb}$, and $\mathrm{Ag}$ are restricted to the solid phase. The inlet mixed fuel stream in this system is composed of $\mathrm{CH} 4$ and $20 \%$ excess oxygen.. Design Spec's on air and oxygen flow consider both free oxygen and that bound in organics.

\begin{tabular}{|l|l|l|l|}
\hline $\begin{array}{l}\text { Block Name } \\
\text { (ASPEN } \\
\text { Type) }\end{array}$ & $\begin{array}{l}\text { Outlet } \\
\text { Temp (F) }\end{array}$ & $\begin{array}{l}\text { Heat Duty } \\
\text { (MMBtu/hr) }\end{array}$ & Description and Assumptions \\
\hline $\begin{array}{l}\text { FEED-SEP } \\
\text { (SEP) }\end{array}$ & 70 & 0 & $\begin{array}{l}\text { 2-way separation of the feed material. SOLIDS stream goes to the } \\
\text { unit representing the vitrifier molten pool, while the organic-type } \\
\text { components go to the unit representing the vitrifier cover gas } \\
\text { region. Five percent of the carbon and sulfur is assumed to end up } \\
\text { trapped in the melt, and 5\% of the solids are assumed to be carried } \\
\text { off with the vapors. }\end{array}$ \\
\hline $\begin{array}{l}\text { PLASMA } \\
\text { RGIBBS) }\end{array}$ & 3000 & +2.933 & $\begin{array}{l}\text { Plasma furnace. Accepts the non-organic portion of the } \\
\text { combustible and non-combustible wastes and metals with fixed } \\
\text { contamination. The organic portion is assumed to be volatilized } \\
\text { by the plasma arc at a lower temperature and released to the } \\
\text { plenum over the melt. Heat duty does not include any losses or } \\
\text { the energy needed to heat and volatilize the organics. } \\
\text { Design Spec SOILH2O: Vary inlet soil feed rate (substream } \\
\text { MIXED, 10\% of total flow) such that it is 1/30 the magnitude of } \\
\text { the SLAG stream (ash-to-gross soil ratio of 2:1). } \\
\text { Design Spec SOILSOL: Vary inlet soil feed rate (substream } \\
\text { CISOLID, 90\% of total flow) such that it is 1/3.333 the magnitude } \\
\text { of the SLAG stream (ash-to-gross soil ratio of 2:1). }\end{array}$ \\
\hline $\begin{array}{l}\text { PLENUM } \\
\text { (RGBBS) }\end{array}$ & 1800 & -5.399 & $\begin{array}{l}\text { 3-way separation of PLASMA products: 100\% of metals exit } \\
\text { system (via stream METAL). Some Fe3O4 is reduced to Fe. All } \\
\text { of the remaining solids go to the slag stream (SLAG). Any gases } \\
\text { produced, as well as 100\% of volatile trace metals, are sent to unit } \\
\text { PLENUM. }\end{array}$ \\
\hline 3000 & 0 & $\begin{array}{l}\text { Plasma furnace plenum area. Organic constituents are volatilized } \\
\text { and released from the melt into the plenum. Oxygen-rich offgas is } \\
\text { recycled into the zone, as well as make-up oxygen, combusting } \\
\text { the vapors. The heat duty does not include any losses. Formation } \\
\text { of CH4 is restricted. } \\
\text { Design Spec OXYGEN: Vary the inlet oxygen flow (stream } \\
\text { OXYGEN) such that the total incoming oxygen is 3.5 times the } \\
\text { outlet oxygen flow (40\% excess). }\end{array}$ \\
\hline
\end{tabular}


Table A-10.

ASSUMPTIONS and ENERGY BALANCES for PLASMA FURNACE W/ $\mathrm{CO}_{2}$ RETENTION, System C-2

GENERAL COMMENTS: RGIBBS units calculate chemical and phase equilibrium with some restrictions as stated. For detailed information refer to the ASPEN PLUS Release 9 input file. Chemical reactions and phase changes for some species are neglected. "Volatile" trace metals $\mathrm{As}, \mathrm{Cd}, \mathrm{Hg}$, and Se are allowed to change phase, while $\mathrm{Ba}, \mathrm{Cr}, \mathrm{Pb}$, and $\mathrm{Ag}$ are restricted to the solid phase. The inlet mixed fuel stream in this system is composed of $\mathrm{CH} 4$ and $20 \%$ excess oxygen. Design Spec's on air and oxygen flow consider both free oxygen and that bound in organics.

\begin{tabular}{|c|c|c|c|}
\hline $\begin{array}{l}\text { Block Name } \\
\text { (ASPEN } \\
\text { Type) } \\
\end{array}$ & $\begin{array}{l}\text { Outlet } \\
\text { Temp (F) }\end{array}$ & $\begin{array}{l}\text { Heat Duty } \\
\text { (MMBtu/hr) }\end{array}$ & Description and Assumptions \\
\hline $\begin{array}{l}\text { SCC } \\
\text { (RGIBBS) }\end{array}$ & 2200 & -0.164 & $\begin{array}{l}\text { Secondary combustion unit. Further combustion of the vapors at a } \\
\text { temperature to assure destruction of PCB's. CH4-fueled with pure } \\
\text { oxygen at } 20 \% \text { excess. Heat losses assumed to be } 5 \% \text { of the } \\
\text { sensible heat of the products. Formation of } \mathrm{CH} 4, \mathrm{C} \text { and } \mathrm{S} \text { is } \\
\text { restricted. } \\
\text { Design Spec QSCC: Vary inlet fuel mixture flow such that heat } \\
\text { duty is } 5 \% \text { of that calculated for unit Q2. } \\
\text { A stream duplicator block used to send a copy of the SCC outlet } \\
\text { stream to the dummy unit Q2. }\end{array}$ \\
\hline $\begin{array}{l}\text { Q2 } \\
\text { (RGIBBS) }\end{array}$ & 68 & -3.283 & $\begin{array}{l}\text { Dummy unit. This calculates chemical equilibrium at } 2200 \mathrm{~F} \text {, } \\
\text { identical to unit SCC, but has an outlet temperature of } 68 \mathrm{~F} \text {, thus } \\
\text { yielding an estimate of the sensible heat of the products of unit } \\
\text { SCC. }\end{array}$ \\
\hline $\begin{array}{l}\text { QUENCH } \\
\text { (RGIBBS) }\end{array}$ & 350 & 0 & $\begin{array}{l}\text { Quenching of the exhaust vapors of the SCC. No losses included. } \\
\text { Formation of } \mathrm{CH} 4, \mathrm{C} \text { and } \mathrm{S} \text { is restricted. } \\
\text { Design Spec QQUENCH: Vary inlet water flow rate (QNCH2O) } \\
\text { to achieve zero heat duty. . }\end{array}$ \\
\hline $\begin{array}{l}\text { BAGHOUSE } \\
\text { (SEP) }\end{array}$ & 350 & 0 & $\begin{array}{l}\text { 3-way separation of QUENCH products, modeling the various } \\
\text { filters. Vapors sent to DRYSCRUB (via QNCHVAP), volatile } \\
\text { trace metals exit system (via HGPLUS), and remainder recycles to } \\
\text { PLASMA (via FLYASH). }\end{array}$ \\
\hline $\begin{array}{l}\text { DRYSCRUB } \\
\text { (RGIBBS) }\end{array}$ & 350 & -2.595 & $\begin{array}{l}\text { Dry scrubber. Chemical equilibrium calculated for conversion of } \\
\mathrm{CaO} \text { to } \mathrm{CaSO} 4 \text { and } \mathrm{CaCl} 2 \text {, with all volatiles in the system } \\
\text { participating. Heat duty does not include any losses. Water used } \\
\text { to slake the lime is not included in the model. } \\
\text { Design Spec SCRUB: Vary the specified flow split of unit SPLIT } \\
\text { such that the inlet } \mathrm{CaO} \text { flow to DRYSCRUB is } 100 \text { times the } \\
\text { outlet } \mathrm{CaO} \text { flow. }\end{array}$ \\
\hline $\begin{array}{l}\text { SCRUBSEP } \\
\text { (SEP) }\end{array}$ & 350 & 0 & $\begin{array}{l}\text { 2-way separation of DRYSCRUB products. Vapors sent to } \mathrm{CO} 2 \text { - } \\
\text { ABS and remainder exits system. }\end{array}$ \\
\hline $\begin{array}{l}\text { CO2-ABS } \\
\text { (RSTOIC) }\end{array}$ & 1200 & -0.271 & $\begin{array}{l}\text { Restricted chemical reaction combining } \mathrm{CO} 2 \text { and } \mathrm{CaO} \text { to form } \\
\mathrm{CaCO} \text {, assuming } 100 \% \text { utilization of the } \mathrm{CO} 2 \text {. Heat duty does } \\
\text { not include any losses. }\end{array}$ \\
\hline $\begin{array}{l}\text { ABSSEP } \\
\text { (SEP) }\end{array}$ & 1200 & 0 & $\begin{array}{l}\text { 2-way separation of } \mathrm{CO} 2-\mathrm{ABS} \text { products. Vapors sent to FLASH. } \\
\text { Remainder, consisting only of } \mathrm{CaCO} \text {, sent to } \mathrm{CO} 2 \text {-DES. }\end{array}$ \\
\hline
\end{tabular}


Table A-10.

ASSUMPTIONS and ENERGY BALANCES for PLASMA FURNACE W/ $\mathrm{CO}_{2}$ RETENTION, System C-2

GENERAL COMMENTS: RGIBBS units calculate chemical and phase equilibrium with some restrictions as stated. For detailed information refer to the ASPEN PLUS Release 9 input file. Chemical reactions and phase changes for some species are neglected. "Volatile" trace metals $\mathrm{As}, \mathrm{Cd}, \mathrm{Hg}$, and $\mathrm{Se}$ are allowed to change phase, while $\mathrm{Ba}, \mathrm{Cr}, \mathrm{Pb}$, and $\mathrm{Ag}$ are restricted to the solid phase. The inlet mixed fuel stream in this system is composed of $\mathrm{CH} 4$ and $20 \%$ excess oxygen.. Design Spec's on air and oxygen flow consider both free oxygen and that bound in organics.

\begin{tabular}{|c|c|c|c|}
\hline $\begin{array}{l}\text { Block Name } \\
\text { (ASPEN } \\
\text { Type) }\end{array}$ & $\begin{array}{l}\text { Outlet } \\
\text { Temp (F) }\end{array}$ & $\begin{array}{l}\text { Heat Duty } \\
\text { (MMBtu/hr) }\end{array}$ & Description and Assumptions \\
\hline $\begin{array}{l}\text { FLASH } \\
\text { (FLASH2) }\end{array}$ & 90 & -4.437 & $\begin{array}{l}1200 \mathrm{~F} \text { inlet stream flashed at } 90 \mathrm{~F} \text { to separate liquid and vapor. } \\
\text { Heat duty does not include any losses. Heat removed by an } \\
\text { unspecified indirect heat exchange process. }\end{array}$ \\
\hline $\begin{array}{l}\text { EX-SPLIT } \\
\text { (FSPLIT) }\end{array}$ & 90 & 0 & $\begin{array}{l}\text { A split of the vapor stream exiting FLASH (predominantly } \\
\text { oxygen), with } 90 \% \text { recycled to PLENUM and } 10 \% \text { exiting system. }\end{array}$ \\
\hline $\begin{array}{l}\text { CO2-DES } \\
\text { (RGIBBS) }\end{array}$ & 1700 & +2.657 & $\begin{array}{l}\text { Desorption of the } \mathrm{CO} 2 \text { from } \mathrm{CaCO} \text {, producing } \mathrm{CaO} \text {. Heat duty } \\
\text { does not include heat losses. }\end{array}$ \\
\hline $\begin{array}{l}\text { CO2-SEP } \\
\text { (SEP) }\end{array}$ & 1700 & -0.613 & $\begin{array}{l}\text { 2-way separation of } \mathrm{CO} \text {-DES products. } \mathrm{CO} 2 \text { is sent to } \\
\text { GASQUENC and remainder, consisting only of } \mathrm{CaO} \text {, sent to } \\
\text { SPLIT. } \mathrm{CaO} \text { (stream CAO-OUT) is flashed to } 68 \mathrm{~F} \text { at outlet to } \\
\text { represent storage of the product before use. }\end{array}$ \\
\hline $\begin{array}{l}\text { SPLIT } \\
\text { (FSPLIT) }\end{array}$ & 1700 & 0 & $\begin{array}{l}\text { A split of the (hot) solids stream from the separator CO2-SEP, } \\
\text { recycling } 90 \% \text { of the } \mathrm{CaO} \text { back to CO2-ABS. The remaining } 10 \% \\
\text { is split between that going to DRYSCRUB for C12 and SO2 } \\
\text { removal ( } 3.3 \%) \text {, and the rest exiting system to stabilization } \\
(6.7 \%) \text {. }\end{array}$ \\
\hline $\begin{array}{l}\text { CAOMIXER } \\
\text { (MIXER) }\end{array}$ & 1547 & 0 & $\begin{array}{l}\text { Mixing of the (hot) recycled } \mathrm{CaO} \text { with make-up } \mathrm{CaO} \text {. } \\
\text { Design } \mathrm{Spec} \mathrm{CAO} \text { : Vary inlet flow of make-up } \mathrm{CaO} \text { to achieve an } \\
\text { equal molar flow rate between the } \mathrm{CO} 2 \text { and the } \mathrm{CaO} \text { as they enter } \\
\text { the absorber (to produce } \mathrm{CaCO} 3 \text { ). }\end{array}$ \\
\hline $\begin{array}{l}\text { GASQUENC } \\
\text { (RGIBBS) }\end{array}$ & 350 & 0 & $\begin{array}{l}\text { Quenching of the } 1700 \text { F CO2 exhaust stream with water. No } \\
\text { losses included. } \\
\text { Design Spec QQUENCH2: Vary inlet water flow rate to achieve } \\
\text { zero heat duty. }\end{array}$ \\
\hline
\end{tabular}


A-28 
Table A-11.

ASSUMPTIONS and ENERGY BALANCES for PLASMA FURNACE W/ STEAM GASIFICATION, System C-3

GENERAL COMMENTS: RGIBBS units calculate chemical and phase equilibrium with some restrictions as stated. For detailed information refer to the ASPEN PLUS Release 9 input file. Chemical reactions and phase changes for some species are neglected. "Volatile" trace metals As, $\mathrm{Cd}, \mathrm{Hg}$, and Se are allowed to change phase, while $\mathrm{Ba}, \mathrm{Cr}, \mathrm{Pb}$, and $\mathrm{Ag}$ are restricted to the solid phase. Design Spec's on air and oxygen flow include both free oxygen and that bound in organics.

\begin{tabular}{|c|c|c|c|}
\hline $\begin{array}{l}\text { Block Name } \\
\text { (ASPEN } \\
\text { Type) }\end{array}$ & $\begin{array}{l}\text { Outlet } \\
\text { Temp (F) }\end{array}$ & $\begin{array}{l}\text { Heat Duty } \\
\text { (MMBtu/hr) }\end{array}$ & Description and Assumptions \\
\hline $\begin{array}{l}\text { FEED-SEP } \\
\text { (SEP) }\end{array}$ & 70 & 0 & $\begin{array}{l}\text { 2-way separation of the feed material. SOLIDS stream goes to the } \\
\text { unit representing the vitrifier molten pool, while the organic-type } \\
\text { components go to the unit representing the vitrifier cover gas } \\
\text { region. Five percent of the carbon and sulfur is assumed to end up } \\
\text { trapped in the melt, and } 5 \% \text { of the solids are assumed to be carried } \\
\text { off with the vapors. }\end{array}$ \\
\hline $\begin{array}{l}\text { PLASMA } \\
\text { (RGIBBS) }\end{array}$ & 3000 & +2.937 & $\begin{array}{l}\text { Plasma furnace. Accepts the non-organic portion of the } \\
\text { combustible and non-combustible wastes and metals with fixed } \\
\text { contamination. The organic portion is assumed to be volatilized } \\
\text { by the plasma arc at a lower temperature and released to the } \\
\text { plenum over the melt. Heat duty does not include any losses or } \\
\text { the energy needed to heat and volatilize the organics. } \\
\text { Design Spec SOILH2O: Vary inlet soil feed rate (substream } \\
\text { MIXE, } 10 \% \text { of total flow) such that it is } 1 / 30 \text { the magnitude of } \\
\text { the SLAG stream (ash-to-gross soil ratio of } 2: 1 \text { ). } \\
\text { Design Spec SOISOL: Vary inlet soil feed rate (substream } \\
\text { CISOLID, } 90 \% \text { of total flow) such that it is } 1 / 3.333 \text { the magnitude } \\
\text { of the SLAG stream (ash-to-gross soil ratio of } 2: 1 \text { ). }\end{array}$ \\
\hline $\begin{array}{l}\text { SLAG-SEP } \\
\text { (SEP) }\end{array}$ & 3000 & 0 & $\begin{array}{l}\text { 3-way separation of PLASMA products: } 100 \% \text { of metals exit } \\
\text { system (via stream METAL). Some Fe } 304 \text { is reduced to Fe. All } \\
\text { of the remaining solids go to the slag stream (SLAG). Any gases } \\
\text { produced, as well as } 100 \% \text { of volatile trace metals, are sent to unit } \\
\text { PLENUM. }\end{array}$ \\
\hline $\begin{array}{l}\text { STEAMGEN } \\
\text { (HEATER) }\end{array}$ & 300 & +0.069 & $\begin{array}{l}\text { Steam Generator. Energy source undefined. Heat duty does not } \\
\text { include losses. }\end{array}$ \\
\hline $\begin{array}{l}\text { PLENUM } \\
\text { (RGIBBS) }\end{array}$ & 1800 & +2.280 & $\begin{array}{l}\text { Plasma furnace plenum area. Organic constituents are volatilized } \\
\text { and released from the melt into the plenum. Steam injected into } \\
\text { the zone to produce synthesis gas (syngas). The heat duty does } \\
\text { not include any losses. Formation of CH4 is restricted. } \\
\text { Design Spec STEAM: Vary inlet water flow in stream WATER } \\
\text { such that total H2O inlet flow (not including soil moisture) equals } \\
6 \text { times H2O outlet flow ( } 20 \% \text { excess). }\end{array}$ \\
\hline $\begin{array}{l}\text { QUENCH } \\
\text { (RGIBBS) }\end{array}$ & 350 & 0 & $\begin{array}{l}\text { Quenching of the syngas stream from the PLENUM. } \\
\text { Formation of CH4, C and } \mathrm{S} \text { is restricted. } \\
\text { Design Spec QQUENCH: Vary inlet water flow rate (QNCH2O) } \\
\text { to achieve zero heat duty. }\end{array}$ \\
\hline
\end{tabular}


Table A-11.

Page 2 of 2

ASSUMPTIONS and ENERGY BALANCES for PLASMA FURNACE W/ STEAM GASIFICATION, System

C-3

GENERAL COMMENTS: RGIBBS units calculate chemical and phase equilibrium with some restrictions as stated. For detailed information refer to the ASPEN PLUS Release 9 input file. Chemical reactions and phase changes for some species are neglected. "Volatile" trace metals As, $\mathrm{Cd}, \mathrm{Hg}$, and $\mathrm{Se}$ are allowed to change phase, while $\mathrm{Ba}, \mathrm{Cr}, \mathrm{Pb}$, and $\mathrm{Ag}$ are restricted to the solid phase. Design Spec's on air and oxygen flow include both free oxygen and that bound in organics.

\begin{tabular}{|l|l|l|l|}
\hline $\begin{array}{l}\text { Block Name } \\
\text { (ASPEN } \\
\text { Type) }\end{array}$ & $\begin{array}{l}\text { Outlet } \\
\text { Temp (F) }\end{array}$ & $\begin{array}{l}\text { Heat Duty } \\
\text { (MMBtu/hr) }\end{array}$ & Description and Assumptions \\
\hline $\begin{array}{l}\text { BAGHOUSE } \\
\text { (SEP) }\end{array}$ & 350 & 0 & $\begin{array}{l}\text { 3-way separation of QUENCH products, modeling the various } \\
\text { filters. Vapors sent to SCRUB (via VAPOR), volatile trace metals } \\
\text { exit system (via HGPLUS), and remainder recycles to GASIFIER } \\
\text { (via FLYASH). }\end{array}$ \\
\hline $\begin{array}{l}\text { SCRUB } \\
\text { (RGIBBS) }\end{array}$ & 120 & -0.835 & $\begin{array}{l}\text { Wet acid scrubber. Formation of CH4, C, and S is restricted. } \\
\text { Design Spec SCRUBH2O: Vary inlet water flow such that inlet } \\
\text { liquid water flow. equals 20 times outlet dissolved salts (5\% TDS), } \\
\text { with a minimum value equal to inlet NaOH flow (50\% soln). } \\
\text { Design Spec NAOH: Vary inlet NaOH flow such that inlet NaOH } \\
\text { flow equals 100 times outlet NaOH flow (1\% excess). }\end{array}$ \\
\hline $\begin{array}{l}\text { L-V-SEP } \\
\text { (FLASH2) }\end{array}$ & 120 & 0 & $\begin{array}{l}\text { 2-way separation of SCRUB products. Flash calculation to } \\
\text { separate liquid and vapor. }\end{array}$ \\
\hline $\begin{array}{l}\text { CATAIYST } \\
\text { (RSTOIC) }\end{array}$ & 1500 & -2.813 & $\begin{array}{l}\text { Catalytic oxidizer. Chemical reactions limited to oxidation of CO, } \\
\text { H2, and CH4: } \\
\text { Design Spec AIR: Vary inlet air flow such that total inlet oxygen } \\
\text { flow equals twice the outlet oxygen flow (100\% excess). }\end{array}$ \\
\hline
\end{tabular}


Table A-12. ASSUMPTIONS AND ENERGY BALANCES for FIXED-HEARTH PYROLYZER W/ $\mathrm{CO}_{2}$ RETENTION, System D-1

GENERAL COMMENTS: RGIBBS units calculate chemical and phase equilibrium with some restrictions, not all stated here. For detailed information refer to the ASPEN PLUS Release 9 input file. Chemical reactions and phase changes for some species are neglected. "Volatile" trace metals $\mathrm{As}, \mathrm{Cd}, \mathrm{Hg}$, and $\mathrm{Se}$ are allowed to change phase, while $\mathrm{Ba}, \mathrm{Cr}, \mathrm{Pb}$, and $\mathrm{Ag}$ are restricted to the solid phase. The pyrolyzer inlet fuel mixture (if used in place of cooling water) is composed of $\mathrm{CH}_{4}$ and $20 \%$ excess oxpgen. Design Spec's on vitrifier and SCC oxygen flows consider both free oxygen and that bound in organics.

\begin{tabular}{|c|c|c|c|}
\hline $\begin{array}{l}\text { Block Name } \\
\text { (ASPEN } \\
\text { Type) } \\
\end{array}$ & $\begin{array}{l}\text { Outlet } \\
\text { Temp (F) }\end{array}$ & $\begin{array}{l}\text { Heat Duty } \\
\text { (MMBtu/hr) }\end{array}$ & Description and Assumptions \\
\hline $\begin{array}{l}\text { PYROLYZR } \\
\text { (RSTOIC) }\end{array}$ & 1200 & -0.062 & $\begin{array}{l}\text { Fixed hearth pyrolyzer. Accepts combustible wastes only. } \\
\text { Directly fired with CH4 if needed, but cooling water has been } \\
\text { used instead to accommodate the assumed } 5 \% \text { heat loss. Model } \\
\text { includes fixed amount of air leakage. Only two chemical } \\
\text { reactions allowed: } 99 \% \text { conversion of carbon to CO, and } 100 \% \\
\text { conversion of CH4 to CO2 and H2O, by use of additional oxygen. } \\
\text { Design Spec OXYGEN: Vary inlet oxygen mass flow (in stream } \\
\text { OXYGEN) such that total inlet oxygen flow equals } 100 \text { times the } \\
\text { outlet oxygen flow (1\% excess). } \\
\text { Design Spec QPYRO: Vary inlet fuel mixture (if heat is needed) } \\
\text { or inlet cooling water (if cooling is needed) such that heat duty is } \\
5 \% \text { of that calculated for unit Q1. }\end{array}$ \\
\hline $\begin{array}{l}\text { DUPL1 } \\
\text { (DUPL) }\end{array}$ & 1200 & 0 & $\begin{array}{l}\text { A stream duplicator block used to send a copy of the PYROLYZR } \\
\text { outlet stream to the dummy unit Q1. }\end{array}$ \\
\hline $\begin{array}{l}\text { Q1 } \\
\text { (HEATER) }\end{array}$ & 68 & -1.235 & $\begin{array}{l}\text { Dummy unit. This calculates simple cooldown of products from } \\
\text { unit PYROLYZR, to a temperature of } 68 \mathrm{~F} \text {, thus yielding an } \\
\text { estimate of their heat content. }\end{array}$ \\
\hline $\begin{array}{l}\text { PYRO-SEP } \\
\text { (SEP) }\end{array}$ & 1200 & -0.027 & $\begin{array}{l}\text { 2-way separation of PYROLYZR products: } 80 \% \text { of "ash" (solid } \\
\text { carbon and sulphur, metals, and inerts) including the solid volatile } \\
\text { trace metals go to VITRIFY (via stream ASH). Remainder, } \\
\text { including } 100 \% \text { of the molten and gaseous volatile trace metals } \\
\text { and the gases and organic constituents, sent to SCC. Stream ASH } \\
\text { is flashed to } 68 \mathrm{~F} \text { to represent storage of material before use. }\end{array}$ \\
\hline $\begin{array}{l}\text { VIRIFY } \\
\text { (RGIBBS) }\end{array}$ & 3000 & +2.571 & $\begin{array}{l}\text { Vitrifier. Accepts non-combustible wastes. Assumed similar to } \\
\text { system B1 vitrifier (use of an oxygen lance assures burnout of } \\
\text { remaining carbon). No heat losses included. Chemical and phase } \\
\text { equilibrium of Fe and Fe } 304 \text { are restricted. } \\
\text { Design Spec O2VIT: Vary the inlet oxygen flow such that total } \\
\text { inlet oxygen flow is } 6 \text { times the outlet oxygen flow ( } 20 \% \text { excess). } \\
\text { Design Spec SOILH2O: Vary inlet soil feed rate (substream } \\
\text { MIXED, 10\% of total flow) such that it is } 1 / 30 \text { the magnitude of } \\
\text { the SLAG stream (ash-to-gross soil ratio of } 2: 1 \text { ). } \\
\text { Design Spec SOILSOL: Vary inlet soil feed rate (substream } \\
\text { CISOLID, } 90 \% \text { of total flow) such that it is } 1 / 3.333 \text { the magnitude } \\
\text { of the SLAG stream (ash-to-gross soil ratio of } 2: 1 \text { ). }\end{array}$ \\
\hline
\end{tabular}


Table A-12.

ASSUMPTIONS AND ENERGY BALANCES for FIXED-HEARTH PYROLYZER W/ $\mathrm{CO}_{2}$ RETENTION, System D-1

GENERAL COMMENTS: RGIBBS units calculate chemical and phase equilibrium with some restrictions, not all stated here. For detailed information refer to the ASPEN PLUS Release 9 input file. Chemical reactions and phase changes for some species are neglected. "Volatile" trace metals $\mathrm{As}, \mathrm{Cd}, \mathrm{Hg}$, and $\mathrm{Se}$ are allowed to change phase, while $\mathrm{Ba}, \mathrm{Cr}, \mathrm{Pb}$, and $\mathrm{Ag}$ are restricted to the solid phase. The pyrolyzer inlet fuel mixture (if used in place of cooling water) is composed of $\mathrm{CH} 4$ and 20\% excess oxpqen. Design Spec's on vitrifier and SCC oxygen flows consider both free oxygen and that bound in organics.

\begin{tabular}{|c|c|c|c|}
\hline $\begin{array}{l}\text { Block Name } \\
\text { (ASPEN } \\
\text { Type) }\end{array}$ & $\begin{array}{l}\text { Outlet } \\
\text { Temp (F) }\end{array}$ & $\begin{array}{l}\text { Heat Duty } \\
\text { (MMBtu/hr) }\end{array}$ & Description and Assumptions \\
\hline $\begin{array}{l}\text { VIT-SEP } \\
\text { (SEP) }\end{array}$ & 3000 & 0 & $\begin{array}{l}\text { 2-way separation of VITRIFY products: } 80 \% \text { of "ash" (solid } \\
\text { carbon and sulphur, metals, and inerts) including the solid volatile } \\
\text { trace metals go to VITRIFY (via stream ASH). Remainder, } \\
\text { including } 100 \% \text { of the molten or gaseous volatile trace metals and } \\
\text { the gases and organic constituents, sent to SCC. NOTE: Actual } \\
\text { system calls for a separate APC system for vitrifier. }\end{array}$ \\
\hline $\begin{array}{l}\text { SCC } \\
\text { (RGIBBS) }\end{array}$ & 2200 & -0.334 & $\begin{array}{l}\text { Secondary combustion unit. CH4 heated or water cooled. Heat } \\
\text { losses assumed to } 5 \% \text { of the sensible heat of the products. } \\
\text { Design Spec QSCC: Vary inlet CH4 flow (if heat is needed) or } \\
\text { water (if cooling is needed) such that heat duty is } 5 \% \text { of that } \\
\text { calculated for dummy unit Q2. } \\
\text { Design Spec O2SCC: Vary the inlet oxygen flow such that total } \\
\text { inlet oxygen flow is } 6 \text { times the outlet oxygen flow ( } 20 \% \text { excess). }\end{array}$ \\
\hline $\begin{array}{l}\text { DUPL2 } \\
\text { (DUPL) }\end{array}$ & 2200 & 0 & $\begin{array}{l}\text { A stream duplicator block used to send a copy of the SCC outlet } \\
\text { stream to the dummy unit Q2. }\end{array}$ \\
\hline $\begin{array}{l}\text { Q2 } \\
\text { (RGIBBS) }\end{array}$ & 68 & -15.791 & $\begin{array}{l}\text { Dummy unit. This calculates chemical equilibrium at } 2200 \mathrm{~F} \text {, } \\
\text { identical to unit SCC, but has an outlet temperature of } 68 \mathrm{~F} \text {, thus } \\
\text { yielding an estimate of the sensible heat of the products of unit } \\
\text { SCC. }\end{array}$ \\
\hline $\begin{array}{l}\text { QUENCH } \\
\text { (RGIBBS) }\end{array}$ & 350 & 0 & $\begin{array}{l}\text { Quenching of the exhaust of the SCC. No losses included. } \\
\text { Formation of } \mathrm{CH} 4, \mathrm{C}, \mathrm{S} \text { and } \mathrm{Cl} 2 \text { is restricted. } \\
\text { Design Spec QQUENCH: Vary inlet water flow rate to achieve } \\
\text { zero heat duty. }\end{array}$ \\
\hline $\begin{array}{l}\text { BAGHOUSE } \\
\text { (SEP) }\end{array}$ & 350 & -0.305 & $\begin{array}{l}\text { 3-way separation of QUENCH products. Vapors sent to } \\
\text { DRYSCRUB, volatile trace metals exit system (via stream } \\
\text { HGPLUS), and remainder (soil + ash) recycles to VITRIFY (via } \\
\text { FLYASH). Stream FLYASH is flashed to } 68 \mathrm{~F} \text { to represent } \\
\text { storage of material before use. (Heat duty may not be accurate.) }\end{array}$ \\
\hline $\begin{array}{l}\text { DRYSCRUB } \\
\text { (RGIBBS) }\end{array}$ & 350 & -0.097 & $\begin{array}{l}\text { Dry scrubber. Chemical equilibrium calculated for conversion of } \\
\mathrm{CaO} \text { to } \mathrm{CaSO} 4 \text { and } \mathrm{CaCl} 2 \text {, with all volatiles in the system } \\
\text { participating. SO2 is fully converted (not included in the product } \\
\text { list). Heat duty does not include any losses. Water used to slake } \\
\text { the lime is not included in the model. } \\
\text { Design Spec SCRUB: Vary the specified fractional flow split of } \\
\text { unit SPLIT (between } 0 \text { and } 0.1 \text { ) such that the inlet } \mathrm{CaO} \text { mole flow } \\
\text { to DRYSCRUB is equal to the inlet mole flow of the } \mathrm{SO} 2 \text { plus } \\
\text { half the inlet mole flow of the HCl. }\end{array}$ \\
\hline
\end{tabular}


Table A-12.

Page 3 of 3

ASSUMPTIONS AND ENERGY BALANCES for FIXED-HEARTH PYROLYZER W/ $\mathrm{CO}_{2}$ RETENTION, System D-1

GENERAL COMMENTS: RGIBBS units calculate chemical and phase equilibrium with some restrictions, not all stated here. For detailed information refer to the ASPEN PLUS Release 9 input file. Chemical reactions and phase changes for some species are neglected. "Volatile" trace metals $\mathrm{As}, \mathrm{Cd}, \mathrm{Hg}$, and Se are allowed to change phase, while $\mathrm{Ba}, \mathrm{Cr}, \mathrm{Pb}$, and $\mathrm{Ag}$ are restricted to the solid phase. The pyrolyzer inlet fuel mixture (if used in place of cooling water) is composed of $\mathrm{CH}_{4}$ and $20 \%$ excess oxvgen. Design Spec's on vitrifier and SCC oxygen flows consider both free oxygen and that bound in organics.

\begin{tabular}{|c|c|c|c|}
\hline $\begin{array}{l}\text { Block Name } \\
\text { (ASPEN } \\
\text { Type) } \\
\end{array}$ & $\begin{array}{l}\text { Outlet } \\
\text { Temp (F) }\end{array}$ & $\begin{array}{l}\text { Heat Duty } \\
\text { (MMBtu/hr) }\end{array}$ & Description and Assumptions \\
\hline $\begin{array}{l}\text { SCRUBSEP } \\
\text { (SEP) }\end{array}$ & 350 & 0 & $\begin{array}{l}\text { 2-way separation of DRYSCRUB products. Vapors sent to CO2- } \\
\text { ABS and remainder exits system. }\end{array}$ \\
\hline $\begin{array}{l}\text { CO2-ABS } \\
\text { (RSTOIC) }\end{array}$ & 1200 & +2.079 & $\begin{array}{l}\text { Restricted chemical reaction combining } \mathrm{CO} 2 \text { and } \mathrm{CaO} \text { to form } \\
\mathrm{CaCO} \text {, assuming } 100 \% \text { utilization of the } \mathrm{CO} 2 \text {. Heat duty does } \\
\text { not include any losses. }\end{array}$ \\
\hline $\begin{array}{l}\text { ABSSEP } \\
\text { (SEP) }\end{array}$ & 1200 & 0 & $\begin{array}{l}\text { 2-way separation of } \mathrm{CO} 2 \text {-ABS products. Vapors sent to FLASH. } \\
\text { Remainder, consisting only of } \mathrm{CaCO} \text {, sent to } \mathrm{CO} \text {-DES. }\end{array}$ \\
\hline $\begin{array}{l}\text { FLASH } \\
\text { (FLASH2) }\end{array}$ & 90 & -11.996 & $\begin{array}{l}1200 \mathrm{~F} \text { inlet stream flashed at } 90 \mathrm{~F} \text { to separate liquid and vapor. } \\
\text { Heat duty does not include any losses. Heat removed by an } \\
\text { unspecified indirect heat exchange process. }\end{array}$ \\
\hline $\begin{array}{l}\text { EX-SPLIT } \\
\text { (FSPLIT) }\end{array}$ & 90 & 0 & $\begin{array}{l}\text { A split of the vapor stream exiting FLASH (predominately } \\
\text { oxygen), with } 90 \% \text { recycled to PLENUM and } 10 \% \text { exiting system. }\end{array}$ \\
\hline $\begin{array}{l}\text { CO2-DES } \\
\text { (RGIBBS) }\end{array}$ & 1700 & +2.444 & $\begin{array}{l}\text { Desorption of the } \mathrm{CO} 2 \text { from } \mathrm{CaCO} 3 \text {, producing } \mathrm{CaO} \text {. Heat duty } \\
\text { does not include heat losses. }\end{array}$ \\
\hline $\begin{array}{l}\text { CO2-SEP } \\
\text { (SEP) }\end{array}$ & 1700 & -0.562 & $\begin{array}{l}\text { 2-way separation of } \mathrm{CO} 2-\mathrm{DES} \text { products. } \mathrm{CO} 2 \text { is sent to } \\
\text { GASQUENC and remainder, consisting only of } \mathrm{CaO} \text {, sent to } \\
\text { SPLIT. CaO (stream CAO-OUT) is flashed to } 68 \mathrm{~F} \text { at outlet to } \\
\text { represent storage of the product before use. }\end{array}$ \\
\hline $\begin{array}{l}\text { SPLIT } \\
\text { (FSPLIT) }\end{array}$ & 68 & 0 & $\begin{array}{l}\text { A split of the solids stream from the separator } \mathrm{CO} 2 \text {-SEP, recycling } \\
90 \% \text { of the } \mathrm{CaO} \text { back to } \mathrm{CO} 2-\mathrm{ABS} \text {. The remaining } 10 \% \text { is split } \\
\text { between that going to DRYSCRUB for } \mathrm{Cl} 2 \text { and } \mathrm{S} \text { removal (3.6\%), } \\
\text { and the rest exiting system to stabilization (6.4\%). }\end{array}$ \\
\hline $\begin{array}{l}\text { CAOMIXER } \\
\text { (MIXER) }\end{array}$ & 68 & 0 & $\begin{array}{l}\text { Mixing of the recycled } \mathrm{CaO} \text { with make-up } \mathrm{CaO} \text {. } \\
\text { Design Spec } \mathrm{CAO} \text { : Vary inlet flow of make-up } \mathrm{CaO} \text { to achieve an } \\
\text { equal molar flow rate between the } \mathrm{CO} 2 \text { and the } \mathrm{CaO} \text { as they enter } \\
\text { the absorber (to produce } \mathrm{CaCO} 3 \text { ). }\end{array}$ \\
\hline $\begin{array}{l}\text { GASQUENC } \\
\text { (RGIBBS) }\end{array}$ & 350 & 0 & $\begin{array}{l}\text { Quenching of the } 1700 \mathrm{~F} \mathrm{CO} 2 \text { exhaust stream with water. No } \\
\text { losses included. } \\
\text { Design Spec QQUENCH2: Vary inlet water flow rate to achieve } \\
\text { zero heat duty. }\end{array}$ \\
\hline $\begin{array}{l}\text { MELTER } \\
\text { (RGIBBS) }\end{array}$ & 3000 & +0.120 & $\begin{array}{l}\text { Metal melter. Accepts metals with fixed contamination. } \\
\text { Electrically heated with heat losses not included. }\end{array}$ \\
\hline
\end{tabular}


A-34 
Table A-13.

ASSUMPTIONS and ENERGY BALANCES for THERMAL DESORPTION, System E-1

Page 1 of 3

GENERAL COMMENTS: RGIBBS units calculate chemical and phase equilibrium with some restrictions, not all stated here. For detailed information refer to the ASPEN PLUS Release 9 input file. Chemical reactions and phase changes for some species are neglected. "Volatile" trace metals $\mathrm{As}, \mathrm{Cd}, \mathrm{Hg}$, and Se are allowed to change phase, while $\mathrm{Ba}, \mathrm{Cr}, \mathrm{Pb}$, and $\mathrm{Ag}$ are restricted to the solid phase. The inlet fuel mixture streams in this system are composed of $\mathrm{CH} 4$ and $20 \%$ excess air. Design Spec's on air and oxygen flow consider both free oxygen and that bound in organics. NOTE: In this model the organic-type products entering the desorber and condenser are not true components but only represent the elemental content of the streams; thus, the splitting off of the "volatile organics" following each unit is done by simply taking a percentage of the total stream flow, based on engineering judgment.

\begin{tabular}{|l|l|l|l|}
\hline $\begin{array}{l}\text { Block Name } \\
\text { (ASPEN } \\
\text { Type) }\end{array}$ & $\begin{array}{l}\text { Outlet } \\
\text { Temp (F) }\end{array}$ & $\begin{array}{l}\text { Heat Duty } \\
\text { (MMBtu/hr) }\end{array}$ & Description and Assumptions \\
\hline $\begin{array}{l}\text { DESORBER } \\
\text { (RGIBBS) }\end{array}$ & 600 & +0.576 & $\begin{array}{l}\text { Thermal desorption unit. Accepts non-combustible wastes. } \\
\text { Heating of the waste releases moisture and volatile organics. } \\
\text { Heated indirectly. Heat losses not included here, but see } \\
\text { HEATER unit below. H2, O2, and H2O restricted from reacting. }\end{array}$ \\
\hline $\begin{array}{l}\text { HEATER } \\
\text { (RGIBBS) }\end{array}$ & 1400 & -0.605 & $\begin{array}{l}\text { Indirect heating of DESORBER. CH4-fueled. } \\
\text { Design Spec QHEATER: Vary inlet fuel mixture flow (stream } \\
\text { FUELMIX1) such that heat output is 5\% greater than that needed } \\
\text { to heat the desorber products, to account for losses. }\end{array}$ \\
\hline $\begin{array}{l}\text { SEPARATR } \\
\text { (SEP) }\end{array}$ & 600 & 0 & $\begin{array}{l}\text { 2-way separation of DESORBER products. The organic-type } \\
\text { products listed in the stream summary are not actual, but only } \\
\text { reflect the elements present. Selection of the volatile fraction is } \\
\text { based on engineering judgment. Assume 80\% of the H2O is } \\
\text { vaporized, the rest being bound in the concrete, etc. Assume 20\% } \\
\text { of all organic-type elements are in a vaporous state (including } \\
\text { equilibrium amount of CH4). Remainder exits system (via stream } \\
\text { SOLmS) for grouting. }\end{array}$ \\
\hline $\begin{array}{l}\text { CONDENSR } \\
\text { (RGIBBS) }\end{array}$ & 80 & -0.364 & $\begin{array}{l}\text { Condensing (actually occurs in 2 stages) of the exhaust "vapors" } \\
\text { ofDESORBER. H2, O2, and H2O restricted from reacting. } \\
\text { Secondary cooling system unspecified. No distinction is made } \\
\text { here between liquid and vapor products (as in models K1 and L1). } \\
\text { Both are merely sent to kiln for combustion. }\end{array}$ \\
\hline
\end{tabular}


Table A-13.

ASSUMPTIONS and ENERGY BALANCES for THERMAL DESORPTION, System E-1

GENERAL COMMENTS: RGIBBS units calculate chemical and phase equilibrium with some restrictions, not all stated here. For detailed information refer to the ASPEN PLUS Release 9 input file. Chemical reactions and phase changes for some species are neglected. "Volatile" trace metals $\mathrm{As}, \mathrm{Cd}, \mathrm{Hg}$, and $\mathrm{Se}$ are allowed to change phase, while $\mathrm{Ba}, \mathrm{Cr}, \mathrm{Pb}$, and $\mathrm{Ag}$ are restricted to the solid phase. The inlet fuel mixture streams in this system are composed of $\mathrm{CH} 4$ and $20 \%$ excess air. Design Spec's on air and oxygen flow consider both free oxygen and that bound in organics. NOTE: In this model the organic-type products entering the desorber and condenser are not true components but only represent the elemental content of the streams; thus, the splitting off of the "volatile organics" following each unit is done by simply taking a percentage of the total stream flow, based on engineering judgment.

\begin{tabular}{|c|c|c|c|}
\hline $\begin{array}{l}\text { Block Name } \\
\text { (ASPEN } \\
\text { Type) }\end{array}$ & $\begin{array}{l}\text { Outlet } \\
\text { Temp (F) }\end{array}$ & $\begin{array}{l}\text { Heat Duty } \\
\text { (MMBtu/hr) }\end{array}$ & Description and Assumptions \\
\hline $\begin{array}{l}\text { KILN } \\
\text { (RGIBBS) }\end{array}$ & 1600 & -0.360 & $\begin{array}{l}\text { Conventional rotary kiln. Accepts combustible wastes. CH4- } \\
\text { fueled with air as oxidant. Heat losses assumed to be } 5 \% \text { of the } \\
\text { sensible heat of the products. Includes fixed air leakage through } \\
\text { seals. Chemical and phase equilibrium of Fe and Fe3O4 are } \\
\text { restricted. } \\
\text { Design Spec QKIIN: Vary either inlet fuel mixture flow (if heat } \\
\text { is needed) or inlet water flow (if cooling is needed) such that heat } \\
\text { duty is } 5 \% \text { of that calculated for unit Q1. } \\
\text { Design Spec OXI: Vary inlet air flow in stream AIR such that } \\
\text { total inlet oxygen flow (minus that used by the CH4) is twice the } \\
\text { outlet oxygen flow (100\% excess). } \\
\text { Design Spec SOIIH2O: Vary inlet soil feed rate (substream } \\
\text { MIXED, } 10 \% \text { of total flow) such that it is } 1 / 30 \text { the magnitude of } \\
\text { the SLAG stream (ash-to-gross soil ratio of } 2: 1 \text { ). } \\
\text { Design Spec SOILSOL: Vary inlet soil feed rate (substream } \\
\text { CISOLID, } 90 \% \text { of total flow) such that it is } 1 / 3.333 \text { the magnitude } \\
\text { of the SLAG stream (ash-to-gross soil ratio of } 2: 1 \text { ). }\end{array}$ \\
\hline $\begin{array}{l}\text { DUPL1 } \\
\text { (DUPL) }\end{array}$ & 1600 & 0 & $\begin{array}{l}\text { A stream duplicator block used to send a copy of the } \mathrm{KIIN} \text { outlet } \\
\text { stream to the dummy unit } \mathrm{Q} 1 \text {. }\end{array}$ \\
\hline $\begin{array}{l}\text { Q1 } \\
\text { (RGIBBS) }\end{array}$ & 68 & -7.209 & $\begin{array}{l}\text { Dummy unit. This calculates chemical equilibrium at } 1600 \mathrm{~F} \text {, } \\
\text { identical to unit KILN, but has an outlet temperature of } 68 \mathrm{~F} \text {, thus } \\
\text { yielding an estimate of the sensible heat of the products of unit } \\
\text { KIIN. }\end{array}$ \\
\hline $\begin{array}{l}\text { KIINSEP } \\
\text { (SEP) }\end{array}$ & 1600 & -0.051 & $\begin{array}{l}\text { 2-way separation of KIIN products: } 80 \% \text { of metals and oxides go } \\
\text { to VITRIFY (via stream ASH). Remainder, including } 100 \% \text { of } \\
\text { volatile trace metals and vapors, sent to SCC. Stream ASH } \\
\text { flashed to } 68 \mathrm{~F} \text { to represent storage of material before use. (Heat } \\
\text { duty may not be accurate.) }\end{array}$ \\
\hline $\begin{array}{l}\text { SCC } \\
\text { (RGIBBS) }\end{array}$ & 2200 & -0.820 & $\begin{array}{l}\text { Secondary combustion unit. CH4-fueled with air as oxidant. Heat } \\
\text { losses assumed to be } 5 \% \text { of the sensible heat of the products. } \\
\text { Chemical and phase equilibrium of } \mathrm{Fe} \text { and } \mathrm{Fe} 3 \mathrm{O} 4 \text { are restricted. } \\
\text { Design Spec QSCC: Vary inlet fuel mixture flow such that heat } \\
\text { duty is } 5 \% \text { of that calculated for unit } \mathrm{Q} 2 \text {. }\end{array}$ \\
\hline $\begin{array}{l}\text { DUPL2 } \\
\text { (DUPL) }\end{array}$ & 2200 & 0 & $\begin{array}{l}\text { A stream duplicator block used to send a copy of the SCC outlet } \\
\text { stream to the dummy unit Q2. }\end{array}$ \\
\hline
\end{tabular}


Table A-13.

ASSUMPTIONS and ENERGY BALANCES for THERMAL DESORPTION, System E-1

GENERAL COMMENTS: RGIBBS units calculate chemical and phase equilibrium with some restrictions, not all stated here. For detailed information refer to the ASPEN PLUS Release 9 input file. Chemical reactions and phase changes for some species are neglected. "Volatile" trace metals $\mathrm{As}, \mathrm{Cd}, \mathrm{Hg}$, and $\mathrm{Se}$ are allowed to change phase, while $\mathrm{Ba}, \mathrm{Cr}, \mathrm{Pb}$, and $\mathrm{Ag}$ are restricted to the solid phase. The inlet fuel mixture streams in this system are composed of $\mathrm{CH} 4$ and $20 \%$ excess air. Design Spec's on air and oxygen flow consider both free oxygen and that bound in organics. NOTE: In this model the organic-type products entering the desorber and condenser are not true components but only represent the elemental content of the streams; thus, the splitting off of the "volatile organics" following each unit is done by simply taking a percentage of the total stream flow, based on engineering judgment.

\begin{tabular}{|c|c|c|c|}
\hline $\begin{array}{l}\text { Block Name } \\
\text { (ASPEN } \\
\text { Type) } \\
\end{array}$ & $\begin{array}{l}\text { Outlet } \\
\text { Temp (F) }\end{array}$ & $\begin{array}{l}\text { Heat Duty } \\
\text { (MMBtu/hr) }\end{array}$ & Description and Assumptions \\
\hline $\begin{array}{l}\text { Q2 } \\
\text { (RGIBBS) }\end{array}$ & 68 & -16.401 & $\begin{array}{l}\text { Dummy unit. This calculates chemical equilibrium at } 2200 \mathrm{~F} \text {, } \\
\text { identical to unit SCC, but has an outlet temperature of } 68 \mathrm{~F} \text {, thus } \\
\text { yielding an estimate of the sensible heat of the products of unit } \\
\text { SCC. }\end{array}$ \\
\hline $\begin{array}{l}\text { QUENCH } \\
\text { (RGBBS) }\end{array}$ & 350 & 0 & $\begin{array}{l}\text { Quenching of the exhaust of the SCC. No losses included. } \\
\text { Formation.of } \mathrm{CH} 4, \mathrm{C}, \mathrm{S} \text { and } \mathrm{Cl} 2 \text { is restricted. Chemical and phase } \\
\text { equilibrium of } \mathrm{Fe} \text { and } \mathrm{Fe} 3 \mathrm{O} 4 \text { are restricted. } \\
\text { Design Spec QQUENCH: Vary inlet water flow rate to achieve } \\
\text { zero heat duty. }\end{array}$ \\
\hline $\begin{array}{l}\text { BAGHOUSE } \\
\text { (SEP) }\end{array}$ & 350 & -0.002 & $\begin{array}{l}\text { 3-way separation of QUENCH products. Vapors sent to SCRUB, } \\
\text { volatile trace metals exit system (via stream HGPLUS), and } \\
\text { remainder (soil + ash) recycles to VITRIFY (via FLYASH). } \\
\text { Stream FLYASH flashed to } 68 \text { F to represent storage of material } \\
\text { before use. (Heat duty may not be accurate.) }\end{array}$ \\
\hline $\begin{array}{l}\text { SCRUB } \\
\text { (RGIBBS) }\end{array}$ & 120 & -14.423 & $\begin{array}{l}\text { Wet acid scrubber. } \\
\text { Design Spec SCRUBH2O: Vary inlet water flow such that inlet } \\
\text { liquid water flow equals } 20 \text { times outlet dissolved salts ( } 5 \% \text { TDS), } \\
\text { with a minimum value equal to inlet } \mathrm{NaOH} \text { flow ( } 50 \% \text { soln). } \\
\text { Design Spec NAOH: Vary inlet } \mathrm{NaOH} \text { flow such that inlet } \mathrm{NaOH} \\
\text { flow equals } 100 \text { times outlet } \mathrm{NaOH} \text { flow ( } 1 \% \text { excess). }\end{array}$ \\
\hline $\begin{array}{l}\text { L-V-SEP } \\
\text { (FLASH2) }\end{array}$ & 120 & 0 & $\begin{array}{l}\text { 2-way separation of SCRUB products. Flash calculation to } \\
\text { separate liquid and vapor. }\end{array}$ \\
\hline $\begin{array}{l}\text { VITRIFY } \\
\text { (RGIBBS) }\end{array}$ & 3000 & +1.561 & $\begin{array}{l}\text { Vitrifier. Heat duty does not include heat losses: Feed material } \\
\text { enters at } 68 \mathrm{~F} \text {. Chemical and phase equilibrium of } \mathrm{Fe} \text { and } \mathrm{Fe} 3 \mathrm{O} 4 \\
\text { are restricted. }\end{array}$ \\
\hline $\begin{array}{l}\text { L-V-SEP2 } \\
\text { (FLASH2) }\end{array}$ & 3000 & 0 & $\begin{array}{l}\text { 2-way separation of VITRIFY products. Flash calculation to } \\
\text { separate liquid and vapor. No vapor expected. }\end{array}$ \\
\hline $\begin{array}{l}\text { MELTER } \\
\text { (RGIBBS) }\end{array}$ & 3000 & +0.120 & $\begin{array}{l}\text { Metal meiter. Accepts metals with fixed contamination. } \\
\text { Electrically heated with heat losses not included. }\end{array}$ \\
\hline
\end{tabular}




$$
\text { A-38 }
$$


Table A-14. ASSUMPTIONS and ENERGY BALANCES for MOLTEN SALT OXIDATION, System F-1

GENERAL COMMENTS: RGIBBS units calculate chemical and phase equilibrium with some restrictions as stated. For detailed information refer to the ASPEN PLUS Release 9 input file. Chemical reactions and phase changes for some species are neglected. "Volatile" trace metals $\mathrm{As}, \mathrm{Cd}, \mathrm{Hg}$, and Se are allowed to change phase, while $\mathrm{Ba}, \mathrm{Cr}, \mathrm{Pb}$, and $\mathrm{Ag}$ are restricted to the solid phase. Inlet fuel mixture stream (FUELMIX2) is composed of CH4 and $20 \%$ excess air (O2 and N2). Design Spec's on air and oxygen flow include both free oxygen and that bound in organics.

\begin{tabular}{|c|c|c|c|}
\hline $\begin{array}{l}\text { Block Name } \\
\text { (ASPEN } \\
\text { Type) }\end{array}$ & $\begin{array}{l}\text { Outlet } \\
\text { Temp (F) }\end{array}$ & $\begin{array}{l}\text { Heat Duty } \\
\text { (MMBtu/hr) }\end{array}$ & Description and Assumptions \\
\hline $\begin{array}{l}\text { MSO } \\
\text { (RGIBBS) }\end{array}$ & 1652 & -3.980 & $\begin{array}{l}\text { Molten salt oxidation. Accepts combustible waste only. Unit } \\
\text { requires heat removal as shown. Losses not included. } \\
\text { Design Spec AIR: Vary inlet air flow (stream AIR) such that total } \\
\text { inlet oxygen inlet flow equals } 6 \text { times outlet flow ( } 20 \% \text { excess). } \\
\text { Design Spec SALT: Vary inlet Na2CO3 flow such that the outlet } \\
\text { solids stream (MSO-BOT) contains } 80 \% \mathrm{Na} 2 \mathrm{CO} 3 \text { and } \mathrm{NaCl} \text {. This } \\
\text { limits the ash content of the MSO unit to } 20 \% \text { to avoid } \\
\text { solidification. }\end{array}$ \\
\hline $\begin{array}{l}\text { MSO-SEP } \\
\text { (SEP) }\end{array}$ & 1652 & -0.312 & $\begin{array}{l}\text { 2-way separation of MSO products. } 100 \% \text { of vapors and volatile } \\
\text { trace metals sent to QUENCH. Remainder is flashed to } 68 \mathrm{~F} \text { and } \\
\text { then sent to SALT-SEP (via MSO-BOT). }\end{array}$ \\
\hline $\begin{array}{l}\text { SALT-SEP } \\
\text { (SEP) }\end{array}$ & 1652 & 0 & $\begin{array}{l}\text { 3-way separation of MSO saits and ash products representing a } \\
\text { dissolving/filtering/drying process. } 90 \% \text { of } \mathrm{Na} 2 \mathrm{CO} 3 \text { recycled to } \\
\text { MSO (via SALT-90), } 10 \% \text { exits system as does the } \mathrm{NaCl} \text { (actually } \\
\text { will be as a solution with added water) (via SALTS). Remaining } \\
\text { MSO products sent to VITRIFY via ASH. }\end{array}$ \\
\hline $\begin{array}{l}\text { QUENCH } \\
\text { (RGIBBS) }\end{array}$ & 350 & 0 & $\begin{array}{l}\text { Quenching of the exhaust vapors of MSO. Formation of } \mathrm{CH} 4, \mathrm{C} \text {, } \\
\text { and } \mathrm{S} \text { is restricted. } \\
\text { Design Spec QQUENCH: Vary inlet water flow rate to achieve } \\
\text { zero heat duty. }\end{array}$ \\
\hline $\begin{array}{l}\text { BAGHOUSE } \\
\text { (SEP) }\end{array}$ & 350 & 0 & $\begin{array}{l}\text { 3-way separation of QUENCH products, modeling the various } \\
\text { filters. Vapors sent to SCRUB, } 100 \% \text { of volatile trace metals exit } \\
\text { system (via HGPLUS), and remainder to VTRIFY. }\end{array}$ \\
\hline $\begin{array}{l}\text { SCRUB } \\
\text { (RGIBBS) }\end{array}$ & 120 & -2.804 & $\begin{array}{l}\text { Wet acid scrubber. } \\
\text { Design Spec SCRUBH2O: Vary inlet water flow such that inlet } \\
\text { liquid water flow equals } 20 \text { times outlet dissolved salts ( } 5 \% \mathrm{TDS} \text { ), } \\
\text { with a minimum value equal to inlet } \mathrm{NaOH} \text { flow ( } 50 \% \text { soln). } \\
\text { Design Spec } \mathrm{NAOH} \text { : Vary inlet } \mathrm{NaOH} \text { flow such that inlet } \mathrm{NaOH} \\
\text { flow equals } 100 \text { times outlet } \mathrm{NaOH} \text { flow ( } 1 \% \text { excess). }\end{array}$ \\
\hline $\begin{array}{l}\text { L-V-SEP } \\
\text { (FLASH2) }\end{array}$ & 120 & 0 & $\begin{array}{l}\text { 2-way separation of SCRUB products. Flash calculation to } \\
\text { separate liquid and vapor. }\end{array}$ \\
\hline
\end{tabular}


Table A-14.

ASSUMPTIONS and ENERGY BALANCES for MOLTEN SALT OXIDATION, System F-1

GENERAL COMMENTS: RGIBBS units calculate chemical and phase equilibrium with some restrictions as stated. For detailed information refer to the ASPEN PLUS Release 9 input file. Chemical reactions and phase changes for some species are neglected. "Volatile" trace metals $\mathrm{As}, \mathrm{Cd}, \mathrm{Hg}$, and Se are allowed to change phase, while $\mathrm{Ba}, \mathrm{Cr}, \mathrm{Pb}$, and $\mathrm{Ag}$ are restricted to the solid phase. Inlet fuel mixture stream (FUELMIX2) is composed of $\mathrm{CH} 4$ and $20 \%$ excess air (O2 and N2). Design Spec's on air and oxygen flow include both free oxygen and that bound in organics.

\begin{tabular}{|c|c|c|c|}
\hline $\begin{array}{l}\text { Block Name } \\
\text { (ASPEN } \\
\text { Type) } \\
\end{array}$ & $\begin{array}{l}\text { Outlet } \\
\text { Temp (F) }\end{array}$ & $\begin{array}{l}\text { Heat Duty } \\
\text { (MMBtu/hr) }\end{array}$ & Description and Assumptions \\
\hline $\begin{array}{l}\text { FEED-SEP } \\
\text { (SEP) }\end{array}$ & 74 & 0 & $\begin{array}{l}\text { 2-way separation of the feed material. SOLIDS stream goes to the } \\
\text { unit representing the vitrifier molten pool, while the organic-type } \\
\text { components go to the unit representing the vitrifier cover gas } \\
\text { region. Five percent of the carbon and sulfur is assumed to end up } \\
\text { trapped in the melt, and } 5 \% \text { of the solids are assumed to be carried } \\
\text { off with the vapors. }\end{array}$ \\
\hline $\begin{array}{l}\text { VITRIFY } \\
\text { (RGIBBS) }\end{array}$ & 3000 & +1.606 & $\begin{array}{l}\text { Virtifier molten pool. Accepts the non-organic portion of the } \\
\text { combustible and non-combustible wastes. The organic portion of } \\
\text { the non-combustible wastes is assumed to be volatilized and } \\
\text { released to the plenum over the melt. Heat duty does not include } \\
\text { any losses or the energy needed to heat and volatilize the organic } \\
\text { portion of the non-combustible wastes. } \\
\text { Design Spec SOILH2O: Vary inlet soil feed rate (substream } \\
\text { MIXED, } 10 \% \text { of total flow) such that it is } 1 / 30 \text { the magnitude of } \\
\text { the SLAG stream (ash-to-gross soil ratio of } 2: 1 \text { ). } \\
\text { Design Spec SOILSOL: Vary inlet soil feed rate (substream } \\
\text { CISOLI, } 90 \% \text { of total flow) such that it is } 1 / 3.333 \text { the magnitude } \\
\text { of the SLAG stream (ash-to-gross soil ratio of } 2: 1 \text { ). }\end{array}$ \\
\hline $\begin{array}{l}\text { VT-SEP } \\
\text { (SEP) }\end{array}$ & 3000 & 0 & $\begin{array}{l}\text { 2-way separation of VITRIFY products: } 100 \% \text { of solids and } \\
\text { liquids exit system (via stream SLAG). Any gases produced, as } \\
\text { well as } 100 \% \text { of volatile trace metals, are sent to unit PLENUM. }\end{array}$ \\
\hline $\begin{array}{l}\text { PLENUM } \\
\text { (RGIBBS) }\end{array}$ & 1800 & +0.736 & $\begin{array}{l}\text { Vitrifier plenum area. Organic constituents are volatilized and } \\
\text { released from the melt into the plenum. Oxygen is injected into } \\
\text { the zone, combusting the vapors. The heat duty does not include } \\
\text { any losses. Formation of CH4 is restricted. } \\
\text { Design Spec OXYGEN: Vary the inlet oxygen flow (stream } \\
\text { OXYGEN) such that the total incoming unbound oxygen is } 6.0 \\
\text { times the outlet oxygen flow ( } 20 \% \text { excess). }\end{array}$ \\
\hline $\begin{array}{l}\text { SCC2 } \\
\text { (RGIBBS) }\end{array}$ & 2200 & -0.068 & $\begin{array}{l}\text { Secondary combustion unit. Further combustion of the vapors at a } \\
\text { temperature to assure destruction of PCB's. CH4-fueled with air } \\
\text { at } 20 \% \text { excess. Heat losses assumed to be } 5 \% \text { of the sensible heat } \\
\text { of the products. } \\
\text { Design Spec QSCC2: Vary inlet fuel mixture flow such that heat } \\
\text { duty is } 5 \% \text { of that calculated for unit Q2. }\end{array}$ \\
\hline $\begin{array}{l}\text { DUPL2 } \\
\text { (DUPL) }\end{array}$ & 2200 & 0 & $\begin{array}{l}\text { A stream duplicator block used to send a copy of the SCC2 outlet } \\
\text { stream to the dummy unit Q2. }\end{array}$ \\
\hline
\end{tabular}


Table A-14.

ASSUMPTIONS and ENERGY BALANCES for MOLTEN SALT OXIDATION, System F-1

GENERAL COMMENTS: RGIBBS units calculate chemical and phase equilibrium with some restrictions as stated. For detailed information refer to the ASPEN PLUS Release 9 input file. Chemical reactions and phase changes for some species are neglected. "Volatile" trace metals $\mathrm{As}, \mathrm{Cd}, \mathrm{Hg}$, and $\mathrm{Se}$ are allowed to change phase, while $\mathrm{Ba}, \mathrm{Cr}, \mathrm{Pb}$, and $\mathrm{Ag}$ are restricted to the solid phase. Inlet fuel mixture stream (FUELMIX2) is composed of $\mathrm{CH} 4$ and $20 \%$ excess air (O2 and N2). Design Spec's on air and oxygen flow include both free oxygen and that bound in organics.

\begin{tabular}{|l|l|l|l|}
\hline \hline $\begin{array}{l}\text { Block Name } \\
\text { (ASPEN } \\
\text { Type) }\end{array}$ & $\begin{array}{l}\text { Outlet } \\
\text { Temp (F) }\end{array}$ & $\begin{array}{l}\text { Heat Duty } \\
\text { (MMBtu/hr) }\end{array}$ & Description and Assumptions \\
\hline $\begin{array}{l}\text { Q2 } \\
\text { (RGIBBS) }\end{array}$ & 68 & -1.357 & $\begin{array}{l}\text { Dummy unit. This calculates chemical equilibrium at 2200 F } \\
\text { identical to unit SCC2, but has an outlet temperature of } 68 \mathrm{~F}, \text { thus } \\
\text { yielding an estimate of the sensible heat of the products of SCC2. }\end{array}$ \\
\hline $\begin{array}{l}\text { QUENCH2 } \\
\text { RGIBBS) }\end{array}$ & 350 & 0 & $\begin{array}{l}\text { Quenching of exhaust products from SCC2. Formation of CH4, } \\
\text { C, and S is restricted. } \\
\text { Design Spec QQUENCH2: Vary inlet water flow rate to achieve } \\
\text { zero heat duty. }\end{array}$ \\
\hline $\begin{array}{l}\text { BAGHSE2 } \\
\text { (SEP) }\end{array}$ & 350 & 0 & $\begin{array}{l}\text { 3-way separation of QUENCH2 products. Vapors exit system, } \\
\text { volatile trace metals exit system (via HGPLUS2), and remainder } \\
\text { (via FLYASH2) recycled to VITRIFY. }\end{array}$ \\
\hline $\begin{array}{l}\text { SCRUB-2 } \\
\text { (RGIBBS) }\end{array}$ & 120 & -1.273 & $\begin{array}{l}\text { Wet acid scrubber. } \\
\text { Design Spec SCRBH2O2: Vary inlet water flow such that inlet } \\
\text { liquid water flow equals 20 times outlet dissolved salts (5\% TDS), } \\
\text { with a minimum value equal to inlet NaOH flow (50\% soln). } \\
\text { Design Spec NAOH-2: Vary inlet NaOH flow such that inlet } \\
\text { NaOH flow equals 100 times outlet NaOH flow (1\% excess). }\end{array}$ \\
\hline $\begin{array}{l}\text { L-V-SEP2 } \\
\text { (FLASH2) }\end{array}$ & 120 & 0 & $\begin{array}{l}\text { 2-way separation of SCRUB products. Flash calculation to } \\
\text { separate liquid and vapor. }\end{array}$ \\
\hline $\begin{array}{l}\text { MELTER } \\
\text { (RGIBBS) }\end{array}$ & 3000 & +0.120 & $\begin{array}{l}\text { Metal melter. Accepts metals with fixed contamination. } \\
\text { Electrically heated with heat losses not included. }\end{array}$ \\
\hline
\end{tabular}




$$
\text { A-42 }
$$


Table A-15.

ASSUMPTIONS and ENERGY BALANCES for MOLTEN METAL DESTRUCTION, System G-1

GENERAL COMMENTS: RGIBBS units calculate chemical and phase equilibrium with some restrictions as stated. For detailed information refer to the ASPEN PLUS Release 9 input file. Chemical reactions and phase changes for some species are neglected. "Volatile" trace metals $\mathrm{As}, \mathrm{Cd}, \mathrm{Hg}$, and $\mathrm{Se}$ are allowed to change phase, while $\mathrm{Ba}, \mathrm{Cr}, \mathrm{Pb}$, and $\mathrm{Ag}$ are restricted to the solid phase. Design Spec's on air and oxygen flow consider both free oxygen and that bound in organics.

\begin{tabular}{|c|c|c|c|}
\hline $\begin{array}{l}\text { Block Name } \\
\text { (ASPEN } \\
\text { Type) } \\
\end{array}$ & $\begin{array}{l}\text { Outlet } \\
\text { Temp (F) }\end{array}$ & $\begin{array}{l}\text { Heat Duty } \\
\text { (MMBtu/hr) }\end{array}$ & Description and Assumptions \\
\hline $\begin{array}{l}\text { MM-CEP } \\
\text { (RGIBBS) }\end{array}$ & 2732 & -5.138 & $\begin{array}{l}\text { Molten metal treatment. Accepts combustible and non- } \\
\text { combustible wastes and metals with fixed contamination. Also } \\
\text { accepts an additional } 468 \mathrm{lbs} / \mathrm{hr} \text { of contaminated metals. Energy } \\
\text { input shown does not include losses. Operating in a reducing } \\
\text { mode, oxygen is supplied at the rate of } 12 \% \text { of the combustible } \\
\text { waste stream (FDWASTEC) on a mass basis. (Negligible amount } \\
\text { of N2 cover gas also is used) Formation of CH4 is restricted. } \\
\text { Reduction of Fe3O4 is allowed. } \\
\text { Design Spec LIME: Vary the inlet mass flow rate of dry lime } \\
\text { (CaO) such that the inlet mole flow rate of lime equals } 90 \% \text { of the } \\
\text { inlet mole flow rate of chlorine (Cl2) in the waste streams ( } 90 \% \\
\text { effective scrubbing), assuming } 100 \% \text { utilization of the CaO. } \\
\text { Design Spec SOILH2O: Vary inlet soil feed rate (substream } \\
\text { MIXED, } 10 \% \text { of total flow) such that it is } 1 / 30 \text { the magnitude of } \\
\text { the SLAG stream (ash-to-gross soil ratio of } 2: 1 \text { ). } \\
\text { Design Spec SOILSOL: Vary inlet soil feed rate (substream } \\
\text { CISOLID, } 90 \% \text { of total flow) such that it is } 1 / 3.333 \text { the magnitude } \\
\text { of the SLAG stream (ash-to-gross soil ratio of } 2: 1 \text { ). }\end{array}$ \\
\hline $\begin{array}{l}\text { MM-SEP } \\
\text { (SEP) }\end{array}$ & 2732 & 0 & $\begin{array}{l}\text { 3-way separation of MM-CEP products. } 100 \% \text { of metals to } \\
\text { stream METAL. } 80 \% \text { of the carbon, sulfur, and metallic oxides } \\
\text { and } 100 \% \text { of molten } \mathrm{CaCl} 2 \text { to stream SLAG. The remainder to } \\
\text { stream OFFGAS. }\end{array}$ \\
\hline $\begin{array}{l}\text { QUENCH } \\
\text { (RGIBBS) }\end{array}$ & 200 & -2.08 & $\begin{array}{l}\text { Indirect cooling of stream OFFGAS. Heat removal shown does } \\
\text { not include losses. Formation of } \mathrm{H} 2 \mathrm{O}, \mathrm{CH} 4, \mathrm{C}, \mathrm{S} \text { and } \mathrm{Cl} \text { is } \\
\text { restricted. }\end{array}$ \\
\hline $\begin{array}{l}\text { SEPRATR } \\
\text { (SEP) }\end{array}$ & 200 & 0 & $\begin{array}{l}\text { 3-way separation of QUENCH products. Fluids sent to } \\
\text { FLUIDSEP, volatile trace metals exit system (via HGPLUS), and } \\
\text { remainder recycle (via SOLIDS) to VITRIFY. }\end{array}$ \\
\hline $\begin{array}{l}\text { FLUIDSEP } \\
\text { (FLASH2) }\end{array}$ & 200 & 0 & Flash calculation. At $200 \mathrm{~F}$ stream is nearly all vapor. \\
\hline
\end{tabular}


Table A-15.

Page 2 of 2

ASSUMPTIONS and ENERGY BALANCES for MOLTEN METAL DESTRUCTION, System G-1

GENERAL COMMENTS: RGIBBS units calculate chemical and phase equilibrium with some restrictions as stated. For detailed information refer to the ASPEN PLUS Release 9 input file. Chemical reactions and phase changes for some species are neglected. "Volatile" trace metals As, $\mathrm{Cd}, \mathrm{Hg}$, and Se are allowed to change phase, while $\mathrm{Ba}, \mathrm{Cr}, \mathrm{Pb}$, and $\mathrm{Ag}$ are restricted to the solid phase. Design Spec's on air and oxygen flow consider both free oxygen and that bound in organics.

\begin{tabular}{|l|l|l|l|}
\hline $\begin{array}{l}\text { Block Name } \\
\text { (ASPEN } \\
\text { Type) }\end{array}$ & $\begin{array}{l}\text { Outlet } \\
\text { Temp (F) }\end{array}$ & $\begin{array}{l}\text { Heat Duty } \\
\text { (MMBtuhr) }\end{array}$ & Description and Assumptions \\
\hline $\begin{array}{l}\text { SCRUB } \\
\text { (RGIBBS) }\end{array}$ & 80 & -0.089 & $\begin{array}{l}\text { Wet acid scrubber. Formation of CH4, C, S and Cl is restricted. } \\
\text { Also, no reaction of CO and CO2 is allowed. Temperature must } \\
\text { be reduced below } 90 \mathrm{~F} \text { to get liquid product. } \\
\text { Design Spec SCRUBH2O: Vary inlet water flow such that inlet } \\
\text { liquid water flow equals 20 times outlet dissolved salts (5\% TDS), } \\
\text { with a minimum value equal to inlet NaOH flow (50\% soln). } \\
\text { Design Spec NAOH: Vary the inlet NaOH flow rate such that it } \\
\text { equals 100 times the outlet NaOH flow (1\% excess). }\end{array}$ \\
\hline $\begin{array}{l}\text { L-V-SEP } \\
\text { (FLASH2) }\end{array}$ & 80 & 0 & $\begin{array}{l}\text { 2-way separation of SCRUB products. Flash calculation to } \\
\text { separate liquid and vapor. }\end{array}$ \\
\hline $\begin{array}{l}\text { CATALYST } \\
\text { (RSTOIC) }\end{array}$ & 1500 & -3.834 & $\begin{array}{l}\text { Catalytic oxidation of hydrogen- and CO-rich exhaust gases. Heat } \\
\text { removal shown does not include losses. } \\
\text { Design Spec AIR: Vary the inlet air flow such that inlet oxygen } \\
\text { flow equals twice outlet oxygen flow (100\% excess). }\end{array}$ \\
\hline
\end{tabular}


Table A-16.

ASSUMPTIONS and ENERGY BALANCES for STEAM GASIFICATION, System H-1

Page 1 of 3

GENERAL COMMENTS: RGIBBS units calculate chemical and phase equilibrium with some restrictions as stated. For detailed information refer to the ASPEN PLUS Release 9 input file. Chemical reactions and phase changes for some species are neglected. "Volatile" trace metals $\mathrm{As}, \mathrm{Cd}, \mathrm{Hg}$, and $\mathrm{Se}$ are allowed to change phase, while $\mathrm{Ba}, \mathrm{Cr}, \mathrm{Pb}$, and $\mathrm{Ag}$ are restricted to the solid phase. Inlet fuel mixture streams (FUELMIX1 and FUELMIX2) are composed of CH4 and $20 \%$ excess air (O2 and N2). Design Spec's on air and oxygen include both free oxygen and that bound in organics.

\begin{tabular}{|c|c|c|c|}
\hline $\begin{array}{l}\text { Block Name } \\
\text { (ASPEN } \\
\text { Type) }\end{array}$ & $\begin{array}{l}\text { Outlet } \\
\text { Temp (F) }\end{array}$ & $\begin{array}{l}\text { Heat Duty } \\
\text { (MMBtu/hr) }\end{array}$ & Description and Assumptions \\
\hline $\begin{array}{l}\text { GASIFIER } \\
\text { (RSTOIC) }\end{array}$ & 1400 & +1.307 & $\begin{array}{l}\text { Steam gasification. Accepts combustible waste. Heat duty does } \\
\text { not included losses, but see HEATER unit below. Reactions are } \\
\text { (a) } 90 \% \text { conversion of carbon to CO and H2, (b) } 100 \% \text { conversion } \\
\text { of chlorine to } \mathrm{HCl} \text {, and } 100 \% \text { conversion of oxygen to equal parts } \\
\mathrm{CO} 2 \text { and } \mathrm{H} 2 \mathrm{O} \text {. } \\
\text { Design Spec STEAM: Vary inlet water flow (via stream STEAM) } \\
\text { such that total inlet water flow equals } 6 \text { times outlet water flow } \\
\text { (20\% excess). This yields a steam-to-carbon ratio of about } 1.5 \text {. }\end{array}$ \\
\hline $\begin{array}{l}\text { STEAMGEN } \\
\text { (HEATER) }\end{array}$ & 900 & +0.645 & $\begin{array}{l}\text { Steam generator. Heats incoming water. Heat duty does not } \\
\text { include losses, but see HEATER unit below. }\end{array}$ \\
\hline $\begin{array}{l}\text { HEATER } \\
\text { (RGIBBS) }\end{array}$ & 1400 & -2.050 & $\begin{array}{l}\text { Indirect heating of GASIFIER and STEAMGEN units, including } \\
5 \% \text { losses. CH4-fueled with } 20 \% \text { excess air. } \\
\text { Design Spec QHEATER: Vary inlet flow rate of air/fuel mixture } \\
\text { (stream FUELMIXI) such that heat output is } 5 \% \text { greater than the } \\
\text { combined heat duty of GASIFIER and STEAMGEN to account } \\
\text { for heat losses in those units. }\end{array}$ \\
\hline $\begin{array}{l}\text { FILTERS } \\
\text { (SEP) }\end{array}$ & 1400 & -0.053 & $\begin{array}{l}\text { 2-way separation of GASIFIER products, modeling a group of } \\
\text { filters. } 100 \% \text { of vapors sent to SCRUB. Remainder flashed to } 68 \\
\text { F and sent to VITRIFY (via stream ASH). }\end{array}$ \\
\hline $\begin{array}{l}\text { SCRUB } \\
\text { (RGIBBS) }\end{array}$ & 160 & -0.049 & $\begin{array}{l}\text { Wet acid scrubber. Formation of } \mathrm{CH} 4, \mathrm{C} \text {, and } \mathrm{S} \text { is restricted. } \\
\text { Design Spec SCRUBH2O: Vary inlet water flow such that inlet } \\
\text { liquid water flow equals } 20 \text { times outlet dissolved salts (5\% TDS), } \\
\text { with a minimum value equal to inlet } \mathrm{NaOH} \text { flow ( } 50 \% \text { soln). } \\
\text { Design Spec } \mathrm{NAOH} \text { : Vary inlet } \mathrm{NaOH} \text { flow such that inlet } \mathrm{NaOH} \\
\text { flow equals } 100 \text { times outlet } \mathrm{NaOH} \text { flow ( } 1 \% \text { excess). }\end{array}$ \\
\hline $\begin{array}{l}\text { L-V-SEP } \\
\text { (FLASH2) }\end{array}$ & 160 & 0 & $\begin{array}{l}\text { 2-way separation of SCRUB products. Flash calculation to } \\
\text { separate liquid and vapor. }\end{array}$ \\
\hline $\begin{array}{l}\text { REHEAT } \\
\text { (RGIBBS) }\end{array}$ & 220 & +0.046 & $\begin{array}{l}\text { Reheat (dry) the vapor stream from L-V-SEP before entry into } \\
\text { CATALYST. Chemical reactions are restricted. }\end{array}$ \\
\hline $\begin{array}{l}\text { CATALYST } \\
\text { (RSTOIC) }\end{array}$ & 1500 & -4.241 & $\begin{array}{l}\text { Catalytic oxidation of hydrogen- and CO-rich exhaust gases. Heat } \\
\text { removal shown does not include losses. } \\
\text { Design Spec AIR: Vary inlet air flow such that total inlet oxygen } \\
\text { flow equals twice outlet oxygen flow ( } 100 \% \text { excess). }\end{array}$ \\
\hline
\end{tabular}


Table A-16.

ASSUMPTIONS and ENERGY BALANCES for STEAM GASIFICATION, System H-1

GENERAL COMMENTS: RGIBBS units calculate chemical and phase equilibrium with some restrictions as stated. For detailed information refer to the ASPEN PLUS Release 9 input file. Chemical reactions and phase changes for some species are neglected. "Volatile" trace metals $\mathrm{As}, \mathrm{Cd}, \mathrm{Hg}$, and $\mathrm{Se}$ are allowed to change phase, while $\mathrm{Ba}, \mathrm{Cr}, \mathrm{Pb}$, and $\mathrm{Ag}$ are restricted to the solid phase. Inlet fuel mixture streams (FUELMIXI and FUELMIX2) are composed of CH4 and $20 \%$ excess air (O2 and N2). Design Spec's on air and oxygen include both free oxygen and that bound in organics.

\begin{tabular}{|c|c|c|c|}
\hline $\begin{array}{l}\text { Block Name } \\
\text { (ASPEN } \\
\text { Type) } \\
\end{array}$ & $\begin{array}{l}\text { Outlet } \\
\text { Temp (F) }\end{array}$ & $\begin{array}{l}\text { Heat Duty } \\
\text { (MMBtu/hr) }\end{array}$ & Description and Assumptions \\
\hline $\begin{array}{l}\text { FEED-SEP } \\
\text { (SEP) }\end{array}$ & 74 & 0 & $\begin{array}{l}\text { 2-way separation of the feed material. SOLIDS stream goes to the } \\
\text { unit representing the vitrifier molten pool, while the organic-type } \\
\text { components go to the unit representing the vitrifier cover gas } \\
\text { region. Five percent of the carbon and sulfur is assumed to end up } \\
\text { trapped in the melt, and } 5 \% \text { of the solids are assumed to be carried } \\
\text { off with the vapors. }\end{array}$ \\
\hline $\begin{array}{l}\text { VITRIFY } \\
\text { (RGIBBS) }\end{array}$ & 3000 & +1.842 & $\begin{array}{l}\text { Virtifier molten pool. Accepts the non-organic portion of the } \\
\text { combustible and non-combustible wastes. The organic portion of } \\
\text { the non-combustible wastes is assumed to be volatilized during } \\
\text { heating and released to the plenum over the melt. Heat duty does } \\
\text { not include any losses or the energy needed to heat and volatilize } \\
\text { the organic portion of the non-combustible wastes. } \\
\text { Design Spec SOILH2O: Vary inlet soil feed rate (substream } \\
\text { MIXED, } 10 \% \text { of total flow) such that it is } 1 / 30 \text { the magnitude of } \\
\text { the SLAG stream (ash-to-gross soil ratio of } 2: 1 \text { ). } \\
\text { Design Spec SOILSOL: Vary inlet soil feed rate (substream } \\
\text { CISOLID, } 90 \% \text { of total flow) such that it is } 1 / 3.333 \text { the magnitude } \\
\text { of the SLAG stream (ash-to-gross soil ratio of } 2: 1 \text { ). }\end{array}$ \\
\hline $\begin{array}{l}\text { VIT-SEP } \\
\text { (SEP) }\end{array}$ & 3000 & 0 & $\begin{array}{l}\text { 2-way separation of VITRIFY products: } 100 \% \text { of solids and } \\
\text { liquids exit system (via stream SLAG). Any gases produced, as } \\
\text { well as } 100 \% \text { of volatile trace metals, are sent to unit PLENUM. }\end{array}$ \\
\hline $\begin{array}{l}\text { PLENUM } \\
\text { (RGBBS) }\end{array}$ & 1800 & +0.335 & $\begin{array}{l}\text { Vitrifier plenum area. Organic constituents are volatilized and } \\
\text { released from the melt into the plenum. Oxygen is injected into } \\
\text { the zone, combusting the vapors. The heat duty does not include } \\
\text { any losses. Formation of CH4 is restricted. } \\
\text { Design Spec OXYGEN: Vary the inlet oxygen flow (stream } \\
\text { OXYGEN) such that the total incoming oxygen is } 6.0 \text { times the } \\
\text { outlet oxygen flow ( } 20 \% \text { excess). }\end{array}$ \\
\hline $\begin{array}{l}\text { SCC2 } \\
\text { (RGIBBS) }\end{array}$ & 2200 & -0.074 & $\begin{array}{l}\text { Secondary combustion unit. Further combustion of the vapors at a } \\
\text { temperature to assure destruction of PCB's. CH4-fueled with air } \\
\text { at } 20 \% \text { excess. Heat losses assumed to be } 5 \% \text { of the sensible heat } \\
\text { of the products. } \\
\text { Design Spec QSCC2: Vary inlet fuel mixture flow such that heat } \\
\text { duty is } 5 \% \text { of that calculated for unit Q2. }\end{array}$ \\
\hline $\begin{array}{l}\text { DUPL2 } \\
\text { (DUPL) }\end{array}$ & 2200 & 0 & $\begin{array}{l}\text { A stream duplicator block used to send a copy of the SCC2 outlet } \\
\text { stream to the dummy unit Q2. }\end{array}$ \\
\hline
\end{tabular}


Table A-16.

ASSUMPTIONS and ENERGY BALANCES for STEAM GASIFICATION, System $\mathrm{H}-1$

GENERAL COMMENTS: RGIBBS units calculate chemical and phase equilibrium with some restrictions as stated. For detailed information refer to the ASPEN PLUS Release 9 input file. Chemical reactions and phase changes for some species are neglected. "Volatile" trace metals $\mathrm{As}, \mathrm{Cd}, \mathrm{Hg}$, and Se are allowed to change phase, while $\mathrm{Ba}, \mathrm{Cr}, \mathrm{Pb}$, and $\mathrm{Ag}$ are restricted to the solid phase. Inlet fuel mixture streams (FUELMIXI and FUELMIX2) are composed of CH4 and $20 \%$ excess air (O2 and N2). Design Spec's on air and oxygen include both free oxygen and that bound in organics.

\begin{tabular}{|l|l|l|l|}
\hline \hline $\begin{array}{l}\text { Block Name } \\
\text { (ASPEN } \\
\text { Type) }\end{array}$ & $\begin{array}{l}\text { Outlet } \\
\text { Temp (F) }\end{array}$ & $\begin{array}{l}\text { Heat Duty } \\
\text { (MMBtu/hr) }\end{array}$ & Description and Assumptions \\
\hline $\begin{array}{l}\text { Q2 } \\
\text { RGIBBS) }\end{array}$ & 68 & -1.482 & $\begin{array}{l}\text { Dummy unit. This calculates chemical equilibrium at 2200 F } \\
\text { identical to unit SCC2, but has an outlet temperature of } 68 \text { F, thus } \\
\text { yielding an estimate of the sensible heat of the products of SCC2. }\end{array}$ \\
\hline $\begin{array}{l}\text { QUENCH2 } \\
\text { (RGIBBS) }\end{array}$ & 350 & 0 & $\begin{array}{l}\text { Quenching of exhaust products from SCC2. Formation of CH4, } \\
\text { C, and S is restricted. } \\
\text { Design Spec QQUENCH2: Vary inlet water flow rate to achieve } \\
\text { zero heat duty. }\end{array}$ \\
\hline $\begin{array}{l}\text { BAGHSE2 } \\
\text { (SEP) }\end{array}$ & 350 & 0 & $\begin{array}{l}\text { 3-way separation of QUENCH2 products. Vapors exit system, } \\
\text { volatile trace metals exit system (via HGPLUS2), and remainder } \\
\text { (via FLYASH2) recycled to VITRIFY. }\end{array}$ \\
\hline $\begin{array}{l}\text { SCRUB-2 } \\
\text { (RGIBBS) }\end{array}$ & 120 & -1.38 & $\begin{array}{l}\text { Wet acid scrubber. } \\
\text { Design Spec SCRBH2O2: Vary inlet water flow such that inlet } \\
\text { liquid water flow equals 20 times outlet dissolved salts (5\% TDS), } \\
\text { with a minimum value equal to inlet NaOH flow (50\% soln). } \\
\text { Design Spec NAOH-2: Vary inlet NaOH flow such that inlet } \\
\text { NaOH flow equals 100 times outlet NaOH flow (1\% excess). }\end{array}$ \\
\hline $\begin{array}{l}\text { L-V-SEP2 } \\
\text { (FLASH2) }\end{array}$ & 120 & 0 & $\begin{array}{l}\text { 2-way separation of SCRUB products. Flash calculation to } \\
\text { separate liquid and vapor. }\end{array}$ \\
\hline $\begin{array}{l}\text { MELTER } \\
\text { (RGIBBS) }\end{array}$ & 3000 & +0.120 & $\begin{array}{l}\text { Metal melter. Accepts metals with fixed contamination. } \\
\text { Electrically heated with heat losses not included. }\end{array}$ \\
\hline
\end{tabular}


Table A-17.

ASSUMPTIONS and ENERGY BALANCES for JOULE-HEATED VITRIFICATION, System J-1

GENERAL COMMENTS: RGIBBS units calculate chemical and phase equilibrium with some restrictions as stated. For detailed information refer to the ASPEN PLUS Release 9 input file. Chemical reactions and phase changes for some species are neglected. "Volatile" trace metals $\mathrm{As}, \mathrm{Cd}, \mathrm{Hg}$, and Se are allowed to change phase, while $\mathrm{Ba}, \mathrm{Cr}, \mathrm{Pb}$, and $\mathrm{Ag}$ are restricted to the solid phase. Inlet fuel mixture stream (FUELMIX1) is composed of $\mathrm{CH} 4$ and $20 \%$ excess air (O2 and N2). Design Spec's on air and oxygen flow include. both free oxygen and that bound in organics.

\begin{tabular}{|c|c|c|c|}
\hline $\begin{array}{l}\text { Block Name } \\
\text { (ASPEN } \\
\text { Type) } \\
\end{array}$ & $\begin{array}{l}\text { Outlet } \\
\text { Temp (F) }\end{array}$ & $\begin{array}{l}\text { Heat Duty } \\
\text { (MMBtu/hr) }\end{array}$ & Description and Assumptions \\
\hline $\begin{array}{l}\text { FEED-SEP } \\
\text { (SEP) }\end{array}$ & 70 & 0 & $\begin{array}{l}\text { 2-way separation of the feed material. SOLIDS stream goes to the } \\
\text { unit representing the vitrifier molten pool, while the organic-type } \\
\text { components go to the unit representing the vitrifier cover gas } \\
\text { region. Five percent of the carbon and sulfur is assumed to end up } \\
\text { trapped in the melt, and } 5 \% \text { of the solids are assumed to be carried } \\
\text { off with the vapors. }\end{array}$ \\
\hline $\begin{array}{l}\text { VITRIFY } \\
\text { (RGIBBS) }\end{array}$ & 3000 & +2.723 & $\begin{array}{l}\text { Virtifier molten pool. Accepts the non-organic portion of the } \\
\text { combustible and non-combustible wastes. The organic portion of } \\
\text { the non-combustible wastes is assumed to be volatilized and } \\
\text { released to the plenum over the melt. Heat duty does not include } \\
\text { any losses or the energy needed to heat and volatilize the organic } \\
\text { portion of the non-combustible wastes. } \\
\text { Design Spec SOILH2O: Vary inlet soil feed rate (substream } \\
\text { MIXED, } 10 \% \text { of total flow) such that it is } 1 / 30 \text { the magnitude of } \\
\text { the SLAG stream (ash-to-gross soil ratio of } 2: 1 \text { ). } \\
\text { Design Spec SOILSOL: Vary inlet soil feed rate (substream } \\
\text { CISOLID, } 90 \% \text { of total flow) such that it is } 1 / 3.333 \text { the magnitude } \\
\text { of the SLAG stream (ash-to-gross soil ratio of } 2: 1 \text { ). }\end{array}$ \\
\hline $\begin{array}{l}\text { VT-SEP } \\
\text { (SEP) }\end{array}$ & 3000 & 0 & $\begin{array}{l}\text { 2-way separation of VITRIFY products: } 100 \% \text { of solids and } \\
\text { liquids exit system (via stream SLAG). Any gases produced, as } \\
\text { well as } 100 \% \text { of volatile trace metals, are sent to unit PLENUM. }\end{array}$ \\
\hline $\begin{array}{l}\text { PLENUM } \\
\text { (RGIBBS) }\end{array}$ & 1800 & -5.353 & $\begin{array}{l}\text { Vitrifier plenum area. Organic constituents are volatilized and } \\
\text { released from the melt into the plenum. Oxygen is injected into } \\
\text { the zone, combusting the vapors. The heat duty does not include } \\
\text { any losses. Formation of CH4 is restricted. } \\
\text { Design Spec OXYGEN: Vary the inlet oxygen flow (stream } \\
\text { OXYGEN) such that the total incoming unbound oxygen is } 6.0 \\
\text { times the outlet oxygen flow ( } 20 \% \text { excess). }\end{array}$ \\
\hline $\begin{array}{l}\text { SCC } \\
\text { (RGIBBS) }\end{array}$ & 2200 & -0.205 & $\begin{array}{l}\text { Secondary combustion unit. Further combustion of the vapors at a } \\
\text { temperature to assure destruction of PCB's. CH4-fueled with air } \\
\text { at } 20 \% \text { excess. Heat losses assumed to be } 5 \% \text { of the sensible heat } \\
\text { of the products. } \\
\text { Design Spec QSCC: Vary inlet fuel mixture flow such that heat } \\
\text { duty is } 5 \% \text { of that calculated for unit Q1. }\end{array}$ \\
\hline $\begin{array}{l}\text { DUPL1 } \\
\text { (DUPL) }\end{array}$ & 2200 & 0 & $\begin{array}{l}\text { A stream duplicator block used to send a copy of the SCC2 outlet } \\
\text { stream to the dummy unit Q1. }\end{array}$ \\
\hline
\end{tabular}


Table A-17.

ASSUMPTIONS and ENERGY BALANCES for JOULE-HEATED VITRIFICATION, System J-1

GENERAL COMMENTS: RGIBBS units calculate chemical and phase equilibrium with some restrictions as stated. For detailed information refer to the ASPEN PLUS Release 9 input file. Chemical reactions and phase changes for some species are neglected. "Volatile" trace metals $\mathrm{As}, \mathrm{Cd}, \mathrm{Hg}$, and $\mathrm{Se}$ are allowed to change phase, while $\mathrm{Ba}, \mathrm{Cr}, \mathrm{Pb}$, and $\mathrm{Ag}$ are restricted to the solid phase. Inlet fuel mixture stream (FUELMXX1) is composed of $\mathrm{CH} 4$ and $20 \%$ excess air (O2 and N2). Design Spec's on air and oxygen flow include both free oxygen and that bound in organics.

\begin{tabular}{|l|l|l|l|}
\hline $\begin{array}{l}\text { Block Name } \\
\text { (ASPEN } \\
\text { Type) }\end{array}$ & $\begin{array}{l}\text { Outlet } \\
\text { Temp (F) }\end{array}$ & $\begin{array}{l}\text { Heat Duty } \\
\text { (MMBtu/hr) }\end{array}$ & Description and Assumptions \\
\hline \hline $\begin{array}{l}\text { Q1 } \\
\text { RGIBBS) }\end{array}$ & 68 & -4.102 & $\begin{array}{l}\text { Dummy unit. This calculates chemical equilibrium at 2200 F } \\
\text { identical to unit SCC, but has an outlet temperature of } 68 \text { F, thus } \\
\text { yielding an estimate of the sensible heat of the products of SCC. }\end{array}$ \\
\hline $\begin{array}{l}\text { QUENCH } \\
\text { RGIBBS) }\end{array}$ & 350 & 0 & $\begin{array}{l}\text { Quenching of exhaust products from SCC. Formation of CH4, C, } \\
\text { and S is restricted. } \\
\text { Design Spec QQUENCH: Vary inlet water flow rate to achieve } \\
\text { zero heat duty. }\end{array}$ \\
\hline $\begin{array}{l}\text { BAGHOUSE } \\
\text { (SEP) }\end{array}$ & 350 & 0 & $\begin{array}{l}\text { 3-way separation of QUENCH products. Vapors exit system, } \\
\text { volatile trace metals exit system (via HGPLUS), and remainder } \\
\text { (via FLYASH) recycled to VITRIFY. }\end{array}$ \\
\hline $\begin{array}{l}\text { SCRUB } \\
\text { (RGIBBS) }\end{array}$ & 120 & -3.78 & $\begin{array}{l}\text { Wet acid scrubber. } \\
\text { Design Spec SCRUBH2O: Vary inlet water flow such that inlet } \\
\text { liquid water flow equals 20 times outlet dissolved salts (5\% TDS), } \\
\text { with a minimum value equal to inlet NaOH flow (50\% soln). } \\
\text { Design Spec NAOH: Vary inlet NaOH flow such that inlet NaOH } \\
\text { flow equals 100 times outlet NaOH flow (1\% excess). }\end{array}$ \\
\hline $\begin{array}{l}\text { L-V-SEP } \\
\text { FLASH2) }\end{array}$ & 120 & 0 & $\begin{array}{l}\text { 2-way separation of SCRUB products. Flash calculation to } \\
\text { separate liquid and vapor. }\end{array}$ \\
\hline $\begin{array}{l}\text { MELTER } \\
\text { RGIBBS) }\end{array}$ & 3000 & +0.120 & $\begin{array}{l}\text { Metal melter. Accepts metals with fixed contamination. } \\
\text { Electrically heated with heat losses not included. }\end{array}$ \\
\hline
\end{tabular}


Table A-18. Page 1 of 3 ASSUMPTIONS and ENERGY BALANCES for THERMAL DESORBER W/MEO, System K-1

GENERAL COMMENTS: RGIBBS units calculate chemical and phase equilibrium with some restrictions as stated. For detailed information refer to the ASPEN PLUS Release 9 input file. Chemical reactions and phase changes for some species are neglected. "Volatile" trace metals $\mathrm{As}, \mathrm{Cd}, \mathrm{Hg}$, and Se are allowed to change phase, while $\mathrm{Ba}, \mathrm{Cr}, \mathrm{Pb}$, and $\mathrm{Ag}$ are restricted to the solid phase. Inlet fuel mixture stream (FUELMIXl) is composed of $\mathrm{CH} 4$ and $20 \%$ excess air (O2 and N2). Design Spec's on oxygen flow include both free oxygen and that bound in organics. NOTE: The organic-type products entering the desorber and condenser are not true components but only represent the elemental content of the streams; thus, the splitting off of the "volatile organics" following each unit is done by simply taking a percentage of the total stream flow, based on engineering judgment.

\begin{tabular}{|l|l|l|l|}
\hline $\begin{array}{l}\text { Block Name } \\
\text { (ASPEN } \\
\text { Type) }\end{array}$ & $\begin{array}{l}\text { Outlet } \\
\text { Temp (F) }\end{array}$ & $\begin{array}{l}\text { Heat Duty } \\
\text { (MMBtu/hr) }\end{array}$ & Description and Assumptions \\
\hline $\begin{array}{l}\text { DESORBER } \\
\text { (RGBBS) }\end{array}$ & 600 & +0.743 & $\begin{array}{l}\text { Thermal desorption unit. Accepts combustible and non- } \\
\text { combustible wastes. Here the combustible waste stream has had } \\
\text { the halogenated and non-halogenated organic liquids and the oils } \\
\text { removed for direct feed into the MEO-UNI. Heating of the } \\
\text { waste releases moisture and volatile organics. Heated indirectly. } \\
\text { Heat losses not included here, but see HEATER unit below. H2, } \\
\text { O2, and H2O restricted from reacting. }\end{array}$ \\
\hline $\begin{array}{l}\text { HEATER } \\
\text { (RGBBBS) }\end{array}$ & 1400 & -0.780 & $\begin{array}{l}\text { Indirect heating of DESORBER. CH4-fueled. } \\
\text { Design Spec QHEATER: Vary inlet fuel mixture flow (stream } \\
\text { FUELMX1) such that heat output is 5\% greater than that needed } \\
\text { to heat the desorber products, to account for losses. }\end{array}$ \\
\hline $\begin{array}{l}\text { SEPRATR } \\
\text { (SEP) }\end{array}$ & 600 & 0 & $\begin{array}{l}\text { 2-way separation of DESORBER products. The organic-type } \\
\text { products listed in the stream summary are not actual, but only } \\
\text { reflect the elements present. Selection of the volatile fraction is } \\
\text { based on engineering judgment. Assume 80\% of the H2O is } \\
\text { vaporized, the rest being bound in the concrete, etc. Assume 20\% } \\
\text { of all organic-type elements are in a vaporous state (including } \\
\text { equilibrium amount of CH4). Remainder exits system (via stream } \\
\text { SOLIDS) for grouting. }\end{array}$ \\
\hline $\begin{array}{l}\text { COND-SEP } \\
\text { (SEP) }\end{array}$ & 80 & 0 & $\begin{array}{l}\text { Condensing (actually occurs in 2 stages) of the exhaust "vapors" } \\
\text { of DESORBER. H2, O2, and H2O restricted from reacting. } \\
\text { Secondary cooling system unspecified. }\end{array}$ \\
\hline $\begin{array}{l}\text { CONDENSR } \\
\text { (RGIBBS) }\end{array}$ & 80 & $\begin{array}{l}\text { 3-way separation of CONDENSR products. Assume largest } \\
\text { fraction (90\%) of ALL components (except volatile trace metals) } \\
\text { reform to liquid state and go to MEO-UNIT. Volatile trace metals } \\
\text { are trapped and exit system (via HGPLUS), and remainder of } \\
\text { products are "vapors" sent to catalytic oxidation. }\end{array}$ \\
\hline
\end{tabular}


Table A-18.

ASSUMPTIONS and ENERGY BALANCES for THERMAL DESORBER W/ MEO, System K-1

GENERAL COMMENTS: RGIBBS units calculate chemical and phase equilibrium with some restrictions as stated. For detailed information refer to the ASPEN PLUS Release 9 input file. Chemical reactions and phase changes for some species are neglected. "Volatile" trace metals $\mathrm{As}, \mathrm{Cd}, \mathrm{Hg}$, and Se are allowed to change phase, while $\mathrm{Ba}, \mathrm{Cr}, \mathrm{Pb}$, and $\mathrm{Ag}$ are restricted to the solid phase. Inlet fuel mixture stream (FUELMIX1) is composed of $\mathrm{CH} 4$ and $20 \%$ excess air (O2 and N2). Design Spec's on oxygen flow include both free oxygen and that bound in organics. NOTE: The organic-type products entering the desorber and condenser are not true components but only represent the elemental content of the streams; thus, the splitting off of the "volatile organics" following each unit is done by simply taking a percentage of the total stream flow, based on engineering judgment.

\begin{tabular}{|c|c|c|c|}
\hline $\begin{array}{l}\text { Block Name } \\
\text { (ASPEN } \\
\text { Type) } \\
\end{array}$ & $\begin{array}{l}\text { Outlet } \\
\text { Temp (F) }\end{array}$ & $\begin{array}{l}\text { Heat Duty } \\
\text { (MMBtu/hr) }\end{array}$ & Description and Assumptions \\
\hline $\begin{array}{l}\text { MEO-UNIT } \\
\text { (RSTOIC) }\end{array}$ & 140 & +1.003 & $\begin{array}{l}\text { Mediated electrochemical oxidation. Accepts as feed the portion } \\
\text { of the combustible waste not fed to the desorber, in addition to the } \\
\text { aqueous liquids waste. Unit requires heat removal as shown. } \\
\text { Losses not included. The makeup electrolyte feed shown includes } \\
\text { mainly water and CoSO4, as } 99 \% \text { of the H2SO4 in the electrolyte } \\
\text { is assumed to be recycled. WATER stream supplies additional } \\
\text { oxygen if not enough in CONDENS8 stream. Only vapor exits } \\
\text { this unit. Allowed reactions are decomposition of all water and } \\
\text { oxidation of all C, CO, CH4, and HCL. (Note that electrolyte } \\
\text { water is specified as a second water component and does not take } \\
\text { part in the reactions.) } \\
\text { Design Spec OXIDANT: Vary inlet water flow stream (WATER) } \\
\text { such that total inlet oxygen flow (O2 and H2O) equals } 6 \text { times } \\
\text { outlet oxygen flow (O2) (20\% excess). } \\
\text { Design Spec.ELECTMX: Vary the electrolyte makeup flow rate } \\
\text { (stream ELECTROL, substream MIXED) such that the makeup } \\
\text { water is } 6.82 \text { times greater than the iniet organic flow rate (i.e., all } \\
\text { but the water in streams FDAQORGS, FDWOLIQS, and } \\
\text { CONDENS8). (See comments within the model as to origin of the } \\
\text { factor } 6.82 \text {.) } \\
\text { Design Spec ELECTSOL: Vary the flow rate of the solids } \\
\text { (CoSO4) portion of the electrolyte makeup stream to maintain the } \\
\text { correct CoSO4/water ratio. }\end{array}$ \\
\hline $\begin{array}{l}\text { MEO-SEP } \\
\text { (SEP) }\end{array}$ & 140 & 0 & $\begin{array}{l}\text { Separation of } \mathrm{H} 2 \text { from other vapors. Hydrogen comes off of } \\
\text { cathode terminal, while other gasses are produced at anode. }\end{array}$ \\
\hline $\begin{array}{l}\text { SCRUB } \\
\text { (RGIBBS) }\end{array}$ & 120 & -0.022 & $\begin{array}{l}\text { Wet acid scrubber. No restriction on formation of products. } \\
\text { Design Spec SCRUBH2O: Vary inlet water flow such that inlet } \\
\text { liquid water flow equals } 20 \text { times outlet dissolved salts ( } 5 \% \mathrm{TDS} \text { ), } \\
\text { with a minimum value equal to inlet } \mathrm{NaOH} \text { flow ( } 50 \% \text { soln). } \\
\text { Design Spec } \mathrm{NAOH} \text { : Vary inlet } \mathrm{NaOH} \text { flow such that inlet } \mathrm{NaOH} \\
\text { flow equals } 100 \text { times outlet } \mathrm{NaOH} \text { flow ( } 1 \% \text { excess). }\end{array}$ \\
\hline $\begin{array}{l}\text { L-V-SEP } \\
\text { (FLASH2) }\end{array}$ & 120 & 0 & $\begin{array}{l}\text { 2-way separation of SCRUB products. Flash calculation to } \\
\text { separate liquid and vapor. }\end{array}$ \\
\hline
\end{tabular}


Table A-18.

ASSUMPTIONS and ENERGY BALANCES for THERMAL DESORBER W/MEO, System K-1

GENERAL COMMENTS: RGIBBS units calculate chemical and phase equilibrium with some restrictions as stated. For detailed information refer to the ASPEN PLUS Release 9 input file. Chemical reactions and phase changes for some species are neglected. "Volatile" trace metals As, $\mathrm{Cd}, \mathrm{Hg}$, and Se are allowed to change phase, while $\mathrm{Ba}, \mathrm{Cr}, \mathrm{Pb}$, and $\mathrm{Ag}$ are restricted to the solid phase. Inlet fuel mixture stream (FUELMIX1) is composed of CH4 and $20 \%$ excess air (O2 and N2). Design Spec's on oxygen flow include both free oxygen and that bound in organics. NOTE: The organic-type products entering the desorber and condenser are not true components but only represent the elemental content of the streams; thus, the splitting off of the "volatile organics" following each unit is done by simply taking a percentage of the total stream flow, based on engineering judgment.

\begin{tabular}{|l|l|l|l|}
\hline $\begin{array}{l}\text { Block Name } \\
\text { (ASPEN } \\
\text { Type) }\end{array}$ & $\begin{array}{l}\text { Outlet } \\
\text { Temp (F) }\end{array}$ & $\begin{array}{l}\text { Heat Duty } \\
\text { (MMBtu/hr) }\end{array}$ & Description and Assumptions \\
\hline $\begin{array}{l}\text { CATALYST } \\
\text { RSTOIC) }\end{array}$ & 1500 & -0.97 & $\begin{array}{l}\text { Catalytic oxidizer. Heat removal required. Losses not included. } \\
\text { Chemical reactions limited to oxidation of C, CO, H2, and CH4. } \\
\text { Design Spec AIR: Vary inlet air flow rate such that total inlet } \\
\text { oxygen flow (O2) equals twice the outlet oxygen flow (100\% } \\
\text { excess). }\end{array}$ \\
\hline $\begin{array}{l}\text { MELTER } \\
\text { (RGIBBS) }\end{array}$ & 3000 & +0.120 & $\begin{array}{l}\text { Metal melter. Accepts metals with fixed contamination. } \\
\text { Electrically heated with heat losses not included. }\end{array}$ \\
\hline
\end{tabular}


A-54 


\section{System L-1: Thermal Desorption and Supercritical Water Oxidation}

Table A-19. ASSUMPTIONS and ENERGY BALANCES for THERMAL DESORBER W/ SCWO, System L-1

GENERAL COMMENTS: RGIBBS units calculate chemical and phase equilibrium with some restrictions as stated. For detailed information refer to the ASPEN PLUS Release 9 input file. Chemical reactions and phase changes for some species are neglected. "Volatile" trace metals $\mathrm{As}, \mathrm{Cd}, \mathrm{Hg}$, and Se are allowed to change phase, while $\mathrm{Ba}, \mathrm{Cr}, \mathrm{Pb}$, and $\mathrm{Ag}$ are restricted to the solid phase. Inlet fuel mixture streams (FUELMIX1 and FUELMIX2) are composed of CH4 and $20 \%$ excess air (O2 and N2). Design Spec's on air and oxygen flow include both free oxygen and that bound in organics. NOTE: In the model the organic-type products entering the desorber and condenser are not true components but only represent the elemental content of the streams; thus, the splitting off of the "volatile organics" following each unit is done by simply taking a percentage of the total stream flow, based on engineering judgment.

\begin{tabular}{|c|c|c|c|}
\hline $\begin{array}{l}\text { Block Name } \\
\text { (ASPEN } \\
\text { Type) }\end{array}$ & $\begin{array}{l}\text { Outlet } \\
\text { Temp (F) }\end{array}$ & $\begin{array}{l}\text { Heat Duty } \\
\text { (MMBtu/hr) }\end{array}$ & Description and Assumptions \\
\hline $\begin{array}{l}\text { DESORBER } \\
\text { (RGIBBS) }\end{array}$ & 600 & +0.743 & $\begin{array}{l}\text { Thermal desorption unit. Accepts combustible and non- } \\
\text { combustible wastes. Here the combustible waste stream has had } \\
\text { the halogenated and non-halogenated organic liquids and the oils } \\
\text { removed for direct feed into the MEO-UNIT. Heating of the } \\
\text { waste releases moisture and volatile organics. Heated indirectly. } \\
\text { Heat losses not included here, but see HEATER unit below. H2, } \\
\mathrm{O} 2 \text {, and } \mathrm{H} 2 \mathrm{O} \text { restricted from reacting. }\end{array}$ \\
\hline $\begin{array}{l}\text { HEATER } \\
\text { (RGIBBS) }\end{array}$ & 1400 & -0.780 & $\begin{array}{l}\text { Indirect heating of DESORBER. CH4-fueled. } \\
\text { Design Spec QHEATER: Vary inlet fuel mixture flow (stream } \\
\text { FUELMIX1) such that heat output is } 5 \% \text { greater than that needed } \\
\text { to heat the desorber products, to account for losses. }\end{array}$ \\
\hline $\begin{array}{l}\text { SEPRATR } \\
\text { (SEP) }\end{array}$ & 600 & 0 & $\begin{array}{l}\text { 2-way separation of DESORBER products. The organic-type } \\
\text { products listed in the stream summary are not actual, but only } \\
\text { reflect the elements present. Selection of the volatile fraction is } \\
\text { based on engineering judgment. Assume } 80 \% \text { of the H2O is } \\
\text { vaporized, the rest being bound in the concrete, etc. Assume } 20 \% \\
\text { of all organic-type elements are in a vaporous state (including } \\
\text { equilibrium amount of CH4). Remainder exits system (via stream } \\
\text { SOLIDS) for grouting. }\end{array}$ \\
\hline $\begin{array}{l}\text { CONDENSR } \\
\text { (RGIBBS) }\end{array}$ & 80 & -0.408 & $\begin{array}{l}\text { Condensing (actually occurs in } 2 \text { stages) of the exhaust "vapors" } \\
\text { of DESORBER. H2, O2, and } \mathrm{H} 2 \mathrm{O} \text { restricted from reacting. } \\
\text { Secondary cooling fluid unspecified. }\end{array}$ \\
\hline $\begin{array}{l}\text { COND-SEP } \\
\text { (SEP) }\end{array}$ & 80 & 0 & $\begin{array}{l}\text { 3-way separation of CONDENSR products. Assume largest } \\
\text { fraction ( } 90 \% \text { ) of ALL components (except volatile trace metals) } \\
\text { reform to liquid state and go to PUMP. Volatile trace metals are } \\
\text { trapped and exit system (via HGPLUS), and remainder of products } \\
\text { are "vapors" sent to catalytic oxidation. }\end{array}$ \\
\hline $\begin{array}{l}\text { 5-STAGEC } \\
\text { (MCOMPR) }\end{array}$ & 80 & $\begin{array}{l}-0.109 \\
+40 \mathrm{HP}\end{array}$ & $\begin{array}{l}\text { 5-stage, isentropic compressor unit with interstage cooling for } \\
\text { compressing of oxygen. No losses included. Pressurizes to } 3200 \\
\text { psia. Assumed mechanical efficiency of } 100 \% \text {, isentropic } \\
\text { efficiency of } 72 \% \text {. }\end{array}$ \\
\hline
\end{tabular}


Table A-19.

ASSUMPTIONS and ENERGY BALANCES for THERMAL DESORBER W/ SCWO, System L-1

GENERAL COMMENTS: RGIBBS units calculate chemical and phase equilibrium with some restrictions as stated. For detailed information refer to the ASPEN PLUS Release 9 input file. Chemical reactions and phase changes for some species are neglected. "Volatile" trace metals $\mathrm{As}, \mathrm{Cd}, \mathrm{Hg}$, and $\mathrm{Se}$ are allowed to change phase, while $\mathrm{Ba}, \mathrm{Cr}, \mathrm{Pb}$, and $\mathrm{Ag}$ are restricted to the solid phase. Inlet fuel mixture streams (FUELMIXI and FUELMIX2) are composed of $\mathrm{CH} 4$ and $20 \%$ excess air (O2 and N2). Design Spec's on air and oxygen flow include both free oxygen and that bound in organics. NOTE: In the model the organic-type products entering the desorber and condenser are not true components but only represent the elemental content of the streams; thus; the splitting off of the "volatile organics" following each unit is done by simply taking a percentage of the total stream flow, based on engineering judgment.

\begin{tabular}{|c|c|c|c|}
\hline $\begin{array}{l}\text { Block Name } \\
\text { (ASPEN } \\
\text { Type) }\end{array}$ & $\begin{array}{l}\text { Outlet } \\
\text { Temp (F) }\end{array}$ & $\begin{array}{l}\text { Heat Duty } \\
\text { (MMBtu/hr) }\end{array}$ & Description and Assumptions \\
\hline $\begin{array}{l}\text { PUMP } \\
\text { (PUMP) }\end{array}$ & 309 & $\begin{array}{l}0 \\
+975 \mathrm{HP} \\
(727 \mathrm{KW})\end{array}$ & $\begin{array}{l}\text { Pump (or group of pumps) for compressing of the liquid feeds and } \\
\text { any liquid produced at each stage of compression. No losses } \\
\text { included. Pressurizes to } 3200 \text { psia. Assumed efficiency of } 68.1 \% \text {. }\end{array}$ \\
\hline $\begin{array}{l}\text { SCWO } \\
\text { (RGIBBS) }\end{array}$ & 800 & 0 & $\begin{array}{l}\text { Super critical water oxidation. No losses included. } \\
\text { Design Spec OXYGEN: Vary inlet oxygen flow rate (stream } \\
\text { OXYGEN) such that total inlet oxygen flow is } 6 \text { times outlet } \\
\text { oxygen flow ( } 20 \% \text { excess). } \\
\text { Design Spec NAOH: Vary inlet NaOH flow such that inlet } \mathrm{NaOH} \\
\text { flow equals } 50 \text { times outlet NaOH flow ( } 2 \% \text { excess). (Note } 1 \% \\
\text { excess, as used elsewhere, caused convergence problems in this } \\
\text { unit) } \\
\text { Design Spec QSCWO: Vary water inlet flow rate such that heat } \\
\text { duty is zero. }\end{array}$ \\
\hline $\begin{array}{l}\text { SCWO-SEP } \\
\text { (SEP) }\end{array}$ & 800 & 0 & $\begin{array}{l}\text { 3-way separation of SCWO products. Assume a recycle stream } \\
\text { back to SCWO (currently set to zero). A salt solution stream } \\
\text { (SALTSOLN) takes out all of the salts (excess } \mathrm{NaOH}, \mathrm{NaCl} \text {, and } \\
\mathrm{NaF} \text { ) at a specified concentration. The remainder of the water, the } \\
\text { gases and the rest go to stream OTHER. } \\
\text { Design Spec SALT: Vary the flow split of the water to achieve } \\
5.67 \text { times as much water as salts ( } \mathrm{NaCl} \text { and } \mathrm{NaF} \text { ) in stream } \\
\text { SALTSOLN (15\% solution). }\end{array}$ \\
\hline $\begin{array}{l}\text { FLASH } \\
\text { (FLASH2) }\end{array}$ & 150 & -4.191 & $\begin{array}{l}\text { 2-way separation of the gases and bottoms in the OTHER stream. } \\
\text { The gases exit the system. The bottoms go to the EVAPOR8R. } \\
\text { The depressurization of stream OTHER from } 3200 \text { psia is } \\
\text { assumed to occur in this unit, hence the large heat removal } \\
\text { required. }\end{array}$ \\
\hline $\begin{array}{l}\text { EVAPOR8R } \\
\text { (FLASH2) }\end{array}$ & 212 & +3.122 & $\begin{array}{l}\text { Heating of the salt solution and bottoms streams to drive off water } \\
\text { and concentrate the solution. The depressurization of stream } \\
\text { SALTSOLN from } 3200 \text { psia is assumed to occur in this unit, } \\
\text { hence the large heat removal required. } \\
\text { Design Spec LIQUOR: Vary the outlet temperature to yield equal } \\
\text { flow rate of salts and water in stream CONCSOLN ( } 50 \% \\
\text { solution). }\end{array}$ \\
\hline $\begin{array}{l}\text { CONDENS2 } \\
\text { (FLASH2) }\end{array}$ & 100 & -3.627 & $\begin{array}{l}\text { Condensing of water vapor from unit EVAPOR8R. Any non- } \\
\text { condensables get sent back to unit FLASH. }\end{array}$ \\
\hline
\end{tabular}


Table A-19.

ASSUMPTIONS and ENERGY BALANCES for THERMAL DESORBER W/ SCWO, System L-1

GENERAL COMMENTS: RGBBS units calculate chemical and phase equilibrium with some restrictions as stated. For detailed information refer to the ASPEN PLUS Release 9 input file. Chemical reactions and phase changes for some species are neglected. "Volatile" trace metals $\mathrm{As}, \mathrm{Cd}, \mathrm{Hg}$, and Se are allowed to change phase, while $\mathrm{Ba}, \mathrm{Cr}, \mathrm{Pb}$, and $\mathrm{Ag}$ are restricted to the solid phase. Inlet fuel mixture streams (FUELMIXl and FUELMIX2) are composed of $\mathrm{CH} 4$ and $20 \%$ excess air (O2 and N2). Design Spec's on air and oxygen flow include both free oxygen and that bound in organics. NOTE: In the model the organic-type products entering the desorber and condenser are not true components but only represent the elemental content of the streams; thus, the splitting off of the "volatile organics" following each unit is done by simply taking a percentage of the total stream flow, based on engineering judgment.

\begin{tabular}{|l|l|l|l|}
\hline $\begin{array}{l}\text { Block Name } \\
\text { (ASPEN } \\
\text { Type) }\end{array}$ & $\begin{array}{l}\text { Outlet } \\
\text { Temp (F) }\end{array}$ & $\begin{array}{l}\text { Heat Duty } \\
\text { (MMBtu/hr) }\end{array}$ & Description and Assumptions \\
\hline \hline $\begin{array}{l}\text { CATALYST } \\
\text { (RSTOIC) }\end{array}$ & 1500 & -0.003 & $\begin{array}{l}\text { Catalytic oxidizer. Heat removal required. Losses not included. } \\
\text { Chemical reactions limited to oxidation of C, CO, H2, and CH4. } \\
\text { Design Spec AR: Vary inlet air flow rate such that total inlet } \\
\text { oxygen flow (O2) equals twice the outlet oxygen flow (100\% } \\
\text { excess). } \\
\text { Metal melter. Accepts metals with fixed contamination. } \\
\text { Electrically heated with heat losses not included. }\end{array}$ \\
\hline
\end{tabular}



Appendix B

\section{ASPEN PLUS Input Files}


B-2 


\section{Appendix B}

\section{ASPEN PLUS Input Files}

NOTE: The following input files show the entire models with all design spec's and other options active. In actually converging the models it is sometimes necessary (and most times advantageous) to selectively turn off design spec's and other options at the early stages of the run. Thus, if one attempts to reproduce the reported results with these input files (if the runs converge at all) they will only be identical to within the tolerances set in the model. The files have also been edited somewhat for this report for clarity and to conserve space.

\section{System A-1}

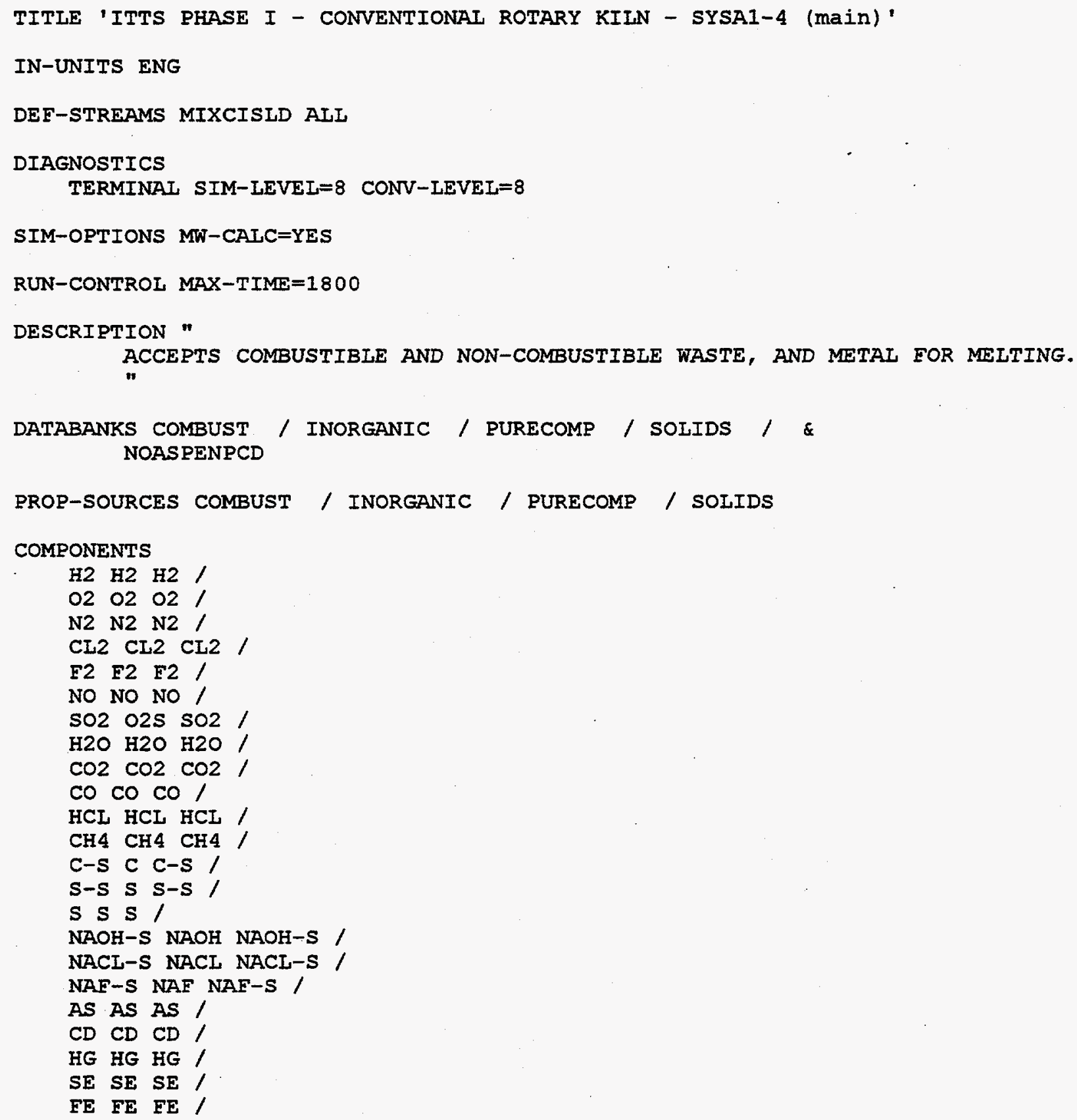


SIO2 SIO2 SIO2 /

AL203 AL203 AL203 /

FE-S EE FE-S /

SIO2-S SIO2 SIO2-S /

AL203-S AL2O3 AL203-S /

AS-S AS AS-S /

$\mathrm{BA}-\mathrm{S} \mathrm{BA} \mathrm{BA}-\mathrm{S} /$

CD-S CD CD-S /

CR-S CR CR-S /

PB-S PB PB-S /

SE-S SE SE-S /

AG-S AG AG-S /

CACO3-S CACO3 CACO3-S /

MGO-S MGO MGO-S /

NA2O-S NA2O NA2O-S /

K2O-S K2O K2O-S I

FE304 FE304 FE304 /

FE 304-S FE304 FE304-S /

CAO-S CAO CAO-S

FLOWSHEET

BLOCK KILN IN=FDWASTEN SOIL FUELMIXI FDWASTEC AIR \& AIRIEAKS COOLH2O OUT $=$ OUT 1

BLOCK KILNSEP IN=KIINOUT OUT=ASH KILNVAP

BLOCK SCC IN=KILNVAP FUELMIX2 OUT=OUT2

BLOCK BAGHOUSE IN=QUENCHED OUT=HGPLUS FLYASH QNCHVAP

BLOCK QUENCH IN=SCCOUT QNCH2O OUT=QUENCHED

BLOCK I-V-SEP IN=SCRUBOUT OUT=SCRUBVAP SCRUBBOT

BLOCK DUPLI IN=OUT1 OUT=KILNOUT DUMIIN

BLOCK $Q I$ IN=DUMIIN OUT=DUMIOUT

BLOCK DUPL2 IN=OUT2 OUT=SCCOUT DUM2IN

BLOCK Q2 IN=DUM2 IN OUT=DUM2OUT

BLOCK VITRIFY IN=FLYASH ASH OUT=VITOUT

BLOCK L-V-SEP2 IN=VITOUT OUT=VITVAP SIAG

BLOCK SCRUB IN=NAOH SCRUBH2O QNCHVAP OUT=SCRUBOUT

BLOCK MELTER IN=FDMETMLT OUT=METAL

PROPERTIES SOLIDS

PROPERTIES IDEAL / RKS-BM

PROP-SET ALI-SUBS TEMP PRES VFRAC UNITS='ATM' SUBSTREAM=ALI

PROP-SET SETI VLSTDMX UNITS='CUFT/HR' SUBSTREAM=MIXED

STREAM AIR

SUBSTREAM MIXED TEMP $=68$ PRES $=1$ <ATM> MASS-FLOW $=5000$

MASS-FRAC $020.233 / \mathrm{N} 20.767$

STREAM AIRLEAKS

SUBSTREAM MIXED TEMP $=68$ PRES $=1$ <ATM> \&

VOLUME-FLOW $=600<$ <UFT $/$ MIN>

MASS-FRAC $020.233 / \mathrm{N} 20.767$

STREAM COOLH2O

SUBSTREAM MIXED TEMP=68 PRES=1 <ATM> MASS-FLOW=1

MASS-FRAC H2O 1

STREAM FDMETMLT

SUBSTREAM MIXED TEMP $=68$ PRES $=1$ <ATM> MASS-FLOW $=1.20$

MASS-FLOW H2 2.42

SUBSTREAM CISOIID TEMP $=68$ PRES $=1$ <ATM> MASS-ELOW $=147.80$

MASS-FLOW C-S $14.44 /$ FE-S $282.02 /$ SIO2-S $0.035 / \&$ 
AL203-S $0.035 / \mathrm{BA}-\mathrm{S} 0.87 / \mathrm{CD}-\mathrm{S} 0.18$

STREAM FDWASTEC

SUBSTREAM MIXED TEMP=68 PRES=1 <ATM>

MASS-FLOW H2 $50.95 /$ O2 $92.76 /$ N2 $0.23 /$ CL2 $53.02 / \&$

E2 $0.11 / \mathrm{H} 2 \mathrm{O} 19.83$

SUBSTREAM CISOLID TEMP $=68$ PRES $=1$ <ATM>

MASS-FLOW C-S $326.28 /$ S-S $0.49 /$ FE-S $7.67 /$ SIO2-S \&

$53.01 /$ AL203-S 53.01/BA-S $0.77 /$ CD-S $0.77 /$ CR-S 1.53

STREAM FDWASTEN

SUBSTREAM MIXED TEMP $=68$ PRES $=1$ <ATM>

MASS-FLOW H2 $0.54 / 021.44 / \mathrm{N} 20.01 / \mathrm{CL} 214.88 / \&$

NO $0.66 / \mathrm{SO} 0.83 / \mathrm{H} 20354.23 /$ HG 0.15

SUBSTREAM CISOLID TEMP $=68$ PRES $=1$ <ATM>

MASS-FLOW C-S $4.77 /$ S-S $0.62 /$ SIO2-S $476.77 / \&$

AL203-S 476.77 / AS-S 0.06 / BA-S $0.04 /$ CD-S $2.63 \&$

/ CR-S $4.95 / \mathrm{PB}-\mathrm{S} 0.17 / \mathrm{SE}-\mathrm{S} 0.03 / \mathrm{AG}-\mathrm{S} 0.03$

STREAM FUELMIXI

SUBSTREAM MIXED TEMP=68 PRES=1 <ATM> MASS-FLOW=1

MASS-FRAC $020.222 / \mathrm{N} 20.732 / \mathrm{CH} 40.046$

STREAM FUELMIX2

SUBSTREAM MIXED TEMP $=68$ PRES $=1$ <ATM > MASS-FLOW=1

MASS-FRAC $020.222 / \mathrm{N} 20.732 / \mathrm{CH} 40.046$

STREAM NAOH

SUBSTREAM MIXED TEMP $=68$ PRES $=1$ <ATM>

SUBSTREAM CISOLID TEMP $=68$ PRES $=1$ <ATM> MASS-FLOW $=80$

MASS-FRAC NAOH-S 1

STREAM OUT 1

SUBSTREAM MIXED TEMP $=2500$ PRES $=1$ <ATM>

MASS-FLOW O2 $1293 /$ N2 $14610 /$ NO $27 / \mathrm{SO} 3 / \mathrm{H} 2 \mathrm{O}_{\text {\& }}$ 1977 / CO2 $2598 / \mathrm{HCL} 70 / \mathrm{FE} 30445$

SUBSTREAM CISOLID TEMP=2500 PRES $=1$ <ATM>

MASS-FLOW SIO2-S 1069 / AL203-S 739 / CR-S 8 / MGO-S \& $18 / \mathrm{NA} 2 \mathrm{O}-\mathrm{S} 10 / \mathrm{K} 2 \mathrm{O}-\mathrm{S} 19 / \mathrm{CAO}-\mathrm{S} 56$

STREAM QNCH2O

SUBSTREAM MIXED TEMP=68 PRES=1 <ATM> MASS-FLOW=1

MASS-FRAC H2O 1

STREAM QUENCHED

SUBSTREAM MIXED TEMP=350 PRES $=1$ <ATM>

MASS-FLOW O2 $1446 /$ N2 $17490 /$ CL2 $29 /$ H2O $16320 / \&$ $\mathrm{CO} 3088 / \mathrm{HCL} 40$

SUBSTREAM CISOLID TEMP $=350$ PRES $=1$ <ATM>

MASS-FLOW SIO2-S 221 / AL2O3-S 148 / CD-S 3.3 / CR-S \& $1.62 /$ MGO-S $3.6 /$ NA2O-S 2 / K2O-S $3.75 /$ FE3O4-S 9

STREAM SCRUBH2O

SUBSTREAM MIXED TEMP $=68$ PRES=1 <ATM> MASS-FLOW=1

MASS-ERAC H2O 1

STREAM SCRUBOUT

SUBSTREAM MIXED TEMP $=120$ PRES $=1$ <ATM>

MASS-FLOW O2 $1452 / \mathrm{N} 217490 / \mathrm{H} 2 \mathrm{O} 373000 / \mathrm{CO} 3088$

SUBSTREAM CISOLID TEMP=120 PRES $=1$ <ATM>

MASS-FLOW NACL-S 112 
STREAM SOII

SUBSTREAM MIXED TEMP=68 PRES=1 <ATM> MASS-ELOW=62.5

MASS-FIOW H2O 0.1

SUBSTREAM CISOLID TEMP $=68$ PRES $=1$ <ATM> MASS-FLOW $=562.5$

MASS-FIOW SIO2-S $0.569 /$ AL203-S $0.102 /$ CACO3-S $0.128 /$ \& MGO-S $0.023 /$ NA2O-S $0.013 /$ K2O-S $0.024 /$ FE3O4-S 0.041

BLOCK BAGHOUSE SEP

FRAC STREAM=HGPLUS SUBSTREAM=MIXED COMPS=H2 O2 N2 CL2 \& F2 NO SO2 H2O CO2 CO HCL CH4 C-S S-S S NAOH-S \& NACL-S NAF-S AS CD HG SE FE SIO2 AL2O3 FE-S SIO2-S \& AL2O3-S AS-S BA-S CD-S CR-S PB-S SE-S AG-S CACO3-S \& MGO-S NA2O-S K2O-S FE304 FE304-S CAO-S FRACS $=0$ O 00 \& $0 \begin{array}{lllllllllllllllllllllllllll}0 & 0 & 0 & 0 & 0 & 0 & 0 & 0 & 0 & 0 & 0 & 0 & 0 & 0 & 1 & 1 & 1 & 1 & 0 & 0\end{array}$ $\begin{array}{lllllllllllllllllll}0 & 0 & 0 & 0 & 1 & 0 & 1 & 0 & 0 & 1 & 0 & 0 & 0 & 0 & 0 & 0 & 0 & 0\end{array}$

FRAC STREAM=HGPLUS SUBSTREAM=CISOLID COMPS=C-S S-S NAOH-S \& NACL-S NAF-S FE-S SIO2-S AL2O3-S AS-S BA-S CD-S CR-S \& PB-S SE-S AG-S CACO3-S MGO-S NA2O-S K2O-S FE3O4-S \& CAO-S FRACS $=0 \begin{array}{llllllllllllllllllll}0 & 0 & 0 & 0 & 0 & 0 & 0 & 1 & 0 & 1 & 0 & 0 & 1 & 0 & 0 & 0 & 0 & 0 & 0 & 0\end{array}$ FRAC STREAM=FLYASH SUBSTREAM=MIXED COMPS=H2 O2 N2 CL2\& F2 NO SO2 H2O CO2 CO HCL CH4 C-S S-S S NAOH-S \& NACL-S NAF-S AS CD HG SE FE SIO2 AL2O3 FE-S SIO2-S \& AL2O3-S AS-S BA-S CD-S CR-S PB-S SE-S AG-S CACO3-S \& MGO-S NA2O-S K2O-S FE304 FE304-S CAO-S FRACS $=0$ O 00 \& $\begin{array}{lllllllllllllllllllllll}0 & 0 & 0 & 0 & 0 & 0 & 0 & 0 & 0 & 1 & 1 & 1 & 1 & 1 & 1 & 0 & 0 & 0 & 0 & 1 & 1 & \&\end{array}$ $\begin{array}{lllllllllllllllllllll}1 & 1 & 1 & 1 & 0 & 1 & 0 & 1 & 1 & 0 & 1 & 1 & 1 & 1 & 1 & 1 & 1 & 1\end{array}$

FRAC STREAM=FLYASH SUBSTREAM=CISOLID COMPS=C-S S-S NAOH-S \& NACL-S NAF-S FE-S SIO2-S AL2O3-S AS-S BA-S CD-S CR-S \& PB-S SE-S AG-S CACO3-S MGO-S NA2O-S K2O-S FE3O4-S \&

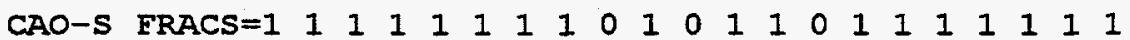

FLASH-SPECS FLYASH TEMP $=68$ PRES $=1$ <ATM>

\section{BLOCK KILNSEP SEP}

FRAC STREAM=ASH SUBSTREAM=MIXED COMPS=H2 O2 N2 CL2 F2 \& NO SO2 H2O CO2 CO HCL CH4 C-S S-S S NAOH-S NACL-S \& NAF-S AS CD HG SE FE SIO2 AL2O3 FE-S SIO2-S AI203-S \& AS-S BA-S CD-S CR-S PB-S SE-S AG-S CACO3-S MGO-S \&

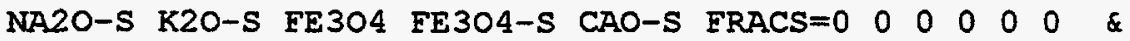
$\begin{array}{lllllllllllllllllll}0 & 0 & 0 & 0 & 0 & 0 & .8 & .8 & 0 & .8 & .8 & .8 & 0 & 0 & 0 & 0 & .8 & .8 & \&\end{array}$ $\begin{array}{llllllllllllllllll}.8 & .8 & .8 & .8 & .8 & .8 & .8 & .8 & .8 & .8 & .8 & .8 & .8 & .8 & .8 & .8 & .8 & .8\end{array}$

FRAC STREAM=ASH SUBSTREAM=CISOLID COMPS=C-S $S-S$ NAOH-S \& NACL-S NAF-S FE-S SIO2-S AL2O3-S AS-S BA-S CD-S CR-S \& PB-S SE-S AG-S CACO3-S MGO-S NA2O-S K2O-S FE304-S \&

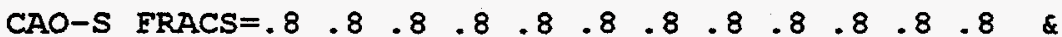
$\begin{array}{llllllllll}.8 & .8 & .8 & .8 & .8 & .8 & .8 & .8 & .8\end{array}$

FIASH-SPECS ASH TEMP $=68$ PRES $=1$ <ATM>

BLOCK L-V-SEP FLASH2

RARAM TEMP $=120$ PRES $=1$ <ATM>

BLOCK I-V-SEP2 FLASH2

PARAM TEMP $=3000$ PRES $=1$ <ATM>

\section{BLOCK KILN RGIBBS}

PARAM TEMP $=1600$ PRES $=1$ <ATM> NPHASE $=2$

$\mathrm{PROD} \mathrm{H} 2 / \mathrm{O} 2 / \mathrm{N} 2 / \mathrm{CL} 2 / \mathrm{E} 2 / \mathrm{NO} / \mathrm{SO} 2 / \mathrm{H} 2 \mathrm{O} /$ \& $\mathrm{CO} 2 / \mathrm{CO} / \mathrm{HCL} / \mathrm{AS} / \mathrm{CD} / \mathrm{HG} / \mathrm{SE} / \mathrm{FE}-\mathrm{S} S \mathrm{SS} \&$ $/$ sI02-S ss / AL203-s ss / As-s ss / BA-S SS / \& CD-S SS / CR-S SS / PB-S SS / SE-S SS / AG-S SS \& / MGO-S SS / NA2O-S SS / K2O-S SS / FE3O4-S SS / \& CAO-S SS

PROD-ERAC FE-S $1 /$ EE304-S 1 
BIOCK MEITER RGIBBS

PARAM TEMP $=3000$ PRES $=1$ <ATM> NPHASE $=2$

PROD H2 / C-S SS / FE / SIO2 / AL2O3 / FE-S SS / \& SIO2-S SS / AL203-S SS / BA-S SS / CD / CD-S SS

BLOCK Q1 RGIBBS

PARAM TEMP=68 PRES=1 <ATM> NPHASE=2 TAPP=1532

PROD H2 / O2 / N2 / CL2 / F2/ NO / SO2/ H2O / \& $\mathrm{CO} 2 / \mathrm{CO} / \mathrm{HCL} / \mathrm{AS} / \mathrm{CD} / \mathrm{HG} / \mathrm{SE} / \mathrm{FE}-\mathrm{S} \mathrm{SS} \&$ / sI02-s sS / AL203-s SS / AS-S SS / BA-s SS / \& CD-S SS / CR-S SS / PB-S SS / SE-S SS / AG-S SS \& / MGO-S SS / NA2O-S SS / K20-S SS / FE304-S SS / \& CAO-S SS

PROD-FRAC FE-S 1 / FE304-S 1

BIOCK Q2 RGIBES

PARAM TEMP=68 PRES=1 <ATM> NPHASE=2 CHEMEQ=YES TAPP=2132

PROD H2 / O2/ N2/ CL2/ F2/ NO / SO2/ H2O/\& $\mathrm{CO} 2 / \mathrm{CO} / \mathrm{HCL} / \mathrm{CH} 4 / \mathrm{S} / \mathrm{AS} / \mathrm{CD} / \mathrm{HG} / \mathrm{SE} \&$ $/$ FE-S SS / SIO2-S SS / AL203-S SS / BA-S SS / \& CR-S SS / PB-S SS / AG-S SS / MGO-S SS / NA2O-S \& SS / K2O-S SS / FE3O4-S SS / CAO-S SS

PROD-FRAC FE-S $1 /$ FE304-S 1

BLOCK QUENCH RGIBBS

PARAM TEMP $=350$ PRES $=1$ <ATM> NPHASE $=2$

PROD H2 / O2 / N2 / E2 / NO / $\mathrm{SO} 2 / \mathrm{H} 2 \mathrm{O} / \mathrm{CO} 2 /$ \& $\mathrm{CO} / \mathrm{HCL} / \mathrm{AS} / \mathrm{CD} / \mathrm{HG} / \mathrm{SE} / \mathrm{FE}-\mathrm{S} \mathrm{SS} / \&$ SIO2-S SS / AL203-S SS / AS-S SS / BA-S SS / \& CD-S SS / CR-S SS / PB-S SS / SE-S SS / AG-S SS \& / MGO-S ss / NA2O-S Ss / K2O-S SS / FE3O4-S Ss / \& CAO-S SS

PROD-ERAC EE-S 1 / FE304-S 1

BIOCK SCC RGIBBS

PARAM TEMP $=2200$ PRES $=1$ <ATM> NPHASE $=2$

PROD H2 / O2 / N2 / CL2 / F2 / NO / SO2 / H2O / \& $\mathrm{CO} 2 / \mathrm{CO} / \mathrm{HCL} / \mathrm{CH} 4 / \mathrm{s} / \mathrm{AS} / \mathrm{CD} / \mathrm{HG} / \mathrm{SE} \&$ / EE-S SS / SIO2-S SS / AL203-S SS / BA-S SS / \& CR-S SS / PB-S SS / AG-S SS / MGO-S SS / NA2O-S \& SS / K20-S SS / EE304-S SS/ CAO-S SS

PROD-FRAC FE-S $1 /$ FE304-S 1

BIOCK SCRUB RGIBBS

PARAM TEMP $=120$ PRES $=1$ <ATM> NPHASE $=2$

PROD H2 / O2 / N2 / CL2 / F2 / NO / SO2 / H2O / $\mathrm{CO} 2$ \& CO / HCL / NAOH-S SS / NACL-S SS / NAF-S SS

\section{BIOCK VITRIFY RGIBBS}

PARAM TEMP $=3000$ PRES $=1$ <ATM> NPHASE=2

PROD H2 / O2 / N2 / $\mathrm{CL} 2 / \mathrm{F} 2 / \mathrm{NO} / \mathrm{SO} 2 / \mathrm{H} 2 \mathrm{O} /$ \& $\mathrm{CO} 2 / \mathrm{CO} / \mathrm{HCL} / \mathrm{C}-\mathrm{S} / \mathrm{S} / \mathrm{AS} / \mathrm{CD} / \mathrm{HG} / \mathrm{SE} \&$ / EE-S SS / SIO2 / AL203 / AS-S SS / BA-S SS / \& CD-S SS / CR-S SS / PB-S SS / SE-S SS / AG-S SS \& / MGO-S ss / NA2O-S SS / K20-s ss / EE304-s ss / \& CAO-S SS

PROD-FRAC FE-S 1 / FE304-S 1

BIOCK DUPL1 DUPL

BIOCK DUPI2 DUPI 
DESIGN-SPEC NAOH

DEFINE CAUIN MASS-FLOW STREAM=NAOH SUBSTREAM=CISOLID\& COMPONENT $=$ NAOH $-S$

DEFINE CAUOUT MASS-FLOW STREAM=SCRUBOUT SUBSTREAM=CISOLID\& COMPONENT $=$ NAOH $-S$

SPEC "CAUIN-100.*CAUOUT" TO "0"

TOL-SPEC "0.01"

VARY STREAM-VAR STREAM=NAOH SUBSTREAM=CISOLID\& VARIABLE=MASS-FLOW

LIMITS "70" "1000"

DESIGN-SPEC OXI

DEFINE AIR MASS-FLOW STREAM=AIR SUBSTREAM=MIXED \& COMPONENT $=02$

DEFINE WC MASS-FLOW STREAM=FDWASTEC SUBSTREAM=MIXED \& COMPONENT $=02$

DEFINE WN MASS-FLOW STREAM=FDWASTEN SUBSTREAM=MIXED \& COMPONENT $=02$

DEFINE F1 MASS-FLOW STREAM=FUELMIX1 SUBSTREAM=MIXED \& COMPONENT $=02$

DEFINE OXOUT MASS-FLOW STREAM=KILNOUT SUBSTREAM=MIXED \& COMPONENT $=02$

DEFINE AL MASS-FLOW STREAM=AIRLEAKS SUBSTREAM=MIXED \& COMPONENT $=02$

SPEC "AI+AIR+WC+WN+(F1/6.0)" TO "2.0*OXOUT"

TOL-SPEC " 1 "

VARY STREAM-VAR STREAM=AIR SUBSTREAM=MIXED \& VARIABLE =MASS-FLOW

LIMITS "1" "10000"

DESIGN-SPEC QKILN

DEFINE QKIIN BLOCK-VAR BLOCK=KILN VARIABLE=QCALC \& SENTENCE $=$ PARAM

DEFINE $Q 1$ BLOCK-VAR BLOCK=Q1 VARIABLE=QCALC SENTENCE=PARAM SPEC "QKILN" TO "0.05*Q1"

TOL-SPEC "10."

VARY STREAM-VAR STREAM=COOLH2O SUBSTREAM=MIXED \& VARIABLE =MASS-FLOW

LIMITS "I" "50000"

DESIGN-SPEC QQUENCH

DEFINE QQNCH BLOCK-VAR BLOCK=QUENCH VARIABLE=QCALC \& SENTENCE = PARAM

SPEC "QQNCH" TO "O"

TOL-SPEC "1."

VARY STREAM-VAR STREAM=QNCH20 SUBSTREAM=MIXED \&

VARIABLE =MASS - FLOW

LIMITS "1" "20000"

DESIGN-SPEC QSCC

DEFINE QSCC BLOCK-VAR BLOCK=SCC VARIABLE=QCAIC \& SENTENCE $=$ PARAM

DEFINE $Q 2$ BLOCK-VAR BLOCK= $Q 2$ VARIABLE=QCALC SENTENCE=PARAM

SPEC "QSCC" TO "0.05*Q2"

TOL-SPEC "10."

VARY STREAM-VAR STREAM=FUELMIX2 SUBSTREAM=MIXED\&

VARIABLE=MASS-FLOW

LIMITS "1" "100000"

DESIGN-SPEC SOILH2O

DEFINE SLAG1 STREAM-VAR STREAM=SLAG SUBSTREAM=MIXED \& VARIABLE=MASS - FLOW 
DEFINE SLAG2 STREAM-VAR STREAM=SIAG SUBSTREAM=CISOLID \& VARIABLE=MASS-FLOW

DEFINE SOIL STREAM-VAR STREAM=SOIL SUBSTREAM=MIXED \& VARIABLE=MASS - ELOW

SPEC "30.*SOIL" TO "SIAG1+SLAG2"

TOL-SPEC "0.01"

VARY STREAM-VAR STREAM=SOIL SUBSTREAM=MIXED \& VARIABIE=MASS - FLOW

LIMITS " 1 " "1000"

DESIGN-SPEC SOILSOL

DEFINE SIAGI STREAM-VAR STREAM=SIAG SUBSTREAM=MIXED \& VARIABLE =MASS - FLOW

DEFINE SLAG2 STREAM-VAR STREAM=SIAG SUBSTREAM=CISOLID \& VARIABLE=MASS - FLOW

DEFINE SOIL STREAM-VAR STREAM=SOIL SUBSTREAM=CISOLID \& VARIABLE=MASS-FIOW

SPEC "3.33333*SOIL" TO "SLAG1+SIAG2"

TOL-SPEC "0.01"

VARY STREAM-VAR STREAM=SOII SUBSTREAM=CISOLID \& VARIABLE=MASS-FLOW

LIMITS "I" "1000"

DESIGN-SPEC SCRUBH2O

DEFINE MASSAI STREAM-VAR STREAM=SCRUBBOT SUBSTREAM=CISOLID \& VARIABLE=MASS-FLOW

DEFINE SCRUB STREAM-VAR STREAM=SCRUBH2O SUBSTREAM=MIXED \& VARIABLE=MASS-FLOW

DEFINE NAOH STREAM-VAR STREAM=NAOH SUBSTREAM=CISOLID \& VARIABLE=MASS - FLOW

SPEC "SCRUB " TO "20*MASSAI"

TOL-SPEC "0.01"

VARY STREAM-VAR STREAM=SCRUBH2O SUBSTREAM=MIXED \& VARIABLE=MASS - FLOW

LIMITS "NAOH" "5000"

CONV-OPTIONS

SECANT MAX-STEP-SIZ $=.05$ BRACKET=YES

STREAM-REPOR NOSORT MOLEFLOW MASSELOW PROPERTIES=SET1 ALL-SUBS 


\section{System A-2}

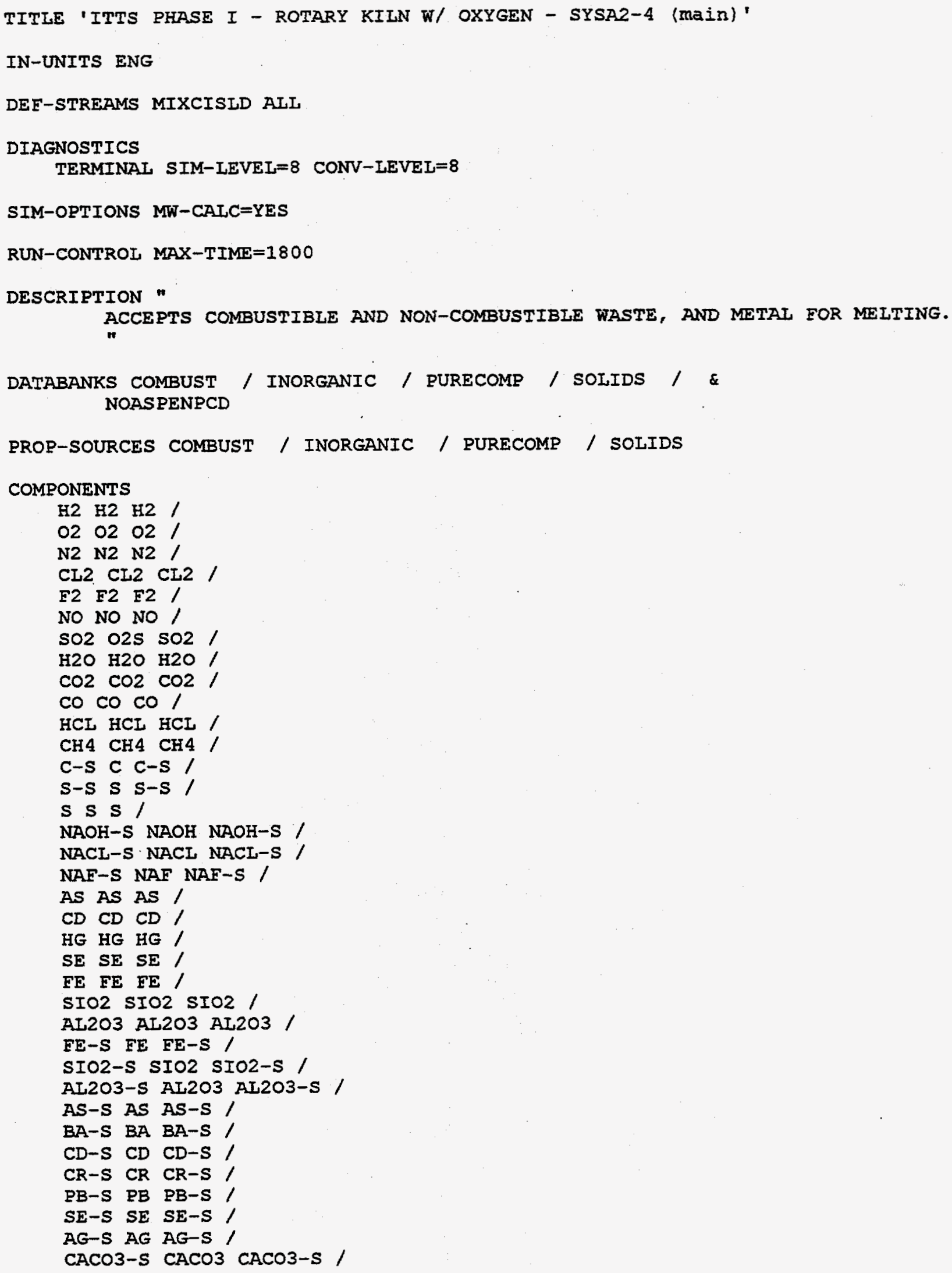


MGO-S MGO MGO-S /

NA2O-S NA2O NA2O-S /

$\mathrm{K} 2 \mathrm{O}-\mathrm{S} \mathrm{K} 2 \mathrm{O} \mathrm{K} 2 \mathrm{O}-\mathrm{S}$ /

FE304 FE304 FE304 /

EE304-S FE304 FE304-S /

CAO-S CAO CAO-S

\section{FLOWSHEET}

BLOCK KILN IN=FDWASTEN SOIL FUELMIXI FDWASTEC OXYGEN \& AIRLEAKS COOLH2O OUT=OUT 1

BLOCK KILNSEP IN=KILNOUT OUT=ASH KILNVAP

BLOCK SCC IN=KIINVAP FUELMIX2 OUT=OUT2

BLOCK BAGHOUSE IN=QUENCHED OUT=HGPLUS FLYASH QNCHVAP

BLOCK QUENCF IN=SCCOUT QNCH2O OUT=QUENCHED

BLOCK I-V-SEP IN=SCRUBOUT OUT=SCRUBVAP SCRUBBOT

BLOCK DUPLI IN=OUTI OUT=KIINOUT DUMIIN

BLOCK $Q 1$ IN=DUMIIN OUT=DUMIOUT

BLOCK DUPL2 IN=OUT2 OUT=SCCOUT DUM2IN

BLOCK 22 IN=DUM2IN OUT=DUM2OUT

BLOCK VITRIFY IN=FLYASH ASH OUT=VITOUT

BLOCK L-V-SEP2 IN=VITOUT OUT=VITVAP SLAG

BLOCK SCRUB IN=NAOH SCRUBH2O QNCHVAP OUT=SCRUBOUT

BLOCK MELTER IN=FDMETMLT OUT=METAL

\section{PROPERTIES SOLIDS \\ PROPERTIES IDEAI / RKS-BM}

PROP-SET ALL-SUBS TEMP PRES VERAC UNITS='ATM' SUBSTREAM=AIL PROP-SET SET1 VLSTDMX UNITS='CUFT/HR' SUBSTREAM=MIXED

STREAM AIRIEAKS

SUBSTREAM MIXED TEMP $=68$ PRES $=1$ <ATM> \& VOLUME-FIOW $=600<C U E T / M I N>$ MASS-ERAC $020.233 / \mathrm{N} 20.767$

STREAM COOLH2O

SUBSTREAM MIXED TEMP=68 PRES=1 <ATM> MASS-FLOW=1

MASS-FRAC H2O 1

STREAM FDMETMLT

SUBSTREAM MIXED TEMP $=68$ PRES $=1$ <ATM> MASS-FLOW $=1.20$

MASS-FLOW H2 2.42

SUBSTREAM CISOLID TEMP $=68$ PRES $=1$ <ATM> MASS-FLOW $=147.80$

MASS-FLOW C-S $14.44 /$ FE-S $282.02 /$ SIO2-S $0.035 / \&$ AL203-S $0.035 /$ BA-S $0.87 / \mathrm{CD}-\mathrm{S} 0.18$

STREAM FDWASTEC

SUBSTREAM MIXED TEMP=68 PRES=1 〈ATM>

MASS-FLOW H2 50.95/O2 $92.76 / \mathrm{N} 20.23 /$ CL2 $53.02 / \&$ F2 $0.11 / \mathrm{H} 2 \mathrm{O} 19.83$

SUBSTREAM CISOLID TEMP $=68$ PRES $=1$ <ATM>

MASS-FLOW C-S $326.28 / \mathrm{S}-\mathrm{S} 0.49 / \mathrm{FE}-\mathrm{S} 7.67 / \mathrm{SIO}-\mathrm{S} \&$ $53.01 /$ AL2O3-S 53.01 / BA-S $0.77 /$ CD-S $0.77 / \&$ CR-S 1.53

STREAM FDWASTEN

SUBSTREAM MIXED TEMP $=68$ PRES $=1$ <ATM>

MASS-ELOW H2 $0.54 / 021.44 / \mathrm{N} 20.01 / \mathrm{CL} 214.88 /$ \& NO $0.66 / \mathrm{SO} 20.83 / \mathrm{H} 2 \mathrm{O} 354.23 / \mathrm{HG} 0.15$

SUBSTREAM CISOLID TEMP $=68$ PRES $=1$ <ATM>

MASS-FLOW C-S $4.77 /$ S-S $0.62 /$ SIO2-S $476.77 / \&$

B-11 
AL203-S 476.77/ AS-S $0.06 /$ BA-S $0.04 /$ CD-S 2.63 \&

$/ \mathrm{CR}-\mathrm{S} 4.95 / \mathrm{PB}-\mathrm{S} 0.17 / \mathrm{SE}-\mathrm{S} 0.03 / \mathrm{AG}-\mathrm{S} 0.03$

STREAM FUELMIX1

SUBSTREAM MIXED TEMP=68 PRES=1 <ATM> MASS-FLOW=1

MASS-ELOW $020.222 /$ CH4 0.046

STREAM FUEIMIX2

SUBSTREAM MIXED TEMP=68 PRES=1 <ATM> MASS-FLOW=2000

MASS-FLOW $020.222 / \mathrm{CH} 40.046$

STREAM NAOH

SUBSTREAM MIXED TEMP=68 PRES=1 <ATM>

SUBSTREAM CISOLID TEMP=68 PRES $=1$ <ATM> MASS-ELOW $=80$

MASS-FRAC NAOH-S 1

STREAM OUT1

SUBSTREAM MIXED TEMP=2500 PRES=1 <ATM>

MASS-FLOW $021293 /$ N2 $14610 /$ NO $27 / \mathrm{SO} 3 / \mathrm{H} 2 \mathrm{O}$ \& 1977 / CO2 $2598 / \mathrm{HCL} 70 / \mathrm{EE} 30445$

SUBSTREAM CISOLID TEMP=2500 PRES=1 〈ATM>

MASS-FLOW SIO2-S 1069 / AL2O3-S $739 /$ CR-S 8 / MGO-S \& $18 /$ NA2O-S $10 / \mathrm{K} 20-\mathrm{S} 19 /$ CAO-S 56

STREAM OXYGEN

SUBSTREAM MIXED TEMP=68 PRES=1 <ATM> MASS-FLOW=2000

MASS-ERAC 021

STREAM QNCH2O

SUBSTREAM MIXED TEMP=68 PRES=1 <ATM> MASS-FLOW=1

MASS-FRAC H2O 1

STREAM QUENCHED

SUBSTREAM MIXED TEMP=350 PRES=1 <ATM>

MASS-FLOW O2 $1446 / \mathrm{N} 217490 / \mathrm{CL} 229 / \mathrm{H} 2016320 /$ \& $\mathrm{CO} 23088 /$ HCL 40

SUBSTREAM CISOLID TEMP $=350$ PRES $=1$ <ATM>

MASS-FLOW SIO2-S $221 /$ AL2O3-S $148 / \mathrm{CD}-\mathrm{S} 3.3 / \mathrm{CR}-\mathrm{S} \&$ $1.62 / \mathrm{MGO}-\mathrm{S} 3.6 / \mathrm{NA} 20-\mathrm{S} 2 / \mathrm{K} 20-\mathrm{S} 3.75 /$ \& EE304-S 9

STREAM SCRUBH2O

SUBSTREAM MIXED TEMP=68 PRES=1 <ATM> MASS-FLOW=1

MASS-FRAC H2O 1

STREAM SCRUBOUT

SUBSTREAM MIXED TEMP $=120$ PRES $=1$ <ATM>

MASS-FLOW O2 $1452 / \mathrm{N} 217490 / \mathrm{H} 20373000 / \mathrm{CO} 3088$

SUBSTREAM CISOLID TEMP=120 PRES $=1$ <ATM>

MASS-FLOW NACL-S 112

STREAM SOIL

SUBSTREAM MIXED TEMP=68 PRES=1 <ATM> MASS-FLOW=62.5

MASS-FLOW H2O 0.1

SUBSTREAM CISOLID TEMP=68 PRES=1 〈ATM> MASS-FLOW=562.5

MASS-FLOW SIO2-S $0.569 /$ AL2O3-S $0.102 /$ CACO3-S $0.128 /$ \& MGO-S $0.023 /$ NA2O-S $0.013 /$ K2O-S $0.024 /$ FE3O4-S \& 0.041

BLOCK BAGHOUSE SEP

ERAC STREAM=HGPLUS SUBSTREAM=MIXED COMPS=H2 O2 N2 CL2 \& F2 NO SO2 H2O CO2 CO HCL CH4 C-S S-S S NAOH-S \& 
NACL-S NAF-S AS CD HG SE FE SIO2 AI2O3 FE-S SIO2-S \& AL203-S AS-S BA-S CD-S CR-S PB-S SE-S AG-S CACO3-S \& MGO-S NA2O-S K2O-S FE304 FE304-S CAO-S FRACS=0 00 \& $\begin{array}{llllllllllllllllllllll}0 & 0 & 0 & 0 & 0 & 0 & 0 & 0 & 0 & 0 & 0 & 0 & 0 & 0 & 0 & 1 & 1 & 1 & 1 & 0 & 0 & \text { \& }\end{array}$ $\begin{array}{llllllllllllllllll}0 & 0 & 0 & 0 & 1 & 0 & 1 & 0 & 0 & 1 & 0 & 0 & 0 & 0 & 0 & 0 & 0 & 0\end{array}$

ERAC STREAM=HGPLUS SUBSTREAM=CISOIID COMPS=C-S S-S NAOH-S \& NACL-S NAF-S FE-S SIO2-S AL2O3-S AS-S BA-S CD-S CR-S \& PB-S SE-S AG-S CACO3-S MGO-S NA2O-S K2O-S EE3O4-S \& CAO-S ERACS $=0 \begin{array}{llllllllllllllllllllll}0 & 0 & 0 & 0 & 0 & 0 & 0 & 1 & 0 & 1 & 0 & 0 & 1 & 0 & 0 & 0 & 0 & 0 & 0 & 0\end{array}$

ERAC STREAM=ELYASH SUBSTREAM=MIXED COMPS=H2 O2 N2 CL2 \& F2 NO SO2 H2O CO2 CO HCL CH4 C-S s-S S NAOH-S \& NACI-S NAF-S AS CD HG SE FE SIO2 AL2O3 FE-S SIO2-S \& AL2O3-S AS-S BA-S CD-S CR-S PB-S SE-S AG-S CACO3-S \& MGO-S NA2O-S K2O-S FE304 FE304-S CAO-S FRACS=0 00 \& $\begin{array}{llllllllllllllllllllll}0 & 0 & 0 & 0 & 0 & 0 & 0 & 0 & 0 & 1 & 1 & 1 & 1 & 1 & 1 & 0 & 0 & 0 & 0 & 1 & 1 & \&\end{array}$ $\begin{array}{llllllllllllllllll}1 & 1 & 1 & 1 & 0 & 1 & 0 & 1 & 1 & 0 & 1 & 1 & 1 & 1 & 1 & 1 & 1 & 1\end{array}$

FRAC STREAM=FLYASH SUBSTREAM=CISOLID COMPS=C-S $S-S$ NAOH-S \& NACL-S NAF-S FE-S SIO2-S AL203-S AS-S BA-S CD-S CR-S \& PB-S SE-S AG-S CACO3-S MGO-S NA2O-S K2O-S EE3O4-S \&

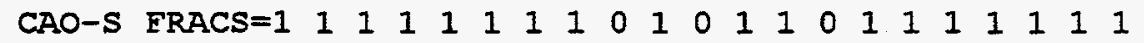

EIASH-SPECS EIYASH TEMP $=68$ PRES $=1$ 〈ATM>

\section{BLOCK KILNSEP SEP}

FRAC STREAM=ASH SUBSTREAM=MIXED COMPS=H2 O2 N2 CL2 F2 \& NO 502 H2O $\mathrm{CO} 2 \mathrm{CO} \mathrm{HCI}$ CH4 C-S S-S S NAOH-S NACL-S \& NAF-S AS CD HG SE FE SIO2 AL2O3 FE-S SIO2-S AI2O3-S \& AS-S BA-S CD-S CR-S PB-S SE-S AG-S CACO3-S MGO-S \&

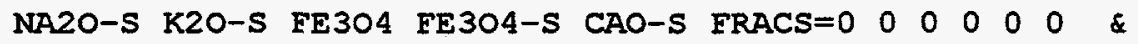
$\begin{array}{lllllllllllllllllll}0 & 0 & 0 & 0 & 0 & 0 & .8 & .8 & 0 & .8 & .8 & .8 & 0 & 0 & 0 & 0 & .8 & .8 & \&\end{array}$ $\begin{array}{llllllllllllllllll}.8 & .8 & .8 & .8 & .8 & .8 & .8 & .8 & .8 & .8 & .8 & .8 & .8 & .8 & .8 & .8 & .8 & .8\end{array}$

FRAC STREAM=ASH SUBSTREAM=CISOLID COMPS=C-S S-S NAOH-S \& NACL-S NAF-S FE-S SIO2-S AL203-S AS-S BA-S CD-S CR-S \& PB-S SE-S AG-S CACO3-S MGO-S NA2O-S K2O-S FE3O4-S \&

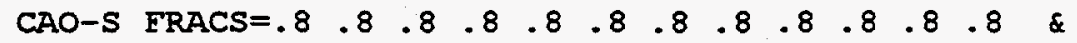
$\begin{array}{lllllllll}.8 & .8 & .8 & .8 & .8 & .8 & .8 & .8 & .8\end{array}$

FIASH-SPECS ASH TEMP $=68$ PRES $=1$ <ATM>

BLOCK L-V-SEP FIASH2

PARAM TEMP=120 PRES=1 〈ATM>

BLOCK L-V-SEP2 FIASH2

PARAM TEMP $=3000$ PRES $=1$ <ATM>

BLOCK KILN RGIBBS

PARAM TEMP $=1600$ PRES $=1$ <ATM> NPHASE $=2$

PROD H2/ O2 / N2/ $\mathrm{CL}_{2} / \mathrm{F} 2 / \mathrm{NO} / \mathrm{SO} 2 / \mathrm{H} 2 \mathrm{O} /$ \& $\mathrm{CO} 2 / \mathrm{CO} / \mathrm{HCL} / \mathrm{AS} / \mathrm{CD} / \mathrm{HG} / \mathrm{SE} / \mathrm{EE}-\mathrm{S} \mathrm{SS} \&$ $/$ sIO2-S ss / AL2O3-s ss / As-s ss / BA-S SS / \& $\mathrm{CD}-\mathrm{S} \mathrm{SS} / \mathrm{CR}-\mathrm{S} \mathrm{SS} / \mathrm{PB}-\mathrm{S} \mathrm{SS} / \mathrm{SE}-\mathrm{S} \mathrm{SS} / \mathrm{AG}-\mathrm{S} \mathrm{SS}$ / MGO-S SS / NA2O-S SS / K20-S SS / FE304-S SS / \& CAO-S SS

PROD-FRAC FE-S $1 /$ FE304-S 1

\section{BLOCK MELTER RGIBBS}

PARAM TEMP $=3000$ PRES=1 <ATM> NPHASE=2

PROD H2 / C-S SS / FE / SIO2 / Al2O3/ FE-S SS / \& SIO2-S SS / AL203-S SS / BA-S SS / CD / CD-S SS

BLOCK Q1 RGIBBS

PARAM TEMP $=68$ PRES $=1$ <ATM> NPHASE $=2$ TAPP=1532

PROD H2 / O2/ $\mathrm{N} 2 / \mathrm{CL} 2 / \mathrm{F} 2 / \mathrm{NO} / \mathrm{SO} 2 / \mathrm{H} 2 \mathrm{O} /$ \& $\mathrm{CO} 2 / \mathrm{CO} / \mathrm{HCL} / \mathrm{AS} / \mathrm{CD} / \mathrm{HG} / \mathrm{SE} / \mathrm{FE}-\mathrm{S}$ SS \& 
/ SIO2-S SS / AL2O3-S sS / AS-S SS / BA-S SS / \& CD-S SS / CR-S SS / PB-S SS / SE-S SS / AG-S SS \& / MGO-S SS / NA2O-S SS / K20-S SS / FE304-S SS / \& CAO-S SS

PROD-FRAC FE-S $1 /$ FE3O4-S 1

BIOCK 22 RGIBBS

PARAM TEMP=68 PRES=1 <ATM> NPHASE=2 CHEMEQ=YES TAPP=2132

$\mathrm{PROD} \mathrm{H} 2 / \mathrm{O} 2 / \mathrm{N} 2 / \mathrm{CL} 2 / \mathrm{F} 2 / \mathrm{NO} / \mathrm{SO} 2 / \mathrm{H} 2 \mathrm{O} /$ \& $\mathrm{CO} 2 / \mathrm{CO} / \mathrm{HCL} / \mathrm{CH} 4 / \mathrm{s} / \mathrm{AS} / \mathrm{CD} / \mathrm{HG} / \mathrm{SE}$ \& $/$ FE-S ss / SI02-s SS / AL203-s SS / BA-S SS / \& CR-S SS / PB-S SS / AG-S SS / MGO-S SS / NA2O-S \& SS / K2O-S SS / FE3O4-S SS / CAO-S SS

PROD-FRAC FE-S $1 /$ FE304-S 1

BLOCK QUENCH RGIBBS

PARAM TEMP $=350$ PRES $=1$ <ATM> NPHASE $=2$

$\mathrm{PROD} \mathrm{H} 2 / \mathrm{O} 2 / \mathrm{N} 2 / \mathrm{F} 2 / \mathrm{NO} / \mathrm{SO} 2 / \mathrm{H} 2 \mathrm{O} / \mathrm{CO} / /$ \& $\mathrm{CO} / \mathrm{HCL} / \mathrm{AS} / \mathrm{CD} / \mathrm{HG} / \mathrm{SE} / \mathrm{FE}-\mathrm{S} \mathrm{SS} / \&$
SIO2-S SS / AL2O3-S SS / AS-S SS/ BA-S SS / \& CD-S SS / CR-S SS / PB-S SS / SE-S SS / AG-S SS \& / MGO-S SS / NA2O-S SS / K20-S SS / EE304-S SS / \& CAO-S SS

PROD-FRAC FE-S $1 /$ FE304-S 1

BLOCK SCC RGIBBS

PARAM TEMP $=2200$ PRES $=1$ <ATM> NPHASE $=2$ MAXIT $=200$

$\mathrm{PROD} \mathrm{H} 2 / \mathrm{O} 2 / \mathrm{N} 2 / \mathrm{CL} 2 / \mathrm{E} 2 / \mathrm{NO} / \mathrm{SO} 2 / \mathrm{H} 2 \mathrm{O} /$ \& $\mathrm{CO} 2 / \mathrm{CO} / \mathrm{HCL} / \mathrm{CH} 4 / \mathrm{s} / \mathrm{AS} / \mathrm{CD} / \mathrm{HG} / \mathrm{SE} \&$ $/$ FE-S SS / SIO2-s SS / AL203-s SS / BA-S SS / \& CR-S SS / PB-S SS / AG-S SS / MGO-S SS / NA2O-S \& SS / K2O-S SS / FE304-S SS / CAO-S SS

PROD-FRAC FE-S $1 /$ FE3O4-S 1

\section{BIOCK SCRUB RGIBBS}

PARAM TEMP=120 PRES=1 <ATM> NPHASE=2

PROD H2 / O2 / N2 / $\mathrm{CL} 2 / \mathrm{F} 2 / \mathrm{NO} / \mathrm{SO} 2 / \mathrm{H} 2 \mathrm{O} /$ \& $\mathrm{CO} 2 / \mathrm{CO} / \mathrm{HCL} / \mathrm{NAOH}-\mathrm{S}$ SS / NACL-S SS / NAF-S SS

BLOCK VITRIFY RGIBBS

PARAM TEMP $=3000$ PRES $=1$ <ATM> NPHASE $=2$

PROD $\mathrm{H} 2 / \mathrm{O} 2 / \mathrm{N} 2 / \mathrm{CL} 2 / \mathrm{E} 2 / \mathrm{NO} / \mathrm{SO} 2 / \mathrm{H} 2 \mathrm{O} /$ \& $\mathrm{CO} 2 / \mathrm{CO} / \mathrm{HCL} / \mathrm{C}-\mathrm{S} / \mathrm{s} / \mathrm{AS} / \mathrm{CD} / \mathrm{HG} / \mathrm{SE}$ \& / FE-S SS / SI02 / AL203/AS-S SS / BA-S SS / \& CD-S SS / CR-S SS / PB-S SS/ SE-S SS/AG-S SS \& / MGO-S Ss / NA2O-S SS / K20-S SS / FE304-S ss / \& CAO-S SS

PROD-FRAC FE-S $1 /$ FE304-S 1

BLOCK DUPL1 DUPL

BIOCK DUPL2 DUPL

DESIGN-SPEC NAOH

DEFINE CAUIN MASS-FLOW STREAM=NAOH SUBSTREAM=CISOLID \& COMPONENT $=\mathrm{NAOH}-\mathrm{S}$

DEFINE CAUOUT MASS-FLOW STREAM=SCRUBOUT SUBSTREAM=CISOIID \& COMPONENT $=$ NAOH $-S$

SPEC "CAUIN-100.*CAUOUT" TO "O"

TOL-SPEC "0.01"

VARY STREAM-VAR STREAM=NAOH SUBSTREAM=CISOIID \& VARIABLE=MASS - FLOW 
LIMITS "70" "1000"

DESIGN-SPEC OXI

DEEINE OXIN MASS-FLOW STREAM=OXYGEN SUBSTREAM=MIXED\& COMPONENT $=02$

DEFINE WC MASS-FLOW STREAM=FDWASTEC SUBSTREAM=MIXED \& COMPONENT $=02$

DEFINE WN MASS-FLOW STREAM=FDWASTEN SUBSTREAM=MIXED \& COMPONENT $=02$

DEFINE F1 MASS-FLOW STREAM=FUELMIX1 SUBSTREAM=MIXED\& COMPONENT $=02$

DEEINE OXOUT MASS-ELOW STREAM=KILNOUT SUBSTREAM=MIXED \& COMPONENT $=02$

DEFINE AL MASS-FLOW STREAM=AIRLEAKS SUBSTREAM=MIXED \& COMPONENT $=02$

SPEC "AL+OXIN+WC+WN+(F1/6.0)" TO "2.0*OXOUT"

TOL-SPEC " 1 "

VARY STREAM-VAR STREAM=OXYGEN SUBSTREAM=MIXED \& VARIABLE=MASS - ELOW

LIMITS " 1 " "10000"

DESIGN-SPEC QKILN

DEFINE QKILN BLOCK-VAR BLOCK=KILN VARIABLE=QCALC \& SENTENCE = PARAM

DEFINE $Q 1$ BLOCK-VAR BLOCK=Q1 VARIABLE=QCALC SENTENCE=PARAM

SPEC "QKILN" TO " $0.05{ }^{*} Q 1$ "

TOL-SPEC "10."

VARY STREAM-VAR STREAM=COOLH2O SUBSTREAM=MIXED \& VARIABLE=MASS-FLOW

LIMITS " 1 " "50000"

DESIGN-SPEC QQUENCH

DEFINE QQNCH BLOCK-VAR BLOCK=QUENCH VARIABLE=QCALC \& SENTENCE = PARAM

SPEC "QQNCH" TO "O"

TOI-SPEC "1."

VARY STREAM-VAR STREAM=QNCH2O SUBSTREAM=MIXED \& VARIABLE $=$ MASS - ELOW

LIMITS " 1 " "20000"

DESIGN-SPEC QSCC

DEFINE QSCC BLOCK-VAR BLOCK=SCC VARIABLE=QCALC\& SENTENCE = PARAM

DEFINE 22 BLOCK-VAR BLOCK= 22 VARIABLE=QCALC SENTENCE=PARAM SPEC "QSCC" TO "0.05* $22 "$

TOL-SPEC "1."

VARY STREAM-VAR STREAM=FUELMIX2 SUBSTREAM=MIXED \& VARIABLE=MASS - FLOW

LIMITS " 1 " "100000"

DESIGN-SPEC SOILH2O

DEFINE SLAGI STREAM-VAR STREAM=SLAG SUBSTREAM=MIXED \& VARIABLE=MASS - FLOW

DEFINE SLAG2 STREAM-VAR STREAM=SLAG SUBSTREAM=CISOIID \& VARIABLE=MASS-FLOW

DEFINE SOIL STREAM-VAR STREAM=SOII SUBSTREAM=MIXED \& VARIABLE=MASS-FLOW

SPEC " 30.*SOIL" TO "SLAG1+SLAG2"

TOL-SPEC "0.01"

VARY STREAM-VAR STREAM=SOIL SUBSTREAM=MIXED \& VARIABLE=MASS-FLOW

IIMITS "1" "1000" 
DESIGN-SPEC SOILSOL

DEFINE SLAG1 STREAM-VAR STREAM=SIAG SUBSTREAM=MIXED \& VARIABLE=MASS $-F L O W$

DEFINE SLAG2 STREAM-VAR STREAM=SLAG SUBSTREAM=CISOLID \& VARIABLE $=$ MASS - FLOW

DEFINE SOII STREAM-VAR STREAM=SOII SUBSTREAM=CISOIID \& VARIABLE=MASS $-F L O W$

SPEC "3.33333*SOIL" TO "SLAGI+SLAG2"

TOL-SPEC "0.01"

VARY STREAM-VAR STREAM=SOIL SUBSTREAM=CISOIID \& VARIABLE=MASS-FLOW

LIMITS "1" "1000"

DESIGN-SPEC SCRUBH2O

DEFINE MASSAL STREAM-VAR STREAM=SCRUBBOT SUBSTREAM=CISOIID \& VARIABLE=MASS-FLOW

DEFINE SCRUB STREAM-VAR STREAM=SCRUBH2O SUBSTREAM=MIXED \& VARIABLE $=$ MASS - FLOW

DEFINE NAOH STREAM-VAR STREAM=NAOH SUBSTREAM=CISOLID \& VARIABLE $=$ MASS - FLOW

SPEC "SCRUB" TO "20*MASSAL"

TOL-SPEC "0.01"

VARY STREAM-VAR STREAM=SCRUBH2O SUBSTREAM=MIXED \& VARIABLE=MASS-FLOW

LIMITS "NAOH" "5000"

CONV-OPTIONS

SECANT MAX-STEP-SIZ $=.05$ BRACKET=YES

STREAM-REPOR NOSORT MOLEFLOW MASSELOW PROPERTIES=SET1 ALL-SUBS 


\section{System A-3}

TITLE 'ITTS PHASE I - ROTARY KILN W/ WET APC - SYSA3-4 (main)'

IN-UNITS ENG

DEF-STREAMS MIXCISLD ALL

DIAGNOSTICS

TERMINAI SIM-LEVEL $=8$ CONV-IEVEI $=8$

SIM-OPTIONS MW-CALC=YES

RUN-CONTROL MAX-TIME $=1800$

DESCRIPTION "

ACCEPTS COMBUSTIBLE AND NON-COMBUSTIBLE WASTE, AND METAL FOR MELTING.

DATABANKS COMBUST
NOASPENPCD

PROP-SOURCES COMBUST / INORGANIC / PURECOMP / SOLIDS

COMPONENTS

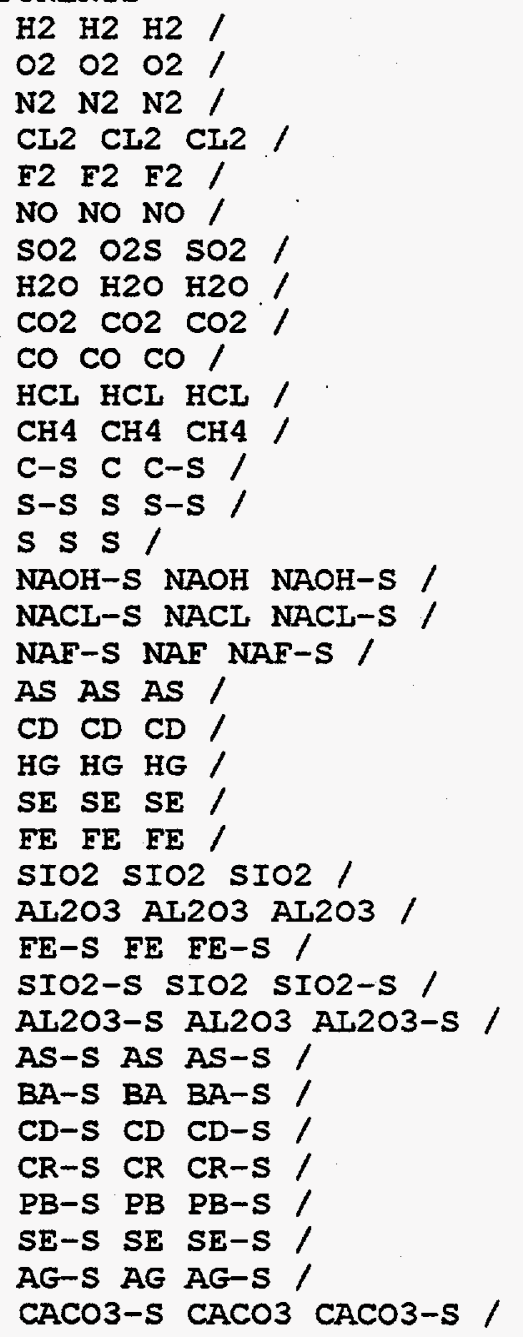




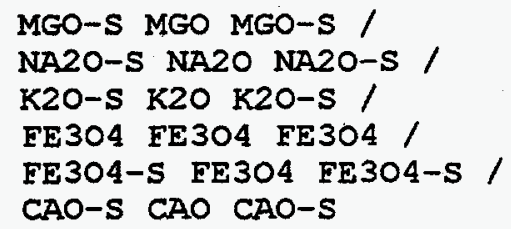


NO $0.66 / \mathrm{SO} 20.83 / \mathrm{H} 20354.23 / \mathrm{HG} 0.15$

SUBSTREAM CISOIID TEMP=68 PRES=1 <ATM>

MASS-ELOW C-S $4.77 /$ S-S $0.62 /$ SIO2-S $476.77 /$ \&

AL203-S 476.77/ AS-S $0.06 /$ BA-S $0.04 /$ CD-S $2.63 \&$

/ CR-S 4.95/ PB-S $0.17 /$ SE-S $0.03 /$ AG-S 0.03

STREAM FUEIMIX1

SUBSTREAM MIXED TEMP=68 PRES=1 <ATM> MASS-ELOW=1

MASS-FRAC O2 $0.222 / \mathrm{N} 20.732 / \mathrm{CH} 40.046$

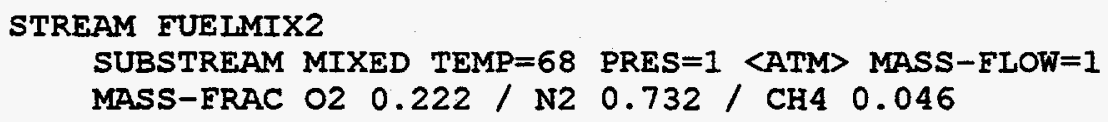

STREAM NAOH

SUBSTREAM MIXED TEMP $=68$ PRES $=1$ <ATM>

SUBSTREAM CISOLID TEMP=68 PRES=1 <ATM> MASS-ELOW=80

MASS-ERAC NAOH-S 1

STREAM OUT1

SUBSTREAM MIXED TEMP=2500 PRES=1 <ATM>

MASS-ELOW O2 $1293 /$ N2 $14610 /$ NO $27 /$ SO2 $3 /$ H2O \&

1977 / CO2 $2598 / \mathrm{HCL} 70 / \mathrm{EE} 30445$

SUBSTREAM CISOLID TEMP=2500 PRES=1 〈ATM>

MASS-FLOW SIO2-S $1069 /$ AL203-S $739 /$ CR-S $8 /$ MGO-S \& $18 /$ NA2O-S $10 /$ K2O-S $19 /$ CAO-S 56

\section{STREAM SCRUBH 20}

SUBSTREAM MIXED TEMP=68 PRES=1 <ATM> MASS-FLOW=1

MASS-ERAC H2O 1

STREAM SCRUBOUT

SUBSTREAM MIXED TEMP=120 PRES=1 <ATM>

MASS-FLOW O2 $1452 / \mathrm{N} 217490 / \mathrm{H} 20373000 / \mathrm{CO} 3088$

SUBSTREAM CISOLID TEMP $=120$ PRES $=1$ <ATM>

MASS-FLOW NACL-S 112

STREAM SOIL

SUBSTREAM MIXED TEMP=68 PRES=1 〈ATM> MASS-FLOW=62.5

MASS-FLOW H2O 0.1

SUBSTREAM CISOLID TEMP=68 PRES=1 <ATM> MASS-FLOW=562.5

MASS-FLOW SIO2-S $0.569 /$ AL2O3-S $0.102 /$ CACO3-S $0.128 /$ \& MGO-S $0.023 /$ NA2O-S $0.013 /$ K20-S $0.024 /$ FE304-S 0.041

BLOCK KILNSEP SEP

FRAC STREAM=ASH SUBSTREAM=MIXED COMPS=H2 O2 N2 CL2 F2 \& NO SO2 H2O $\mathrm{CO} 2 \mathrm{CO} H C L$ CH4 C-S S-s S NAOH-S NACL-S \& NAF-S AS CD HG SE FE SIO2 AL2O3 FE-S SIO2-S AL2O3-S \& AS-S BA-S CD-S CR-S PB-S SE-S AG-S CACO3-S MGO-S \& NA2O-S K2O-S FE304 FE304-S CAO-S FRACS $=0$ O 000 O $\begin{array}{lllllllllllllllllll}0 & 0 & 0 & 0 & 0 & 0 & .8 & .8 & 0 & .8 & .8 & .8 & 0 & 0 & 0 & 0 & .8 & .8 & \&\end{array}$ $\begin{array}{llllllllllllllll}.8 & .8 & .8 & .8 & .8 & .8 & .8 & .8 & .8 & .8 & .8 & .8 & .8 & .8 & .8 & \&\end{array}$ $.8 \quad .8 \quad .8$

FRAC STREAM=ASH SUBSTREAM=CISOLID COMPS=C-S S-S NAOH-S \& NACL-S NAF-S FE-S SIO2-S AL2O3-S AS-S BA-S CD-S CR-S \& PB-S SE-S AG-S CACO3-S MGO-S NA2O-S K2O-S FE3O4-S \&

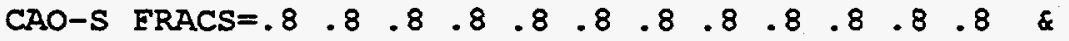
$\begin{array}{lllllllll}.8 & .8 & .8 & .8 & .8 & .8 & .8 & .8 & .8\end{array}$

FLASH-SPECS ASH TEMP $=68$ PRES $=1$ <ATM>

BLOCK SOLIDSEP SEP

FRAC STREAM=HGPLUS SUBSTREAM=MIXED COMPS=H2 O2 N2 CL2 \& 
F2 NO SO2 H2O CO2 CO HCL CH4 C-S S-S S NAOH-S \& NACL-S NAF-S AS CD HG SE FE SIO2 AL2O3 FE-S SIO2-S \& AL2O3-S AS-S BA-S CD-S CR-S PB-S SE-S AG-S CACO3-S \& MGO-S NA2O-S K2O-S FE3O4 FE304-S CAO-S FRACS $=0$ O 0 O \& $\begin{array}{lllllllllllllllllllllll}0 & 0 & 0 & 0 & 0 & 0 & 0 & 0 & 0 & 0 & 0 & 0 & 0 & 0 & 0 & 1 & 1 & 1 & 1 & 0 & 0 & \&\end{array}$

$\begin{array}{lllllllllllllllllllllllll}0 & 0 & 0 & 0 & 1 & 0 & 1 & 0 & 0 & 1 & 0 & 0 & 0 & 0 & 0 & 0 & 0 & 0\end{array}$

FRAC STREAM=HGPLUS SUBSTREAM=CISOLID COMPS=C-S $S-S$ NAOH-S NACL-S NAF-S FE-S SIO2-S AL2O3-S AS-S BA-S CD-S CR-S \& PB-S SE-S AG-S CACO3-S MGO-S NA2O-S K2O-S FE3O4-S \& CAO-S FRACS $=0 \begin{array}{lllllllllllllllllllll}0 & 0 & 0 & 0 & 0 & 0 & 0 & 1 & 0 & 1 & 0 & 0 & 1 & 0 & 0 & 0 & 0 & 0 & 0 & 0\end{array}$ FRAC STREAM=FLYASH SUBSTREAM=MIXED COMPS=H2 O2 N2 CL2 \& F2 NO SO2 H2O CO2 CO HCL CH4 C-S S-S S NAOH-S \& NACI-S NAF-S AS CD HG SE FE SIO2 AL2O3 FE-S SIO2-S \& AL203-S AS-S BA-S CD-S CR-S PB-S SE-S AG-S CACO3-S \& MGO-S NA2O-S K2O-S FE3O4 FE3O4-S CAO-S FRACS $=0$ O 0 \& \& $0 \begin{array}{lllllllllllllllllllll:}0 & 0 & 0 & 0 & 0 & 0 & 0 & 0 & 1 & 1 & 1 & 0 & 0 & 0 & 0 & 0 & 0 & 0 & 1 & 1\end{array}$ $\begin{array}{lllllllllllllllllllllll}1 & 1 & 1 & 1 & 0 & 1 & 0 & 1 & 1 & 0 & 1 & 1 & 1 & 1 & 1 & 1 & 1 & 1\end{array}$

FRAC STREAM=FLYASH SUBSTREAM=CISOLID COMPS=C-S S-S NAOH-S \& NACL-S NAF-S FE-S SIO2-S AL203-S AS-S BA-S CD-S CR-S \& PB-S SE-S AG-S CACO3-S MGO-S NA2O-S K2O-S FE3O4-S \&

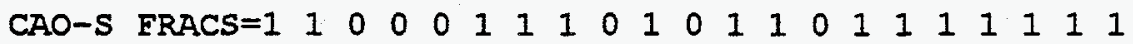

BIOCK L-V-SEP FLASH2

PARAM TEMP $=120$ PRES $=1$ <ATM>

BLOCK I-V-SEP2 FLASH2

PARAM TEMP $=3000$ PRES $=1$ <ATM>

BLOCK KILN RGIBBS

PARAM TEMP $=1600$ PRES $=1$ <ATM> NPHASE $=2$

PROD H2 / O2 / N2 / CL2 / F2 / NO / SO2 / H2O / \& $\mathrm{CO} 2 / \mathrm{CO} / \mathrm{HCL} / \mathrm{AS} / \mathrm{CD} / \mathrm{HG} / \mathrm{SE} / \mathrm{FE}-\mathrm{S} S \mathrm{SS} \&$ / SIO2-S SS / AL2O3-S SS / AS-S SS / BA-S SS / \& CD-S SS / CR-S SS / PB-S SS / SE-S SS / AG-S SS \& / MGO-S SS / NA2O-S SS / K20-S SS / FE304-S SS / \& CAO-S SS

PROD-FRAC FE-S 1 / FE3O4-S 1

BLOCK MELTER RGIBBS

PARAM TEMP $=3000$ PRES $=1$ <ATM $>$ NPHASE $=2$

PROD H2 / C-S SS / FE / SIO2 / AL2O3 / FE-S SS / \& SIO2-S SS / AL2O3-S SS / BA-S SS / CD / CD-S SS

BLOCK Q1 RGIBBS

PARAM TEMP $=68$ PRES $=1$ <ATM> NPHASE $=2$ TAPP $=1532$

PROD H2 / O2/ N2/ CL2/ 2 2/ NO / SO2/ H2O / \& $\mathrm{CO} 2 / \mathrm{CO} / \mathrm{HCL} / \mathrm{AS} / \mathrm{CD} / \mathrm{HG} / \mathrm{SE} / \mathrm{FE}-\mathrm{S} S \mathrm{SS} \&$ / SIO2-S SS / AL203-s SS / AS-S SS / BA-S SS / \& CD-S SS / CR-S SS / PB-S SS / SE-S SS / AG-S SS \& / MGO-S SS / NA2O-S SS / K2O-S SS / FE3O4-S SS / \& CAO-S SS

PROD-FRAC FE-S 1 / FE3O4-S 1

BLOCK Q2 RGIBBS

PARAM TEMP $=68$ PRES $=1$ <ATM $>$ NPHASE $=2$ CHEMEQ $=Y E S$ TAPP $=2132$

$\mathrm{PROD} \mathrm{H} 2 / \mathrm{O} 2 / \mathrm{N} 2 / \mathrm{CL} 2 / \mathrm{F} 2 / \mathrm{NO} / \mathrm{SO} 2 / \mathrm{H} 2 \mathrm{O} / \&$ $\mathrm{CO} 2 / \mathrm{CO} / \mathrm{HCL} / \mathrm{CH} 4 / \mathrm{S} / \mathrm{AS} / \mathrm{CD} / \mathrm{HG} / \mathrm{SE} \&$ / FE-S SS / SIO2-S SS / AL203-s ss / BA-S SS / \& CR-S SS / PB-S SS / AG-S SS / MGO-S SS / NA2O-S \& SS / K2O-S SS / FE 304-S SS / CAO-S SS

PROD-ERAC FE-S $1 /$ FE3O4-S 1 
BIOCK SCC RGIBBS

PARAM TEMP $=2200$ PRES $=1$ <ATM> NPHASE=2 MAXIT $=100$

PROD H2/ O2/ $\mathrm{N} 2 / \mathrm{CL} 2 / \mathrm{F} 2 / \mathrm{NO} / \mathrm{SO} 2 / \mathrm{H} 2 \mathrm{O} /$ \& $\mathrm{CO} 2 / \mathrm{CO} / \mathrm{HCL} / \mathrm{CH} 4 / \mathrm{S} / \mathrm{AS} / \mathrm{CD} / \mathrm{HG} / \mathrm{SE}$ \& / FE-S SS / SIO2-S SS / AL203-S SS / BA-S SS / \& CR-S SS / PB-S SS / AG-S SS / MGO-S SS / NA2O-S \& SS / K2O-S SS / FE304-S SS / CAO-S SS

PROD-FRAC FE-S $1 /$ FE304-S 1

BLOCK SCRUB RGIBBS

PARAM TEMP $=120$ PRES $=1$ <ATM> NPHASE $=2$

$\mathrm{PROD} \mathrm{H} 2 / \mathrm{O} 2 / \mathrm{N} 2 / \mathrm{CL} 2 / \mathrm{F} 2 / \mathrm{NO} / \mathrm{SO} 2 / \mathrm{H} 2 \mathrm{O} /$ \& $\mathrm{CO} 2 / \mathrm{CO} / \mathrm{HCL} / \mathrm{NAOH}-\mathrm{S}$ sS / NACL-S SS / NAF-S \& SS / AS / CD / HG / SE / EE-S SS / SIO2-S SS / \& AL203-S SS / AS-S SS / BA-S SS / CD-S SS / CR-S \& $\mathrm{SS} / \mathrm{PB}-\mathrm{S} \mathrm{SS} / \mathrm{SE}-\mathrm{S}$ SS/AG-S SS/MGO-S SS/\& NA20-S SS / K20-S SS / FE304-s Ss / CAO-S SS

\section{BLOCK VITRIFY RGIBBS}

PARAM TEMP $=3000$ PRES $=1$ <ATM> NPHASE $=2$

$\mathrm{PROD} H 2 / \mathrm{O} 2 / \mathrm{N} 2 / \mathrm{CL} 2 / \mathrm{F} 2 / \mathrm{NO} / \mathrm{SO} 2 / \mathrm{H} 2 \mathrm{O} /$ \& $\mathrm{CO} 2 / \mathrm{CO} / \mathrm{HCL} / \mathrm{C}-\mathrm{S} / \mathrm{S} / \mathrm{AS} / \mathrm{CD} / \mathrm{HG} / \mathrm{SE}$ \& / FE-S SS / SIO2 / AL203 / As-S ss / BA-S ss / \& CD-S SS / CR-S SS / PB-S SS / SE-S SS / AG-S SS \& / MGO-S SS / NA2O-S SS / K2O-S SS / FE304-S SS / \& CAO-S SS

PROD-FRAC EE-S 1 / FE304-S 1

BIOCK-OPTION RESTART=NO

BLOCK DUPL1 DUPPI

BLOCK DUPL2 DUPI.

DESIGN-SPEC NAOH

DEFINE CAUIN MASS-FLOW STREAM=NAOH SUBSTREAM=CISOLID \& COMPONENT $=$ NAOH $-S$

DEFINE CAUOUT MASS-FLOW STREAM=SCRUBOUT SUBSTREAM=CISOLID \& COMPONENT $=$ NAOH $-S$

SPEC "CAUIN-100.*CAUOUT" TO "O"

TOL-SPEC "0.01"

VARY STREAM-VAR STREAM=NAOH SUBSTREAM=CISOLID \& VARIABLE=MASS - ELOW

LIMITS "70" "1000"

DESIGN-SPEC OXI

DEFINE AIR MASS-FLOW STREAM=AIR SUBSTREAM=MIXED \& COMPONENT $=02$

DEFINE WC MASS-FLOW STREAM=EDWASTEC SUBSTREAM=MIXED \& COMPONENT $=02$

DEFINE WN MASS-FLOW STREAM=FDWASTEN SUBSTREAM=MIXED \& COMPONENT $=02$

DEFINE F1 MASS-FLOW STREAM=FUEIMIX1 SUBSTREAM=MIXED \& COMPONENT $=02$

DEFINE OXOUT MASS-FLOW STREAM=KILNOUT SUBSTREAM=MIXED \& COMPONENT $=02$

DEFINE AL MASS-FLOW STREAM=AIRLEAKS SUBSTREAM=MIXED \& COMPONENT $=02$

SPEC "AL+AIR+WC+WN+ $(F 1 / 6.0)$ " TO "2.0*OXOUT"

TOL-SPEC "I "

VARY STREAM-VAR STREAM=AIR SUBSTREAM=MIXED \& VARIABLE=MASS-FLOW

LIMITS " 1 " "10000" 


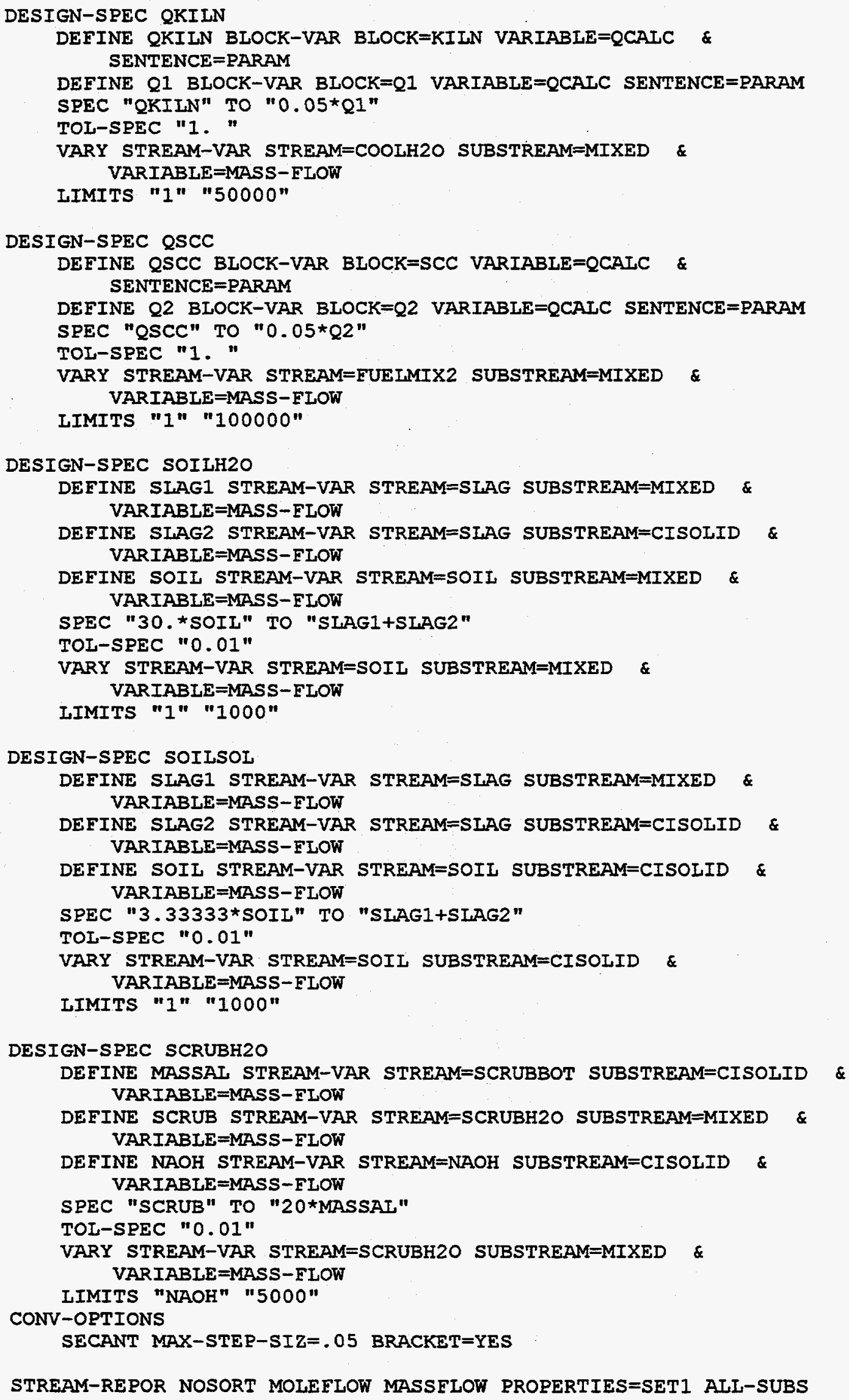




\section{System A-4}

TITLE 'ITTS PHASE I - ROTARY KILN W/ CO2 RETENTION - MODEL A4-3 '

IN-UNITS ENG

DEF-STREAMS MIXCISID ALI

DIAGNOSTICS

TERMINAI SIM-IEVEL=8 CONV-LEVEL $=8$

RUN-CONTROI MAX-TIME $=1800$

DATABANKS COMBUST / INORGANIC / PURECOMP / SOLIDS / \& NOASPENPCD

PROP-SOURCES COMBUST . / INORGANIC / PURECOMP / SOLIDS

COMPONENTS

$\mathrm{H} 2 \mathrm{H} 2 \mathrm{H} 2 /$

$020202 \%$

N2 N2 N2 /

$\mathrm{CL} 2 \mathrm{CL} 2 \mathrm{CI} 2 /$

F2 F2 F2/

NO NO NO /

SO2 025 so2 /

$\mathrm{H} 2 \mathrm{O}$ H2O H2O /

$\mathrm{CO} 2 \mathrm{CO} 2 \mathrm{CO} 2 /$

$\mathrm{CO} \mathrm{CO} \mathrm{CO} /$

HCI HCI HCL /

$\mathrm{CH} 4 \mathrm{CH} 4 \mathrm{CH} 4 /$

C-S C C-S /

s-s $s$ s-s/

$\mathbf{s} \mathbf{s} /$

NAOH-S NAOH NAOH-S /

NACL-S NACL NACL-S /

NAF-S NAF NAF-S /

AS AS AS /

$C D C D C D /$

HG HG HG /

SE SE SE /

FE FE FE /

SIO2 SIO2 SIO2 /

AL203 AL203 AL2O3 /

EE-S FE FE-S /

SIO2-S SIO2 SIO2-S /

AL203-S AL2O3 AL203-S /

AS-S AS AS-S /

BA-S BA BA-S /

CD-S CD CD-S /

CR-S CR CR-S /

PB-S PB PB-S /

SE-S SE SE-S /

AG-S AG AG-S /

CACO3-S CACO3 CACO3-S /

MGO-S MGO MGO-S /

NA2O-S NA2O NA2O-S /

K2O-S K2O K2O-S /

FE304 FE304 FE304/

FE304-S FE3O4 FE304-S /

CAO-S CAO CAO-S / 
CACL2-S CACL2 CACL2-S /

CASO4-S CASO4 CASO4-S

FLOWSHEET

BLOCK KIIN IN=FDWASTEN SOIL FUEIMIXI FDWASTEC OXYGEN \& AIRLEAKS COOLH2O EX-RECYC OUT=OUT1

BIOCK KIINSEP IN=KIINOUT OUT=ASH KIINVAP

BIOCK SCC IN=KILNVAP FUEIMIX2 OUT=OUT2

BLOCK BAGHOUSE IN=QUENCHED OUT=HGPLUS FLYASH QNCHVAP

BLOCK QUENCH IN=SCCOUT QNCH2O OUT=QUENCHED

BLOCK DUPL1 IN=OUT1 OUT=KIINOUT DUM1IN

BLOCK Q1 IN=DUMIIN OUT=DUM1OUT

BLOCK DUPL2 IN=OUT2 OUT=SCCOUT DUM2IN

BLOCK 22 IN=DUM2IN OUT=DUM2OUT

BLOCK VITRIFY IN=FLYASH ASH OUT=VITOUT

BLOCK VIT-SEP IN=VITOUT OUT=VIT-VAP SLAG

BIOCK SCRUBSEP IN=SCRUBOUT OUT=SCRUBVAP SCRUBBOT

BIOCK ABSSEP IN=ABSORBED OUT=ABSGAS ABSSOL

BLOCK CO2-DES IN=ABSSOL OUT=CO2-DES

BIOCK EIASH IN=ABSGAS OUT=EXHAUST H2OOUT

BLOCK CO2-SEP IN=CO2-DES OUT=CO2 CAO-OUT

BLOCK SPLIT IN=CAO-OUT OUT=RECYCLE CAOSCRUB SPENTCAO

BIOCK CAOMIXER IN=RECYCLE CAO-IN OUT=CAOMIXED

BLOCK DRYSCRUB IN=CAOSCRUB QNCHVAP OUT=SCRUBOUT

BLOCK GASQUENC IN=QNCH2O-2 CO2 OUT=CO2OUT

BLOCK CO2-ABS IN=SCRUBVAP CAOMIXED OUT=ABSORBED

BLOCK EX-SPIIT IN=EXHAUST OUT=OFFGAS EX-RECYC

BLOCK MEITER IN=FDMETMLT OUT=METAI

PROPERTIES SOLIDS

PROPERTIES IDEAL / RKS-BM

PROP-SET ALI-SUBS TEMP PRES VFRAC UNITS='ATM' SUBSTREAM=ALI

PROP-SET SET1 VISTDMX UNITS='CUFT/HR' SUBSTREAM=MIXED

STREAM AIRIEAKS

SUBSTREAM MIXED TEMP $=68$ PRES $=1$ <ATM> \&

VOIUME-FLOW $=600$ <CUET/MIN>

MASS-FRAC $020.233 / \mathrm{N} 20.767$

STREAM CAO-IN

SUBSTREAM CISOLID TEMP=68 PRES=1 <ATM> MASS-FLOW=373.0501

MASS-FRAC CAO-S 1

STREAM COOLH2O

SUBSTREAM MIXED TEMP=68 PRES=1 <ATM> MASS-FLOW=1

MASS-FRAC H2O 1

STREAM FDMETMIT

SUBSTREAM MIXED TEMP=68 PRES=1 <ATM> MASS-FLOW=1.20

MASS-ELOW H2 2.42

SUBSTREAM CISOLID TEMP=68 PRES=1 <ATM> MASS-FLOW=147.80

MASS-ELOW C-S $14.44 /$ FE-S $282.02 /$ SIO2-S $0.035 /$ \&

AL203-S $0.035 / \mathrm{BA}-\mathrm{S} 0.87 / \mathrm{CD}-\mathrm{S} 0.18$

STREAM FDWASTEC

SUBSTREAM MIXED TEMP=68 PRES=1 <ATM>

MASS-ELOW H2 $50.95 / 0292.76 / \mathrm{N} 20.23 / \mathrm{CL} 253.02 / \&$ F2 $0.11 / \mathrm{H} 2019.83$

SUBSTREAM CISOLID TEMP $=68$ PRES $=1$ <ATM>

MASS-ELOW C-S $326.28 /$ S-S $0.49 /$ FE-S $7.67 /$ SIO2-S \& 
53.01/ AL203-S 53.01/ BA-S $0.77 /$ CD-S $0.77 / \&$ CR-S 1.53

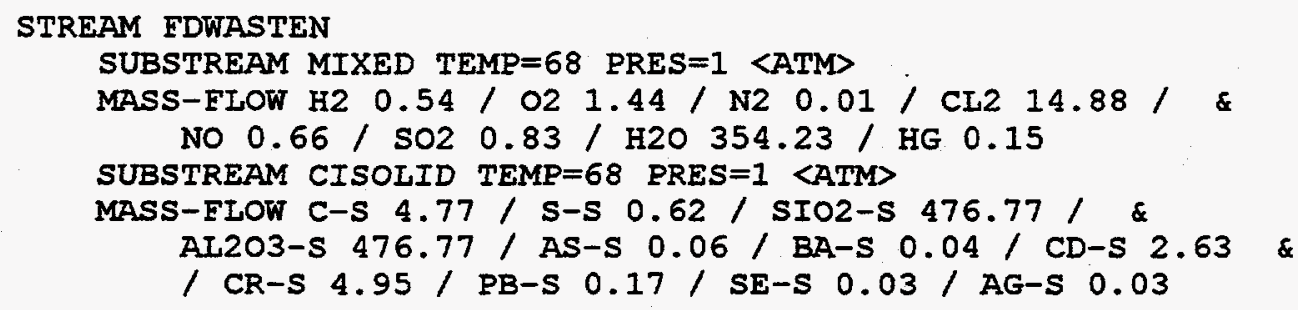

STREAM FUEIMIX1

SUBSTREAM MIXED TEMP=68 PRES=1 <ATM> MASS-FLOW=1481.360

MASS-FIOW $020.222 / \mathrm{CH} 40.046$

STREAM EUEIMIX2

SUBSTREAM MIXED TEMP=68 PRES=1 <ATM> MASS-FLOW=2043.100

MASS-FLOW O2 $0.222 /$ CH4 0.046

STREAM OUT1

SUBSTREAM MIXED TEMP $=2500$ PRES $=1$ <ATM>

MASS-FLOW $021293 / \mathrm{N} 214610 /$ NO $27 /$ SO2 $3 / \mathrm{H} 2 \mathrm{O}$ \& $1977 / \mathrm{CO} 2598 / \mathrm{HCL} 70 / \mathrm{FE} 30445$

SUBSTREAM CISOLID TEMP $=2500$ PRES $=1$ <ATM>

MASS-FLOW SIO2-S $1069 /$ AL2O3-S $739 /$ CR-S $8 /$ MGO-S \& $18 /$ NA2O-S $10 /$ K2O-S $19 /$ CAO-S 56

STREAM OXYGEN

SUBSTREAM MIXED TEMP=68 PRES=1 <ATM> MASS-FLOW=206.4140 MASS-ERAC O2 1

STREAM QNCH2O

SUBSTREAM MIXED TEMP=68 PRES=1 <ATM> MASS-FLOW=13281.81 MASS-ERAC H2O 1

STREAM QNCH2O-2

SUBSTREAM MIXED TEMP=68 PRES=1 <ATM> MASS-FLOW=915.7221 MASS-FRAC H2O 1

STREAM QUENCHED

SUBSTREAM MIXED TEMP=350 PRES=1 <ATM>

MASS-ELOW O2 $1446 /$ N2 $17490 /$ CL2 $29 / \mathrm{H} 2016320 /$ \& $\mathrm{CO} 23088 / \mathrm{HCL} 40$

SUBSTREAM CISOLID TEMP $=350$ PRES $=1$ <ATM>

MASS-FIOW SIO2-S $221 /$ AL2O3-S $148 /$ CD-S $3.3 /$ CR-S \& $1.62 /$ MGO-S $3.6 /$ NA2O-S $2 / \mathrm{K} 20-\mathrm{S} 3.75 /$ \& EE304-5 9

STREAM SOIL

SUBSTREAM MIXED TEMP=68 PRES=1 <ATM> MASS-FLOW=49.84335

MASS-ELOW H2O 0.1

SUBSTREAM CISOLID TEMP=68 PRES=1 <ATM> MASS-FLOW=448.59

MASS-FLOW SIO2-S $0.569 /$ AL203-S $0.102 /$ CACO3-S $0.128 /$ \& MGO-S $0.023 /$ NA2O-S $0.013 /$ K2O-S $0.024 /$ FE3O4-S 0.041

BIOCK CAOMIXER MIXER

PARAM PRES $=1$ <ATM> NPHASE $=3$

BLOCK EX-SPLIT FSPIIT

FRAC EX-RECYC 0.90 
BLOCK SPIIT FSPLIT

FRAC RECYCLE 0.9 / CAOSCRUB 0.0154191

BLOCK ABSSEP SEP

FRAC STREAM=ABSSOI SUBSTREAM=MIXED COMPS=H2 O2 N2 CL2 \& F2 NO SO2 H2O CO2 CO HCL CH4 C-S S-S S NAOH-S \& NACL-S NAF-S AS CD HG SE FE SIO2 AL2O3 FE-S SIO2-S \& AL203-S AS-S BA-S CD-S CR-S PB-S SE-S AG-S CACO3-S \& MGO-S NA2O-S K2O-S FE3O4 FE3O4-S CAO-S CACL2-S CASO4-S \& FRACS $=0 \begin{array}{lllllllllllllllllllll}0 & 0 & 0 & 0 & 0 & 0 & 0 & 0 & 0 & 0 & 0 & 0 & 1 & 1 & 1 & 1 & 1 & 1 & \&\end{array}$ $\begin{array}{lllllllllllllllllllllllll}1 & 1 & 1 & 1 & 1 & 1 & 1 & 1 & 1 & 1 & 1 & 1 & 1 & 1 & 1 & 1 & 1 & 1 & 1 & 1 & 1 & \varepsilon\end{array}$ $\begin{array}{lllll}1 & 1 & 1 & 1 & 1\end{array}$

FRAC STREAM=ABSSOL SUBSTREAM=CISOLID COMPS=C-S $S-S$ NAOH-S \& NACL-S NAF-S FE-S SIO2-S AL2O3-S AS-S BA-S CD-S CR-S \& PB-S SE-S AG-S CACO3-S MGO-S NA2O-S K2O-S FE3O4-S\&

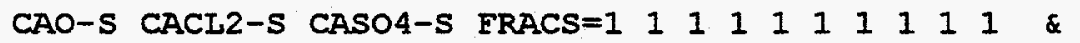
$\begin{array}{lllllllllllllll}1 & 1 & 1 & 1 & 1 & 1 & 1 & 1 & 1 & 1 & 1 & 1 & 1\end{array}$

BLOCK BAGHOUSE SEP

FRAC STREAM=HGPLUS SUBSTREAM=MIXED COMPS=H2 O2 N2 CL2 \& F2 NO SO2 H2O CO2 CO HCL CH4 C-S S-S S NAOH-S \& NACL-S NAF-S AS CD HG SE FE SIO2 AL2O3 FE-S SIO2-S \& AL2O3-S AS-S BA-S CD-S CR-S PB-S SE-S AG-S CACO3-S \& MGO-S NA2O-S K2O-S FE3O4 FE304-S CAO-S FRACS $=0$ O 0 \& $0 \begin{array}{lllllllllllllllllllll}0 & 0 & 0 & 0 & 0 & 0 & 0 & 0 & 0 & 0 & 0 & 0 & 0 & 0 & 1 & 1 & 1 & 1 & 0 & 0 & \&\end{array}$ $\begin{array}{llllllllllllllllll}0 & 0 & 0 & 0 & 1 & 0 & 1 & 0 & 0 & 1 & 0 & 0 & 0 & 0 & 0 & 0 & 0 & 0\end{array}$

FRAC STREAM=HGPLUS SUBSTREAM=CISOIID COMPS=C-S S-S NAOH-S \& NACL-S NAF-S FE-S SIO2-S AL2O3-S AS-S BA-S CD-S CR-S \& PB-S SE-S AG-S CACO3-S MGO-S NA2O-S K2O-S FE3O4-S \&

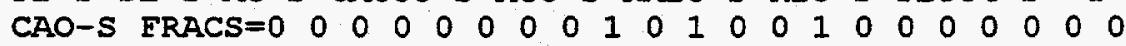

FRAC STREAM=FLYASH SUBSTREAM=MIXED COMPS=H2 O2 N2 CL2 \& F2 NO SO2 H2O CO2 CO HCL CH4 C-S S-S S NAOH-S \& NACL-S NAF-S AS CD HG SE FE SIO2 AL2O3 FE-S SIO2-S \& AL2O3-S AS-S BA-S CD-S CR-S PB-S SE-S AG-S CACO3-S \& MGO-S NA2O-S K2O-S FE304 FE304-S CAO-S FRACS $=0$ O 00 \& $\begin{array}{lllllllllllllllllllllll}0 & 0 & 0 & 0 & 0 & 0 & 0 & 0 & 0 & 1 & 1 & 1 & 1 & 1 & 1 & 0 & 0 & 0 & 0 & 1 & 1 & \&\end{array}$ $\begin{array}{lllllllllllllllllllllllllll}1 & 1 & 1 & 1 & 0 & 1 & 0 & 1 & 1 & 0 & 1 & 1 & 1 & 1 & 1 & 1 & 1 & 1\end{array}$

FRAC STREAM=FLYASH SUBSTREAM=CISOLID COMPS=C-S S-S NAOH-S\& NACL-S NAF-S FE-S SIO2-S AL2O3-S AS-S BA-S CD-S CR-S \& PB-S SE-S AG-S CACO3-S MGO-S NA2O-S K2O-S FE3O4-S \&

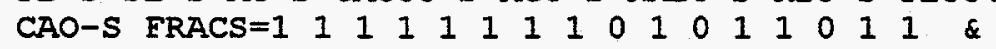
$\begin{array}{lllll}1 & 1 & 1 & 1 & 1\end{array}$

FLASH-SPECS FLYASH TEMP=68 PRES=1 <ATM>

BLOCK CO2-SEP SEP

FRAC STREAM=CO2 SUBSTREAM=MIXED COMPS=H2 O2 N2 CL2 F2 \& NO SO2 $\mathrm{H} 2 \mathrm{O} \mathrm{CO} 2$ CO HCL CH4 C-S S-S S NAOH-S NACL-S \& NAF-S AS CD HG SE FE SIO2 AL2O3 FE-S SIO2-S AL2O3-S \& AS-S BA-S CD-S CR-S PB-S SE-S AG-S CACO3-S MGO-S \& NA2O-S K2O-S FE3O4 FE3O4-S CAO-S CACL2-S CASO4-S \& FRACS $=0 \begin{array}{llllllllllllllllll}0 & 0 & 0 & 0 & 0 & 0 & 0 & 0 & 1 & 0 & 0 & 0 & 0 & 0 & 0 & 0 & 0 & 0\end{array}$ $0 \begin{array}{lllllllllllllllllllllllll}0 & 0 & 0 & 0 & 0 & 0 & 0 & 0 & 0 & 0 & 0 & 0 & 0 & 0 & 0 & 0 & 0 & 0 & 0 & 0 & 0 & 0 & 0 & 0 & 0\end{array}$

FRAC STREAM=CO2 SUBSTREAM=CISOLID COMPS=C-S $S-S$ NAOH-S \& NACL-S NAF-S FE-S SIO2-S AL203-S AS-S BA-S CD-S CR-S \& PB-S SE-S AG-S CACO3-S MGO-S NA2O-S K2O-S FE3O4-S \& CAO-S CACL2-S CASO4-S FRACS $=0 \begin{array}{llllllllllll}0 & 0 & 0 & 0 & 0 & 0 & 0 & 0 & 0\end{array}$ 0000000000000000

FLASH-SPECS CAO-OUT TEMP $=68$ PRES $=1$ <ATM>

BLOCK KIINSEP SEP

FRAC STREAM=ASH SUBSTREAM=MIXED COMPS=H2 O2 N2 CL2 F2 \& NO $\mathrm{SO} 2 \mathrm{H} 2 \mathrm{O} \mathrm{CO} 2 \mathrm{CO} \mathrm{HCL}$ CH4 C-S S-S S NAOH-S NACL-S \& 
NAF-S AS CD HG SE FE SIO2 AL2O3 FE-S SIO2-S AL2O3-S \& AS-S BA-S CD-S CR-S PB-S SE-S AG-S CACO3-S MGO-S \&

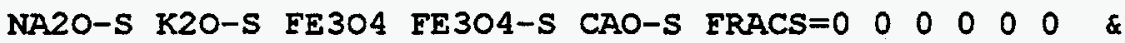
$\begin{array}{lllllllllllllllllll}0 & 0 & 0 & 0 & 0 & 0 & .8 & .8 & 0 & .8 & .8 & .8 & 0 & 0 & 0 & 0 & .8 & .8 & \&\end{array}$ $\begin{array}{llllllllllllllllll}.8 & .8 & .8 & .8 & .8 & .8 & .8 & .8 & .8 & .8 & .8 & .8 & .8 & .8 & .8 & .8 & .8 & .8\end{array}$

FRAC STREAM=ASH SUBSTREAM=CISOLID COMPS=C-S S-S NAOH-S \& NACL-S NAF-S FE-S SIO2-S AL2O3-S AS-S BA-S CD-S CR-S \& PB-S SE-S AG-S CACO3-S MGO-S NA20-S K2O-S FE3O4-S \&

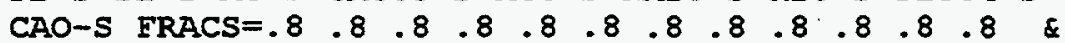
$\begin{array}{llllllllll}.8 & .8 & .8 & .8 & .8 & .8 & .8 & .8 & .8\end{array}$

FIASH-SPECS ASH TEMP $=68$ PRES $=1$ <ATM>

\section{BLOCK SCRUBSEP SEP}

FRAC STREAM=SCRUBBOT SUBSTREAM=MIXED COMPS=H2 O2 N2 CI2 \& F2 NO SO2 H2O CO2 CO HCI CH4 C-s s-s s NAOH-S \& NACL-S NAF-S AS CD HG SE FE SIO2 AI2O3 FE-S SIO2-S \& AL2O3-S AS-S BA-S CD-S CR-S PB-S SE-S AG-S CACO3-S \& MGO-S NA2O-S K2O-S FE304 FE304-S CAO-S CACL2-S CASO4-S \& FRACS $=0 \begin{array}{llllllllllllllllll}0 & 0 & 0 & 0 & 0 & 0 & 0 & 0 & 0 & 0 & 0 & 1 & 1 & 0 & 1 & 1 & 1\end{array}$ $\begin{array}{llllllllllllllllllllllllll}1 & 1 & 1 & 1 & 1 & 1 & 1 & 1 & 1 & 1 & 1 & 1 & 1 & 1 & 1 & 1 & 1 & 1 & 1 & 1 & 1 & 1 & 1 & 1 & 1 & 1\end{array}$

ERAC STREAM $=S C R U B B O T$ SUBSTREAM $=C I S O L I D$ COMPS $=C-S \mathrm{~S}-S \&$ NAOH-S NACI-S NAF-S FE-S SIO2-S AL2O3-S AS-S BA-S \& CD-S CR-S PB-S SE-S AG-S CACO3-S MGO-S NA2O-S K2O-S \&

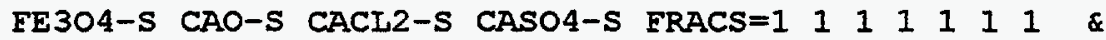
$\begin{array}{lllllllllllllllll} & 1 & 1 & 1 & 1 & 1 & 1 & 1 & 1 & 1 & 1 & 1 & 1 & 1 & 1 & 1\end{array}$

BLOCK VIT-SEP SEP

ERAC STREAM=VIT-VAP SUBSTREAM=MIXED COMPS=H2 O2 N2 CL2 \& F2 NO SO2 H2O CO2 CO HCL CH4 C-S s-s s NAOH-S \& NACL-S NAF-S AS CD HG SE FE SIO2 AL2O3 FE-S SIO2-S \& AL2O3-S AS-S BA-S CD-S CR-S PB-S SE-S AG-S CACO3-S \& MGO-S NA2O-S K2O-S FE304 FE304-S CAO-S ERACS=1 11 \& $\begin{array}{llllllllllllllllllllll}1 & 1 & 1 & 1 & 1 & 1 & 1 & 1 & 1 & 0 & 0 & 0 & 0 & 0 & 0 & 0 & 0 & 0 & 0 & 0 & 0 & \&\end{array}$

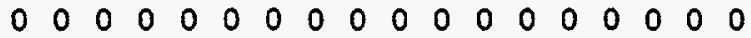

FRAC STREAM=VIT-VAP SUBSTREAM=CISOLID COMPS=C-S $\mathrm{S}-\mathrm{S}$ \& NAOH-S NACL-S NAF-S EE-S SIO2-S AI2O3-S AS-S BA-S \& CD-S CR-S PB-S SE-S AG-S CACO3-S MGO-S NA2O-S K2O-S \&

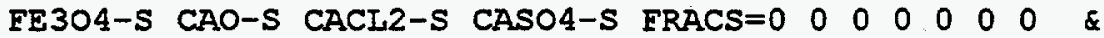
$0 \begin{array}{lllllllllllllll}0 & 0 & 0 & 0 & 0 & 0 & 0 & 0 & 0 & 0 & 0 & 0 & 0 & 0 & 0\end{array}$

BLOCK FLASH FIASH2 PARAM TEMP $=90$ PRES $=1$ <ATM> TOL $=0.000001$ PROPERTIES IDEAL

BLOCK CO2-ABS RSTOIC

PARAM TEMP $=1200$ PRES $=1$ <ATM> TOL $=0.000001$

STOIC 1 MIXED CO2 - 1 / CISOLID CAO-S - $1 /$ CACO3-S 1

CONV 1 MIXED CO2 1

BLOCK CO2-DES RGIBBS

PARAM TEMP $=1700$ PRES $=1$ <ATM> NPHASE $=2$

PROD CO2 / CAO-S SS

PROPERTIES IDEAL

\section{BLOCK DRYSCRUB RGIBBS}

PARAM TEMP $=350$ PRES $=1$ <ATM> NPHASE $=2$ MAXIT $=500$ TOL $=0.0001$

PROD H2 / O2 / N2 / $\mathrm{F} 2 / \mathrm{NO} / \mathrm{H} 2 \mathrm{O} / \mathrm{CO} 2 / \mathrm{CO} / \&$ HCL / CH4 / CAO-S SS / CACL2-S SS / CASO4-S SS

BLOCK GASQUENC RGIBBS

PARAM TEMP $=350$ PRES=1 <ATM> NPHASE $=2$

PROD H2 / O2/ $\mathrm{H} 2 \mathrm{O} / \mathrm{CO} 2 / \mathrm{CO} / \mathrm{C}-\mathrm{S}$ SS 
BLOCK KILN RGIBBS

DESCRIPTION "change tolerance to converge initially"

PARAM TEMP $=1600$ PRES $=1$ <ATM> NPHASE=2 MAXIT $=100$ \& TOI $=0.000001$

PROD H2 / O2 / N2 / CL2 / F2 / SO2 / H2O / CO2 / \& CO / HCL / AS / CD / HG / SE / FE-S SS / \& SIO2-s SS / AI2O3-s SS / AS-s ss / BA-S ss / \& CD-S SS / CR-S SS / PB-S SS / SE-S SS / AG-S SS \& / MGO-S SS / NA2O-S SS / K2O-S SS / FE3O4-S SS / \& CAO-S SS

PROD-FRAC FE-S $1 /$ FE304-S 1

\section{BLOCK MELTER RGIBBS}

PARAM TEMP $=3000$ PRES $=1$ <ATM> NPHASE $=2$ MAXIT $=500$

PROD H2 / C-S SS / FE / SIO2 / AL2O3 / FE-S SS / \& SIO2-S SS / AL2O3-S SS / BA-S SS / CD / CD-S SS

\section{BLOCK 01 RGIBBS}

PARAM TEMP=68 PRES=1 <ATM $>$ NPHASE $=2$ TAPP $=1532 \cdot \mathrm{TOL}=0.000001$

$\mathrm{PROD} \mathrm{H} 2 / \mathrm{O} 2 / \mathrm{N} 2 / \mathrm{CL} 2 / \mathrm{F} 2 / \mathrm{NO} / \mathrm{SO} 2 / \mathrm{H} 2 \mathrm{O} / \&$ $\mathrm{CO} 2 / \mathrm{CO} / \mathrm{HCL} / \mathrm{AS} / \mathrm{CD} / \mathrm{HG} / \mathrm{SE} / \mathrm{FE}-\mathrm{S}$ SS \& / SIO2-S SS / AL2O3-S SS / AS-S SS / BA-S SS / \& CD-S SS / CR-S SS / PB-S SS / SE-S SS / AG-S SS \& / MGO-S SS / NA2O-S SS / K2O-S SS / FE3O4-S SS / \& CAO-S SS

PROD-FRAC FE-S $1 /$ FE304-S 1

BLOCK Q2 RGIBBS

PARAM TEMP=68 PRES=1 <ATM> NPHASE=2 TAPP=2132 TOL=0.000001

PROD H2 / O2 / N2 / CL2 / 2 2 / NO / SO2 / H2O / \& $\mathrm{CO} 2$ / $\mathrm{CO} / \mathrm{HCL} / \mathrm{CH} 4 / \mathrm{S} / \mathrm{AS} / \mathrm{CD} / \mathrm{HG} / \mathrm{SE} \&$ / FE-S SS / SIO2-S SS / AL203-s SS / BA-S SS / \& CR-S SS / PB-S SS / AG-S SS / MGO-S SS / NA2O-S \& SS / K2O-S SS / FE3O4-S SS / CAO-S SS

PROD-FRAC FE-S 1 / FE304-S 1

\section{BLOCK QUENCH RGIBBS}

PARAM TEMP $=350$ PRES $=1$ <ATM $>$ NPHASE $=2$ MAXIT $=500$ TOI $=0.0001$ $\mathrm{PROD} \mathrm{H} 2 / \mathrm{O} 2 / \mathrm{N} 2 / \mathrm{F} 2 / \mathrm{NO} / \mathrm{SO} 2 / \mathrm{H} 2 \mathrm{O} / \mathrm{CO} 2 / \&$ CO / HCL / AS / CD / HG / SE / FE-S SS / \& SIO2-S SS / AL2O3-S SS / AS-S SS / BA-S SS / \& CD-S SS / CR-S SS / PB-S SS / SE-S SS / AG-S SS \& / MGO-S SS / NA2O-S SS / K2O-S SS / FE304-S SS / CAO-S SS

PROD-FRAC FE-S $1 /$ FE304-S 1

\section{BLOCK SCC RGIBBS}

PARAM TEMP $=2200$ PRES $=1$ <ATM $>$ NPHASE $=2$ TOL $=0.000001$ PROD H2 / O2 / N2 / CL2 / F2 / NO / SO2 / H2O / \& $\mathrm{CO} 2 / \mathrm{CO} / \mathrm{HCL} / \mathrm{S} / \mathrm{AS} / \mathrm{CD} / \mathrm{HG} / \mathrm{SE} / \&$ FE-S SS / SIO2-S SS / AL2O3-S SS / BA-S SS / \& CR-S SS / PB-S SS / AG-S SS / MGO-S SS / NA2O-S \& SS / K2O-S SS / FE304-S SS / CAO-S SS

PROD-FRAC EE-S 1 / FE3O4-S 1

BIOCK-OPTION RESTART $=$ NO

\section{BLOCK VITRIFY RGIBBS}

PARAM TEMP $=3000$ PRES $=1$ <ATM> NPHASE $=2$ TOL $=0.000001$ PROD H2 / O2 / N2 / CL2 / $\mathrm{E} 2$ / NO / SO2 / $\mathrm{H} 20$ / \& $\mathrm{CO} / \mathrm{CO} / \mathrm{HCL} / \mathrm{C}-\mathrm{s} \mathrm{Ss} / \mathrm{s} / \mathrm{AS} / \mathrm{CD} / \mathrm{HG} / \&$ $\mathrm{SE} / \mathrm{FE}-\mathrm{S}$ SS / SIO2 / AL2O3 / AS-S SS / BA-S SS \& $/ \mathrm{CD}-\mathrm{S}$ SS / CR-S SS / $\mathrm{PB}-\mathrm{S}$ SS / SE-S SS / AG-S \& 
SS / MGO-S SS / NA20-S SS / K20-S SS / FE304-S \&

SS / CAO-S SS

PROD-FRAC FE-S 1 / FE304-S 1

BLOCK-OPTION RESTART=NO

BLOCK DUPII DUPL

BLOCK DUPL2 DUPL

DESIGN-SPEC CAO

DEFINE CO2CON MOLE-FLOW STREAM=SCRUBVAP SUBSTREAM=MIXED \& COMPONENT $=\mathrm{CO} 2$

DEFINE CAOMXD MOLE-FLOW STREAM=CAOMIXED SUBSTREAM=CISOLID \& COMPONENT $=$ CAO-S

SPEC "CO2CON" TO "CAOMXD"

TOL-SPEC "O.1"

VARY STREAM-VAR STREAM=CAO-IN SUBSTREAM=CISOLID \& VARIABLE =MASS - FLOW

IIMITS "1" "10000"

DESIGN-SPEC OXYGEN

DEFINE OXIN MASS-FLOW STREAM=OXYGEN SUBSTREAM=MIXED \& COMPONENT $=02$

DEFINE WC MASS-FLOW STREAM=FDWASTEC SUBSTREAM=MIXED \& COMPONENT $=02$

DEEINE WN MASS-ELOW STREAM=FDWASTEN SUBSTREAM=MIXED \& COMPONENT $=02$

DEFINE E1 MASS-FLOW STREAM=FUELMIX1 SUBSTREAM=MIXED \& COMPONENT $=02$

DEFINE AI MASS-FIOW STREAM=AIRLEAKS SUBSTREAM=MIXED \& COMPONENT $=02$

DEFINE EX MASS-FLOW STREAM=EX-RECYC SUBSTREAM=MIXED \& COMPONENT $=02$

DEFINE OXOUT MASS-FLOW STREAM=KILNOUT SUBSTREAM=MIXED \& COMPONENT $=02$

SPEC "AL+OXIN+WC+WN+(E1/6.0)+EX" TO "OXOUT*2.0"

TOL-SPEC "O.1"

VARY STREAM-VAR STREAM=OXYGEN SUBSTREAM=MIXED \& VARIABLE=MASS-FLOW

LIMITS "1 " $100000 "$

DESIGN-SPEC QKIINI

DEFINE QKILN BIOCK-VAR BLOCK=KIIN VARIABLE=QCALC \& SENTENCE = PARAM

DEFINE $Q 1$ BLOCK-VAR BLOCK=Q1 VARIABLE=QCALC SENTENCE=PARAM

SPEC "QKILN" TO "0.05* $Q 1 "$

TOI-SPEC " 1 ."

VARY STREAM-VAR STREAM=FUEIMIX1 SUBSTREAM=MIXED \& VARIABLE=MASS-FLOW

LIMITS "1" "50000"

DESIGN-SPEC QQUENCH

DEFINE QQNCH BLOCK-VAR BLOCK=QUENCH VARIABLE=QCALC \& SENTENCE = PARAM

SPEC "QQNCH" TO "O"

TOL-SPEC "1"

VARY STREAM-VAR STREAM=QNCH20 SUBSTREAM=MIXED \& VARIABLE=MASS - LLOW

LIMITS "1" "100000"

DESIGN-SPEC QQUENCH2

DEFINE QGASQU BLOCK-VAR BLOCK=GASQUENC VARIABLE=QCAIC \& 


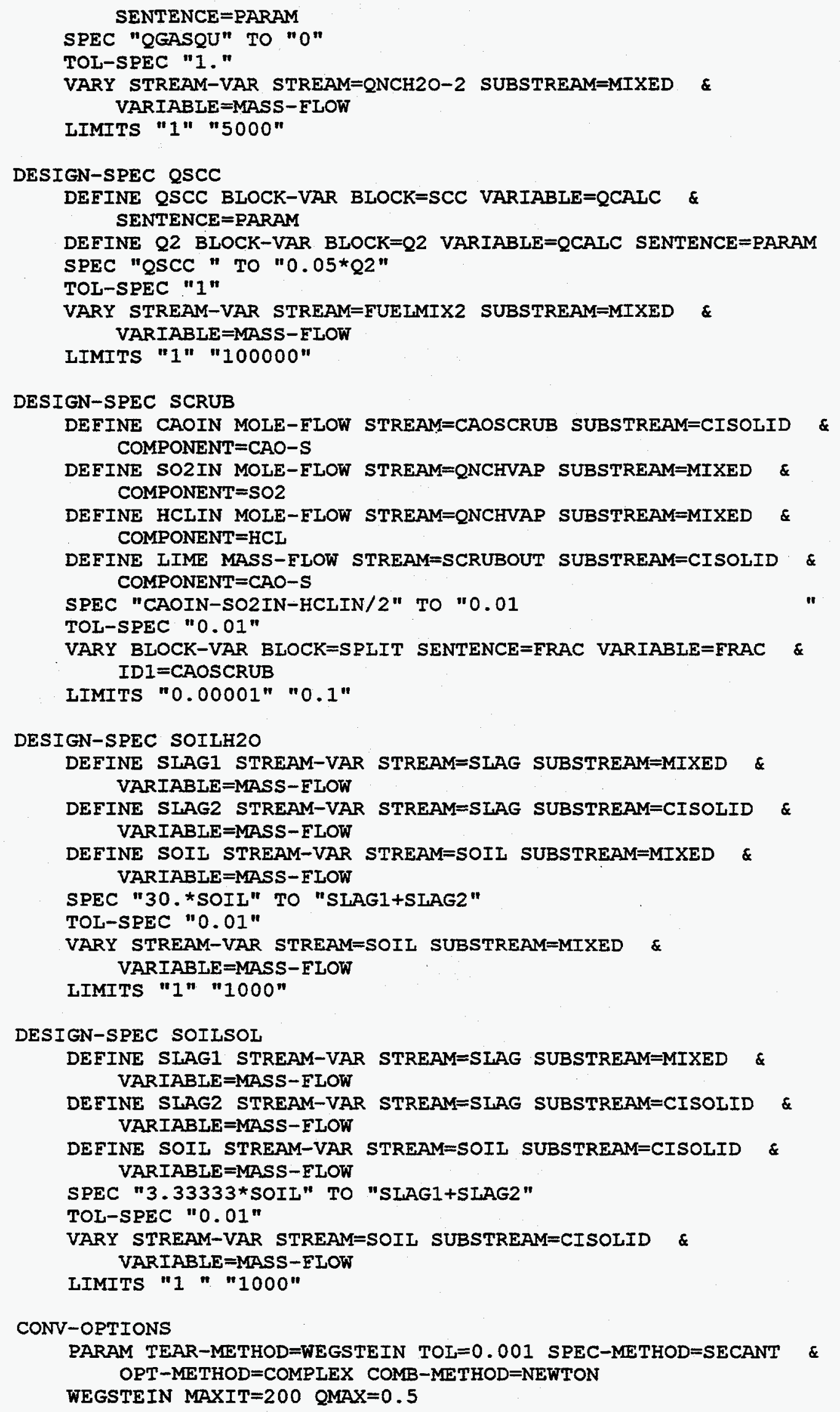


SECANT MAX-STEP-SIZ $=.05$ BRACKET $=$ NO

REPORT NOCOSTBLOCK NOUNITS NOUTIIITIES NOECONOMIC

STREAM-REPOR NOSORT MOLEFLOW MASSFLOW PROPERTIES=SET1 ALL-SUBS 


\section{System A-5}

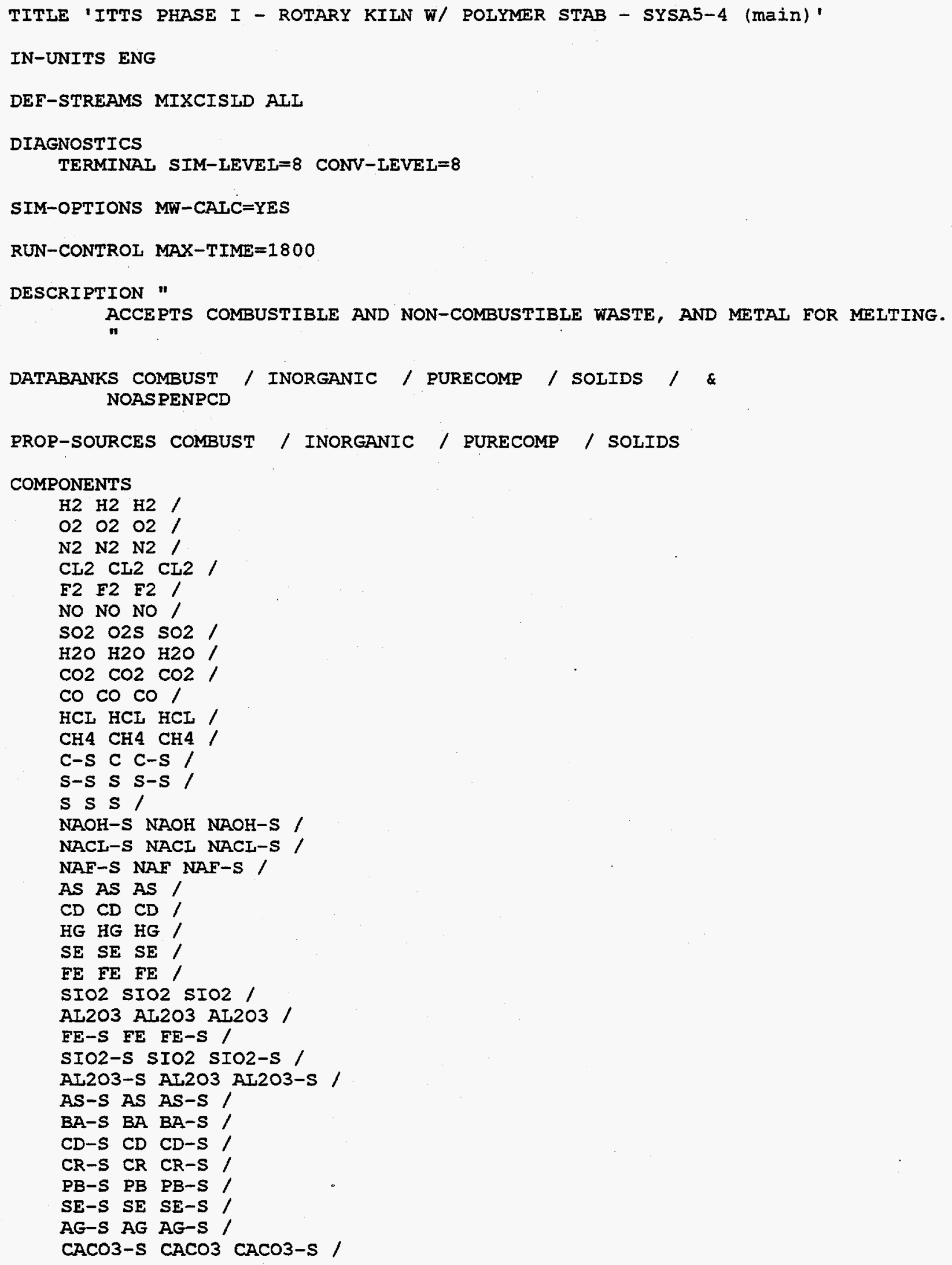


MGO-S MGO MGO-S /

NA2O-S NA2O NA2O-S /

K2O-S K2O K2O-S /

FE304 FE304 FE304/

FE304-S FE304 EE304-S /

CAO-S CAO CAO-S

FLOWSHEET

BLOCK KILN IN=AIRIEAKS AIR FDWASTEC FUELMIX1 COOLF2O * FDWASTEN OUT $=$ OUT 1

BLOCK KILNSEP IN=KILNOUT OUT=ASH KILNVAP

BLOCK SCC IN=KILNVAP FUELMIX2 OUT=OUT2

BLOCK BAGHOUSE IN=QUENCHED OUT=HGPLUS FLYASH QNCHVAP

BLOCK QUENCH IN=SCCOUT QNCH2O OUT=QUENCHED

BLOCK I-V-SEP IN=SCRUBOUT OUT=SCRUBVAP SCRUBBOT

BLOCK DUPL1 IN=OUT1 OUT=KIINOUT DUMIIN

BIOCK 21 IN=DUMIIN OUT=DUM1OUT

BLOCK DUPL2 IN=OUT2 OUT=SCCOUT DUM2IN

BLOCK 22 IN=DUM2IN OUT=DUM2OUT

BLOCK SCRUB IN=NAOH SCRUBH2O QNCHVAP OUT=SCRUBOUT

BLOCK MELTER IN=FDMETMLT OUT=METAL

PROPERTIES SOLIDS

PROPERTIES IDEAI / RKS-BM

PROP-SET ALL-SUBS TEMP PRES VFRAC UNITS='ATM' SUBSTREAM=ALL

PROP-SET SET1 VLSTDMX UNITS='CUFT/HR' SUBSTREAM=MIXED

STREAM AIR

SUBSTREAM MIXED TEMP=68 PRES=1 <ATM> MASS-FLOW=7877.802

MASS-FRAC $020.233 / \mathrm{N} 20.767$

STREAM AIRLEAKS

SUBSTREAM MIXED TEMP $=68$ PRES=1 <ATM> \&

VOLUME-FLOW $=600$ 〈CUFT/MIN>

MASS-FRAC $020.233 / \mathrm{N} 20.767$

STREAM COOLH2O

SUBSTREAM MIXED TEMP=68 PRES=1 <ATM> MASS-ELOW=662.536

MASS-FRAC H2O 1

STREAM FDMETMLT

SUBSTREAM MIXED TEMP=68 PRES $=1$ <ATM> MASS-FLOW=1.20

MASS-FLOW H2 2.42

SUBSTREAM CISOLID TEMP $=68$ PRES $=1$ <ATM> MASS-FLOW $=147.80$

MASS-FLOW C-S 14.44 / FE-S $282.02 /$ SIO2-S $0.035 / \&$

AL203-S $0.035 / \mathrm{BA}-\mathrm{S} 0.87 / \mathrm{CD}-\mathrm{S} 0.18$

STREAM FDWASTEC

SUBSTREAM MIXED TEMP $=68$ PRES $=1$ <ATM>

MASS-FLOW H2 $50.95 /$ O2 $92.76 /$ N2 $0.23 /$ CL2 $53.02 /$ \& F2 $0.11 / \mathrm{H} 2019.83$

SUBSTREAM CISOLID TEMP=68 PRES=1 <ATM>

MASS-FLOW C-S $326.28 /$ S-S $0.49 /$ FE-S $7.67 /$ SIO2-S \&

$53.01 /$ AL2O3-S 53.01/ BA-S $0.77 /$ CD-S $0.77 /$ CR-S 1.53

STREAM FDWASTEN

SUBSTREAM MIXED TEMP=68 PRES=1 <ATM>

MASS-FLOW H2 $0.54 / 021.44 /$ N2 $0.01 /$ CL2 $14.88 /$ \&

NO $0.66 / \mathrm{SO} 20.83 / \mathrm{H} 2 \mathrm{O} 354.23 / \mathrm{HG} 0.15$

SUBSTREAM CISOLID TEMP=68 PRES $=1$ <ATM> 
MASS-FLOW C-S $4.77 /$ S-S $0.62 /$ SIO2-S $476.77 / \&$

AL203-S 476.77/ AS-S $0.06 / \mathrm{BA}-\mathrm{S} 0.04 / \mathrm{CD}-\mathrm{S} 2.63$ \&

/ CR-S $4.95 / \mathrm{PB}-\mathrm{S} 0.17 / \mathrm{SE}-\mathrm{S} 0.03 / \mathrm{AG}-\mathrm{S} 0.03$

STREAM FUELMIXI

SUBSTREAM MIXED TEMP=68 PRES=1 <ATM> MASS-FLOW=1

MASS-FRAC $020.222 / \mathrm{N} 20.732 / \mathrm{CH} 40.046$

STREAM FUELMIX2

SUBSTREAM MIXED TEMP=68 PRES=1 <ATM> MASS-FLOW=9213.910

MASS-FRAC $020.222 / \mathrm{N} 20.732 / \mathrm{CH} 40.046$

STREAM NAOH

SUBSTREAM MIXED TEMP=68 PRES=1 <ATM>

SUBSTREAM CISOLID TEMP=68 PRES=1 <ATM> MASS-ELOW=77.611

MASS-FRAC NAOH-S 1

STREAM OUT1

SUBSTREAM MIXED TEMP=2500 PRES=1 <ATM>

MASS-FLOW $021293 /$ N2 $14610 /$ NO $27 /$ SO2 $3 /$ H2O \&

1977 / CO2 $2598 / \mathrm{HCL} 70$ / FE304 45

SUBSTREAM CISOLID TEMP $=2500$ PRES $=1$ <ATM>

MASS-FLOW SIO2-S 1069 / AL203-S $739 /$ CR-S 8 / MGO-S \& $18 /$ NA2O-S $10 /$ K2O-S $19 /$ CAO-S 56

STREAM QNCH2O

SUBSTREAM MIXED TEMP=68 PRES=1 <ATM> MASS-FLOW=10354.180

MASS-FRAC H2O 1

STREAM QUENCHED

SUBSTREAM MIXED TEMP $=350$ PRES $=1$ <ATM>

MASS-FLOW O2 $1446 / \mathrm{N} 217490 /$ CL2 $29 / \mathrm{H} 2016320 /$ \& CO2 $3088 /$ HCI 40

SUBSTREAM CISOLID TEMP $=350$ PRES $=1$ <ATM>

MASS-FLOW SIO2-S $221 /$ AL2O3-S $148 /$ CD-S $3.3 /$ CR-S \&

$1.62 /$ MGO-S $3.6 /$ NA2O-S $2 /$ K2O-S $3.75 /$ EE3O4-S 9

STREAM SCRUBH 20

SUBSTREAM MIXED TEMP=68 PRES=1 <ATM> MASS-FLOW=1

MASS-FRAC H2O 1

STREAM SCRUBOUT

SUBSTREAM MIXED TEMP=120 PRES=1 〈ATM>

MASS-FLOW O2 $1452 / \mathrm{N} 217490 / \mathrm{H} 20373000 / \mathrm{CO} 3088$

SUBSTREAM CISOIID TEMP $=120$ PRES=1 <ATM>

MASS-ELOW NACL-S 112

BLOCK BAGHOUSE SEP

FRAC STREAM=HGPLUS SUBSTREAM=MIXED COMPS=H2 O2 N2 CL2 \& F2 NO SO2 H2O CO2 CO HCL CH4 C-S s-s s NAOH-S \& NACI-S NAF-S AS CD HG SE FE SIO2 AL2O3 FE-S SIO2-S \& AL2O3-S AS-S BA-S CD-S CR-S PB-S SE-S AG-S CACO3-S \& MGO-S NA2O-S K2O-S FE304 FE304-S CAO-S FRACS=0 0 O \& $0 \begin{array}{lllllllllllllllllllll}0 & 0 & 0 & 0 & 0 & 0 & 0 & 0 & 0 & 0 & 0 & 0 & 0 & 0 & 1 & 1 & 1 & 1 & 0 & 0 & \text { \& }\end{array}$

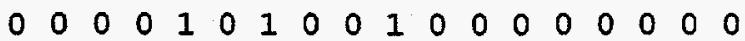

FRAC STREAM=HGPIUS SUBSTREAM=CISOIID COMPS=C-S S-S NAOH-S \& NACL-S NAF-S FE-S SIO2-S AL203-S AS-S BA-S CD-S CR-S \& PB-S SE-S AG-S CACO3-S MGO-S NA2O-S K2O-S FE3O4-S \&

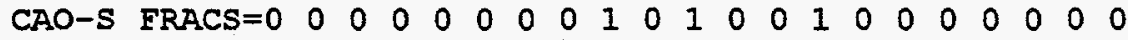
FRAC STREAM=ELYASH SUBSTREAM=MIXED COMPS=H2 O2 N2 CL2 \& F2 NO SO2 H2O CO2 CO HCL CH4 C-S S-S S NAOH-S \& NACL-S NAF-S AS CD HG SE FE SIO2 AL2O3 FE-S SIO2-S \& 
AI203-S AS-S BA-S CD-S CR-S PB-S SE-S AG-S CACO3-S \& MGO-S NA2O-S K2O-S FE304 FE304-S CAO-S FRACS=0 0 O \& $\begin{array}{llllllllllllllllllllll}0 & 0 & 0 & 0 & 0 & 0 & 0 & 0 & 0 & 1 & 1 & 1 & 1 & 1 & 1 & 0 & 0 & 0 & 0 & 1 & 1 & \text { \& }\end{array}$

$\begin{array}{llllllllllllllllll}1 & 1 & 1 & 1 & 0 & 1 & 0 & 1 & 1 & 0 & 1 & 1 & 1 & 1 & 1 & 1 & 1 & 1\end{array}$

FRAC STREAM=FLYASH SUBSTREAM=CISOLID COMPS=C-S S-S NAOH-S \& NACI-S NAF-S FE-S SIO2-S AI203-S AS-S BA-S CD-S CR-S \& PB-S SE-S AG-S CACO3-S MGO-S NA2O-S K2O-S EE3O4-S \&

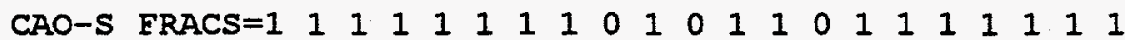

BLOCK KIINSEP SEP

FRAC STREAM=ASH SUBSTREAM=MIXED COMPS=H2 O2 N2 CL2 F2 \& NO $\mathrm{SO} 2 \mathrm{H} 2 \mathrm{O} \mathrm{CO} 2 \mathrm{CO} \mathrm{HCL} \mathrm{CH} 4 \mathrm{C}-\mathrm{s}$ s-s $s$ NAOH-S NACL-S \& NAF-S AS CD HG SE EE SIO2 AL2O3 FE-S SIO2-S AL2O3-S \& AS-S BA-S CD-S CR-S PB-S SE-S AG-S CACO3-S MGO-S \& NA20-S K20-S FE304 EE304-S CAO-S FRACS=0 0000000 \& $\begin{array}{lllllllllllllllllll}0 & 0 & 0 & 0 & 0 & 0 & .8 & .8 & 0 & .8 & .8 & .8 & 0 & 0 & 0 & 0 & .8 & .8 & \&\end{array}$ $\begin{array}{llllllllllllllllll}.8 & .8 & .8 & .8 & .8 & .8 & .8 & .8 & .8 & .8 & .8 & .8 & .8 & .8 & .8 & .8 & .8 & .8\end{array}$

ERAC STREAM=ASH SUBSTREAM=CISOIID COMPS=C-S $S-S$ NAOH-S \& NACI-S NAF-S FE-S SIO2-S AL203-S AS-S BA-S CD-S CR-S \& PB-S SE-S AG-S CACO3-S MGO-S NA2O-S K2O-S FE304-S \&

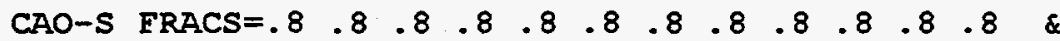
$\begin{array}{llllllllll}.8 & .8 & .8 & .8 & .8 & .8 & .8 & .8 & .8\end{array}$

BLOCK I-V-SEP FLASH2

PARAM TEMP $=120$ PRES $=1$ <ATM>

BLOCK .KILN RGIBBS

PARAM TEMP $=1600$ PRES $=1$ <ATM> NPHASE $=2$

PROD H2/.02/ N2/ CL2/ F2/ NO / SO2/ H2O / \& $\mathrm{CO} 2 / \mathrm{CO} / \mathrm{HCL} / \mathrm{AS} / \mathrm{CD} / \mathrm{HG} / \mathrm{SE} / \mathrm{FE}-\mathrm{S} \mathrm{SS}$ \& / SIO2-S SS / AI203-S SS / AS-S SS / BA-S SS / \& CD-S SS/CR-S SS/ PB-S SS/. SE-S SS/AG-S SS \& / MGO-S SS / NA2O-S SS / K20-S SS / FE304-S SS / CAO-S SS

PROD-FRAC FE-S 1

BLOCK MELTER RGIBBS

PARAM TEMP $=3000$ PRES $=1$ <ATM> NPHASE $=2$

PROD H2 / C-S SS / FE / SIO2 / AI203/ FE-S SS / \& sI02-S SS / AL2O3-s ss / BA-s SS / CD / CD-S ss

BLOCK 21 RGIBBS

PARAM TEMP $=68$ PRES $=1$ <ATM> NPHASE $=2$ TAPP $=1532$

PROD H2 / O2 / N2 / CL2 / E2 / NO / SO2 / H2O / \& $\mathrm{CO} 2 / \mathrm{CO} / \mathrm{HCL} / \mathrm{AS} / \mathrm{CD} / \mathrm{HG} / \mathrm{SE} / \mathrm{FE}-\mathrm{S}$ SS \& / sI02-s ss / AL2O3-S SS / As-s SS / BA-S SS / \& CD-S SS / CR-S SS / PB-S SS / SE-S SS / AG-S SS \& / MGO-S SS / NA2O-S SS / K20-S SS / FE304-S SS / \& CAO-S SS

PROD-FRAC FE-S 1

\section{BIOCK Q2 RGIBBS}

PARAM TEMP $=68$ PRES $=1$ <ATM> NPHASE=2 CHEMEQ $=Y E S$ TAPP=2132

$\mathrm{PROD} \mathrm{H} 2 / \mathrm{O} 2 / \mathrm{N} 2 / \mathrm{CL} 2 / \mathrm{F} 2 / \mathrm{NO} / \mathrm{SO} 2 / \mathrm{H} 2 \mathrm{O} / \&$ $\mathrm{CO} 2 / \mathrm{CO} / \mathrm{HCL} / \mathrm{CH} 4 / \mathrm{s} / \mathrm{AS} / \mathrm{CD} / \mathrm{HG} / \mathrm{SE} \&$ $/$ EE-S SS / SIO2-S SS / AL203-S SS / BA-S SS / \& CR-S SS / PB-S SS / AG-S SS / MGO-S SS / NA2O-S \& SS / K20-S SS / FE304-S SS / CAO-S SS

PROD-FRAC FE-S $1 /$ FE3O4-S 1

BLOCK QUENCH RGIBBS

PARAM TEMP $=350$ PRES $=1$ <ATM> NPHASE $=2$

PROD $\mathrm{H} 2 / \mathrm{O} 2 / \mathrm{N} 2 / \mathrm{F} 2 / \mathrm{NO} / \mathrm{SO} 2 / \mathrm{H} 2 \mathrm{O} / \mathrm{CO} / / \&$ 


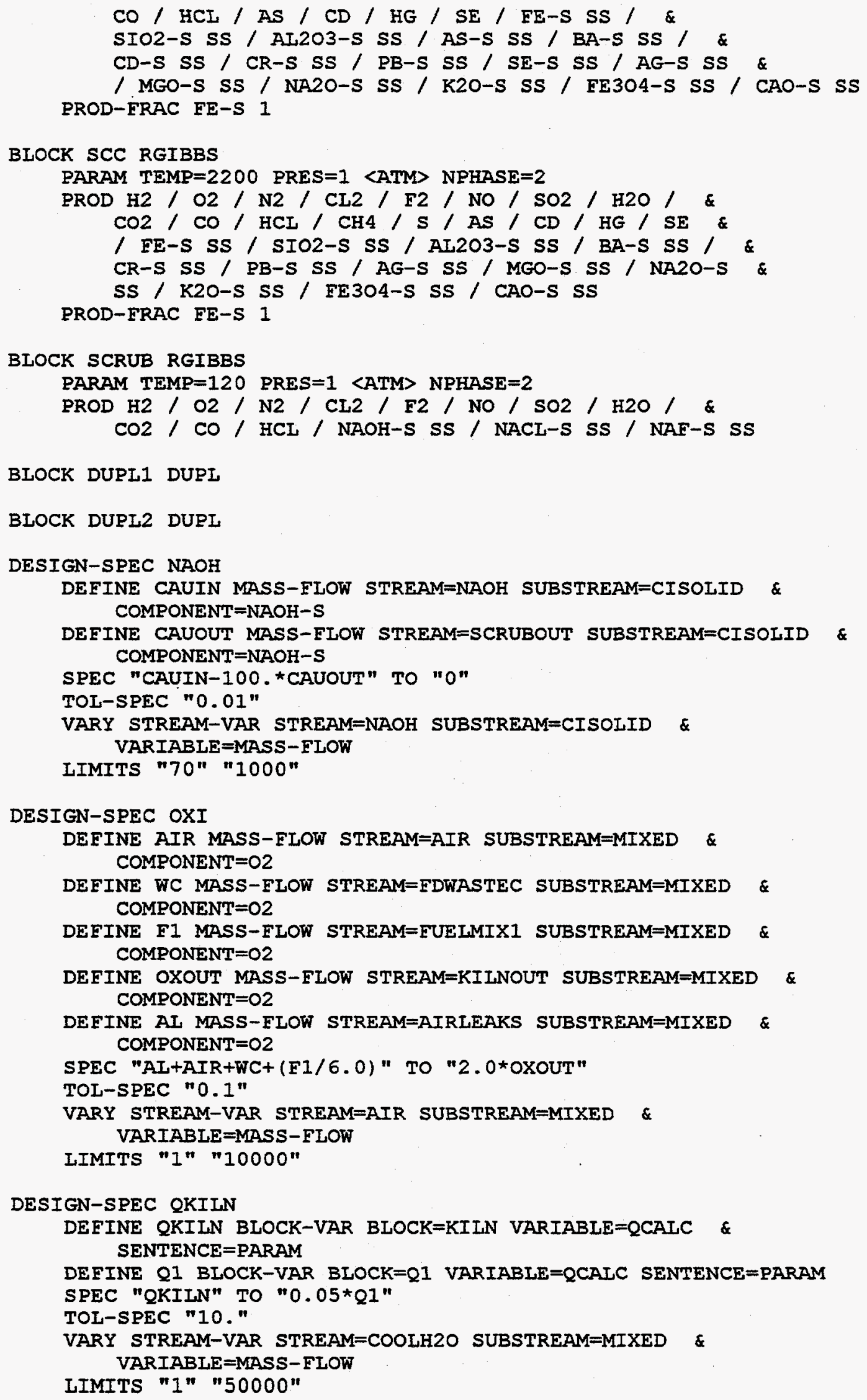

BLOCK DUPL1 DUPL

BLOCK DUPL2 DUPL

DESIGN-SPEC NAOH

DEFINE CAUIN MASS-FLOW STREAM=NAOH SUBSTREAM=CISOLID \& COMPONENT $=\mathrm{NAOH}-\mathrm{S}$

DEFINE CAUOUT MASS-FLOW STREAM=SCRUBOUT SUBSTREAM=CISOLID\& COMPONENT $=\mathrm{NAOH}-\mathrm{S}$ 


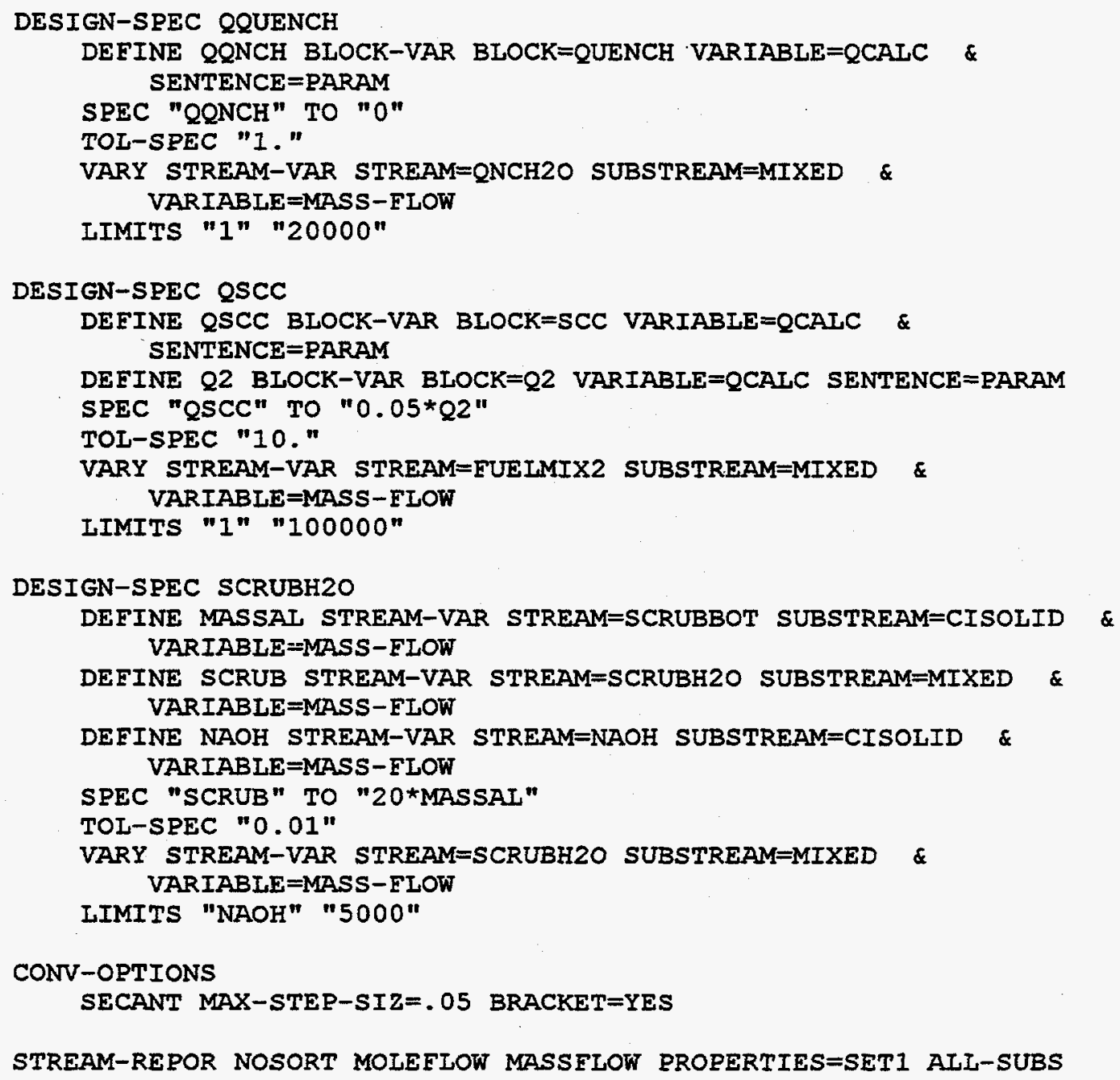

STREAM-REPOR NOSORT MOLEFLOW MASSFLOW PROPERTIES=SET1 ALL-SUBS 


\section{System A-6}

TITLE \& 'ITTS PHASE I - ROTARY KILN W/ MAX RECYCIING - SYSA6-4 (main)'

IN-UNITS ENG

DEF-STREAMS MIXCISLD ALL

DIAGNOSTICS

TERMINAI SIM-LEVEL=8 CONV-IEVEI=8

SIM-OPTIONS MW-CALC=YES

RUN-CONTROI MAX-TIME $=1800$

DESCRIPTION "

ACCEPTS COMBUSTIBLE AND NON-COMBUSTIBLE WASTE, AND METAL FOR MELTING. ASSUME 140 IBS/HR OF METAL GETS RECYCIED (OUT OF ABOUT 147) INSTEAD OF GOING TO KIIN.

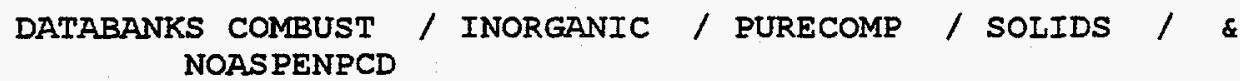


AG-S AG AG-S/

CACO3-S CACO3 CACO3-S /

MGO-S MGO MGO-S /

NA2O-S NA2O NA2O-S /

K2O-S K2O K2O-S /

EE304 FE304 FE304 /

FE304-S FE304 FE304-S /

CAO-S CAO CAO-S

FLOWSHEET

BLOCK KIIN IN=SOIL FUEIMIX1 AIR AIRLEAKS COOLH2O FDWASTE \& OUT $=$ OUT 1

BLOCK KILNSEP IN=KILNOUT OUT=ASH KILNVAP

BLOCK SCC IN=KILNVAP FUEIMIX2 OUT=OUT2

BIOCK BAGHOUSE IN=QUENCHED OUT=HGPLUS FLYASH QNCHVAP

BLOCK QUENCH IN=SCCOUT QNCH2O OUT=QUENCHED

BLOCK L-V-SEP IN=SCRUBOUT OUT=SCRUBVAP SCRUBBOT

BLOCK DURL1 IN=OUT1 OUT=KILNOUT DUM1IN

BLOCK $Q 1$ IN=DUMIIN OUT=DUMIOUT

BLOCK DUPL2 IN=OUT2 OUT=SCCOUT DUM2IN

BLOCK Q2 IN=DUM2IN OUT=DUM2OUT

BLOCK VITRIFY IN=FLYASH ASH OUT=VITOUT

BLOCK I-V-SEP2 IN=VITOUT OUT=VITVAP SLAG

BLOCK SCRUB IN=NAOH QNCHVAP USEDNAOH SCRUBH2O OUT= SCRUBOUT

BLOCK METALSEP IN=FDWASTEN FDWASTEC OUT=CASTMET FDWASTE

BLOCK SALT-SEP IN=PRODUCTS OUT=USEDNAOH RECYCLE HCL-SOLN \& GAS-SALT

BLOCK SPIITTER IN=SCRUBBOT OUT=PRODUCTS

BLOCK MELTER IN=FDMETMLT OUT=METAI

PROPERTIES SOLIDS

PROPERTIES IDEAI / RKS-BM

PROP-SET AIL-SUBS TEMP PRES VERAC UNITS='ATM' SUBSTREAM=ALL

PROP-SET SET1 VOLFLMX UNITS='CUFT/HR' SUBSTREAM=AIL

STREAM AIR

SUBSTREAM MIXED TEMP=68 PRES=1 <ATM> MASS-FLOW=7858.798

MASS-FRAC $020.233 / \mathrm{N} 20.767$

STREAM AIRLEAKS

SUBSTREAM MIXED TEMP=68 PRES=1 <ATM> \&

VOLUME-FLOW $=600<\mathrm{CUFT} / \mathrm{MIN}>$

MASS-FRAC O2 $0.233 / \mathrm{N} 20.767$

STREAM COOLH2O

SUBSTREAM MIXED TEMP=68 PRES=1 <ATM> MASS-FIOW=1

MASS-FRAC H2O 1

STREAM FDMETMLT

SUBSTREAM MIXED TEMP=68 PRES=1 <ATM> MASS-FLOW=1.20

MASS-FLOW H2 2.42

SUBSTREAM CISOLID TEMP=68 PRES=1 <ATM> MASS-ELOW=147.80

MASS-FLOW C-S $14.44 /$ FE-S $282.02 /$ SIO2-S $0.035 /$ \&

AL203-S $0.035 /$ BA-S $0.87 /$ CD-S 0.18

STREAM FDWASTEC

SUBSTREAM MIXED TEMP=68 PRES=1 <ATM>

MASS-FLOW H2 $50.95 /$ O2 $92.76 / \mathrm{N} 20.23 / \mathrm{CL} 253.02 /$ \&

F2 $0.11 / \mathrm{H} 2019.83$

SUBSTREAM CISOLID TEMP=68 PRES=1 <ATM> 
MASS-FLOW C-S $326.28 / \mathrm{S}-\mathrm{S} 0.49 / \mathrm{FE}-\mathrm{S} 7.67 /$ SIO2-S \& $53.01 / \mathrm{AL} 203-\mathrm{S} 53.01 / \mathrm{BA}-\mathrm{S} 0.77 / \mathrm{CD}-\mathrm{S} 0.77 / \mathrm{CR}-\mathrm{S} 1.53$

\section{STREAM FDWASTEN}

SUBSTREAM MIXED TEMP $=68$ PRES $=1$ <ATM>

MASS-FLOW H2 $0.54 / 021.44 / \mathrm{N} 20.01 /$ CL2 $14.88 /$ \&

NO $0.66 / \mathrm{sO} 20.83 / \mathrm{H} 20354.23 / \mathrm{HG} 0.15$

SUBSTREAM CISOLID TEMP=68 PRES=1 〈ATM>

MASS-FLOW C-S $4.77 /$ S-S $0.62 /$ SIO2-S $476.77 / \&$

AL203-S $476.77 /$ AS-S $0.06 /$ BA-S $0.04 / \mathrm{CD}-\mathrm{S} 2.63 \&$

/ CR-S $4.95 /$ PB-S $0.17 /$ SE-S $0.03 /$ AG-S 0.03

STREAM EUELMIX1

SUBSTREAM MIXED TEMP=68 PRES=1 <ATM> MASS-FIOW=1

MASS-FRAC $020.222 / \mathrm{N} 20.732 / \mathrm{CH} 40.046$

STREAM FUELMIX2

SUBSTREAM MIXED TEMP=68 PRES=1 <ATM> MASS-FLOW=1

MASS-FRAC $020.222 / \mathrm{N} 20.732 / \mathrm{CH} 40.046$

STREAM NAOH

SUBSTREAM MIXED TEMP $=68$ PRES=1 <ATM>

SUBSTREAM CISOLID TEMP=68 PRES=1 <ATM> MASS-FLOW=0. 8

MASS-FRAC NAOH-S 1

STREAM OUT1

SUBSTREAM MIXED TEMP=2500 PRES=1 <ATM>

MASS-FLOW O2 $1293 /$ N2 $14610 /$ NO $27 /$ SO2 $3 / \mathrm{H} 2 \mathrm{O}$ \& 1977 / CO2 $2598 / \mathrm{HCL} 70 /$ FE304 45

SUBSTREAM CISOIID TEMP $=2500$ PRES $=1$ <ATM>

MASS-FLOW SIO2-S 1069 / AL203-S $739 /$ CR-S 8 / MGO-S \& $18 /$ NA2O-S $10 / \mathrm{K} 20-\mathrm{S} 19 / \mathrm{CAO}-\mathrm{S} 56$

STREAM QNCH2O

SUBSTREAM MIXED TEMP=68 PRES=1 <ATM> MASS-FLOW=10224

MASS-ERAC H2O 1

STREAM QUENCHED

SUBSTREAM MIXED TEMP=350 PRES=1 <ATM>

MASS-FLOW O2 $1446 /$ N2 $17490 /$ CL2 $29 /$ H2O 16320 / \& $\mathrm{CO} 3088 / \mathrm{HCL} 40$

SUBSTREAM CISOIID TEMP=350 PRES $=1$ <ATM>

MASS-FLOW SIO2-S $221 /$ AL2O3-S $148 /$ CD-S $3.3 / \mathrm{CR}-\mathrm{S}$ \&

$1.62 / \mathrm{MGO}-\mathrm{S} 3.6 / \mathrm{NA} 20-\mathrm{S} 2 / \mathrm{K} 20-\mathrm{S} 3.75 /$ FE3O4-S 9

STREAM SCRUBH2O

SUBSTREAM MIXED TEMP=68 PRES=1 <ATM> MASS-FLOW=2

MASS-FRAC H2O 1

STREAM SCRUBOUT

SUBSTREAM MIXED TEMP=120 PRES $=1$ <ATM>

MASS-FIOW O2 1452 / N2 $17490 / \mathrm{H} 20373000 / \mathrm{CO} 3088$

SUBSTREAM CISOIID TEMP=120 PRES=1 <ATM>

MASS-FLOW NACL-S 112

STREAM SOIL

SUBSTREAM MIXED TEMP=68 PRES=1 <ATM> MASS-ELOW=50

MASS-FLOW H2O 0.1

SUBSTREAM CISOLID TEMP=68 PRES $=1$ <ATM> MASS-ELOW=450

MASS-FLOW SIO2-S $0.569 /$ AL203-S $0.102 /$ CACO3-S $0.128 /$ \& MGO-S $0.023 /$ NA2O-S $0.013 /$ K2O-S $0.024 /$ FE304-S 0.041 
BLOCK BAGHOUSE SEP

FRAC STREAM=HGPLUS SUBSTREAM=MIXED COMPS=H2 O2 N2 CL2 \& F2 NO SO2 H2O CO2 CO HCL CH4 C-s s-s s NAOH-S \& NACI-S NAF-S AS CD HG SE FE SIO2 AL2O3 EE-S SIO2-S AL203-S AS-S BA-S CD-S CR-S PB-S SE-S AG-S CACO3-S MGO-S NA2O-S K2O-S FE304 FE304-S CAO-S FRACS $=0$ O 0 $\begin{array}{llllllllllllllllllllll}0 & 0 & 0 & 0 & 0 & 0 & 0 & 0 & 0 & 0 & 0 & 0 & 0 & 0 & 0 & 1 & 1 & 1 & 1 & 0 & 0 & \&\end{array}$ $0 \begin{array}{llllllllllllllllll}0 & 0 & 0 & 1 & 0 & 1 & 0 & 0 & 1 & 0 & 0 & 0 & 0 & 0 & 0 & 0 & 0\end{array}$

FRAC STREAM=HGPLUS SUBSTREAM=CISOLID COMPS=C-S $S-S$ NAOH-S \& NACL-S NAF-S FE-S SIO2-S AL2O3-S AS-S BA-S CD-S CR-S \& PB-S SE-S AG-S CACO3-S MGO-S NA2O-S K2O-S FE3O4-S \& CAO-S FRACS $=0 \begin{array}{lllllllllllllllllllll}0 & 0 & 0 & 0 & 0 & 0 & 0 & 1 & 0 & 1 & 0 & 0 & 1 & 0 & 0 & 0 & 0 & 0 & 0 & 0\end{array}$

FRAC STREAM=FLYASH SUBSTREAM=MIXED COMPS=H2 O2 N2 CL2 \& F2 NO SO2 H2O $\mathrm{CO} 2 \mathrm{CO}$ HCL CH4 C-S S-S S NAOH-S \& NACL-S NAF-S AS CD HG SE FE SIO2 AL2O3 FE-S SIO2-S \& AI203-S AS-S BA-S CD-S CR-S PB-S SE-S AG-S CACO3-S \& MGO-S NA2O-S K2O-S FE304 FE304-S CAO-S FRACS=0 00 \& $\begin{array}{llllllllllllllllllllll}0 & 0 & 0 & 0 & 0 & 0 & 0 & 0 & 0 & 1 & 1 & 1 & 1 & 1 & 1 & 0 & 0 & 0 & 0 & 1 & 1 & \text { \& }\end{array}$ $\begin{array}{llllllllllllllllll}1 & 1 & 1 & 1 & 0 & 1 & 0 & 1 & 1 & 0 & 1 & 1 & 1 & 1 & 1 & 1 & 1 & 1\end{array}$

FRAC STREAM=FLYASH SUBSTREAM=CISOLID COMPS=C-S S-S NAOH-S \& NACL-S NAF-S FE-S SIO2-S AL203-S AS-S BA-S CD-S CR-S \& PB-S SE-S AG-S CACO3-S MGO-S NA2O-S K2O-S FE3O4-S \&

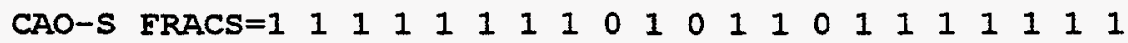

FLASH-SPECS FLYASH TEMP $=68$ PRES $=1$ <ATMP

\section{BLOCK KIINSEP SEP}

FRAC STREAM=ASH SUBSTREAM=MIXED COMPS=H2 O2 N2 CL2 F2 \& NO SO2 H2O CO2 CO HCL CH4 C-S S-S S NAOH-S NACL-S \& NAF-S AS CD HG SE FE SIO2 AL2O3 FE-S SIO2-S AL2O3-S \& AS-S BA-S CD-S CR-S PB-S SE-S AG-S CACO3-S MGO-S \&

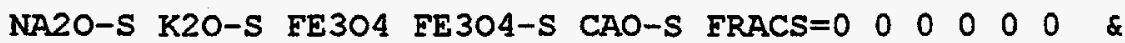
$\begin{array}{lllllllllllllllllll}0 & 0 & 0 & 0 & 0 & 0 & .8 & .8 & 0 & .8 & .8 & .8 & 0 & 0 & 0 & 0 & .8 & .8 & \&\end{array}$ $\begin{array}{lllllllllllllllllll}.8 & .8 & .8 & .8 & .8 & .8 & .8 & .8 & .8 & .8 & .8 & .8 & .8 & .8 & .8 & .8 & .8 & .8\end{array}$

FRAC STREAM=ASH SUBSTREAM=CISOLID COMPS $=C-S$ S $S$ NAOH $S$ \& NACL-S NAF-S FE-S SIO2-S AI203-S AS-S BA-S CD-S CR-S \& PB-S SE-S AG-S CACO3-S MGO-S NA2O-S K2O-S EE3O4-S \&

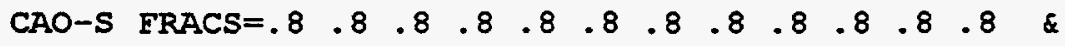
$\begin{array}{llllllllll}.8 & .8 & .8 & .8 & .8 & .8 & .8 & .8 & .8\end{array}$

FIAASH-SPECS ASH TEMP $=68$ PRES $=1<$ ATM $>$

\section{BLOCK METALSEP SEP}

FRAC STREAM=CASTMET SUBSTREAM=MIXED COMPS=H2 O2 N2 CL2 F2 NO SO2 H2O CO2 CO HCL CH4 C-S s-s s NAOH-S \& NACL-S NAF-S AS CD HG SE FE SIO2 AL2O3 FE-S SIO2-S AL203-S AS-S BA-S CD-S CR-S PB-S SE-S AG-S CACO3-S MGO-S NA2O-S K2O-S FE3O4 FE304-S CAO-S FRACS $=0 \quad 0 \quad 0$ $0 \begin{array}{lllllllllllllllllllll}0 & 0 & 0 & 0 & 0 & 0 & 0 & 0 & 0 & 0 & 0 & 0 & 0 & 0 & 0 & 0 & 0 & 0 & 0 & 0 & \text { \& }\end{array}$ $0 \begin{array}{lllllllllllllllll}0 & 0 & 0 & 0 & 0 & 0 & 0 & 0 & 0 & 0 & 0 & 0 & 0 & 0 & 0 & 0 & 0\end{array}$

FRAC STREAM=CASTMET SUBSTREAM=CISOLID COMPS=C-S S-S \& NAOH-S NACL-S NAF-S FE-S SIO2-S AI2O3-S AS-S BA-S \& CD-S CR-S PB-S SE-S AG-S CACO3-S MGO-S NA.2O-S K2O-S \& FE 304-S CAO-S FRACS $=0 \begin{array}{llllllllllllll}0 & 0 & 0 & 0 & 0 & 1 & 0 & 0 & 0 & 0 & 0 & 0 & 0 & \text { \& }\end{array}$ $\begin{array}{llllllll}0 & 0 & 0 & 0 & 0 & 0 & 0 & 0\end{array}$

FIASH-SPECS CASTMET TEMP $=68$ PRES $=1$ <ATM>

FLASH-SPECS FDWASTE TEMP=68 PRES=1 <ATM>

\section{BLOCK SALT-SEP SEP}

FRAC STREAM=USEDNAOH SUBSTREAM=MIXED COMPS=H2 O2 N2 CL2 \& F2 NO SO2 H2O CO2 CO HCI CH4 C-S S-S S NAOH-S \& NACI-S NAF-S AS CD HG SE FE SIO2 AL2O3 EE-S SIO2-S AL2O3-S AS-S BA-S CD-S CR-S PB-S SE-S AG-S CACO3-S MGO-S NA2O-S K2O-S FE304 FE304-S CAO-S FRACS $=0 \quad 0$ $\boldsymbol{\alpha}$ 
$\begin{array}{llllllllllllllllllllll}0 & 0 & 0 & 0 & 0 & 0 & 0 & 0 & 0 & 0 & 0 & 0 & 0.98 & 0 & 0 & 0 & 0 & 0 & 0 & 0 & \&\end{array}$

$0 \begin{array}{llllllllllllllllll}0 & 0 & 0 & 0 & 0 & 0 & 0 & 0 & 0 & 0 & 0 & 0 & 0 & 0 & 0 & 0 & 0 & 0\end{array}$

FRAC STREAM=USEDNAOH SUBSTREAM=CISOIID COMPS=C-S S-S \&

NAOH-S NACL-S NAF-S FE-S STO2-S AI2O3-S AS-S BA-S \&

CD-S CR-S PB-S SE-S AG-S CACO3-S MGO-S NA2O-S K2O-S \&

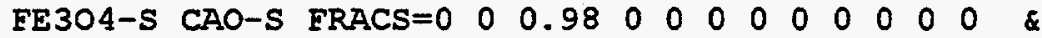

$0 \begin{array}{lllllllll}0 & 0 & 0 & 0 & 0 & 0 & 0 & 0 & 0\end{array}$

FRAC STREAM=HCL-SOLN SUBSTREAM=MIXED COMPS=H2 O2 N2 CL2 \& F2 NO SO2 H2O CO2 CO HCL CH4 C-S s-s S NAOH-S \& NACI-S NAF-S AS CD HG SE FE SIO2 AL2O3 FE-S SIO2-S \& AL203-S AS-S BA-S CD-S CR-S PB-S SE-S AG-S CACO3-S \& MGO-S NA2O-S K2O-S FE304 FE304-S CAO-S FRACS $=0$ O 0 \& $0 \begin{array}{lllllllllllllllllllll}0 & 0 & 0 & 0 & 0 & 0 & 1 & 0 & 0 & 0 & 0 & 0 & 0 & 0 & 0 & 0 & 0 & 0 & 0 & 0 & \text { \& }\end{array}$ $0 \begin{array}{lllllllllllllllll}0 & 0 & 0 & 0 & 0 & 0 & 0 & 0 & 0 & 0 & 0 & 0 & 0 & 0 & 0 & 0 & 0\end{array}$

FRAC STREAM=HCL-SOIN SUBSTREAM=CISOLID COMPS=C-S S-S \& NAOH-S NACL-S NAF-S FE-S SIO2-S AL2O3-S AS-S BA-S \& CD-S CR-S PB-S SE-S AG-S CACO3-S MGO-S NA2O-S K2O-S \& FE304-S CAO-S FRACS $=0 \begin{array}{lllllllllllll}0 & 0 & 0 & 0 & 0 & 0 & 0 & 0 & 0 & 0 & 0 & 0\end{array}$ $\begin{array}{llllllll}0 & 0 & 0 & 0 & 0 & 0 & 0 & 0\end{array}$

FRAC STREAM=GAS-SAIT SUBSTREAM=MIXED COMPS=H2 O2 N2 CL2 \& F2 NO SO2 H2O CO2 CO HCL CH4 C-S S-S S NAOH-S \& NACL-S NAF-S AS CD HG SE FE SIO2 AL2O3 FE-S SIO2-S \& AL2O3-S AS-S BA-S CD-S CR-S PB-S SE-S AG-S CACO3-S \& MGO-S NA20-S K20-S FE304 FE304-S CAO-S FRACS=1 111 \& $\begin{array}{llllllllllllllllllllll} & 1 & 1 & 1 & 1 & 0 & 1 & 1 & 0 & 1 & 1 & 1 & 1 & 0 & 1 & 1 & 1 & 1 & 1 & 1 & 1 & 1\end{array}$ $\begin{array}{llllllllllllllllll}1 & 1 & 1 & 1 & 1 & 1 & 1 & 1 & 1 & 1 & 1 & 1 & 1 & 1 & 1 & 1 & 1 & 1\end{array}$

FRAC STREAM=GAS-SAIT SUBSTREAM=CISOLID COMPS=C-S $S-S$ \& NAOH-S NACL-S NAF-S FE-S SIO2-S AL2O3-S AS-S BA-S \& CD-S CR-S PB-S SE-S AG-S CACO3-S MGO-S NA2O-S K2O-S \&

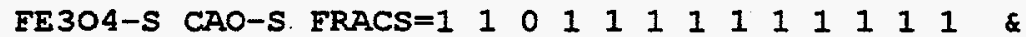
$\begin{array}{llllllll}1 & 1 & 1 & 1 & 1 & 1 & 1 & 1\end{array}$

FLASH-SPECS USEDNAOH TEMP $=68$ PRES $=1$ <ATM> FLASH-SPECS HCL-SOLN TEMP=68 PRES=1 <ATM> FLASH-SPECS RECYCLE TEMP=68 PRES=1 〈ATM>

BLOCK I-V-SEP FLASH2

PARAM TEMP $=120$ PRES $=1$ <ATM>

BIOCK I-V-SEP2 FLASH2

PARAM TEMP $=3000$ PRES $=1$ 〈ATM〉

BLOCK SPLITTER RSTOIC

PARAM TEMP $=221$ PRES $=1$ <ATM>

STOIC 1 CISOLID NACL-S $-1 /$ MIXED H2O $-1 / \mathrm{HCL} 1 /$ \& CISOLID NAOH-S 1

CONV 1 CISOLID NACI-S 1

BLOCK KILN RGIBBS

PARAM TEMP $=1600$ PRES $=1$ <ATM> NPHASE $=2$ MAXIT $=500$

PROD H2/. O2/ N2/ $\mathrm{CL} 2 / \mathrm{F} 2 / \mathrm{NO} / \mathrm{SO} 2 / \mathrm{H} 2 \mathrm{O} / \&$ $\mathrm{CO} 2 / \mathrm{CO} / \mathrm{HCL} / \mathrm{AS} / \mathrm{CD} / \mathrm{HG} / \mathrm{SE} / \mathrm{SIO} 2-\mathrm{S} \&$ ss / AI203-s ss / As-s ss / BA-s ss / CD-s ss / \& CR-S SS / PB-S SS/ SE-S SS/AG-S SS / MGO-S \& SS / NA2O-S SS / K2O-S SS / FE304-S SS / CAO-S SS BLOCK-OPTION RESTART=NO

BLOCK MELTER RGIBBS

PARAM TEMP $=3000$ PRES=1 <ATM> NPHASE=2

PROD H2 / C-S SS / FE / SIO2 / AL2O3/ FE-S SS / \& SIO2-S SS/ AL2O3-S SS/ BA-S SS/ CD/ CD-S SS

BIOCK $Q 1$ RGIBBS 
PARAM TEMP=68 PRES=1 <ATM> NPHASE=2 TAPP=1532

$\mathrm{PROD} \mathrm{H} 2 / \mathrm{O} 2 / \mathrm{N} 2 / \mathrm{CL} 2 / \mathrm{F} 2 / \mathrm{NO} / \mathrm{SO} 2 / \mathrm{H} 2 \mathrm{O} /$ \& $\mathrm{CO} 2 / \mathrm{CO} / \mathrm{HCL} / \mathrm{AS} / \mathrm{CD} / \mathrm{HG} / \mathrm{SE} / \mathrm{SIO2}-\mathrm{S}$ \& SS / AL203-S SS / AS-S SS / BA-S SS / CD-S SS / \& CR-S SS / PB-S SS / SE-S SS / AG-S SS / MGO-S \& SS / NA2O-S SS / K2O-S SS / FE304-S SS / CAO-S SS BLOCK-OPTION RESTART $=$ NO

BIOCK Q2 RGIBBS

PARAM TEMP=68 PRES=1 <ATM> NPHASE=2 CHEMEQ=YES TAPP=2132 PROD H2 / O2 / N2 / CL2 / E2 / NO $/ \mathrm{SO} 2 / \mathrm{H} 2 \mathrm{O} /$ \& $\mathrm{CO} 2 / \mathrm{CO} / \mathrm{HCL} / \mathrm{CH} 4 / \mathrm{s} / \mathrm{AS} / \mathrm{CD} / \mathrm{HG} / \mathrm{SE}$ \& / SIO2-S SS / AL2O3-S SS / BA-S SS / CR-S SS / \& PB-S SS / AG-S SS / MGO-S SS / NA2O-S SS / K2O-S \& SS / FE304-S SS / CAO-S SS

BLOCK-OPTION RESTART=NO

BLOCK QUENCH RGIBBS

PARAM TEMP $=350$ PRES $=1$ <ATM> NPHASE $=2$ MAXIT $=500$

PROD H2 / O2 / N2 / F2 / NO / SO2 / H2O / $\mathrm{CO} 2 / \&$ $\mathrm{CO} / \mathrm{HCL} / \mathrm{AS} / \mathrm{CD} / \mathrm{HG} / \mathrm{SE} / \mathrm{SIO2-S} \mathrm{SS} / \&$ AI203-s ss / As-s Ss / BA-s SS / CD-S SS / CR-s \& SS / $\mathrm{PB}-\mathrm{S}$ SS / SE-S SS / AG-S SS / MGO-S SS / \& NA2O-S SS / K20-S SS / FE304-S SS / CAO-S SS

BLOCK-OPTION RESTART=NO

BLOCK SCC RGIBBS

PARAM TEMP $=2200$ PRES=1 <ATM> NPHASE=2

$\mathrm{PROD} \mathrm{H} 2 / \mathrm{O} 2 / \mathrm{N} 2 / \mathrm{CL} 2 / \mathrm{F} 2 / \mathrm{NO} / \mathrm{SO} 2 / \mathrm{H} 2 \mathrm{O} /$ \& $\mathrm{CO} 2 / \mathrm{CO} / \mathrm{HCL} / \mathrm{CH} 4 / \mathrm{S} / \mathrm{AS} / \mathrm{CD} / \mathrm{HG} / \mathrm{SE}$ \& / SIO2-S sS / AL203-S sS / BA-S sS / CR-S SS / \& PB-S SS / AG-S SS / MGO-S SS / NA2O-S SS / K2O-S \& SS / EE3O4-S SS / CAO-S SS

BLOCK-OPTION RESTART $=$ NO

BLOCK SCRUB RGIBBS

PARAM TEMP=120 PRES=1 <ATM> NPHASE $=2$ MAXIT $=500$

PROD $\mathrm{H} 2 / \mathrm{O} 2 / \mathrm{N} 2 / \mathrm{CL} 2 / \mathrm{SO} 2 / \mathrm{H} 20 / \mathrm{CO} 2 / \mathrm{CO} / \&$

HCL / NAOH-S SS / NACL-S SS / NAF-S SS

BLOCK-OPTION RESTART=NO

BLOCK VITRIFY RGIBBS

PARAM TEMP $=3000$ PRES $=1$ <ATM> NPHASE $=2$

PROD H2 / O2 / N2 / CL2 / E2 / NO / SO2 / H2O / \& $\mathrm{CO} 2 / \mathrm{CO} / \mathrm{HCL} / \mathrm{C}-\mathrm{S} / \mathrm{S} / \mathrm{AS} / \mathrm{CD} / \mathrm{HG} / \mathrm{SE} \&$ / SI02/AL203/AS-S ss / BA-s ss / CD-S SS / \& CR-S SS / PB-S SS / SE-S SS / AG-S SS / MGO-S \& SS / NA2O-S SS / K2O-S SS / FE3O4-S SS / CAO-S SS BLOCK-OPTION RESTART=NO

BLOCK DUPL1 DUPL

BLOCK DUPL2 DUPL

DESIGN-SPEC HCL

DEEINE HCL MASS-FLOW STREAM=HCL-SOLN SUBSTREAM=MIXED \& COMPONENT $=H C L$

DEFINE H2O MASS-FLOW STREAM=HCL-SOLN SUBSTREAM=MIXED \& COMPONENT $=\mathrm{H} 2 \mathrm{O}$

SPEC "HCL-H2O" TO "O"

TOL-SPEC "0.01"

VARY BLOCK-VAR BLOCK=SALT-SEP SENTENCE=FRAC VARIABLE=FRACS \& 


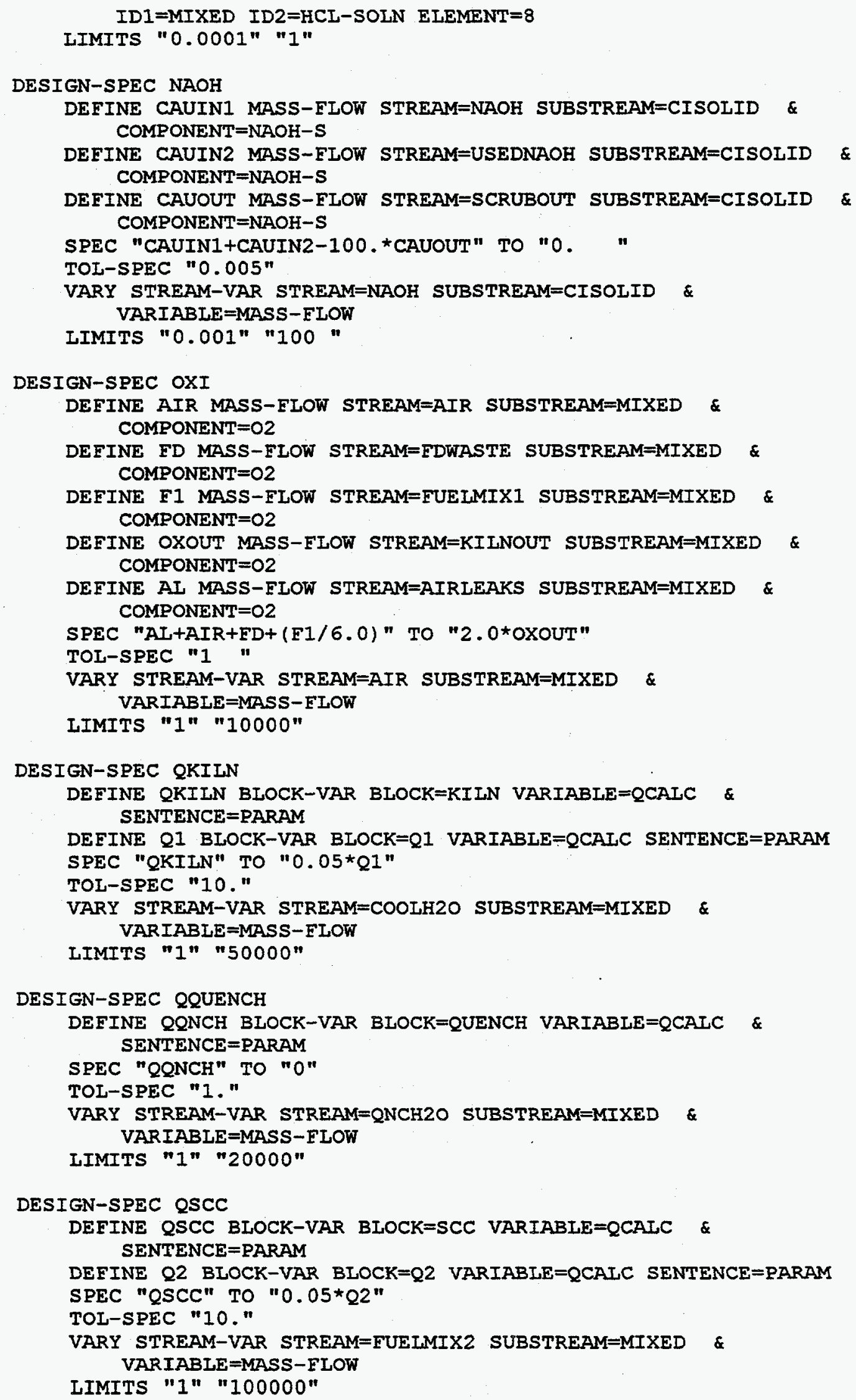


DESIGN-SPEC SCRUBH2O

DEFINE MASSAL STREAM-VAR STREAM=SCRUBBOT SUBSTREAM=CISOLID VARIABLE=MASS-FLOW

DEFINE SCRUB STREAM-VAR STREAM=SCRUBH2O SUBSTREAM=MIXED \& VARIABLE = MASS - FLOW

DEFINE NAOH STREAM-VAR STREAM=NAOH SUBSTREAM=MIXED \& VARIABLE=MASS - ELOW

SPEC "SCRUB" TO "20*MASSAL"

TOL-SPEC "0.01"

VARY STREAM-VAR STREAM=SCRUBH2O SUBSTREAM=MIXED \& VARIABLE =MASS- ELOW

LIMITS "NAOH" "5000"

DESIGN-SPEC SOILH2O

DEEINE SIAGI STREAM-VAR STREAM=SIAAG SUBSTREAM=MIXED \& VARIABIEE=MASS - FLOW

DEFINE SLAG2 STREAM-VAR STREAM=SIAG SUBSTREAM=CISOLID \& VARIABLE=MASS-ELOW

DEFINE SOIL STREAM-VAR STREAM=SOIL SUBSTREAM=MIXED \& VARIABLE =MASS-FLOW

SPEC "30.* SOIL" TO "SIAG1+SIAG2"

TOL-SPEC "0.01"

VARY STREAM-VAR STREAM=SOIL SUBSTREAM=MIXED \& VARIABLE =MASS-FLOW

LIMITS "1" "1000"

DESIGN-SPEC SOILSOL

DEFINE SIAGI STREAM-VAR STREAM=SLAG SUBSTREAM=MIXED \& VARIABLE =MASS-FLOW

DEEINE SIAG2 STREAM-VAR STREAM=SLAG SUBSTREAM=CISOLID \& VARIABLE =MASS-FLOW

DEEINE SOII STREAM-VAR STREAM=SOII SUBSTREAM=CISOLID \& VARIABLE=MASS - ELOW

SPEC "3.33333*SOIL" TO "SLAGI+SLAG2"

TOI-SPEC "0.01"

VARY STREAM-VAR STREAM=SOIL SUBSTREAM=CISOIID \& VARIABLE=MASS- ELOW

LIMITS "I" "1000"

CONV-OPTIONS

PARAM TOI $=0.01$

SECANT MAX-STEP-SIZ $=.05$ BRACKET=YES

STREAM-REPOR NOSORT MOLEFLOW MASSFLOW PROPERTIES=SET1 ALL-SUBS 


\section{System A-7}

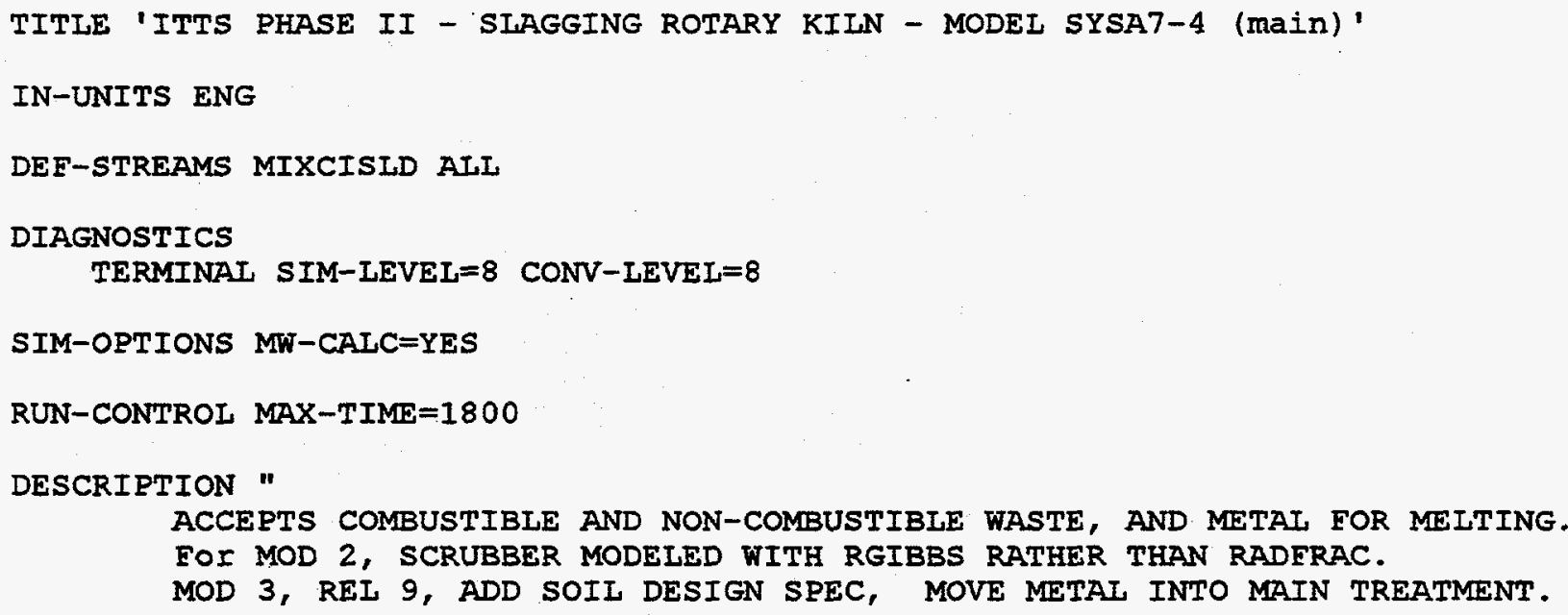




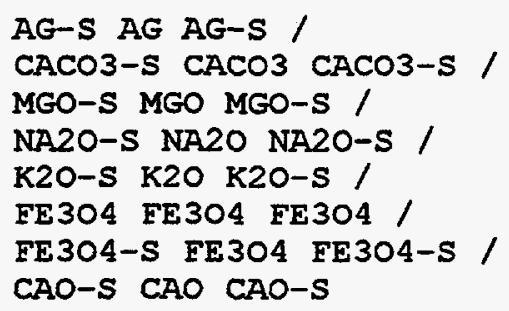

STREAM FUELMIXI 
SUBSTREAM MIXED TEMP $=68$ PRES $=1$ <ATM> MASS-FLOW $=12786.567$

MASS-FLOW $020.222 / \mathrm{N} 20.767 / \mathrm{CH} 40.046$

STREAM FUELMIX2

SUBSTREAM MIXED TEMP=68 PRES=1 <ATM> MASS-FLOW $=4526.77$

MASS-FLOW $020.222 / \mathrm{N} 20.732 / \mathrm{CH} 40.046$

STREAM NAOH

SUBSTREAM MIXED TEMP $=68$ PRES $=1$ <ATM>

SUBSTREAM CISOLID TEMP=68 PRES=1 <ATM> MASS-FLOW=77.611

MASS-FLOW NAOH-S 1

STREAM OUT1

SUBSTREAM MIXED TEMP $=2500$ PRES $=1$ <ATM>

MASS-FLOW O2 1293 / N2 14610 / NO 27 / SO2 3 / H2O \& 1977 / CO2 2598 / HCL 70 / FE304 45

SUBSTREAM CISOLID TEMP $=2500$ PRES $=1$ <ATM>

MASS-FLOW SIO2-S 1069 / AL2O3-S 739 / CR-S 8 / MGO-S \&

$18 /$ NA2O-S $10 /$ K2O-S 19 / CAO-S 56

STREAM QNCH2O

SUBSTREAM MIXED TEMP=68 PRES=1 <ATM> MASS-ELOW=15424.714

MASS-FLOW H2O 1

STREAM QUENCHED

SUBSTREAM MIXED TEMP $=350$ PRES $=1$ <ATM>

MASS-FLOW O2 1446 / N2 17490 / CL2 29 / H2O $16320 /$ \& $\mathrm{CO} 23088$ / HCL 40

SUBSTREAM CISOIID TEMP $=350$ PRES $=1$ <ATM>

MASS-FLOW SIO2-S 221 / AL2O3-S 148 / CD-S 3.3 / CR-S \& $1.62 / \mathrm{MGO}-\mathrm{S} 3.6 / \mathrm{NA} 2 \mathrm{O}-\mathrm{S} 2 / \mathrm{K} 20-\mathrm{S} 3.75 / \mathrm{FE} 304-\mathrm{S} 9$

STREAM SCRUBH2O

SUBSTREAM MIXED TEMP=68 PRES=1 <ATM> MASS-FLOW=78

MASS-FLOW H2O 1

STREAM SCRUBOUT

SUBSTREAM MIXED TEMP $=120$ PRES $=1$ <ATM>

MASS-FLOW 021452 / N2 17490 / H2O $373000 / \mathrm{CO} 3088$

SUBSTREAM CISOLID TEMP $=120$ PRES $=1$ <ATM>

MASS-FLOW NACL-S 112

STREAM SOII

SUBSTREAM MIXED TEMP $=68$ PRES=1 <ATM> MASS-FLOW=56.359

MASS-FLOW H2O 0.1

SUBSTREAM CISOLID TEMP=68 PRES=1 <ATM> MASS-FLOW=507.231

MASS-FLOW SIO2-S $0.569 /$ AL2O3-S $0.102 /$ CACO3-S $0.128 / \&$ MGO-S $0.023 / \mathrm{NA2O-S} 0.013 / \mathrm{K} 20-\mathrm{S} 0.024 / \mathrm{FE} 304-\mathrm{S} 0.041$

BLOCK BAGHOUSE SEP

FRAC STREAM=HGPLUS SUBSTREAM=MIXED COMPS=H2 O2 N2 CL2 \& F2 NO SO2 H2O CO2 CO HCL CH4 C-S S-S S NAOH-S \& NACL-S NAF-S AS CD HG SE FE SIO2 AL2O3 FE-S SIO2-S \& AL2O3-S AS-S BA-S CD-S CR-S PB-S SE-S AG-S CACO3-S \& MGO-S NA2O-S K2O-S FE3O4 FE304-S CAO-S FRACS $=0 \quad 0 \quad 0 \quad \&$ $0 \begin{array}{lllllllllllllllllllll}0 & 0 & 0 & 0 & 0 & 0 & 0 & 0 & 0 & 0 & 0 & 0 & 0 & 0 & 1 & 1 & 1 & 1 & 0 & 0 & \&\end{array}$ $0 \begin{array}{lllllllllllllllllllllllll}0 & 0 & 0 & 0 & 1 & 0 & 1 & 0 & 0 & 1 & 0 & 0 & 0 & 0 & 0 & 0 & 0 & 0\end{array}$

FRAC STREAM=HǴPLUS SUBSTREAM=CISOLID COMPS=C-S S-S NAOH-S \& NACL-S NAF-S FE-S SIO2-S AL203-S AS-S BA-S CD-S CR-S \& PB-S SE-S AG-S CACO3-S MGO-S NA2O-S K2O-S FE3O4-S \& CAO-S FRACS $=0 \begin{array}{lllllllllllllllllllll}0 & 0 & 0 & 0 & 0 & 0 & 0 & 1 & 0 & 1 & 0 & 0 & 1 & 0 & 0 & 0 & 0 & 0 & 0 & 0\end{array}$

FRAC STREAM=FLYASH SUBSTREAM=MIXED COMPS=H2 O2 N2 CI2 \& 
F2 NO SO2 H2O CO2 CO HCI CH4 C-S S-S S NAOH-S \& NACI-S NAF-S AS CD HG SE FE SIO2 AI2O3 FE-S SIO2-S \& AL2O3-S AS-S BA-S CD-S CR-S PB-S SE-S AG-S CACO3-S \& MGO-S NA2O-S K2O-S FE304 FE304-S CAO-S FRACS=0 000 \& $0 \begin{array}{lllllllllllllllllllll}0 & 0 & 0 & 0 & 0 & 0 & 0 & 0 & 0 & 1 & 1 & 1 & 1 & 1 & 1 & 0 & 0 & 0 & 0 & 1 & 1\end{array}$ $\begin{array}{llllllllllllllllllllllll}1 & 1 & 1 & 1 & 0 & 1 & 0 & 1 & 1 & 0 & 1 & 1 & 1 & 1 & 1 & 1 & 1 & 1\end{array}$

FRAC STREAM=FLYASH SUBSTREAM=CISOLID COMPS=C-S S-S NAOH-S \& NACI-S NAF-S FE-S SIO2-S AL2O3-S AS-S BA-S CD-S CR-S \& PB-S SE-S AG-S CACO3-S MGO-S NA2O-S K2O-S FE3O4-S \&

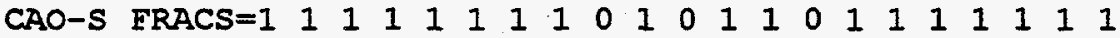

BLOCK KILNSEP SEP

FRAC STREAM=SLAG SUBSTREAM=MIXED COMPS=H2 O2 N2 CL2 F2 \& NO SO2 H2O CO2 CO HCL CH4 C-S S-S S NAOH-S NACL-S \& NAF-S AS CD HG SE FE SIO2 AI2O3 FE-S SIO2-S AI203-S \& AS-S BA-S CD-S CR-S PB-S SE-S AG-S CACO3-S MGO-S \&

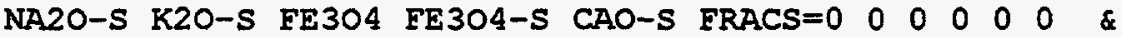
$\begin{array}{lllllllllllllllllll}0 & 0 & 0 & 0 & 0 & 0 & .8 & .8 & 0 & .8 & .8 & .8 & 0 & 0 & 0 & 0 & .8 & .8 & \alpha\end{array}$ $\begin{array}{llllllllllllllllllll}.8 & .8 & .8 & .8 & .8 & .8 & .8 & .8 & .8 & .8 & .8 & .8 & .8 & .8 & .8 & .8 & .8 & .8\end{array}$

FRAC STREAM=SLAG SUBSTREAM=CISOLID COMPS=C-S S-S NAOH-S \& NACL-S NAF-S FE-S SIO2-S AL2O3-S AS-S BA-S CD-S CR-S \& PB-S SE-S AG-S CACO3-S MGO-S NA2O-S K2O-S FE3O4-S \&

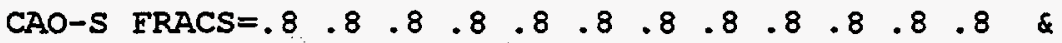
$\begin{array}{llllllllll}.8 & .8 & .8 & .8 & .8 & .8 & .8 & .8 & .8 & .8\end{array}$

BLOCK L-V-SEP FLASH2

PARAM TEMP $=120$ PRES $=1$ <ATM>

BLOCK KILN RGIBBS

PARAM TEMP $=2500$ PRES $=1$ <ATM $>$ NPHASE $=2$ MAXIT $=500$

PROD H2 / O2/ N2 / CL2 / F2 / NO / SO2 / H2O / \& $\mathrm{CO} 2 / \mathrm{CO} / \mathrm{HCL} / \mathrm{AS} / \mathrm{CD} / \mathrm{HG} / \mathrm{SE} / \mathrm{SIO} 2 / \&$ AL203 / FE-S SS / AS-S SS / BA-S SS / CD-S SS / \& CR-S SS / PB-S SS / SE-S SS / AG-S SS / MGO-S \& SS / NA2O-S SS / K2O-S SS / FE304-S SS / CAO-S SS PROD-FRAC FE-S $1 /$ FE304-S 1 BLOCK-OPTION RESTART $=$ NO

BLOCK $Q 1$ RGIBBS

PARAM TEMP=68 PRES=1 <ATM> NPHASE=2 TAPP=2432 MAXIT=500

$\mathrm{PROD} \mathrm{H} 2 / \mathrm{O} 2 / \mathrm{N} 2 / \mathrm{CL} 2 / \mathrm{E} 2 / \mathrm{NO} / \mathrm{sO} 2 / \mathrm{H} 2 \mathrm{O} / \&$ $\mathrm{CO} 2 / \mathrm{CO} / \mathrm{HCL} / \mathrm{AS} / \mathrm{CD} / \mathrm{HG} / \mathrm{SE} / \mathrm{SIO} 2 / \&$ AL203 / FE-S SS / AS-S SS / BA-S SS / CD-S SS / \& CR-S SS / PB-S SS / SE-S SS / AG-S SS / MGO-S \& SS / NA2O-S SS / K2O-S SS / FE304-S SS / CAO-S SS PROD-FRAC FE-S $1 /$ FE3O4-S 1

BLOCK Q2 RGIBBS

PARAM TEMP=68 PRES=1 <ATM> NPHASE=2 CHEMEQ=YES TAPP=2432 \& MAXIT $=60$

PROD H2 / O2 / N2 / CL2 / $\mathrm{F} 2 / \mathrm{NO} / \mathrm{SO} / \mathrm{H} 2 \mathrm{O} / \&$ $\mathrm{CO} 2 / \mathrm{CO} / \mathrm{HCL} / \mathrm{CH} 4 / \mathrm{S} / \mathrm{AS} / \mathrm{CD} / \mathrm{HG} / \mathrm{SE}$ \& / SIO2 / AL2O3 / FE-S SS / BA-S SS / CR-S SS / \& PB-S SS / AG-S SS / MGO-S SS / NA2O-S SS / K2O-S \& SS / FE304-S SS / CAO-S SS

PROD-FRAC FE-S 1 / FE304-S 1

\section{BLOCK OUENCH RGIBBS}

PARAM TEMP $=350$ PRES $=1$ <ATM> NPHASE $=2$

PROD H2 / O2 / N2 / CL2 / F2 / NO / $\mathrm{SO} 2 / \mathrm{H} 2 \mathrm{O} / \&$ $\mathrm{CO} 2 / \mathrm{CO} / \mathrm{HCL} / \mathrm{AS} / \mathrm{CD} / \mathrm{HG} / \mathrm{SE} / \mathrm{FE}-\mathrm{S}$ SS \& / SIO2-S SS / AL2O3-S SS / AS-S SS / BA-S SS / \& 
CD-S SS / CR-S SS / PB-S SS / SE-S SS / AG-S SS \&

/ MGO-S SS / NA2O-S SS / K20-S SS / FE304-S SS / \& CAO-S SS

PROD-FRAC EE-S $1 /$ FE304-S 1

BIOCK SCC RGIBBS

PARAM TEMP $=2500$ PRES $=1$ <ATM> NPHASE $=2$

PROD H2 / O2/ N2/ $\mathrm{CL} 2 / \mathrm{F} 2 / \mathrm{NO} / \mathrm{sO} / \mathrm{H} 2 \mathrm{O} /$ \& $\mathrm{CO} 2 / \mathrm{CO} / \mathrm{HCL} / \mathrm{CH} 4 / \mathrm{S} / \mathrm{AS} / \mathrm{CD} / \mathrm{HG} / \mathrm{SE} \&$ / SIO2/AL203/ $/ \mathrm{FE}-\mathrm{S}$ SS / BA-S SS / CR-S SS / \& PB-S SS / AG-S SS / MGO-S SS / NA2O-S SS / K2O-S \& SS / FE304-S SS / CAO-S SS

PROD-FRAC FE-S $1 /$ FE304-S 1

BLOCK SCRUB RGIBBS

PARAM TEMP=120 PRES=1 <ATM> NPHASE=2 MAXIT $=300$

PROD H2 / O2/ N2/ CL2/ $2 /$ NO / SO2 / H2O/ \& $\mathrm{CO} 2 / \mathrm{CO} / \mathrm{HCL} / \mathrm{NAOH}-\mathrm{S}$ SS / NACL-S SS / NAF-S SS

BLOCK DUPL1 DUPI

BLOCK DUPL2 DUPL

DESIGN-SPEC NAOH

DEFINE CAUIN MASS-FLOW STREAM=NAOH SUBSTREAM=CISOLID \& COMPONENT $=\mathrm{NAOH}-\mathrm{S}$

DEFINE CAUOUT MASS-FLOW STREAM=SCRUBOUT SUBSTREAM=CISOLID \& COMPONENT $=$ NAOH $-S$

SPEC "CAUIN-100.*CAUOUT" TO "O"

TOI-SPEC "0.01"

VARY STREAM-VAR STREAM=NAOH SUBSTREAM=CISOLID \& VARIABLE=MASS-FLOW

LIMITS "1" "500"

DESIGN-SPEC OXI

DEFINE AIR MASS-FLOW STREAM=AIR SUBSTREAM=MIXED \& COMPONENT $=02$

DEFINE WC MASS-FLOW STREAM=FDWASTEC SUBSTREAM=MIXED \& COMPONENT $=02$

DEFINE WN MASS-FLOW STREAM=FDWASTEN SUBSTREAM=MIXED * COMPONENT $=02$

DEEINE E1 MASS-ELOW STREAM=FUEIMIX1 SUBSTREAM=MIXED \& COMPONENT $=02$

DEFINE OXOUT MASS-FLOW STREAM=KILNOUT SUBSTREAM=MIXED \& COMPONENT $=02$

DEEINE AI MASS-FLOW STREAM=AIRIEAKS SUBSTREAM=MIXED \& COMPONENT $=02$

SPEC "AL+AIR+WC+WN+(F1/6.0)" TO "2.0*OXOUT"

TOL-SPEC ". I"

VARY STREAM-VAR STREAM=ATR SUBSTREAM=MTXED \& VARIABLE =MASS-FLOW

IIMITS " 1 " "10000"

DESIGN-SPEC OKILN

DEFINE QKILN BLOCK-VAR BLOCK=KILN VARIABLE=QCALC \& SENTENCE=PARAM

DEFINE Q1 BLOCK-VAR BLOCK=Q1. VARIABLE=QCALC SENTENCE=PARAM SPEC "OKILN" TO "0.05*Q1"

TOL-SPEC "I. "

VARY STREAM-VAR STREAM=FUELMIX1 SUBSTREAM=MIXED \& VARIABLE=MASS - FLOW

LIMITS "1" "50000" 
DESIGN-SPEC QQUENCH

DEFINE QQNCH BLOCK-VAR BLOCK=QUENCH VARIABLE=QCALC \&

SENTENCE = PARAM

SPEC "QQNCH" TO "O"

TOL-SPEC "1."

VARY STREAM-VAR STREAM=QNCH2O SUBSTREAM=MIXED \& VARIABLE =MASS-FIOW

LIMITS "1" "20000"

DESIGN-SPEC QSCC

DEEINE QSCC BLOCK-VAR BLOCK=SCC VARIABLE=QCALC \&

SENTENCE=PARAM

DEEINE Q2 BLOCK-VAR BLOCK=Q2 VARIABLE=QCALC SENTENCE=PARAM

SPEC "QSCC" TO "0.05*Q2"

TOL-SPEC "10."

VARY STREAM-VAR STREAM=FUELMIX2 SUBSTREAM=MIXED \& VARIABIE=MASS-FLOW

IIMITS "1" "10000"

DESIGN-SPEC SCRUBH2O

DEEINE MASSAL STREAM-VAR STREAM=SCRUBBOT SUBSTREAM=CISOLID \& VARIABLE $=$ MASS $-E L O W$

DEEINE SCRUB STREAM-VAR STREAM=SCRUBH2O SUBSTREAM=MIXED \& VARIABLE=MASS-ELOW

DEFINE NAOH STREAM-VAR STREAM=NAOH SUBSTREAM=CISOLID \& VARIABLE=MASS - FLOW

SPEC "SCRUB" TO "20*MASSAI"

TOL-SPEC "0.01"

VARY STREAM-VAR STREAM=SCRUBH2O SUBSTREAM=MIXED \& VARIABLE=MASS-ELOW

LIMITS "NAOH" "5000"

DESIGN-SPEC SOILH 20

DEEINE SLAGI STREAM-VAR STREAM=SIAG SUBSTREAM=MIXED \& VARIABLE=MASS - ELOW

DEFINE SLAG2 STREAM-VAR STREAM=SLAG SUBSTREAM=CISOLID \& VARIABLE =MASS-ELOW

DEEINE SOIL STREAM-VAR STREAM=SOII SUBSTREAM=MIXED \& VARIABLE =MASS - FLOW

SPEC "30.* SOIL" TO "SLAG1+SIAG2"

TOL-SPEC "0.01"

VARY STREAM-VAR STREAM=SOIL SUBSTREAM=MIXED \& VARIABLE =MASS - ELOW

LIMITS " 1 " "1000"

DESIGN-SPEC SOILSOL

DEEINE SLAGI STREAM-VAR STREAM=SLAG SUBSTREAM=MIXED \& VARIABLE $=$ MASS - FIOW

DEFINE SLAG2 STREAM-VAR STREAM=SIAG SUBSTREAM=CISOIID \& VARIABLE =MASS-FLOW

DEEINE SOIL STREAM-VAR STREAM=SOII SUBSTREAM=CISOLID \& VARIABLE=MASS-FLOW

SPEC " 3.33333 *SOIL" TO "SIAG1+SIAG2"

TOL-SPEC "0.01"

VARY STREAM-VAR STREAM=SOII SUBSTREAM=CISOIID \& VARIABLE=MASS-ELOW

IIMITS "I" "I000"

CONV-ORTIONS

SECANT MAX-STEP-SIZ $=.05$ BRACKET=YES

CONVERGENCE BROQKIIN BROYDEN 
SPEC QKIIN

STREAM-REPOR NOSORT MOLEFLOW MASSFLOW PROPERTIES=SET1 ALI-SUBS 


\section{System B-1}

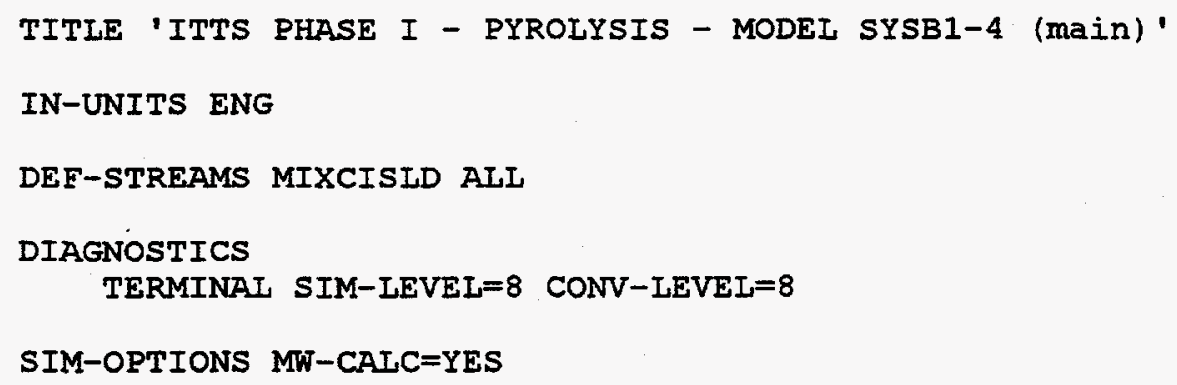


EE304-S EE304 EE304-S /

CAO-S CAO CAO-S

FLOWSHEET

BLOCK PYRO-SEP IN=PYROOUT OUT=PYROVAP ASH

BLOCK BAGHOUSE IN=QUENCHED OUT=HGPIUS FLYASH QNCHVAP

BLOCK QUENCH IN=SCCOUT QNCH 20 OUT=QUENCHED

BLOCK I-V-SEP IN=SCRUBOUT OUT=SCRUBVAP SCRUBBOT

BIOCK VITRIFY IN=FLYASH ASH FDWASTEN SOIL O2-VIT OUT $=\&$ VITOUT

BLOCK VIT-SEP IN=VITOUT OUT=VITVAP SLAG

BLOCK PYROLYZR IN=AIRLEAKS FDWASTEC OXYGEN OUT=PYROOUT

BLOCK SCC IN=PYROVAP VITVAP O2-SCC OUT=SCCOUT

BLOCK SCRUB IN=SCRUBH2O NAOH QNCHVAP OUT=SCRUBOUT

BLOCK MELTER IN=FDMETMLT OUT=METAL

PROPERTIES SOLIDS

PROPERTIES IDEAL / RKS-BM

PROP-SET ALI-SUBS TEMP PRES VFRAC UNITS='ATM' SUBSTREAM=ALI

STREAM AIRIEAKS

SUBSTREAM MIXED TEMP $=68$ PRES $=1$ <ATM> MASS-FLOW $=224$

MASS-FRAC $020.233 / \mathrm{N} 20.767$

STREAM FDMETMLT

SUBSTREAM MIXED TEMP $=68$ PRES $=1$ <ATM> MASS-FLOW $=1.20$

MASS-FLOW H2 2.42

SUBSTREAM CISOLID TEMP $=68$ PRES $=1$ <ATM> MASS-FLOW=147.80

MASS-FLOW C-S $14.44 /$ FE-S $282.02 /$ SIO2-S $0.035 / \&$

AL203-S $0.035 / \mathrm{BA}-\mathrm{S} 0.87 / \mathrm{CD}-\mathrm{S} 0.18$

STREAM FDWASTEC

SUBSTREAM MIXED TEMP $=68$ PRES $=1$ <ATM>

MASS-FLOW H2 50.95/ O2 $92.76 / \mathrm{N} 20.23 / \mathrm{CL} 253.02 / \&$ F2 $0.11 / \mathrm{H} 2019.83$

SUBSTREAM CISOLID TEMP $=68$ PRES=1 <ATM>

MASS-FLOW C-S 326.28 / S-S $0.49 /$ FE-S $7.67 /$ SIO2-S \&

$53.01 /$ AL2O3-S 53.01/ BA-S $0.77 /$ CD-S $0.77 /$ CR-S 1.53

STREAM FDWASTEN

SUBSTREAM MIXED TEMP=68 PRES=1 <ATM>

MASS-FLOW H2 $0.54 / 021.44$ / N2 $0.01 / \mathrm{CL} 214.88 / \&$

NO $0.66 / \mathrm{SO} 0.83 / \mathrm{H} 20354.23 / \mathrm{HG} 0.15$

SUBSTREAM CISOLID TEMP $=68$ PRES $=1$ <ATM>

MASS-FLOW C-S 4.77 / S-S 0.62 / SIO2-S 476.77 / \&

AL203-S 476.77 / AS-S $0.06 /$ BA-S $0.04 / \mathrm{CD}-\mathrm{S} 2.63$ \&

/ CR-S 4.95 / PB-S $0.17 /$ SE-S $0.03 /$ AG-S 0.03

STREAM NAOH

SUBSTREAM MIXED TEMP=68 PRES=1 <ATM>

SUBSTREAM CISOLID TEMP=68 PRES $=1$ <ATM> MASS-FLOW=77.611

MASS-FRAC NAOH-S 1

STREAM 02-SCC

SUBSTREAM MIXED TEMP=68 PRES=1 <ATM> MASS-ELOW=833.774

MASS-FRAC O2 1

STREAM O2-VIT

SUBSTREAM MIXED TEMP=68 PRES=1 <ATM> MASS-ELOW=22.936

MASS-FRAC 021 
STREAM OXYGEN

SUBSTREAM MIXED TEMP=68 PRES=1 <ATM> MASS-FLOW=289.513

MASS-FRAC 021

STREAM QNCH2O

SUBSTREAM MIXED TEMP=68 PRES=1 〈ATM> MASS-FLOW=1410.762

MASS-FRAC H2O 1

STREAM SCRUBH 20

SUBSTREAM MIXED TEMP=68 PRES=1 <ATM> MASS-FLOW=78

MASS-FLOW H2O 1

STREAM SCRUBOUT

SUBSTREAM MIXED TEMP=120 PRES=1 <ATM>

MASS-FLOW O2 $1452 / \mathrm{N} 217490 / \mathrm{H} 20373000 / \mathrm{CO} 3088$

SUBSTREAM CISOLID TEMP=120 PRES $=1$ <ATM>

MASS-FIOW NACL-S 112

STREAM SOIL

SUBSTREAM MIXED TEMP=68 PRES=1 <ATM> MASS-FLOW=49.8413

MASS-FLOW H2O 0.1

SUBSTREAM CISOLID TEMP=68 PRES=1 <ATM> MASS-FLOW $=448.572$

MASS-FLOW SIO2-S $0.569 /$ AL203-S $0.102 /$ CACO3-S $0.128 / \&$ . MGO-S $0.023 /$ NA2O-S $0.013 /$ K2O-S $0.024 /$ FE3O4-S 0.041

BLOCK BAGHOUSE SEP

ERAC STREAM=HGPIUS SUBSTREAM=MIXED COMPS=H2 O2 N2 CL2 \& E2 NO SO2 H2O $\mathrm{CO} 2 \mathrm{CO} H C L \mathrm{CH} 4 \mathrm{C}-\mathrm{S} S-\mathrm{S} S \mathrm{NAOH}-\mathrm{S} \&$ NACL-S NAF-S AS CD HG SE FE SIO2 AL2O3 FE-S SIO2-S \& AL2O3-S AS-S BA-S CD-S CR-S PB-S SE-S AG-S CACO3-S \& MGO-S NA2O-S K2O-S FE304 FE304-S CAO-S FRACS $=0 \quad 0 \quad 0$ \& $\begin{array}{llllllllllllllllllllll}0 & 0 & 0 & 0 & 0 & 0 & 0 & 0 & 0 & 0 & 0 & 0 & 0 & 0 & 0 & 1 & 1 & 1 & 1 & 0 & 0 & \&\end{array}$ $\begin{array}{llllllllllllllllll}0 & 0 & 0 & 0 & 1 & 0 & 1 & 0 & 0 & 1 & 0 & 0 & 0 & 0 & 0 & 0 & 0 & 0\end{array}$

FRAC STREAM=HGPLUS SUBSTREAM=CISOIID COMPS=C-S S-S NAOH-S \& NACL-S NAF-S EE-S SIO2-S AI2O3-S AS-S BA-S CD-S CR-S \& PB-S SE-S AG-S CACO3-S MGO-S NA2O-S K2O-S FE3O4-S \& CAO-S FRACS $=0 \begin{array}{lllllllllllllllllllll}0 & 0 & 0 & 0 & 0 & 0 & 0 & 0 & 1 & 0 & 1 & 0 & 0 & 1 & 0 & 0 & 0 & 0 & 0 & 0 & 0\end{array}$

ERAC STREAM=FLYASH SUBSTREAM=MIXED COMPS=H2 O2 N2 CL2 \& F2 NO SO2 H2O $\mathrm{CO} 2 \mathrm{CO} \mathrm{HCI} \mathrm{CH} 4 \mathrm{C}-\mathrm{S}$ s-S $\mathrm{S}$ NAOH-S \& NACL-S NAF-S AS CD HG SE EE SIO2 AL2O3 FE-S SIO2-S \& AL2O3-S AS-S BA-S CD-S CR-S PB-S SE-S AG-S CACO3-S \& MGO-S NA2O-S K2O-S FE304 FE304-S CAO-S FRACS $=0$ O 0 \& $\begin{array}{llllllllllllllllllllll}0 & 0 & 0 & 0 & 0 & 0 & 0 & 0 & 0 & 1 & 1 & 1 & 1 & 1 & 1 & 0 & 0 & 0 & 0 & 1 & 1 & \varepsilon\end{array}$ $\begin{array}{llllllllllllllllll}1 & 1 & 1 & 1 & 0 & 1 & 0 & 1 & 1 & 0 & 1 & 1 & 1 & 1 & 1 & 1 & 1 & 1\end{array}$

ERAC STREAM=FLYASH SUBSTREAM=CISOIID COMPS=C-S S-S NAOH-S \& NACL-S NAF-S FE-S SIO2-S AL2O3-S AS-S BA-S CD-S CR-S \& PB-S SE-S AG-S CACO3-S MGO-S NA2O-S K2O-S FE3O4-S \&

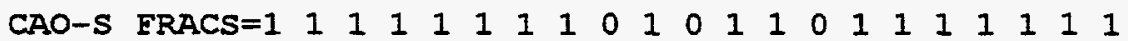
FLASH-SPECS FLYASH TEMP=68 PRES=1 〈ATM> NPHASE=1

\section{BIOCK PYRO-SEP SEP}

FRAC STREAM=ASH SUBSTREAM=MIXED COMPS=H2 O2 N2 CL2 F2 \& NO SO2 H2O CO2 CO HCL CH4 C-S s-S S NAOH-S NACL-S \& NAF-S AS CD HG SE FE SIO2 AL2O3 FE-S SIO2-S AL203-S \& AS-S BA-S CD-S CR-S PB-S SE-S AG-S CACO3-S MGO-S \&

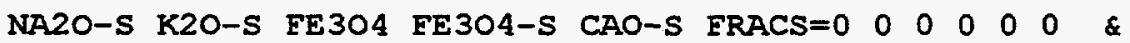
$\begin{array}{llllllllllllllllll}0 & 0 & 0 & 0 & 0 & 0 & .8 & .8 & 0 & .8 & .8 & .8 & 0 & 0 & 0 & 0 & .8 & .8\end{array}$ $\begin{array}{llllllllllllllllll}.8 & .8 & .8 & .8 & .8 & .8 & .8 & .8 & .8 & .8 & .8 & .8 & .8 & .8 & .8 & .8 & .8 & .8\end{array}$

FRAC STREAM=ASH SUBSTREAM=CISOIID COMPS=C-S $\mathbf{s}-\mathrm{S}$ NAOH-S \& NACL-S NAF-S FE-S SIO2-S AI203-S AS-S BA-S CD-S CR-S \& PB-S SE-S AG-S CACO3-S MGO-S NA2O-S K2O-S FE3O4-S \&

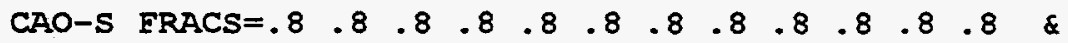


$\begin{array}{llllllllll}.8 & .8 & .8 & .8 & .8 & .8 & .8 & .8 & .8\end{array}$

FLASH-SPECS ASH TEMP $=68$ PRES $=1$ <ATM>

BLOCK VIT-SEP SEP

FRAC STREAM=VITVAP SUBSTREAM=MIXED COMPS=H2 O2 N2 CL2 \& F2 NO SO2 H2O $\mathrm{CO} 2 \mathrm{CO} \mathrm{HCI}$ CH4 c-s s-s s NAOH-S \& NACL-S NAF-S AS CD HG SE FE SIO2 AI2O3 FE-S SIO2-S \& AL203-S AS-S BA-S CD-S CR-S PB-S SE-S AG-S CACO3-S \& MGO-S NA2O-S K2O-S FE304 EE304-S CAO-S ERACS=1 11 \& $\begin{array}{llllllllllllllllllllll}1 & 1 & 1 & 1 & 1 & 1 & 1 & 1 & 1 & 0 & 0 & 0 & 0 & 0 & 0 & 1 & 1 & 1 & 1 & 0 & 0 & \text { \& }\end{array}$

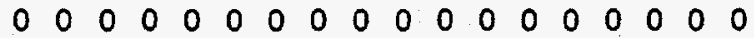

FRAC STREAM=VITVAP SUBSTREAM=CISOLID COMPS=C-S S-S NAOH-S \& NACI-S NAF-S EE-S SIO2-S AL203-S AS-S BA-S CD-S CR-S \& PB-S SE-S AG-S CACO3-S MGO-S NA2O-S K2O-S FE304-S \& CAO-S ERACS $=0 \begin{array}{llllllllllllllllllll}0 & 0 & 0 & 0 & 0 & 0 & 0 & 0 & 0 & 0 & 0 & 0 & 0 & 0 & 0 & 0 & 0 & 0 & 0 & 0\end{array}$

BLOCK L-V-SEP FLASH2

PARAM TEMP=120 PRES=1 〈ATM>

BIOCK PYROIYZR RSTOIC

PARAM TEMP $=1200$ PRES $=1 \quad\langle$ ATM $>$

STOIC 1 CISOLID C-S $-1 /$ MIXED O2 -0.5/ CO 1

CONV 1 CISOLID C-S 0.99

BIOCK MELTER RGIBBS

PARAM TEMP $=3000$ PRES $=1$ <ATM> NPHASE=2

PROD H2 / C-S SS/ FE / SIO2/AL2O3/ FE-S SS / \& SIO2-S SS / AL2O3-S SS / BA-S SS / CD / CD-S SS

BLOCK QUENCH RGIBBS

PARAM TEMP $=350$ PRES=1 <ATM> NPHASE=2 MAXIT=100

$\mathrm{PROD} \mathrm{H} 2 / \mathrm{O} 2 / \mathrm{N} 2 / \mathrm{F} 2 / \mathrm{NO} / \mathrm{SO} / \mathrm{H} 2 \mathrm{O} / \mathrm{CO} 2 / \&$ $\mathrm{CO} / \mathrm{HCL} / \mathrm{AS} / \mathrm{CD} / \mathrm{HG} / \mathrm{SE} / \mathrm{EE}-\mathrm{S}$ SS / \& SIO2-S SS / AL203-S SS / AS-S SS / BA-S SS / \& CD-S SS / CR-S SS / PB-S SS / SE-S SS / AG-S SS \& / MGO-S SS / NA2O-S SS / K2O-S SS / CAO-S SS

BLOCK-OPTION RESTART=NO

\section{BLOCK SCC RGIBBS}

PARAM TEMP $=2200$ PRES $=1$ <ATM> NPHASE $=2$

$\mathrm{PROD} \mathrm{H} 2 / \mathrm{O} 2 / \mathrm{N} 2 / \mathrm{CL} 2 / \mathrm{E} 2 / \mathrm{NO} / \mathrm{SO} 2 / \mathrm{H} 2 \mathrm{O} / \&$ $\mathrm{CO} 2 / \mathrm{CO} / \mathrm{HCL} / \mathrm{CH} 4 / \mathrm{s} / \mathrm{AS} / \mathrm{CD} / \mathrm{HG} / \mathrm{SE} \&$ $/$ EE-S SS / SIO2-S sS / AI203-s Ss / BA-s SS / \& CR-S SS / PB-S SS / AG-S SS / MGO-S SS/ NA20-S \& SS / K2O-S SS / CAO-S SS

BLOCK-OPTION RESTART=NO

BIOCK SCRUB RGIBBS

PARAM TEMP $=120$ PRES $=1$ <ATM> NPHASE=2

PROD H2/ $02 / \mathrm{N} 2 / \mathrm{CL} 2 / \mathrm{F} 2 / \mathrm{NO} / \mathrm{SO} 2 / \mathrm{H} 2 \mathrm{O} /$ \& $\mathrm{CO} 2 / \mathrm{CO} / \mathrm{HCL} / \mathrm{NAOH}-\mathrm{S}$ sS / NACL-S SS / NAF-S SS

BLOCK VITRIFY RGIBBS

PARAM TEMP=3000 PRES $=1$ <ATM> NPHASE $=2$ MAXIT $=500$ TOL $=0.00001$

PROD H2 / O2 / N2 / CL2 / $2 / \mathrm{NO} / \mathrm{SO} 2 / \mathrm{H} 2 \mathrm{O} /$ \&

$\mathrm{CO} / \mathrm{CO} / \mathrm{HCL} / \mathrm{C}-\mathrm{s} \mathrm{SS} / \mathrm{s} / \mathrm{AS} / \mathrm{CD} / \mathrm{HG} / \&$ SE / EE-S SS / SIO2 / AL203/AS-S SS / BA-S SS \& / CD-S SS / CR-S SS / PB-S SS / SE-S SS / AG-S \& ss / MGO-S SS/ NA2O-S SS / K20-S SS/ FE304-S \& SS / CAO-S SS

PROD-FRAC EE-S $1 /$ FE304-S 1

BLOCK-OPTION RESTART $=$ NO 
DESIGN-SPEC NAOH

DEFINE CAUIN MASS-FLOW STREAM=NAOH SUBSTREAM=CISOLID \& COMPONENT $=$ NAOH $-S$

DEFINE CAUOUT MASS-FLOW STREAM=SCRUBOUT SUBSTREAM=CISOIID \& COMPONENT $=\mathrm{NAOH}-S$

SPEC "CAUIN-100.*CAUOUT" TO "0."

TOL-SPEC "0.01"

VARY STREAM-VAR STREAM=NAOH SUBSTREAM=CISOLID \& VARIABLE=MASS-FLOW

LIMITS "1" "1000"

DESIGN-SPEC O2-SCC

DEEINE OXIN1 MASS-ELOW STREAM=02-SCC SUBSTREAM=MIXED \& COMPONENT $=02$

DEFINE OXIN2 MASS-FLOW STREAM=VITVAP SUBSTREAM=MIXED \& COMPONENT $=02$

DEFINE OXIN3 MASS-FLOW STREAM=PYROVAP SUBSTREAM=MIXED \& COMPONENT $=02$

DEFINE OXOUT MASS-FLOW STREAM=SCCOUT SUBSTREAM=MIXED \& COMPONENT $=02$

SPEC "OXIN1+OXIN2+OXIN3" TO "51.0*OXOUT"

TOL-SPEC "0.00I"

VARY STREAM-VAR STREAM=02-SCC SUBSTREAM=MIXED \& VARIABIE $=$ MASS-FLOW

LIMITS "I" "100000"

DESIGN-SPEC O2-VIT

DEFINE OXIN1 MASS-FLOW STREAM=O2-VIT SUBSTREAM=MIXED \& COMPONENT $=02$

DEFINE OXIN2 MASS-FLOW STREAM=EDWASTEN SUBSTREAM=MIXED \& COMPONENT $=02$

DEFINE OXIN3 MASS-FLOW STREAM=SOIL SUBSTREAM=MIXED \& COMPONENT $=02$

DEFINE OXOUT MASS-FLOW STREAM=VITOUT SUBSTREAM=MIXED \& COMPONENT $=02$

SPEC "OXIN1+OXIN2+OXIN3" TO "6.*OXOUT"

TOL-SPEC "0.01"

VARY STREAM-VAR STREAM=02-VIT SUBSTREAM=MIXED \& VARIABIE=MASS-FLOW

LIMITS " 1 " "1000"

DESIGN-SPEC OXYGEN

DEEINE OXOUT MOLE-FLOW STREAM=PYROOUT SUBSTREAM=MIXED \& COMPONENT $=02$

DEEINE COUT MOLE-FLOW STREAM=PYROOUT SUBSTREAM=CISOLID \& COMPONENT $=\mathrm{C}-\mathrm{S}$

SPEC "COUT-2.0*OXOUT" TO "0.01"

TOI-SPEC "0.001"

VARY STREAM-VAR STREAM=OXYGEN SUBSTREAM=MIXED \& VARIABIE=MASS-FIOW

LIMITS " 1 " " 1000 "

DESIGN-SPEC QQUENCH

DEFINE QQNCH BLOCK-VAR BLOCK=QUENCH VARIABLE=QCALC \& SENTENCE = PARAM

SPEC "QQNCH" TO "O"

TOL-SPEC "0.10"

VARY STREAM-VAR STREAM=QNCH2O SUBSTREAM=MIXED \& VARIABLE=MASS - FLOW

LIMITS "I" "90000"

DESIGN-SPEC SCRUBH2O 
DEFINE MASSAL STREAM-VAR STREAM=SCRUBBOT SUBSTREAM=CISOLID\& VARIABLE=MASS- FIOW

DEFINE SCRUB STREAM-VAR STREAM=SCRUBH 20 SUBSTREAM=MIXED \& VARIABLE=MASS - FLOW

DEFINE NAOH STREAM-VAR STREAM=NAOH SUBSTREAM=CISOLID\& VARIABLE = MASS-FLOW

SPEC "SCRUB" TO "20*MASSAL"

TOL-SPEC "0.01"

VARY STREAM-VAR STREAM=SCRUBH2O SUBSTREAM=MIXED \& VARIABIE =MASS-FLOW

LIMITS "NAOH" "5000"

DESIGN-SPEC SOILH2O

DEFINE SLAGI STREAM-VAR STREAM=SLAG SUBSTREAM=MIXED \& VARIABLE=MASS-FLOW

DEFINE SLAG2 STREAM-VAR STREAM=SLAG SUBSTREAM=CISOLID \& VARIABLE=MASS-FLOW

DEFINE SOIL STREAM-VAR STREAM=SOIL SUBSTREAM=MIXED\& VARIABLE $=$ MASS-FLOW

SPEC "30.* SOIL" TO "SLAGI+SLAG2"

TOL-SPEC "0.01"

VARY STREAM-VAR STREAM=SOIL SUBSTREAM=MIXED \& VARIABLE=MASS-FLOW

IIMITS " 1 " "1000"

DESIGN-SPEC SOILSOI

DEFINE SLAGI STREAM-VAR STREAM=SLAG SUBSTREAM=MIXED \& VARIABLE $=$ MASS - FLOW

DEFINE SLAG2 STREAM-VAR STREAM=SIAG SUBSTREAM=CISOLID \& VARIABLE =MASS-FLOW

DEFINE SOIL STREAM-VAR STREAM=SOIL SUBSTREAM=CISOLID \& VARIABLE $=$ MASS - FLOW

SPEC "3.33333*SOIL" TO "SLAGI+SIAG2"

TOL-SPEC "0.01"

VARY STREAM-VAR STREAM=SOII SUBSTREAM=CISOIID \& VARIABLE=MASS-FLOW

LIMITS "1" "1000"

CONV-OPTIONS

PARAM TOL $=0.001$ SPEC-ME $T H O D=B R O Y D E N$

WEGSTEIN MAXIT $=500$

SECANT MAXIT $=300$ MAX-STEP-SIZ=.05 BRACKET=YES

CONVERGENCE C-1 SECANT

SPEC NAOH 0.01

CONVERGENCE BROY2 BROYDEN

SPEC SOILH2O / SOILSOL

CONVERGENCE NEWTOXI NEWTON

SPEC O2-VIT

STREAM-REPOR NOSORT MOLEFLOW MASSELOW PROPERTIES=ALL-SUBS 


\section{System C-1}

TITLE 'ITTS PHASE I - PLASMA FURNACE - MODEL SYSCI-4 (main)'

IN-UNITS ENG

DEF-STREAMS MIXCISID ALI

DIAGNOSTICS

TERMINAL SIM-LEVEL $=8$ CONV-LEVE $L=8$

RUN-CONTROL MAX-TIME $=1800$

DATABANKS COMBUST / INORGANIC / PURECOMP / SOLIDS / \& NOASPENPCD

PROP-SOURCES COMBUST / INORGANIC / PURECOMP / SOLIDS

COMPONENTS

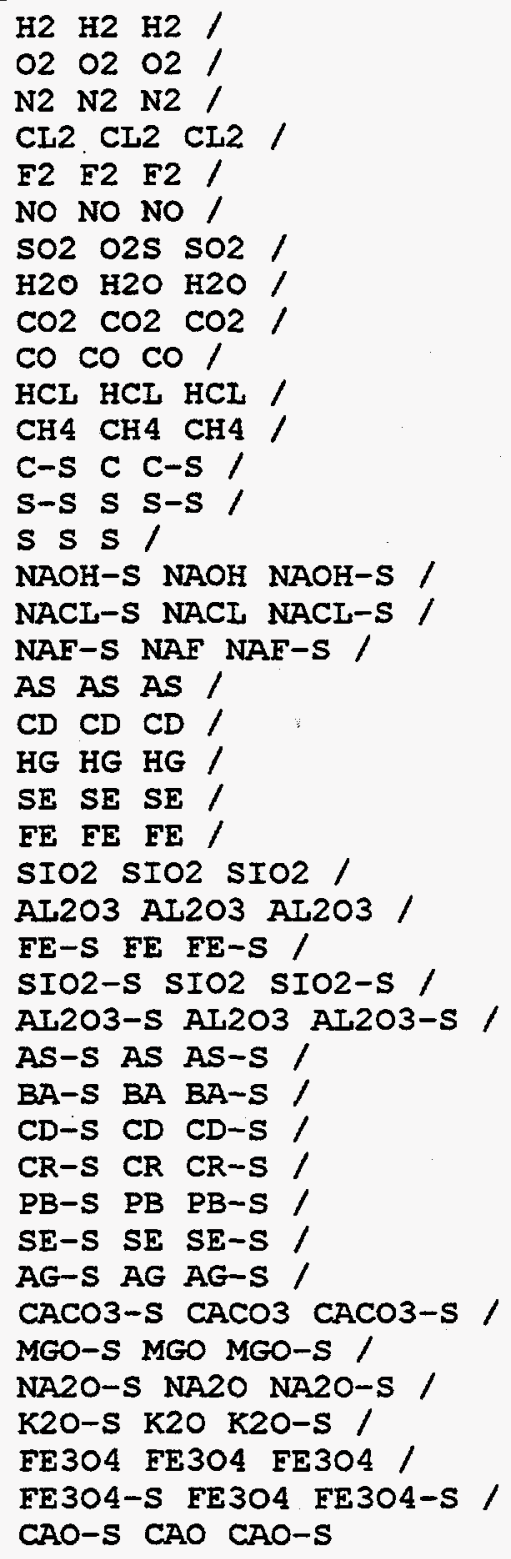


FLOWSHEET

BLOCK PLASMA IN=SOLIDS OUT=PLASOUT

BLOCK PLAS-SEP IN=PLASOUT OUT=METAL PLASVAP SLAG

BLOCK SCC IN=AIR2 EFFLUENT OUT=SCCOUT

BLOCK BAGHOUSE IN=QUENCHED OUT=QNCHVAP HGPLUS FLYASH

BLOCK L-V-SEP IN=SCRUBOUT OUT=SCRUBVAP SCRUBBOT

BLOCK SCRUB IN=QNCHVAP SCRUBH2O NAOH OUT=SCRUBOUT

BLOCK QUENCH IN=SCCOUT QNCH2O OUT=QUENCHED

BLOCK FEED-SEP IN=SOIL FDWASTEC FDWASTEN FDMETMLT FLYASH \& OUT=SOLIDS ORGANICS

BLOCK PLENUM IN=ORGANICS AIR PLASVAP OUT=EFFLUENT

PROPERTIES SOLIDS

PROPERTIES IDEAI / RKS-BM

PROP-SET ALL-SUBS TEMP PRES VFRAC UNITS='ATM' SUBSTREAM=ALL

STREAM AIR

SUBSTREAM MIXED TEMP $=68$ PRES $=1$ <ATM> MASS-FLOW $=6238.97$

MASS-FRAC $020.233 / \mathrm{N} 20.767$

STREAM AIR2

SUBSTREAM MIXED TEMP $=68$ PRES $=1$ <ATM> MASS-FLOW $=1.001$

MASS-FRAC $020.233 / \mathrm{N} 20.767$

STREAM FDMETMLT

SUBSTREAM MIXED TEMP=68 PRES $=1$ <ATM> MASS-FLOW=1.20

MASS-FLOW H2 2.42

SUBSTREAM CISOLID TEMP=68 PRES $=1$ <ATM> MASS-FLOW $=147.80$

MASS-FLOW C-S $14.44 / \mathrm{FE}-\mathrm{S} 282.02 / \mathrm{SIO}-\mathrm{S} 0.035 /$ \&

AL203-S $0.035 / \mathrm{BA}-\mathrm{S} 0.87$ / CD-S 0.18

STREAM FDWASTEC

SUBSTREAM MIXED TEMP=68 PRES=1 <ATM>

MASS-FLOW H2 50.95/ O2 $92.76 / \mathrm{N} 20.23 / \mathrm{CL} 253.02 /$ \& F2 0.11 / H2O 19.83

SUBSTREAM CISOLID TEMP $=68$ PRES $=1$ 〈ATM>

MASS-FIOW C-S $326.28 /$ S-S $0.49 /$ FE-S $7.67 /$ SIO2-S \&

$53.01 /$ AL203-S 53.01 / BA-S 0.77 / CD-S 0.77 / CR-S 1.53

STREAM FDWASTEN

SUBSTREAM MIXED TEMP $=68$ PRES $=1$ <ATM>

MASS-FLOW H2 $0.54 / 021.44 / \mathrm{N} 20.01 / \mathrm{CL} 214.88 /$ \&

NO $0.66 / \mathrm{SO} 0.83 / \mathrm{H} 20354.23 / \mathrm{HG} 0.15$

SUBSTREAM CISOLID TEMP $=68$ PRES $=1$ <ATM>

MASS-FLOW C-S 4.77 / S-S $0.62 /$ SIO2-S $476.77 / \&$

AL203-S 476.77 / AS-S $0.06 /$ BA-S $0.04 /$ CD-S $2.63 \&$

/ CR-S $4.95 /$ PB-S $0.17 /$ SE-S $0.03 /$ AG-S 0.03

STREAM NAOH

SUBSTREAM CISOLID TEMP=68 PRES=1 <ATM> MASS-FLOW=77.611

MASS-FRAC NAOH-S 1

STREAM PIASOUT

SUBSTREAM MIXED TEMP $=3000$ PRES $=1$ <ATM>

MASS-FLOW H2 51 / N2 4 / CL2 53 / H2O $375 /$ CO 784 \&

/ FE $148 /$ SIO2 $1012 /$ AL2O3 725

SUBSTREAM CISOLID TEMP=3000 PRES=1 <ATM>

MASS-FLOW C-S $0 /$ S-S $0 /$ SIO2-S 1

STREAM QNCH2O

SUBSTREAM MIXED TEMP=68 PRES=1 <ATM> MASS-FLOW=3548.898 
MASS-FLOW H2O 1

STREAM SCRUBH2O

SUBSTREAM MIXED TEMP=68 PRES=1 <ATM> MASS-FLOW=1

MASS-FRAC H2O I

STREAM SOIL

SUBSTREAM MIXED TEMP=68 PRES=1 <ATM> MASS-FLOW=48.1832

MASS-FLOW H2O .100

SUBSTREAM CISOLID TEMP=68 PRES=1 <ATM> MASS-FLOW=433.65

MASS-FLOW SIO2-S $0.569 /$ AL203-S $0.102 /$ CACO3-S $0.128 /$ \& MGO-S $0.023 /$ NA2O-S $0.013 /$ K2O-S $0.024 /$ FE304-S \& 0.041

BLOCK BAGHOUSE SEP

FRAC STREAM=HGPLUS SUBSTREAM=MIXED COMPS=H2 O2 N2 CL2 \& F2 NO SO2 H2O CO2 CO HCI CH4 C-s s-s s NAOH-S \& NACL-S NAF-S AS CD HG SE EE SIO2 AL203 FE-S SIO2-S \& AL2O3-S AS-S BA-S CD-S CR-S PB-S SE-S AG-S CACO3-S \& MGO-S NA2O-S K2O-S FE304 FE304-S CAO-S FRACS $=0$ O 0 \& $\begin{array}{llllllllllllllllllllll}0 & 0 & 0 & 0 & 0 & 0 & 0 & 0 & 0 & 0 & 0 & 0 & 0 & 0 & 0 & 1 & 1 & 1 & 1 & 0 & 0 & \&\end{array}$ $0 \begin{array}{lllllllllllllllll}0 & 0 & 0 & 1 & 0 & 1 & 0 & 0 & 1 & 0 & 0 & 0 & 0 & 0 & 0 & 0 & 0\end{array}$

FRAC STREAM=HGPLUS SUBSTREAM=CISOLID COMPS=C-S $S-S$ NAOH-S \& NACL-S NAF-S FE-S SIO2-S AI203-S AS-S BA-S CD-S CR-S \& PB-S SE-S AG-S CACO3-S MGO-S NA2O-S K2O-S FE3O4-S \&

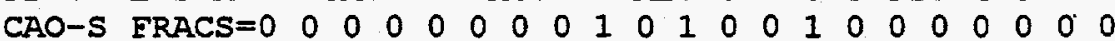

FRAC STREAM=ELYASH SUBSTREAM=MIXED COMPS=H2 O2 N2 CL2 \& F2 NO SO2 H2O CO2 CO HCL CH4 C-S S-S S NAOH-S \& NACL-S NAF-S AS CD HG SE FE SIO2 AL2O3 FE-S SIO2-S \& AL203-S AS-S BA-S CD-S CR-S PB-S SE-S AG-S CACO3-S \& MGO-S NA2O-S K2O-S FE304 FE304-S CAO-S FRACS=0 00 \& $\begin{array}{lllllllllllllllllllllll}0 & 0 & 0 & 0 & 0 & 0 & 0 & 0 & 0 & 1 & 1 & 1 & 1 & 1 & 1 & 0 & 0 & 0 & 0 & 1 & 1 & \&\end{array}$ $\begin{array}{llllllllllllllllll}1 & 1 & 1 & 1 & 0 & 1 & 0 & 1 & 1 & 0 & 1 & 1 & 1 & 1 & 1 & 1 & 1 & 1\end{array}$

ERAC STREAM=FLYASH SUBSTREAM=CISOLID COMPS=C-S $s-S$ NAOH-S \& NACL-S NAF-S EE-S SIO2-S AL2O3-S AS-S BA-S CD-S CR-S \& PB-S SE-S AG-S CACO3-S MGO-S NA2O-S K2O-S FE3O4-S \&

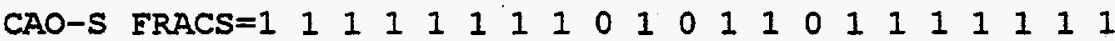
FLASH-SPECS FLYASH TEMP $=68$ PRES $=1$ <ATM>

BIOCK FEED-SEP SEP

ERAC STREAM=SOLIDS SUBSTREAM=MIXED COMPS=H2 O2 N2 CL2 \& F2 NO SO2 H2O CO2 CO HCL CH4 C-S S-S S NAOH-S \& NACI-S NAF-S AS CD HG SE FE SIO2 AL2O3 FE-S SIO2-S \& AL2O3-S AS-S BA-S CD-S CR-S PB-S SE-S AG-S CACO3-S \& MGO-S NA2O-S K2O-S FE304 FE304-S CAO-S FRACS=0 000 \& $\begin{array}{lllllllllllllllllll}0 & 0 & 0 & 0 & 0 & 0 & 0 & 0 & 0 & .05 & .05 & .05 & .95 & .95 & .95 & .95 & \&\end{array}$ $\begin{array}{lllllllllllll}.95 & .95 & .95 & .95 & .95 & .95 & .95 & .95 & .95 & .95 & .95 & .95 & \&\end{array}$ $\begin{array}{llllllllllll}.95 & .95 & .95 & .95 & .95 & .95 & .95 & .95 & .95 & .95 & .95\end{array}$

FRAC STREAM=SOLIDS SUBSTREAM=CISOIID COMPS=C-S $S-S$ NAOH-S \& NACL-S NAF-S FE-S SIO2-S AL2O3-S AS-S BA-S CD-S CR-S \& PB-S SE-S AG-S CACO3-S MGO-S NA2O-S K2O-S FE304-S \&

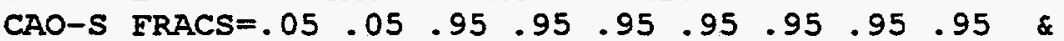
$\begin{array}{llllllllllllll}.95 & .95 & .95 & .95 & .95 & .95 & .95 & .95 & .95 & .95 & .95 & .95\end{array}$

FIASH-SPECS SOLIDS TEMP $=68$ PRES $=1$ <ATM>

FI.ASH-SPECS ORGANICS TEMP=68. PRES $=1$ <ATM>

BLOCK PLAS-SEP SEP

FRAC STREAM=METAI SUBSTREAM=MIXED COMPS=H2 O2 N2 CL2 F2 \& NO SO2 H2O CO2 CO HCL CH 4 C-S S-S S NAOH-S NACL-S \& NAF-S AS CD HG SE FE SIO2 AI2O3 FE-S SIO2-S AL2O3-S * AS-S BA-S CD-S CR-S PB-S SE-S AG-S CACO3-S MGO-S \& NA2O-S K20-S FE304 FE304-S CAO-S FRACS $=0 \begin{array}{llllll}0 & 0 & 0 & 0 & 0\end{array}$ 
$\begin{array}{llllllllllllllllllllll}0 & 0 & 0 & 0 & 0 & 0 & 0 & 0 & 0 & 0 & 0 & 0 & 0 & 0 & 0 & 0 & 1 & 0 & 0 & 1 & 0 & \&\end{array}$

$\begin{array}{lllllllllllllll}0 & 0 & 1 & 0 & 1 & 1 & 0 & 1 & 0 & 0 & 0 & 0 & 0 & 0 & 0\end{array}$

FRAC STREAM=METAL SUBSTREAM=CISOLID COMPS=C-S $S-S$ NAOH-S \& NACI-S NAF-S FE-S SIO2-S AL2O3-S AS-S BA-S CD-S CR-S \& PB-S SE-S AG-S CACO3-S MGO-S NA2O-S K2O-S FE3O4-S \& CAO-S FRACS $=0 \begin{array}{lllllllllllllllllllll}0 & 0 & 0 & 0 & 0 & 1 & 0 & 0 & 0 & 1 & 0 & 1 & 1 & 0 & 1 & 0 & 0 & 0 & 0 & 0 & 0\end{array}$ FRAC STREAM=SLAG SUBSTREAM=MIXED COMPS=H2 O2 N2 CL2 F2 \& NO SO2 H2O CO2 CO HCL CH4 C-s s-s s NAOH-S NACL-S \& NAF-S AS CD HG SE FE SIO2 AL2O3 FE-S SIO2-S AL2O3-S \& AS-S BA-S CD-S CR-S PB-S SE-S AG-S CACO3-S MGO-S \& NA2O-S K2O-S FE304 FE304-S CAO-S FRACS $=0 \begin{array}{llllll}0 & 0 & 0 & 0 & 0 & \&\end{array}$ $\begin{array}{llllllllllllllllllllll}0 & 0 & 0 & 0 & 0 & 0 & 1 & 1 & 1 & 1 & 1 & 1 & 0 & 0 & 0 & 0 & 0 & 1 & 1 & 0 & 1 & \&\end{array}$ $\begin{array}{lllllllllllllll}1 & 0 & 0 & 0 & 0 & 0 & 0 & 0 & 1 & 1 & 1 & 1 & 1 & 1 & 1\end{array}$

FRAC STREAM=SLAG SUBSTREAM=CISOIID COMPS=C-S S-S NAOH-S \& NACL-S NAF-S FE-S SIO2-S AI2O3-S AS-S BA-S CD-S CR-S \& PB-S SE-S AG-S CACO3-S MGO-S NA2O-S K2O-S FE3O4-S \&

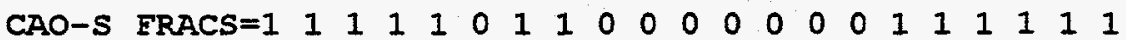

BLOCK L-V-SEP FLASH2

PARAM TEMP=120 PRES=1 <ATM>

BLOCK PIASMA RGIBBS

PARAM TEMP $=3000$ PRES $=1$ <ATM> NPHASE $=2$ CHEMEQ $=Y E S$

PROD H2 / O2/ N2 / CL2 / E2 / NO / SO2/ H2O \& \& $\mathrm{CO} 2 / \mathrm{CO} / \mathrm{HCL} / \mathrm{CH}_{4} / \mathrm{C}-\mathrm{S} \mathrm{SS} / \mathrm{S} / \mathrm{AS} / \mathrm{CD} / \&$ $\mathrm{HG} / \mathrm{SE} / \mathrm{EE} / \mathrm{SIO2} / \mathrm{AL} 203$ / BA-S SS / CR-S \& SS / PB-S SS / AG-S SS / MGO-S SS / NA2O-S SS / \& K2O-S SS / FE3O4 / CAO-S SS

\section{BLOCK PLENUM RGIBBS}

PARAM TEMP $=1800$ PRES $=1$ 〈ATM> NPHASE $=2$

PROD H2 / O2/ N2 / CL2 / F2 / NO / SO2 / H2O / \& $\mathrm{CO} 2 / \mathrm{CO} / \mathrm{HCL} / \mathrm{C}-\mathrm{s}$ SS / S-S SS / s / As / \& $\mathrm{CD} / \mathrm{HG} / \mathrm{SE} / \mathrm{EE}-\mathrm{S} \mathrm{SS} / \mathrm{SIO2-S} \mathrm{SS} / \mathrm{AL203-S} \mathrm{SS} \&$ $/ \mathrm{BA}-\mathrm{S}$ SS / CR-S SS/ PB-S SS/ AG-S SS / MGO-S \& SS / NA2O-S SS / K2O-S SS / FE304-S SS / CAO-S SS

\section{BLOCK QUENCH RGIBBS}

PARAM TEMP $=350$ PRES $=1$ <ATM> NPHASE $=2$

PROD H2 / O2 / N2 / F2 / NO / SO2 / H2O / $\mathrm{CO} / \&$ CO / HCL / AS / CD / HG / SE / FE-S SS / \& SIO2-S SS / AL2O3-S SS / AS-S SS / BA-S SS / \& CD-S SS / CR-S SS / PB-S SS / SE-S SS / AG-S SS \& / MGO-S SS / NA2O-S SS / K20-S SS / FE304-S sS / CAO-S ss

BLOCK SCC RGIBBS

PARAM TEMP $=2200$ PRES=1 <ATM> NPHASE=2 MAXIT=100 TOL $=0.00001$

PROD H2 / O2 / N2 / CL2 / E2 / NO / SO2 / H2O / \& $\mathrm{CO} 2 / \mathrm{CO} / \mathrm{HCL} / \mathrm{AS} / \mathrm{CD} / \mathrm{HG} / \mathrm{SE} / \mathrm{EE}-\mathrm{S} S \mathrm{~S}$ \& / SIO2-S SS / AL2O3-S SS / BA-S SS / CR-S SS / \& $\mathrm{PB}-\mathrm{S} S \mathrm{SS} / \mathrm{AG}-\mathrm{S} \mathrm{SS} / \mathrm{MGO}-\mathrm{S} \mathrm{SS} / \mathrm{NA2O} \mathrm{S} \mathrm{SS} / \mathrm{K} 2 \mathrm{O}-\mathrm{S} \&$ SS / EE304-S SS / CAO-S SS

BLOCK SCRUB RGIBBS

PARAM TEMP=120 PRES=1 <ATM> NPHASE=2 MAXIT $=500$

PROD H2 / O2 / N2 / CL2 / F2 / NO / SO2 / H2O / \& $\mathrm{CO} 2 / \mathrm{CO} / \mathrm{HCL} / \mathrm{NAOH}-\mathrm{S}$ SS / NACL-S ss / NAF-S ss

DESIGN-SPEC AIR

DEEINE OXIN1 MASS-FIOW STREAM=AIR SUBSTREAM=MIXED \& COMPONENT $=02$

DEEINE OXIN2 MASS-FLOW STREAM=ORGANICS SUBSTREAM=MIXED \& 
COMPONENT $=02$

DEFINE OXOUT MASS-ELOW STREAM=EFFLUENT SUBSTREAM=MIXED \& COMPONENT $=02$

SPEC "OXIN1+OXIN2" TO "6.0*OXOUT"

TOL-SPEC "0.1"

VARY STREAM-VAR STREAM=AIR SUBSTREAM=MIXED \& VARIABLE=MASS-FLOW

LIMITS "1" "10000"

DESIGN-SPEC AIR2

DEFINE OXIN1 MASS-FLOW STREAM=AIR2 SUBSTREAM=MIXED \& COMPONENT $=02$

DEFINE OXIN2 MASS-FLOW STREAM=EFELUENT SUBSTREAM=MIXED \& COMPONENT $=02$

DEFINE OXOUT MASS-FLOW STREAM=SCCOUT SUBSTREAM=MIXED \& COMPONENT $=02$

SPEC "OXIN1+OXIN2" TO "6.0*OXOUT"

TOL-SPEC "0.1"

VARY STREAM-VAR STREAM=AIR2 SUBSTREAM=MIXED \& VARIABLE =MASS-FLOW

LIMITS "1" "100000"

DESIGN-SPEC NAOH

DEFINE CAUIN MASS-FLOW STREAM=NAOH SUBSTREAM=CISOLID \& COMPONENT $=$ NAOH-S

DEFINE CAUOUT MASS-FLOW STREAM=SCRUBOUT SUBSTREAM=CISOLID \& COMPONENT $=$ NAOH $-S$

SPEC "CAUIN" TO "100.*CAUOUT"

TOL-SPEC "0.01"

VARY STREAM-VAR STREAM=NAOH SUBSTREAM=CISOLID\& VARIABLE $=$ MASS - FLOW

LIMITS "I" "1000"

DESIGN-SPEC QQUENCH

DEFINE QQUENC BLOCK-VAR BLOCK=QUENCH VARIABIE=QCALC \& SENTENCE $=$ PARAM

SPEC "QQUENC" TO "O"

TOL-SPEC "1

VARY STREAM-VAR STREAM=QNCH2O SUBSTREAM=MIXED \& VARIABLE=MASS-FLOW

LIMITS " 1 " "100000"

DESIGN-SPEC SCRUBH2O

DEFINE MASSAI STREAM-VAR STREAM=SCRUBBOT SUBSTREAM=CISOLID \& VARIABLE=MASS - FLOW

DEFINE SCRUB STREAM-VAR STREAM=SCRUBH2O SUBSTREAM=MIXED \& VARIABLE=MASS-FIOW

DEEINE NAOH STREAM-VAR STREAM=NAOH SUBSTREAM=CISOIID \& VARIABI, $=$ MASS - FLOW

SPEC "SCRUB" TO "20*MASSAL"

TOL-SPEC "0.01"

VARY STREAM-VAR STREAM=SCRUBH2O SUBSTREAM=MIXED \& VARIABLE =MASS-FLOW

LIMITS "NAOH" "5000"

DESIGN-SPEC SOILH2O

DEFINE SIAGI STREAM-VAR STREAM=SLAG SUBSTREAM=MIXED VAR IABLE =MASS - FLOW

DEFINE SIAG2 STREAM-VAR STREAM=SIAAG SUBSTREAM=CISOLID \& VARIABLE=MASS - FLOW

DEEINE SOII STREAM-VAR STREAM=SOIL SUBSTREAM=MIXED \& VARIABLE =MASS-FLOW 


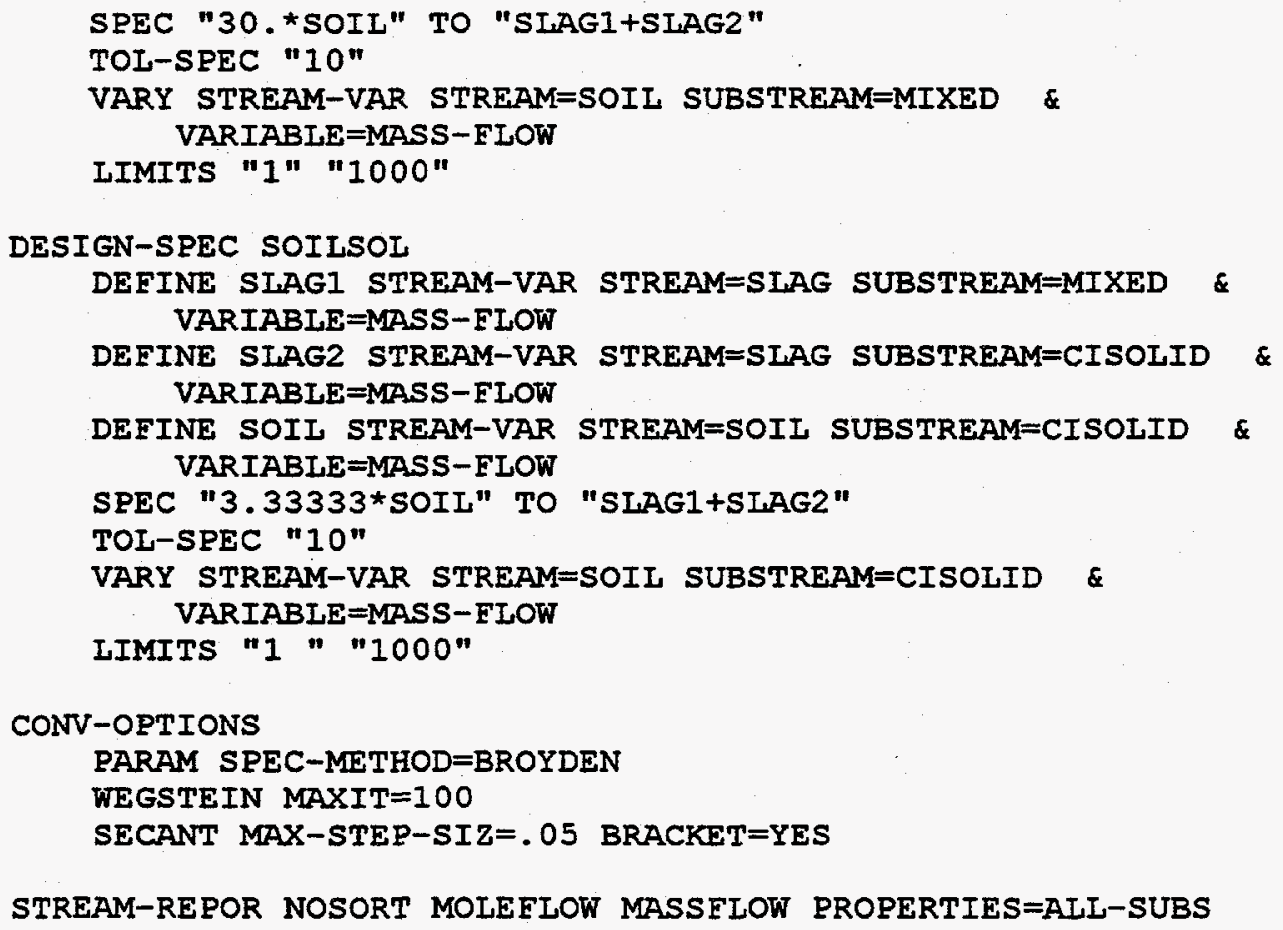




\section{System C-2}

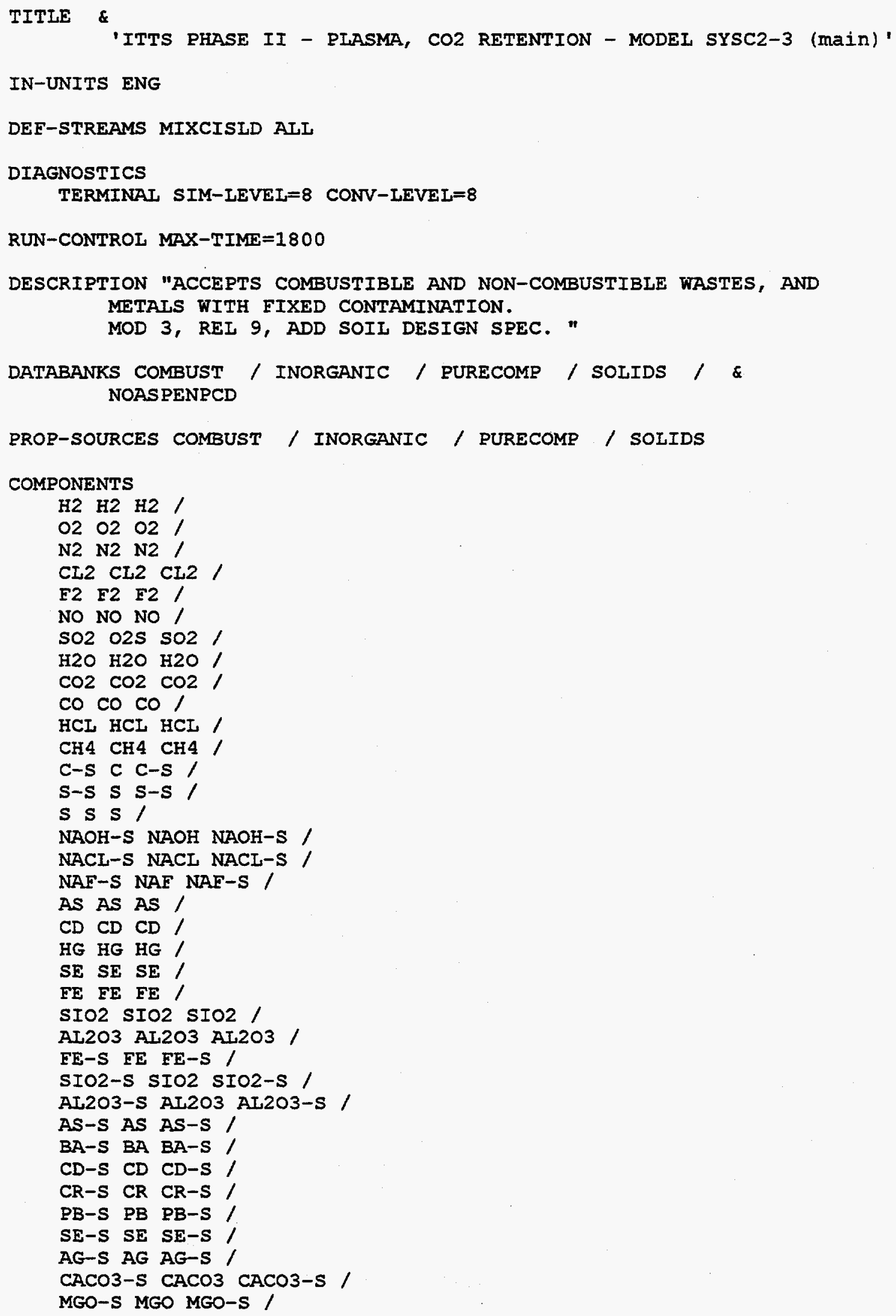




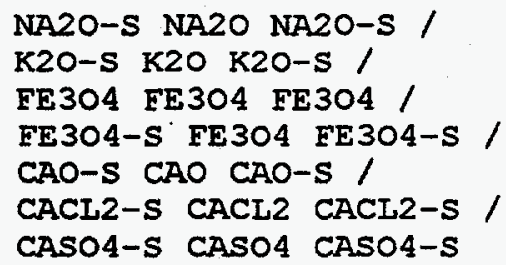

FLOWSHEET

BLOCK PLASMA IN=SOLIDS OUT=PIASOUT

BIOCK PLAS-SEP IN=PLASOUT OUT=METAL PLASVAP SIAG

BLOCK SCC IN=FUELMIX2 EFFLUENT OUT=OUT2

BIOCK BAGHOUSE IN=QUENCHED OUT=QNCHVAP HGPLUS FLYASH

BLOCK SCRUBSEP IN=SCRUBOUT OUT=SCRUBVAP SCRUBBOT

BLOCK ABSSEP IN=ABSORBED OUT=ABSGAS ABSSOL

BLOCK CO2-DES IN=ABSSOL OUT=CO2-DES

BLOCK ELASH IN=ABSGAS OUT=EXHAUST H2OOUT

BLOCK CO2-SEP IN=CO2-DES OUT $=\mathrm{CO} 2$ CAO-OUT

BLOCK SRLIT IN=CAO-OUT OUT=RECYCLE CAOSCRUB SPENTCAO

BLOCK CAOMIXER IN=RECYCIE CAO-IN OUT=CAOMIXED

BLOCK DRYSCRUB IN=CAOSCRUB QNCHVAP OUT=SCRUBOUT

BIOCK QUENCH IN=SCCOUT QNCH2O OUT=QUENCHED

BLOCK DUPL2 IN=OUT2 OUT=SCCOUT DUM2IN

BLOCK Q2 IN=DUM2IN OUT=DUM2OUT

BLOCK GASQUENC IN=QNCH2O-2 CO2 OUT=CO2OUT

BLOCK CO2-ABS IN=SCRUBVAP CAOMIXED OUT=ABSORBED

BLOCK EX-SPLIT IN=EXHAUST OUT=EX-RECYC OFFGAS

BLOCK EEED-SEP IN=SOIL FDWASTEC FDWASTEN FDMETMIT FLYASH \& OUT=SOLIDS ORGANICS

BLOCK PLENUM IN=ORGANICS OXYGEN EX-RECYC PLASVAP OUT= \& EFELUENT

PROPERTIES SOLIDS

PROPERTIES IDEAI / RKS-BM

PROP-SET SET1 VLSTDMX UNITS='CUET/HR' SUBSTREAM=MIXED

STREAM CAO-IN

SUBSTREAM CISOIID TEMP $=68$ PRES $=1$ <ATM> MASS-ELOW=172

MASS-FIOW CAO-S 1

STREAM FDMETMLT

SUBSTREAM MIXED TEMP=68 PRES=1 <ATM> MASS-FLOW=1.20

MASS-FIOW H2 2.42

SUBSTREAM CISOLID TEMP=68 PRES=1 <ATM> MASS-FLOW=147.80

MASS-FLOW C-S $14.44 /$ EE-S $282.02 /$ SIO2-S $0.035 /$ \& AL203-S $0.035 /$ BA-S $0.87 /$ CD-S 0.18

STREAM EDWASTEC

SUBSTREAM MIXED TEMP $=68$ PRES $=1$ <ATM>

MASS-FLOW H2 $50.95 / 0292.76 / \mathrm{N} 20.23 / \mathrm{CL} 253.02 /$ \& E2 $0.11 / \mathrm{H} 2019.83$

SUBSTREAM CISOLID TEMP $=68$ PRES $=1$ <ATM>

MASS-FLOW C-S $326.28 / \mathrm{S}-\mathrm{S} 0.49 /$ FE-S $7.67 /$ SIO2-S \& $53.01 / \mathrm{AL} 203-\mathrm{S} 53.01 / \mathrm{BA}-\mathrm{S} 0.77 / \mathrm{CD}-\mathrm{S} 0.77 / \&$ CR-S 1.53

STREAM FDWASTEN

SUBSTREAM MIXED TEMP $=68$. PRES $=1$ <ATM>

MASS-FLOW H2 $0.54 / 021.44 / \mathrm{N} 20.01 / \mathrm{CL} 214.88 /$ \&

NO $0.66 / \mathrm{SO} 0.83 / \mathrm{H} 20354.23 / \mathrm{HG} 0.15$

SUBSTREAM CISOIID TEMP=68 PRES=1 <ATM> 
MASS-FLOW C-S $4.77 /$ S-S $0.62 /$ SIO2-S $476.77 /$ \&

AL203-S $476.77 /$ AS-S $0.06 /$ BA-S $0.04 /$ CD-S 2.63 \&

/ CR-S $4.95 / \mathrm{PB}-\mathrm{S} 0.17 / \mathrm{SE}-\mathrm{S} 0.03 / \mathrm{AG}-\mathrm{S} 0.03$

STREAM EUELMIX2

SUBSTREAM MIXED TEMP=68 PRES=1 <ATM> MASS-FLOW=10

MASS-FLOW $02.0 .222 /$ CH4 0.046

STREAM OXYGEN

SUBSTREAM MIXED TEMP=68 PRES=1 <ATM> MASS-FLOW=100

MASS-FLOW 021

STREAM PLASOUT

SUBSTREAM MIXED TEMP=3000 PRES=1 <ATM>

MASS-FLOW H2 $51 / \mathrm{N} 24 /$ CL2 $53 / \mathrm{H} 2 \mathrm{O} 375 /$ CO 784 \&

/ EE $148 /$ SIO2 $1012 /$ AL2O3 725

SUBSTREAM CISOIID TEMP $=3000$ PRES $=1$ <ATM>

MASS-ELOW C-S $0 /$ S-S $0 /$ SIO2-S 1

STREAM QNCH2O

SUBSTREAM MIXED TEMP=68 PRES=1 <ATM> MASS-FLOW=5675

MASS-FLOW H2O 1

STREAM QNCH2O-2

SUBSTREAM MIXED TEMP=68 PRES=1 <ATM> MASS-FLOW=100

MASS-FLOW H2O 1

STREAM SOII

SUBSTREAM MIXED TEMP=68 PRES=1 <ATM> MASS-FLOW=62.5

MASS-FLOW H2O . 100

SUBSTREAM CISOIID TEMP=68 PRES=1 <ATM> MASS-FLOW=562.5

MASS-FLOW SIO2-S $0.569 /$ AL2O3-S $0.102 /$ CACO3-S $0.128 /$ \& MGO-S $0.023 /$ NA2O-S $0.013 /$ K2O-S $0.024 /$ FE 304-S 0.041

BLOCK CAOMIXER MIXER

PARAM PRES $=1$ <ATM> NPHASE $=3$

BLOCK EX-SPLIT FSPLIT

FRAC EX-RECYC 0.90

BLOCK SPLIT FSPIIT

FRAC RECYCLE $0.90 /$ CAOSCRUB 0.09

BLOCK ABSSEP SEP

FRAC STREAM=ABSSOL SUBSTREAM=MIXED COMPS=H2 O2 N2 CL2 \&

F2 NO SO2 H2O CO2 CO HCL CH4 C-S S-S S NAOH-S \&

NACL-S NAF-S AS CD HG SE EE SIO2 AL2O3 FE-S SIO2-S \&

AL203-S AS-S BA-S CD-S CR-S PB-S SE-S AG-S CACO3-S \&

MGO-S NA2O-S K2O-S FE304 FE304-S CAO-S CACL2-S CASO4-S FRACS $=0 \begin{array}{lllllllllllllllllll}0 & 0 & 0 & 0 & 0 & 0 & 0 & 0 & 0 & 0 & 0 & 0 & 1 & 1 & 1 & 1 & 1 & 1\end{array}$ \&

$\begin{array}{lllllllllllllllllllllllllll} & 1 & 1 & 1 & 1 & 1 & 1 & 1 & 1 & 1 & 1 & 1 & 1 & 1 & 1 & 1 & 1 & 1 & 1 & 1 & 1 & 1 & 1 & 1 & 1 & 1\end{array}$

FRAC STREAM=ABSSOI SUBSTREAM=CISOLID COMPS=C-S $S-S$ NAOH-S \& NACL-S NAF-S EE-S SIO2-S AI2O3-S AS-S BA-S CD-S CR-S \& PB-S SE-S AG-S CACO3-S MGO-S NA2O-S K2O-S FE3O4-S \& CAO-S CACI2-S CASO4-S FRACS=1 1 $\begin{array}{llllllllllllll}1 & 1 & 1 & 1 & 1 & 1 & 1 & 1 & 1 & 1 & 1 & 1 & 1\end{array}$

BLOCK BAGHOUSE SEP

FRAC STREAM=HGPLUS SUBSTREAM=MIXED COMPS $=\mathrm{H} 2$ O2 N2 CL2 \& E2 NO SO2 H2O CO2 CO HCL CH4 C-S S-s S NAOH-S \& NACL-S NAF-S AS CD HG SE FE SIO2 AL2O3 FE-S SIO2-S \& AI203-S AS-S BA-S CD-S CR-S PB-S SE-S AG-S CACO3-S \& 
MGO-S NA2O-S K2O-S FE3O4 FE304-S CAO-S CACL2-S CASO4-S \& FRACS $=0 \begin{array}{llllllllllllllllll}0 & 0 & 0 & 0 & 0 & 0 & 0 & 0 & 0 & 0 & 0 & 0 & 0 & 0 & 0 & 0 & 0 & \&\end{array}$ $\begin{array}{llllllllllllllllllllllllll}1 & 1 & 1 & 1 & 0 & 0 & 0 & 0 & 0 & 0 & 1 & 0 & 1 & 0 & 0 & 1 & 0 & 0 & 0 & 0 & 0 & 0 & 0 & 0 & 0 & 0\end{array}$

FRAC STREAM=HGPLUS SUBSTREAM=CISOLID COMPS=C-S S-S NAOH-S \& NACL-S NAF-S FE-S SIO2-S AL2O3-S AS-S BA-S CD-S CR-S \& PB-S SE-S AG-S CACO3-S MGO-S NA2O-S K2O-S FE3O4-S \& CAO-S CACL2-S CASO4-S ERACS $=0 \begin{array}{lllllllllll}0 & 0 & 0 & 0 & 0 & 0 & 0 & 0 & 1 & 0 & \&\end{array}$ 1000100000000000

FRAC STREAM=FLYASH SUBSTREAM=MIXED COMPS=H2 O2 N2 CL2 \& F2 NO SO2 H2O CO2 CO HCL CH4 C-S S-S S NAOH-S \& NACL-S NAF-S AS CD HG SE FE SIO2 AL2O3 FE-S SIO2-S \& AL2O3-S AS-S BA-S CD-S CR-S PB-S SE-S AG-S CACO3-S \& MGO-S NA2O-S K2O-S FE304 FE304-S CAO-S CACL2-S CASO4-S \& FRACS $=0 \begin{array}{lllllllllllllllllllll}0 & 0 & 0 & 0 & 0 & 0 & 0 & 0 & 0 & 0 & 0 & 0 & 1 & 1 & 1 & 1 & 1 & 1 & \alpha\end{array}$ $\begin{array}{llllllllllllllllllllllllll}0 & 0 & 0 & 0 & 1 & 1 & 1 & 1 & 1 & 1 & 0 & 1 & 0 & 1 & 1 & 0 & 1 & 1 & 1 & 1 & 1 & 1 & 1 & 1 & 1 & 1\end{array}$

FRAC STREAM=FLYASH SUBSTREAM=CISOLID COMPS=C-S S-S NAOH-S \& NACL-S NAF-S FE-S SIO2-S AL2O3-S AS-S BA-S CD-S CR-S \& PB-S SE-S AG-S CACO3-S MGO-S NA2O-S K2O-S FE3O4-S \&

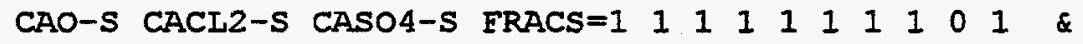
$0 \begin{array}{llllllllllllllllll}0 & 1 & 0 & 1 & 1 & 1 & 1 & 1 & 1 & 1 & 1 & 1\end{array}$

BLOCK CO2-SEP SEP

FRAC STREAM $=\mathrm{CO} 2$ SUBSTREAM=MIXED COMPS=H2 O2 N2 CL2 F2 \& NO SO2 H2O CO2 CO HCL CH4 C-S S-S S NAOH-S NACL-S \& NAF-S AS CD HG SE FE SIO2 AL2O3 FE-S SIO2-S AL2O3-S \& AS-S BA-S CD-S CR-S PB-S SE-S AG-S CACO3-S MGO-S \& NA2O-S K2O-S FE3O4 FE304-S CAO-S CACL2-S CASO4-S \& FRACS $=0 \begin{array}{llllllllllllllllll}0 & 0 & 0 & 0 & 0 & 0 & 0 & 1 & 0 & 0 & 0 & 0 & 0 & 0 & 0 & 0 & 0 & \&\end{array}$ $0 \begin{array}{lllllllllllllllllllllllll}0 & 0 & 0 & 0 & 0 & 0 & 0 & 0 & 0 & 0 & 0 & 0 & 0 & 0 & 0 & 0 & 0 & 0 & 0 & 0 & 0 & 0 & 0 & 0 & 0\end{array}$

FRAC STREAM=CO2 SUBSTREAM=CISOLID COMPS=C-S S-S NAOH-S \& NACL-S NAF-S FE-S SIO2-S AL203-S AS-S BA-S CD-S CR-S \& PB-S SE-S AG-S CACO3-S MGO-S NA2O-S K2O-S FE3O4-S \& CAO-S CACL2-S CASO4-S FRACS $=0 \begin{array}{lllllllllll}0 & 0 & 0 & 0 & 0 & 0 & 0 & 0 & 0 & \&\end{array}$

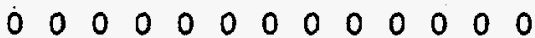

FLASH-SPECS CAO-OUT TEMP=68 PRES $=1$ <ATM>

BLOCK FEED-SEP SEP

FRAC STREAM=SOIIDS SUBSTREAM=MIXED COMPS=H2 O2 N2 CL2 \& E2 NO SO2 H2O CO2 CO HCL CH4 C-S S-S S NAOH-S \& NACL-S NAF-S AS CD HG SE FE SIO2 AI2O3 FE-S SIO2-S \& AL2O3-S AS-S BA-S CD-S CR-S PB-S SE-S AG-S CACO3-S \& MGO-S NA2O-S K2O-S FE304 FE304-S CAO-S CACI2-S CASO4-S \& FRACS $=0 \begin{array}{lllllllllllllllll}0 & 0 & 0 & 0 & 0 & 0 & 0 & 0 & 0 & 0 & 0 & .05 & .05 & .05 & .95 & \text { \& }\end{array}$ $\begin{array}{llllllllllll}.95 & .95 & .95 & .95 & .95 & .95 & .95 & .95 & .95 & .95 & .95 & .95\end{array}$ $\begin{array}{lllllllllllllllll}.95 & .95 & .95 & .95 & .95 & .95 & .95 & .95 & .95 & .95 & .95 & .95 & .95 & .95 & .95 & .95\end{array}$

FRAC STREAM=SOIIDS SUBSTREAM=CISOLID COMPS=C-S S-S NAOH-S \& NACI-S NAF-S EE-S SIO2-S AI203-S AS-S BA-S CD-S CR-S \& PB-S SE-S AG-S CACO3-S MGO-S NA2O-S K2O-S FE304-S \& CAO-S CACL2-S CASO4-S ERACS $=.05 \quad .05 \quad .95 \quad .95 \quad .95 \quad .95 \quad \&$

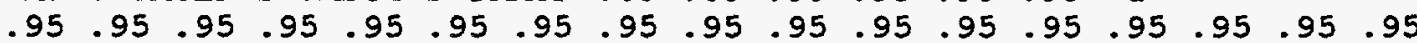

BLOCK PIAS-SEP SEP

FRAC STREAM=METAL SUBSTREAM=MIXED COMPS=H2 O2 N2 CL2 F2 \& NO $\mathrm{SO} 2 \mathrm{H} 2 \mathrm{O} \mathrm{CO} 2 \mathrm{CO} H C L$ CH4 C-S S-S S NAOH-S NACI-S \& NAF-S AS CD HG SE FE SIO2 AL203 FE-S SIO2-S AL2O3-S \& AS-S BA-S CD-S CR-S PB-S SE-S AG-S CACO3-S MGO-S \& NA2O-S K2O-S EE304 FE304-S CAO-S CACL2-S CASO4-S \& ERACS $=0 \begin{array}{llllllllllllllllll}0 & 0 & 0 & 0 & 0 & 0 & 0 & 0 & 0 & 0 & 0 & 0 & 0 & 0 & 0 & 0 & 0 & 0\end{array}$ $\begin{array}{lllllllllllllllllllllllllll}0 & 0 & 0 & 0 & 1 & 0 & 0 & 1 & 0 & 0 & 0 & 1 & 0 & 1 & 1 & 0 & 1 & 0 & 0 & 0 & 0 & 0 & 0 & 0 & 0 & 0\end{array}$

FRAC STREAM=METAL SUBSTREAM=CISOLID COMPS=C-S S-S NAOH-S \& NACL-S NAF-S FE-S SIO2-S AL203-S AS-S BA-S CD-S CR-S \& PB-S SE-S AG-S CACO3-S MGO-S NA2O-S K2O-S FE3O4-S \& 
CAO-S CACL2-S CASO4-S FRACS $=0 \begin{array}{lllllllllll}0 & 0 & 0 & 0 & 0 & 1 & 0 & 0 & 0 & 1 & \&\end{array}$ $\begin{array}{llllllllllllll}0 & 1 & 1 & 0 & 1 & 0 & 0 & 0 & 0 & 0 & 0 & 0 & 0\end{array}$

FRAC STREAM=SLAG SUBSTREAM=MIXED COMPS=H2 O2 N2 CL2 F2 \& NO SO2 H2O CO2 CO HCL CH4 C-S s-S S NAOH-S NACL-S \& NAF-S AS CD HG SE FE SIO2 AI2O3 FE-S SIO2-S AL2O3-S \& AS-S BA-S CD-S CR-S PB-S SE-S AG-S CACO3-S MGO-S \& NA2O-S K2O-S FE304 EE304-S CAO-S CACL2-S CASO4-S \& FRACS $=0 \begin{array}{llllllllllllllllll}0 & 0 & 0 & 0 & 0 & 0 & 0 & 0 & 0 & 0 & 0 & 0 & 1 & 1 & 1 & 1 & 1 & 1\end{array}$ $\begin{array}{llllllllllllllllllllllllll}0 & 0 & 0 & 0 & 0 & 1 & 1 & 0 & 1 & 1 & 0 & 0 & 0 & 0 & 0 & 0 & 0 & 1 & 1 & 1 & 1 & 1 & 1 & 1 & 1 & 1\end{array}$

FRAC STREAM=SLAG SUBSTREAM=CISOLID COMPS=C-S $5-S$ NAOH-S \& NACL-S NAF-S FE-S SIO2-S AL2O3-S AS-S BA-S CD-S CR-S \& PB-S SE-S AG-S CACO3-S MGO-S NA2O-S K2O-S EE3O4-S \&

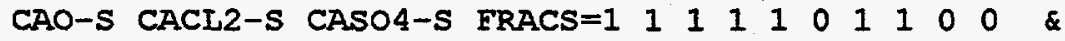
$\begin{array}{llllllllllllllllllllll}0 & 0 & 0 & 0 & 0 & 1 & 1 & 1 & 1 & 1 & 1 & 1 & 1\end{array}$

\section{BLOCK SCRUBSEP SEP}

FRAC STREAM=SCRUBBOT SUBSTREAM=MIXED COMPS=H2 O2 N2 CL2 \& F2 NO SO2 H2O CO2 CO HCL CH4 C-s s-s S NAOH-S \& NACL-S NAF-S AS CD HG SE FE SIO2 AL2O3 FE-S SIO2-S \& AL203-S AS-S BA-S CD-S CR-S PB-S SE-S AG-S CACO3-S \& MGO-S NA2O-S K2O-S EE3O4 FE304-S CAO-S CACL2-S CASO4-S \& FRACS $=0 \begin{array}{lllllllllllllllllll}0 & 0 & 0 & 0 & 0 & 0 & 0 & 0 & 0 & 0 & 0 & 0 & 1 & 1 & 0 & 1 & 1 & 1 & \&\end{array}$ $\begin{array}{llllllllllllllllllllllllll}1 & 1 & 1 & 1 & 1 & 1 & 1 & 1 & 1 & 1 & 1 & 1 & 1 & 1 & 1 & 1 & 1 & 1 & 1 & 1 & 1 & 1 & 1 & 1 & 1 & 1\end{array}$

FRAC STREAM=SCRUBBOT SUBSTREAM=CISOLID COMPS=C-S S-S * NAOH-S NACL-S NAF-S FE-S SIO2-S AI2O3-S AS-S BA-S \& CD-S CR-S PB-S SE-S AG-S CACO3-S MGO-S NA2O-S K2O-S \& FE304-S CAO-S CACI2-S CASO4-S ERACS=1 111111111 $\begin{array}{llllllllllllllll}1 & 1 & 1 & 1 & 1 & 1 & 1 & 1 & 1 & 1 & 1 & 1 & 1 & 1 & 1 & 1\end{array}$

BLOCK FLASH FLASH2

PARAM TEMP=90 PRES $=1$ <ATM>

PROPERTIES IDEAL

BIOCK CO2-ABS RSTOIC

PARAM TEMP $=1200$ PRES $=1$ <ATM> MAXIT $=200$

STOIC 1 MIXED CO2 - 1 / CISOLID CAO-S - 1 / CACO3-S 1

CONV 1 MIXED $\mathrm{CO} 21$

BLOCK CO2-DES RGIBBS

PARAM TEMP=1700 PRES $=1$ <ATM> NPHASE=2

PROD CO2 / CAO-S SS

PROPERTIES IDEAL

BLOCK DRYSCRUB RGIBBS

PARAM TEMP $=350$ PRES $=1$ <ATM N NPHASE $=2$ MAXIT $=500$

PROD H2 / O2/ N2/ $\mathrm{CL} 2 / \mathrm{F} 2 / \mathrm{NO} / \mathrm{SO} 2 / \mathrm{H} 2 \mathrm{O} /$ \& $\mathrm{CO} 2 / \mathrm{CO} / \mathrm{HCI} / \mathrm{CH} 4 / \mathrm{CASO} 4-\mathrm{S}$ sS / CACL2-S SS / CAO-S SS

BLOCK GASQUENC RGIBBS

PARAM TEMP $=350$ PRES=1 <ATM> NPHASE=2 MAXIT=500

PROD H2 / O2 / H2O / $\mathrm{CO} 2 / \mathrm{CO} / \mathrm{C}-\mathrm{S}$ SS

PROD-FRAC H2 $1 / \mathrm{O} 21 / \mathrm{H} 2 \mathrm{O} 1 / \mathrm{CO} 21$

BLOCK PLASMA RGIBBS

PARAM TEMP $=3000$ PRES $=1$ <ATM> NPHASE=2 CHEMEQ=YES

PROD H2 / O2 / N2 / $\mathrm{CI} 2 / \mathrm{F} 2 / \mathrm{NO} / \mathrm{SO} 2 / \mathrm{H} 2 \mathrm{O} /$ \& $\mathrm{CO} 2 / \mathrm{CO} / \mathrm{HCL} / \mathrm{CH} 4 / \mathrm{C}-\mathrm{s} \mathrm{ss} / \mathrm{s} / \mathrm{As} / \mathrm{CD} / \&$ $\mathrm{HG} / \mathrm{SE} / \mathrm{FE} / \mathrm{SIO} / \mathrm{AL} 203 / \mathrm{BA}-\mathrm{S} \mathrm{SS} / \mathrm{CR}-\mathrm{S}$ \& ss / $\mathrm{PB}-\mathrm{S}$ SS / AG-S ss / MGO-S ss / NA2O-S Ss / \& K2O-S SS / FE3O4 / CAO-S SS

BLOCK PIENUM RGIBBS 
PARAM TEMP $=1800$ PRES $=1$ <ATM> NPHASE $=2$

PROD H2 / O2 / N2 / CL2 / F2 / NO / SO2 / H2O / \&

$\mathrm{CO} 2 / \mathrm{CO} / \mathrm{HCL} / \mathrm{C}-\mathrm{s}$ ss / s-s ss / s / As / \& $\mathrm{CD} / \mathrm{HG} / \mathrm{SE} / \mathrm{FE}-\mathrm{S}$ SS / SIO2-S SS / AL2O3-S SS \& / BA-S SS / CR-S SS / PB-S SS / AG-S SS / MGO-S \& SS / NA2O-S SS / K2O-S SS / FE304-S SS / CAO-S SS

BLOCK Q2 RGIBBS

PARAM TEMP $=68$ PRES $=1$ <ATM> NPHASE $=2$ TAPP $=2132$

PROD H2 / O2 / N2 / CL2 / F2 / NO / SO2 / H2O / \& $\mathrm{CO} 2 / \mathrm{CO} / \mathrm{HCL} / \mathrm{AS} / \mathrm{CD} / \mathrm{HG} / \mathrm{SE} / \mathrm{FE}-\mathrm{S} \mathrm{SS} \&$ / SIO2-S SS / AL2O3-S SS / BA-S SS / CR-S SS / \& PB-S SS / AG-S SS / MGO-S SS / NA2O-S SS / K2O-S \& SS / FE3O4-S SS / CAO-S SS

BLOCK QUENCH RGIBBS

PARAM TEMP $=350$ PRES $=1$ <ATM> NPHASE $=2$

PROD H2 / O2 / N2 / CL2 / F2 / NO / SO2 / H2O / \& $\mathrm{CO} 2 / \mathrm{CO} / \mathrm{HCL} / \mathrm{AS} / \mathrm{CD} / \mathrm{HG} / \mathrm{SE} / \mathrm{FE}-\mathrm{S} \mathrm{SS} \&$ / SIO2-S SS / AL203-S SS / AS-S SS / BA-S SS / \& CD-S SS / CR-S SS / PB-S SS / SE-S SS / AG-S SS \& / MGO-S SS / NA2O-S SS / K2O-S SS / FE3O4-S SS / CAO-S SS

BIOCK SCC RGIBBS

PARAM TEMP $=2200$ PRES $=1$ <ATM> NPHASE $=2$ MAXIT $=100$

PROD H2 / O2 / N2 / CL2 / $\mathrm{E} 2 / \mathrm{NO} / \mathrm{SO} / \mathrm{H} 2 \mathrm{O} / \&$ $\mathrm{CO} 2 / \mathrm{CO} / \mathrm{HCL} / \mathrm{AS} / \mathrm{CD} / \mathrm{HG} / \mathrm{SE} / \mathrm{FE}-\mathrm{S} S \mathrm{SS} \&$ / SIO2-S SS / AL203-S SS / BA-S SS / CR-S SS / \& PB-S SS / AG-S SS / MGO-S SS / NA2O-S SS / K2O-S \& SS / FE3O4-S SS / CAO-S SS

BLOCK DUPL2 DUPL

DESIGN-SPEC CAO

DEFINE CO2CON MOLE-FLOW STREAM=SCRUBVAP SUBSTREAM=MIXED \& COMPONENT $=\mathrm{CO} 2$

DEFINE CAOMXD MOLE-FLOW STREAM=CAOMIXED SUBSTREAM=CISOLID \& COMPONENT $=$ CAO-S

SPEC "CO2CON" TO "CAOMXD"

TOL-SPEC "0.1"

VARY STREAM-VAR STREAM=CAO-IN SUBSTREAM=CISOLID \& VARIABLE =MASS - FLOW

LIMITS "1" "10000"

DESIGN-SPEC OXYGEN

DEFINE OXIN1 MASS-FLOW STREAM=OXYGEN SUBSTREAM=MIXED \& COMPONENT $=02$

DEFINE OXIN2 MASS-FLOW STREAM=EX-RECYC SUBSTREAM=MIXED \& COMPONENT $=02$

DEFINE OXIN3 MASS-FLOW STREAM=ORGANICS SUBSTREAM=MIXED\& COMPONENT $=02$

DEFINE OXOUT MASS-FLOW STREAM=EFELUENT SUBSTREAM=MIXED \& COMPONENT $=02$

SPEC "OXIN1+OXIN2+OXIN3" TO "3.5*OXOUT"

TOL-SPEC "0.1"

VARY STREAM-VAR STREAM=OXYGEN SUBSTREAM=MIXED \& VARIABLE $=$ MASS - FLOW

IIMITS " 1 " "100000"

DESIGN-SPEC QQUENCH

DEFINE QQUENC BLOCK-VAR BLOCK=QUENCH VARIABLE=QCALC \& SENTENCE $=$ PARAM 


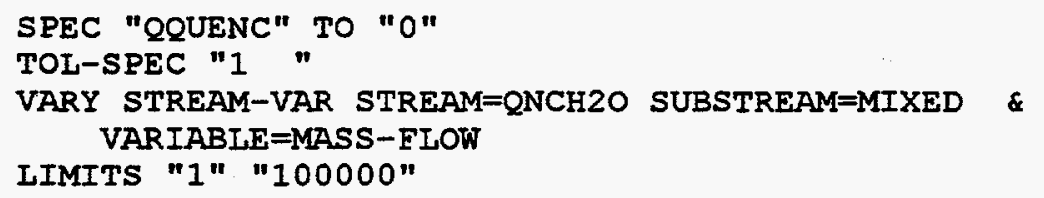

DESIGN-SPEC SOIIH2O

DEFINE SIAGI STREAM-VAR STREAM=SIAG SUBSTREAM=MIXED \& VARIABLE =MASS - FLOW

DEFINE SIAG2 STREAM-VAR STREAM=SIAG SUBSTREAM=CISOLID \& VARIABLE =MASS - FLOW

DEFINE SOIL STREAM-VAR STREAM=SOIL SUBSTREAM=MIXED \& VARIABLE=MASS-FLOW

SPEC "30. "SOIL" TO "SLAG1+SIAG2"

TOL-SPEC " 10 "

VARY STREAM-VAR STREAM=SOIL SUBSTREAM=MIXED \& VARIABLE =MASS - FLOW

LIMITS "1" "1000"

DESIGN-SPEC SOILSOL

DEFINE SLAG1 STREAM-VAR STREAM=SLAG SUBSTREAM=MIXED \& VARIABLE = MASS - FLOW

DEFINE SLAG2 STREAM-VAR STREAM=SIAG SUBSTREAM=CISOIID \& VARIABLE=MASS - ELOW

DEEINE SOIL STREAM-VAR STREAM=SOIL SUBSTREAM=CISOLID \& VARIABLE=MASS - FLOW

SPEC "3.33333*SOIL" TO "SLAGI+SLAG2"

TOL-SPEC "10"

VARY STREAM-VAR STREAM=SOIL SUBSTREAM=CISOLID \& VARIABLE =MASS- FLOW

LIMITS " 1 " "1000" 
CONV-OPTIONS

WEGSTEIN MAXIT $=100$

SECANT MAX-STEP-SIZ $=.05$ BRACKET $=$ YES

STREAM-REPOR NOSORT MOLEFLOW MASSFLOW PROPERTIES=SETI 


\section{System C-3}

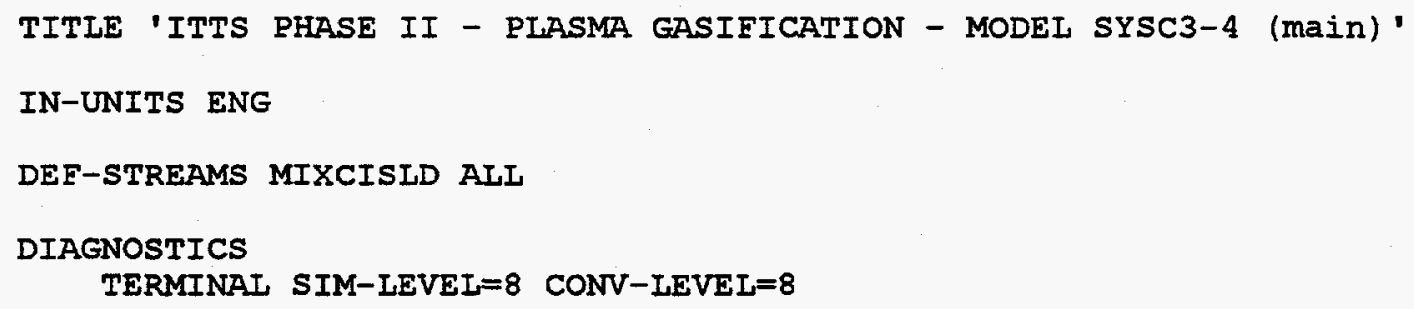


K2O-S K2O K2O-S /

FE304 FE304 FE304 /

FE304-S FE304 FE304-S /

CAO-S CAO CAO-S

FLOWSHEET

BLOCK BAGHOUSE IN=QUENCHED OUT=VAPOR FLYASH HGPLUS

BLOCK SLAG-SEP IN=EFFLUENT OUT=METAL GASVAP SLAG

BLOCK CATALYST IN=AIR SCRUBVAP OUT=EXHAUST

BLOCK QUENCH IN=QNCH2O SYNGAS OUT=QUENCHED

BLOCK SCRUB IN=SCRUBH2O NAOH VAPOR OUT=SCRUBOUT

BLOCK I-V-SEP IN=SCRUBOUT OUT=SCRUBVAP SCRUBBOT

BLOCK STEAMGEN IN=WATER OUT=STEAM

BLOCK PLENUM IN=STEAM GASVAP ORGANICS OUT=SYNGAS

BLOCK FEED-SEP IN=FLYASH FDWASTEC FDWASTEN FDMETMLT SOIL \& OUT=SOLIDS ORGANICS

BLOCK GASIFIER IN=SOLIDS OUT=EEFLUENT

PROPERTIES SOLIDS

PROPERTIES IDEAL / RKS-BM

PROP-SET AIL-SUBS TEMP PRES VFRAC VOLFLMX UNITS='ATM' \& SUBSTREAM $=A L I$

STREAM AIR

SUBSTREAM MIXED TEMP $=68$ PRES $=1$ <ATM> MASS- FLOW $=10250$

MASS-FRAC $020.233 /$ N2 0.767

STREAM FDMETMLT

SUBSTREAM MIXED TEMP $=68$ PRES $=1$ <ATM> MASS-FLOW $=1.20$

MASS-FLOW H2 2.42

SUBSTREAM CISOLID TEMP=68 PRES=1 <ATM> MASS- $E L O W=147.8$

MASS-FLOW C-S $14.44 /$ FE-S $282.02 /$ SIO2-S $0.035 / \&$

AL203-S $0.035 / \mathrm{BA}-\mathrm{S} 0.87 / \mathrm{CD}-\mathrm{S} 0.18$

STREAM FDWASTEC

SUBSTREAM MIXED TEMP $=68$ PRES $=1$ <ATM>

MASS-FLOW H2 $50.95 / O 292.76 / \mathrm{N} 20.23 /$ CL2 $53.02 / \&$

F2 $0.11 / \mathrm{H} 2019.83$

SUBSTREAM CISOIID TEMP $=68$ PRES $=1$ <ATM>

MASS-FLOW C-S $326.28 /$ S-S $0.49 /$ FE-S $7.67 /$ SIO2-S \&

$53.01 /$ AL203-S 53.01/ BA-S 0.77 / CD-S 0.77 / CR-S 1.53

STREAM FDWASTEN

SUBSTREAM MIXED TEMP $=68$ PRES=1 <ATM>

MASS-FIOW H2 $0.54 / 021.44 /$ N2 $0.01 /$ CL2 $14.88 / \&$

NO $0.66 /$ SO2 $0.83 /$ H2O $354.23 /$ HG 0.15

SUBSTREAM CISOLID TEMP $=68$ PRES $=1$ <ATM>

MASS-FLOW C-S $4.77 / \mathrm{S}-\mathrm{S} 0.62 /$ SIO2-S $476.77 / \&$

AL203-S 476.77 / AS-S $0.06 /$ BA-S $0.04 /$ CD-S 2.63 \&

/ CR-S $4.95 /$ PB-S $0.17 /$ SE-S $0.03 /$ AG-S 0.03

STREAM NAOH

SUBSTREAM CISOIID TEMP=68 PRES=1 <ATM> MASS-FLOW=77.611

MASS-FLOW NAOH-S 1

STREAM QNCH2O

SUBSTREAM MIXED TEMP=68 PRES=1 <ATM> MASS-ELOW=1189

MASS-FLOW H2O 1

STREAM SCRUBH2O

SUBSTREAM MIXED TEMP $=68$ PRES $=1$ <ATM> MASS-FLOW $=100$

B-74 
MASS-FLOW H2O 1

STREAM SOIL

SUBSTREAM MIXED TEMP=68 PRES=1 <ATM> MASS-FLOW=48.39

MASS-FLOW H2O 62.5

SUBSTREAM CISOLID TEMP=68 PRES=1 <ATM> MASS-FLOW=435.5

MASS-FLOW SIO2-S 355.6 / AL203-S 63.75 / CACO3-S $80 / \&$ MGO-S $14.38 / \mathrm{NA2O}-\mathrm{S} 8.125 / \mathrm{K} 20-\mathrm{S} 15 / \mathrm{FE} 304-\mathrm{S} \&$ 25.63

\section{STREAM WATER}

SUBSTREAM MIXED TEMP=68 PRES=1 <ATM> MASS-FLOW=59.57

MASS-FLOW H2O 1

BLOCK BAGHOUSE SEP

FRAC STREAM=FLYASH SUBSTREAM=MIXED COMPS=H2 O2 N2 CL2 \& F2 NO SO2 H2O CO2 CO HCL CH4 C-S S-S S NAOH-S \& NACL-S NAF-S AS CD HG SE FE SIO2 AL2O3 FE-S SIO2-S \& AL203-S AS-S BA-S CD-S CR-S PB-S SE-S AG-S CACO3-S \& MGO-S NA2O-S K2O-S FE304 FE304-S CAO-S FRACS $=0$ O 00 \& $\begin{array}{lllllllllllllllllllllll}0 & 0 & 0 & 0 & 0 & 0 & 0 & 0 & 0 & 1 & 1 & 1 & 1 & 1 & 1 & 0 & 0 & 0 & 0 & 1 & 1 & \&\end{array}$ $\begin{array}{llllllllllllllllllllllll}1 & 1 & 1 & 1 & 0 & 1 & 0 & 1 & 1 & 0 & 1 & 1 & 1 & 1 & 1 & 1 & 1 & 1\end{array}$

FRAC STREAM=ELYASH SUBSTREAM=CISOLID COMPS=C-S S-S NAOH-S \& NACL-S NAF-S FE-S SIO2-S AL2O3-S AS-S BA-S CD-S CR-S \& PB-S SE-S AG-S CACO3-S MGO-S NA2O-S K2O-S FE3O4-S \&

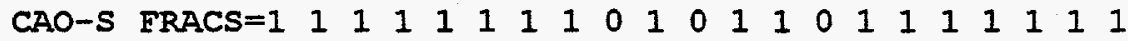

FRAC STREAM=HGPLUS SUBSTREAM=MIXED COMPS=H2 O2 N2 CL2 \& F2 NO SO2 H2O CO2 CO HCL CH4 C-S S-S S NAOH-S \& NACL-S NAF-S AS CD HG SE FE SIO2 AL2O3 FE-S SIO2-S \& AL203-S AS-S BA-S CD-S CR-S PB-S SE-S AG-S CACO3-S \& MGO-S NA2O-S K2O-S FE304 FE304-S CAO-S FRACS $=0$ O 0 O \& $0 \begin{array}{lllllllllllllllllllllllllll}0 & 0 & 0 & 0 & 0 & 0 & 0 & 0 & 0 & 0 & 0 & 0 & 0 & 0 & 1 & 1 & 1 & 1 & 0 & 0 & \text { \& }\end{array}$ $\begin{array}{lllllllllllllllllll}0 & 0 & 0 & 0 & 1 & 0 & 1 & 0 & 0 & 1 & 0 & 0 & 0 & 0 & 0 & 0 & 0 & 0\end{array}$

FRAC STREAM=HGPLUS SUBSTREAM=CISOLID COMPS=C-S $S-S$ NAOH-S\& NACL-S NAF-S FE-S SIO2-S AL2O3-S AS-S BA-S CD-S CR-S \& PB-S SE-S AG-S CACO3-S MGO-S NA2O-S K2O-S EE3O4-S \& CAO-S FRACS $=0 \begin{array}{lllllllllllllllllllll}0 & 0 & 0 & 0 & 0 & 0 & 0 & 1 & 0 & 1 & 0 & 0 & 1 & 0 & 0 & 0 & 0 & 0 & 0 & 0\end{array}$

\section{BLOCK FEED-SEP SEP}

FRAC STREAM=SOLIDS SUBSTREAM=MIXED COMPS=H2 O2 N2 CL2 \& F2 NO SO2 H2O CO2 CO HCL CH4 C-S S-S S NAOH-S \& NACL-S NAF-S AS CD HG SE FE SIO2 AL2O3 FE-S SIO2-S \& AL203-S AS-S BA-S CD-S CR-S PB-S SE-S AG-S CACO3-S \& MGO-S NA2O-S K2O-S FE304 FE304-S CAO-S FRACS $=0$ O 0 \& $\begin{array}{llllllllllllllllll}0 & 0 & 0 & 0 & 0 & 0 & 0 & 0 & 0 & .05 & .05 & .05 & .95 & .95 & .95 & .95 & \&\end{array}$

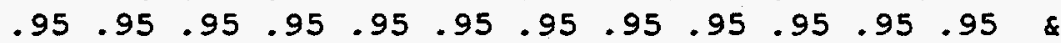
$\begin{array}{lllllllllll}.95 & .95 & .95 & .95 & .95 & .95 & .95 & .95 & .95 & .95 & .95\end{array}$

FRAC STREAM=SOLIDS SUBSTREAM=CISOLID COMPS=C-S $S-S$ NAOH-S \& NACI-S NAF-S FE-S SIO2-S AL203-S AS-S BA-S CD-S CR-S \& PB-S SE-S AG-S CACO3-S MGO-S NA2O-S K2O-S FE304-S \&

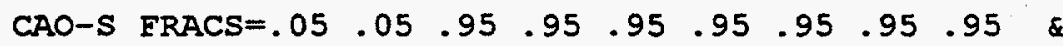

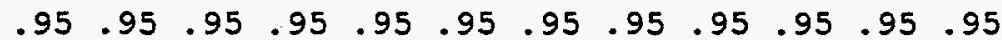

\section{BLOCK SLAG-SEP SEP}

FRAC STREAM=METAI SUBSTREAM=MIXED COMPS=H2 O2 N2 CL2 F2 \& NO $\mathrm{SO} 2 \mathrm{H} 2 \mathrm{O} \mathrm{CO} 2$ CO HCL CH4 C-S S-S S NAOH-S NACL-S \& NAF-S AS CD HG SE FE SIO2 AL2O3 FE-S SIO2-S AL2O3-S \& AS-S BA-S CD-S CR-S PB-S SE-S AG-S CACO3-S MGO-S \& NA2O-S K2O-S FE304 FE304-S CAO-S FRACS $=0$ O 00 O 00 O \& $0 \begin{array}{lllllllllllllllllllll}0 & 0 & 0 & 0 & 0 & 0 & 0 & 0 & 0 & 0 & 0 & 0 & 0 & 0 & 0 & 1 & 0 & 0 & 1 & 0 & \&\end{array}$ $\begin{array}{llllllllllllllllllll}0 & 0 & 1 & 0 & 1 & 1 & 0 & 1 & 0 & 0 & 0 & 0 & 0 & 0 & 0\end{array}$

FRAC STREAM=METAL SUBSTREAM=CISOLID COMPS=C-S S-S NAOH-S \& 
NACL-S NAF-S FE-S SIO2-S AI203-S AS-S BA-S CD-S CR-S PB-S SE-S AG-S CACO3-S MGO-S NA2O-S K20-S EE304-S \& CAO-S FRACS $=0 \begin{array}{lllllllllllllllllllll}0 & 0 & 0 & 0 & 0 & 1 & 0 & 0 & 0 & 1 & 0 & 1 & 1 & 0 & 1 & 0 & 0 & 0 & 0 & 0 & 0\end{array}$ FRAC STREAM=SIAG SUBSTREAM=MIXED COMPS=H2 $02 \quad \mathrm{~N} 2 \quad \mathrm{CL} 2$ F2 \& NO SO2 H2O CO2 CO HCL CH4 C-S s-s S NAOH-S NACL-S \& NAF-S AS CD HG SE FE SIO2 AL2O3 FE-S SIO2-S AL203-S \& AS-S BA-S CD-S CR-S PB-S SE-S AG-S CACO3-S MGO-S \& NA20-S K20-S FE304 FE304-S CAO-S FRACS=0 00 O 00 O 0 \& $\begin{array}{llllllllllllllllllllll}0 & 0 & 0 & 0 & 0 & 0 & 1 & 1 & 1 & 1 & 1 & 1 & 0 & 0 & 0 & 0 & 0 & 1 & 1 & 0 & 1 & \&\end{array}$ $\begin{array}{lllllllllllllll}1 & 0 & 0 & 0 & 0 & 0 & 0 & 0 & 1 & 1 & 1 & 1 & 1 & 1 & 1\end{array}$

FRAC STREAM=SLAG SUBSTREAM=CISOLID COMPS=C-S $\mathbf{S}-\mathbf{S}$ NAOH-S \& NACL-S NAF-S FE-S SIO2-S AL203-S AS-S BA-S CD-S CR-S \& PB-S SE-S AG-S CACO3-S MGO-S NA2O-S K2O-S EE3O4-S \&

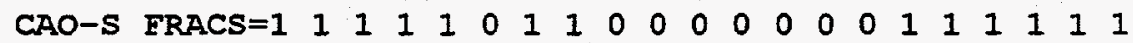

BLOCK STEAMGEN HEATER

PARAM TEMP $=300$ PRES $=1$ <ATM>

BLOCK I-V-SEP FIASH2

PARAM TEMP $=120$ PRES $=1$ <ATM>

BLOCK CATALYST RSTOIC

PARAM TEMP $=1500$ PRES $=1$ <ATM>

STOIC 1 MIXED CO $-1 / 02-0.5 / \mathrm{CO} 21$

STOIC 2 MIXED H2 $-1 / \mathrm{O} 2-0.5 / \mathrm{H} 2 \mathrm{O} 1$

STOIC 3 MIXED O2 $-2 / \mathrm{CH} 4-1 / \mathrm{H} 2 \mathrm{O} 2 / \mathrm{CO} 21$

CONV 1 MIXED CO 1

CONV 2 MIXED H2 1

CONV 3 MIXED CH4 1

BLOCK GASIFIER RGIBBS

PARAM TEMP $=3000$ PRES $=1$ <ATM> NPHASE $=2$

PROD H2 / O2 / N2/ CL2/ F2/ NO / SO2/ $\mathrm{H} 2 \mathrm{O} / \&$ $\mathrm{CO} 2 / \mathrm{CO} / \mathrm{HCL} / \mathrm{CH} 4 / \mathrm{C}-\mathrm{s}$ ss / s-s ss / $\mathrm{s} /$ \& $\mathrm{AS} / \mathrm{CD} / \mathrm{HG} / \mathrm{SE} / \mathrm{EE} / \mathrm{SIO} 2 / \mathrm{AL} 203 / \mathrm{BA}-\mathrm{S}$ \& SS / CR-S SS / $\mathrm{PB}-\mathrm{S}$ SS / AG-S SS / MGO-S SS / \& NA2O-S SS / K2O-S SS / FE304/ CAO-S SS

BLOCK PLENUM RGIBBS

PARAM TEMP $=1800$ PRES $=1$ <ATM> NPHASE $=2$

PROD H2 / O2 / N2 / CL2 / E2 / NO / SO2 / H2O / \& $\mathrm{CO} 2 / \mathrm{CO} / \mathrm{HCL} / \mathrm{C}-\mathrm{s}$ ss / s-s ss / s / As / \& $\mathrm{CD} / \mathrm{HG} / \mathrm{SE} / \mathrm{FE}-\mathrm{s}$ s $/$ sIO2-S ss / AL203-s SS \& $/ \mathrm{BA}-\mathrm{S}$ SS / CR-S SS/ $\mathrm{PB}-\mathrm{S}$ SS / AG-S SS/ MGO-S \& SS / NA2O-S SS / K2O-S SS / FE3O4-S SS / CAO-S SS

BLOCK QUENCH RGIBBS

PARAM TEMP $=350$ PRES $=1$ <ATM> NPHASE $=2$

PROD H2 / O2 / N2 / CL2 / E2 / NO / sO2 / H2O / \& $\mathrm{CO} 2 / \mathrm{CO} / \mathrm{HCL} / \mathrm{AS} / \mathrm{CD} / \mathrm{HG} / \mathrm{SE} / \mathrm{FE}-\mathrm{S} S \mathrm{SS}$ / SIO2-S SS / AL2O3-S SS / As-s ss / BA-S SS / \& CD-S SS / CR-S SS / PB-S SS / SE-S SS / AG-S SS \& / MGO-S SS / NA2O-S SS/ K20-S SS/ FE304-s ss / \& CAO-S SS

BLOCK SCRUB RGIBBS

PARAM TEMP $=120$ PRES=1 $\angle A T M>N P H A S E=2$

PROD H2 / O2 / N2 / $\mathrm{Cl} 2 / \mathrm{E} 2 / \mathrm{NO} / \mathrm{SO} 2 / \mathrm{H} 2 \mathrm{O} /$ \& $\mathrm{CO} 2 / \mathrm{CO} / \mathrm{HCL} / \mathrm{NAOH}-\mathrm{S}$ SS / NACI-S SS / NAF-S SS

DESIGN-SPEC AIR

DEFINE OXINI MASS-ELOW STREAM=AIR SUBSTREAM=MIXED \& 
COMPONENT $=02$

DEFINE OXIN2 MASS-FLOW STREAM=SCRUBVAP SUBSTREAM=MIXED \& COMPONENT $=02$

DEFINE OXOUT MASS-FLOW STREAM=EXHAUST SUBSTREAM=MIXED \& COMPONENT $=02$

SPEC "OXIN1+OXIN2" TO "OXOUT*2"

TOL-SPEC ".01"

VARY STREAM-VAR STREAM=AIR SUBSTREAM=MIXED \& VARIABLE=MASS-FIOW

LIMITS "1" "20000"

DESIGN-SPEC NAOH

DEFINE CAUIN MASS-FLOW STREAM=NAOH SUBSTREAM=CISOLID \& COMPONENT $=$ NAOH $-S$

DEFINE CAUOUT MASS-FLOW STREAM=SCRUBOUT SUBSTREAM=CISOLID\& COMPONENT $=$ NAOH $-S$

SPEC "CAUIN" TO "CAUOUT 100 "

TOL-SPEC "0.1"

VARY STREAM-VAR STREAM=NAOH SUBSTREAM=CISOLID\& VARIABLE=MASS-FLOW

LIMITS "1" "100"

DESIGN-SPEC QQUENCH

DEFINE QQUENC BLOCK-VAR BLOCK=QUENCH VARIABLE $=Q C A L C \quad \&$ SENTENCE $=$ PARAM

SPEC "QQUENC" TO "O"

TOL-SPEC "1."

VARY STREAM-VAR STREAM=QNCH2O SUBSTREAM=MIXED\&

VARIABLE $=$ MASS-FLOW

IIMITS "1" "10000"

DESIGN-SPEC SCRUBH 20

DEFINE MASSAL STREAM-VAR STREAM=SCRUBBOT SUBSTREAM=CISOLID \& VARIABLE =MASS-FLOW

DEFINE SCRUB STREAM-VAR STREAM=SCRUBH2O SUBSTREAM=MIXED \& VARIABLE=MASS - FLOW

DEFINE NAOH STREAM-VAR STREAM=NAOH SUBSTREAM=CISOIID \& VARIABLE = MASS-FLOW

SPEC "SCRUB" TO "20*MASSAL"

TOL-SPEC "0.01"

VARY STREAM-VAR STREAM=SCRUBH2O SUBSTREAM=MIXED \& VARIABLE=MASS-FIOW

LIMITS "NAOH" "5000"

DESIGN-SPEC SOILH2O

DEFINE SLAG1 STREAM-VAR STREAM=SLAG SUBSTREAM=MIXED \& VARIABLE =MASS -FLOW

DEFINE SLAG2 STREAM-VAR STREAM=SLAG SUBSTREAM=CISOLID \& VARIABLE = MASS - FLOW

DEFINE SOIL STREAM-VAR STREAM=SOIL SUBSTREAM=MIXED \& VARIABLE=MASS-FLOW

SPEC "30.* SOIL" TO "SLAG1+SLAG2"

TOI-SPEC "0.01"

VARY STREAM-VAR STREAM=SOIL SUBSTREAM=MIXED \& VARIABLE = MASS - FLOW

LIMITS " 1 " "1000"

DESIGN-SPEC SOILSOI

DEFINE SIAGI STREAM-VAR STREAM=SLAG SUBSTREAM=MIXED \& VARIABLE =MASS - FLOW

DEFINE SLAG2 STREAM-VAR STREAM=SIAG SUBSTREAM=CISOLID \& VARIABLE=MASS - FLOW 
DEFINE SOIL STREAM-VAR STREAM=SOIL SUBSTREAM=CISOIID \& VARIABLE = MASS - ELOW

SPEC "3.33333*SOIL" TO "SLAGI+SLAG2"

TOL-SPEC "0.01"

VARY STREAM-VAR STREAM=SOII SUBSTREAM=CISOLID \& VARIABLE $=$ MASS-FLOW

LIMITS "1" "1000"

\section{DESIGN-SPEC STEAM}

DEFINE H2OIN1 MASS-FLOW STREAM=STEAM SUBSTREAM=MIXED \& COMPONENT $=\mathrm{H} 2 \mathrm{O}$

DEFINE H2OIN2 MASS-FLOW STREAM=ORGANICS SUBSTREAM=MIXED \& COMPONENT $=\mathrm{H} 2 \mathrm{O}$

DEFINE H2OOUT MASS-FLOW STREAM=SYNGAS SUBSTREAM=MIXED \& COMPONENT $=\mathrm{H} 2 \mathrm{O}$

SPEC "H2OIN1+H2OIN2" TO "H2OOUT*6.0"

TOI-SPEC "0.1"

VARY STREAM-VAR STREAM=WATER SUBSTREAM=MIXED\& VARIABLE=MASS-FLOW

IIMITS "1" "100000"

CONV-OPTIONS

SECANT MAX-STEP-SIZ $=.05$ BRACKET=YES

STREAM-REPOR NOSORT MOLEFLOW MASSFLOW PROPERTIES=ALL-SUBS 


\section{System D-1}

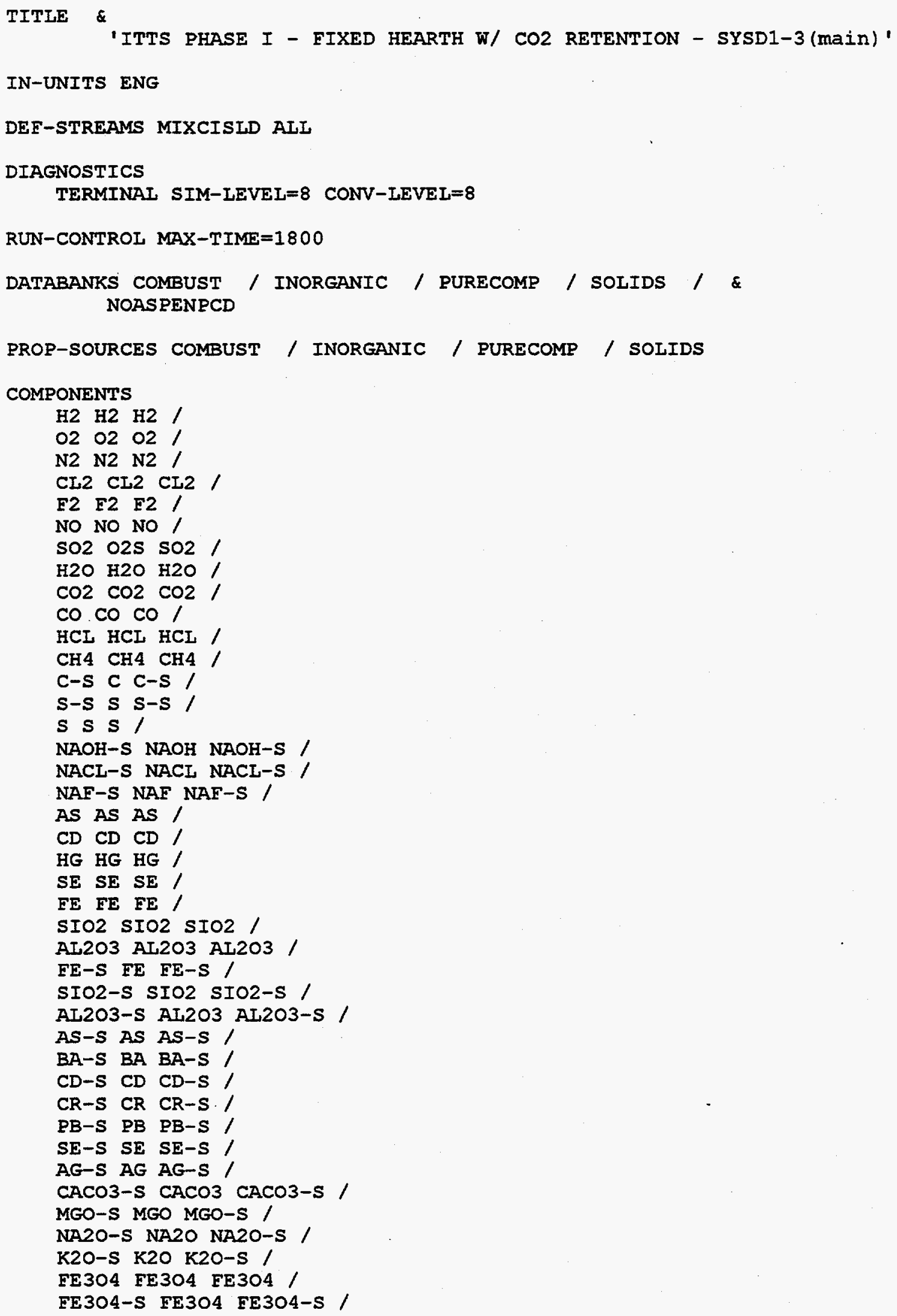


CAO-S CAO CAO-S /

CACL2-S CACL2 CACL2-S /

CASO4-S CASO4 CASO4-S

FLOWSHEET

BLOCK VITRIFY IN=FLYASH FDWASTEN SOIL ASH O2-VIT OUT $=\approx$ VITOUT

BLOCK SCC IN=FUEL-CH4 PYROVAP O2-SCC VITVAP COOLH2O OUT $=\approx$ OUT2

BLOCK BAGHOUSE IN=QUENCHED OUT=QNCHVAP HGPLUS FLYASH

BLOCK SCRUBSEP IN=SCRUBOUT OUT=SCRUBVAP SCRUBBOT

BLOCK ABSSEP IN=ABSORBED OUT=ABSSOL ABSGAS

BLOCK CO2-DES IN=ABSSOI OUT=CO2-DES

BLOCK FLASH IN=ABSGAS OUT=EXHAUST H2OOUT

BLOCK CO2-SEP IN=CO2-DES OUT=CO2 CAO-OUT

BLOCK SPIIT IN=CAO-OUT OUT=RECYCLE CAOSCRUB SPENTCAO

BLOCK CAOMIXER IN=RECYCLE CAO-IN OUT=CAOMIXED

BLOCK DRYSCRUB IN=CAOSCRUB QNCHVAP OUT=SCRUBOUT

BLOCK QUENCH IN=SCCOUT QNCH2O OUT=QUENCHED

BLOCK DUPL2 IN=OUT2 OUT=SCCOUT DUM2IN

BLOCK Q2 IN=DUM2IN OUT=DUM2OUT

BLOCK GASQUENC IN=QNCH2O-2 $\mathrm{CO} 2$ OUT=CO2OUT

BIOCK CO2-ABS IN=SCRUBVAP CAOMIXED OUT=ABSORBED

BLOCK EX-SPLIT IN=EXHAUST OUT=OFFGAS EX-RECYC

BLOCK MELTER IN=FDMETMLT OUT=METAL

BLOCK PYROLYZR IN=AIRLEAKS OXYGEN FDWASTEC EX-RECYC \& PYROH 20 OUT $=O U T 1$

BLOCK PYRO-SEP IN=PYROOUT OUT=ASH PYROVAP

BLOCK DUPLI IN=OUT 1 OUT=PYROOUT DUMIIN

BLOCK $Q 1$ IN=DUM1IN OUT=DUM1OUT

BLOCK VIT-SEP IN=VITOUT OUT=VITVAP SLAG

PROPERTIES SOIIDS

PROPERTIES IDEAL / RKS-BM

PROP-SET ALL-SUBS TEMP PRES VERAC UNITS='ATM' SUBSTREAM=ALL

PROP-SET SET1 VLSTDMX UNITS='CUFT/HR' SUBSTREAM=MIXED

STREAM AIRLEAKS

SUBSTREAM MIXED TEMP=68 PRES=1 <ATM> MASS-FLOW=224

MASS-FRAC $020.233 / \mathrm{N} 20.767$

STREAM CAO-IN

SUBSTREAM CISOIID TEMP $=68$ PRES $=1$ <ATM> MASS-FLOW=172

MASS-FRAC CAO-S 1

STREAM COOLH2O

SUBSTREAM MIXED TEMP=68 PRES=1 <ATM> MASS-FLOW=1

MASS-FLOW H2O 1

STREAM FDMETMLT

SUBSTREAM MIXED TEMP=68 PRES $=1$ <ATM> MASS-FLOW $=1.20$

MASS-FIOW H2 2.42

SUBSTREAM CISOLID TEMP $=68$ PRES $=1$ <ATM> MASS-FLOW $=147.80$

MASS-FLOW C-S $14.44 /$ FE-S $282.02 /$ SIO2-S $0.035 /$ \&

AL203-S $0.035 /$ BA-S $0.87 /$ CD-S 0.18

STREAM FDWASTEC

SUBSTREAM MIXED TEMP=68 PRES $=1$ <ATM>

MASS-FLOW H2 $50.95 / 0292.76 / \mathrm{N} 20.23 / \mathrm{CL} 253.02 /$ \& F2 $0.11 / \mathrm{H} 2019.83$ 
SUBSTREAM CISOLID TEMP $=68$ PRES $=1$ <ATM>

MASS-ELOW C-S $326.28 /$ S-S $0.49 /$ FE-S $7.67 /$ SIO2-S \&

$53.01 /$ AI203-S 53.01/ BA-S $0.77 / \mathrm{CD}-\mathrm{S} 0.77 /$ \&

CR-S 1.53

STREAM FDWASTEN

SUBSTREAM MIXED TEMP=68 PRES=1 <ATM>

MASS-FLOW H2 $0.54 / 021.44 / \mathrm{N} 20.01 /$ CL2 $14.88 /$ \&

NO $0.66 /$ SO2 $0.83 / \mathrm{H} 20354.23 /$ HG 0.15

SUBSTREAM CISOIID TEMP $=68$ PRES $=1$ <ATM>

MASS-FLOW C-S $4.77 /$ s-S $0.62 /$ SIO2-S $476.77 /$ \&

AL203-S 476.77/ AS-S $0.06 /$ BA-S $0.04 /$ CD-S 2.63 \&

/ CR-S $4.95 / \mathrm{PB}-\mathrm{S} 0.17 / \mathrm{SE}-\mathrm{S} 0.03 / \mathrm{AG}-\mathrm{S} 0.03$

STREAM FUEL-CH4

SUBSTREAM MIXED TEMP=68 PRES=1 <ATM> MASS-FLOW=1

MASS-FRAC CH4 1

STREAM O2-SCC

SUBSTREAM MIXED TEMP=68 PRES=1 <ATM> MASS-FLOW=2000

MASS-FRAC $02 \cdot 1$

STREAM O2-VIT

SUBSTREAM MIXED TEMP=68 PRES=1 <ATM> MASS-FLOW=1

MASS-FRAC O2 1

STREAM OXYGEN

SUBSTREAM MIXED TEMP=68 PRES=1 <ATM> MASS-FLOW=1

MASS-FRAC 021

STREAM PYROH2O

SUBSTREAM MIXED TEMP=68 PRES=1 <ATM> MASS-FLOW=10000

MASS-FLOW H2O 1

STREAM ONCH2O

SUBSTREAM MIXED TEMP=68 PRES=1 <ATM> MASS-FLOW=4430

MASS-ERAC H2O 1

STREAM QNCH2O-2

SUBSTREAM MIXED TEMP=68 PRES=1 <ATM> MASS-FLOW=100

MASS-FRAC H2O 1

STREAM SOIL

SUBSTREAM MIXED TEMP=68 PRES=1 <ATM> MASS-FLOW=62.5

MASS-FIOW H2O . 100

SUBSTREAM CISOLID TEMP $=68$ PRES $=1$ <ATM> MASS-FLOW=562.5

MASS-FIOW SIO2-S $0.569 /$ AL203-S $0.102 /$ CACO3-S $0.128 /$ \& MGO-S $0.023 /$ NA2O-S $0.013 /$ K2O-S $0.024 /$ FE304-S 0.041

BLOCK CAOMIXER MIXER

PARAM PRES $=1$ <ATM> NPHASE $=2$

BLOCK EX-SPLIT FSPIIT

ERAC EX-RECYC 0.90

BLOCK SPLIT ESPIIT

ERAC RECYCIE $0.90 /$ CAOSCRUB 0.015

BLOCK ABSSEP SEP

FRAC STREAM=ABSSOI SUBSTREAM=MIXED COMPS=H2 O2 N2 CI2 \& F2 NO SO2 H2O CO2 CO HCL CH4 C-S S-S S NAOH-S \& NACI-S NAF-S AS CD HG SE FE SIO2 AL2O3 FE-S SIO2-S \&

B-81 
AL203-S AS-S BA-S CD-S CR-S PB-S SE-S AG-S CACO3-S \& MGO-S NA2O-S K2O-S FE304 FE3O4-S CAO-S CACL2-S CASO4-S \& ERACS $=0 \begin{array}{lllllllllllllllllll}0 & 0 & 0 & 0 & 0 & 0 & 0 & 0 & 0 & 0 & 0 & 1 & 1 & 1 & 1 & 1 & 1 & \&\end{array}$ $\begin{array}{llllllllllllllllllllllllll}1 & 1 & 1 & 1 & 1 & 1 & 1 & 1 & 1 & 1 & 1 & 1 & 1 & 1 & 1 & 1 & 1 & 1 & 1 & 1 & 1 & 1 & 1 & 1 & 1 & 1\end{array}$

FRAC STREAM =ABSSOI SUBSTREAM=CISOLID COMPS=C-S $S-S$ NAOH-S \& NACL-S NAF-S FE-S SIO2-S AI203-S AS-S BA-S CD-S CR-S \& PB-S SE-S AG-S CACO3-S MGO-S NA2O-S K2O-S EE3O4-S \&

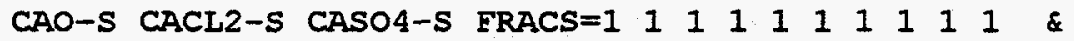
$\begin{array}{llllllllllllll}1 & 1 & 1 & 1 & 1 & 1 & 1 & 1 & 1 & 1 & 1 & 1 & 1\end{array}$

\section{BLOCK BAGHOUSE SEP}

FRAC STREAM=HGPLUS SUBSTREAM=MIXED COMPS=H2 O2 N2 CL2 \& F2 NO SO2 H2O CO2 CO HCL CH4 C-S S-S S NAOH-S \& NACI-S NAF-S AS CD HG SE FE SIO2 AI2O3 FE-S SIO2-S \& AL2O3-S AS-S BA-S CD-S CR-S PB-S SE-S AG-S CACO3-S \& MGO-S NA2O-S K2O-S FE304 FE304-S CAO-S CACL2-S CASO4-S \& FRACS $=0 \begin{array}{llllllllllllllllll}0 & 0 & 0 & 0 & 0 & 0 & 0 & 0 & 0 & 0 & 0 & 0 & 0 & 0 & 0 & 0 & 0 & \&\end{array}$ $\begin{array}{llllllllllllllllllllllllll}1 & 1 & 1 & 1 & 0 & 0 & 0 & 0 & 0 & 0 & 1 & 0 & 1 & 0 & 0 & 1 & 0 & 0 & 0 & 0 & 0 & 0 & 0 & 0 & 0 & 0\end{array}$

FRAC STREAM=HGPLUS SUBSTREAM=CISOLID COMPS=C-S $S-S$ NAOH-S \& NACI-S NAF-S FE-S SIO2-S AL2O3-S AS-S BA-S CD-S CR-S \& PB-S SE-S AG-S CACO3-S MGO-S NA2O-S K2O-S FE304-S \& CAO-S CACI2-S CASO4-S FRACS $=\begin{array}{lllllllllll}0 & 0 & 0 & 0 & 0 & 0 & 0 & 0 & 1 & 0 & \&\end{array}$ 1000011000000000000

FRAC STREAM=FLYASH SUBSTREAM=MIXED COMPS=H2 O2 N2 CL2 \& F2. NO SO2 H2O CO2 CO HCL CH4 C-S S-S S NAOH-S \& NACI-S NAF-S AS CD HG SE FE SIO2 AL2O3 EE-S SIO2-S \& AL203-S AS-S BA-S CD-S CR-S PB-S SE-S AG-S CACO3-S \& MGO-S NA2O-S K2O-S EE304 FE304-S CAO-S CACL2-S CASO4-S \& FRACS $=\begin{array}{lllllllllllllllllll}0 & 0 & 0 & 0 & 0 & 0 & 0 & 0 & 0 & 0 & 0 & 0 & 1 & 1 & 1 & 1 & 1 & 1\end{array}$ $\begin{array}{llllllllllllllllllllllllll}0 & 0 & 0 & 0 & 1 & 1 & 1 & 1 & 1 & 1 & 0 & 1 & 0 & 1 & 1 & 0 & 1 & 1 & 1 & 1 & 1 & 1 & 1 & 1 & 1 & 1\end{array}$

FRAC STREAM=FLYASH SUBSTREAM=CISOIID COMPS=C-S $S-S$ NAOH-S \& NACL-S NAF-S FE-S SIO2-S AL2O3-S AS-S BA-S CD-S CR-S \& PB-S SE-S AG-S CACO3-S MGO-S NA2O-S K2O-S FE304-S \&

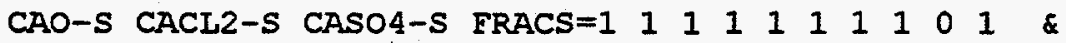
$\begin{array}{llllllllllllll}0 & 1 & 1 & 0 & 1 & 1 & 1 & 1 & 1 & 1 & 1 & 1 & 1\end{array}$

FLASH-SPECS FLYASH TEMP=68 PRES=1 <ATM>

BIOCK CO2-SEP SEP

ERAC STREAM=CO2 SUBSTREAM=MIXED COMPS=H2 O2 N2 CL2 F2 \& NO SO2 H2O CO2 CO HCL CH4 C-S S-S S NAOH-S NACL-S \& NAF-S AS CD HG SE FE SIO2 AL2O3 FE-S SIO2-S AL2O3-S \& AS-S BA-S CD-S CR-S PB-S SE-S AG-S CACO3-S MGO-S \& NA2O-S K2O-S FE3O4 FE3O4-S CAO-S CACL2-S CASO4-S \& ERACS $=0 \begin{array}{lllllllllllllllllll}0 & 0 & 0 & 0 & 0 & 0 & 0 & 0 & 1 & 0 & 0 & 0 & 0 & 0 & 0 & 0 & 0 & 0 & \&\end{array}$ $0 \begin{array}{lllllllllllllllllllllllll}0 & 0 & 0 & 0 & 0 & 0 & 0 & 0 & 0 & 0 & 0 & 0 & 0 & 0 & 0 & 0 & 0 & 0 & 0 & 0 & 0 & 0 & 0 & 0 & 0\end{array}$

FRAC STREAM=CO2 SUBSTREAM=CISOLID COMPS=C-S S-S NAOH-S \& NACI-S NAF-S FE-S SIO2-S AL2O3-S AS-S BA-S CD-S CR-S \& PB-S SE-S AG-S CACO3-S MGO-S NA2O-S K2O-S FE3O4-S \& CAO-S CACL2-S CASO4-S ERACS $=0 \begin{array}{llllllllll}0 & 0 & 0 & 0 & 0 & 0 & 0 & 0 & 0 & 0\end{array}$ $0 \begin{array}{lllllllllllll}0 & 0 & 0 & 0 & 0 & 0 & 0 & 0 & 0 & 0 & 0 & 0\end{array}$

FLASH-SPECS CAO-OUT TEMP=68 PRES=1 〈ATM>

\section{BLOCK PYRO-SEP SEP}

FRAC STREAM=ASH SUBSTREAM=MIXED COMPS=H2 O2 N2 CL2 F2 \& NO SO2 H2O CO2 CO HCL CH4 C-S S-S S NAOH-S NACI-S \& NAF-S AS CD HG SE FE SIO2 AL203 FE-S SIO2-S AL2O3-S \& AS-S BA-S CD-S CR-S PB-S SE-S AG-S CACO3-S MGO-S \& NA2O-S K2O-S FE304 FE304-S CAO-S CACL2-S CASO4-S \& FRACS $=0 \begin{array}{lllllllllllllllll}0 & 0 & 0 & 0 & 0 & 0 & 0 & 0 & 0 & 0 & 0 & 0 & .8 & .8 & 0 & .8 & .8\end{array}$ $\begin{array}{lllllllllllllllll}.8 & 0 & 0 & 0 & 0 & .8 & .8 & .8 & .8 & .8 & .8 & .8 & .8 & .8 & .8 & .8 & 8\end{array}$ $\begin{array}{llllllllllll}.8 & .8 & .8 & .8 & .8 & .8 & .8 & .8 & .8 & .8 & .8\end{array}$

FRAC STREAM=ASH SUBSTREAM=CISOLID COMPS=C-S $s-S$ NAOH-S \& 
NACI-S NAF-S FE-S SIO2-S AI203-S AS-S BA-S CD-S CR-S \& PB-S SE-S AG-S CACO3-S MGO-S NA2O-S K2O-S FE304-S \&

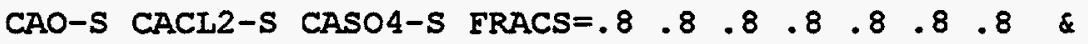
$\begin{array}{llllllllllllllll}.8 & .8 & .8 & .8 & .8 & .8 & .8 & .8 & .8 & .8 & .8 & .8 & .8 & .8 & .8 & .8\end{array}$

FLASH-SPECS ASH TEMP $=68$ PRES $=1$ <ATM>

BLOCK SCRUBSEP SEP

FRAC STREAM=SCRUBBOT SUBSTREAM=MIXED COMPS=H2 O2 N2 CL2 \& F2 NO SO2 H2O CO2 CO HCL CH4 C-S s-s S NAOH-S \& NACL-S NAF-S AS CD HG SE FE SIO2 AI2O3 FE-S SIO2-S \& AL203-S AS-S BA-S CD-S CR-S PB-S SE-S AG-S CACO3-S \& MGO-S NA2O-S K2O-S FE3O4 FE3O4-S CAO-S CACL2-S CASO4-S \& ERACS $=0 \begin{array}{lllllllllllllllllll}0 & 0 & 0 & 0 & 0 & 0 & 0 & 0 & 0 & 0 & 0 & 0 & 1 & 1 & 0 & 1 & 1 & 1\end{array}$ $\begin{array}{lllllllllllllllllllllllllll}1 & 1 & 1 & 1 & 1 & 1 & 1 & 1 & 1 & 1 & 1 & 1 & 1 & 1 & 1 & 1 & 1 & 1 & 1 & 1 & 1 & 1 & 1 & 1 & 1 & 1\end{array}$

FRAC STREAM=SCRUBBOT SUBSTREAM=CISOLID COMPS $=\mathrm{C}-\mathrm{S}$ S-S \& NAOH-S NACI-S NAF-S FE-S SIO2-S AI2O3-S AS-S BA-S \& CD-S CR-S PB-S SE-S AG-S CACO3-S MGO-S NA2O-S K2O-S \& FE3O4-S CAO-S CACL2-S CASO4-S FRACS=1 111111111 \& $\begin{array}{lllllllllllllllll}1 & 1 & 1 & 1 & 1 & 1 & 1 & 1 & 1 & 1 & 1 & 1 & 1 & 1 & 1 & 1\end{array}$

BLOCK VIT-SEP SEP

ERAC STREAM=VITVAP SUBSTREAM=MIXED COMPS=H2 O2 N2 CL2 \& F2 NO SO2 H2O CO2 CO HCI CH4 C-S S-S S NAOH-S \& NACL-S NAF-S AS CD HG SE EE SIO2 AL2O3 FE-S SIO2-S \& AL2O3-S AS-S BA-S CD-S CR-S PB-S SE-S AG-S CACO3-S \& MGO-S NA2O-S K2O-S FE304 FE304-S CAO-S CACL2-S CASO4-S \&

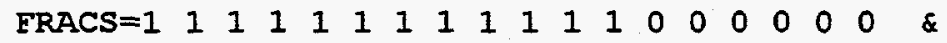
$\begin{array}{lllllllllllllllllllllllllll}1 & 1 & 1 & 1 & 0 & 0 & 0 & 0 & 0 & 0 & 0 & 0 & 0 & 0 & 0 & 0 & 0 & 0 & 0 & 0 & 0 & 0 & 0 & 0 & 0 & 0\end{array}$

FRAC STREAM=VITVAP SUBSTREAM=CISOLID COMPS=C-S $S-S$ NAOH-S \& NACL-S NAF-S FE-S SIO2-S AL2O3-S AS-S BA-S CD-S CR-S \& PB-S SE-S AG-S CACO3-S MGO-S NA2O-S K2O-S FE304-S \& CAO-S CACL2-S CASO4-S FRACS $=0 \begin{array}{llllllllll}0 & 0 & 0 & 0 & 0 & 0 & 0 & 0 & 0 & 0\end{array}$ $\begin{array}{lllllllllllll}0 & 0 & 0 & 0 & 0 & 0 & 0 & 0 & 0 & 0 & 0 & 0 & 0\end{array}$

BLOCK Q1 HEATER

PARAM TEMP=68 PRES=1 <ATM>

BLOCK FLASH FLASH2

PARAM TEMP $=90$ PRES $=1$ <ATM> TOL $=0.000001$

PROPERTIES IDEAI

BLOCK CO2-ABS RSTOIC

PARAM TEMP=1200 PRES=1 <ATM> NPHASE=1 MAXIT=200 \& TOL $=0.000001$

STOIC 1 MIXED CO2 - 1 / CISOLID CAO-S - $1 /$ CACO3-S 1

CONV 1 MIXED CO2 1

BIOCK-OPTION RESTART $=$ NO

BLOCK PYROLYZR RSTOIC

PARAM TEMP $=1200$ PRES $=1$ 〈ATM>

STOIC 1 CISOLID C-S - 1 / MIXED O2 -0.5/ CO 1

STOIC 2 MIXED O2-2/ CH4 -1/ H2O $2 / \mathrm{CO} 21$

CONV 1 CISOLID C-S 0.99

CONV 2 MIXED CH 41

BLOCK CO2-DES RGIBBS

PARAM TEME $=1700$ PRES=1 <ATM> NPHASE=2

PROD $\mathrm{CO} 2 /$ CAO-S SS

PROPERTIES IDEAL

BLOCK DRYSCRUB RGIBBS

PARAM TEMP $=350$ PRES $=1$ <ATM> NPHASE=2 MAXIT=500 
PROD H2 / O2 / N2 / CL2 / F2 / NO / $\mathrm{H} 2 \mathrm{O} / \mathrm{CO} 2 / \&$ CASO4-S SS / CACL2-S SS / CAO-S SS

BLOCK GASQUENC RGIBBS

PARAM TEMP $=350$ PRES $=1$ <ATM $>$ NPHASE $=2$ MAXIT $=500$

PROD H2 / O2 / $\mathrm{H} 2 \mathrm{O} / \mathrm{CO} / \mathrm{CO} / \mathrm{C}-\mathrm{S} \mathrm{SS}$

PROD-FRAC H2 $1 / \mathrm{O} 21 / \mathrm{H} 2 \mathrm{O} 1 / \mathrm{CO} 21$

BLOCK MELTER RGIBBS

PARAM TEMP $=3000$ PRES=1 〈ATM> NPHASE $=2$

PROD H2 / C-S SS / FE / SIO2 / AL2O3 / FE-S SS / \& SIO2-S SS / AI203-S SS / BA-S SS / CD / CD-S SS

\section{BLOCK Q2 RGIBBS}

PARAM TEMP $=68$ PRES $=1$ <ATM> NPHASE $=2$ TAPP $=2132$

PROD H2 / O2 / N2 / CL2 / F2 / NO / SO2 / H2O / \& $\mathrm{CO} 2 / \mathrm{CO} / \mathrm{HCL} / \mathrm{CH} 4 / \mathrm{S} / \mathrm{AS} / \mathrm{CD} / \mathrm{HG} / \mathrm{SE} \&$ / FE-S SS / SIO2-S SS / AL2O3-S SS / AS-S SS / \& $\mathrm{BA}-\mathrm{S}$ SS / CD-S SS / CR-S SS / PB-S SS / SE-S SS \& / AG-S SS / MGO-S SS / NA2O-S SS / K2O-S SS / CAO-S SS

BLOCK QUENCH RGIBBS

PARAM TEMP $=350$ PRES $=1$ <ATM> NPHASE $=2$

PROD H2 / O2 / N2 / $\mathrm{F} 2 / \mathrm{NO} / \mathrm{SO} 2 / \mathrm{H} 2 \mathrm{O} / \mathrm{CO} 2 / \&$ $\mathrm{CO} / \mathrm{HCL} / \mathrm{AS} / \mathrm{CD} / \mathrm{HG} / \mathrm{SE} / \mathrm{FE}-\mathrm{S} \mathrm{SS} / \&$ SIO2-S SS / AL2O3-S SS / AS-S SS / BA-S SS / \& CD-S SS / CR-S SS / PB-S SS / SE-S SS / AG-S SS \& / MGO-S SS / NA2O-S SS / K20-S SS / FE304-S SS / CAO-S SS

BLOCK SCC RGIBBS

PARAM TEMP $=2200$ PRES $=1$ <ATM $>$ NPHASE $=2$ MAXIT $=150$

PROD H2 / O2 / N2 / CL2 / F2 / NO / SO2 / H2O / \& $\mathrm{CO} 2 / \mathrm{CO} / \mathrm{HCL} / \mathrm{CH} 4 / \mathrm{S} / \mathrm{AS} / \mathrm{CD} / \mathrm{HG} / \mathrm{SE} \&$ / FE-S Ss / SIO2-S SS / AL203-s ss / AS-S SS / \& $\mathrm{BA}-\mathrm{S}$ SS / CD-S SS / CR-S SS / PB-S SS / SE-S SS \& / AG-S SS / MGO-S SS / NA2O-S SS / K2O-S SS / CAO-S SS

BLOCK VITRIEY RGIBBS

PARAM TEMP $=3000$ PRES=1 <ATM> NPHASE=2 CHEMEQ=YES MAXIT $=500 \&$ TOL $=0.00001$

PROD H2 / O2 / N2 / CL2 / $22 /$ NO / SO2 / H2O / \& $\mathrm{CO} 2 / \mathrm{CO} / \mathrm{HCL} / \mathrm{C}-\mathrm{S} \mathrm{SS} / \mathrm{s} / \mathrm{AS} / \mathrm{CD} / \mathrm{HG} / \&$ SE / FE-S SS / SIO2 / AL2O3 / AS-S SS / BA-S SS \& / CD-S SS / CR-S SS / $\mathrm{PB}-\mathrm{S}$ SS / SE-S SS / AG-S \& SS / MGO-S SS / NA2O-S SS / K20-S SS / FE3O4-S \& SS / CAO-S SS

PROD-FRAC FE-S 1 / FE3O4-S 1

BLOCK-OPTION RESTART $=$ YES

BLOCK DUPLI DUPL

BLOCK DUPL2 DUPL

DESIGN-SPEC CAO

DEFINE CO2CON MOLE-FLOW STREAM=SCRUBVAP SUBSTREAM=MIXED \& COMPONENT $=\mathrm{CO} 2$

DEFINE CAOMXD MOLE-FLOW STREAM=CAOMIXED SUBSTREAM=CISOLID \& COMPONENT $=$ CAO-S

SPEC "CO2CON" TO "CAOMXD"

TOL-SPEC "0.01"

VARY STREAM-VAR STREAM=CAO-IN SUBSTREAM=CISOLID \& VARIABLE $=$ MASS-FLOW 
LIMITS "1" "10000"

DESIGN-SPEC O2SCC

DEFINE OXIN1 MASS-FLOW STREAM=02-SCC SUBSTREAM=MIXED \& COMPONENT $=02$

DEFINE OXIN2 MASS-FLOW STREAM=PYROVAP SUBSTREAM=MIXED \& COMPONENT $=02$

DEFINE OXIN3 MASS-ELOW STREAM=VITVAP SUBSTREAM=MIXED \& COMPONENT $=02$

DEEINE OXOUT MASS-FLOW STREAM=SCCOUT SUBSTREAM=MIXED \& COMPONENT $=02$

SPEC "OXIN1+OXIN2+OXIN3" TO "6.0*OXOUT"

TOL-SPEC "0.01"

VARY STREAM-VAR STREAM=02-SCC SUBSTREAM=MIXED \& VARIABLE=MASS-FLOW

IIMITS "1" "100000"

DESIGN-SPEC O2VIT

DEFINE OX1 MASS-FLOW STREAM=EDWASTEN SUBSTREAM=MIXED \& COMPONENT $=02$

DEFINE OX2 MASS-FLOW STREAM=02-VIT SUBSTREAM=MIXED \& COMPONENT $=02$

DEFINE OX3 MASS-ELOW STREAM=SOII SUBSTREAM=MIXED \& COMPONENT $=02$

DEFINE OXOUT MASS-FLOW STREAM=VITOUT SUBSTREAM=MIXED \& COMPONENT $=02$

SPEC "OX1+OX2+OX3" TO "6.0*OXOUT"

TOL-SPEC "0.05"

VARY STREAM-VAR STREAM=O2-VIT SUBSTREAM=MIXED \& VARIABLE =MASS - FLOW

IIMITS " 1 " " $10000 "$

DESIGN-SPEC OXYGEN

DEFINE OXI MASS-FLOW STREAM=OXYGEN SUBSTREAM=MIXED \& COMPONENT $=02$

DEFINE OX2 MASS-FLOW STREAM=EX-RECYC SUBSTREAM=MIXED \& COMPONENT $=02$

DEFINE OX3 MASS-FLOW STREAM=FDWASTEC SUBSTREAM=MIXED \& COMPONENT $=02$

DEFINE OX4 MASS-ELOW STREAM=AIRLEAKS SUBSTREAM=MIXED \& COMPONENT $=02$

DEFINE OX5 MASS-FLOW STREAM=PYROH20 SUBSTREAM=MIXED \& COMPONENT $=02$

DEFINE OXOUT MASS-ELOW STREAM=PYROOUT SUBSTREAM=MIXED \& COMPONENT $=02$

SPEC "OX $1+0 \times 2+0 \times 3+0 \times 4+(0 \times 5 / 6)$ " TO "100.*OXOUT"

TOL-SPEC "0.05"

VARY STREAM-VAR STREAM=OXYGEN SUBSTREAM=MIXED \& VARIABLE=MASS - FLOW

IIMITS "1" "100000"

DESIGN-SPEC QPYRO

DEFINE QPYRO BLOCK-VAR BLOCK=PYROLYZR VARIABLE=QCALC \& SENTENCE = PARAM

DEFINE Q1 BLOCK-VAR BLOCK=Q1 VARIABLE=QCALC SENTENCE=PARAM

SPEC "QPYRO" TO "0.05*Q1"

TOL-SPEC "1."

VARY STREAM-VAR STREAM=PYROH20 SUBSTREAM=MIXED \& VARIABLE=MASS-FIOW

LIMITS " 1 " "10000"

DESIGN-SPEC QQUENCH 
DEFINE QQUENC BLOCK-VAR BLOCK=QUENCH VARIABLE=QCALC \& SENTENCE $=$ PARAM

SPEC "QQUENC" TO "O"

TOI-SPEC " 1

VARY STREAM-VAR STREAM=QNCH2O SUBSTREAM=MIXED \& VARIABLE=MASS-FLOW

LIMITS " 1 " "100000"

DESTIGN-SPEC QQUENCH2

DEFINE QGASQU BLOCK-VAR BLOCK=GASQUENC VARIABLE=QCALC \& SENTENCE $=$ PARAM

SPEC "QGASQU" TO "O"

TOL-SPEC "1."

VARY STREAM-VAR STREAM $=Q N C H 2 O-2$ SUBSTREAM=MIXED \& VARIABLE=MASS-FLOW

LIMITS "1" "5000"

DESIGN-SPEC QSCC

DEFINE QSCC BLOCK-VAR BLOCK=SCC VARIABLE=QCALC \& SENTENCE $=$ PARAM

DEFINE $Q 2$ BLOCK-VAR BLOCK=Q2 VARIABLE=QCALC SENTENCE=PARAM SPEC "OSCC " TO " $0.05 * Q 2$ "

TOI-SPEC "1."

VARY STREAM-VAR STREAM=COOLH2O SUBSTREAM=MIXED \& VARIABLE=MASS - FLOW

LIMITS " 1 " " $100000 "$

DESIGN-SPEC SCRUB

DEFINE CAOIN MOLE-FLOW STREAM=CAOSCRUB SUBSTREAM=CISOLID COMPONENT $=$ CAO-S

DEFINE SO2IN MOLE-FLOW STREAM=QNCHVAP SUBSTREAM=MIXED \& COMPONENT $=\mathrm{SO} 2$

DEFINE HCLIN MOLE-FLOW STREAM=QNCHVAP SUBSTREAM=MIXED\& COMPONENT $=$ HCL

SPEC "CAOIN-SO2IN-HCLIN/2" TO "0.01"

TOL-SPEC " 0.002 "

VARY BLOCK-VAR BLOCK=SPLIT SENTENCE=FRAC VARIABLE=FRAC \& IDI $=$ CAOSCRUB

LIMITS "0.00001" "0.10"

DESIGN-SPEC SOILH2O

DEFINE SLAGI STREAM-VAR STREAM=SLAG SUBSTREAM=MIXED \& VARIABLE = MASS-FLOW

DEFINE SLAG2 STREAM-VAR STREAM=SLAG SUBSTREAM=CISOLID \& VARIABLE $=$ MASS - FLOW

DEFINE SOIL STREAM-VAR STREAM=SOIL SUBSTREAM=MIXED \& VARIABLE =MASS-FLOW

SPEC "30.*SOIL" TO "SLAG1+SLAG2"

TOL-SPEC " 10 "

VARY STREAM-VAR STREAM=SOIL SUBSTREAM=MIXED\& VARIABLE=MASS - FLOW

IIMITS " 1 " "1000"

DESIGN-SPEC SOILSOL

DEFINE SLAGI STREAM-VAR STREAM=SLAG SUBSTREAM=MIXED \& VARIABLE=MASS-FLOW

DEFINE SLAG2 STREAM-VAR STREAM=SIAG SUBSTREAM=CISOLID \& VARIABLE=MASS-FLOW

DEFINE SOIL STREAM-VAR STREAM=SOIL SUBSTREAM=CISOLID\& VARIABLE $=$ MASS-FLOW

SPEC " 3.33333 *SOIL" TO "SIAGI+SLAG2"

TOL-SPEC "10" 
VARY STREAM-VAR STREAM=SOII SUBSTREAM=CISOIID \& VARIABLE=MASS-FLOW

LIMITS "1" "1000"

\section{CONV-OPTIONS}

PARAM TOL $=0.001$ OPT-METHOD=COMPLEX MSPEC-METHOD=NEWTON

WEGSTEIN MAXIT $=200$ QMAX $=0.5$

SECANT MAX-STEP-SIZ=.05 BRACKET=NO

CONVERGENCE C-1 NEWTON

SPEC O2SCC

CONVERGENCE C-2 NEWTON

SPEC QPYRO

STREAM-REPOR NOSORT MOLEELOW MASSELOW PROPERTIES=SET1 ALL-SUBS 


\section{System E-1}

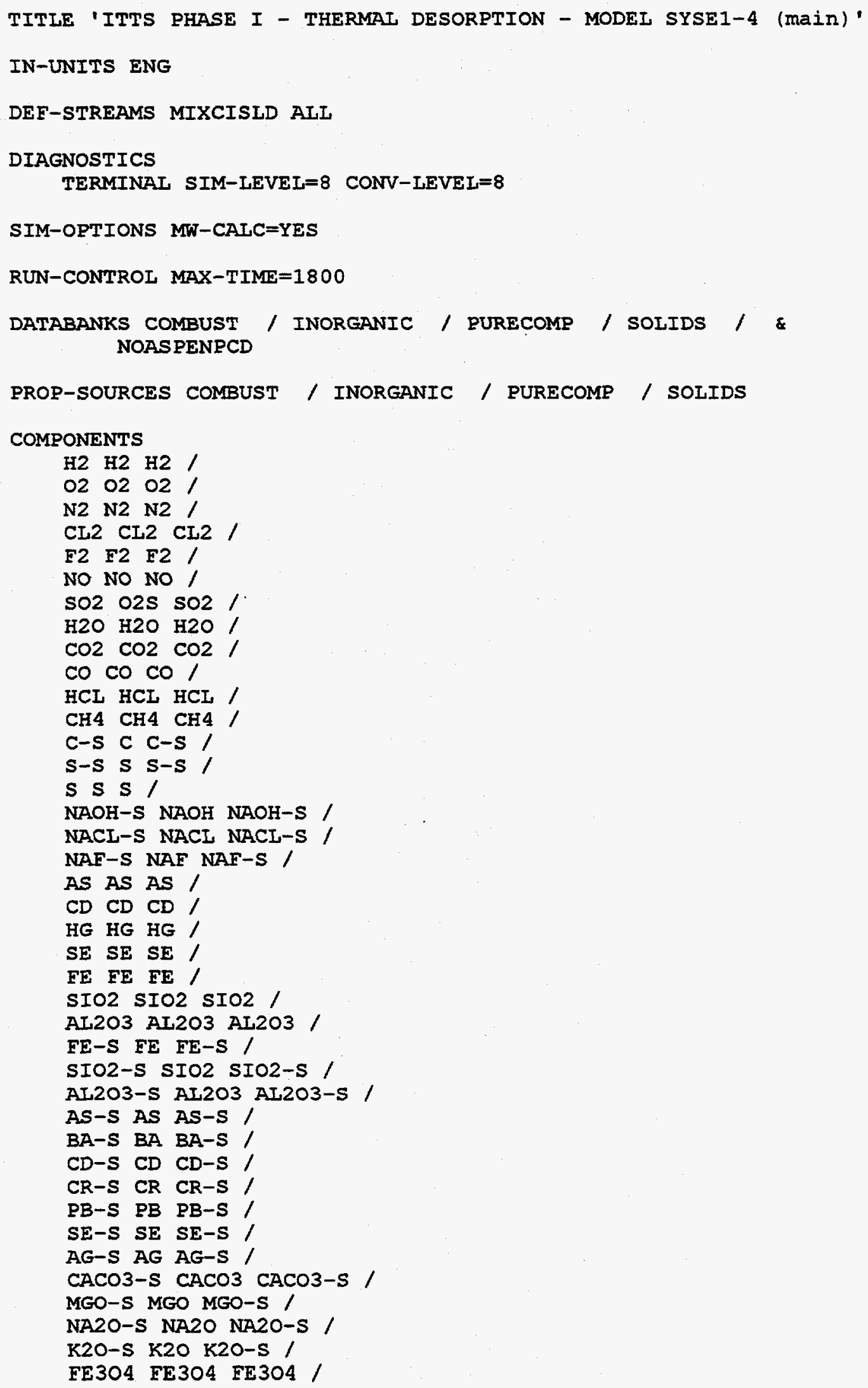


FE304-S FE304 FE304-S /

CAO-S CAO CAO-S

\section{FLOWSHEET}

BLOCK KILN IN=SOIL FUELMIX1 FDWASTEC AIR AIRLEAKS COOLH2O \& CONDENSD OUT $=$ OUT 1

BIOCK KIINSEP IN=KILNOUT OUT=ASH KIINVAP

BIOCK SCC IN=KIINVAP FUEIMIX2 OUT=OUT2

BIOCK BAGHOUSE IN=QUENCHED OUT=HGPLUS ELYASH QNCHVAP

BLOCK QUENCH IN=SCCOUT QNCH2O OUT=QUENCHED

BLOCK L-V-SEP IN=SCRUBOUT OUT=SCRUBVAP SCRUBBOT

BLOCK DUPL1 IN=OUT1 OUT=KIINOUT DUM1IN

BLOCK $Q 1$ IN=DUM1IN OUT=DUM1OUT

BLOCK DUPL2 IN=OUT2 OUT=SCCOUT DUM2IN

BLOCK $Q 2$ IN=DUM2IN OUT=DUM2OUT

BLOCK VITRIFY IN=FLYASH ASH OUT=VITOUT

BLOCK SCRUB IN=QNCHVAP NAOH SCRUBH2O OUT=SCRUBOUT

BLOCK DESORBER . IN=FDWASTEN OUT=DESORBED

BIOCK CONDENSR IN=DESRBVAP OUT=CONDENSD

BLOCK SEPARATR IN=DESORBED OUT=DESRBVAP SOLIDS

BLOCK MELTER IN=FDMETMLT OUT=METAI

BLOCK I-V-SEP2 IN=VITOUT OUT=VIT-VAP SLAG

BLOCK HEATER IN=FUELMIX3 OUT=EXHAUST3

PROPERTIES SOLIDS

PROPERTIES IDEAI / RKS-BM

PROP-SET ALL-SUBS TEMP PRES VFRAC UNITS='ATM' SUBSTREAM=ALL

PROP-SET PSLIQ CPMX HMX UNITS='BTU/LB-R' 'J/KG-K' 'BTU/LB' \&

' $\mathrm{J} / \mathrm{KG}$ ' SUBSTREAM $=$ MIXED PHASE=L

PROP-SET PSSOL CPMX HMX UNITS='BTU/LB-R' 'J/KG-K' 'BTU/LB' \&

' $\mathrm{J} / \mathrm{KG}$ ' SUBSTREAM=CISOLID PHASE=S

PROP-SET PSVAP CPMX HMX UNITS='BTU/LB-R' 'J/KG-K' 'BTU/LB' \& ' $\mathrm{J} / \mathrm{KG}$ ' SUBSTREAM=MIXED PHASE=V

STREAM AIR

SUBSTREAM MIXED TEMP=68 PRES=1 <ATM> MASS-ELOW=7766.243

MASS-FRAC $020.233 / \mathrm{N} 20.767$

STREAM AIRLEAKS

SUBSTREAM MIXED TEMP=68 PRES=1 <ATM> \&

VOLUME-FLOW $=600<\mathrm{CUFT} / \mathrm{MIN}>$

MASS-ERAC O2 $0.233 / \mathrm{N} 20.767$

STREAM COOLH2O

SUBSTREAM MIXED TEMP=68 PRES=1 <ATM> MASS-FLOW=909.895

MASS-ERAC H2O 1

STREAM FDMETMLT

SUBSTREAM MIXED TEMP=68 PRES=1 <ATM> MASS-FLOW=1.20

MASS-FLOW H2 2.42

SUBSTREAM CISOIID TEMP=68 PRES $=1$ <ATM> MASS-FLOW $=147.80$

MASS-ELOW C-S $14.44 /$ FE-S $282.02 /$ SIO2-S $0.035 /$ \&

AL203-S $0.035 /$ BA-S $0.87 /$ CD-S 0.18

STREAM FDWASTEC

SUBSTREAM MIXED TEMP=68 PRES=1 <ATM>

MASS-FLOW H2 $50.95 / 0292.76 / \mathrm{N} 20.23 / \mathrm{CL} 2.53 .02 /$ \& F2 $0.11 / \mathrm{H} 2019.83$ 
SUBSTREAM CISOLID TEMP=68 PRES $=1$ <ATM>

MASS-FLOW C-S $326.28 /$ S-S 0.49 / FE-S $7.67 /$ SIO2-S \& $53.01 /$ AL203-S 53.01/ BA-S $0.77 /$ CD-S $0.77 /$ CR-S 1.53

STREAM EDWASTEN

SUBSTREAM MIXED TEMP=68 PRES=1 <ATM>

MASS-FLOW H2 $0.54 / 021.44 /$ N2 $0.01 /$ CL2 $14.88 /$ \&

NO $0.66 / \mathrm{SO} 20.83 / \mathrm{H} 20354.23 / \mathrm{HG} 0.15$

SUBSTREAM CISOLID TEMP $=68$ PRES=1 <ATM>

MASS-FLOW C-S $4.77 /$ S-S $0.62 /$ SIO2-S $476.77 /$ \&

AL203-S $476.77 /$ AS-S $0.06 / \mathrm{BA}-\mathrm{S} 0.04 / \mathrm{CD}-\mathrm{S} 2.63$ \&

/ CR-S $4.95 /$ PB-S $0.17 /$ SE-S $0.03 /$ AG-S 0.03

STREAM FUELMIX1

SUBSTREAM MIXED TEMP=68 PRES=1 <ATM> MASS-FLOW=1

MASS-FRAC 020.222 / N2 $0.732 / \mathrm{CH} 40.046$

STREAM FUELMIX2

SUBSTREAM MIXED TEMP=68 PRES=1 <ATM> MASS-FLOW=9285.13

MASS-FRAC $020.222 / \mathrm{N} 20.732 / \mathrm{CH} 40.046$

STREAM FUELMIX3

SUBSTREAM MIXED TEMP=68 PRES=1 〈ATM> MASS-FLOW=994.065

MASS-FLOW $020.222 / \mathrm{N} 20.732 / \mathrm{CH} 40.046$

STREAM NAOH

SUBSTREAM MIXED TEMP $=68$ PRES $=1$ <ATM>

SUBSTREAM CISOIID TEMP=68 PRES=1 <ATM> MASS-FLOW=64.046

MASS-FLOW NAOH-S 1

STREAM QNCH2O

SUBSTREAM MIXED TEMP=68 PRES=1 <ATM> MASS-FLOW=10407.226

MASS-FRAC H2O 1

STREAM SCRUBH2O

SUBSTREAM MIXED TEMP=68 PRES=1 <ATM> MASS-FLOW=1

MASS-ERAC H2O 1

STREAM SOII

SUBSTREAM MIXED TEMP=68 PRES=1 <ATM> MASS-ELOW=5.37917

MASS-FLOW H2O 0.1

SUBSTREAM CISOIID TEMP=68 PRES $=1$ <ATM> MASS-FLOW=48.4125

MASS-FLOW SIO2-S $0.569 /$ AL2O3-S $0.102 /$ CACO3-S $0.128 / \&$ MGO-S $0.023 / \mathrm{NA2O} 0.013 / \mathrm{K} 20-\mathrm{S} 0.024 / \mathrm{FE} 304-\mathrm{S} 0.041$

BLOCK BAGHOUSE SEP

FRAC STREAM=HGPLUS SUBSTREAM=MIXED COMPS=H2 O2 N2 CL2 \& F2 NO SO2 H2O CO2 CO HCL CH4 C-S S-S S NAOH-S \& NACL-S NAF-S AS CD HG SE FE SIO2 AL2O3 FE-S SIO2-S \& AL2O3-S AS-S BA-S CD-S CR-S PB-S SE-S AG-S CACO3-S \& MGO-S NA2O-S K2O-S FE304 FE304-S CAO-S FRACS $=0$ O 0 \&

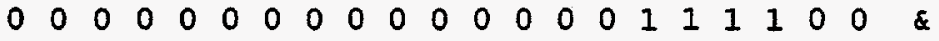
$\begin{array}{lllllllllllllllllll}0 & 0 & 0 & 0 & 1 & 0 & 1 & 0 & 0 & 1 & 0 & 0 & 0 & 0 & 0 & 0 & 0 & 0\end{array}$

FRAC STREAM=HGPLUS SUBSTREAM=CISOLID COMPS=C-S S-S NAOH-S \& NACL-S NAF-S FE-S SIO2-S AL2O3-S AS-S BA-S CD-S CR-S \& PB-S SE-S AG-S CACO3-S MGO-S NA2O-S K2O-S FE3O4-S \& CAO-S FRACS $=0 \begin{array}{lllllllllllllllllllllllllll}0 & 0 & 0 & 0 & 0 & 0 & 0 & 1 & 0 & 1 & 0 & 0 & 1 & 0 & 0 & 0 & 0 & 0 & 0 & 0\end{array}$

FRAC STREAM=FLYASH SUBSTREAM=MIXED COMPS=H2 O2 N2 CL2 \& F2 NO SO2 H2O CO2 CO HCL CH4 C-S S-S S NAOH-S \& NACL-S NAF-S AS CD HG SE FE SIO2 AL2O3 FE-S SIO2-S \& AL2O3-S AS-S BA-S CD-S CR-S PB-S SE-S AG-S CACO3-S \& MGO-S NA2O-S K2O-S FE304 FE304-S CAO-S FRACS $=0$ O 0 \& \& 
$\begin{array}{llllllllllllllllllllll}0 & 0 & 0 & 0 & 0 & 0 & 0 & 0 & 0 & 1 & 1 & 1 & 1 & 1 & 1 & 0 & 0 & 0 & 0 & 1 & 1 & \&\end{array}$

$\begin{array}{llllllllllllllllll}1 & 1 & 1 & 1 & 0 & 1 & 0 & 1 & 1 & 0 & 1 & 1 & 1 & 1 & 1 & 1 & 1 & 1\end{array}$

FRAC STREAM=FLYASH SUBSTREAM=CISOIID COMPS=C-S S-S NAOH-S \& NACL-S NAF-S FE-S SIO2-S AL203-S AS-S BA-S CD-S CR-S \& PB-S SE-S AG-S CACO3-S MGO-S NA2O-S K2O-S FE3O4-S \&

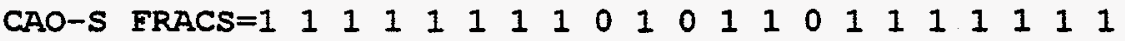
ELASH-SPECS FLYASH TEMP=68 PRES=1 <ATM>

\section{BLOCK KIINSEP SEP}

FRAC STREAM=ASH SUBSTREAM=MIXED COMPS=H2 O2 N2 CL2 F2 \& NO SO2 H2O CO2 CO HCL CH4 C-s s-s S NAOH-S NACL-S \& NAF-S AS CD HG SE FE SIO2 AL2O3 FE-S SIO2-S AL2O3-S \& AS-S BA-S CD-S CR-S PB-S SE-S AG-S CACO3-S MGO-S \&

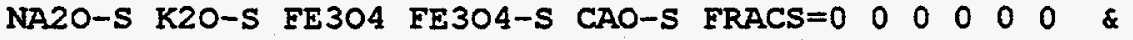
$\begin{array}{lllllllllllllllllll}0 & 0 & 0 & 0 & 0 & 0 & .8 & .8 & 0 & .8 & .8 & .8 & 0 & 0 & 0 & 0 & .8 & .8\end{array}$ \& $\begin{array}{llllllllllllllllll}.8 & .8 & .8 & .8 & .8 & .8 & .8 & .8 & .8 & .8 & .8 & .8 & .8 & .8 & .8 & .8 & .8 & .8\end{array}$

FRAC STREAM=ASH SUBSTREAM=CISOLID COMPS=C-S S-S NAOH-S \& NACL-S NAF-S FE-S SIO2-S AL203-S AS-S BA-S CD-S CR-S \& PB-S SE-S AG-S CACO3-S MGO-S NA2O-S K2O-S FE304-S \&

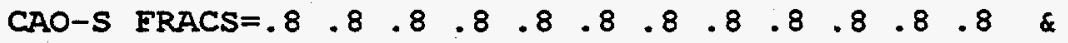
$\begin{array}{lllllllll}.8 & .8 & .8 & .8 & .8 & .8 & .8 & .8 & .8\end{array}$

FLASH-SPECS ASH TEMP $=68$ PRES $=1$ 〈ATM>

BLOCK SEPARATR SEP

FRAC STREAM=SOLIDS SUBSTREAM=MIXED COMPS=H 2 O2 N2 CL2 \& F2 NO SO2 H2O CO2 CO HCL CH4 C-S S-S S NAOH-S \& NACL-S NAF-S AS CD HG SE FE SIO2 AL2O3 FE-S SIO2-S \& AI203-S AS-S BA-S CD-S CR-S PB-S SE-S AG-S CACO3-S \& MGO-S NA2O-S K2O-S FE304 FE304-S CAO-S FRACS $=.8$. 8 \& $\begin{array}{lllllllllllllllllll}.8 & .8 & .8 & .8 & .8 & .2 & .8 & .8 & .8 & .8 & .8 & 1 & 1 & 1 & 1 & 1 & 0 & \&\end{array}$ $\begin{array}{llllllllllllllllllllllll}0 & 0 & 0 & 1 & 1 & 1 & 1 & 1 & 1 & 1 & 1 & 1 & 1 & 1 & 1 & 1 & 1 & 1 & 1 & 1 & 1 & 1 & 1\end{array}$

FRAC STREAM=SOLIDS SUBSTREAM=CISOLID COMPS=C-S S-S NAOH-S \& NACL-S NAF-S FE-S SIO2-S AL2O3-S AS-S BA-S CD-S CR-S \& PB-S SE-S AG-S CACO3-S MGO-S NA2O-S K2O-S FE304-S \&

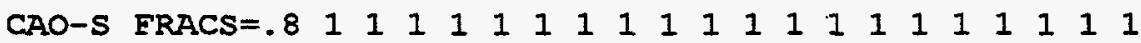

BLOCK L-V-SEP FLASH2

PARAM TEMP=120 PRES=1 <ATM>

BLOCK I-V-SEP2 EIASH2

PARAM TEMP $=3000$ PRES $=1$ <ATM>

BLOCK CONDENSR RGIBBS

PARAM TEMP $=80$ PRES $=1$ <ATM> NPHASE $=2$

PROD H2/ O2/ N2/ CL2/ $\mathrm{F} 2 / \mathrm{NO} / \mathrm{SO} 2 / \mathrm{H} 2 \mathrm{O} /$ \& $\mathrm{C}-\mathrm{s}$ SS / S-S SS/ $/ \mathrm{AS} / \mathrm{CD} / \mathrm{HG} / \mathrm{SE} / \&$ FE-S SS / SIO2-S SS / AI203-S SS / As-S SS / \& $\mathrm{BA}-\mathrm{S} \mathrm{SS} / \mathrm{CD}-\mathrm{S} \mathrm{SS} / \mathrm{CR}-\mathrm{S} \mathrm{SS} / \mathrm{PB}-\mathrm{S} \mathrm{SS} / \mathrm{SE}-\mathrm{S} \mathrm{SS} / \mathrm{AG}-\mathrm{S} \mathrm{SS}$ PROD-FRAC H2 $1 / \mathrm{O} 21 / \mathrm{H} 2 \mathrm{O} 1$

\section{BLOCK DESORBER RGIBBS}

PARAM TEMP $=600$ PRES $=1$ <ATM> NPHASE $=2$

PROD $\mathrm{H} 2 / \mathrm{O} 2 / \mathrm{N} 2 / \mathrm{CL} 2 / \mathrm{F} 2 / \mathrm{NO} / \mathrm{SO} 2 / \mathrm{H} 2 \mathrm{O} /$ \& $\mathrm{C}-\mathrm{s} \mathrm{s} / \mathrm{s}-\mathrm{s} \mathrm{s} / \mathrm{s} / \mathrm{As} / \mathrm{CD} / \mathrm{HG} / \mathrm{sE} / \&$ EE-S ss / SIO2-S SS / AL203-S SS / As-S SS / \& $\mathrm{BA}-\mathrm{S}$ SS / CD-S SS / CR-S SS / PB-S SS / SE-S SS / AG-S SS PROD-FRAC H2 $1 / 021 / \mathrm{H} 2 \mathrm{O} 1$

BLOCK HEATER RGIBBS

PARAM TEMP=1400 PRES=1 <ATM> NPHASE=2

BIOCK KILN RGIBBS 
PARAM TEMP $=1600$ PRES $=1$ <ATM> NRHASE $=2$ MAXIT $=500$

PROD H2 / O2 / N2 / CL2 / F2 / NO / SO2 / H2O / \& $\mathrm{CO} 2 / \mathrm{CO} / \mathrm{HCL} / \mathrm{AS} / \mathrm{CD} / \mathrm{HG} / \mathrm{SE} / \mathrm{FE}-\mathrm{S}$ SS \& / SIO2-S SS / AL2O3-S SS / AS-S SS / BA-S SS / \& CD-S SS / CR-S SS / PB-S SS / SE-S SS / AG-S SS \& / MGO-S SS / NA2O-S SS / K20-S SS / FE3O4-S SS / CAO-S SS

PROD-FRAC FE-S 1 / FE3O4-S 1

BLOCK-OPTION RESTART=NO

BIOCK MELTER RGIBBS

PARAM TEMP $=3000$ PRES $=1$ <ATM> NPHASE $=2$

PROD H2 / C-S SS / FE / SIO2 / AL2O3 / FE-S SS / \& SIO2-S SS / AL2O3-S SS / BA-S SS / CD / CD-S SS

\section{BLOCK Q1 RGIBBS}

PARAM TEMP $=68$ PRES $=1$ <ATM> NPHASE=2 TAPP=1532 MAXIT $=500$

PROD $\mathrm{H} 2 / \mathrm{O} 2 / \mathrm{N} 2 / \mathrm{CL} 2 / \mathrm{F} 2 / \mathrm{NO} / \mathrm{SO} 2 / \mathrm{H} 2 \mathrm{O} / \&$ $\mathrm{CO} 2 / \mathrm{CO} / \mathrm{HCL} / \mathrm{AS} / \mathrm{CD} / \mathrm{HG} / \mathrm{SE} / \mathrm{EE}-\mathrm{S} S \mathrm{SS} \&$ / SIO2-S SS / AL2O3-S SS / AS-S SS / BA-S SS / \& CD-S SS / CR-S SS / PB-S SS / SE-S SS / AG-S SS \& / MGO-S SS / NA2O-S SS / K2O-S SS / FE304-S SS / CAO-S SS PROD-FRAC FE-S $1 /$ FE304-S 1 BLOCK-OPTION RESTART $=$ NO

BLOCK Q2 RGIBBS

PARAM TEMP=68 PRES=1 <ATM> NPHASE=2 CHEMEQ=YES TAPP=2132 \& MAXIT $=60$

PROD 02 / N2 / CL2 / $\mathrm{F} 2 / \mathrm{NO} / \mathrm{SO} 2 / \mathrm{H} 2 \mathrm{O} / \mathrm{CO} 2 / \&$ $\mathrm{CO} / \mathrm{HCL} / \mathrm{CH} 4 / \mathrm{S} / \mathrm{AS} / \mathrm{CD} / \mathrm{HG} / \mathrm{SE} / \&$ FE-S SS / SIO2-S SS / AL203-s SS / BA-S SS / \& CR-S SS / PB-S SS / AG-S SS / MGO-S SS / NA2O-S \& SS / K2O-S SS / FE3O4-S SS / CAO-S SS

PROD-FRAC EE-S 1 / FE304-S 1

BLOCK-OPTION RESTART $=$ NO

BLOCK QUENCH RGIBBS

PARAM TEMP $=350$ PRES $=1$ <ATM > NPHASE $=2$

PROD H2 / O2 / N2 / $\mathrm{F} 2 / \mathrm{NO} / \mathrm{SO} 2 / \mathrm{H} 2 \mathrm{O} / \mathrm{CO} 2 / \&$ $\mathrm{CO} / \mathrm{HCL} / \mathrm{AS} / \mathrm{CD} / \mathrm{HG} / \mathrm{SE} / \mathrm{FE}-\mathrm{S} \mathrm{SS} / \&$ SIO2-S SS / AL203-S SS / AS-S SS / BA-S SS / \& CD-S SS / CR-S SS / PB-S SS / SE-S SS / AG-S SS \& / MGO-S SS / NA2O-S SS / K2O-S SS / FE3O4-S SS / CAO-S SS PROD-FRAC FE-S $1 /$ FE3O4-S 1 BLOCK-OPTION RESTART $=$ NO

BLOCK SCC RGIBBS

PARAM TEMP $=2200$ PRES $=1$ <ATM> NPHASE $=2$

PROD H2 / O2 / N2 / CL2 / 2 2 / NO / SO2 / H2O / \& $\mathrm{CO} 2$ / $\mathrm{CO} / \mathrm{HCL} / \mathrm{CH} 4 / \mathrm{S} / \mathrm{AS} / \mathrm{CD} / \mathrm{HG} / \mathrm{SE} \&$ $/$ FE-s SS / SIO2-S SS / AL2O3-S SS / BA-S SS / \& CR-S SS / PB-S SS / AG-S SS / MGO-S SS / NA2O-S \& SS / K2O-S SS / FE3O4-S SS / CAO-S SS

PROD-FRAC FE-S 1 / FE3O4-S 1

BIOCK-OPTION RESTART $=$ NO

BLOCK SCRUB RGIBBS

PARAM TEMP $=120$ PRES $=1$ <ATM> NPHASE $=2$

PROD H2 / O2 / N2 / CL2 / E2 / NO / SO2 / H2O / \& $\mathrm{CO} 2 / \mathrm{CO} / \mathrm{HCL} / \mathrm{NAOH}-\mathrm{S}$ SS / NACL-S SS / NAF-S SS

BLOCK VITRIFY RGIBBS

PARAM TEMP=3000 PRES=1 <ATM> NPHASE $=2$ 


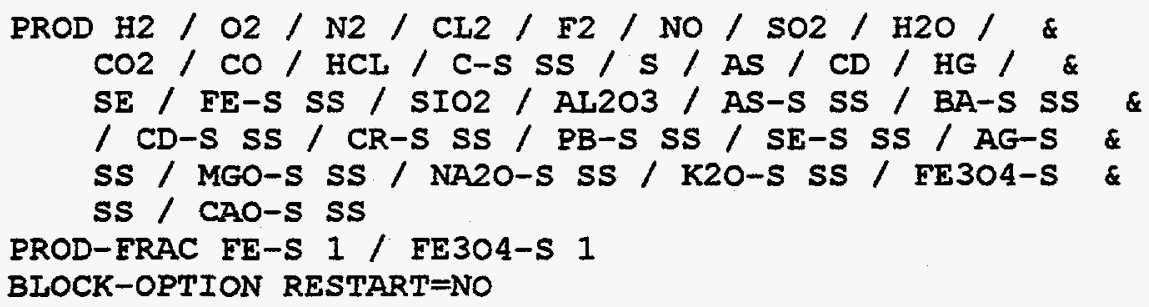

BLOCK DUPL1 DUPL

BLOCK DUPL2 DUPI

DESIGN-SPEC NAOH

DEFINE CAUIN MASS-FLOW STREAM=NAOH SUBSTREAM=CISOLID \& COMPONENT $=$ NAOH $-S$

DEFINE CAUOUT MASS-FLOW' STREAM=SCRUBOUT SUBSTREAM=CISOLID\& COMPONENT $=\mathrm{NAOH}-\mathrm{S}$

SPEC "CAUIN-100.*CAUOUT" TO "O"

TOL-SPEC "0.01"

VARY STREAM-VAR STREAM=NAOH SUBSTREAM=CISOIID \& VARIABLE=MASS - FLOW

LIMITS " 1 " "5000"

DESIGN-SPEC OXI

DEFINE AIR MASS-FLOW STREAM=AIR SUBSTREAM=MIXED \& COMPONENT $=02$

DEFINE WC MASS-FLOW STREAM=FDWASTEC SUBSTREAM=MIXED \& COMPONENT $=02$

DEFINE F1 MASS-FLOW STREAM=FUELMIX1 SUBSTREAM=MIXED \& COMPONENT $=02$

DEFINE OXOUT MASS-FLOW STREAM=KILNOUT SUBSTREAM=MIXED \& COMPONENT $=02$

DEFINE AI MASS-FLOW STREAM=AIRLEAKS SUBSTREAM=MIXED \& COMPONENT $=02$

SPEC "AL+AIR+WC+(F1/6.0)" TO "2.0*OXOUT"

TOL-SPEC "0.1"

VARY STREAM-VAR STREAM=AIR SUBSTREAM=MIXED \& VARIABLE=MASS-FLOW

LIMITS "1" "10000"

DESIGN-SPEC QHEATER

DEFINE QIN BLOCK-VAR BLOCK=DESORBER VARIABLE=QCALC \& SENTENCE= PARAM

DEFINE QOUT BLOCK-VAR BLOCK=HEATER VARIABLE=QCALC \& SENTENCE= PARAM

SPEC "1.05*QIN+QOUT" TO "O"

TOI-SPEC "1."

VARY STREAM-VAR STREAM=FUELMIX3 SUBSTREAM=MIXED \& VARIABLE=MASS - FLOW

LIMITS "1" "10000"

DESIGN-SPEC QKILN

DEFINE QKILN BLOCK-VAR BLOCK=KILN VARIABLE=QCALC\& SENTENCE $=$ PARAM

DEFINE $Q 1$ BLOCK-VAR BLOCK=Q1 VARIABLE=QCALC SENTENCE=PARAM SPEC "QKIIN" TO "0.05*Q1"

TOL-SPEC "1."

VARY STREAM-VAR STREAM=COOLH2O SUBSTREAM=MIXED \& VARIABLE=MASS-FLOW

LIMITS "1" "50000" 
DESIGN-SPEC QQUENCH

DEFINE QQNCH BLOCK-VAR BLOCK=QUENCH VARIABLE=QCALC\& SENTENCE = PARAM

SPEC "QQNCH" TO "O"

TOL-SPEC "1."

VARY STREAM-VAR STREAM=QNCH2O SUBSTREAM=MIXED \& VARIABLE=MASS-FLOW

LIMITS " 1 " "1000000"

DESIGN-SPEC QSCC

DEEINE QSCC BLOCK-VAR BLOCK=SCC VARIABLE=QCALC \& SENTENCE $=$ PARAM

DEFINE $Q 2$ BLOCK-VAR BLOCK=Q2 VARIABLE=QCALC SENTENCE=PARAM

SPEC "QSCC" TO "0.05*Q2"

TOL-SPEC " 1 ."

VARY STREAM-VAR STREAM=FUELMIX2 SUBSTREAM=MIXED \& VARIABLE=MASS-FLOW

LIMITS "1" "100000"

DESIGN-SPEC SCRUBH2O

DEFINE MASSAL STREAM-VAR STREAM=SCRUBBOT SUBSTREAM=CISOLID \& VARIABIE =MASS-FLOW

DEFINE SCRUB STREAM-VAR STREAM=SCRUBH2O SUBSTREAM=MIXED \& VARIABLE=MASS-FIOW

DEFINE NAOH STREAM-VAR STREAM=NAOH SUBSTREAM=CISOLID $\propto$ VARIABLE $=$ MASS - FLOW

SPEC "SCRUB" TO "20*MASSAL"

TOL-SPEC "0.01"

VARY STREAM-VAR STREAM=SCRUBH2O SUBSTREAM=MIXED \& VARIABLE=MASS-FLOW

IIMITS "NAOH" "5000"

DESIGN-SPEC SOILH2O

DEFINE SLAGI STREAM-VAR STREAM=SLAG SUBSTREAM=MIXED \& VARIABLE =MASS $-F I O W$

DEFINE SLAG2 STREAM-VAR STREAM=SLAG SUBSTREAM=CISOLID \& VARIABLE=MASS-FLOW

DEFINE SOIL STREAM-VAR STREAM=SOIL SUBSTREAM=MIXED\& VARIABLE = MASS - FIOW

SPEC "30.* SOIL" TO "SLAG1+SLAG2"

TOL-SPEC "0.01"

VARY STREAM-VAR STREAM=SOIL SUBSTREAM=MIXED \& VARIABLE=MASS-ELOW

LIMITS " 1 " "1000"

DESIGN-SPEC SOILSOL

DEFINE SIAGI STREAM-VAR STREAM=SLAG SUBSTREAM=MIXED \& VARIABLE =MASS-FLOW

DEFINE SIAG2 STREAM-VAR STREAM=SLAG SUBSTREAM=CISOLID \& VARIABLE =MASS-FLOW

DEFINE SOIL STREAM-VAR STREAM=SOIL SUBSTREAM=CISOIID \& VARIABLE=MASS-FLOW

SPEC "3.33333*SOIL" TO "SIAG1+SLAG2"

TOL-SPEC "0.01"

VARY STREAM-VAR STREAM=SOIL SUBSTREAM=CISOLID \& VARIABLE =MASS-FLOW

LIMITS "1" "1000"

CONV-OPTIONS

SECANT MAX-STEP-SIZ $=.05$ BRACKET=YES

STREAM-REPOR NOSORT MOLEFLOW MASSFLOW PROPERTIES=ALL-SUBS 


\section{System F-1}

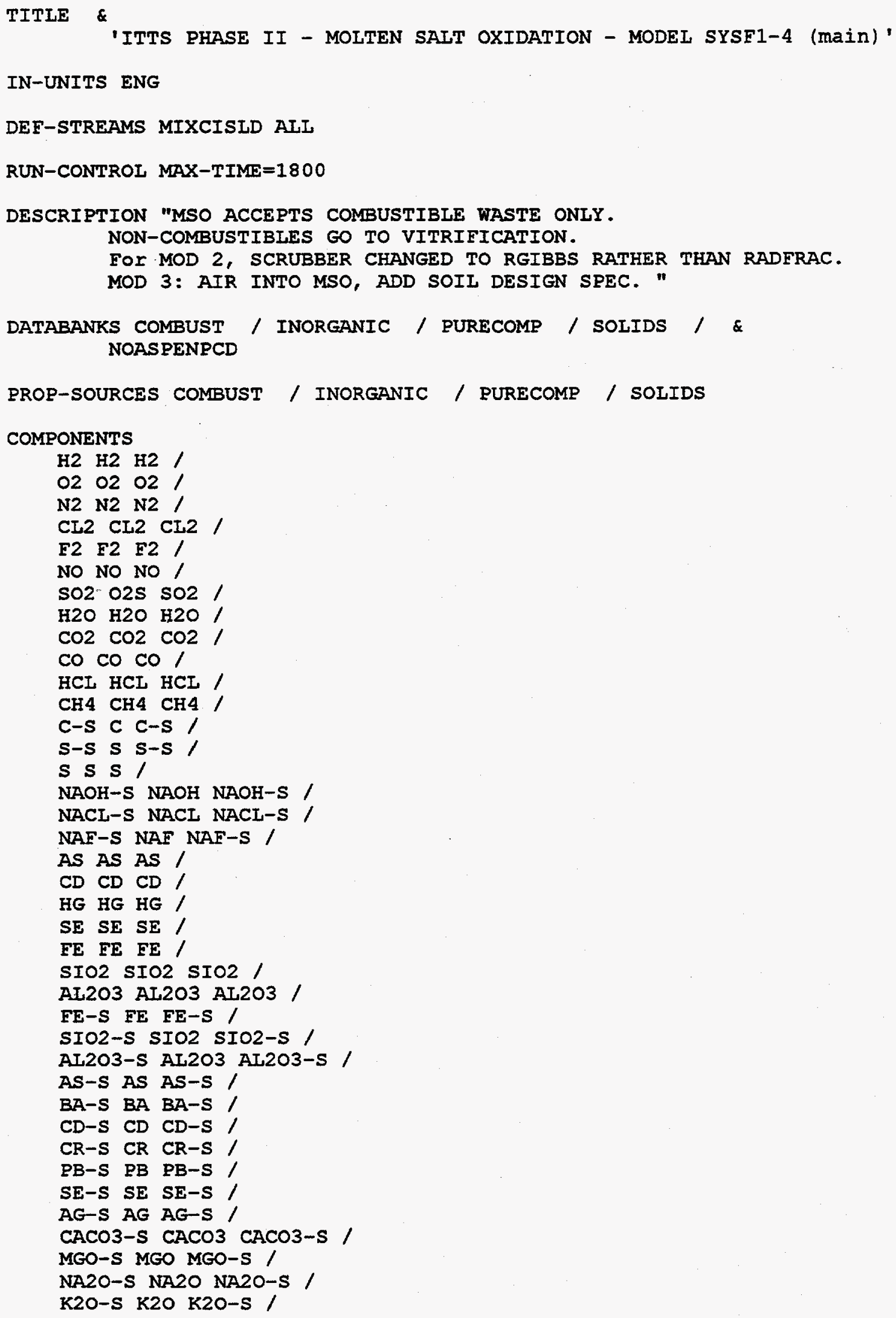


FE304 FE304 FE304 /

FE304-S FE304 FE304-S /

CAO-S CAO CAO-S /

$\mathrm{NA} 2 \mathrm{CO} 3 \mathrm{NA} 2 \mathrm{CO} 3 \mathrm{NA} 2 \mathrm{CO} 3 /$

$\mathrm{NA2SO} 4 \mathrm{NA} 2 \mathrm{SO} 4 \mathrm{NA2SO} 4 /$

$\mathrm{H} 2 \mathrm{SO} 4 \mathrm{H} 2 \mathrm{SO} 4 \mathrm{H} 2 \mathrm{SO} 4$ /

$\mathrm{NA} 2 \mathrm{CO} 3-\mathrm{S}$ NA2 $\mathrm{CO} 3$ NA2 $\mathrm{CO} 3-\mathrm{S} /$

NA2SO4-S NA2SO4 NA2SO4-S

FLOWSHEET

BLOCK VITRIFY IN=SOLIDS OUT=VITOUT

BLOCK VIT-SEP IN=VITOUT OUT=VITVAP SLAG

BLOCK SCC2 IN=FUELMIX2 EFFLUENT OUT=OUT2

BLOCK QUENCH IN=QNCH2O OFFGAS OUT=QUENCHED

BLOCK BAGHOUSE IN=QUENCHED OUT=FLYASH HGPLUS VAPOR

BLOCK $Q 2$ IN=DUM2IN OUT=DUM2OUT

BLOCK DUPL2 IN=OUT2 OUT=SCCOUT2 DUM2IN

BLOCK MELTER IN=FDMETMLT OUT=METAL

BLOCK MSO IN=AIR FDWASTEC SALT-IN SALT-90 OUT=MSO-OUT

BLOCK MSO-SEP IN=MSO-OUT OUT=MSO-BOT OFFGAS

BLOCK SALT-SEP IN=MSO-BOT OUT=SALTS ASH SALT-90

BLOCK SCRUB IN=NAOH SCRUBH2O VAPOR OUT=SCRUBOUT

BLOCK I-V-SEP IN=SCRUBOUT OUT=SCRUBVAP SCRUBBOT

BLOCK QUENCH2 IN=SCCOUT2 ONCH2O-2 OUT=QUENCHD2

BLOCK BAGHSE2 IN=QUENCHD2 OUT=FILTGAS2 HGPLUS2 FLYASH2

BLOCK FEED-SEP IN=ASH FLYASH SOIL FDWASTEN FLYASH2 OUT= \& SOLIDS ORGANICS

BLOCK PLENUM IN=ORGANICS VITVAP OXYGEN OUT=EFFLUENT

BLOCK I-V-SEP2 IN=SCRBOUT2 OUT=SCRBVAP2 SCRBBOT2

BLOCK SCRUB-2 IN=SCRBH2O2 NAOH-2 FILTGAS2 OUT=SCRBOUT2

PROPERTIES SOIIDS

PROPERTIES IDEAL / RKS-BM

PRO'P-SET AIL-SUBS TEMP PRES VERAC UNITS='ATM' SUBSTREAM=ALL

STREAM AIR

SUBSTREAM MIXED TEMP=68.0 PRES=1 <ATM> MASS-FLOW=6120

MASS-FRAC $020.233 / \mathrm{N} 20.767$

STREAM EDMETMLT

SUBSTREAM MIXED TEMP=68 PRES=1 <ATM> MASS-ELOW=1.20

MASS-FLOW H2 1.20

SUBSTREAM CISOLID TEMP=67 PRES=1 <ATM> MASS-FLOW=147.8

MASS-FLOW C-S $14.44 /$ EE-S $282.02 /$ SIO2-S $0.035 /$ \&

AL203-S $0.035 / \mathrm{BA}-\mathrm{S} 0.87 / \mathrm{CD}-\mathrm{S} 0.18$

STREAM EDWASTEC

SUBSTREAM MIXED TEMP=68 PRES=1 <ATM>

MASS-FIOW H2 $50.95 / 0292.76 / \mathrm{N} 20.23 / \mathrm{CL} 253.02 /$ \&

F2 $0.11 / \mathrm{H} 2019.83$

SUBSTREAM CISOIID TEMP=68 PRES=1 <ATM>

MASS-FLOW C-S $326.28 /$ S-S $0.49 /$ FE-S $7.67 /$ SIO2-S \&

$53.01 / \mathrm{AL203-S} 53.01 / \mathrm{BA}-\mathrm{S} 0.77 / \mathrm{CD}-\mathrm{S} 0.77 / \mathrm{CR}-\mathrm{S} 1.53$

STREAM FDWASTEN

SUBSTREAM MIXED TEMP $=68$ PRES $=1$ <ATM>

MASS-FLOW H2 $0.54 / 021.44 /$ N2 $0.01 /$ CL2 $14.88 / \&$

NO $0.66 / \mathrm{SO} 0.83 / \mathrm{H} 20354.23 /$ HG 0.15

SUBSTREAM CISOIID TEMP $=68$ PRES $=1$ <ATM>

MASS-EIOW C-S $4.77 /$ S-S $0.62 /$ SIO2-S $476.77 / \&$

AI203-S 476.77/ AS-S $0.06 / \mathrm{BA}-\mathrm{S} 0.04 / \mathrm{CD}-\mathrm{S} 2.63$ \& 


$$
\text { / CR-S } 4.95 / \mathrm{PB}-\mathrm{S} 0.17 / \mathrm{SE}-\mathrm{S} 0.03 / \mathrm{AG}-\mathrm{S} 0.03
$$

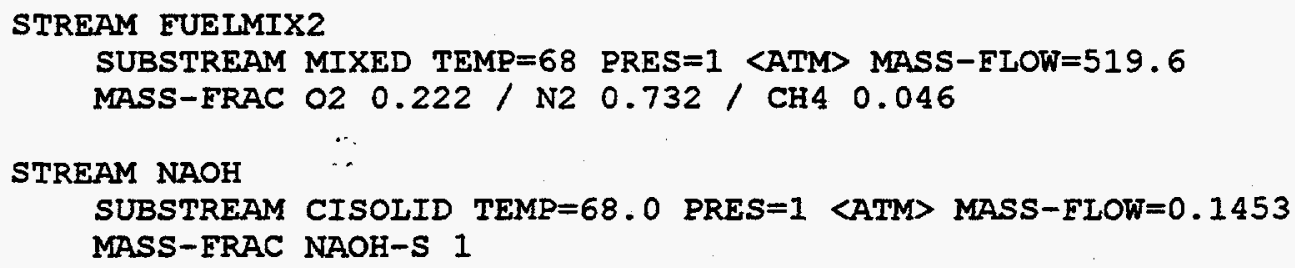

BLOCK BAGHOUSE SEP

FRAC STREAM=FIYASH SUBSTREAM=MIXED COMPS=H2 O2 N2 CL2 \& F2 NO SO2 H2O CO2 CO HCL CH4 C-S s-s S NAOH-S \& NACL-S NAF-S AS CD HG SE FE SIO2 AL2O3 FE-S SIO2-S \& AI203-S AS-S BA-S CD-S CR-S PB-S SE-S AG-S CACO3-S \& MGO-S NA2O-S K2O-S FE3O4 FE3O4-S CAO-S NA2CO3 NA2SO4 \&

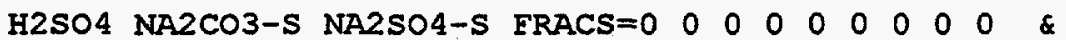
$\begin{array}{llllllllllllllllllllll}0 & 0 & 0 & 1 & 1 & 1 & 1 & 1 & 1 & 0 & 0 & 0 & 0 & 1 & 1 & 1 & 1 & 1 & 1 & 0 & 1 & \&\end{array}$ $\begin{array}{lllllllllllllllll}0 & 1 & 1 & 0 & 1 & 1 & 1 & 1 & 1 & 1 & 1 & 1 & 1 & 1 & 0 & 1 & 1\end{array}$

FRAC STREAM=FLYASH SUBSTREAM=CISOLID COMPS=C-S $S-S$ NAOH-S \& NACL-S NAF-S FE-S SIO2-S AI2O3-S AS-S BA-S CD-S CR-S \& PB-S SE-S AG-S CACO3-S MGO-S NA2O-S K2O-S FE3O4-S \&

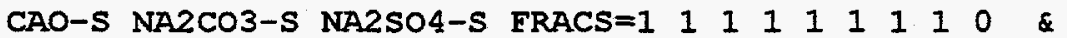
$\begin{array}{llllllllllllllllll}1 & 0 & 1 & 1 & 0 & 1 & 1 & 1 & 1 & 1 & 1 & 1 & 1 & 1\end{array}$

FRAC STREAM=HGPLUS SUBSTREAM=MIXED COMPS=H2 O2 N2 CL2 \& F2 NO SO2 H2O CO2 CO HCL CH4 C-S S-S S NAOH-S \& NACL-S NAF-S AS CD HG SE FE SIO2 AI2O3 FE-S SIO2-S \& 
AL2O3-S AS-S BA-S CD-S CR-S PB-S SE-S AG-S CACO3-S \& MGO-S NA2O-S K2O-S FE3O4 FE3O4-S CAO-S NA2CO3 NA2SO4 \& H2SO4 NA2CO3-S NA2SO4-S FRACS $=0 \begin{array}{lllllllll}0 & 0 & 0 & 0 & 0 & 0 & 0 & 0 & \&\end{array}$ $0 \begin{array}{llllllllllllllllllllll}0 & 0 & 0 & 0 & 0 & 0 & 0 & 0 & 0 & 1 & 1 & 1 & 1 & 0 & 0 & 0 & 0 & 0 & 0 & 1 & 0 & \&\end{array}$

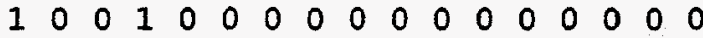

FRAC STREAM=HGPLUS SUBSTREAM=CISOLID COMPS=C-S S-S NAOH-S \& NACL-S NAF-S FE-S SIO2-S AL2O3-S AS-S BA-S CD-S CR-S \& PB-S SE-S AG-S CACO3-S MGO-S NA2O-S K2O-S FE3O4-S \& CAO-S NA2CO3-S NA2SO4-S FRACS $=0 \begin{array}{llllllllll}0 & 0 & 0 & 0 & 0 & 0 & 0 & 1 & \&\end{array}$ 01000100000000000

BLOCK BAGHSE2 SEP

FRAC STREAM=FILTGAS2 SUBSTREAM=MIXED COMPS=H2 O2 N2 CL2 \& F2 NO SO2 H2O CO2 CO HCI CH4 C-s S-S S NAOH-S \& NACL-S NAF-S AS CD HG SE FE SIO2 AL2O3 FE-S SIO2-S \& AL2O3-S AS-S BA-S CD-S CR-S PB-S SE-S AG-S CACO3-S \& MGO-S NA2O-S K2O-S FE3O4 FE304-S CAO-S NA2CO3 NA2SO4\& H2SO4 NA2CO3-S NA2SO4-S FRACS=1 11 lllllllllllll $\begin{array}{llllllllllllllllllllll}1 & 1 & 1 & 0 & 0 & 0 & 0 & 0 & 0 & 0 & 0 & 0 & 0 & 0 & 0 & 0 & 0 & 0 & 0 & 0 & 0 & \&\end{array}$ $0 \begin{array}{lllllllllllllllll}0 & 0 & 0 & 0 & 0 & 0 & 0 & 0 & 0 & 0 & 0 & 0 & 0 & 1 & 0 & 0\end{array}$

FRAC STREAM=FILTGAS2 SUBSTREAM=CISOIID COMPS=C-S $S-S \&$ NAOH-S NACI-S NAF-S FE-S SIO2-S AL2O3-S AS-S BA-S \& CD-S CR-S PB-S SE-S AG-S CACO3-S MGO-S NA2O-S K2O-S \& FE 304-S CAO-S NA2CO3-S NA2SO4-S FRACS $=0 \begin{array}{lllllll}0 & 0 & 0 & 0 & 0\end{array}$

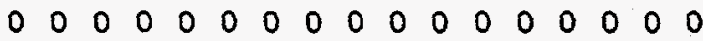

FRAC STREAM=HGPLUS2 SUBSTREAM=MIXED COMPS=H2 O2 N2 CL2 \& F2 NO SO2 H2O CO2 CO HCL CH4 C-S S-S S NAOH-S \& NACL-S NAF-S AS CD HG SE FE SIO2 AL2O3 FE-S SIO2-S \& AL203-S AS-S BA-S CD-S CR-S PB-S SE-S AG-S CACO3-S \& MGO-S NA2O-S K2O-S FE3O4 FE304-S CAO-S NA2CO3 NA2SO4\&

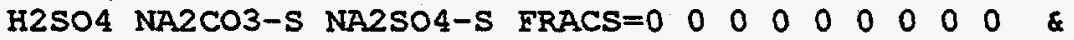
$\begin{array}{lllllllllllllllllllllll}0 & 0 & 0 & 0 & 0 & 0 & 0 & 0 & 0 & 1 & 1 & 1 & 1 & 0 & 0 & 0 & 0 & 0 & 0 & 1 & 0 & \&\end{array}$

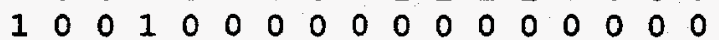

FRAC STREAM=HGPLUS2 SUBSTREAM=CISOLID COMPS=C-S $S-S \&$ NAOH-S NACL-S NAF-S FE-S SIO2-S AL2O3-S AS-S BA-S \& CD-S CR-S PB-S SE-S AG-S CACO3-S MGO-S NA2O-S K2O-S \& FE3O4-S CAO-S NA2CO3-S NA2SO4-S FRACS $=0 \begin{array}{lllllll}0 & 0 & 0 & 0 & 0\end{array}$ $0 \begin{array}{lllllllllllllllll}0 & 1 & 0 & 1 & 0 & 0 & 1 & 0 & 0 & 0 & 0 & 0 & 0 & 0 & 0 & 0\end{array}$

BLOCK FEED-SEP SEP

FRAC STREAM=SOLIDS SUBSTREAM=MIXED COMPS=H2 O2 N2 CL2 \& F2 NO SO2 H2O CO2 CO HCL CH4 C-S S-S S NAOH-S \& NACL-S NAF-S AS CD HG SE FE SIO2 AL2O3 FE-S SIO2-S \& AL203-S AS-S BA-S CD-S CR-S PB-S SE-S AG-S CACO3-S \& MGO-S NA2O-S K2O-S FE3O4 FE3O4-S CAO-S NA2CO3 NA2SO4 \&

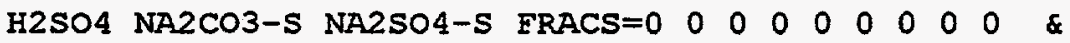
$\begin{array}{llllllllllllll}0 & 0 & 0 & .05 & .05 & .05 & .95 & .95 & .95 & .95 & .95 & .95 & .95 & 8\end{array}$ $\begin{array}{llllllllllllll}.95 & .95 & .95 & .95 & .95 & .95 & .95 & .95 & .95 & .95 & .95 & .95 & \&\end{array}$

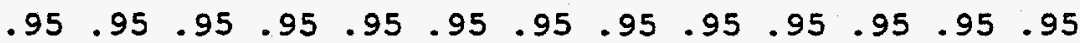

FRAC STREAM=SOLIDS SUBSTREAM =CISOLID COMPS=C-S $S-S$ NAOH-S \& NACL-S NAF-S FE-S SIO2-S AL2O3-S AS-S BA-S CD-S CR-S \& PB-S SE-S AG-S CACO3-S MGO-S NA2O-S K2O-S FE3O4-S \& CAO-S NA2CO3-S NA2SO4-S FRACS $=.05 \quad .05 \quad .95 \quad .95 \quad .95 \quad \&$ $\begin{array}{lllllllllllll}.95 & .95 & .95 & .95 & .95 & .95 & .95 & .95 & .95 & .95 & .95 & .95 & 8\end{array}$ $\begin{array}{llllll}.95 & .95 & .95 & .95 & .95 & .95\end{array}$

BLOCK MSO-SEP SEP

FRAC STREAM=OFFGAS SUBSTREAM=MIXED COMPS=H2 O2 N2 CL2 * F2 NO SO2 H2O CO2 CO HCL CH4 C-S S-S S NAOH-S \& NACL-S NAF-S AS CD HG SE FE SIO2 AL2O3 FE-S SIO2-S \& AL2O3-S AS-S BA-S CD-S CR-S PB-S SE-S AG-S CACO3-S \& MGO-S NA2O-S K2O-S FE3O4 FE3O4-S CAO-S NA2CO3 NA2SO4\& 


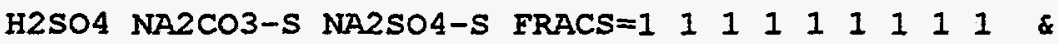
$\begin{array}{llllllllllllllllllllll}1 & 1 & 1 & 0 & 0 & 1 & 0 & 0 & 0 & 1 & 1 & 1 & 1 & 0 & 0 & 0 & 0 & 0 & 0 & 1 & 0 & \&\end{array}$ $\begin{array}{lllllllllllllllll}1 & 0 & 0 & 1 & 0 & 0 & 0 & 0 & 0 & 0 & 0 & 0 & 0 & 0 & 1 & 0 & 0\end{array}$

FRAC STREAM=OFFGAS SUBSTREAM=CISOLID COMPS=C-S S-S NAOH-S \& NACL-S NAF-S EE-S SIO2-S AL203-S AS-S BA-S CD-S CR-S \& PB-S SE-S PF-S CACO3-S MGO-S NA2O-S K2O-S FE3O4-S \&

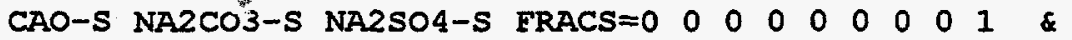
$\begin{array}{lllllllllllllll}0 & 1 & 0 & 0 & 1 & 0 & 0 & 0 & 0 & 0 & 0 & 0 & 0 & 0\end{array}$

FLASH-SPECS MSO-BOT TEMP=68 PRES=1 〈ATM>

\section{BLOCK SALT-SEP SEP}

FRAC STREAM=ASH SUBSTREAM=MIXED COMPS=H2 O2 N2 CL2 F2 \& NO SO2 H2O $\mathrm{CO} 2 \mathrm{CO} H C L$ CH4 C-S s-S S NAOH-S NACL-S \& NAF-S AS CD HG SE FE SIO2 AL2O3 FE-S SIO2-S AI2O3-S \& AS-S BA-S CD-S CR-S PB-S SE-S AG-S CACO3-S MGO-S \& NA2O-S K2O-S FE3O4 FE3O4-S CAO-S NA2CO3 NA2SO4 H2SO4 \&

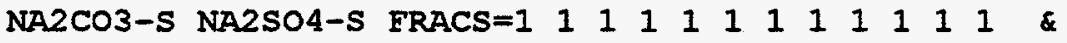
$\begin{array}{lllllllllllllllllllllll}1 & 1 & 1 & 1 & 0 & 0 & 1 & 1 & 1 & 1 & 1 & 1 & 1 & 1 & 1 & 1 & 1 & 1 & 1 & 1 & 1 & \&\end{array}$ $\begin{array}{lllllllllllllll}1 & 1 & 1 & 1 & 1 & 1 & 1 & 1 & 1 & 0 & 1 & 1 & 0 & 1\end{array}$

ERAC STREAM=ASH SUBSTREAM=CISOLID COMPS=C-S S-S NAOH-S \& NACL-S NAF-S FE-S SIO2-S AL203-S AS-S BA-S CD-S CR-S \& PB-S SE-S AG-S CACO3-S MGO-S NA2O-S K2O-S FE3O4-S \&

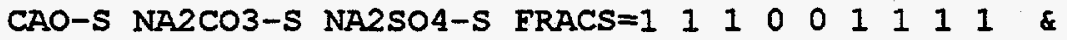
$\begin{array}{lllllllllllllll}1 & 1 & 1 & 1 & 1 & 1 & 1 & 1 & 1 & 1 & 1 & 1 & 0 & 1\end{array}$

FRAC STREAM=SAIT-90 SUBSTREAM=MIXED COMPS=H2 O2 N2 CL2 \& E2 NO SO2 H2O CO2 CO HCL CH4 C-S S-S S NAOH-S \& NACL-S NAF-S AS CD HG SE EE SIO2 AL2O3 FE-S SIO2-S \& AI203-S AS-S BA-S CD-S CR-S PB-S SE-S AG-S CACO3-S \& MGO-S NA2O-S K2O-S FE3O4 FE3O4-S CAO-S NA2CO3 NA2SO4 \&

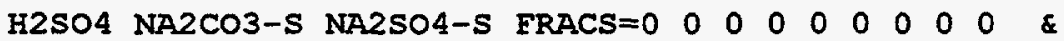
$\begin{array}{llllllllllllllllllllll}0 & 0 & 0 & 0 & 0 & 0 & 0 & 0 & 0 & 0 & 0 & 0 & 0 & 0 & 0 & 0 & 0 & 0 & 0 & 0 & 0 & \&\end{array}$ $\begin{array}{lllllllllllllllllll}0 & 0 & 0 & 0 & 0 & 0 & 0 & 0 & 0 & 0 & 0 & 0 & 0.90 & 0 & 0 & 0.90 & 0\end{array}$

FRAC STREAM=SAIT-90 SUBSTREAM=CISTOLID COMPS=C-S S-S \& NAOH-S NACL-S NAF-S FE-S SIO2-S AL2O3-S AS-S BA-S \& CD-S CR-S PB-S SE-S AG-S CACO3-S MGO-S NA2O-S K2O-S \&

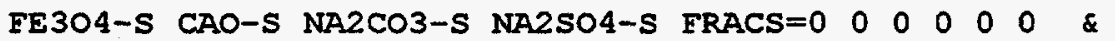
$0 \begin{array}{llllllllllllllll}0 & 0 & 0 & 0 & 0 & 0 & 0 & 0 & 0 & 0 & 0 & 0 & 0 & 0 & 0.90 & 0\end{array}$

BLOCK VIT-SEP SEP

FRAC STREAM=SIAG SUBSTREAM=MIXED COMPS=H2 O2 N2 CL2 F2 \& NO SO2 H2O CO2 CO HCL CH4 C-S S-S 5 NAOH-S NACL-S \& NAF-S AS CD HG SE FE SIO2 AL203 FE-S SIO2-S AL203-S \& AS-S BA-S CD-S CR-S PB-S SE-S AG-S CACO3-S MGO-S \& NA2O-S K2O-S FE3O4 FE3O4-S CAO-S NA2CO3 NA2SO4 H2SO4 \& NA2 $203-S$ NA2SO4-S FRACS $=0 \begin{array}{llllllllllll}0 & 0 & 0 & 0 & 0 & 0 & 0 & 0 & 0 & 0 & 0 & 0\end{array}$ $\begin{array}{llllllllllllllllllllll}1 & 1 & 1 & 1 & 1 & 1 & 0 & 0 & 0 & 0 & 1 & 1 & 1 & 1 & 1 & 1 & 1 & 1 & 1 & 1 & 1 & \&\end{array}$ $\begin{array}{llllllllllllll}1 & 1 & 1 & 1 & 1 & 1 & 1 & 1 & 1 & 1 & 1 & 1 & 1 & 1\end{array}$

FRAC STREAM=SLAG SUBSTREAM=CISOLID COMPS=C-S $\$-S$ NAOH-S \& NACL-S NAF-S FE-S SIO2-S AL2O3-S AS-S BA-S CD-S CR-S \& PB-S SE-S AG-S CACO3-S MGO-S NA2O-S K2O-S FE3O4-S \&

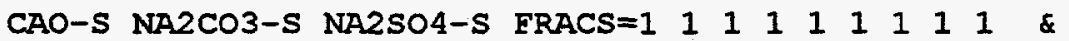
$\begin{array}{lllllllllllllll}1 & 1 & 1 & 1 & 1 & 1 & 1 & 1 & 1 & 1 & 1 & 1 & 1 & 1\end{array}$

BLOCK I-V-SEP FLASH2

PARAM TEMP $=120$ PRES $=1$ <ATM>

BIOCK L-V-SEP2 FLASH2 PARAM TEMP $=120$ PRES $=1$ <ATM>

BLOCK MELTER RGIBBS PARAM TEMP=3000 PRES=1 <ATM> NPHASE=2 VAPOR=YES MAXIT=500 PROD H2 / C-S SS / FE / SIO2 / AL2O3/ FE-S SS / \& 
BIOCK MSO RGIBBS

PARAM TEMP $=1652.0$ PRES=1 <ATM> NPHASE=2 MAXIT $=100$

PROD H2 / O2 / N2 / CL2 / F2 / NO / SO2 / H2O / \& $\mathrm{CO} 2 / \mathrm{CO} / \mathrm{HCL} / \mathrm{CH} 4 / \mathrm{s} / \mathrm{NAOH}-\mathrm{s}$ ss / NACL-S \& SS / NAF-S SS / AS / CD / HG / SE / FE / SIO2 \& / AL203 / FE-S SS / SIO2-S SS / AL203-S SS / \& AS-S SS / BA-S SS / CD-S SS / CR-S SS / PB-S SS \& / SE-S SS / AG-S SS / NA20-S SS / EE304 / \& FE3O4-S SS / NA2CO3 / NA2SO4 / H2SO4 / NA2CO3-S SS \& / NA2SO4-S SS

BLOCK PLENUM RGIBBS

PARAM TEMP $=1800$ PRES $=1$ <ATM> NPHASE $=2$

PROD $\mathrm{H} 2 / \mathrm{O} 2 / \mathrm{N} 2 / \mathrm{CL}_{2} / \mathrm{F} 2 / \mathrm{NO} / \mathrm{sO} 2 / \mathrm{H} 2 \mathrm{O} /$ \& $\mathrm{CO} 2 / \mathrm{CO} / \mathrm{HCL} / \mathrm{C}-\mathrm{s}$ ss / s-s ss/s/As/ \& $\mathrm{CD} / \mathrm{HG} / \mathrm{SE} / \mathrm{FE}-\mathrm{S}$ SS / SIO2-S SS / AL203-S ss \& / BA-S SS / CR-S SS / PB-S SS / AG-S SS / MGO-S \& SS / NA2O-S SS / K2O-S SS / FE3O4-S SS / CAO-S SS

BLOCK Q2 RGIBBS

PARAM TEMP=68 PRES=1 <ATM> NPHASE=2 TAPP=2132

PROD H2 / O2/ N2/ CL2/ F2/ NO/ SO2/ H2O / \& $\mathrm{CO} 2 / \mathrm{CO} / \mathrm{HCL} / \mathrm{AS} / \mathrm{CD} / \mathrm{HG} / \mathrm{SE} / \mathrm{FE}-\mathrm{S} \mathrm{SS} \&$ / SIO2-S SS / AL2O3-S SS / BA-S SS / CR-S SS / \&

PB-S SS / SE-S SS / AG-S SS / MGO-S SS / NA2O-S \& SS / K2O-S SS / FE304-S SS / CAO-S SS

BLOCK QUENCH RGIBBS

PARAM TEMP $=350$ PRES $=1$ <ATM> NPHASE $=2$ MAXIT $=500$

PROD H2 / O2 / N2 / CL2 / SO2 / H2O / $\mathrm{CO} 2 / \mathrm{CO} / \mathrm{HCL} / \mathrm{CD}$

BLOCK QUENCH2 RGIBBS

PARAM TEMP $=350$ PRES $=1$ <ATM> NPHASE $=2$

$\mathrm{PROD} \mathrm{H} 2 / \mathrm{O} 2 / \mathrm{N} 2 / \mathrm{CL} 2 / \mathrm{F} 2 / \mathrm{NO} / \mathrm{SO} 2 / \mathrm{H} 2 \mathrm{O} /$ \& $\mathrm{CO} 2 / \mathrm{CO} / \mathrm{HCL} / \mathrm{AS} / \mathrm{CD} / \mathrm{HG} / \mathrm{SE} / \mathrm{FE}-\mathrm{S} \mathrm{SS} \&$ / sI02-S SS / AL203-s Ss / As-s ss / BA-s SS / \& CD-S SS / CR-S SS / PB-S SS / SE-S SS / AG-S SS \& $/$ MGO-S SS / NA20-S SS / K20-S SS / FE3O4-S SS/ CAO-S SS

BLOCK SCC2 RGIBBS

PARAM TEMP $=2200$ PRES=1 <ATM> NPHASE=2

PROD H2 M/O2 M/ N2 M/ CL2 M/ F2 M/ NO M / \& $\mathrm{SO} 2 \mathrm{M} / \mathrm{H} 2 \mathrm{O} \mathrm{M} / \mathrm{CO} 2 \mathrm{M} / \mathrm{CO} \mathrm{M} / \mathrm{HCI} / \mathrm{AS} / \mathrm{CD}$ \& / HG / SE / FE-S SS / SIO2-S SS / AL203-S SS / \& BA-S SS / CR-S SS / PB-S SS / AG-S SS / MGO-S \& SS / NA2O-S SS / K2O-S SS / FE304-S SS / CAO-S SS

BLOCK SCRUB RGIBBS

PARAM TEMP=120 PRES=1 <ATM> NPHASE $=2$ MAXIT $=500$

PROD H2 / O2 / N2 / $\mathrm{CL} 2 / \mathrm{E} 2 / \mathrm{NO} / \mathrm{SO} 2 / \mathrm{H} 2 \mathrm{O} /$ \& $\mathrm{CO} 2 / \mathrm{CO} / \mathrm{HCL} / \mathrm{C}-\mathrm{S}$ ss / s-s Ss / NAOH-S SS / NACL-S SS

BLOCK SCRUB-2 RGIBBS

PARAM TEMP $=120$ PRES $=1$ <ATM> NPHASE=2 MAXIT=500

PROD H2 / O2 / N2/ CL2/ $2 / / \mathrm{NO} / \mathrm{SO} 2 / \mathrm{H} 2 \mathrm{O} /$ \& $\mathrm{CO} 2 / \mathrm{CO} / \mathrm{HCL} / \mathrm{C}-\mathrm{s}$ ss / s-s ss / NAOH-s ss / NACL-s s's

BLOCK VITRIEY RGIBBS

PARAM TEMP $=3000$ PRES=1 <ATM> NPHASE=2 MAXIT=50

$\mathrm{PROD} \mathrm{H} 2 / \mathrm{O} 2 / \mathrm{N} 2 / \mathrm{CL} 2 / \mathrm{F} 2 / \mathrm{NO} / \mathrm{SO} 2 / \mathrm{H} 2 \mathrm{O} /$ \& 


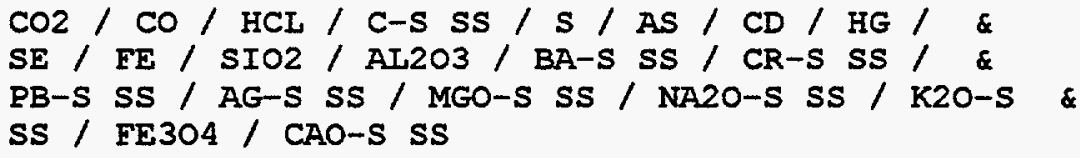

BLOCK DUPL2 DUPI

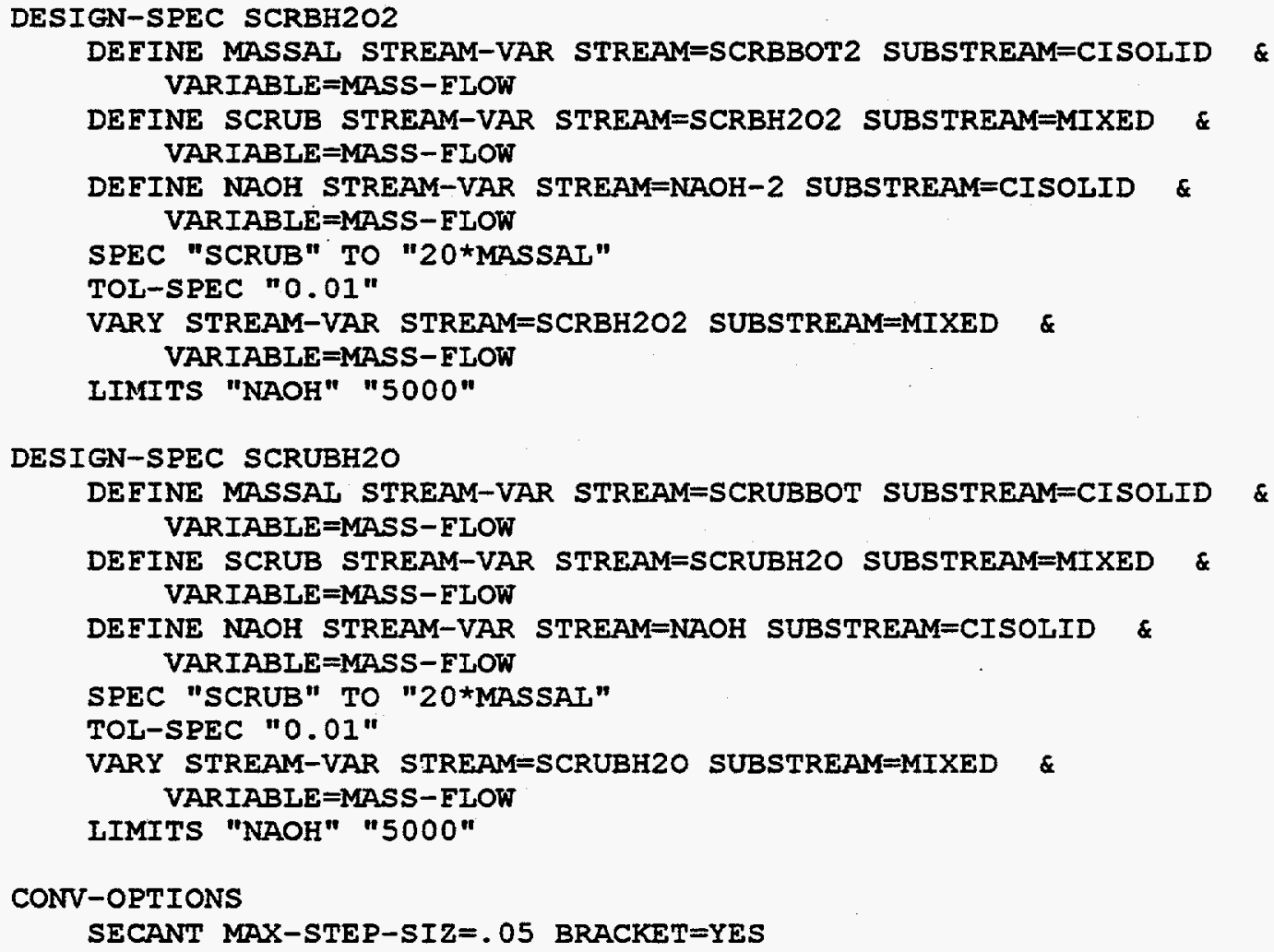

STREAM-REPOR NOSORT MOLEELOW MASSELOW PROPERTIES=AIL-SUBS 


\section{System G-1}

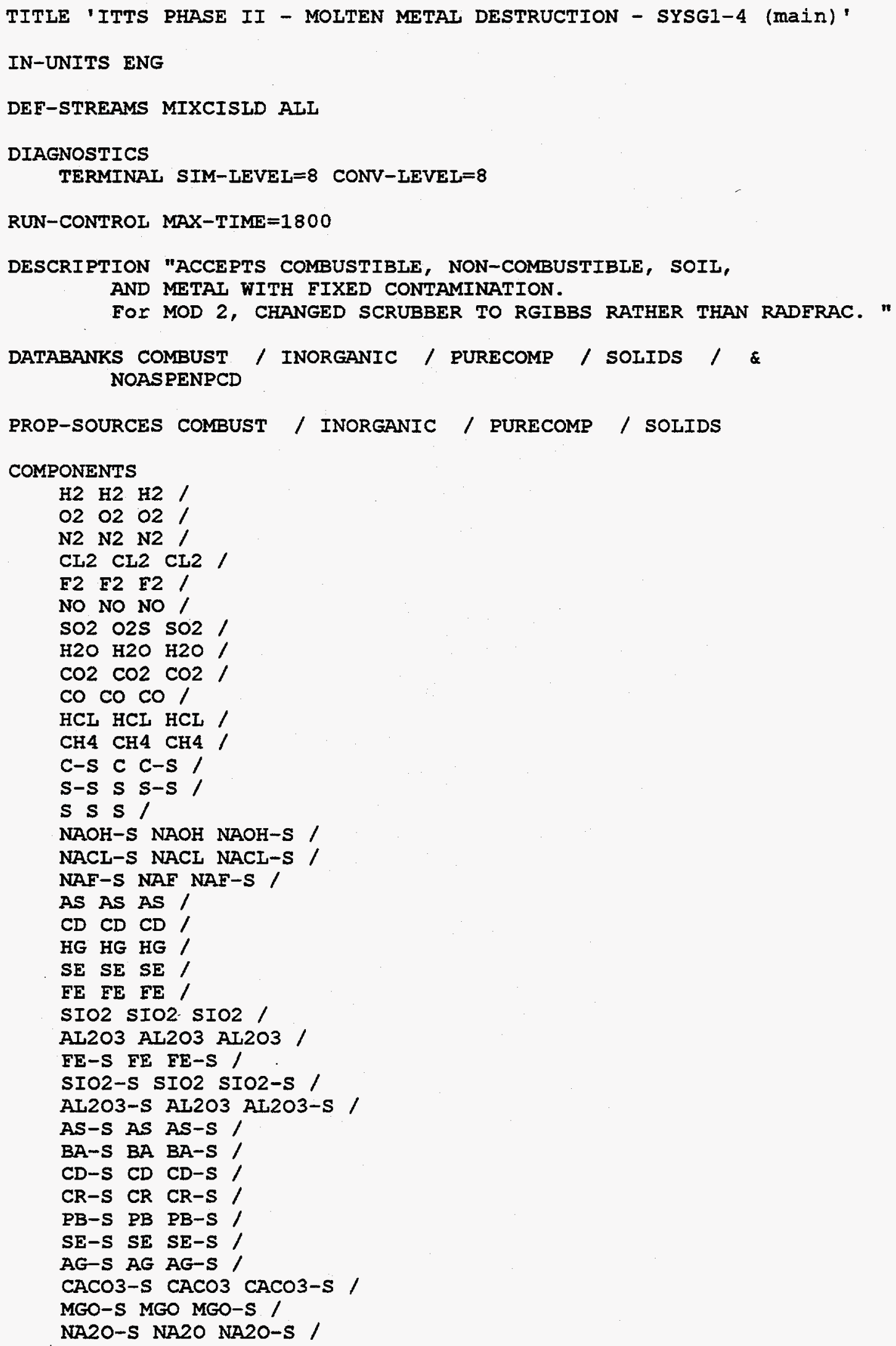


STREAM NAOH

SUBSTREAM CISOID TEMP=68 PRES=1 <ATM> MASS-FLOW=77

MASS-FRAC NAOH-S 1

STREAM OXYGEN

SUBSTREAM MIXED TEMP=68 PRES=1 <ATM> MASS-FLOW=79.25 \&

MAXIT $=300$

MASS-FRAC 021

STREAM SCRUBH2O

SUBSTREAM MIXED TEMP=68 PRES=1 <ATM> MASS-FLOW=100

MASS-FRAC H2O 1

STREAM SOIL

SUBSTREAM MIXED TEMP=68 PRES=1 <ATM> MASS-ELOW=62.5

MASS-FLOW H2O . 100

SUBSTREAM CISOLID TEMP=68 PRES=1 <ATM> MASS-FLOW=562.5

MASS-FLOW SIO2-S $0.569 /$ AL203-S $0.102 /$ CACO3-S $0.128 / \&$ MGO-S 0.023 / NA2O-S $0.013 / \mathrm{K} 20-S 0.024$ / FE3O4-S 0.041

BLOCK MM-SEP SEP

PARAM MAXIT $=100$ TOL $=0.001$

FRAC STREAM=METAL SUBSTREAM=MIXED COMPS=H2 O2 N2 CL2 F2 \& NO SO2 H2O CO2 CO HCL CH4 C-S S-S S NAOH-S NACL-S \& NAF-S AS CD HG SE FE SIO2 AL2O3 FE-S SIO2-S AL2O3-S \& AS-S BA-S CD-S CR-S PB-S SE-S AG-S CACO3-S MGO-S \& NA2O-S K2O-S FE3O4 FE3O4-S CAO-S CACL2-S CASO4-S \& FRACS $=0 \begin{array}{lllllllllllllllllll}0 & 0 & 0 & 0 & 0 & 0 & 0 & 0 & 0 & 0 & 0 & 0 & 0 & 0 & 0 & 0 & 0 & 0 & \&\end{array}$ $\begin{array}{llllllllllllllllllllllllll}0 & 0 & 0 & 0 & 1 & 0 & 0 & 1 & 0 & 0 & 1 & 1 & 1 & 1 & 1 & 1 & 1 & 0 & 0 & 0 & 0 & 0 & 0 & 0 & 0 & 0\end{array}$

FRAC STREAM=METAI SUBSTREAM=CISOLID COMPS=C-S S-S NAOH-S\& NACL-S NAF-S FE-S SIO2-S AL2O3-S AS-S BA-S CD-S CR-S \& PB-S SE-S AG-S CACO3-S MGO-S NA2O-S K2O-S FE304-S \& CAO-S CACL2-S CASO4-S FRACS $=0 \begin{array}{llllllllllll}0 & 0 & 0 & 0 & 0 & 1 & 0 & 0 & 1 & 1 & \&\end{array}$ $\begin{array}{llllllllllllll}1 & 1 & 1 & 1 & 1 & 0 & 0 & 0 & 0 & 0 & 0 & 0 & 0\end{array}$

FRAC STREAM=SIAG SUBSTREAM=MIXED COMPS=H2 O2 N2 CL2 F2 \& NO SO2 H2O CO2 CO HCL CH4 C-S $s-s$ S NAOH-S NACL-S \& NAF-S AS CD HG SE FE SIO2 AL2O3 FE-S SIO2-S AL2O3-S \& AS-S BA-S CD-S CR-S PB-S SE-S AG-S CACO3-S MGO-S \& NA2O-S K2O-S FE3O4 FE3O4-S CAO-S CACL2-S CASO4-S CACL2 \& FRACS $=0 \begin{array}{lllllllllllllllllll}0 & 0 & 0 & 0 & 0 & 0 & 0 & 0 & 0 & 0 & 0 & .8 & .8 & 0 & .8 & .8 & \&\end{array}$ $\begin{array}{llllllllllllllllllll}.8 & 0 & 0 & 0 & 0 & 0 & .8 & .8 & 0 & .8 & .8 & 0 & 0 & 0 & 0 & 0 & 0 & 0 & .8 & \&\end{array}$ $\begin{array}{llllllllll}.8 & .8 & .8 & .8 & .8 & .8 & .8 & .8 & 1\end{array}$

FRAC STREAM=SLAG SUBSTREAM=CISOLID COMPS=C-S S-S NAOH-S \& NACI-S NAF-S FE-S SIO2-S AL2O3-S AS-S BA-S CD-S CR-S \& PB-S SE-S AG-S CACO3-S MGO-S NA2O-S K2O-S FE3O4-S \&

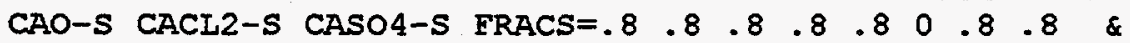
$\begin{array}{lllllllllllllllll}0 & 0 & 0 & 0 & 0 & 0 & 0 & .8 & .8 & .8 & .8 & .8 & .8 & .8 & .8\end{array}$

PROPERTIES SOIIDS

BLOCK SEPARATR SEP

FRAC STREAM=SOIIDS SUBSTREAM=MIXED COMPS=H2 O2 N2 CL2 \& F2 NO SO2 H2O CO2 CO HCL CH4 C-S S-S S NAOH-S \& NACL-S NAF-S AS CD HG SE FE SIO2 AL2O3 FE-S SIO2-S \& AL2O3-S AS-S BA-S CD-S CR-S PB-S SE-S AG-S CACO3-S \& MGO-S NA2O-S K2O-S FE3O4 FE3O4-S CAO-S CACL2-S CASO4-S \& CAO CACL2 FRACS $=0 \begin{array}{llllllllllllll}0 & 0 & 0 & 0 & 0 & 0 & 0 & 0 & 0 & 0 & 0 & 1 & 1\end{array}$ $\begin{array}{llllllllllllllllllllllll}1 & 0 & 0 & 0 & 0 & 0 & 0 & 0 & 1 & 1 & 1 & 1 & 1 & 1 & 0 & 1 & 0 & 1 & 1 & 0 & 1 & 1\end{array}$ $\begin{array}{llllllllllll}1 & 1 & 1 & 1 & 1 & 1 & 1 & 1 & 1 & 1 & 1\end{array}$

FRAC STREAM=SOLIDS SUBSTREAM=CISOLID COMPS=C-S S-S NAOH-S \& NACL-S NAF-S FE-S SIO2-S AI2O3-S AS-S BA-S CD-S CR-S \& PB-S SE-S AG-S CACO3-S MGO-S NA2O-S K2O-S FE3O4-S \&

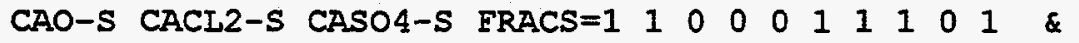


$\begin{array}{llllllllllllll}0 & 1 & 1 & 0 & 1 & 1 & 1 & 1 & 1 & 1 & 1 & 1 & 1\end{array}$

FRAC STREAM=HGPLUS SUBSTREAM=MIXED COMPS=H2 O2 N2 CL2 \& F2 NO SO2 H2O CO2 CO HCL CH4 C-S S-S S NAOH-S \& NACL-S NAF-S AS CD HG SE FE SIO2 AL2O3 FE-S SIO2-S \& AL2O3-S AS-S BA-S CD-S CR-S PB-S SE-S AG-S CACO3-S \& MGO-S NA2O-S K2O-S FE304 FE304-S CAO-S CACL2-S CASO4-S \&

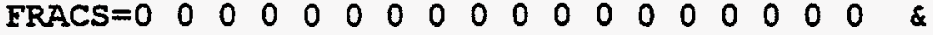
$\begin{array}{llllllllllllllllllllllllll}1 & 1 & 1 & 1 & 0 & 0 & 0 & 0 & 0 & 0 & 1 & 0 & 1 & 0 & 0 & 1 & 0 & 0 & 0 & 0 & 0 & 0 & 0 & 0 & 0 & 0\end{array}$

FRAC STREAM=HGPLUS SUBSTREAM=CISOIID COMPS=C-S S-S NAOH-S NACL-S NAF-S FE-S SIO2-S AL203-S AS-S BA-S CD-S CR-S \& PB-S SE-S AG-S CACO3-S MGO-S NA2O-S K2O-S FE3O4-S \& CAO-S CACL2-S CASO4-S ERACS $=0 \begin{array}{llllllllll}0 & 0 & 0 & 0 & 0 & 0 & 0 & 0 & 1 & 0\end{array}$ $\begin{array}{llllllllllllll}1 & 0 & 0 & 1 & 0 & 0 & 0 & 0 & 0 & 0 & 0 & 0 & 0\end{array}$

BLOCK FLUIDSEP FLASH2

PARAM TEMP=200 PRES $=1<$ ATM $>$

BLOCK L-V-SEP FLASH2

PARAM TEMP $=80$ PRES $=1$ <ATM>

BLOCK CATALYST RSTOIC

PARAM TEMP $=1500$ RRES $=1$ <ATM>

STOIC 1 MIXED CO $-1 / 02-.5 / \mathrm{CO} 21$

STOIC 2 MIXED H2 $-1 / \mathrm{O} 2-.5 / \mathrm{H} 2 \mathrm{O} 1$

CONV 1 MIXED CO 1

CONV 2 MIXED H2 1

BLOCK MM-CEP RGIBBS

PARAM TEMP=2732 PRES=1 <ATM> NPHASE=2 MAXIT $=300$

$\mathrm{PROD} \mathrm{H} 2 / \mathrm{O} 2 / \mathrm{N} 2 / \mathrm{CI} 2 / \mathrm{E} 2 / \mathrm{NO} / \mathrm{SO} 2 / \mathrm{H} 2 \mathrm{O} / \&$ $\mathrm{CO} 2 / \mathrm{CO} / \mathrm{HCL} / \mathrm{C}-\mathrm{s}$ ss $/ \mathrm{s}-\mathrm{s}$ ss $/ \mathrm{s} / \mathrm{NAOH}-\mathrm{s} \&$ $\mathrm{sS} / \mathrm{NACL}-\mathrm{S} \mathrm{sS} / \mathrm{NAF}-\mathrm{S}$ sS / AS / $\mathrm{CD} / \mathrm{HG} / \mathrm{SE}$ \& / SIO2 / AL2O3 / FE-S SS / BA-S SS / CR-S SS / \& PB-S SS / AG-S SS / MGO-S SS / NA2O-S SS / K20-S \& SS / FE304-S SS / CASO4-S SS / CAO-S SS / CACL2-S \& sS / CACL2

BLOCK QUENCH RGIBBS

PARAM TEMP=200 PRES=1 <ATM> NPHASE=2 MAXIT=500

PROD H2 / O2 / N2 / F2 / NO / SO2 / $\mathrm{H} 2 \mathrm{O} / \mathrm{CO} 2 / \&$ $\mathrm{CO} / \mathrm{HCL} / \mathrm{NAOH}-\mathrm{S}$ SS / NACL-S Ss / NAF-S SS /\& AS / CD / HG / SE / FE-S SS / SIO2-S SS / \& AL203-S SS / AS-S SS / BA-S SS / CD-S SS / CR-S \& SS / PB-S SS / SE-S SS / AG-S SS / MGO-S SS / \& NA20-s sS / K20-S SS/ FE304-S SS / CAO-S SS / \& CACI2-S SS / CASO4-S SS

PROD-FRAC H2O 0.9999

BIOCK-OPTION RESTART=NO

BLOCK SCRUB RGIBBS

PARAM TEMP $=80$ PRES $=1$ <ATM> NPHASE $=2$ MAXIT $=500$

PROD H2/ O2/ N2/ $\mathrm{F} 2 / \mathrm{NO} / \mathrm{SO} 2 / \mathrm{H} 2 \mathrm{O} / \mathrm{CO} 2 / \&$ CO / HCL / NACL-S SS / NAF-S SS / NAOH-S SS PROD-FRAC CO $1 / \mathrm{CO} 1$

DESIGN-SPEC AIR

DEFINE OXIN1 MASS-FLOW STREAM=AIR SUBSTREAM=MIXED \& COMPONENT $=02$

DEFINE OXIN2 MASS-FLOW STREAM=SCRUBVAP SUBSTREAM=MIXED \& COMPONENT $=02$

DEFINE OXOUT MASS-FLOW STREAM=EXHAUST SUBSTREAM=MIXED \& COMPONENT $=02$ 
SPEC "OXIN1+OXIN2-2.0*OXOUT" TO "O"

TOL-SPEC "0.1"

VARY STREAM-VAR STREAM=AIR SUBSTREAM=MIXED \&

VARIABLE=MASS-FLOW

LIMITS "1" "1000000"

DESIGN-SPEC IIME

DEFINE CAOIN MOLE-FLOW STREAM=IIME SUBSTREAM=CISOLID \& COMPONENT $=$ CAO-S

DEFINE CL2INC MOLE-FLOW STREAM=FDWASTEC SUBSTREAM=MIXED \& COMPONENT $=\mathrm{CL} 2$

DEFINE CL2INN MOLE-FLOW STREAM=FDWASTEN SUBSTREAM=MIXED \& COMPONENT $=$ CL2

SPEC "CAOIN" TO "0.9* (CL2INC+CL2INN) "

TOL-SPEC "0.01"

VARY STREAM-VAR STREAM=LIME SUBSTREAM=CISOLID\&

VARIABLE=MASS-FLOW

LIMITS "1" "1000"

DESIGN-SPEC NAOH

DEFINE CAUIN MASS-FLOW STREAM=NAOH SUBSTREAM=CISOIID\& COMPONENT $=\mathrm{NAOH}-\mathrm{S}$

DEFINE CAUOUT MASS-FLOW STREAM=SCRUBOUT SUBSTREAM=CISOLID \& COMPONENT $=\mathrm{NAOH}-\mathrm{S}$

SPEC "CAUIN" TO "CAUOUT* $100 "$

TOL-SPEC "0.1"

VARY STREAM-VAR STREAM=NAOH SUBSTREAM=CISOLID\& VARIABLE = MASS - FLOW

LIMITS "13" "100"

DESIGN-SPEC SCRUBH2O

DEFINE MASSAL STREAM-VAR STREAM=SCRUBBOT SUBSTREAM=CISOLID\& VARIABLE =MASS -FLOW

DEFINE SCRUB STREAM-VAR STREAM=SCRUBH2O SUBSTREAM=MIXED \& VARIABLE=MASS-FLOW

DEFINE NAOH STREAM-VAR STREAM=NAOH SUBSTREAM=CISOLID\& VARIABLE=MASS - FLOW

SPEC "SCRUB" TO "20*MASSAL"

TOL-SPEC "0.01"

VARY STREAM-VAR STREAM $=$ SCRUBH2O SUBSTREAM=MIXED\& VARTABLE=MASS-FLOW

LIMITS "NAOH" "5000"

DESIGN-SPEC SOILH2O

DEFINE SLAG1 STREAM-VAR STREAM=SLAG SUBSTREAM=MIXED \& VARIABLE =MASS-FLOW

DEFINE SLAG2 STREAM-VAR STREAM=SLAG SUBSTREAM=CISOLID \& VARIABLE =MASS- FLOW

DEFINE SOII STREAM-VAR STREAM=SOIL SUBSTREAM=MIXED \& VARIABLE =MASS-FLOW

SPEC "30.*SOIL" TO "SIAG1+SLAG2"

TOL-SPEC "0.10"

VARY STREAM-VAR STREAM=SOIL SUBSTREAM=MIXED \& VARIABIE $=$ MASS $-F L O W$

LIMITS "1" "1000"

DESIGN-SPEC SOIISOL

DEFINE SLAG1 STREAM-VAR STREAM=SLAG SUBSTREAM=MIXED \& VAR I ABLE = MASS $-F L O W$

DEFINE SLAG2 STREAM-VAR STREAM=SLAG SUBSTREAM=CISOLID \& VARIABLE=MASS-FLOW

DEFINE SOII STREAM-VAR STREAM=SOIL SUBSTREAM=CISOLID \& 
VARIABLE $=$ MASS-FLOW

SPEC "3.33333*SOIL" TO "SLAGI+SLAG2"

TOI-SPEC "0.10"

VARY STREAM-VAR STREAM=SOIL SUBSTREAM=CISOLID\& VARIABLE=MASS-FLOW

LIMITS " 1 " "1000"

CONV-OPTIONS

PARAM TEAR-METHOD=BROYDEN

SECANT MAXIT=75 MAX-STEP-SIZ=.06 BRACKET=YES

STREAM-REPOR NOSORT MOLEELOW MASSFLOW PROPERTIES=ALL-SUBS 


\section{System H-1}

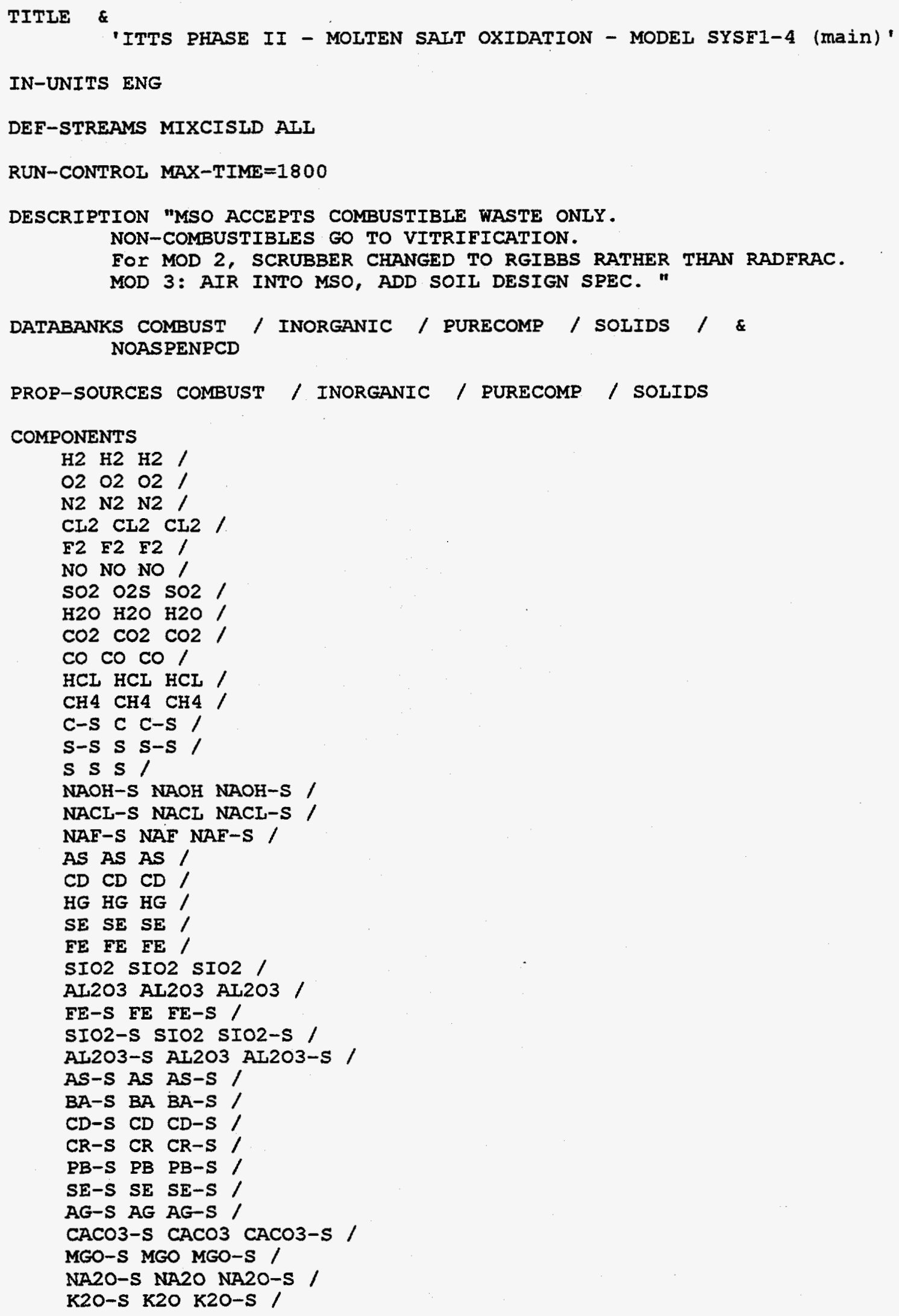


FE304 FE304 FE304 /

FE304-S FE304 FE304-S /

CAO-S CAO CAO-S

FLOWSHEET

BLOCK VITRIFY IN=SOIIDS OUT=VITOUT

BLOCK VIT-SEP IN=VITOUT OUT=VITVAP SLAG

BLOCK SCC2 IN=FUELMIX2 EFFLUENT OUT=OUT2

BLOCK 22 IN=DUM2IN OUT=DUM2OUT

BLOCK DUPL2 IN=OUT2 OUT=SCCOUT2 DUM2IN

BLOCK MELTER IN=FDMETMLT OUT=METAL

BLOCK FILTERS IN=GASOUT OUT=SYNGAS ASH

BLOCK SCRUB IN=NAOH SCRUBH2O SYNGAS OUT=SCRUBOUT

BLOCK L-V-SEP IN=SCRUBOUT OUT=SCRUBVAP SCRUBBOT

BLOCK QUENCH2 IN=SCCOUT2 QNCH2O-2 OUT=QUENCHD2

BLOCK BAGHSE2 IN=QUENCHD2 OUT=FILTGAS2 HGPLUS2 FLYASH2

BLOCK FEED-SEP IN=SOIL FDWASTEN FLYASH2 ASH OUT=SOLIDS ORGANICS

BLOCK PLENUM IN=ORGANICS VITVAP OXYGEN OUT=EFFLUENT

BLOCK L-V-SEP2 IN=SCRBOUT2 OUT=SCRBVAP2 SCRBBOT2

BLOCK SCRUB-2 IN=SCRBH2O2 NAOH-2 FILTGAS2 OUT=SCRBOUT2

BLOCK HEATER IN=FUELMIX1 OUT=EXHAUST1

BLOCK STEAMGEN IN=WATER OUT=STEAM

BLOCK CATALYST IN=HOTVAP AIR OUT=EXHAUST2

BLOCK REHEAT IN=SCRUBVAP OUT=HOTVAP

BLOCK GASIFIER IN=FDWASTEC STEAM OUT=GASOUT

PROPERTIES SOLIDS

PROPERTIES IDEAI / RKS-BM

PROP-SET ALL-SUBS TEMP PRES VERAC UNITS='ATM' SUBSTREAM=ALL

STREAM AIR

SUBSTREAM MIXED TEMP $=68.0$ PRES $=1$ <ATM> MASS-FLOW $=6000$

MASS-FRAC $020.233 / \mathrm{N} 20.767$

STREAM FDMETMLT

SUBSTREAM MIXED TEMP $=68$ PRES $=1$ <ATM> MASS-FLOW $=1.20$

MASS-FLOW H2 1.20

SUBSTREAM CISOLID TEMP $=67$ PRES $=1$ <ATM> MASS-FLOW $=147.8$

MASS-FLOW C-S 14.44 / FE-S $282.02 /$ SIO2-S $0.035 / \&$

AL203-S $0.035 / \mathrm{BA}-\mathrm{S} 0.87 / \mathrm{CD}-\mathrm{S} 0.18$

STREAM FDWASTEC

SUBSTREAM MIXED TEMP $=68$ PRES $=1$ <ATM>

MASS-FLOW H2 $50.95 /$ O2 $92.76 / \mathrm{N} 20.23 / \mathrm{CL} 253.02 /$ \& F2 $0.11 / \mathrm{H} 2019.83$

SUBSTREAM CISOLID TEMP $=68$ PRES $=1$ 〈ATM>

MASS-FLOW C-S $326.28 /$ S-S $0.49 /$ FE-S $7.67 /$ SIO2-S\&

$53.01 /$ AL2O3-S 53.01/ BA-S $0.77 /$ CD-S $0.77 /$ CR-S 1.53

STREAM FDWASTEN

SUBSTREAM MIXED TEMP $=68$ PRES $=1$ <ATM>

MASS-FLOW H2 $0.54 / 021.44 / \mathrm{N} 20.01 / \mathrm{CL} 214.88 / \&$

NO $0.66 / \mathrm{SO} 20.83 / \mathrm{H} 20354.23$ / HG 0.15

SUBSTREAM CISOLID TEMP $=68$ PRES $=1$ <ATM>

MASS-FLOW C-S $4.77 /$ S-S $0.62 /$ SIO2-S $476.77 /$ \&

AL203-S 476.77 / AS-S 0.06 / BA-S 0.04 / CD-S 2.63 \&

/ CR-S $4.95 /$ PB-S $0.17 /$ SE-S $0.03 /$ AG-S 0.03 


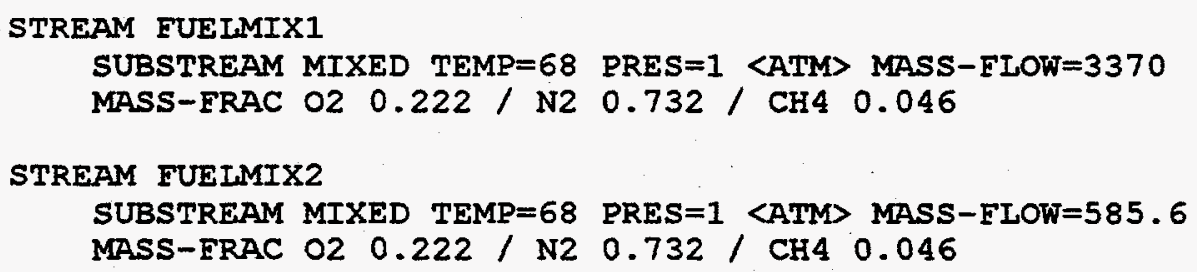

BLOCK BAGHSE2 SEP

FRAC STREAM=FILTGAS2 SUBSTREAM=MIXED COMPS=H2 O2 N2 CL2 \&

F2 NO SO2 H2O CO2 CO HCL CH4 C-S S-S S NAOH-S \& NACL-S NAF-S AS CD HG SE FE SIO2 AI2O3 FE-S SIO2-S \& AL2O3-S AS-S BA-S CD-S CR-S PB-S SE-S AG-S CACO3-S \& MGO-S NA2O-S K2O-S FE304 FE304-S CAO-S FRACS=1 11 \& $\begin{array}{llllllllllllllllllllll}1 & 1 & 1 & 1 & 1 & 1 & 1 & 1 & 1 & 0 & 0 & 0 & 0 & 0 & 0 & 0 & 0 & 0 & 0 & 0 & 0 & \&\end{array}$ $0 \begin{array}{lllllllllllllllll}0 & 0 & 0 & 0 & 0 & 0 & 0 & 0 & 0 & 0 & 0 & 0 & 0 & 0 & 0 & 0 & 0\end{array}$

ERAC STREAM=EIITGAS2 SUBSTREAM=CISOLID COMPS=C-s $s-5$ \& NAOH-S NACL-S NAF-S FE-S SIO2-S AI203-S AS-S BA-S \& CD-S CR-S PB-S SE-S AG-S CACO3-S MGO-S NA2O-S K2O-S \& FE 304-S CAO-S ERACS $=0 \begin{array}{lllllllllllll}0 & 0 & 0 & 0 & 0 & 0 & 0 & 0 & 0 & 0 & 0 & 0 & 0\end{array}$ $\begin{array}{llllllll}0 & 0 & 0 & 0 & 0 & 0 & 0 & 0\end{array}$

ERAC STREAM=HGPIUS2 SUBSTREAM=MIXED COMPS=H2 O2 N2 CL2 \& F2 NO SO2 H2O $\mathrm{CO} 2 \mathrm{CO}$ HCL CH4 C-S S-S S NAOH-S \& NACI-S NAF-S AS CD HG SE FE SIO2 AL2O3 FE-S SIO2-S \& AL2O3-S AS-S BA-S CD-S CR-S PB-S SE-S AG-S CACO3-S \& MGO-S NA2O-S K2O-S FE304 FE304-S CAO-S FRACS $=0 \quad 0 \quad 0$ \& 
$\begin{array}{llllllllllllllllllllll}0 & 0 & 0 & 0 & 0 & 0 & 0 & 0 & 0 & 0 & 0 & 0 & 0 & 0 & 0 & 1 & 1 & 1 & 1 & 0 & 0 & \alpha\end{array}$

$\begin{array}{llllllllllllllllll}0 & 0 & 0 & 0 & 1 & 0 & 1 & 0 & 0 & 1 & 0 & 0 & 0 & 0 & 0 & 0 & 0 & 0\end{array}$

FRAC STREAM=HGPLUS2 SUBSTREAM=CISOLID COMPS=C-S S-S \& NAOH-S NACL-S NAF-S FE-S SIO2-S AL2O3-S AS-S BA-S \& CD-S CR-S PB-S SE-S AG-S CACO3-S MGO-S NA2O-S K2O-S \& FE304-S CAO-S FRACS $=0 \begin{array}{llllllllllllll}0 & 0 & 0 & 0 & 0 & 0 & 0 & 1 & 0 & 1 & 0 & 0 & \&\end{array}$ $\begin{array}{llllllll}1 & 0 & 0 & 0 & 0 & 0 & 0 & 0\end{array}$

BLOCK FEED-SEP SEP

FRAC STREAM=SOLIDS SUBSTREAM=MIXED COMPS=H2 O2 N2 CI2 \& F2 NO SO2 H2O CO2 CO HCL CH4 C-S S-S S NAOH-S \& NACL-S NAF-S AS CD HG SE FE SIO2 AI203 FE-S SIO2-S \& AL203-S AS-S BA-S CD-S CR-S PB-S SE-S AG-S CACO3-S \& MGO-S NA2O-S K2O-S FE304 EE304-S CAO-S FRACS=0 00 \& $\begin{array}{lllllllllllllllllll}0 & 0 & 0 & 0 & 0 & 0 & 0 & 0 & 0 & .05 & .05 & .05 & .95 & .95 & .95 & .95 & \&\end{array}$ $\begin{array}{llllllllllllll}.95 & .95 & .95 & .95 & .95 & .95 & .95 & .95 & .95 & .95 & .95 & .95 & 8\end{array}$ $\begin{array}{lllllllllll}.95 & .95 & .95 & .95 & .95 & .95 & .95 & .95 & .95 & .95 & .95\end{array}$

FRAC STREAM=SOLIDS SUBSTREAM=CISOIID COMPS=C-S $S-S$ NAOH-S \& NACL-S NAF-S FE-S SIO2-S AL203-S AS-S BA-S CD-S CR-S \& PB-S SE-S AG-S CACO3-S MGO-S NA2O-S K2O-S FE304-S \&

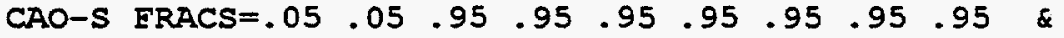
$\begin{array}{lllllllllllll}.95 & .95 & .95 & .95 & .95 & .95 & .95 & .95 & .95 & .95 & .95 & .95\end{array}$

BIOCK FIITERS SEP

ERAC STREAM=SYNGAS SUBSTREAM=MIXED COMPS=H2 O2 N2 CL2 \& F2 NO SO2 H2O CO2 CO HCL CH4 C-S S-S S NAOH-S \& NACL-S NAF-S AS CD HG SE EE SIO2 AL2O3 FE-S SIO2-S \& AL203-S AS-S BA-S CD-S CR-S PB-S SE-S AG-S CACO3-S \& MGO-S NA2O-S K2O-S FE304 FE304-S CAO-S FRACS=1 11 \& $\begin{array}{llllllllllllllllllllll}1 & 1 & 1 & 1 & 1 & 1 & 1 & 1 & 1 & 0 & 0 & 0 & 0 & 0 & 0 & 0 & 0 & 0 & 0 & 0 & 0 & \&\end{array}$ $\begin{array}{lllllllllllllllllll}0 & 0 & 0 & 0 & 0 & 0 & 0 & 0 & 0 & 0 & 0 & 0 & 0 & 0 & 0 & 0 & 0 & 0\end{array}$

FRAC STREAM=SYNGAS SUBSTREAM=CISOIID COMPS=C-S $S-S$ NAOH-S \& NACL-S NAF-S FE-S SIO2-S AL2O3-S AS-S BA-S CD-S CR-S \& PB-S SE-S AG-S CACO3-S MGO-S NA2O-S K2O-S FE3O4-S \& CAO-S ERACS $=0 \begin{array}{lllllllllllllllllllll}0 & 0 & 0 & 0 & 0 & 0 & 0 & 0 & 0 & 0 & 0 & 0 & 0 & 0 & 0 & 0 & 0 & 0 & 0 & 0\end{array}$ FLASH-SPECS ASH TEMP $=68$ PRES $=1$ <ATM>

\section{BLOCK VIT-SEP SEP}

FRAC STREAM=SIAG SUBSTREAM=MIXED COMPS=H2 O2 N2 CL2 F2 \& NO SO2 H2O CO2 CO HCL CH4 C-S S-S S NAOH-S NACI-S \& NAF-S AS CD HG SE FE SIO2 AL2O3 FE-S SIO2-S AL2O3-S \& AS-S BA-S CD-S CR-S PB-S SE-S AG-S CACO3-S MGO-S \&

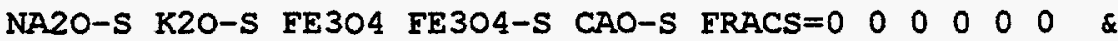
$\begin{array}{llllllllllllllllllllll}0 & 0 & 0 & 0 & 0 & 0 & 1 & 1 & 1 & 1 & 1 & 1 & 0 & 0 & 0 & 0 & 1 & 1 & 1 & 1 & 1 & 1\end{array}$ $\begin{array}{llllllllllllllll}1 & 1 & 1 & 1 & 1 & 1 & 1 & 1 & 1 & 1 & 1 & 1 & 1 & 1 & 1\end{array}$

FRAC STREAM=SLAG SUBSTREAM=CISOLID COMPS=C-S S-S NAOH-S \& NACI-S NAF-S FE-S SIO2-S AL2O3-S AS-S BA-S CD-S CR-S \& PB-S SE-S AG-S CACO3-S MGO-S NA2O-S K2O-S FE304-S \&

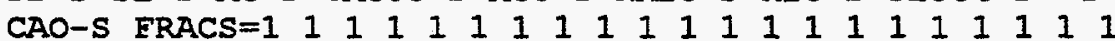

BLOCK STEAMGEN HEATER PARAM TEMP $=900$ PRES=1 〈ATM>

BLOCK I-V-SEP FLASH2

PARAM TEMP $=160$ PRES $=1$ 〈ATM>

BLOCK L-V-SEP2 FIASH2

PARAM TEMP=120 PRES=1 〈ATM>

BLOCK CATALYST RSTOIC

PARAM TEMP $=1500$ PRES $=1$ <ATM>

STOIC 1 MIXED CO $-1 / 02-0.5 /$ CO2 1 
STOIC 2 MIXED H2 $-1 / \mathrm{O} 2-0.5 / \mathrm{H} 2 \mathrm{O} 1$

STOIC 3 MIXED CH4 - / O2 -2/ H2O $2 / \mathrm{CO} 21$

CONV 1 MIXED CO 1

CONV 2 MIXED H2 1

CONV 3 MIXED CH4 1

BLOCK GASIFIER RSTOIC

PARAM TEMP $=1400$ PRES $=1$ <ATM>

STOIC 1 CISOLID C-S - 1 / MIXED H2O - I / H2 $1 /$ CO 1

STOIC 2 MIXED H2 $-1 /$ CL2 $-1 /$ HCL 2

STOIC 3 MIXED O2 $-1 / \mathrm{CO}-2 / \mathrm{CO} 22$

STOIC 4 MIXED O2 $-1 / \mathrm{H} 2-2 / \mathrm{H} 2 \mathrm{O} 2$

CONV 1 CISOLID C-S .90

CONV 2 MIXED CL2 1

CONV 3 MIXED 020.5

CONV 4 MIXED $02 \quad 0.5$

BLOCK HEATER RGIBBS

PARAM TEMP $=1400$ PRES $=1$ <ATM> NPHASE $=2$

PROD H2 / $\mathrm{O} 2 / \mathrm{N} 2 / \mathrm{NO} / \mathrm{H} 2 \mathrm{O} / \mathrm{CO} 2 / \mathrm{CO} / \mathrm{CH} 4$ / C-S SS

BIOCK MELTER RGIBBS

PARAM TEMP $=3000$ PRES=1 <ATM> NPHASE=2 VAPOR=YES MAXIT $=500$

PROD H2 / C-S SS / FE / SIO2 / AL2O3 / FE-S SS / \& SIO2-S SS / AL2O3-S SS / BA-S SS / CD / CD-S SS

BLOCK. PLENUM RGIBBS

PARAM TEMP $=1800$ PRES $=1$ <ATM> NPHASE $=2$

$\mathrm{PROD} \mathrm{H} 2 / \mathrm{O} 2 / \mathrm{N} 2 / \mathrm{CL} 2 / \mathrm{F} 2 / \mathrm{NO} / \mathrm{SO} 2 / \mathrm{H} 2 \mathrm{O} / \&$

$\mathrm{CO} 2 / \mathrm{CO} / \mathrm{HCL} / \mathrm{C}-\mathrm{s}$ ss / s-s ss / s / As / \&

CD / HG / SE / FE-S SS / SIO2-S SS / AL2O3-S SS \&

/ $\mathrm{BA}-\mathrm{S}$ SS / CR-S SS / PB-S SS / AG-S SS / MGO-S \&

SS / NA2O-S SS / K2O-S SS / FE304-S SS / CAO-S SS

BLOCK 22 RGIBBS

PARAM TEMP $=68$ PRES=1 <ATM> NPHASE $=2$ TAPP $=2132$

PROD H2 / O2 / N2 / $\mathrm{CL} 2 / \mathrm{F} 2 / \mathrm{NO} / \mathrm{SO} 2 / \mathrm{H} 2 \mathrm{O} / \&$ $\mathrm{CO} 2 / \mathrm{CO} / \mathrm{HCL} / \mathrm{AS} / \mathrm{CD} / \mathrm{HG} / \mathrm{SE} / \mathrm{FE}-\mathrm{S} \mathrm{SS} \&$ / SI02-S SS / AL2O3-S SS / BA-S SS / CR-S SS / \& PB-S SS / AG-S SS / MGO-S SS / NA2O-S SS / K2O-S \& SS / FE3O4-S SS / CAO-S SS

PROD-FRAC SE 1

BLOCK QUENCH2 RGIBBS

PARAM TEMP $=350$ PRES $=1$ <ATM $>$ NPHASE $=2$ MAXIT $=500$

PROD H2 / O2 / N2 / CL2 / F2 / NO / SO2 / H2O / \& $\mathrm{CO} 2 / \mathrm{CO} / \mathrm{HCL} / \mathrm{AS} / \mathrm{CD} / \mathrm{HG} / \mathrm{SE} / \mathrm{FE}-\mathrm{S} \mathrm{SS} \&$ / SIO2-S SS / AL2O3-S SS / AS-S SS / BA-S SS / \& CD-S SS / CR-S SS / PB-S SS / SE-S SS / AG-S SS \& / MGO-S SS / NA2O-S SS / K20-S SS / FE3O4-S SS / \& CAO-S SS

BLOCK REHEAT RGIBBS

PARAM TEMP $=220$ PRES $=1$ <ATM> NPHASE $=2$ CHEMEQ $=\mathrm{YES}$

PROD H2 / O2 / N2 / SO2 / $\mathrm{H} 2 \mathrm{O} / \mathrm{CO} 2 / \mathrm{CO}$

PROD-FRAC O2 $1 / \mathrm{H} 2 \mathrm{O} 1 / \mathrm{CO} 1$

BLOCK SCC2 RGIBBS

PARAM TEMP $=2200$ PRES $=1$ <ATM> NPHASE $=2$

PROD H2 $M / O 2 \mathrm{M} / \mathrm{N} 2 \mathrm{M} / \mathrm{CL} 2 \mathrm{M} / \mathrm{F} 2 \mathrm{M} / \mathrm{NO} \mathrm{M} / \&$ $\mathrm{SO} 2 \mathrm{M} / \mathrm{H} 20 \mathrm{M} / \mathrm{CO} 2 \mathrm{M} / \mathrm{CO} \mathrm{M} / \mathrm{HCL} / \mathrm{AS} / \mathrm{CD}$ / HG / SE / FE-S SS / SIO2-S SS / AL2O3-S SS / \& 
BA-S SS / CR-S SS / PB-S SS / AG-S SS / MGO-S \&

SS / NA2O-S SS / K2O-S SS / FE304-S SS / CAO-S SS

BLOCK SCRUB RGIBBS

PARAM TEMP $=160$ PRES $=1$ <ATM> NPHASE=2 MAXIT=500

PROD $\mathrm{H} 2 / \mathrm{O} 2 / \mathrm{N} 2 / \mathrm{CL} 2 / \mathrm{F} 2 / \mathrm{NO} / \mathrm{SO} 2 / \mathrm{H} 2 \mathrm{O} / \&$

$\mathrm{CO} 2 / \mathrm{CO} / \mathrm{HCL} / \mathrm{NAOH}-\mathrm{S}$ SS / NACL-S SS / NAF-S \&

SS

PROD-FRAC $\mathrm{CO} 2^{-1} 1 \mathrm{CO} 1 / \mathrm{SO} 21$

BLOCK SCRUB-2 RGIBBS

PARAM TEMP $=120$ PRES $=1$ <ATM> NPHASE $=2$ MAXIT $=500$

PROD H2 / O2/ N2/ CL2/ F2/ NO / SO2/ H2O/\& $\mathrm{CO} 2 / \mathrm{CO} / \mathrm{HCL} / \mathrm{C}-\mathrm{s}$ ss / s-s ss / NAOH-S ss / \& NACL-S SS

BLOCK VITRIFY RGIBBS

PARAM TEMP $=3000$ PRES $=1$ <ATM> NPHASE $=2$ MAXIT $=50$

$\mathrm{PROD} \mathrm{H} 2 / \mathrm{O} 2 / \mathrm{N} 2 / \mathrm{CL} 2 / \mathrm{F} 2 / \mathrm{NO} / \mathrm{SO} 2 / \mathrm{H} 2 \mathrm{O} / \&$ $\mathrm{CO} 2 / \mathrm{CO} / \mathrm{HCL} / \mathrm{C}-\mathrm{S} \mathrm{SS} / \mathrm{s} / \mathrm{AS} / \mathrm{CD} / \mathrm{HG} /$ \& $\mathrm{SE} / \mathrm{EE} / \mathrm{SIO} 2 / \mathrm{AL} 203 / \mathrm{BA}-\mathrm{S} \mathrm{SS} / \mathrm{CR}-\mathrm{S} \mathrm{SS} / \&$ PB-S SS / AG-S SS / MGO-S SS / NA2O-S SS / K20-S \& SS / FE $304 /$ CAO-S SS

BLOCK DUPL2 DUPI

DESIGN-SPEC SCRBH 202

DEFINE MASSAI STREAM-VAR STREAM=SCRBBOT2 SUBSTREAM=CISOLID \& VARIABLE=MASS-FLOW

DEFINE SCRUB STREAM-VAR STREAM=SCRBH2O2 SUBSTREAM=MIXED \& VARIABLE =MASS - ELOW

DEFINE NAOH STREAM-VAR STREAM=NAOH-2 SUBSTREAM=CISOLID \& VARIABLE=MASS-FLOW

SPEC "SCRUB" TO "20*MASSAL"

TOL-SPEC "0.01"

VARY STREAM-VAR STREAM=SCRBH2O2 SUBSTREAM=MIXED \& VARIABLE=MASS-FLOW

LIMITS "NAOH" "5000"

DESIGN-SPEC SCRUBH2O

DEFINE MASSAL STREAM-VAR STREAM=SCRUBBOT SUBSTREAM=CISOLID \& VARIABIE=MASS-FLOW

DEEINE SCRUB STREAM-VAR STREAM=SCRUBH2O SUBSTREAM=MIXED \& VARIABIE =MASS-FLOW

DEFINE NAOH STREAM-VAR STREAM=NAOH SUBSTREAM=CISOLID \& VARIABLE=MASS-ELOW

SPEC "SCRUB" TO "20*MASSAL"

TOL-SPEC "0.0I"

VARY STREAM-VAR STREAM=SCRUBH2O SUBSTREAM=MIXED \& VARIABLE=MASS-FLOW

LIMITS "NAOH" "5000"

CONV-OPTIONS

SECANT MAX-STEP-SIZ=.05 BRACKET=YES

STREAM-REPOR NOSORT MOLEFLOW MASSFLOW PROPERTIES=ALL-SUBS 


\section{System J-1}

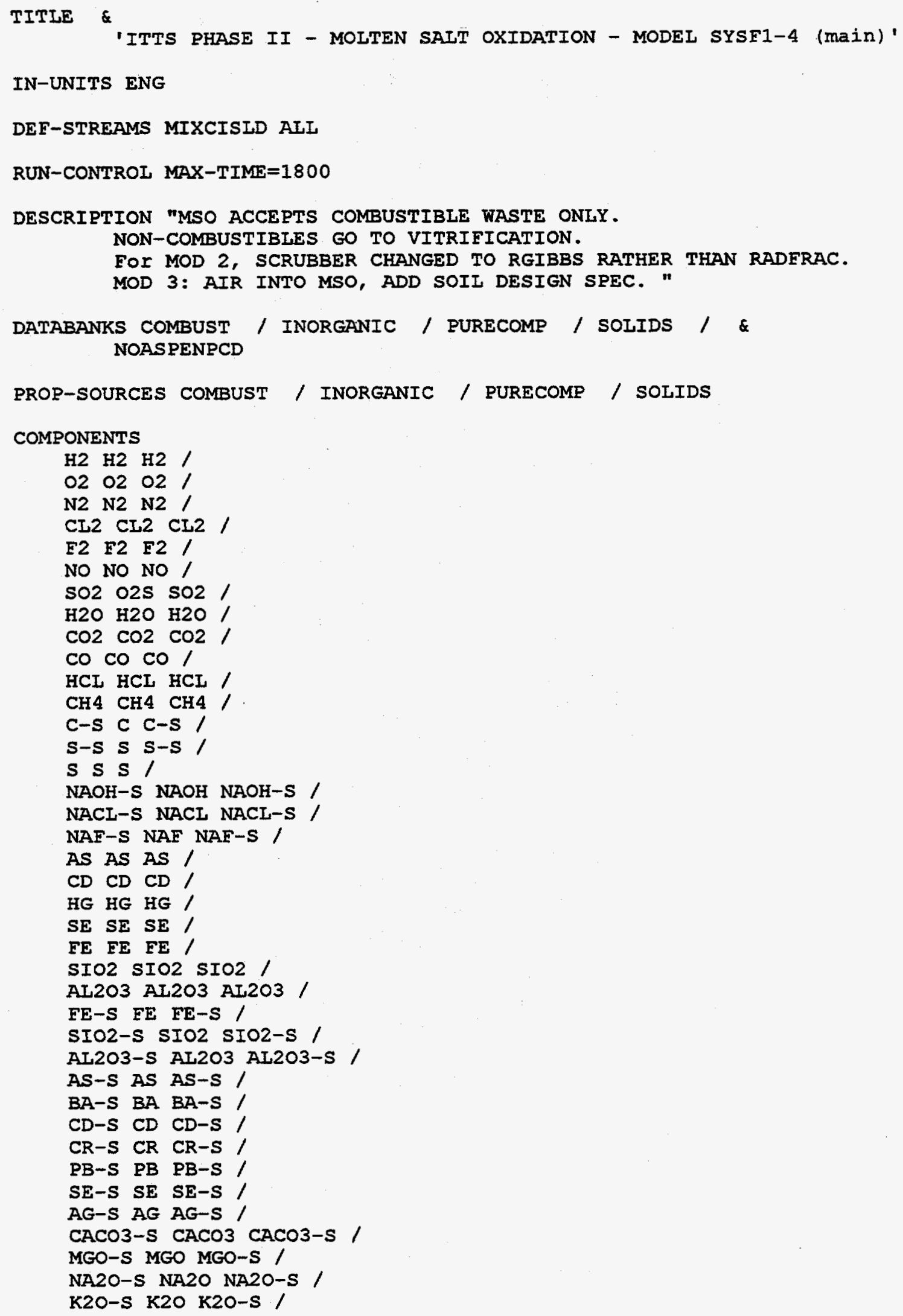




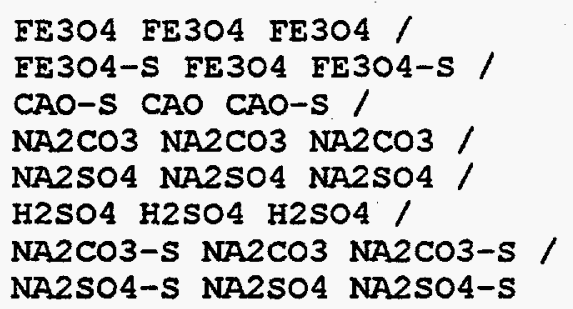

\section{FLOWSHEET}

BLOCK VITRIFY IN=SOLIDS OUT=VITOUT

BLOCK VIT-SEP IN=VITOUT OUT=VITVAP SLAG

BLOCK SCC IN=FUEIMIX1 EFFLUENT OUT=OUT1

BLOCK Q1 IN=DUM1IN OUT=DUM1OUT

BLOCK DUPL1 IN=OUT1 OUT=SCCOUT DUMIIN

BLOCK MELTER IN=FDMETMLT OUT=METAI

BLOCK QUENCH IN=SCCOUT $Q N C H 2 O$ OUT=QUENCHED

BLOCK BAGHOUSE IN=QUENCHED OUT=FILTGAS HGPLUS FLYASH

BLOCK FEED-SEP IN=SOIL FDWASTEN FLYASH FDWASTEC OUT $=$ \& SOLIDS ORGANICS

BLOCK PLENUM IN=ORGANICS VITVAP OXYGEN OUT=EFFLUENT

BLOCK L-V-SEP IN=SCRUBOUT OUT=SCRUBVAP SCRUBBOT

BLOCK SCRUB IN=SCRUBH2O NAOH FILTGAS OUT=SCRUBOUT

PROPERTIES SOLIDS

PROPERTIES IDEAL / RKS-BM

PROP-SET AIL-SUBS TEMP PRES VERAC UNITS='ATM' SUBSTREAM=AII

STREAM FDMETMLT

SUBSTREAM MIXED TEMP=68 PRES $=1$ <ATM> MASS-FLOW=1.20

MASS-FLOW H2 1.20

SUBSTREAM CISOLID TEMP=67 PRES=1 <ATM> MASS-FLOW=147.8

MASS-ELOW C-S $14.44 /$ FE-S $282.02 /$ SIO2-S $0.035 /$ \&

AL203-S $0.035 /$ BA-S $0.87 /$ CD-S 0.18

\section{STREAM FDWASTEC}

SUBSTREAM MIXED TEMP=68 PRES $=1$ 〈ATM>

MASS-FLOW H2 $50.95 / 0292.76 / \mathrm{N} 20.23 /$ CL2 $53.02 /$ \& E2 $0.11 / \mathrm{H} 2 \mathrm{O} 19.83$

SUBSTREAM CISOLID TEMP=68 PRES $=1$ 〈ATM>

MASS-FLOW C-S $326.28 /$ S-S $0.49 /$ FE-S $7.67 /$ SIO2-S \&

$53.01 /$ AL203-S 53.01/ BA-S $0.77 /$ CD-S $0.77 /$ CR-S 1.53

STREAM FDWASTEN

SUBSTREAM MIXED TEMP=68 PRES=1 〈ATM>

MASS-FLOW H2 $0.54 / 021.44 / \mathrm{N} 20.01 / \mathrm{CL} 214.88 / \&$

NO $0.66 / \mathrm{sO} 20.83 / \mathrm{H} 20354.23 / \mathrm{HG} 0.15$

SUBSTREAM CISOLID TEMP $=68$ PRES $=1$ <ATM>

MASS-FLOW C-S $4.77 /$ S-S $0.62 /$ SIO2-S $476.77 /$ \&

AL203-S $476.77 /$ As-S $0.06 /$ BA-S $0.04 / \mathrm{CD}-\mathrm{S} 2.63$

$/ \mathrm{CR}-\mathrm{S} 4.95 / \mathrm{PB}-\mathrm{S} 0.17 / \mathrm{SE}-\mathrm{S} 0.03 / \mathrm{AG}-\mathrm{S} 0.03$

STREAM ELYASH

SUBSTREAM MIXED TEMP=350 PRES=1 <ATM>

SUBSTREAM CISOLID TEMP $=350$ PRES $=1$ <ATM>

MASS-FLOW SIO2-S 43 / AI203-S 31 / NA2O-S 1 / FE304-S \&

$2 /$ CAO-S 2

STREAM FUELMIX1

SUBSTREAM MIXED TEMP=68 PRES=1 <ATM> MASS-FLOW=1742

MASS-ERAC O2 $0.222 / \mathrm{N} 20.732 / \mathrm{CH} 40.046$ 
STREAM NAOH

SUBSTREAM CISOLID TEMP=68 PRES=1 <ATM> MASS-ELOW=77.38

MASS-FRAC NAOH-S 1

STREAM OXYGEN

SUBSTREAM MIXED TEMP=68 PRES=1 <ATM> MASS-FLOW=1414

MASS-FRAC O2 1

STREAM QNCH2O

SUBSTREAM MIXED TEMP=68 PRES=1 〈ATM> MASS-FLOW=2293

MASS-ERAC H2O 1

STREAM SCRUBH2O

SUBSTREAM MIXED TEMP=68 PRES=1 <ATM> MASS-ELOW=5000

MASS-ERAC H2O 1

STREAM SOIL

SUBSTREAM MIXED TEMP=68 PRES=1 <ATM> MASS-ELOW=49.81

MASS-FLOW H2O . 100

SUBSTREAM CISOLID TEMP=68 PRES=1 <ATM> MASS-FLOW=448.3

MASS-FLOW SIO2-S $0.569 /$ AL2O3-S $0.102 /$ CACO3-S $0.128 /$ \& MGO-S $0.023 /$ NA2O-S $0.013 /$ K20-S $0.024 /$ FE3O4-S 0.041

BIOCK BAGHOUSE SEP

FRAC STREAM=FILTGAS SUBSTREAM=MIXED COMPS=H2 O2 N2 CL2 \& F2 NO SO2 H2O CO2 CO HCL CH4 C-S S-S S NAOH-S \& NACL-S NAF-S AS CD HG SE FE SIO2 AL203 FE-S SIO2-S \& AL203-S AS-S BA-S CD-S CR-S PB-S SE-S AG-S CACO3-S \& MGO-S NA2O-S K2O-S EE3O4 FE3O4-S CAO-S NA2CO3 NA2SO4 \&

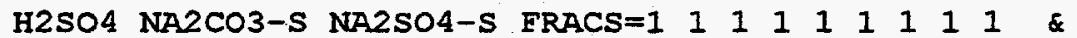
$\begin{array}{llllllllllllllllllllll}1 & 1 & 1 & 0 & 0 & 0 & 0 & 0 & 0 & 0 & 0 & 0 & 0 & 0 & 0 & 0 & 0 & 0 & 0 & 0 & 0 & \&\end{array}$ $\begin{array}{lllllllllllllllll}0 & 0 & 0 & 0 & 0 & 0 & 0 & 0 & 0 & 0 & 0 & 0 & 0 & 0 & 1 & 0 & 0\end{array}$

FRAC STREAM=FILTGAS SUBSTREAM=CISOLID COMPS=C-S S-S \& NAOH-S NACL-S NAF-S FE-S SIO2-S AL2O3-S AS-S BA-S CD-S CR-S PB-S SE-S AG-S CACO3-S MGO-S NA2O-S K2O-S \&

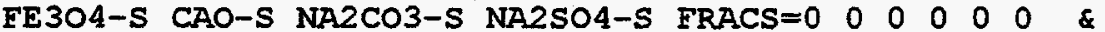
$0 \begin{array}{lllllllllllllllll}0 & 0 & 0 & 0 & 0 & 0 & 0 & 0 & 0 & 0 & 0 & 0 & 0 & 0 & 0 & 0\end{array}$

FRAC STREAM=HGPLUS SUBSTREAM=MIXED COMPS=H2 O2 N2 CL2 \& F2 NO SO2 H2O CO2 CO HCL CH4 C-S S-S S NAOH-S \& NACL-S NAF-S AS CD HG SE FE SIO2 AL2O3 FE-S SIO2-S \& AL203-S AS-S BA-S CD-S CR-S PB-S SE-S AG-S CACO3-S \& MGO-S NA2O-S K2O-S FE3O4 FE3O4-S CAO-S NA2CO3 NA2SO4 \&

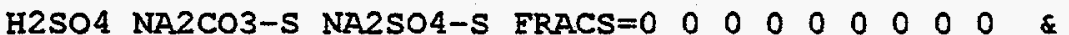
$\begin{array}{llllllllllllllllllllll}0 & 0 & 0 & 0 & 0 & 0 & 0 & 0 & 0 & 1 & 1 & 1 & 1 & 0 & 0 & 0 & 0 & 0 & 0 & 1 & 0 & \&\end{array}$

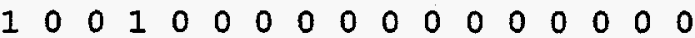

FRAC STREAM=HGPLUS SUBSTREAM=CISOLID COMPS=C-S $S-S$ NAOH-S \& NACL-S NAF-S FE-S SIO2-S AL2O3-S AS-S BA-S CD-S CR-S \& PB-S SE-S AG-S CACO3-S MGO-S NA2O-S K2O-S FE304-S \&

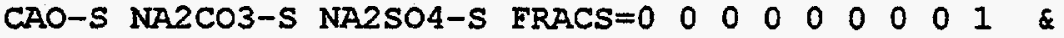
$\begin{array}{lllllllllllllll}0 & 1 & 0 & 0 & 1 & 0 & 0 & 0 & 0 & 0 & 0 & 0 & 0 & 0\end{array}$

BIOCK FEED-SEP SEP

ERAC STREAM=SOLIDS SUBSTREAM=MIXED COMPS=H2 O2 N2 CL2 \& F2 NO SO2 H2O $\mathrm{CO} 2 \mathrm{CO} \mathrm{HCL} \mathrm{CH} 4 \mathrm{C}-\mathrm{S}$ S-S S NAOH-S \& NACL-S NAF-S AS CD HG SE FE SIO2 AI2O3 FE-S SIO2-S \& AL203-S AS-S BA-S CD-S CR-S PB-S SE-S AG-S CACO3-S \& MGO-S NA2O-S K2O-S FE3O4 FE304-S CAO-S NA2CO3 NA2SO4 \&

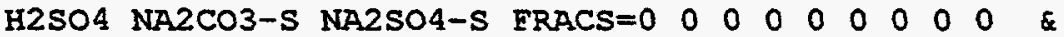
$\begin{array}{llllllllllllll}0 & 0 & 0 & .05 & .05 & .05 & .95 & .95 & .95 & .95 & .95 & .95 & .95 & .95\end{array}$ $\begin{array}{llllllllllll}.95 & .95 & .95 & .95 & .95 & .95 & .95 & .95 & .95 & .95 & .95 & .95\end{array}$

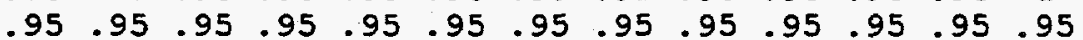

FRAC STREAM=SOLIDS SUBSTREAM=CISOLID COMPS=C-S $S-S$ NAOH-S \&

B-116 
NACL-S NAF-S FE-S SIO2-S AL2O3-S AS-S BA-S CD-S CR-S \& PB-S SE-S AG-S CACO3-S MGO-S NA2O-S K2O-S FE304-S \& CAO-S NA2CO3-S NA2SO4-S ERACS $=.05 \quad .05 \quad .95 \quad .95 \quad .95$ \& $\begin{array}{lllllllllllll}.95 & .95 & .95 & .95 & .95 & .95 & .95 & .95 & .95 & .95 & .95 & .95\end{array}$ $\begin{array}{llllll}.95 & .95 & .95 & .95 & .95 & .95\end{array}$

BLOCK VIT-SEP SEP

FRAC STREAM=SLAG SUBSTREAM=MIXED COMPS=H2 O2 N2 CL2 F2 \& NO SO2 H2O CO2 CO HCL CH4 C-S $\$$ NAF-S AS CD HG SE FE SIO2 AL2O3 FE-S SIO2-S AL2O3-S \& AS-S BA-S CD-S CR-S PB-S SE-S AG-S CACO3-S MGO-S \& NA2O-S K2O-S EE3O4 FE3O4-S CAO-S NA2CO3 NA2SO4 H2SO4 \&

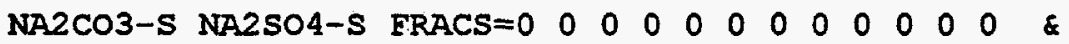
$\begin{array}{llllllllllllllllllllll}1 & 1 & 1 & 1 & 1 & 1 & 0 & 0 & 0 & 0 & 1 & 1 & 1 & 1 & 1 & 1 & 1 & 1 & 1 & 1 & 1 & \text { \& }\end{array}$ $\begin{array}{lllllllllllllll}1 & 1 & 1 & 1 & 1 & 1 & 1 & 1 & 1 & 1 & 1 & 1 & 1 & 1\end{array}$

FRAC STREAM=SIAG SUBSTREAM=CISOLID COMPS=C-S S-S NAOH-S \& NACL-S NAF-S FE-S SIO2-S AI203-S AS-S BA-S CD-S CR-S \& PB-S SE-S AG-S CACO3-S MGO-S NA2O-S K2O-S FE304-S \& CAO-S NA2CO3-S NA2SO4-S FRACS=1 $1111111111111 \%$ \& $\begin{array}{lllllllllllllll}1 & 1 & 1 & 1 & 1 & 1 & 1 & 1 & 1 & 1 & 1 & 1 & 1 & 1\end{array}$

BLOCK L-V-SEP FIASH2

PARAM TEMP $=120$ PRES $=1$ <ATM>

BLOCK MELTER RGIBBS

PARAM TEMP=3000 PRES=1 <ATMS NPHASE=2 VAPOR=YES MAXIT=500

PROD H2 / C-S SS / FE / SIO2 / AI203 / FE-S SS / \& SIO2-S SS / AL2O3-S SS / BA-S SS / CD / CD-S SS

BLOCK PLENUM RGIBBS

PARAM TEMP $=1800$ PRES $=1$ <ATM> NPHASE $=2$

$\mathrm{PROD} \mathrm{H} 2 / \mathrm{O} 2 / \mathrm{N} 2 / \mathrm{CL} 2 / \mathrm{F} 2 / \mathrm{NO} / \mathrm{SO} 2 / \mathrm{H} 2 \mathrm{O} /$ \& $\mathrm{CO} 2 / \mathrm{CO} / \mathrm{HCL} / \mathrm{C}-\mathrm{s} \mathrm{ss} / \mathrm{s}-\mathrm{s} \mathrm{ss} / \mathrm{s} / \mathrm{As} / \&$ CD / HG / SE / EE-S SS / SIO2-S SS / AI203-S SS \& $/ \mathrm{BA}-\mathrm{s}$ ss / CR-S ss / $\mathrm{PB}-\mathrm{s}$ ss / AG-S SS/MGO-S \& SS / NA2O-S SS / K2O-S SS / FE304-S SS / CAO-S SS

\section{BLOCK $Q 1$ RGIBBS}

PARAM TEMP $=68$ PRES $=1$ <ATM> NPHASE=2 TAPP=2132

$\mathrm{PROD} \mathrm{H} 2 / \mathrm{O} 2 / \mathrm{N} 2 / \mathrm{CL} 2 / \mathrm{F} 2 / \mathrm{NO} / \mathrm{SO} 2 / \mathrm{H} 2 \mathrm{O} /$ \& $\mathrm{CO} 2 / \mathrm{CO} / \mathrm{HCL} / \mathrm{AS} / \mathrm{CD} / \mathrm{HG} / \mathrm{SE} / \mathrm{FE}-\mathrm{S} \mathrm{SS} \&$ / SIO2-S SS / AL203-S SS / BA-S SS / CR-S SS / \& $\mathrm{PB}-\mathrm{S}$ SS / AG-S SS / MGO-S SS / NA2O-S SS/ $\mathrm{K} 2 \mathrm{O}-\mathrm{S}$ \& SS / FE304-S SS / CAO-S SS

\section{BLOCK QUENCH RGIBBS}

PARAM TEMP $=350$ PRES $=1$ <ATM> NPHASE $=2$

PROD H2 / O2 / N2 / $\mathrm{CL} 2 / \mathrm{F} 2 / \mathrm{NO} / \mathrm{SO} 2 / \mathrm{H} 2 \mathrm{O} / \&$ $\mathrm{CO} 2 / \mathrm{CO} / \mathrm{HCL} / \mathrm{AS} / \mathrm{CD} / \mathrm{HG} / \mathrm{SE} / \mathrm{EE}-\mathrm{S} \mathrm{SS} \&$ / SIO2-S SS / AL2O3-S SS / AS-S SS / BA-S SS / \& CD-S SS / CR-S SS / $\mathrm{PB}-\mathrm{S}$ SS/ SE-S SS/AG-S SS \& $/$ MGO-S SS / NA20-S SS / K20-S SS / FE304-S SS / CAO-S SS

\section{BLOCK SCC RGIBBS}

PARAM TEMP=2200 PRES=1 <ATM> NPHASE=2

PROD H2 M / O2 M / N2 M / CL2 M/ F2 M/ NO M / \& $\mathrm{SO} 2 \mathrm{M} / \mathrm{H} 20 \mathrm{M} / \mathrm{CO} 2 \mathrm{M} / \mathrm{CO} \mathrm{M} / \mathrm{HCL} / \mathrm{AS} / \mathrm{CD}$ \& $/ \mathrm{HG} / \mathrm{SE} / \mathrm{FE}-\mathrm{S}$ SS/SIO2-S SS/AL203-S SS / \& $\mathrm{BA}-\mathrm{S}$ SS / CR-S SS / $\mathrm{PB}-\mathrm{S}$ ss / AG-S SS / MGO-S \& SS / NA2O-S SS / K2O-S SS / FE304-S SS / CAO-S \& SS 
BLOCK SCRUB RGIBBS

PARAM TEMP=120 PRES=1 <ATM> NPHASE $=2$ MAXIT $=500$

$\mathrm{PROD} \mathrm{H} 2 / \mathrm{O} 2 / \mathrm{N} 2 / \mathrm{CL} 2 / \mathrm{F} 2 / \mathrm{NO} / \mathrm{SO} 2 / \mathrm{H} 2 \mathrm{O} / \&$ $\mathrm{CO} 2 / \mathrm{CO} / \mathrm{HCL} / \mathrm{C}-\mathrm{S}$ SS / S-S SS / NAOH-S SS / \& NACL-S SS

BLOCK VITRIFY RGIBBS

PARAM TEMP $=3000$ PRES=1 <ATM> NPHASE=2 MAXIT $=50$

PROD H2 / O2 / N2 / CL2 / E2 / NO / SO2 / H2O / \& $\mathrm{CO} 2 / \mathrm{CO} / \mathrm{HCL} / \mathrm{C}-\mathrm{s} \mathrm{sS} / \mathrm{s} / \mathrm{AS} / \mathrm{CD} / \mathrm{HG} / \&$ $\mathrm{SE} / \mathrm{FE} / \mathrm{SIO} 2 / \mathrm{AL} 203 / \mathrm{BA}-\mathrm{S}$ SS / CR-S SS / \& PB-S SS / AG-S SS / MGO-S SS / NA2O-S SS / K2O-S \& SS / FE $304 /$ CAO-S SS

BIOCK DUPLI DUPI

DESIGN-SPEC NAOH

DEFINE CAUIN MASS-FLOW STREAM=NAOH SUBSTREAM=CISOLID \& COMPONENT $=$ NAOH $-S$

DEEINE CAUOUT MASS-FLOW STREAM=SCRUBOUT SUBSTREAM=CISOLID \& COMPONENT $=$ NAOH $-S$

SPEC "CAUIN-100.*CAUOUT" TO "O"

TOL-SPEC "0.01"

VARY STREAM-VAR STREAM $=$ NAOH SUBSTREAM=CISOLID \& VARIABIE=MASS-FLOW

LIMITS "1" "1000"

DESIGN-SPEC OXYGEN

DEFINE OXIN1 MASS-FLOW STREAM=OXYGEN SUBSTREAM=MIXED \& COMPONENT $=02$

DEEINE OXIN2 MASS-FLOW STREAM=ORGANICS SUBSTREAM=MIXED \& COMPONENT $=02$

DEEINE OXOUT MASS-ELOW STREAM=EFFLUENT SUBSTREAM=MIXED \& COMPONENT $=02$

SPEC "OXIN1+OXIN2" TO "6.0*OXOUT"

TOI-SPEC "0.1"

VARY STREAM-VAR STREAM=OXYGEN SUBSTREAM=MIXED \& VARIABLE=MASS-ELOW

LIMITS " 1 " "100000"

DESIGN-SPEC QQUENCH

DEFINE QQNCH2 BLOCK-VAR BLOCK=QUENCH VARIABIE=QCALC\& SENTENCE = PARAM

SPEC "QQNCH2" TO "O"

TOL-SPEC " 1 ."

VARY STREAM-VAR STREAM=QNCH2O SUBSTREAM=MIXED \& VARIABLE=MASS-ELOW

LIMITS "1" "50000"

DESIGN-SPEC QSCC

DEFINE QSCC2 BLOCK-VAR BLOCK=SCC VARIABLE=QCALC \& SENTENCE=PARAM

DEFINE $Q 2$ BLOCK-VAR BLOCK=Q1 VARIABLE=QCAIC SENTENCE=PARAM SPEC "QSCC2" TO "0.05* $22 "$

TOL-SPEC "1."

VARY STREAM-VAR STREAM=FUEIMIX1 SUBSTREAM=MIXED \& VARIABLE=MASS-ELOW

LIMITS "1" "10000"

DESIGN-SPEC SCRUBH2O

DEEINE MASSAL STREAM-VAR STREAM=SCRUBBOT SUBSTREAM=CISOLID * VARIABIE $=$ MASS-FLOW 
DEFINE SCRUB STREAM-VAR STREAM=SCRUBH2O SUBSTREAM=MIXED

VARIABLE=MASS-FLOW

DEFINE NAOH STREAM-VAR STREAM=NAOH SUBSTREAM=CISOIID \& VARIABLE =MASS-FLOW

SPEC "SCRUB" TO "20*MASSAL"

TOL-SPEC "0.01"

VARY STREAM-VAR STREAM=SCRUBH2O SUBSTREAM=MIXED \& VARIABLE=MASS-FLOW

IIMITS "NAOH" "5000"

DESIGN-SPEC SOILH2O

DEFINE SLAGI STREAM-VAR STREAM=SIAG SUBSTREAM=MIXED VARIABLE=MASS-FIOW

DEFINE SLAG2 STREAM-VAR STREAM=SIAG SUBSTREAM=CISOLID \& VARIABLE=MASS- FLOW

DEFINE SOIL STREAM-VAR STREAM=SOIL SUBSTREAM=MIXED \& VARIABLE=MASS - FLOW

SPEC "30.* SOIL" TO "SLAGI+SLAG2"

TOL-SPEC "0.01"

VARY STREAM-VAR STREAM=SOIL SUBSTREAM=MIXED \& VARIABLE=MASS-ELOW

LIMITS " 1 " "1000"

DESIGN-SPEC SOILSOL

DEFINE SIAGI STREAM-VAR STREAM=SIAG SUBSTREAM=MIXED \& VARIABLE=MASS-FLOW

DEFINE SIAG2 STREAM-VAR STREAM=SIAG SUBSTREAM=CISOLID \& VARIABLE=MASS - FLOW

DEFINE SOIL STREAM-VAR STREAM=SOIL SUBSTREAM=CISOLID \& VARIABIE =MASS-FLOW

SPEC "3.33333*SOIL" TO "SLAGI+SLAG2"

TOL-SPEC "0.01"

VARY STREAM-VAR STREAM=SOIL SUBSTREAM=CISOIID \& VARIABLE=MASS-FLOW

LIMITS "1" "1000"

CONV-OPTIONS

SECANT MAX-STEP-SIZ=.05 BRACKET=YES

STREAM-REPOR NOSORT MOLEELOW MASSELOW PROPERTIES=ALL-SUBS 


\section{System K-1}

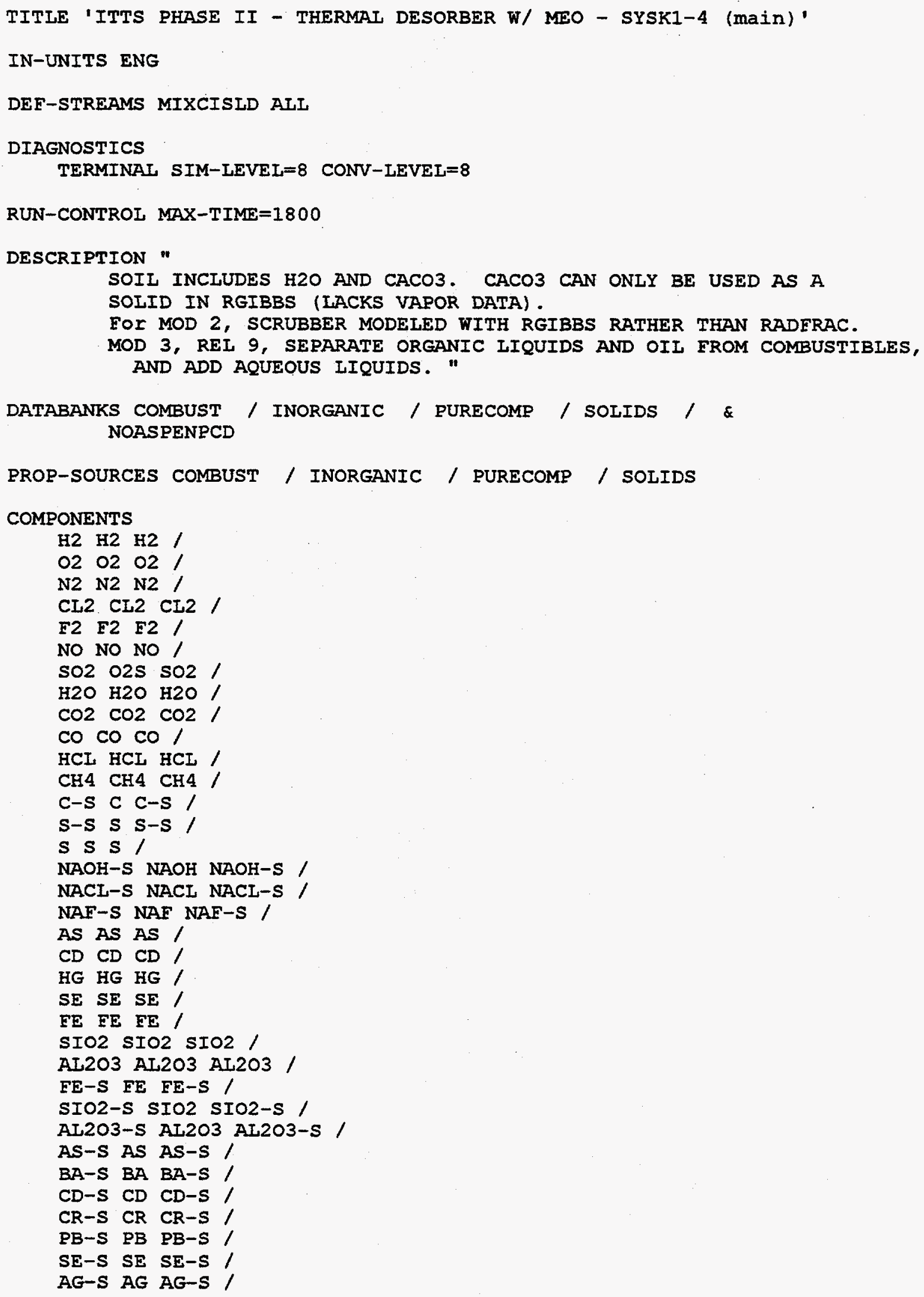




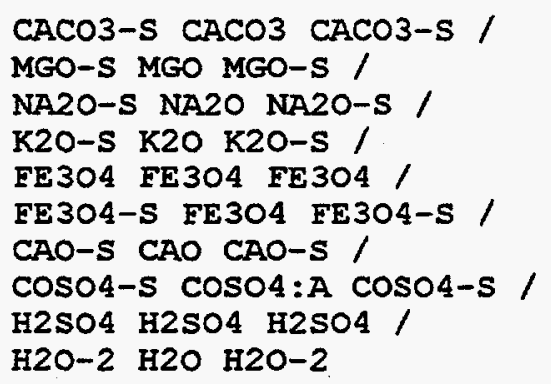

BLOCK SEPRATR IN=DESORBED OUT=SOLIDS KILNVAP

BLOCK L-V-SEP IN=SCRUBOUT OUT=SCRUBVAP SCRUBBOT

BLOCK COND-SEP IN=COOLED OUT=CONDENS 8 VAPORS HGPLUS

BLOCK SCRUB IN=NAOH SCRUBH2O GASES OUT=SCRUBOUT

BLOCK MELTER IN=FDMETMLT OUT=METAL

BLOCK MEO-UNIT IN=WATER FDWOLIQS CONDENS8 FDAQORGS \& ELECTROI H2SO4 OUT=MEOGAS

BLOCK CATALYST IN=VAPORS AIR HYDROGEN SCRUBVAP OUT $=\approx$ EXHAUST2

BLOCK MEO-SEP IN=MEOGAS OUT=GASES HYDROGEN H2SO4 COSO4\& H2O-2

BLOCK HEATER IN=FUELMIX1 OUT=EXHAUST1

BLOCK DESORBER IN=FDWASTEN FDWC-ORG OUT=DESORBED

BLOCK CONDENSR IN=KILNVAP OUT=COOLED

PROPERTIES SOLIDS

PROPERTIES IDEAI / RKS-BM

PROP-SET ALL-SUBS TEMP PRES VFRAC UNITS='ATM' SUBSTREAM=ALI

STREAM AIR

SUBSTREAM MIXED TEMP=68 PRES=1 <ATM> MASS-FLOW=2960.2

MASS-FLOW $020.233 /$ N2 0.767

STREAM ELECTROL

SUBSTREAM MIXED TEMP=68 PRES=1 <ATM>

MASS-FLOW H2SO4 $1.05903 / \mathrm{H} 20-2953.13$

SUBSTREAM CISOLID TEMP=68 PRES=1 <ATM>

MASS-FLOW COSO4-S 82.0751

STREAM FDAQORGS

SUBSTREAM MIXED TEMP $=68$ PRES $=1$ <ATM>

MASS-FLOW H2 $0.02 / \mathrm{CL} 20.13 / \mathrm{H} 2 \mathrm{O} 15.44$

SUBSTREAM CISOLID TEMP $=68$ PRES=1 <ATM>

MASS-FLOW C-S 0.17

STREAM FDMETMLT

SUBSTREAM MIXED TEMP=68 PRES=1 <ATM> MASS-FLOW=1.2

MASS-FLOW H2 2.42

SUBSTREAM CISOLID TEMP $=68$ PRES $=1$ <ATM> MASS-FLOW=147.8

MASS-FLOW C-S 14.44/ FE-S $282.02 /$ SIO2-S $0.035 / \&$

AL203-S $0.035 /$ BA-S $0.87 /$ CD-S 0.18

STREAM FDWASTEN

SUBSTREAM MIXED TEMP $=68$ PRES $=1$ <ATM>

MASS-FLOW H2 $0.54 / 021.44 / \mathrm{N} 20.01 / \mathrm{CL} 214.88 / \&$ NO $0.66 / \mathrm{SO} 0.83 / \mathrm{H} 20354.23 / \mathrm{HG} 0.15$

SUBSTREAM CISOLID TEMP=68 PRES $=1$ <ATM>

MASS-FLOW C-S $4.77 /$ S-S $0.62 /$ SIO2-S $476.77 /$ \&

AL203-S $476.77 /$ AS-S $0.06 /$ BA-S $0.04 /$ CD-S 2.63 


\section{STREAM FDWC-ORG}

SUBSTREAM MIXED TEMP $=68$ PRES $=1$ <ATM>

MASS-FLOW H2 $47.21 / 0291.70 / \mathrm{N} 20.23 / \mathrm{CL} 235.12 /$ \&

F2 $0.11 / \mathrm{H} 2019.24$

SUBSTREAM CISOLID TEMP=68 PRES $=1$ <ATM>

MASS-ELOW C-S $299.15 /$ S-S $0.39 /$ EE-S $7.67 /$ SIO2-S \& $52.91 / \mathrm{AL} 203-\mathrm{S} 52.91 / \mathrm{BA}-\mathrm{S} 0.77 / \mathrm{CD}-\mathrm{S} 0.77 / \mathrm{CR}-\mathrm{S} 1.53$

\section{STREAM FDWOLIQS}

SUBSTREAM MIXED TEMP $=68$ PRES $=1$ <ATM>

MASS-FLOW H2 $3.74 / 021.06 /$ CL2 $17.90 /$ H2O 0.59

SUBSTREAM CISOLID TEMP $=68$ PRES $=1$ <ATM>

MASS-FLOW C-S $27.13 /$ S-S $0.10 /$ SIO2-S $0.10 /$ AL203-S 0.10

STREAM FUETMIXI

SUBSTREAM MIXED TEMP=68 PRES=1 <ATM> MASS-FLOW=1282.9

MASS-FLOW $020.222 / \mathrm{N} 20.732 / \mathrm{CH} 40.046$

STREAM H2SO4

SUBSTREAM MIXED TEMP=140 PRES=1 <ATM>

MASS-FLOW H2SO4 103.863

STREAM NAOH

SUBSTREAM MIXED TEMP=68 PRES=1 <ATM>

SUBSTREAM CISOLID TEMP=68 PRES=1 <ATM> MASS-FLOW=30.8449 MASS-ELOW NAOH-S 1

STREAM SCRUBH2O

SUBSTREAM MIXED TEMP=68 PRES=1 〈ATM>

MASS-ELOW H2O 1

STREAM WATER

SUBSTREAM MIXED TEMP=68 PRES=1 <ATM> MASS-FLOW=. 1

MASS-ELOW H2O 1

BLOCK COND-SEP SEP

FRAC STREAM=VAPORS SUBSTREAM=MIXED COMPS=H2 O2 N2 CL2 \&

F2 NO SO2 H2O CO2 CO HCL CH4 C-S S-S S NAOH-S \& NACL-S NAF-S AS CD HG SE FE SIO2 AL2O3 FE-S SIO2-S \& AL203-S AS-S BA-S CD-S CR-S PB-S SE-S AG-S CACO3-S \& MGO-S NA20-S K2O-S FE304 FE304-S CAO-S FRACS $=.1 .1$ \& $\begin{array}{lllllllllllllllll}.1 & .1 & .1 & .1 & .1 & .1 & .1 & .1 & .1 & .1 & .1 & .1 & .1 & .1 & .1 & 1\end{array}$

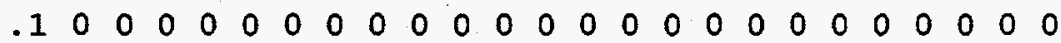

ERAC STREAM=VAPORS SUBSTREAM=CISOLID COMPS=C-S S-S NAOH-S \& NACL-S NAF-S FE-S SIO2-S AI203-S AS-S BA-S CD-S CR-S \& PB-S SE-S AG-S CACO3-S MGO-S NA2O-S K2O-S FE3O4-S \&

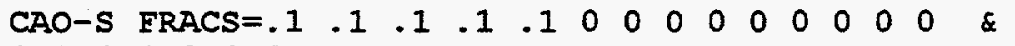
$\begin{array}{lllllll}0 & 0 & 0 & 0 & 0 & 0 & 0\end{array}$

FRAC STREAM=HGPLUS SUBSTREAM=MIXED COMPS=H2 O2 N2 CL2 \& E2 NO SO2 H2O CO2 CO HCL CH4 C-S S-S S NAOH-S \& NACL-S NAF-S AS CD HG SE FE SIO2 AL2O3 FE-S SIO2-S \& AI203-S AS-S BA-S CD-S CR-S PB-S SE-S AG-S CACO3-S \& MGO-S NA2O-S K2O-S FE304 EE304-S CAO-S FRACS $=0$ O 0 \& $\begin{array}{llllllllllllllllllllll}0 & 0 & 0 & 0 & 0 & 0 & 0 & 0 & 0 & 0 & 0 & 0 & 0 & 0 & 0 & 1 & 1 & 1 & 1 & 0 & 0 & \text { \& }\end{array}$ $\begin{array}{llllllllllllllllll}0 & 0 & 0 & 0 & 1 & 0 & 1 & 0 & 0 & 1 & 0 & 0 & 0 & 0 & 0 & 0 & 0 & 0\end{array}$

FRAC STREAM=HGPLUS SUBSTREAM=CISOLID COMPS=C-S $S-S$ NAOH-S \& NACL-S NAF-S FE-S SIO2-S AL203-S AS-S BA-S CD-S CR-S \& PB-S SE-S AG-S CACO3-S MGO-S NA2O-S K2O-S FE3O4-S \& CAO-S FRACS $=0 \begin{array}{llllllllllllllllllllll}0 & 0 & 0 & 0 & 0 & 0 & 0 & 1 & 0 & 1 & 0 & 0 & 1 & 0 & 0 & 0 & 0 & 0 & 0 & 0\end{array}$ 
BLOCK MEO-SEP SEP

FRAC STREAM=GASES SUBSTREAM=MIXED COMPS=H2 O2 N2 CL2 F2 \& NO SO2 H2O CO2 CO HCL CH4 C-S S-S S NAOH-S NACL-S \& NAF-S AS CD HG SE FE SIO2 AL2O3 FE-S SIO2-S AL2O3-S \& AS-S BA-S CD-S CR-S PB-S SE-S AG-S CACO3-S MGO-S \& NA2O-S K2O-S FE3O4 FE3O4-S CAO-S COSO4-S H2SO4 H2O-2 \& ERACS $=0 \begin{array}{llllllllllllllllll} & 1 & 1 & 1 & 1 & 1 & 1 & 1 & 1 & 1 & 1 & 1 & 1 & 1 & 1 & 1 & 1 & 1\end{array}$ $\begin{array}{llllllllllllllllllllllllllll}1 & 1 & 1 & 1 & 1 & 1 & 1 & 1 & 1 & 1 & 1 & 1 & 1 & 1 & 1 & 1 & 1 & 1 & 1 & 1 & 1 & 1 & 1 & 1 & 0 & 0 & 0\end{array}$

FRAC STREAM=GASES SUBSTREAM=CISOLID COMPS=C-S S-S NAOH-S \& NACL-S NAF-S FE-S SIO2-S AL2O3-S AS-S BA-S CD-S CR-S \& PB-S SE-S AG-S CACO3-S MGO-S NA2O-S K2O-S FE3O4-S \& CAO-S COSO4-S FRACS= $1 \begin{array}{lllllllllllll}1 & 1 & 1 & 1 & 1 & 1 & 1 & 1 & 1 & 1 & 1 & 1 & \text { \& }\end{array}$ $\begin{array}{llllllllll}1 & 1 & 1 & 1 & 1 & 1 & 1 & 1 & 0\end{array}$

FRAC STREAM=HYDROGEN SUBSTREAM=MIXED COMPS=H2 O2 N2 CL2 \& E2 NO SO2 H2O CO2 CO HCL CH4 C-S S-S S NAOH-S \& NACL-S NAF-S AS CD HG SE EE SIO2 AL2O3 FE-S SIO2-S \& AL2O3-S AS-S BA-S CD-S CR-S PB-S SE-S AG-S CACO3-S \& MGO-S NA2O-S K2O-S FE3O4 EE3O4-S CAO-S COSO4-S H2SO4 \&

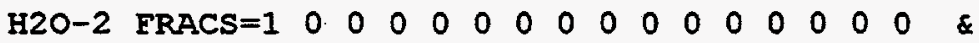
$0 \begin{array}{lllllllllllllllllllll}0 & 0 & 0 & 0 & 0 & 0 & 0 & 0 & 0 & 0 & 0 & 0 & 0 & 0 & 0 & 0 & 0 & 0 & 0 & 0 & \&\end{array}$ $\begin{array}{llllllll}0 & 0 & 0 & 0 & 0 & 0 & 0 & 0\end{array}$

FRAC STREAM=HYDROGEN SUBSTREAM=CISOLID COMPS=C-S S-S \& NAOH-S NACL-S NAF-S FE-S SIO2-S AL2O3-S AS-S BA-S \& CD-S CR-S PB-S SE-S AG-S CACO3-S MGO-S NA2O-S K2O-S \& FE304-S CAO-S COSO4-S FRACS $=0 \begin{array}{lllllllllll}0 & 0 & 0 & 0 & 0 & 0 & 0 & 0 & 0 & 0 & \text { \& }\end{array}$ $0 \begin{array}{lllllllllll}0 & 0 & 0 & 0 & 0 & 0 & 0 & 0 & 0 & 0 & 0\end{array}$

FRAC STREAM=H2SO4 SUBSTREAM=MIXED COMPS=H2 O2 N2 CL2 F2 \& NO SO2 H2O CO2 CO HCL CH4 C-S S-S S NAOH-S NACL-S \& NAF-S AS CD HG SE FE SIO2 AL203 FE-S SIO2-S AL2O3-S \& AS-S BA-S CD-S CR-S PB-S SE-S AG-S CACO3-S MGO-S \& NA2O-S K2O-S FE3O4 EE3O4-S CAO-S COSO4-S H2SO4 H2O-2 \& ERACS $=0 \begin{array}{lllllllllllllllllll}0 & 0 & 0 & 0 & 0 & 0 & 0 & 0 & 0 & 0 & 0 & 0 & 0 & 0 & 0 & 0 & 0 & 0 & \text { \& }\end{array}$ $0 \begin{array}{llllllllllllllllllll}0 & 0 & 0 & 0 & 0 & 0 & 0 & 0 & 0 & 0 & 0 & 0 & 0 & 0 & 0 & 0 & 0 & 0 & 0 & 0\end{array}$ $\begin{array}{llllllll}0 & 0 & 0 & 0 & 0.99 & 0\end{array}$

FRAC STREAM=H2SO4 SUBSTREAM=CISOLID COMPS=C-S S-S NAOH-S NACI-S NAF-S FE-S SIO2-S AL2O3-S AS-S BA-S CD-S CR-S PB-S SE-S AG-S CACO3-S MGO-S NA2O-S K2O-S FE3O4-S\&

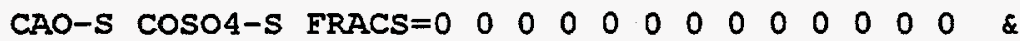
$\begin{array}{llllllllll}0 & 0 & 0 & 0 & 0 & 0 & 0 & 0 & 0\end{array}$

FRAC STREAM=COSO 4 SUBSTREAM=MIXED COMPS=H2 O2 N2 CL2 E2 \& NO SO2 H2O CO2 CO HCI CH4 C-S $5-s$ s NAOH-S NACL-S \& NAF-S AS CD HG SE FE SIO2 AI2O3 FE-S SIO2-S AI2O3-S \& AS-S BA-S CD-S CR-S PB-S SE-S AG-S CACO3-S MGO-S \& NA2O-S K2O-S FE3O4 FE3O4-S CAO-S COSO4-S H2SO4 H2O-2 \& FRACS $=0 \begin{array}{llllllllllllllllll}0 & 0 & 0 & 0 & 0 & 0 & 0 & 0 & 0 & 0 & 0 & 0 & 0 & 0 & 0 & 0 & 0 & 0\end{array}$ $0 \begin{array}{llllllllllllllllllllllllll}0 & 0 & 0 & 0 & 0 & 0 & 0 & 0 & 0 & 0 & 0 & 0 & 0 & 0 & 0 & 0 & 0 & 0 & 0 & 0 & 0 & 0 & 0 & 1 & 0 & 0\end{array}$

FRAC STREAM=COSO 4 SUBSTREAM=CISOLID COMPS=C-S $S-S$ NAOH-S \& NACL-S NAF-S FE-S SIO2-S AL2O3-S AS-S BA-S CD-S CR-S \& PB-S SE-S AG-S CACO3-S MGO-S NA2O-S K2O-S FE3O4-S \& CAO-S COSO4-S FRACS $=0 \begin{array}{lllllllllllll}0 & 0 & 0 & 0 & 0 & 0 & 0 & 0 & 0 & 0 & 0 & 0\end{array}$ $\begin{array}{llllllllll}0 & 0 & 0 & 0 & 0 & 0 & 0 & 0 & 1\end{array}$

FIASH-SPECS COSO 4 KODE $=0$

BIOCK SEPRATR SEP

FRAC STREAM=SOLIDS SUBSTREAM=MIXED COMPS=H2 O2 N2 CL2 \& F2 NO SO2 H2O CO2 CO HCI CH4 C-S s-S S NAOH-S \& NACL-S NAF-S AS CD HG SE FE SIO2 AL2O3 FE-S SIO2-S \& AL2O3-S AS-S BA-S CD-S CR-S PB-S SE-S AG-S CACO3-S \& MGO-S NA2O-S K2O-S FE304 FE304-S CAO-S H2O-2 FRACS $=.8$ \& $\begin{array}{llllllllllllllllll}.8 & .8 & .8 & .8 & .8 & .8 & .2 & .8 & .8 & .8 & .8 & .8 & 1 & 1 & 1 & 1 & 1 & \&\end{array}$ $\begin{array}{llllllllllllllllllllllllll}0 & 0 & 0 & 0 & 1 & 1 & 1 & 1 & 1 & 1 & 1 & 1 & 1 & 1 & 1 & 1 & 1 & 1 & 1 & 1 & 1 & 1 & 1 & 1 & 0\end{array}$

FRAC STREAM=SOIIDS SUBSTREAM=CISOLID COMPS=C-S $S-S$ NAOH-S \& 
NACI-S NAF-S FE-S SIO2-S AL2O3-S AS-S BA-S CD-S CR-S \& PB-S SE-S AG-S CACO3-S MGO-S NA2O-S K2O-S FE3O4-S \&

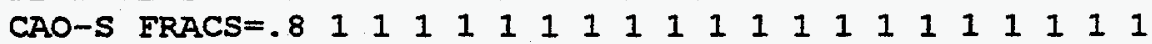

BLOCK L-V-SEP FLASH2

PARAM TEMP=120 PRES=1 <ATM>

BLOCK CATALYST RSTOIC

PARAM TEMP $=1500$ PRES $=1$ <ATM

STOIC 1 MIXED CO $-1 / 02-0.5 / \mathrm{CO} 1$

STOIC 2 MIXED CH4 $-1 / \mathrm{O} 2-2 / \mathrm{H} 2 \mathrm{O} 2 / \mathrm{CO} 21$

STOIC 3 MIXED H2 - $1 /$ O2 $-0.5 / \mathrm{H} 2 \mathrm{O} 1$

STOIC 4 MIXED O2 - 1 / CISOLID C-S -1/ MIXED CO2 1

CONV 1 MIXED CO 1

CONV 2 MIXED CH4 1

CONV 3 MIXED H2 1

CONV 4 CISOLID C-S 1

BLOCK MEO-UNIT RSTOIC

PARAM TEMP $=140$ PRES $=1$ <ATM

STOIC 1 MIXED CO $-1 / 02-0.5 / \mathrm{CO} 1$

STOIC 2 MIXED H2O $-1 / \mathrm{H} 21 / \mathrm{O} 20.5$

STOIC 3 MIXED CH4 -1/ O2 $-1 / \mathrm{CO} 21 / \mathrm{H} 22$

STOIC 4 MIXED HCL $-2 / \mathrm{H} 2$ I / CL2 1

STOIC 5 MIXED $02-1 /$ CISOLID C-S $-1 /$ MIXED CO2 1

CONV 1 MIXED CO 1

CONV 2 MIXED H2O 1

CONV 3 MIXED CH4 1

CONV 4 MIXED HCL 1

CONV 5 CISOLID C-S 1

BLOCK CONDENSR RGIBBS

PARAM TEMP $=80$ RRES=1 <ATM> NPHASE $=2$

PROD H2 / O2 / N2 / CL2 / F2 / NO / SO2 / H2O / \&

C-s SS / S-s SS / S / AS / CD / HG / SE / \&

FE-S SS / SIO2-S SS / AL2O3-S SS / AS-S SS / \&

BA-S SS / CD-S SS / CR-S SS / PB-S SS / SE-S SS / AG-S SS

PROD-FRAC H2 1/O21/ H2O 1

BLOCK DESORBER RGIBBS

PARAM TEMP $=600$ PRES $=1$ <ATM> NPHASE $=2$ CHEMEQ $=Y E S$

PROD H2 / O2 / N2 / CL2 / F2 / NO / SO2 / H2O / \&

$\mathrm{C}-\mathrm{S} S \mathrm{SS} / \mathrm{S}-\mathrm{S} \mathrm{SS} / \mathrm{S} / \mathrm{AS} / \mathrm{CD} / \mathrm{HG} / \mathrm{SE} / \&$

FE-S SS / SIO2-S SS / AL2O3-S SS / AS-S SS / \&

BA-S SS / CD-S SS / CR-S SS / PB-S SS / SE-S SS / AG-S SS

PROD-FRAC H2O $1 / \mathrm{O} 21 / \mathrm{H} 21$

BLOCK HEATER RGIBBS

PARAM TEMP=1400 PRES=1 $\angle A T M>$

PROD H2 / O2 / $\mathrm{N} 2 / \mathrm{NO} / \mathrm{H} 2 \mathrm{O} / \mathrm{CO} 2 / \mathrm{CO}$

BLOCK MELTER RGIBBS

PARAM TEMP $=3000$ PRES $=1$ <ATM> NPHASE $=2$ VAPOR=YES

PROD H2 M / C-S SS / FE / SIO2 / AL2O3 / FE-S SS / \& SIO2-S SS / AL2O3-S SS / BA-S SS / CD / CD-S SS

BLOCK SCRUB RGIBBS

PARAM TEMP=120 PRES=1 <ATM> NPHASE=2 CHEMEQ=YES MAXIT $=500$ \& NPSOL $=0$

PROD O2 / N2 / NO / SO2 / H2O / $\mathrm{CO} 2$ / HCL / S-S \& SS / NAOH-S SS / NACL-S SS / NAF-S SS / SIO2-S \&

SS / AL2O3-S SS 
DESIGN-SPEC AIR

DEEINE OXIN1 MASS-ELOW STREAM=AIR SUBSTREAM=MIXED \& COMPONENT $=02$

DEFINE OXIN2 MASS-FIOW STREAM=VAPORS SUBSTREAM=MIXED \& COMPONENT $=02$

DEFINE OXIN3 MASS-FLOW STREAM=SCRUBVAP SUBSTREAM=MIXED \& COMPONENT $=02$

DEEINE OXOUT MASS-ELOW STREAM=EXHAUST2 SUBSTREAM=MIXED \& COMPONENT $=02$

SPEC "OXIN1+OXIN2+OXIN3" TO "2.0*OXOUT"

TOL-SPEC " 0.01 "

VARY STREAM-VAR STREAM=AIR SUBSTREAM=MIXED \& VARIABLE=MASS- FLOW

IIMITS "I" "10000"

DESIGN-SPEC ELECTMIX

DEEINE AI STREAM-VAR STREAM=FDAQORGS SUBSTREAM=MIXED \& VARIABLE =MASS-ELOW

DEFINE A2 STREAM-VAR STREAM=FDAQORGS SUBSTREAM=CISOLID \& VARIABLE=MASS - ELOW

DEFINE A MASS-FIOW STREAM=FDAQORGS SUBSTREAM=MIXED \& COMPONENT $=\mathrm{H} 20$

DEEINE II STREAM-VAR STREAM=FDWOLIQS SUBSTREAM=MIXED \& VARIABLE=MASS - FLOW

DEEINE I2 STREAM-VAR STREAM=FDWOLIQS SUBSTREAM=CISOLID \& VARIABLE=MASS - FLOW

DEFINE I MASS-FLOW STREAM=FDWOLIQS SUBSTREAM=MIXED \& COMPONENT $=\mathrm{H} 2 \mathrm{O}$

DEFINE CI STREAM-VAR STREAM=CONDENS\& SUBSTREAM=MIXED \& VARIABLE = MASS - ELOW

DEFINE C2 STREAM-VAR STREAM=CONDENS8 SUBSTREAM=CISOLID \& VARIABIE=MASS-FLOW

DEFINE C MASS-FLOW STREAM=CONDENS8 SUBSTREAM=MIXED \& COMPONENT $=\mathrm{H} 2 \mathrm{O}$

DEFINE ELECT STREAM-VAR STREAM=ELECTROL SUBSTREAM=MIXED \& VARIABLE =MASS $-\mathrm{ELOW}$

SPEC "A1+A2+L1+L2+C1+C2-A-I-C " TO "ELECT/6.82"

TOL-SPEC "0.01"

VARY STREAM-VAR STREAM=ELECTROI SUBSTREAM=MIXED \& VARIABLE=MASS-ELOW

LIMITS "0.1" "10000"

DESIGN-SPEC ELECTSOI

DEFINE MIX STREAM-VAR STREAM=ELECTROI SUBSTREAM=MIXED \& VARIABLE=MASS-ELOW

DEEINE SOL STREAM-VAR STREAM=ELECTROL SUBSTREAM=CISOLID \& VARIABLE=MASS - FLOW

SPEC "SOL" TO "MIX*77.5/901."

TOI-SPEC "0.01"

VARY STREAM-VAR STREAM=ELECTROL SUBSTREAM=CISOIID \& VARIABLE=MASS - ELOW

LIMITS "I" "1000"

DESIGN-SPEC NAOH

DEEINE CAUIN MASS-ELOW STREAM=NAOH SUBSTREAM=CISOLID \& COMPONENT $=$ NAOH $-S$

DEFINE CAUOUT MASS-FLOW STREAM=SCRUBOUT SUBSTREAM=CISOIID \& COMPONENT $=$ NAOH $-S$

SPEC "CAUIN" TO "CAUOUT*100."

TOL-SPEC "0.01"

VARY STREAM-VAR STREAM=NAOH SUBSTREAM=CISOLID \& VARIABLE=MASS - FLOW 


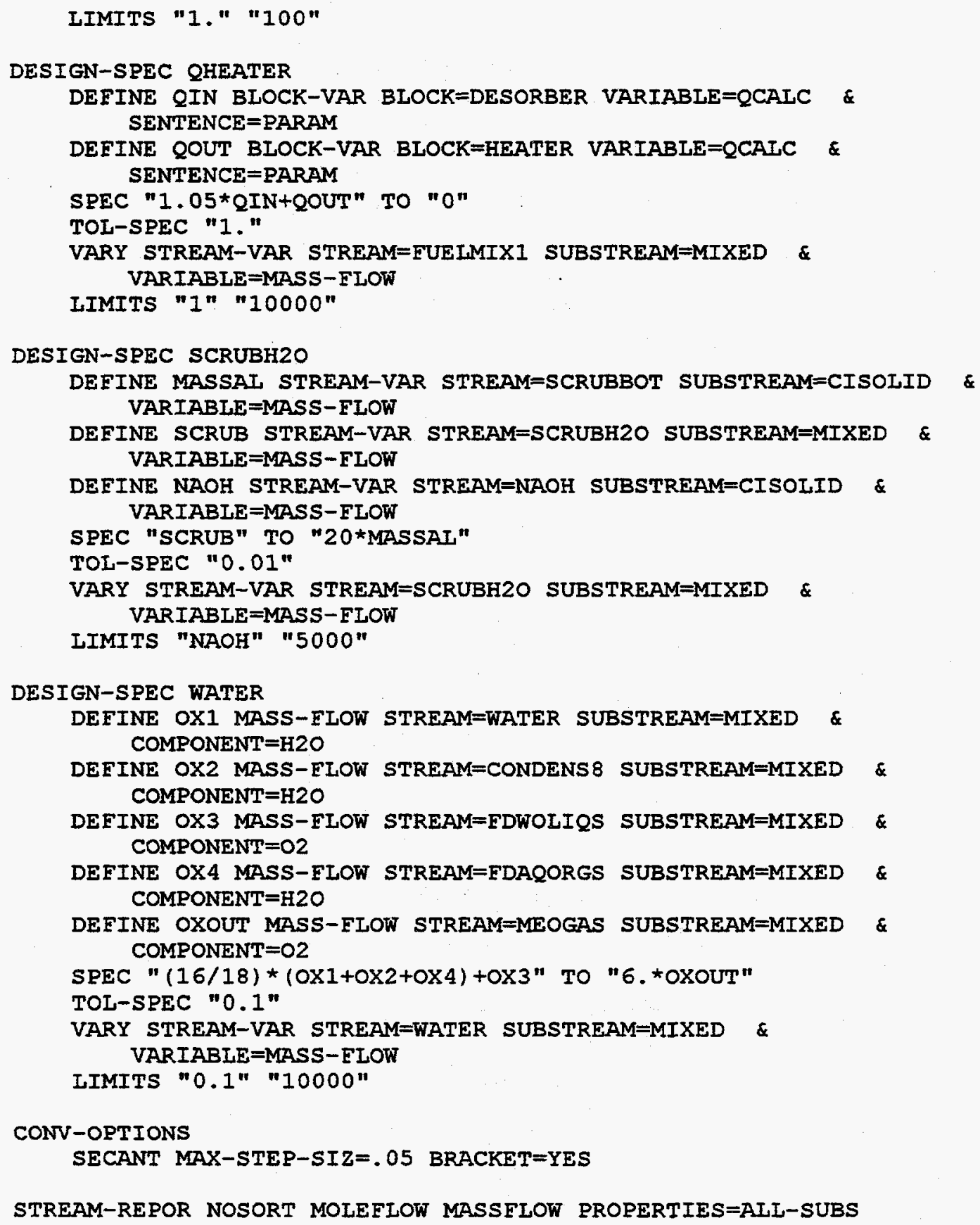




\section{System L-1}

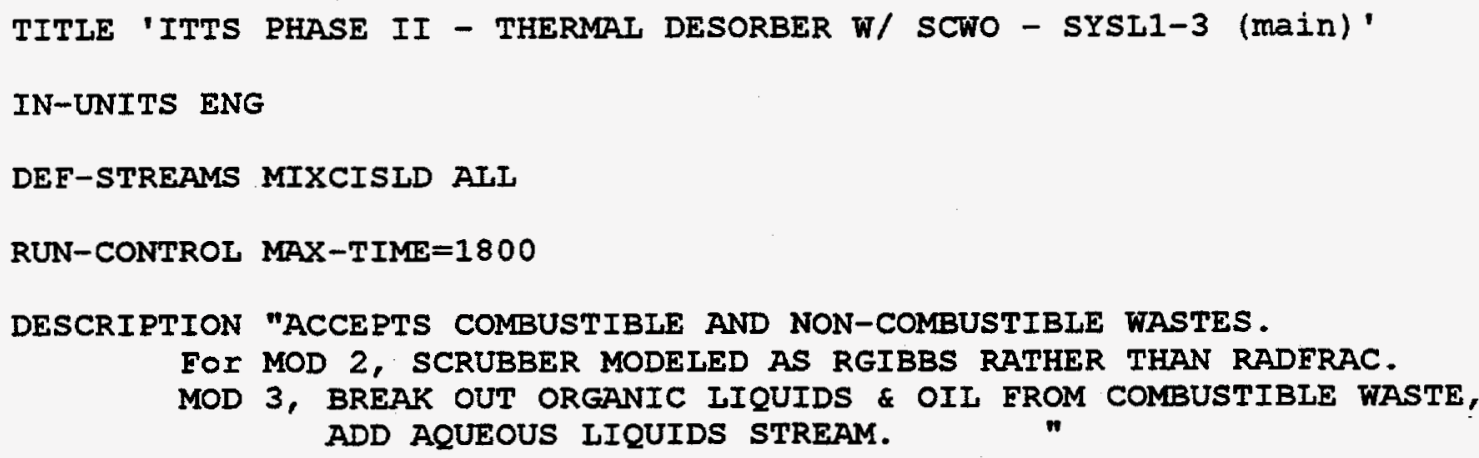
ADD AQUEOUS IIQUIDS STREAM.

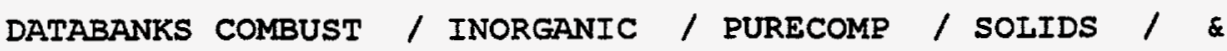


FE304-S FE304 FE304-S /

CAO-S CAO CAO-S

HENRY-COMPS GLOBAL CO2 CL2 SO2 HCL

FLOWSHEET

BLOCK SEPRATR IN=DESORBED OUT=SOLIDS KILNVAP

BIOCK SCWO-SEP IN=SCWOPROD OUT=SALTSOLN RECYCLE OTHER

BLOCK FLASH IN=OTHER NON-COND OUT=OFFGAS BOTTOMS

BLOCK SCWO IN=COMPRESS IIQUID RECYCLE OUT=SCWOPROD

BLOCK DESORBER IN=FDWC-ORG FDWASTEN OUT=DESORBED

BLOCK MELTER IN=FDMETMLT OUT=METAL

BLOCK 5-STAGEC IN=OXYGEN OUT=COMPRESS

BLOCK PUMP IN=CONDENS 8 FDAQORGS FDWOLIQS WATER NAOH OUT $=\&$

LIQUID

BLOCK CONDENSR IN=KILNVAP OUT=COOLED

BLOCK COND-SEP IN=COOLED OUT=VAPORS CONDENS 8 HGPLUS

BLOCK CATALYST IN=VAPORS AIR OUT=EXHAUST2

BLOCK EVAPOR8R IN=SALTSOLN BOTTOMS OUT=VAPOR2 CONCSOLN

BLOCK CONDENS2 IN=VAPOR2 OUT=NON-COND CONDENSD

BLOCK HEATER IN=FUELMIX1 OUT=EXHAUST1

PROPERTIES SOLIDS

PROPERTIES IDEAI / PENG-ROB / RKS-BM

PROP-DATA PRKIJ-1

IN-UNITS ENG

PROP-LIST PRKIJ

BPVAI H2 N2 . 1030000

BPVAL H2 $\mathrm{CO} 2-.1622000$

BPVAL H2 CO .0919000

BPVAL H2 CH4 .0156000

BPVAI $02 \mathrm{~N} 2-.0119000$

BPVAI N2 $\mathrm{CO} 2-.0170000$

BPVAI N2 CO .0307000

BPVAL N2 CH4 .0311000

BPVAL N2 H2 . 1030000

BPVAL N2 $02-.0119000$

BPVAL N2 $\mathrm{SO} 2.0800000$

BPVAI SO2 N2 .0800000

BPVAI SO2 CH4 .1356000

BPVAI H2O CO2 .1200000

BPVAL CO2 N2 -.0170000

BPVAL CO2 CH4 .0919000

BPVAI $\mathrm{CO} 2 \mathrm{H} 2-.1622000$

BPVAL CO2 H2O .1200000

BPVAL CO N2 .0307000

BPVAL CO CH4 .0300000

BPVAL CO H2 .0919000

BPVAL CH4 N2 .0311000

BPVAL CH4 CO2 .0919000

BPVAL CH4 CO .0300000

BPVAL CH4 H2 .0156000

BPVAL CH4 SO2 .1356000

PROP-DATA HENRY-1

IN-UNITS ENG

PROP-LIST HENRY

BPVAI CL2 H2O - $116.97814371 .51619 .18540-4.9559 \mathrm{E}-3$ 49.73000103 .7300

BPVAL SO2 H2O $17.90421-5171.328-.3028800 \quad 0.049 .73000$ \& 235.1300 
BPVAL CO2 H2O $175.2762-15734.79-21.66900 \quad 6.12550 \mathrm{E}-4$ \& $31.73000 \quad 175.7300$

BPVAI HCL H2O $-49.781402187 .0008 .370700-5.3294 E-3 \quad \&$ $-4.00000068 .00000$

PROP-SET ALI-SUBS TEMP PRES VERAC UNITS='ATM' SUBSTREAM=ALI

STREAM AIR

SUBSTREAM MIXED TEMP=68 PRES=1 <ATM> MASS-FLOW=10000

MASS-ELOW O2 $0.233 / \mathrm{N} 20.767$

STREAM FDAQORGS

SUBSTREAM MIXED TEMP $=68$ PRES $=1$ <ATM>

MASS-FLOW H2 $0.02 /$ CL2 $0.13 /$ H2O 15.44

SUBSTREAM CISOLID TEMP $=68$ PRES $=1$ <ATM>

MASS-ELOW C-S 0.17

STREAM FDMETMLT

SUBSTREAM MIXED TEMP=68 PRES=1 <ATM> MASS-FLOW=1.20

MASS-FLOW H2 2.42

SUBSTREAM CISOLID TEMP=68 PRES=1 <ATM> MASS-FLOW=147.80

MASS-FLOW C-S $14.44 /$ FE-S $282.02 /$ SIO2-S $0.035 / \&$

AL203-S $0.035 /$ BA-S $0.87 /$ CD-S 0.18

STREAM FDWASTEN

SUBSTREAM MIXED TEMP=68 PRES $=1$ <ATM>

MASS-ELOW H2 $0.54 / 021.44 / \mathrm{N} 20.01 /$ CL2 $14.88 / \&$

NO $0.66 / \mathrm{SO} 20.83 / \mathrm{H} 20354.23 / \mathrm{HG} 0.15$

SUBSTREAM CISOIID TEMP=68 PRES $=1$ <ATM>

MASS-ELOW C-S $4.77 /$ S-S $0.62 /$ SIO2-S $476.77 / \&$

AL203-S $476.77 /$ AS-S $0.06 / \mathrm{BA}-\mathrm{S} 0.04 / \mathrm{CD}-\mathrm{S} 2.63$ \&

/ CR-S $4.95 /$ PB-S $0.17 /$ SE-S $0.03 /$ AG-S 0.03

STREAM FDWC-ORG

SUBSTREAM MIXED TEMP $=68$ PRES $=1$ <ATM>

MASS-FLOW H2 $47.21 / 0291.70 / \mathrm{N} 20.23 /$ CL2 $35.12 /$ \& F2 $0.11 / \mathrm{H} 2 \mathrm{O} 19.24$

SUBSTREAM CISOLID TEMP $=68$ PRES $=1$ <ATM>

MASS-ELOW C-S $299.15 /$ S-S $0.39 /$ FE-S $7.67 /$ SIO2-S \& $52.91 /$ AL2O3-S 52.91/ BA-S $0.77 / \mathrm{CD}-\mathrm{S} 0.77 / \mathrm{CR}-\mathrm{S} 1.53$

STREAM FDWOLIOS

SUBSTREAM MIXED TEMP=68 PRES=1 <ATM>

MASS-FLOW H2 $3.74 / 021.06 /$ CL2 $17.90 / \mathrm{H} 200.59$

SUBSTREAM CISOLID TEMP $=68$ PRES $=1$ <ATM>

MASS-FLOW C-S $27.13 /$ S-S $0.10 /$ SIO2-S $0.10 /$ AL203-S 0.10

STREAM FUEIMIX1

SUBSTREAM MIXED TEMP=68 PRES=1 <ATM>

MASS-FLOW $02.222 / \mathrm{N} 2.732 / \mathrm{CH} 4.046$

STREAM NAOH

SUBSTREAM CISOLID TEMP=68 PRES=1 <ATM> MASS-FLOW=31

MASS-ELOW NAOH-S 1

STREAM OXYGEN

SUBSTREAM MIXED TEMP=68 PRES=1 <ATM> MASS-FLOW=352

MASS-ELOW O2 1

STREAM WATER

SUBSTREAM MIXED TEMP=68 PRES=1 <ATM> MASS-FLOW=2842

MASS-FLOW H2O 1 
BIOCK COND-SEP SEP

FRAC STREAM=VAPORS SUBSTREAM=MIXED COMPS=H2 O2 N2 CL2 \& F2 NO SO2 H2O CO2 CO HCL CH4 C-S S-S S NAOH-S \& NACL-S NAF-S AS CD HG SE EE SIO2 AL2O3 EE-S SIO2-S \& AL203-S AS-S BA-S CD-S CR-S PB-S SE-S AG-S CACO3-S \& MGO-S NA20-S K20-S EE304 EE304-S CAO-S ERACS $=.1 .1$ \& $\begin{array}{lllllllllllllllll}.1 & .1 & .1 & .1 & .1 & .1 & .1 & .1 & .1 & .1 & .1 & .1 & .1 & .1 & .1 & \text { \& }\end{array}$

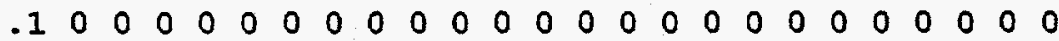

FRAC STREAM=VAPORS SUBSTREAM=CISOLID COMPS=C-S S-S NAOH-S \& NACL-S NAF-S FE-S SIO2-S AI2O3-S AS-S BA-S CD-S CR-S \& PB-S SE-S AG-S CACO3-S MGO-S NA2O-S K2O-S FE304-S \&

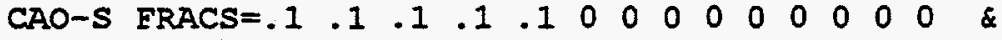
$\begin{array}{lllllll}0 & 0 & 0 & 0 & 0 & 0 & 0\end{array}$

FRAC STREAM=HGPLUS SUBSTREAM=MIXED COMPS=H2 O2 N2 CL2 \& E2 NO SO2 H2O $\mathrm{CO} 2 \mathrm{CO}$ HCL CH4 C-S S-S S NAOH-S \& NACI-S NAF-S AS CD HG SE FE SIO2 AL203 FE-S SIO2-S \& AL2O3-S AS-S BA-S CD-S CR-S PB-S SE-S AG-S CACO3-S \& MGO-S NA2O-S K20-S FE304 FE304-S CAO-S FRACS $=0$ 0 0 \& $0 \begin{array}{lllllllllllllllllllll}0 & 0 & 0 & 0 & 0 & 0 & 0 & 0 & 0 & 0 & 0 & 0 & 0 & 0 & 1 & 1 & 1 & 1 & 0 & 0 & \text { \& }\end{array}$ $0 \begin{array}{llllllllllllllllll}0 & 0 & 0 & 0 & 1 & 0 & 1 & 0 & 0 & 1 & 0 & 0 & 0 & 0 & 0 & 0 & 0 & 0\end{array}$

ERAC STREAM=HGPLUS SUBSTREAM=CISOIID COMPS=C-S S-S NAOH-S \& NACI-S NAF-S EE-S SIO2-S AL203-S AS-S BA-S CD-S CR-S \& PB-S SE-S AG-S CACO3-S MGO-S NA2O-S K2O-S FE3O4-S \& CAO-S FRACS $=0 \begin{array}{llllllllllllllllll}0 & 0 & 0 & 0 & 0 & 0 & 0 & 0 & 1 & 0 & 1 & 0 & 0 & 1 & 0 & 0 & \&\end{array}$ $\begin{array}{lllll}0 & 0 & 0 & 0 & 0\end{array}$

BLOCK SCWO-SEP SEP

FRAC STREAM=SALTSOLN SUBSTREAM=MIXED COMPS=H2 O2 N2 CL2 \& F2 NO SO2 H2O CO2 CO HCL CH4 C-S S-S S NAOH-S \& NACI-S NAF-S AS CD HG SE FE SIO2 AI2O3 FE-S SIO2-S \& AI203-S AS-S BA-S CD-S CR-S PB-S SE-S AG-S CACO3-S \& MGO-S NA2O-S K2O-S FE304 FE304-S CAO-S FRACS=0 00 \& $\begin{array}{llllllllllllllllllll}0 & 0 & 0 & 0 & 0.999 & 0 & 0 & 0 & 0 & 0 & 0 & 0 & 1 & 1 & 1 & 0 & 0 & 0 & 0\end{array}$ $0 \begin{array}{lllllllllllllllllll}0 & 0 & 0 & 0 & 0 & 0 & 0 & 0 & 0 & 0 & 0 & 0 & 0 & 0 & 0 & 0 & 0 & 0 & 0\end{array}$

ERAC STREAM=SALTSOLN SUBSTREAM=CISOLID COMPS=C-S S-S \& NAOH-S NACL-S NAF-S FE-S SIO2-S AI203-S AS-S BA-S \& CD-S CR-S PB-S SE-S AG-S CACO3-S MGO-S NA2O-S K2O-S \& FE304-S CAO-S FRACS $=0 \begin{array}{llllllllllllll}0 & 0 & 1 & 1 & 1 & 0 & 0 & 0 & 0 & 0 & 0 & 0 & 0 & \&\end{array}$ $\begin{array}{llllllll}0 & 0 & 0 & 0 & 0 & 0 & 0 & 0\end{array}$

FRAC STREAM=RECYCLE SUBSTREAM=MIXED COMPS=H2 O2 N2 CL2 \& F2 NO SO2 H2O CO2 CO HCL CH4 C-S s-s s NAOH-S \& NACL-S NAF-S AS CD HG SE FE SIO2 AL2O3 FE-S SIO2-S \& AL2O3-S AS-S BA-S CD-S CR-S PB-S SE-S AG-S CACO3-S \& MGO-S NA2O-S K2O-S FE304 FE304-S CAO-S FRACS $=0 \quad 0 \quad 0 \quad \&$ $\begin{array}{lllllllllllllllllllll}0 & 0 & 0 & 0 & 0.001 & 0 & 0 & 0 & 0 & 0 & 0 & 0 & 0 & 0 & 0 & 0 & 0 & 0 & 0 & \&\end{array}$

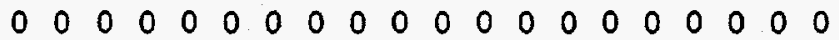

FRAC STREAM=RECYCLE SUBSTREAM=CISOLID COMPS=C-S S-S \& NAOH-S NACL-S NAF-S FE-S SIO2-S AL2O3-S AS-S BA-S \& CD-S CR-S PB-S SE-S AG-S CACO3-S MGO-S NA2O-S K2O-S \& FE304-S CAO-S ERACS $=0 \begin{array}{llllllllllll}0 & 0 & 0 & 0 & 0 & 0 & 0 & 0 & 0 & 0 & 0 & 0\end{array}$ $\begin{array}{lllllllll}0 & 0 & 0 & 0 & 0 & 0 & 0 & 0\end{array}$

BLOCK SEPRATR SEP

FRAC STREAM=SOLIDS SUBSTREAM=MIXED COMPS=H 02 N2 CL2 \& F2 NO SO2 H2O $\mathrm{CO} 2 \mathrm{CO} \mathrm{HCI} C H 4 \mathrm{C}-\mathrm{S}$ S-S S NAOH-S \& NACI-S NAF-S AS CD HG SE FE SIO2 AL2O3 FE-S SIO2-S \& AL203-S AS-S BA-S CD-S CR-S PB-S SE-S AG-S CACO3-S \& MGO-S NA20-S K2O-S FE304 FE304-S CAO-S FRACS $=.8 .8$ \& $\begin{array}{llllllllllllllllll}.8 & .8 & .8 & .8 & .8 & 0.2 & .8 & .8 & .8 & .8 & .8 & 1 & 1 & 1 & 1 & 1 & 0 & \alpha\end{array}$ $\begin{array}{llllllllllllllllllllll}0 & 0 & 0 & 1 & 1 & 1 & 1 & 1 & 1 & 1 & 1 & 1 & 1 & 1 & 1 & 1 & 1 & 1 & 1 & 1 & 1 & \&\end{array}$ 11 
ERAC STREAM=SOLIDS SUBSTREAM=CISOLID COMPS=C-S S-S NAOH-S \& NACI-S NAF-S FE-S SIO2-S AL2O3-S AS-S BA-S CD-S CR-S \& PB-S SE-S AG-S CACO3-S MGO-S NA2O-S K2O-S FE3O4-S \&

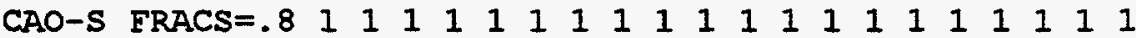

BLOCK CONDENS2 FLASH2

PARAM TEMP $=100$ PRES $=1$ <ATM>

BLOCK EVAPOR8R FLASH2

PARAM PRES $=1$ <ATM> VFRAC $=0.5$ MAXIT $=500$

PROPERTIES SOLIDS

BLOCK FLASH FLASH2

PARAM TEMP $=150$ PRES $=1$ <ATM>

PROPERTIES SOIIDS

BLOCK CATALYST RSTOIC

PARAM TEMP $=1500$ PRES $=1$ <ATM>

STOIC 1 MIXED CO $-1 / \mathrm{O} 2-0.5 / \mathrm{CO} 21$

STOIC 2 MIXED CH4 - $1 / \mathrm{O} 2-2 / \mathrm{H} 2 \mathrm{O} 2 / \mathrm{CO} 21$

STOIC 3 MIXED H2 $-1 / \mathrm{O} 2-0.5 / \mathrm{H} 2 \mathrm{O} 1$

STOIC 4 MIXED O2 -1/ CISOLID C-S -1/ MIXED CO2 1

CONV 1 MIXED CO 1

CONV 2 MIXED CH4 1

CONV 3 MIXED H2 1

CONV 4 CISOLID C-S 1

BLOCK CONDENSR RGIBBS

PARAM TEMP=80 PRES=1 <ATM> NPHASE $=2$ CHEMEQ $Q=Y E S$ MAXIT $=100$

PROD H2 / O2 / N2 / CL2 / F2 / NO / SO2 / H2O / \& C-S SS / S-S SS / S / AS / CD / HG / FE-S SS \& / SIO2-S SS / AL203-s SS / AS-S SS / BA-S SS / \& CD-S SS / CR-S SS / PB-S SS / SE-S SS / AG-S SS

PROD-FRAC H2 $1 / \mathrm{O} 21 / \mathrm{H} 2 \mathrm{O} 1$

BIOCK DESORBER RGIBBS

PARAM TEMP $=600$ PRES $=1$ <ATM> NPHASE $=2$ CHEMEQ $=Y E S$ MAXIT $=200$

PROD H2 / O2 / N2 / CL2 / F2 / NO / SO2 / H2O / \& $\mathrm{C}-\mathrm{s}$ sS / S-s SS / $\mathrm{s} / \mathrm{AS} / \mathrm{CD} / \mathrm{HG} / \mathrm{SE} /$ \& FE-S SS / SIO2-S SS / AL2O3-S SS / AS-S SS / \& BA-S SS / CD-S SS / CR-S SS / PB-S SS / SE-S SS / AG-S SS PROD-FRAC H2 $1 / \mathrm{O} 21 / \mathrm{H} 2 \mathrm{O} 1$

BIOCK HEATER RGIBBS

PARAM TEMP=1400 PRES=1 <ATM> NPHASE=2

BLOCK MELTER RGIBBS

PARAM TEMP $=3000$ PRES=1 <ATM> NPHASE=3 VAPOR=YES

PROD H2 / C-S SS / FE / SIO2 / AL203/ FE-S SS / \& SIO2-S SS / AL203-S SS / BA-S SS / CD / CD-S SS

BLOCK SCWO RGIBBS

PARAM TEMP $=800$ PRES $=3200$ NPHASE $=2$ MAXIT $=100$

PROD H2 M / O2 M / N2 M / CL2 / F2 / NO / SO2 / \& $\mathrm{H} 2 \mathrm{O} / \mathrm{CO} 2 / \mathrm{CO} / \mathrm{HCL} / \mathrm{NAOH}-\mathrm{S}$ SS / NACL-S SS / \& NAF-S SS / SIO2-S SS / AL2O3-S SS

PROPERTIES PENG-ROB

BLOCK PUMP PUMP

PARAM PRES $=3200$ NPHASE $=2$ MAXIT $=200$

PROPERTIES PENG-ROB 
BLOCK 5-STAGEC MCOMPR

PARAM NSTAGE=5 TYPE=ISENTROPIC PRES=3200 COMPR-NPHASE=2 \& MAXIT $=200$

FEEDS OXYGEN 1

PRODUCTS COMPRESS 5

COOLER-SPECS 1 TEMP=80

PROPERTIES PENG-ROB

DESIGN-SPEC AIR

DEFINE OXIN1 MASS-ELOW STREAM=AIR SUBSTREAM=MIXED COMPONENT $=02$

DEFINE OXIN2 MASS-FIOW STREAM=VAPORS SUBSTREAM=MIXED \& COMPONENT $=02$

DEFINE OXOUT MASS-FLOW STREAM=EXHAUST2 SUBSTREAM=MIXED \& COMPONENT $=02$

SPEC "OXIN1+OXIN2-2. *OXOUT" TO "O"

TOL-SPEC "0.01"

VARY STREAM-VAR STREAM=AIR SUBSTREAM=MIXED \& VARIABIE =MASS-ELOW

IIMITS "I" " 10000 "

DESIGN-SPEC IIQUOR

DEFINE H2O MASS-ELOW STREAM=CONCSOIN SUBSTREAM=MIXED \& COMPONENT $=\mathrm{H} 2 \mathrm{O}$

DEFINE SAITS STREAM-VAR STREAM=CONCSOLN SUBSTREAM=CISOLID \& VARIABLE=MASS-ELOW

SPEC "H2O-SALTS" TO "O"

TOL-SPEC "0.01"

VARY BLOCK-VAR BLOCK=EVAPOR8R VARIABLE=VERAC SENTENCE=PARAM

LIMITS "0 " " "

DESIGN-SPEC NAOH

DEFINE CAUIN MASS-FIOW STREAM=NAOH SUBSTREAM=CISOLID \& COMPONENT $=\mathrm{NAOH}-\mathrm{S}$

DEEINE CAUOUT MASS-FLOW STREAM=SCWORROD SUBSTREAM=CISOLID \& COMPONENT $=$ NAOH $-S$

SPEC "CAUIN" TO "CAUOUT*50."

TOL-SPEC "0.01"

VARY STREAM-VAR STREAM $=$ NAOH SUBSTREAM $=$ CISOLID \&

VARIABLE=MASS - FLOW

LIMITS "29" "100"

DESIGN-SPEC OXYGEN

DEFINE OXIN1 MASS-FIOW STREAM=COMPRESS SUBSTREAM=MIXED \& COMPONENT $=02$

DEFINE OXIN2 MASS-FLOW STREAM=LIQUID SUBSTREAM=MIXED \& COMPONENT $=02$

DEEINE OXOUT MASS-FLOW STREAM=SCWOPROD SUBSTREAM=MIXED \& COMPONENT $=02$

SPEC "OXIN1+OXIN2" TO "6.0*OXOUT"

TOL-SPEC "0.01"

VARY STREAM-VAR STREAM=OXYGEN SUBSTREAM=MIXED \& VARIABIE =MASS - FLOW

LIMITS "1" "1000"

DESIGN-SPEC QHEATER

DEFINE QIN BLOCK-VAR BLOCK=DESORBER VARIABIE=QCALC \& SENTENCE=PARAM

DEFINE QOUT BLOCK-VAR BLOCK=HEATER VARIABLE=QCALC \& SENTENCE=PARAM

SPEC "1.05*QIN+QOUT" TO "O"

TOL-SPEC "1." 
VARY STREAM-VAR STREAM=FUEIMIXI SUBSTREAM=MIXED \& VARIABIE $=$ MASS - FLOW

IIMITS "1" "10000"

DESIGN-SPEC QSCWO

DEFINE QSCWO BIOCK-VAR BLOCK=SCWO VARIABLE=QCALC \& SENTENCE =PARAM

SPEC "QSCWO" TO "O"

TOL-SPEC "1."

VARY STREAM-VAR STREAM=WATER SUBSTREAM=MIXED \& VARIABLE=MASS-ELOW

LIMITS "1000" "10000"

DESIGN-SPEC SAIT

DEFINE NACL MASS-FLOW STREAM=SALTSOLN SUBSTREAM=CISOLID \& COMPONENT $=$ NACL $-S$

DEFINE NAF MASS-FLOW STREAM=SALTSOLN SUBSTREAM=CISOLID \& COMPONENT $=$ NAF-S

DEFINE WATER MASS-FLOW STREAM=SALTSOLN SUBSTREAM=MIXED \& COMPONENT $=\mathrm{H} 2 \mathrm{O}$

SPEC "5.67* (NACL+NAE)" TO "WATER"

TOI-SPEC "0.05"

VARY BLOCK-VAR BLOCK=SCWO-SEP SENTENCE=FRAC VARIABLE=FRACS \& ID1=MIXED ID2=SALTSOLN ELEMENT $=8$

IIMITS "0.01" "1."

CONV-OPTIONS

PARAM CHECKSEQ $=$ NO

SECANT MAXIT $=500$ MAX-STEP-SIZ $=.05$ BRACKET $=$ YES

CONVERGENCE CVI WEGSTEIN

TEAR RECYCLE

CONVERGENCE CV2 SECANT

SPEC AIR

CONVERGENCE CV7 SECANT SPEC SAIT

STREAM-REPOR NOSORT MOLEFLOW MASSFLOW PRORERTIES=ALI-SUBS 
Appendix C

ASPEN PLUS Stream Summaries

C-1 
C-2 
System A-1

C-3 